\title{
Historical Data Analysis Supporting the Data Quality Objectives for the INL Site Environmental Soil Monitoring Program
}

February 2017

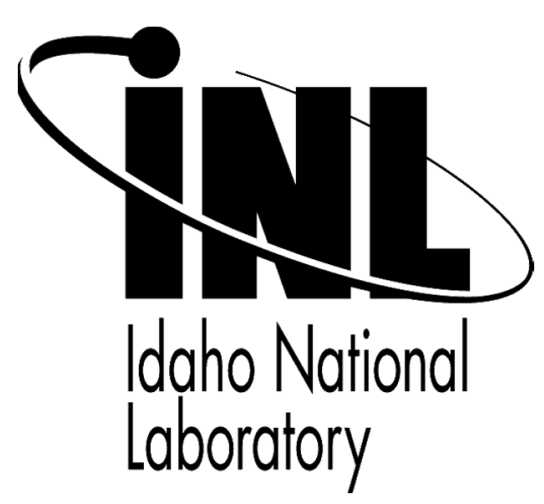




\section{DISCLAIMER}

This information was prepared as an account of work sponsored by an agency of the U.S. Government. Neither the U.S. Government nor any agency thereof, nor any of their employees, makes any warranty, expressed or implied, or assumes any legal liability or responsibility for the accuracy, completeness, or usefulness, of any information, apparatus, product, or process disclosed, or represents that its use would not infringe privately owned rights. References herein to any specific commercial product, process, or service by trade name, trade mark, manufacturer, or otherwise, does not necessarily constitute or imply its endorsement, recommendation, or favoring by the U.S. Government or any agency thereof. The views and opinions of authors expressed herein do not necessarily state or reflect those of the U.S. Government or any agency thereof. 
INL/INT-15-37431

Revision 0

\section{Historical Data Analysis Supporting the Data Quality Objectives for the INL Site Environmental Soil Monitoring Program}

February

Idaho National Laboratory Idaho Falls, Idaho 83415 



\section{Historical Data Analysis Supporting the Data Quality Objectives for the INL Site Environmental Soil Monitoring Program}

INL/NT-15-37431

Revision 0

February 2017

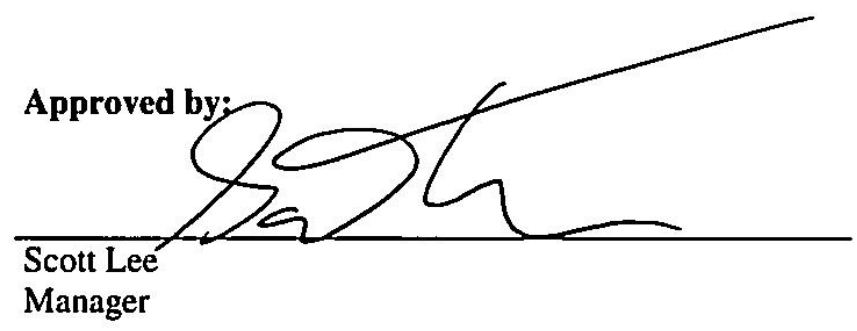

$\frac{2-8-17}{\text { Date }}$ 



\begin{abstract}
This document represents the initial evaluation and soil monitoring proposed by Battelle Energy Alliance, LLC (BEA) in 2015. The evaluation included analyses of historical soil monitoring data and soil inventories, current emission estimates, and modeled potential deposition/accumulation patterns. The initially proposed monitoring included a 5-year rotation of in-situ gamma measurements augmented by soil sampling with laboratory analyses near each major active and some inactive facilities. It also proposed rotational in-situ gamma measurements and soil sampling at two centrally located onsite air monitoring locations coinciding with sampling at the traditional offsite soil monitoring locations. The chosen alternative includes only physical soil sampling with laboratory analysis and only at the Radioactive Waste Management Complex (RWMC), the two air monitors and the offsite locations as documented in Data Quality Objectives Supporting the Environmental Soil Monitoring Program for the Idaho National Laboratory (INL) Site, INL/EXT-15-34909, Revision 0, February 2016. The data and evaluations in this document are valid for comparisons with future soil data that may be collected in many INL site locations.
\end{abstract}




\section{CONTENTS}

ABSTRACT vii

ACRONYMS xiii

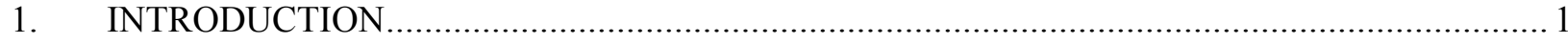

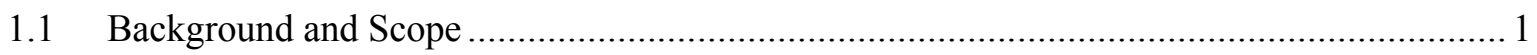

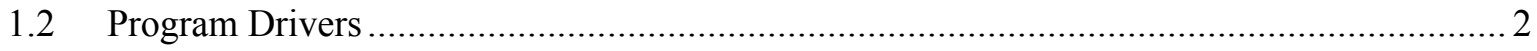

1.3 Data Quality Objectives Process ..................................................................... 2

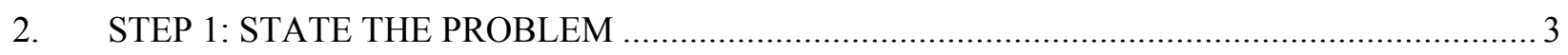

3. STEP 2: IDENTIFY THE GOALS OF THE STUDY …................................................... 3

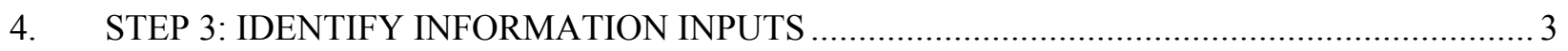

5. STEP 4: DEFINE THE BOUNDARIES OF STUDY …......................................................... 4

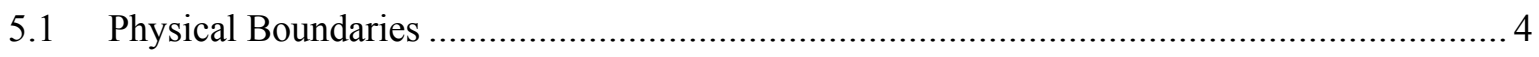

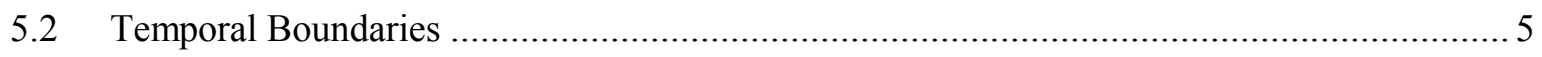

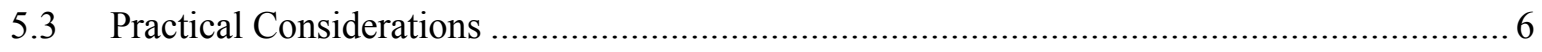

6. STEP 5: DEVELOP THE ANALYTICAL APPROACH .......................................................... 7

7. STEP 6: SPECIFY PERFORMANCE OR ACCEPTANCE CRITERIA...................................... 9

8. STEP 7: DEVELOP THE DETAILED PLAN FOR OBTAINING DATA ............................... 9

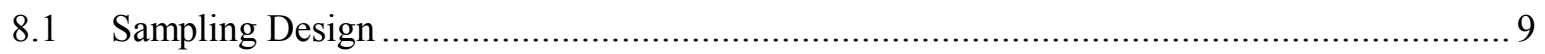

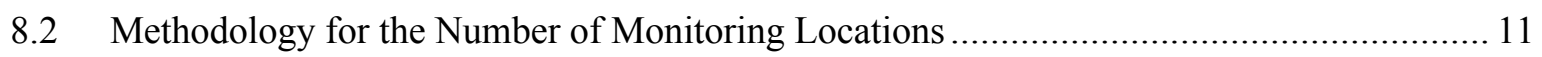

8.3 Methodology for Soil Monitoring Location Selection ..................................................... 12

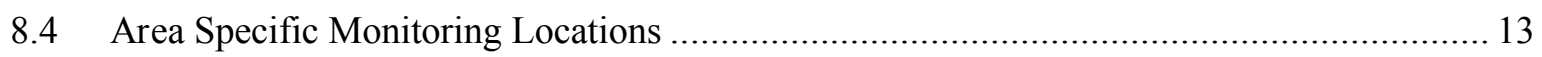

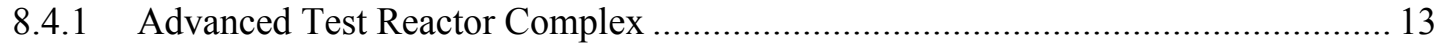

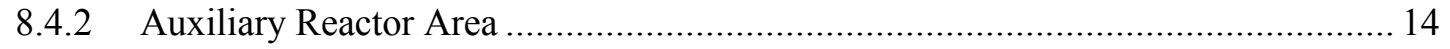

8.4.3 Idaho Nuclear Technology and Engineering Center ............................................ 15

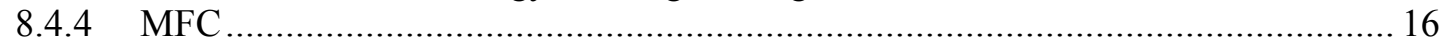

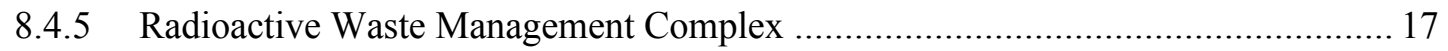

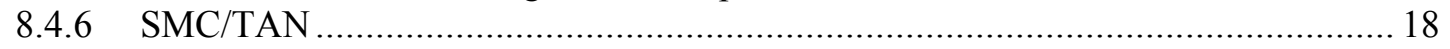

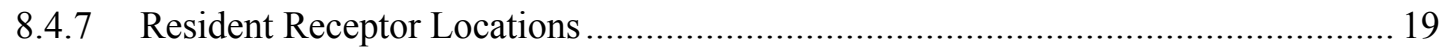

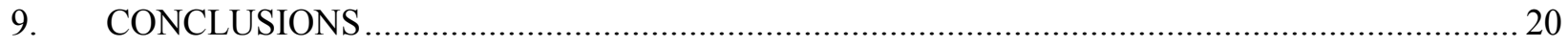

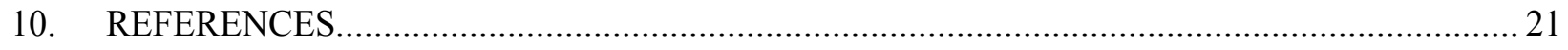

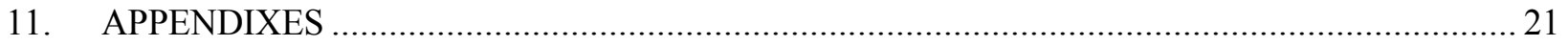




\section{FIGURES}

Figure 1. Map of the INL Site showing the locations of major facilities. ........................................ 6

Figure 2. Region within 50 miles of INL Site facilities showing Census divisions used in the 50 -mile population dose calculation. 7

Figure 3. Composite sampling grid.

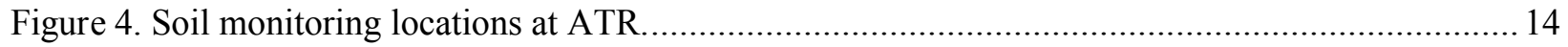

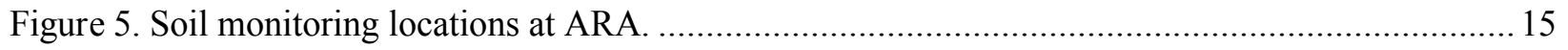

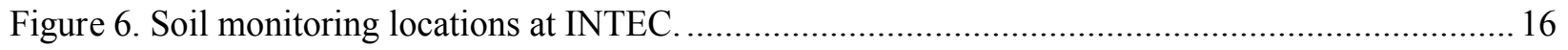

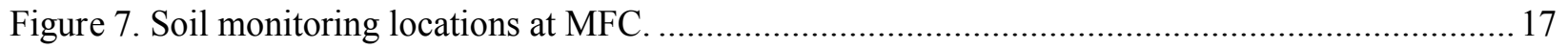

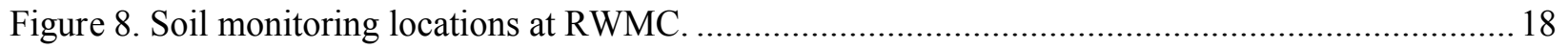

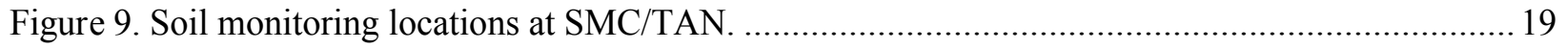

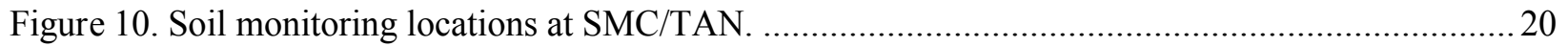

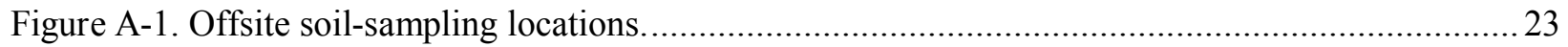

Figure A-2. Areal activity of Cs-137 in offsite surface soil sampled from 1975 through 2014. Each column represents the geometric average of all locations for that year. ........................26

Figure A-3. Areal activity of Sr-90 in offsite surface soil sampled from 1975 through 2014. Each column represents the geometric average of all locations for that year.................................2 27

Figure A-4. Cs-137/Sr-90 ratios in offsite surface soil shown as a function of time. ........................... 27

Figure A-5. Scatterplot of individual measurements of Cs-137 in surface soil samples collected at locations off the INL Site from 2000 to 2014. A trend line and $95 \%$ confidence limits are shown.

Figure A-6. Scatterplot of individual measurements of Sr-90 in surface soil samples collected at locations off the INL site from 2000 to 2014. A trend line and $95 \%$ confidence limits

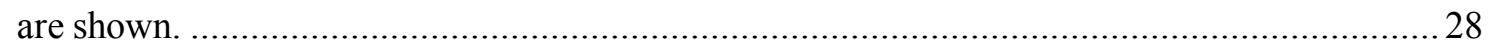

Figure A-7. Mean Cs-137 concentrations in upper and lower soil layers.............................................29

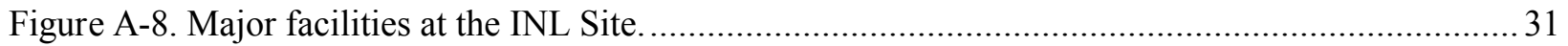

Figure A-9. Unit deposition values $\left(\mathrm{hr} \mathrm{m}^{-2} \times 10^{9}\right)$ for particles 1 micron in diameter released continuously from the ATR Complex TRA-770 stack for 1 year.

Figure A-10. Unit deposition values $\left(\mathrm{hr} \mathrm{m}^{-2} \times 10^{9}\right)$ for particles 1 micron in diameter released continuously at ground level from the ATR Complex for 1 year.

Figure A-11. Unit deposition values $\left(\mathrm{hr} \mathrm{m}^{-2} \times 10^{9}\right)$ for particles 1 micron in diameter released continuously at ground level from INTEC for 1 year.

Figure A-12. Unit deposition values $\left(\mathrm{hr} \mathrm{m}^{-2} \times 10^{9}\right)$ for particles 1 micron in diameter released continuously from the MFC-764 stack for 1 year......

Figure A-13. Unit deposition values $\left(\mathrm{hr} \mathrm{m}^{-2} \times 10^{9}\right)$ for particles 1 micron in diameter released continuously at ground level from MFC for 1 year.

Figure A-14. Unit deposition values $\left(\mathrm{hr} \mathrm{m}^{-2} \times 10^{9}\right)$ for particles 1 micron in diameter released continuously at ground level from the RWMC for 1 year. 
Figure A-15. Major process which affected radionuclide concentrations in surface soil. 36

Figure A-16. Soil sampling grid.

\section{TABLES}

Table 1. Radionuclides that will be measured under the soil monitoring program................................ 4

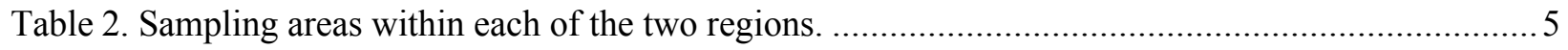

Table 3. Background levels for near-facility and distant areas. ..................................................... 8

Table A-1. Radionuclides that are important in terms of radiological dose based on the 20072013 NESHAP reports (Rood and Sondrup 2014). 22

Table A-2. Summary statistics for RESL and current data for offsite samples collected at depths of $0-5 \mathrm{~cm}$ and $5-10 \mathrm{~cm}(\mathrm{pCi} / \mathrm{g})$. .25

Table A-3. Key radionuclide background concentrations in offsite soil as of 2014. The means and upper confidence limits were calculated with all data regardless if they were above the detection level.

Table A-4. Estimated time (years) to achieve build-up concentration in surface soil for $1 \mu \mathrm{m}$ particles released continuously for 1 year from a stack (TRA-770 stack) at the Advanced Test Reactor Complex. ${ }^{a}$ The top five results are highlighted. Two of the sampler locations, Van Buren and EFS, have replicate samplers (BEA-VAN and ESER-VAN, BEA-EFS, and ESER-EFS).

Table A-5. Estimated time (years) to achieve build-up concentration in surface soil for $30 \mu \mathrm{m}$ particles released continuously for 1 year at ground level from the Advanced Test Reactor Complex. ${ }^{a}$ The shortest five time periods are highlighted in yellow. Two of the sampler locations, Van Buren and EFS, have replicate samplers (BEA-VAN and ESER-VAN, BEA-EFS, and ESER-EFS).

Table A-6. Estimated time (years) to achieve build-up concentration in surface soil for $10-\mu \mathrm{m}$ particles released continuously for 1 year at ground level from Idaho Nuclear Technology and Engineering Complex. ${ }^{\text {a }}$ The top five results are highlighted in yellow. Two of the sampler locations, Van Buren and EFS, have replicate samplers (BEA-VAN and ESER-VAN, BEA-EFS, and ESER-EFS).

Table A-7. Estimated time (years) to achieve build-up concentration in surface soil for $1 \mu \mathrm{m}$ particles released continuously for 1 year from a stack (MFC-764 stack) at the Materials and Fuel Complex. The top five results are highlighted. Two of the sampler locations, MFC Parking Lots and MFC Guard Shack, are proposed.

Table A-8. Estimated time (years) to achieve build-up concentration in surface soil for $30 \mu \mathrm{m}$ particles released continuously for 1 year at ground level from the Materials and Fuels Complex. The shortest five time periods are highlighted in yellow. Two of the sampler locations, MFC Parking Lots and MFC Guard Shack, are proposed.

Table A-9. Estimated time (years) to achieve build-up concentration in surface soil for $30 \mu \mathrm{m}$ particles released continuously for 1 year at ground level from the Radioactive Waste Management Complex. The five shortest time periods are highlighted in yellow.

Table B-1. Summary statistics for data collected at ARA. .55

Table B-2. Summary statistics for data collected at ATR .56 


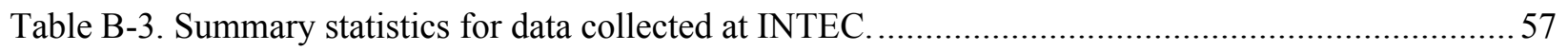

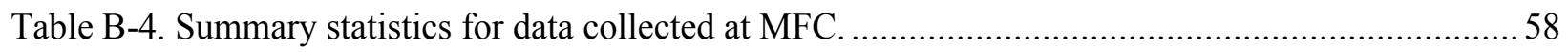

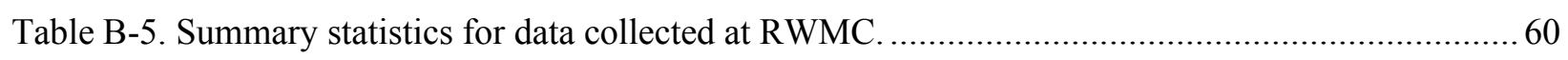

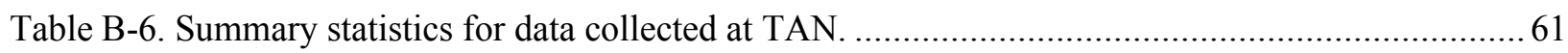

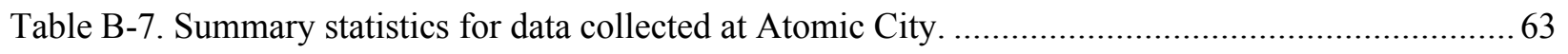

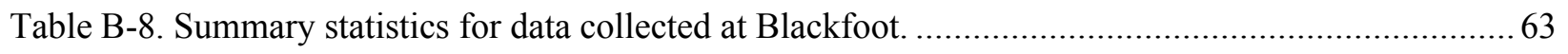

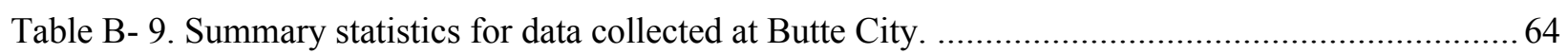

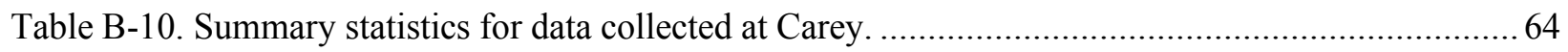

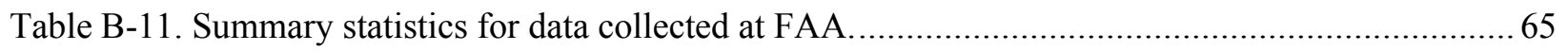

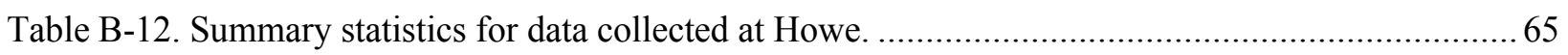

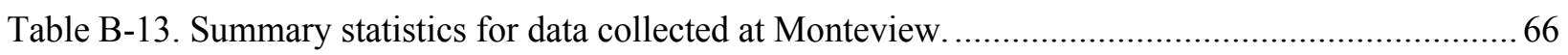

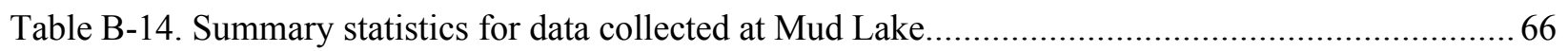

Table B-15. Summary statistics for data collected at Blue Dome/Birch Creek Hydro. ........................... 67

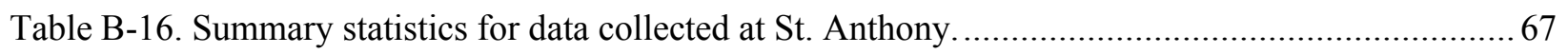

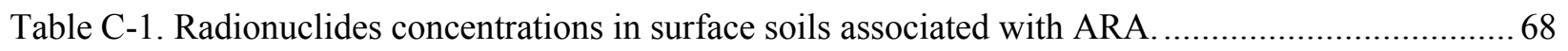

Table C-2. Radionuclides concentrations in surface soils associated with ATR. ............................... 118

Table C-3. Radionuclides concentrations in surface soils associated with INTEC............................. 137

Table C-4. Radionuclides concentrations in surface soils associated with MFC . ................................204

Table C-5. Radionuclides concentrations in surface soils associated with RWMC.............................223

Table C-6. Radionuclides concentrations in surface soils associated with TAN/SMC ....................... 267

Table C-7. Radionuclides concentrations in surface soils associated with Atomic City......................283

Table C-8. Radionuclides concentrations in surface soils associated with Blackfoot. ..........................284

Table C-9. Radionuclides concentrations in surface soils associated with Butte City. ......................... 286

Table C-10. Radionuclides concentrations in surface soils associated with Carey .............................. 288

Table C-11. Radionuclides concentrations in surface soils associated with FAA. ..............................290

Table C-13. Radionuclides concentrations in surface soils associated with Howe. .............................. 292

Table C-14. Radionuclides concentrations in surface soils associated with Monteview.......................294

Table C-15. Radionuclides concentrations in surface soils associated with Mud Lake. ....................... 296

Table C-16. Radionuclides concentrations in surface soils associated with Blue Dome/Birch

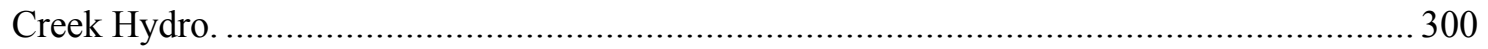

Table C-17. Radionuclides concentrations in surface soils associated with St. Anthony ....................... 302 


\section{ACRONYMS}

ARA Airborne Radiation Area

ATR Advanced Test Reactor

CERCLA Comprehensive Environmental Response, Compensation, and Liability Act

DOE Department of Energy

DQO data quality objective

DS decision statement

EFS Experimental Field Station

EPA Environmental Protection Agency

ICDF INL CERCLA Disposal Facility

IET Initial Engine Test

INL Idaho National Laboratory

INTEC Idaho Nuclear Technology and Engineering Center

LOFT Loss of Fluid Test

MFC Materials and Fuels Complex

NESHAPs National Emission Standards for Hazardous Air Pollutants

PSQ principal study question

RESL Radiological and Environmental Sciences Laboratory

REST Highway 20-26 Big Lost River rest area

RWMC Radioactive Waste Management Complex

SMC Specific Manufacturing Capability

TSF Technical Support Facility

UTL upper tolerance limit

WRRTF Water Reactor Research Test Facility 


\title{
Historical Data Analysis Supporting the Data Quality Objectives for the INL Site Environmental Soil Monitoring Program
}

\author{
1. INTRODUCTION
}

Soil provides an integrating media that can account for contaminants released to the atmosphere, either directly in gaseous effluents or indirectly from resuspension of onsite contamination or through liquid effluents released to a stream that is subsequently used for irrigation (DOE 2015). Soil sampling is a useful approach to determine the accumulation of initially airborne radionuclides that have been deposited on the ground but generally serves a supplementary role in environmental surveillance monitoring programs (Gallegos 1995, Hardy and Krey 1971, EML 1997). However, soil sampling is of questionable value in attempting to estimate small increments of deposition over a period of a few years or less and it is not recommended as a routine method of environmental monitoring except in preoperational surveys (EML 1997). Soil sampling and analysis should be used to evaluate the long-term accumulation trends to estimate environmental radionuclide inventories (DOE 2015).

The purpose of the Environmental Soil Monitoring Program at Idaho National Laboratory (INL) is to monitor levels of radionuclides in soils within INL Site boundaries and surrounding areas. The program involves determining long-term accumulation trends and establishing and maintaining baseline inventories of radionuclides in the soils around INL facilities and in areas within 50 miles of the INL Site boundary.

This document presents the development of the data quality objectives (DQOs) for the INL Soil Monitoring Program. This DQO follows the Environmental Protection Agency (EPA) DQO process (EPA 2006). The DQO process is used to clarify objectives, define the type of data, and specify the limits on the likelihood of making potential decision errors that will be used as the basis for establishing the quality and quantity of data needed to support decision-making.

\subsection{Background and Scope}

The INL Site has a long history of operation that includes various large-scale experiments, and large user facilities, such as reactors. The primary sources of soil contamination were the operation of reactors, radioactive material management, processes such as calcining, and unplanned releases to native soil, such when the Subsurface Disposal Area of the Radioactive Waste Management Complex (RWMC) was flooded in 1962 and 1969. Soil sampling has been performed at INL Site for decades to evaluate facility contributions to environmental levels of manmade radionuclides. In 1970, the Department of Energy (DOE) Radiological and Environmental Sciences Laboratory (RESL) established a routine program for collecting surface soils $(0-5$ and $5-10 \mathrm{~cm})$ on and around INL Site. At that time, RESL established extensive onsite soil sampling grids outside facilities. Offsite locations were also established by RESL during this process to serve as background sites. Between 1970 and 1978 RESL sampled these onsite grids extensively, and then reduced the onsite sampling frequency to a 7-year rotation (see Appendix A) and offsite to every 2 years. RESL analyzed all samples (on and offsite) for gamma-emitting radionuclides. In addition, the surface component $(0-5 \mathrm{~cm})$ of the offsite samples was analyzed for $\mathrm{Sr}-90$ and alpha emitting radionuclides (Am-241 and isotopes of plutonium). However, current operations are significantly reduced, many of the Comprehensive Environmental Response, Compensation, and Liability Act (CERCLA) sites have been remediated, and all but one reactor has been shut down. In addition, years of sampling and analyses that characterized the nature and extent of the contamination in these areas show slowly declining trends in the concentrations of short-lived manmade radionuclides. 
While the soil program will continue monitoring locations that were historically contaminated from INL Site activities on a rotational basis, the current basis for sampling soil as listed in the DOE Handbook for Environmental Radiological Effluent Monitoring and Environmental Surveillance (DOE 2015) is to "evaluate the long term accumulation trends and to estimate environmental radionuclide inventories." As such, these historically contaminated areas (as well as current emissions from active facilities) are viewed as source terms to be evaluated for possible impacts to the environment as modeled at INL Site locations with the highest deposition potential. Even in the locations of highest potential deposition, modeling results show little chance for detectable accumulations. The Handbook also states that sampling points for environmental monitoring should be located in areas that are susceptible to contamination and that one of the primary purposes of monitoring is to "characterize routine and non-routine releases of radioactive material." Thus, areas where radiological activities take place will be sampled to maintain a baseline inventory for those areas. Baseline inventories will also be determined for other areas where it is deemed practicable.

\subsection{Program Drivers}

Sampling of soil is performed on and around the INL Site to meet the following requirements and criteria for environmental surveillance of DOE facilities:

- DOE Order 458.1, "Radiation Protection of the Public and the Environment"

- "DOE Handbook Environmental Radiological Effluent Monitoring and Environmental Surveillance" (DOE 2015), which updates and supersedes Environmental Regulatory Guide for Radiological Effluent Monitoring and Environmental Surveillance (DOE/EH-0173t) (DOE 1991).

Other key drivers of the soil surveillance program include stakeholder inputs and values.

Each facility is responsible for monitoring within the facility boundaries so those areas are not addressed by this monitoring program.

Onsite air monitoring is conducted at effective fixed positions based on projections made by CALPUFF, 114a non-steady-state Lagrangian puff dispersion model (Rood and Sondrup 2014). The DOE Handbook (DOE 2015) states, "Where possible, soil sampling locations should be selected to coincide with air sampling stations, since the comparability of data may be important in achieving the objectives of the overall environmental sampling program." Therefore, to support the air surveillance program and comply with the Handbook, soil data should be obtained in the vicinity of air monitoring locations that are in the vicinity of the areas that are monitored under this program (predetermined with CALPUFF) that will most likely be affected by a planned or unplanned release from INL activities. Soil data will be collected on and around the INL Site at frequencies and locations evaluated using historical measurements and results of modeled deposition of radionuclides released by INL Site activities. In addition, soil sampling will be conducted at offsite areas that are not expected to be impacted by INL Site releases to characterize the distribution and fate of naturally occurring and fall-out radionuclides in soil to estimate the contribution to onsite soil measurements. Data from offsite locations will also be used to develop and maintain a baseline inventory for those locations.

\subsection{Data Quality Objectives Process}

The DQOs are discussed in the context of the DQO process as defined by Guidance for the Data Quality Objectives Process (EPA 2006). The EPA developed this process to ensure that the type, quantity, and quality of data used in decision-making are appropriate for the intended application. The DQO process includes seven steps, each of which has specific outputs. Each of the following sections corresponds to a step in the DQO process, and the output for each step is provided as appropriate. The DQOs do the following:

1. Clarify the study objective. 
2. Define the most appropriate type of data to collect.

3. Determine the most appropriate conditions from which to collect the data.

4. Specify tolerable limits on decision errors that will be used as a basis for establishing the quantity and quality of data needed to support the decision(s) to be made using the data.

The DQOs for the Environmental Soil Monitoring Program are discussed in the rest of this document.

\section{STEP 1: STATE THE PROBLEM}

INL Site activities have the potential to increase radionuclide concentrations in soils inside the INL Site boundaries and in the areas surrounding INL Site. Research activities at the INL Site involve anthropogenic radioactive materials; therefore, there is a potential for soil contamination within INL Site boundaries and in the areas surrounding INL Site. Thus, the INL Site monitors radionuclide concentrations to evaluate long-term accumulation trends and to estimate environmental radionuclide inventories. Radiological research has been conducted at the INL Site since the 1950s. Years of environmental monitoring data show that manmade radionuclide contaminated soil of INL origin exists near onsite emission points, that shorter-lived radionuclides (Cs-137 and Sr-90) continue to show a slow but steady decrease, and that manmade radionuclides detected offsite are present due to worldwide fallout unrelated to INL activities. Radionuclides also occur naturally and natural radionuclide concentrations vary in the areas surrounding the INL Site. Therefore, the ongoing soil surveillance program determines baseline concentrations in each localized area so that a baseline radionuclide inventory can be established and maintained for those sites.

The problem statement addressed by these DQOs is to determine the long-term deposition of radionuclides from INL activities in the soils in and surrounding the INL Site and to characterize the radionuclide inventory in the soils for key areas within the INL boundaries and in the surrounding areas.

\section{STEP 2: IDENTIFY THE GOALS OF THE STUDY}

The second step in the DQO process is to identify the decisions and the potential actions that will be affected by the data collected. This is done by specifying principal study questions (PSQs), alternative actions that could result from resolution of the PSQs, and combining the PSQs and alternative actions into decision statements (DSs). This monitoring effort is designed to answer two PSQs:

PSQ1: What is the baseline inventory of radionuclides of interest in the soils for the areas under investigation?

DS1: Determine the concentration of radionuclides of interest in the soil and use monitoring data to update the baseline over time.

PSQ2: What is the long-term deposition of radionuclides in soils due to INL activities?

DS2: Use air models to determine the most likely locations of deposition of radionuclides due to INL activities and monitor those soils to determine the long-term deposition due to Site activities.

\section{STEP 3: IDENTIFY INFORMATION INPUTS}

Inputs needed to resolve the DSs include the following:

- Identification of radionuclides of interest for soils in INL and surrounding areas

- Quantification of the radionuclide concentrations in the soils within the region being studied

- Historical radionuclide concentrations in the soils for the region being studied

- Pertinent historical information for each area 
- Wind patterns within the region to identify the area most likely to be exposed to radioactive fallout from Site activities

- Identification of possible source terms of radionuclides

- Identification of areas that may have increased inventories due to past activities

- Location of population centers in the regional vicinity of the Site

- Locations of areas within the Site boundaries where planned or unplanned releases may occur due to INL activities.

The historical information obtained from the area of interest will be used to establish localized baseline radionuclide inventories. It is known that the natural level of radiation can vary considerably within the region that is being examined. Therefore, the historical radionuclide concentration information and Site history information will be obtained and analyzed to determine the appropriate background levels for each localized region and to establish a baseline for each area. This will provide the necessary basis for determining the effect of a planned or unplanned release in soils potentially affected by such a release. Site information for each area can shed light on the measured radionuclide concentrations in the area to ensure that measurements are consistent with what is expected based on historical measurements.

Table 1 shows the radionuclides that are of primary concern under this monitoring effort. Appendix A provides the justification for the selection. The radionuclide I-129 will also be investigated. However, it is not currently emitted by any Site activities and the inventory at the Site is constant. Thus, it is not addressed in these DQOs because they are concerned with radionuclides that may affect soils due to a release or long-term deposition.

Table 1. Radionuclides that will be measured under the soil monitoring program.

\begin{tabular}{|c|c|}
\hline \multicolumn{2}{|c|}{ Radionuclides } \\
\hline Am-241 & Sr-90 \\
\hline Cs-137 & Pu-238 \\
\hline Pu-239/240 & \\
\hline
\end{tabular}

\section{STEP 4: DEFINE THE BOUNDARIES OF STUDY 5.1 Physical Boundaries}

The physical boundaries of the study include areas within the Site boundary and the 50-mile radius surrounding the INL Site per DOE guidance (DOE 2015). This area of interest has been divided into three regions based on proximity to source terms and the public:

- Near Facility Region

- Mid-Range Region

- Distant Region, limited to a 50-mile radius of the INL Site. 
Table 2. Sampling areas within each of the two regions.

\begin{tabular}{|lll|}
\hline \multicolumn{1}{|c|}{ Near Facility Region } & \multicolumn{1}{c|}{ Mid-Range Region } & Distant Region \\
ATR & Rest Area & Frenchman's Cabin (new site) \\
ARA & EFS & Howe \\
INTEC & & Monteview \\
MFC & Mud Lake \\
RWMC & Atomic City \\
TAN/SMC & Blackfoot \\
& Federal Aviation Administration \\
& Tower \\
& Butte City \\
& Blue Dome/Birch Creek Hydro \\
& Carey (Craters of the Moon) \\
& St. Anthony (Rexburg) \\
& Selected Ambient air monitoring \\
& locations \\
\hline
\end{tabular}

Sampling locations are selected using the following guiding criteria:

- Areas where a planned or unplanned release is most likely to occur

- Areas of likely deposition of radionuclides from source terms based on air deposition modeling

- Areas where an air monitoring location exists

- Areas with increased radionuclide inventories due to past activities

- Areas where historical data are available.

\subsection{Temporal Boundaries}

The temporal boundaries for soil monitoring are encompassed by the time between soil sample collections at a location, which may have been as early as the 1970s, up until soil monitoring is no longer measured under this program. Soil monitoring will continue as long as the Site is operational under DOE. This is likely to be long as 100 years into the future. The number of monitoring locations at a specific sampling area and the length of time that a particular sampling location is measured can vary depending on changes in conditions or activities in that particular sampling location.

The DOE Handbook states "Environmental surveillance measurements may be performed occasionally when potential dose is low, but should be performed at least every 5 years" (DOE 2015). Based on historical data and deposition modeling included in "Data Quality Objectives Supporting the Environmental Soil Monitoring Program for the INL Site" (INL 2015), the annual sampling frequency is technically unjustifiable for soils because examination of 40 years of data shows that concentrations of radionuclides in these soils are aged fallout products. The longer-lived radionuclide concentrations should remain constant, unless soils are disturbed. Cesium-137 and Sr-90 will continue to decrease with time. The decreasing trends are not statistically observable over short time periods (i.e., every 2 years), but rather require longer periods to distinguish the trends from natural variability in soil samples. In addition, atmospheric dispersion modeling of current releases shows that offsite soil concentrations from INL sources will never be distinguishable from background. For this reason, a 5-year sampling regime has been selected to maintain baseline and confirm observed trends in each region. When monitoring global fallout, short-term changes in radiation concentrations are generally small compared to the variability in the local radionuclide distribution (EML 1997). If a major unplanned release is detected on the INL Site, 
select locations on the Site boundary may be sampled in response, if it is believed, through modeling or air monitoring, that the location may be affected.

\subsection{Practical Considerations}

Practical constraints on collecting data are access to the property where samples are to be collected, the lack of undisturbed surface soil (e.g., some air monitor locations), the cost of sampling and analysis, and the time needed to collect and analyze samples. Soil sampling locations have been identified at INL for over 40 years and those same locations will be used to determine where samples will be collected under this monitoring effort. Soil sampling locations should be placed at points corresponding to air sampling locations to allow for comparability of data in areas that may be affected by a planned or unplanned release. However, some air monitors are located in areas that do not have soil or that are not associated with areas that are examined under this monitoring program. Samples will not be collected at these locations.

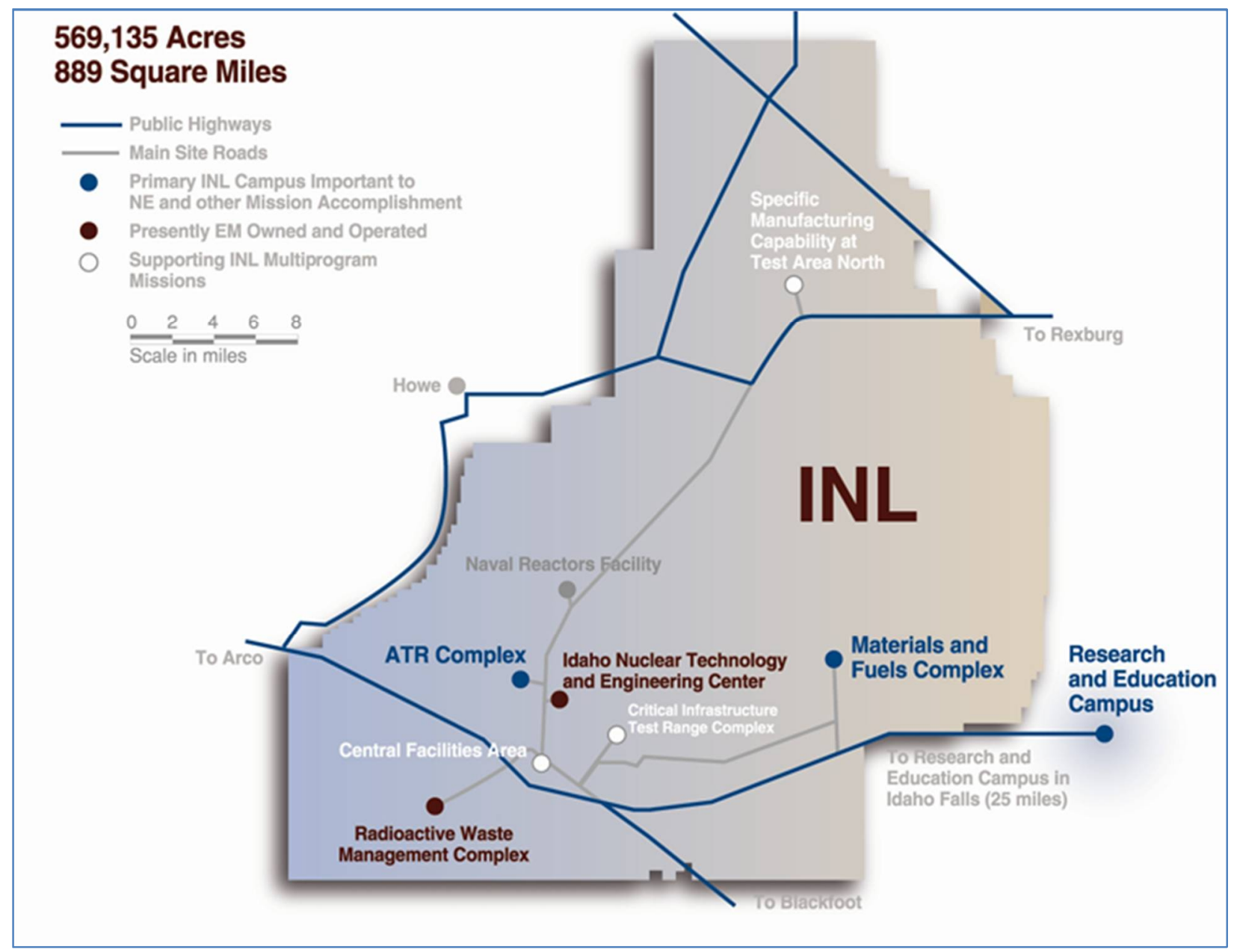

Figure 1. Map of the INL Site showing the locations of major facilities. 


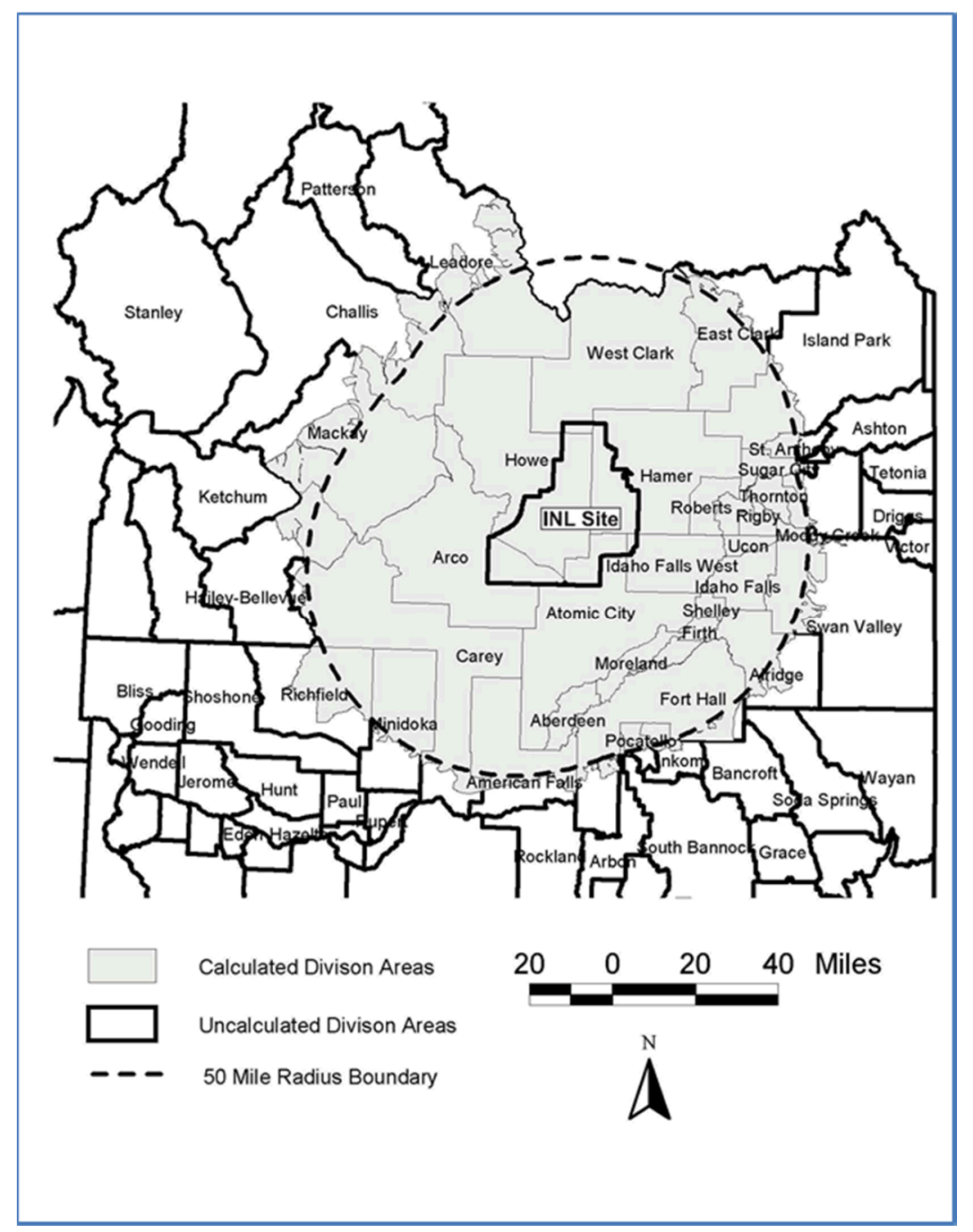

Figure 2. Region within 50 miles of INL Site facilities showing Census divisions used in the 50-mile population dose calculation.

\section{STEP 5: DEVELOP THE ANALYTICAL APPROACH}

Step 5 defines the analytical approach, which requires that the population parameters that will be used for decision making are defined as well as the action levels and appropriate estimators. The analytical approach is varied and involved. A comprehensive discussion of the approach is dependent on the statistical methods that are used to develop action limits and analyze data as it is obtained. Appendix C contains the details of the statistical analysis involved in the analytical approach while this section provides a high-level overview of the approach.

The first PSQ defined in Section 3 pertains to establishing a baseline radionuclide inventory for the soils associated with each of the near-facility regions and distant regions. This baseline provides a frame of reference in the case of a planned or unplanned release to determine the effect of the release on the soils. A background level will be computed for each near-facility and distant region to establish the baseline. Individual measurements obtained during sampling events can also be compared to the background level to alert the monitoring program when there is potentially high measurement. The background level assumes a parameter of the 99th percentile of all radionuclide concentrations in the soil. Thus, the 99\%/95\% upper tolerance limit (UTL) will be used as the estimate for the background level. 
This is the level such that $99 \%$ of the concentrations will be less than the background level with $95 \%$ confidence. The data obtained from each sampling event will be compared to the appropriate set of background levels to determine if any of the measurements exceed the background level. If the background level is exceeded, the sample will be further investigated to assess the reason for the larger value. It is assumed that $1 \%$ of the concentrations will exceed the background level. Thus, a single measurement that exceeds the background level does not necessarily indicate an unusually high amount of that radionuclide in the area. Once the data obtained from a sampling event have been examined and compared to the background levels, the background level will be updated using the new data to ensure that the baseline profile remains current. Table 3 shows the background levels for each of the areas in the near-facility and distant region. These background levels were computed using historical data that is listed in Appendix D.

Table 3. Background levels for near-facility and distant areas.

\begin{tabular}{|c|c|c|c|c|c|}
\hline Radionuclide & $\begin{array}{r}\text { Am-241 } \\
(\mathrm{pCi} / \mathrm{g}) \\
\end{array}$ & $\begin{array}{l}\mathrm{Cs}-137 \\
(\mathrm{pCi} / \mathrm{g})\end{array}$ & $\begin{array}{l}\mathrm{Pu}-238 \\
\text { (pCi/g) } \\
\end{array}$ & $\begin{array}{c}\mathrm{Pu}-239 / 240 \\
(\mathrm{pCi} / \mathrm{g})\end{array}$ & $\begin{array}{c}\mathrm{Sr}-90 \\
(\mathrm{pCi} / \mathrm{g})\end{array}$ \\
\hline \multicolumn{6}{|l|}{ INL Sites } \\
\hline ARA & 0.401 & 133 & 0.025 & 0.0577 & 57 \\
\hline ATR & 0.49 & 223 & 0.0116 & 0.0728 & 1.349 \\
\hline INTEC & 0.9 & 40 & 0.387 & 0.73 & 14.9 \\
\hline $\mathrm{MFC}$ & 0.008 & 1.99 & 0.01 & 0.0487 & 0.953 \\
\hline RWMC & 8.4 & 3.54 & 0.058 & 2.57 & 2.47 \\
\hline TAN & 0.086 & 23.8 & 0.014 & 0.029 & 1.754 \\
\hline \multicolumn{6}{|l|}{ Distant Sites } \\
\hline Atomic City & 0.0278 & 1.012 & 0.0227 & 0.0573 & 0.734 \\
\hline Blackfoot & 0.0405 & 2.697 & 0.154 & 0.239 & 0.398 \\
\hline Butte City & 0.0942 & 1.248 & 0.0337 & 0.0487 & 0.56 \\
\hline Carey & 0.0556 & 0.963 & 0.0447 & 0.0671 & 0.534 \\
\hline FAA & 0.0356 & 1.623 & 0.0743 & 0.0829 & 0.806 \\
\hline Frenchman's Cabin & \multicolumn{5}{|c|}{ Insufficient data to compute a background value } \\
\hline Howe & 0.01 & 0.7 & 0.0119 & 0.0353 & 0.67 \\
\hline Monteview & $0.0194 \mathrm{c}$ & 1.11 & 0.035 & 0.0477 & 0.268 \\
\hline Mud Lake & 0.0875 & 0.624 & 0.0514 & 0.0892 & 0.335 \\
\hline $\begin{array}{l}\text { Blue Dome/Birch } \\
\text { Creek Hydro }\end{array}$ & 0.0268 & 1.583 & $0.0144 \mathrm{c}$ & 0.0677 & 0.911 \\
\hline St. Anthony & 0.0422 & 1.758 & 0.0857 & 0.0954 & 0.948 \\
\hline
\end{tabular}

Data are collected at the mid-range areas, Highway 20-26 Big Lost River rest area at Milepost 265 (REST) and Experimental Field Station (EFS), to determine long-term deposition of radionuclides. A trend analysis will be performed on data obtained from these locations to assess the deposition over time. Historical data are not available for REST or EFS, so trend analysis will begin when sufficient data are available. 


\section{STEP 6: SPECIFY PERFORMANCE OR ACCEPTANCE CRITERIA}

This step is where decision rules and estimation uncertainty are defined. The purpose of the soils monitoring effort is to obtain acceptable estimates of the radionuclide inventory in areas within the INL boundaries and in the surrounding areas. Thus, the analytical plan is developed for estimation rather than decision making. The level of confidence for the background doses was chosen to be $95 \%$ and the other uncertainties were minimized by ensuring that sufficient data are collected at each area and that best sampling practices are implemented. Although every sampling project has a chance of committing a decision error, this program has numerous checks and balances in place such as historical data, sampling conducted under other programs, and monitoring of other media such as air and biota. These additional safeguards ensure that the chance of missing an authentic increase in radiation from INL is negligible.

\section{STEP 7: DEVELOP THE DETAILED PLAN FOR OBTAINING DATA}

Step 7 of the DQO process is where a detailed plan is laid out for obtaining data of sufficient quality to answer the primary questions of the study. It is important that the data are collected in a manner that meets the requirements of the statistical methods that will be used to analyze the data. This study obtains data from several areas both within INL boundaries and in surrounding areas. The location, history, and purpose of each of these areas differ so data requirements are not the same for all areas. Areas that are adjacent to facilities where radiological activities are taking place require sampling to ensure that the baseline inventory for those areas is adequately defined in the case of a planned or unplanned release. Areas that are not in the vicinity of radiological activities correspondingly require fewer sampling locations to maintain a baseline and examine long-term deposition.

This section outlines the general reasoning behind the number of monitoring locations at each area, the frequency of sampling, the methodology for selecting sampling locations, and lastly provides a detailed explanation for selecting sampling locations at the Site, at Idaho Falls facilities, and in the regional area.

\subsection{Sampling Design}

Statistical methods typically require that sampling locations are determined using a probability sampling design. That is a random method is used to determine sampling locations. Practical constraints are incorporated in location selection, such as predominant wind directions, accessibility, and facility features, while still maintaining a sufficient amount of randomness to meet the requirements of the statistical methods. Data have been collected over years from the same locations at many of these areas and previous sampling locations are taken into account when determining future sampling locations to ensure continuity and optimal information.

The most basic probability sampling method is simple random sampling. It is the method upon which the others are built. A simple random sample is where every possible sample is identified and then a random number generator is used to select the sample locations that will be used. No human bias is involved in sample location selection. It is important to note that haphazard sampling is acutely biased in nature and is not random sampling.

Another sampling method is systematic random sampling. Systematic random sampling is where a system, such as fixed intervals along a line or on a systematic grid, is used to select sample locations. The starting location is determined by random selection. Thus, the system is not random, but the placement is; the method is still a probability sampling method. This sampling design is applicable when uniform coverage of an area is desired. It is also often easier to implement a systematic method in the field than a non-systematic design. 
Composite sampling is a method where sample locations are determined by a random sampling method and 2-5 samples are mixed together and one representative aliquot is collected from the composite and analyzed. Samples that are composited together should be close to each other so that as much of the information in regard to variability across the area being sampled can be preserved. Composite sampling has several benefits. This method provides more accurate averages at a lower cost because the information from two to five samples can be obtained for the cost of one analytical sample. Composites are helpful with soil sampling because soil is expressly heterogeneous. Compositing samples allow the data user to gain a more accurate profile of the area in question. However, composite samples mask some of the variability, which must be considered during data analysis. It is essential that measures such as UTLs that are computed from composite samples are compared with other composite samples.

A method that is often used with soil is MULTI INCREMENT ${ }^{\circledR}$ sampling. This sampling method was developed by Envirostat and uses the principles developed by Pierre Gy for his Theory of Sampling method (Gy 1979). MULTI INCREMENT sampling is often mistaken for composite sampling, but they are very different in implementation and benefits. A MULTI INCREMENT sampling design is one where the sampling area is clearly defined and decision units are identified. Decision units are areas where a decision would be made and often covers a large area. A grid is placed over each decision unit and many small samples are collected systematically over the grid. All of the samples are combined and thoroughly mixed to ensure the soil is homogenous. One representative sample is collected from the homogenized soils and is analyzed. MULTI INCREMENT sampling is designed to determine the mean concentration of a particular analyte over a specific area and it is remarkably effective at determining the mean. However, the variability of the concentrations in that area cannot be measured and thus MULTI INCREMENT sampling cannot determine if an individual measurement from the area in question is within the normal range of concentrations.

The primary goals of the soil monitoring programs are to measure long-term deposition and to maintain a baseline for different areas inside the Site and in the surrounding areas. The baseline profile is defined by UTLs that can be compared to individual measurements to determine if those individual measurements are within the normal range of concentrations for that area. Thus, MULTI INCREMENT sampling cannot be used to meet the objectives of the monitoring program. A combination of systematic sampling and composite sampling will be used for this program. Each of the areas within Site boundaries that are to be monitored to maintain a baseline inventory have sampling locations that have been identified for many years. It is unnecessary to collect samples at all of these locations to establish and maintain a background profile. Thus, a systematic random sampling method will be used to determine which sample locations will be included under the program. Section 8.4 outlines the methodology that is used for each of the six near-facility regions in detail.

A composite sample consisting of five individual samples will be used at each sampling locations. This will minimize the heterogeneity at each sampling location. Five samples will be collected at the corners of a $10 \times 10$ meter square and a sample will be collected in the middle. All five samples will be well mixed and a single composite sample will be analyzed (see Figure 3). This compositing methodology will be used at all sampling locations regardless of location or purpose. 


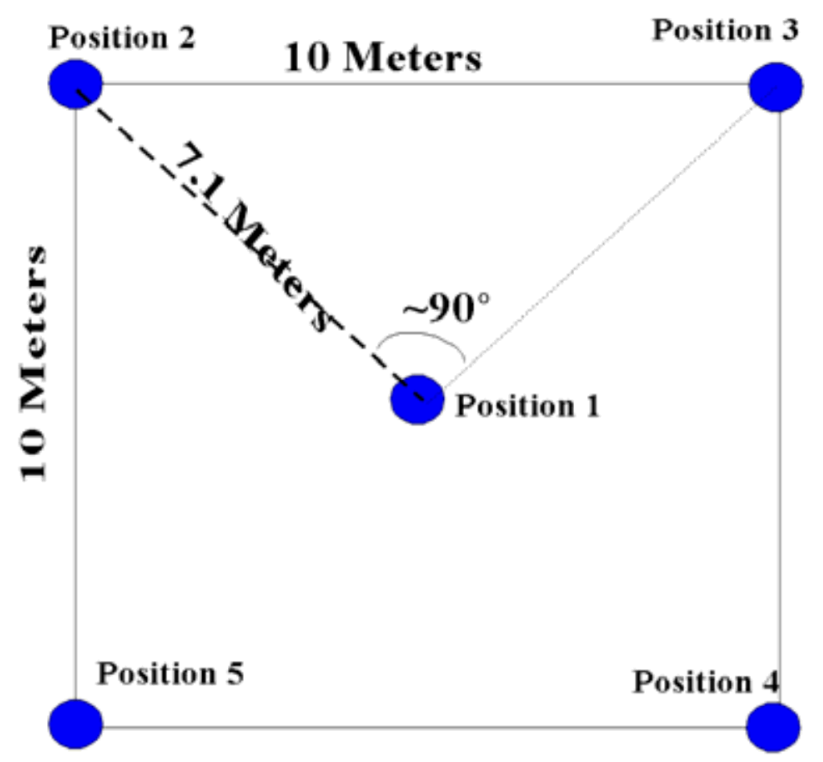

Figure 3. Composite sampling grid.

\subsection{Methodology for the Number of Monitoring Locations}

This subsection outlines the methodology for determining the number of monitoring locations at a specific area and for the frequency of sample collection. The general guidelines for each type of area are discussed along with area features that assist in determining the sampling locations and the number samples that are needed in a specific area.

The soil monitoring program establishes the baseline inventory of radionuclides in the soils within INL boundaries and in the surrounding areas as well as determines the long-term deposition of radionuclides in the soils due to INL activities. The number of samples needed to perform an analysis is dependent on the statistical methods used to analyze the data and make decisions. Some statistical methods, such as the upper confidence limit, have formulas associated with them to aid the data user in determining how many samples are required to meet certain quality criteria. However, UTLs will be used to compute baseline inventories with the monitoring data and UTLs do not have sample size equations associated with them to aid in determining an appropriate sample size. Thus, other criteria must be used to determine the appropriate sample size. Characteristics, such as variability of the data, history, and current activities associated with the area being sampled, and the size of the area, are all taken into account when determining the number of samples needed and the location of those samples.

Because the primary concern is about areas where a planned or unplanned release from INL may occur, facilities within the Site that perform radiological activities are where sampling will be most concentrated. There are also areas within the Site boundaries and outside of the Site boundaries that are not in close proximity to radiological activities, but are located where the public may be affected. Hence, establishing a baseline radiological inventory of the soils in those areas is of interest in the case of an event. Fewer monitoring locations are needed in these areas because of their distance from the potential sources of INL radiation. The sample size criterion considers the proximity of an area to radiological activities when determining the minimum number of samples to be collected.

The criteria for determining sample size considers the proximity of the areas to radiological activities, the ability to compute appropriate statistical measures, the variability in radionuclide concentrations across the area exhibited in historical data, and the physical characteristics of the area. Areas are separated into three main categories: near facility, mid-range, and distant areas. Near facility areas are facilities within the Site boundaries where radiological activities are taking place or have taken place. Not all such 
locations were selected for sampling. A facility was included in the set of sites to be sampled if radiological activities are taking place or if previous activities may have resulted in higher concentrations of radionuclides in the soils than in surrounding areas. Mid-range areas will be sampled only to assess long-term deposition. Distant areas may be along the Site boundary or outside of the Site boundary. These include areas where no radiological activities take place but where the public may be present. A baseline radiological inventory will also be developed for these areas; however, fewer sampling locations are required to attain and maintain a baseline for the distant areas than for the near facility areas.

Large facility areas require a minimum of $8-15$ sampling locations, not including duplicates. This is because a UTL will need to be computed and maintained for the area every 5 years to ensure it remains relevant because it is possible that radiation inventory could change at those locations due to facility activities. Site characteristics or other motivators may indicate that more than eight monitoring locations are warranted. Historical data were examined to aid in determining the number of samples needed from a particular area. If the variability for a facility is large more sample locations were selected.

Mid-range areas only require a single composite sample consisting of five grab samples. As with the near-facility areas, it may be warranted to identify more than five sampling locations around the area due to physical characteristics of the area or other reasons.

Distant areas may be maintained with a single composite sample consisting of five grab samples. Fewer samples are required at such areas because of their distance from an INL activity-based source. However, background levels can be maintained with one sample location because of the availability of historical data. It is possible to use data acquired over time to compute area-specific background doses because the doses at the distant locations have not previously exhibited evidence of increased dose from INL radiological activities.

The Handbook guidance allows for surveillance monitoring as infrequently as every 5 years if the projected annual effective dose to the public is less than 0.1 mrem per year, which is the case for INL. Because the focus of soil monitoring is to establish and maintain a baseline inventory and to assess long-term deposition, sampling on a 5-year rotation schedule is adequate to meet project requirements.

\subsection{Methodology for Soil Monitoring Location Selection}

The previous section provides a minimum number of monitoring locations for each type of area, but it does not indicate where the soil sampling locations should be located or how to determine whether more than the minimum number is warranted. This section provides guidelines for incorporating this approach in determining the location of specific sampling locations for an area. The guidelines consist of knowledge of the location of sources of external radiation that are to be monitored, natural conditions that may affect the spread of radionuclides, the location of air monitoring locations, the area of elevated simulated air concentrations, and stakeholder concern.

Proximity to potential source of radiation. One of the most important factors in determining the location of sampling locations is where the potential sources of a radiation release are located. The monitoring program does not measure inside of the outer fence of any INL facility; the INL Radiological Control program is responsible for monitoring those areas. Therefore, this monitoring program places many of the sampling locations near the fence surrounding these facilities to ensure the areas that are most likely to be affected by a release are measured.

Prevailing wind directions. The Handbook guidance states that sampling should occur in the prevailing wind directions. In most INL locations the predominant wind direction is from the southwest, but there is also a significant component of wind from the northeast. For this reason sample locations will be placed both to the northeast and the southwest of near-facility areas. These soil sampling locations are placed close to the outer facility fence lines and extend as far as the wind may carry particulates originating from the Site. The stacks at the Materials and Fuels Complex (MFC), Idaho Nuclear Technology and Engineering Center (INTEC), and Advanced Test Reactor (ATR) Complex, which are 
the stacks with potential radioactive emissions that are evaluated annually for National Emission Standards for Hazardous Air Pollutants (NESHAPs) require that sampling extends further from the Site boundaries than at other near-facility sites. The estimated distances result from simulations using the Gaussian plume model and area-specific wind rose information averaged from 3 years (2006-2008). These simulations are summarized in Appendix A.

Low-Volume Air Monitoring Locations. Sampling locations were identified at low-volume air monitoring locations when possible for data integration purposes. This provides measured air concentrations and direct radiation doses that can be compared to simulated air concentrations that are predicted for the Annual Site Environmental Reports. As an example, the offsite regional monitoring location direct radiation measurements are averaged and compared to the calculated effective dose from natural background sources (see Table 7-6 in DOE-ID [2014]). The ability to collocate sampling locations with existing air monitors is limited by proximity of soil to the air monitor and to the availability of air monitors within areas where soil monitoring is beneficial.

Stakeholder concerns. In some cases, soil sampling locations were determined to alleviate concerns by public stakeholders. This is generally the case for soil sampling located away from active INL facilities and in regional population centers. Monitoring at these locations provides a baseline radionuclide inventory that can be referenced in the case of a planned or unplanned release.

Soil samples will be collected from surface soils only. The primary purpose is to maintain a background inventory of area that can be used to assess the effect of a planned or unplanned release. The impact of a release would be most evident on the surface soils, so sampling of subsurface soils is of little value in achieving the goals outlined in these DQOs.

\subsection{Area Specific Monitoring Locations}

This subsection discusses the specific sampling locations for each of the areas. The characteristics of the facility that affect the placement and number of soil sampling locations are discussed and shown in Figures 4 through 10. Each figure shows the soil sampling locations that are selected in this DQO for use in soil monitoring. These locations are designated with a maroon triangle. The areas that are addressed in this subsection are ATR Complex, Auxiliary Reactor Area (ARA), INTEC, MFC, RWMC, Specific Manufacturing Capability (SMC), and Resident Receptor locations. Historical data collected from the 1970s through 2014 were examined to determine concentrations variability and other characteristics that influenced the location and number of monitoring locations that were selected. Findings from the historical data are addressed in each facility subsection.

\subsubsection{Advanced Test Reactor Complex}

Historical data collected near the ATR complex was examined to select the sampling locations that will be used for monitoring that facility. Historical samples were collected in the area northeast of ATR and also Northeast and Southwest of the Warm Waste Pond. Examination of concentrations observed in those samples from the 1970s through 2014 shows that radionuclide concentrations are highest in the northeast transect through the center of the Warm Waste Pond. The highest concentrations were observed at sample Location 4.2, which is along the northeast boundary of the pond. The highest concentration near ATR is at sample Location 4.5. 
Sample locations are numbered from -2.2 through 8.3 near the pond and from A1.2 through A5.5 near ATR. A systematic sampling approach that samples every fifth sample location was developed for the samples near the ponds and every third sample was selected near the main facility. A random number generator selected 3 as a random start location for the pond and 1 for the main facility. Thus, the first selected sample is -1.3 and then every fifth sample was selected thereafter. This resulted in seven monitoring locations being selected by this method. A few of the sample locations are in the ATR ponds. When this occurred alternative sampling locations were selected that were near the original ones. Two other locations were identified as having higher concentrations of radionuclides than other methods so they were also selected as soil monitoring locations to ensure that these areas are adequately characterized. These locations are 4.2 and A4.5. The total number of monitoring locations at ATR is 14. This provides sufficient data to compute a UTL while assuring areas with higher radionuclide concentrations are adequately monitored.

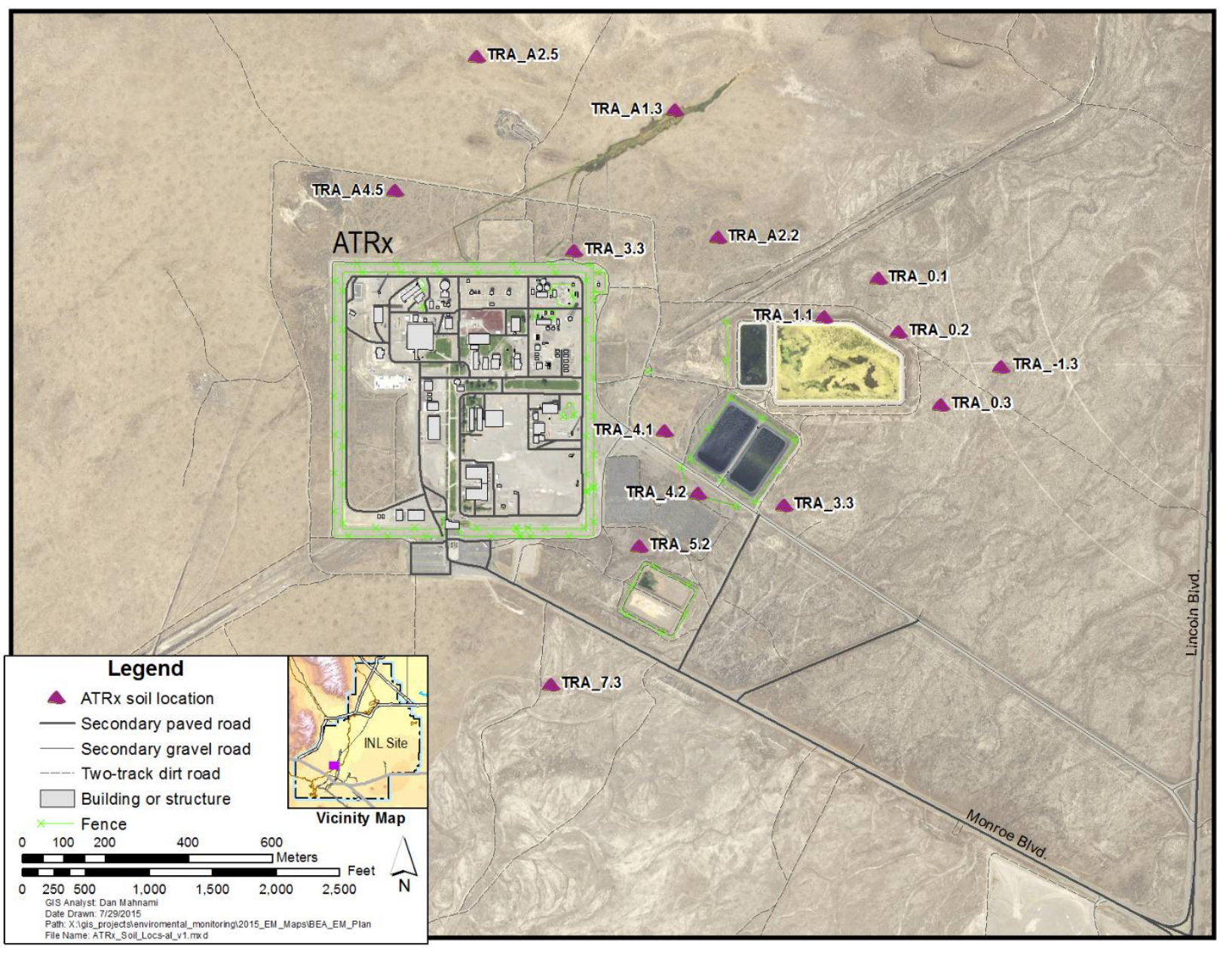

Figure 4. Soil monitoring locations at ATR.

\subsubsection{Auxiliary Reactor Area}

The sample locations at ARA are located radially from the facility. Prior to the year 2000 sample locations were identified by an angle and a distance from the center. After 2000, samples were identified by number. Historical data were assessed to determine where the highest concentrations of radionuclides were in the area and examine patterns of distribution. This is also where the burial ground for SL-1 is located. It was found that the highest concentrations were seen along the northeast border of the facility and to the south and east of SL-1. A modified systematic random sampling plan was used to select 
monitoring locations. A random start of 2 was determined and every eighth sample number was selected for inclusion. Several additional points were selected along the northeast boarder of ARA and in the vicinity south and east of SL-1 to ensure that these areas are adequately documented. This resulted in 15 monitoring locations for ARA. Locations are shown on the map in Figure 5.

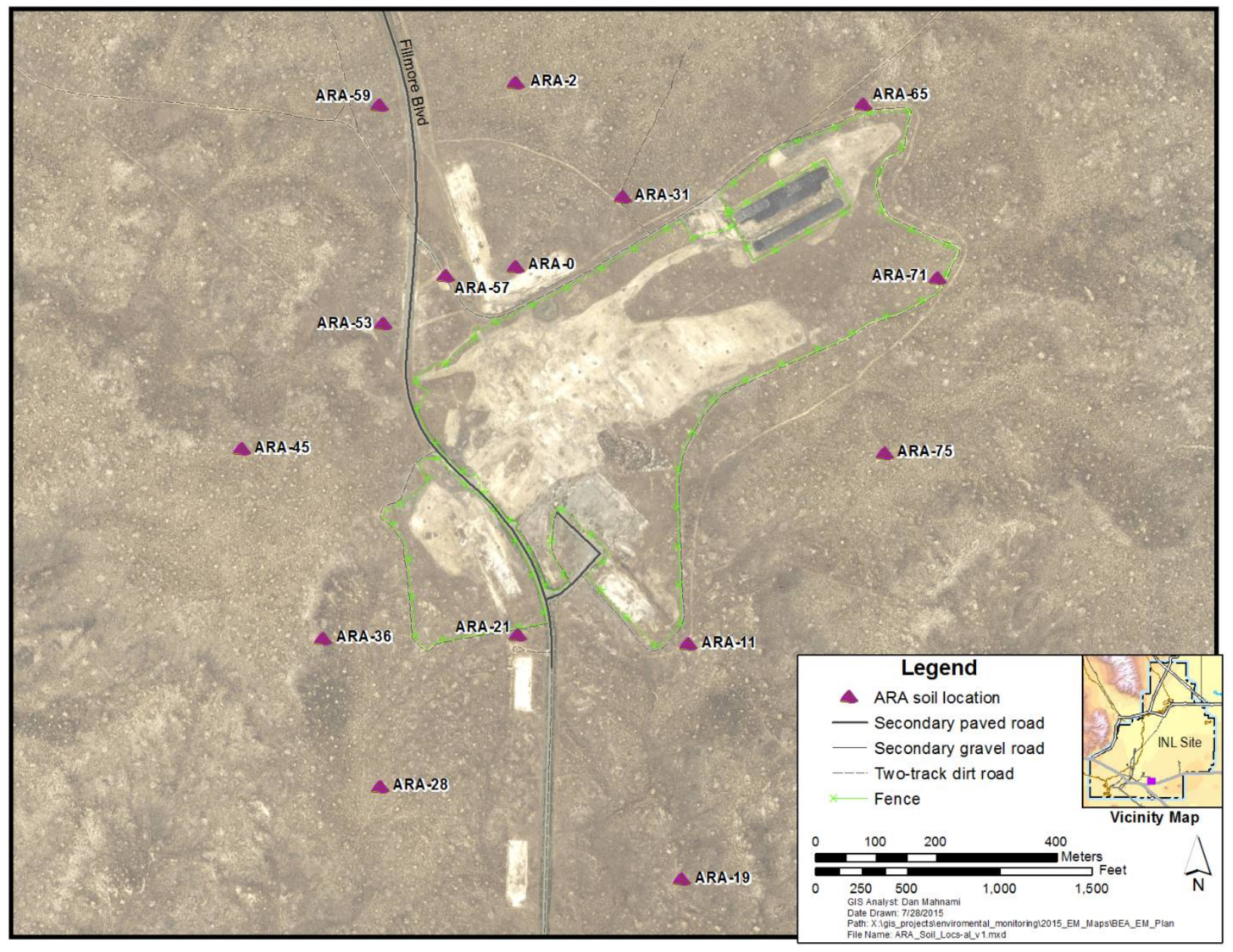

Figure 5. Soil monitoring locations at ARA.

\subsubsection{Idaho Nuclear Technology and Engineering Center}

The RESL program used an extensive grid to sample the soils surrounding INTEC. Although sampling locations are identified for every point on the grid not all of the locations were sampled. However, sufficient sampling was conducted to form a clear picture of where the radionuclides are present in the highest concentrations. Data collected from the 1970s through 2014 show that the concentrations of radionuclides are higher northeast and southwest of the facility. Concentrations are higher close to the facility than they are further away from the facility. The area southwest of INTEC and within about $200 \mathrm{ft}$ of the facility boundary shows the highest concentrations in the area.

A systematic design was used to select sampling locations. A grid was placed on the sampling area that divided that sample locations into groups of 16 samples $(4 \times 4$ squares). A random number generator selected 12 as the random start location. So, every twelfth sampling location within each $4 \times 4$ square was selected as a monitoring location. The main grid does not start with location C120, which is the most northwestern sample point. It was moved to fit the pattern where radionuclides were observed. The northwest corner of the main sampling grid is Location C96. Some of the squares were not sampled 
because they were not close to areas where radionuclides were observed. As stated previously, higher concentrations of radionuclides were observed in the area southwest of the facility. Four additional monitoring locations (B29, B36, B50, and B92) were selected from this area to ensure that the area is adequately characterized. Some of the selected samples were in areas that are now within INL CERCLA Disposal Facility (ICDF), in a parking lot, or are otherwise inaccessible. When this occurred, an alternative monitoring location was selected that was close to the original selection. Figure 6 shows the selected soil monitoring locations.

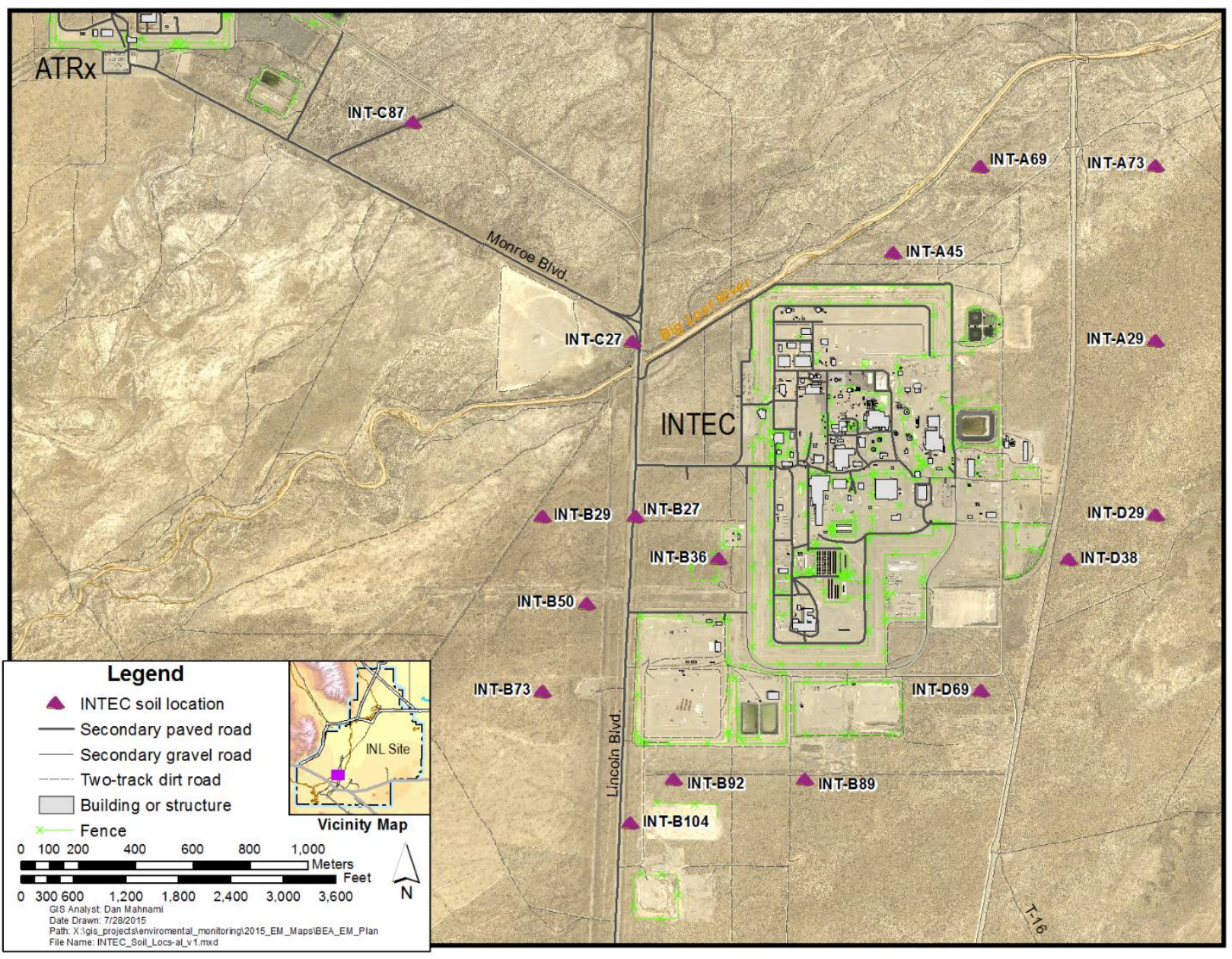

Figure 6. Soil monitoring locations at INTEC.

\subsubsection{MFC}

Samples were collected from MFC surrounding the main facility and surrounding TREAT. Four sample locations are located around TREAT and 18 sample locations are located around the main facility. The main facility locations are predominantly northeast and southwest of the facility. Historical data collected from the 1970s through 2014 were examined to determine trends in radionuclide concentrations. The historical data show that some of the some of the radionuclides have higher concentrations at TRT-2 and TRT-4 while others have higher concentrations at TRT-1 and TRT-3. The highest concentrations around the main facility were seen at locations EBR II-6, EBR II-11, and EBR II-15. EBR II-6 and EBR II-11 are close to the north side of the fence.

Because higher concentrations of radionuclides were observed in all four sampling locations around TREAT, it is proposed that all four of those locations are retained as monitoring locations. The three 
locations where higher radionuclide concentrations were observed are also included as monitoring locations. A systematic random sampling design was used to select samples from the north side of the main facility and then from the south side of the main facility. The sample numbers that were not already selected were lined up and a random starting point between 1 and 3 was chosen. A random number generator selected 1 for the southern samples and 3 for the northern samples. The selected soil monitoring locations are shown in Figure 7. Some of the sampling areas were not accessible. When this occurred alternative sampling locations that were near the originally selected locations were chosen as monitoring locations.

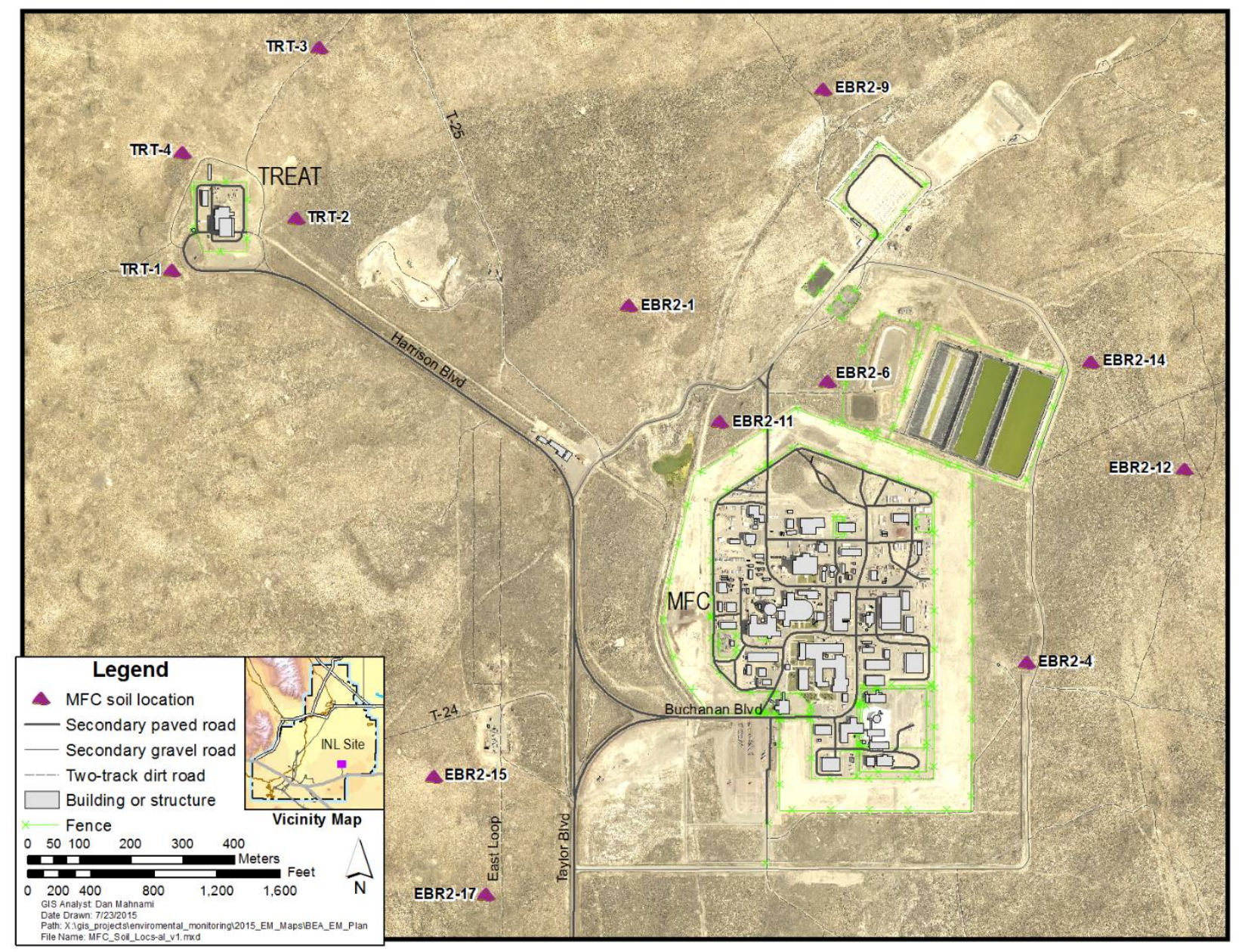

Figure 7. Soil monitoring locations at MFC.

\subsubsection{Radioactive Waste Management Complex}

Historical data are RWMC were concentrated southwest and northeast of the facility. The data show that the highest concentrations of radionuclides are along the north fence and at locations RW2-1, RW3-1, and RW3-2. A systematic random sampling design was used to determine which of these locations will be used as monitoring locations. It was determined to sample every third location so the sample identifier numbers were lined up from smallest number to largest number and every third location was selected. A random number generator selected the first sample as the random start location. Because the north and west sides of the fence have much higher concentrations of radionuclides than the other locations, Locations RW2-1, RW2-4, RW3-1, RW3-2, RW5-4, RW5-5, and RW5-8 are included as monitoring locations in addition to those selected through systematic random sampling. Figure 8 shows the monitoring locations. 


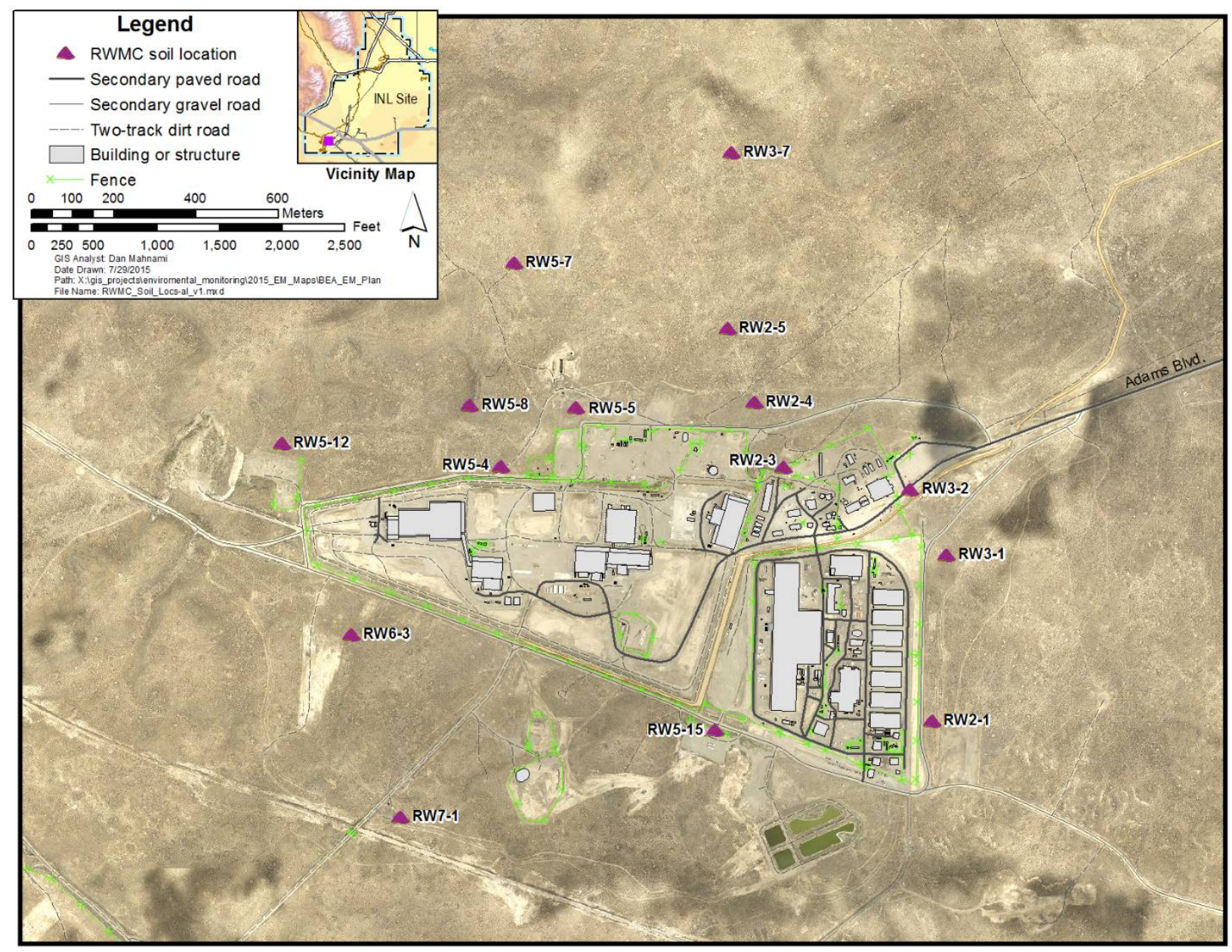

Figure 8. Soil monitoring locations at RWMC.

\subsubsection{SMC/TAN}

Historical data at the SMC/TAN complex was examined to select the sampling locations that will be used for monitoring that facility. Historical samples were collected in the areas surrounding Initial Engine Test (IET), Loss-of-Fluid Test (LOFT), Technical Support Facility (TSF), and Water Reactor Research Test Facility (WRRTF). The distribution of sample locations is widespread. However, the concentrations of radionuclides are rather low. Historical data were used to determine the sampling locations in each for the four main areas that had the highest concentrations of radionuclides. Two locations were selected from both IET and WRRTF. Three locations were selected from LOFT. Nine sampling locations are identified near TSF. A systematic random sample was used to select three samples from that area. The sample locations were ordered by number and a random number generator selected 2 as the random start. Figure 9 shows the selected monitoring locations. 


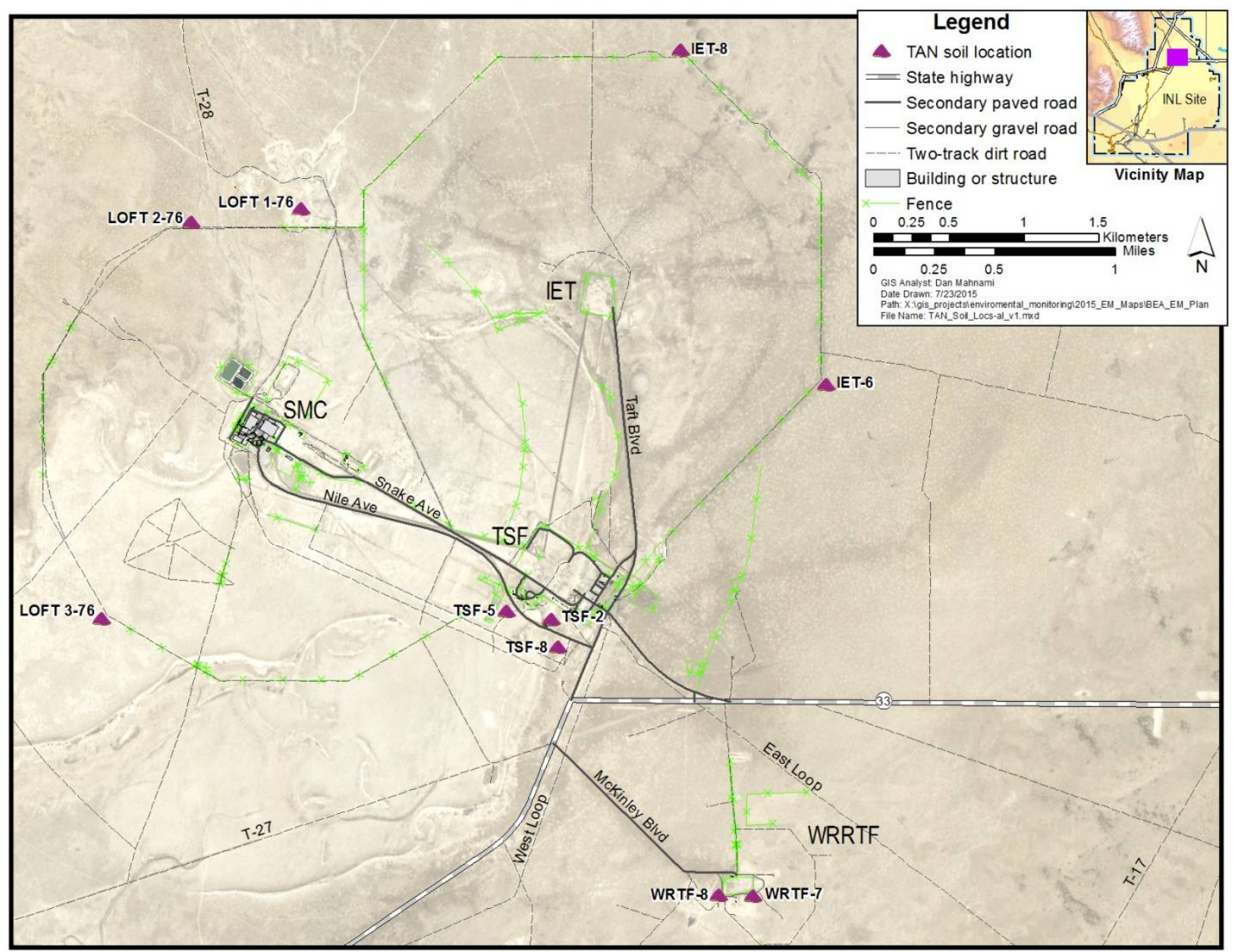

Figure 9. Soil monitoring locations at SMC/TAN.

\subsubsection{Resident Receptor Locations}

Eleven distant areas and two mid-range areas are monitored under this the soil monitoring program. One composite sample comprised of five grab samples is collected from each of these locations during each sampling event. Data collected over time is used to compute a baseline inventory for each area. Because extensive historical data exists for most of these areas the same sampling location will continue to be used to ensure that there is data comparability over time. Figure 10 shows the distant sampling locations and the mid-range sample locations. 


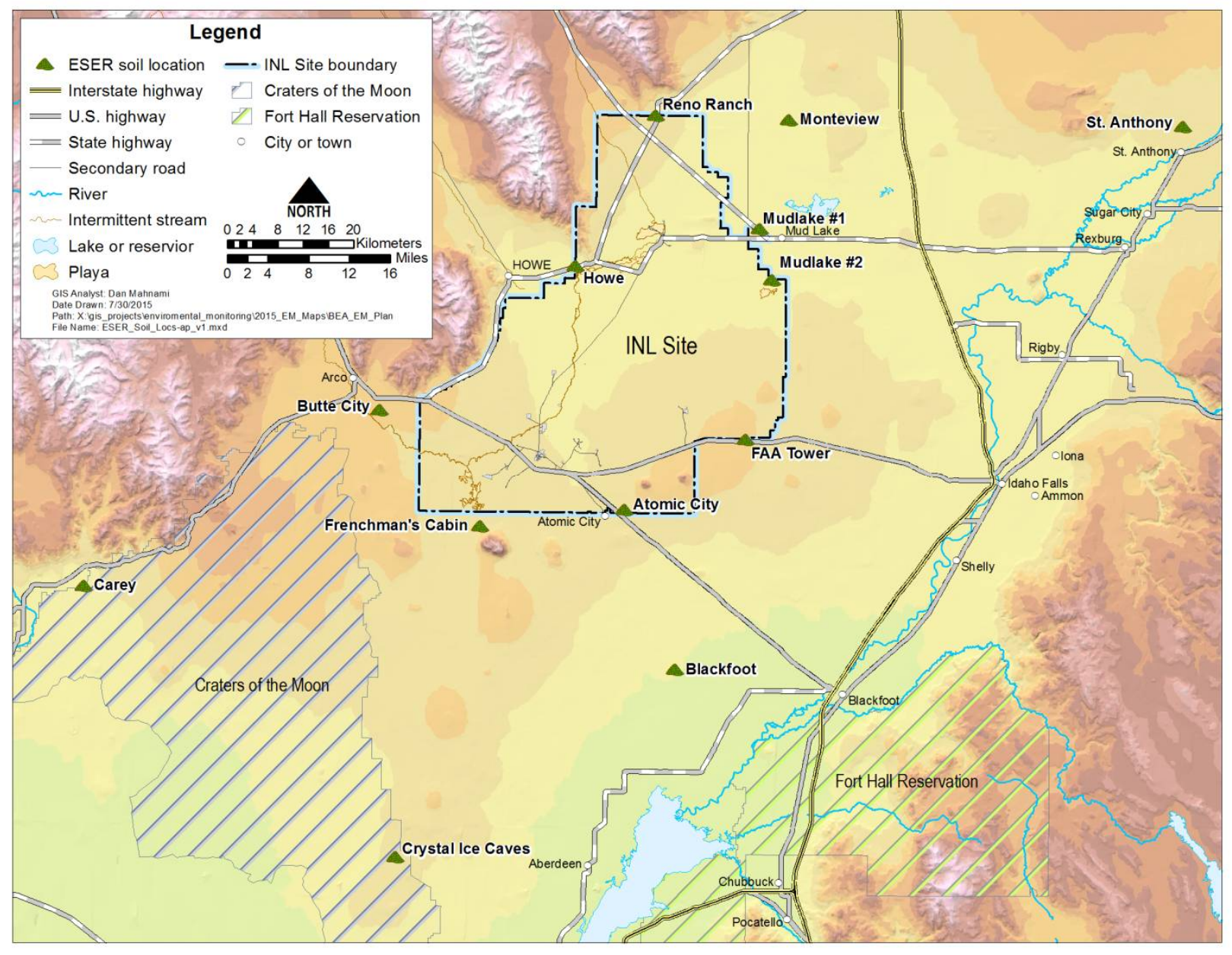

Figure 10. Soil monitoring locations at SMC/TAN.

\section{CONCLUSIONS}

As previously mentioned, this document represents the initial evaluation and soil monitoring proposed by BEA in 2015. The evaluation included analyses of historical soil monitoring data and soil inventories, current emission estimates, and modeled potential deposition/accumulation patterns. The initially proposed monitoring included a 5-year rotation of in-situ gamma measurements augmented by soil sampling with laboratory analyses near each major active and some inactive facilities. It also proposed rotational in-situ gamma measurements and soil sampling at two centrally located onsite air monitoring locations coinciding with sampling at the traditional offsite soil monitoring locations. The preferred alternative selected by DOE includes only physical soil sampling with laboratory analysis and only at RWMC, the two air monitors, and the offsite locations, as documented in Data Quality Objectives Supporting the Environmental Soil Monitoring Program for the INL Site, INL/EXT-15-34909, Revision 0 , February 2016. The data and evaluations in this document are valid for comparisons with future soil data that may be collected in many INL Site locations. This document provides a technical explanation and justification for the process used to select sampling locations, analyze results, and make decisions based on acquired data. The number and location of soil sampling locations at each area may change as INL activities change. However, as long as changes are made within the guidelines set out in this document, such changes will not be in violation of the DQO parameters. Additional information is available in the INL 2014 report, Technical Basis for Environmental Monitoring and Surveillance at the Idaho National Laboratory Site, DOE/ID-11485, February 2014. 


\section{REFERENCES}

DOE, 2015, DOE Handbook, "Environmental Radiological Effluent Monitoring and Environmental Surveillance,” DOE-HDBK-1216-2015.

DOE Order 458.1, 2011, "Radiation Protection of the Public and Environment," U.S. Department of Energy, February 2011.

INL, 2014, Technical Basis for Environmental Monitoring and Surveillance at the Idaho National Laboratory Site, DOE/ID-11485, February 2014.

INL, 2016, Data Quality Objectives Supporting the Environmental Soil Monitoring Program for the INL Site, INL/EXT-15-34909, February 2016.

Rood, A. S. and A. J. Sondrup, 2015, Quantitative Assessment of Detection Frequency for the INL Ambient Air Monitoring Network, INL/EXT-14-33194, March 2015.

Jessmore, P. J., L. A. Lopez, and T. J. Haney, 1994, Compilation and Evaluation of the Idaho National Engineering Laboratory Radiological and Environmental Sciences Laboratory Surface Soil Sample Data for Use in Operable Unit 10-06 Baseline Risk Assessment, EGG-ER-11227, September 1994.

Gy, P. M., 1979, Sampling of Particulate Materials: Theory and Practice, Elsevier Scientific Publishing Company, 1979.

Rood, et al., 1996, Executive Summary for Background Dose Equivalent Rates and Surficial Soil Metal and Radionuclide Concentrations for the Idaho National Engineering Laboratory, INEL-94/0250, Rev. 1, September 1996.

Morris, R. C., 2000, Radioecology of Iodine-129 in the Sagebrush-Steppe Ecosystem, Environmental Science \& Research Foundation, Inc., Annual Technical Report to DOE-ID, ESRF-037, June 2000.

\section{APPENDIXES}

Appendix A, "Historical Information and Air Modeling Results that Determine Radionuclides of Interest and Soil Sample Locations"

Appendix B, "Statistical Analysis Associated with Soil Monitoring Data Quality Objectives"

Appendix C, "Data Used for Decision Limits" 


\section{Appendix A}

\section{Historical Information and Air Modeling Results that Determine Radionuclides of Interest and Soil Sample Locations}

This appendix lists and describes the sources used for determine the radionuclides of interest for soil monitoring and where soil samples should be collected. The type of information is described that is needed to meet performance acceptance criteria and provides direction for sampling and analysis methods.

\section{A-1. RADIONUCLIDES OF INTEREST}

Air is considered to be the most critical pathway from the Idaho National Laboratory (INL) Site to offsite receptors (DOE 2015, DOE/ID 2014a, and NCRP 2010). Surface soil can become contaminated with radionuclides by deposition of airborne particles released from INL Site activities.

Information on current radiological effluents is contained in the most recent INL Site NESHAP report, National Emission Standards for Hazardous Air Pollutants-Calendar Year 2013 INL Report for Radionuclides, referred to hereafter as the NESHAPs Report (DOE-ID 2014b). Using data from the 2007-2013 NESHAP reports, the radionuclides that contribute at least $1 \%$ of the total estimated dose (for a total of $97 \%$ of the total estimated dose) over the 7-year period are summarized in Table A-1. Estimated doses to the Maximally Exposed Individual (MEI) during this time period ranged from 0.093 mrem to $0.131 \mathrm{mrem}$.

Table A-1. Radionuclides that are important in terms of radiological dose based on the 2007-2013 NESHAP reports (Rood and Sondrup 2014).

\begin{tabular}{|c|c|}
\hline Radionuclide $^{\mathrm{a}}$ & Percent of total dose $(2007-2013)^{b}$ \\
\hline H-3 (vapor) & $25.22 \%$ \\
\hline $\mathrm{Pu}-239 / 240$ & $19.78 \%$ \\
\hline Cs-137 & $18.13 \%$ \\
\hline Sr-90 & $12.49 \%$ \\
\hline Am-241 & $8.20 \%$ \\
\hline Ar-41 (gas) & $7.22 \%$ \\
\hline $\mathrm{I}-129$ & $4.54 \%$ \\
\hline $\mathrm{Pu}-238$ & $1.51 \%$ \\
\hline Total & $97.09 \%$ \\
\hline
\end{tabular}

Of the radionuclides shown in Table A-1, Am-241, Cs-137, Sr-90, I-129, and isotopes of plutonium are released in particulate form and could be deposited on surface soil. Once in the soil, radionuclides are available for direct exposure, uptake by plants, resuspension of particles, and mixing from human activities, such as agriculture. Cesium-137, Am-241, Pu-238, and Pu-239/240 were also were identified by Radiological and Environmental Sciences Laboratory (RESL) in areas on the INL Site potentially contaminated with radionuclides in excess of offsite background levels (Jessmore et al., 1994). 
In addition to radionuclides that are specific to a particular operation or facility, naturally occurring (e.g., the uranium and thorium decay chains and beryllium [Be-7]) and fall-out radionuclides can be expected in soil samples."

To establish background levels of natural and fallout radioactivity in surface soil and to assess any potential buildup of radioactivity offsite from INL Site operations, soil samples have been collected from undisturbed distant and boundary locations every year since 1970, except 1972, 1977, and 1979 (DOE/ID 1981). A biennial sampling program was established in 1978 and included offsite locations from Idaho Falls, Blackfoot, Pocatello, Crystal Ice Caves, Craters of the Moon, and St. Anthony. Boundary locations included Reno Ranch, Monteview, Mud Lake (two stations), Atomic City, Arco, and Howe. (A rotating 7-year schedule was used to sample onsite soils around major INL Site facilities.) In 1984, Pocatello and Idaho Falls were removed from the routine sampling program. In 1984, three samples (Mud Lake No. 1, Mud Lake No. 2, and Crystal Ice Caves) were excluded from the data set because they appeared to be uncharacteristically low and may have been influenced by disturbance (vehicular traffic, farming, etc.). These sampling locations and the location at Monteview were re-evaluated and moved to more representative undisturbed locations in 1986. The sampling site at the Federal Aviation Administration (FAA) Tower was added to the boundary group in 1986. Collection of soil at Frenchman's Cabin, the location of the MEI, began in 2010. Current offsite soil sampling locations are shown in Figure A-1.

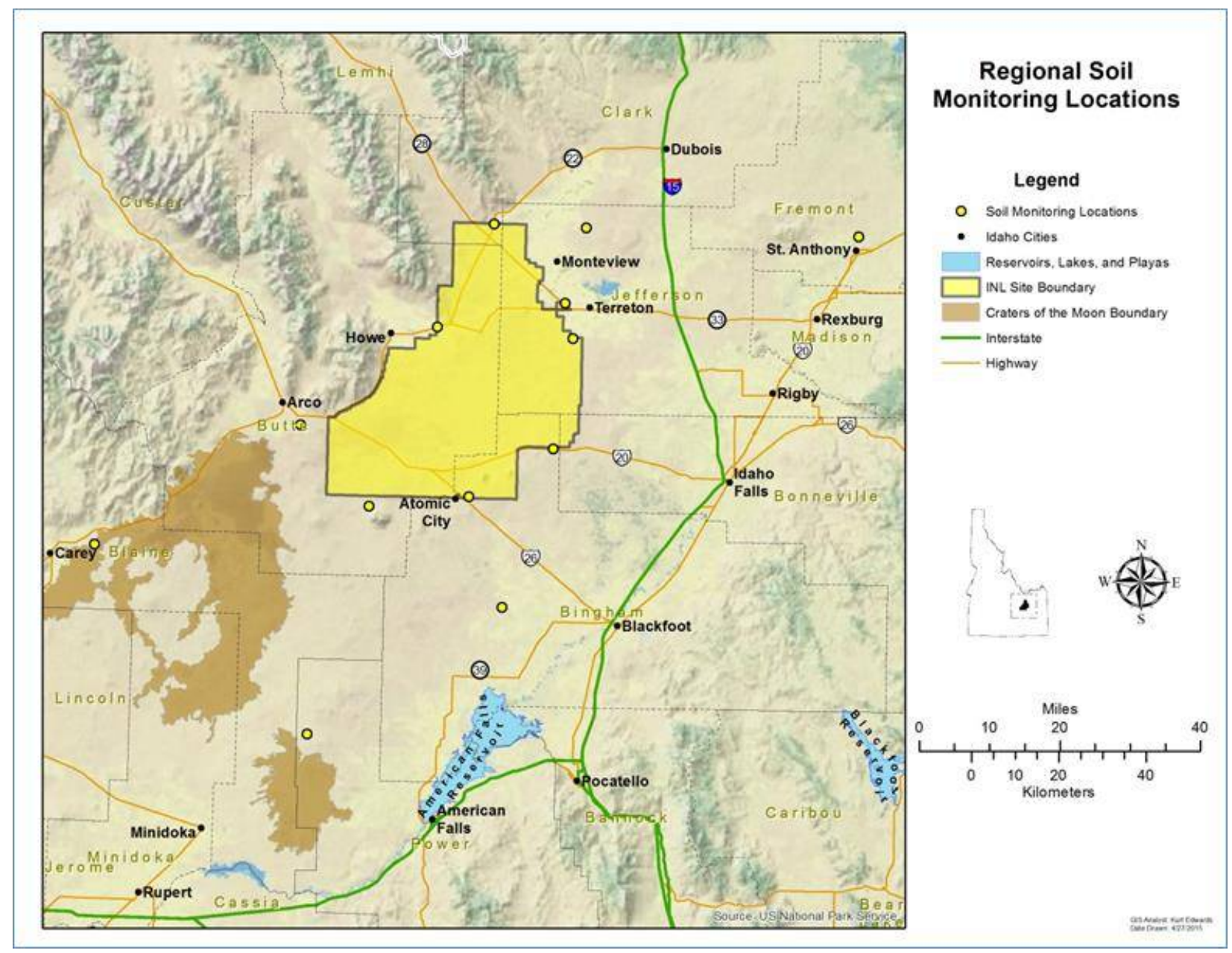

Figure A-1. Offsite soil-sampling locations. 
Soil samples collected in 1970, 1971, and 1973 represented a composite of five cores of soil from $1-\mathrm{m}^{2}$ area. Each core was a cylinder $10 \mathrm{~cm}$ in diameter and $5 \mathrm{~cm}$ in depth. In all other years, the five cores were collected from a $100-\mathrm{m}^{2}$ area. A number of samples were also collected from the 5- to 10-cm depth. The core method provides a useful measure of the concentration of deposited material in soil related to the amount per unit area and allows for the estimation of the inventory of the material deposited over a given area (EML Procedures Manual, HASL-300, 28th Edition, available at https://www.orau.org/ptp/PTP\%20Library/library/DOE/eml/hasl300/HASL300TOC.htm).

Soils were dried and sieved with a 35-mesh size so that only soil particles less than $500 \mu \mathrm{m}$ in diameter were analyzed. All soil samples were analyzed for gamma-emitting radionuclides. Most surface soil samples ( $0-5 \mathrm{~cm}$ in depth) were also analyzed for $\mathrm{Sr}-90$ and alpha-emitting radionuclides.

Iodine-129 has not been detected in surface outside of the INL Site because it is not routinely analyzed for due to the expense of analysis. According to annual site environmental reports (Hoff et. al., 1991, DOE 1991, Hoff et al., 1992, Hoff et al., 1993, Mitchell 1994, Mitchell et al., 1995, Mitchell et al., 1996) approximately 3.6 Ci of I-129 were released through 1995 from a 69-m stack located at Idaho Nuclear Technology and Engineering Center (INTEC). From 1996 through 1999, the Environmental Science and Research Foundation (ESRF) sampled sagebrush, grass-forb, litter, small mammals, and four soil compartments to estimate the total I-129 inventory on the INL Site (Morris 2000). The results were never published (Morris and Soto, unpublished data) but were shared with the Environmental Surveillance Education and Research (ESER) program (Morris 2003). The ESRF collected three samples each of four soil layers, litter, sage brush, grass, and forbs, and small mammal samples at each of 16 locations on and near the INL Site for I-129 analysis. Although a preliminary study, they estimated that most of the current inventory of I-129 on the INL Site resides southwest of INTEC, highest in litter and lowest in deep soil. They also approximated that around $6 \%$ of the total inventory released to the environment remains at the INL Site. They hypothesized that the rest was transported offsite after release from the stack. The I-129 concentration in forbs and grasses collected just south of the INL boundary and north of the Big Southern Butte, where the highest air concentrations have been modeled to occur offsite (Rood and Sondrup 2014), averaged $1.8 \mathrm{pCi} / \mathrm{kg}$. Iodine-129 concentrations in upper soil at the same location averaged $1.2 \mathrm{pCi} / \mathrm{kg}$.

No particular trend is indicated in the graphs of $\mathrm{Am}-241, \mathrm{Pu}-238$, and $\mathrm{Pu} 239 / 240$ concentrations in surface soil $(0-5 \mathrm{~cm})$ over time (DOE 2014). This is consistent with the long half-lives of these radionuclides and the relative immobility of these radionuclides in undisturbed soils. A study of soil profiles of plutonium and americium in Nevada Test Site soils indicate that $95 \%$ of these radionuclides dispersed as fallout to desert environment had remained in the top $5 \mathrm{~cm}$ of soil (Romney et al., 1987). This is not surprising given the high hydraulic conductivity of these radionuclides and low amount of rainfall in the region. Any downward migration of particles and the contaminants attached to them is fundamentally influenced by gravity, such as when particles fall into open pore spaces (Till et al., 2008), making it less susceptible to resuspension.

Table A-2 presents summary statistics for results of offsite soil samples collected initially by RESL from 1975 through 1998 and subsequently by contractors at primarily the same locations through 2014 (biennially since 1978). The surface data do not suggest any concentration changes in Am-241 and plutonium isotopes over time; therefore, the data do not indicate any accumulation over time from INL Site operations. However, concentrations of Cs-137 and Sr-90 show that these radionuclides have decreased over time in surface soil. For this reason, only the latest results for Cs-137 and Sr-90 are shown in Table 2 as representative of current concentrations. In addition, all radionuclides except $\mathrm{Pu}-238$ were shown through t-tests to have activities that depend on depth (Rood et al., 1995). That is, concentrations decrease with depth. Cesium-137 was analyzed for in surface $(0-5 \mathrm{~cm})$ and deeper soil. 
Table A-2. Summary statistics for RESL and current data for offsite samples collected at depths of 0$5 \mathrm{~cm}$ and $5-10 \mathrm{~cm}(\mathrm{pCi} / \mathrm{g})$.

\begin{tabular}{|c|c|c|c|c|c|c|c|}
\hline Radionuclide & Year & $\begin{array}{l}\text { Sample depth } \\
(\mathrm{cm})\end{array}$ & $\mathbf{N}$ & Minimum & Maximum & Mean & $\begin{array}{c}\text { Upper } 95 \% \\
\text { confidence limit }\end{array}$ \\
\hline \multirow{2}{*}{ Am-241a } & $1975-2014$ & $0-5$ & 257 & $-0.0003 \pm 0.002$ & $0.029 \pm 0.002$ & 0.006 & 0.021 \\
\hline & $1992 b$ & $5-10$ & 18 & $-0.001 \pm 0.002$ & $0.01 \pm 0.004$ & 0.002 & 0.0035 \\
\hline \multirow[t]{2}{*}{ Cs-137c } & 2014 & $0-5$ & 13 & $0.16 \pm 0.001$ & $0.64 \pm 0.04$ & 0.37 & 0.44 \\
\hline & 2014 & $5-10$ & 96 & $-0.01 \pm 0.01$ & $0.88 \pm 0.01$ & 0.16 & 0.015 \\
\hline \multirow[t]{2}{*}{ Pu-238a } & $1975-2014$ & $0-5$ & 276 & $-0.0015 \pm 0.0012$ & $0.012 \pm 0.006$ & 0.003 & 0.014 \\
\hline & $1992 b$ & $5-10$ & 20 & $-0.000035 \pm 0.0004$ & $0.0062 \pm 0.0001$ & 0.0011 & 0.0019 \\
\hline \multirow[t]{2}{*}{ Pu-239/240a } & $1975-2014$ & $0-5$ & 275 & $0.0002 \pm 0.0008$ & $0.27 \pm 0.003$ & 0.018 & 0.057 \\
\hline & $1992 b$ & $5-10$ & 20 & $0.00 \pm 0.0012$ & $0.029 \pm 0.0009$ & 0.0048 & 0.0078 \\
\hline Sr-90c & 2014 & $0-5$ & 13 & $0.0001 \pm 0.03$ & $0.036 \pm 0.05$ & 0.12 & 0.16 \\
\hline \multicolumn{8}{|c|}{$\begin{array}{l}\text { a. Geometric means and upper confidence limits were calculated for } \mathrm{Am}-241, \mathrm{Pu}-238 \text {, and } \mathrm{Pu}-239 / 240 \text { by combining data sets from } 1975 \text { to } \\
\text { 2014. Historic data sets were reported by RESL with geometric means and confidence limits because the results were typically } \\
\text { lognormally distributed. More current data were also evaluated as having lognormal distributions to compare and combine all results. } \\
\text { b. Results shown for Am-241, Pu-238, and Pu-239/240 for the } 5-10 \mathrm{~cm} \text { depth were measured in samples collected during a special study in } \\
\text { 1992. No samples collected at this depth since then have been analyzed for these radionuclides. } \\
\text { c. Arithmetic means and upper confidence limits were calculated for Sr-90 and Cs-137 using } 2014 \text { data. These data were determined to be } \\
\text { normally distributed. }\end{array}$} \\
\hline
\end{tabular}

Most of the activity is in the top 5-cm layer-about $85 \%$ of the Cs-137 and $\mathrm{Pu}-239$, according to studies conducted by RESL (DOE/ID 1976). Contemporary research at the INL Site using in-situ gammaray spectrometry shows that the depth profile of Cs-137 in regional and INL Site soils is exponential, with the majority of activity in the upper 0-5 cm layer (Walker 2000, Giles et al. 2008). Walker (2008) compared in-situ and conventional soil collection techniques and found that an average $90 \%$ of Cs-137 collected at an undisturbed location (Reno Ranch) was in the first 3 centimeters of soil. The majority of Cs-137 in two samples collected on the INL Site was found in the first 5 centimeters of soil. Walker (2008) concluded that soil sampling to a depth of 5 centimeters corresponds to averaging over $90 \%$ of the total Cs-137 inventory in a soil column.

Further analysis of Cs-137 and Sr-90 concentrations in surface soil samples collected from 1975 through 2014 demonstrate the decreasing trends in the radionuclides. Cesium-137 was detected (above 3s) in most surface soil samples and Sr-90 was detected in about half of the samples collected. Statistical comparisons of geometric mean surface concentrations $\left(\mathrm{nCi} / \mathrm{m}^{2}\right)$ of the radionuclides in distant and boundary locations have not indicated any differences due to location. There are large uncertainties associated with the estimated averages due to the highly unreproducible nature of sampling soil, a heterogeneous medium.

A plot of average areal Cs-137 concentrations over time, from 1975 through 2014, indicates an exponential rate of decrease in areal concentration that is indicative of radioactive decay (Figure A-2). The data were plotted as $\mathrm{nCi} / \mathrm{m}^{2}$, as recommended in HASL-300, which states that “...The most useful measures of the concentration of deposited material in soil relate to the amount per unit area. Therefore, sampling is carried out in such a way that the weight of the material collected can be directly related to the area sampled and the depth of the sampling. The analytical results from a weighed aliquot of the soil sample can then be readily related to area concentration." (HASL-300 recommendations on soil sampling may be found at https://www.orau.org/ptp/PTP\%20Library/library/DOE/eml/hasl300/sampling.pdf.) 


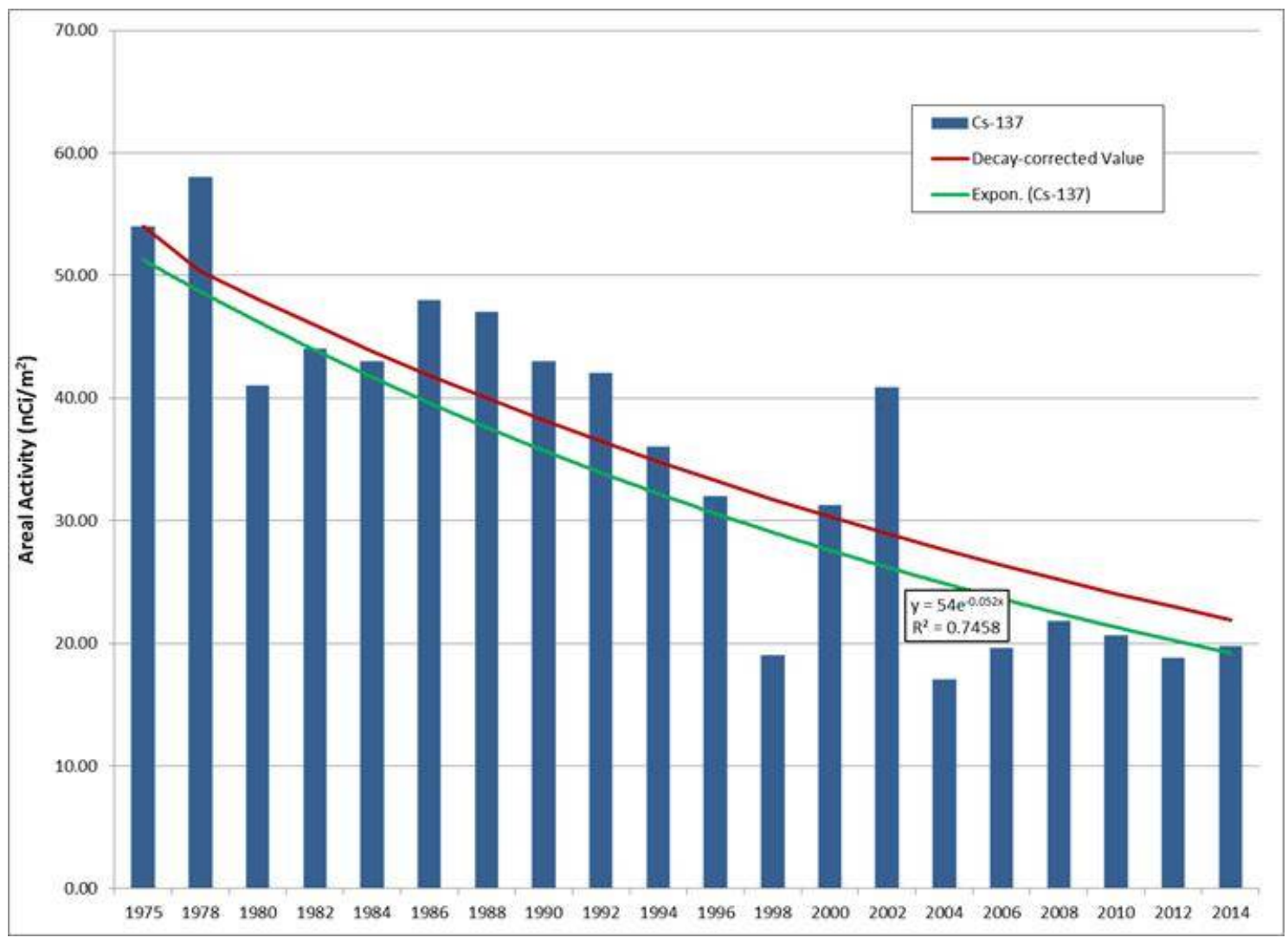

Figure A-2. Areal activity of Cs-137 in offsite surface soil sampled from 1975 through 2014. Each column represents the geometric average of all locations for that year.

Mean annual concentrations of Sr-90 in surface over time appear to decrease at a rate which exceeds that projected for radioactive decay (Figure A-3). Sr-90 is more mobile than Cs-137 in alkaline soils (Schulz 1965) and the accelerated decrease may be due to other processes in the soil, such as movement into other soil compartments or uptake by plants. This is further illustrated by the Cs-137/Sr-90 ratio that increases with time (Figure A-4). These decreasing trends indicate that the source of Cs-137 and Sr-90 is not from INL Site operations and is most likely derived from worldwide fallout activity. It is interesting to note that the trends observed in the Cs-137 and Sr-90 can only be observed over a long period of time (decades) and not over relatively short periods (i.e., years). This is because radionuclide measurements are highly variable because of the inhomogeneous distribution of radioactivity in soil due to variable patterns of deposition, differences in geography, and other environmental factors. The highly variable nature of soil is illustrated in Figures A-5 and A-6, which shows scatterplots of individual results of analyses for of Cs-137 and Sr-90 in offsite soil samples collected from 2000 through 2014. 


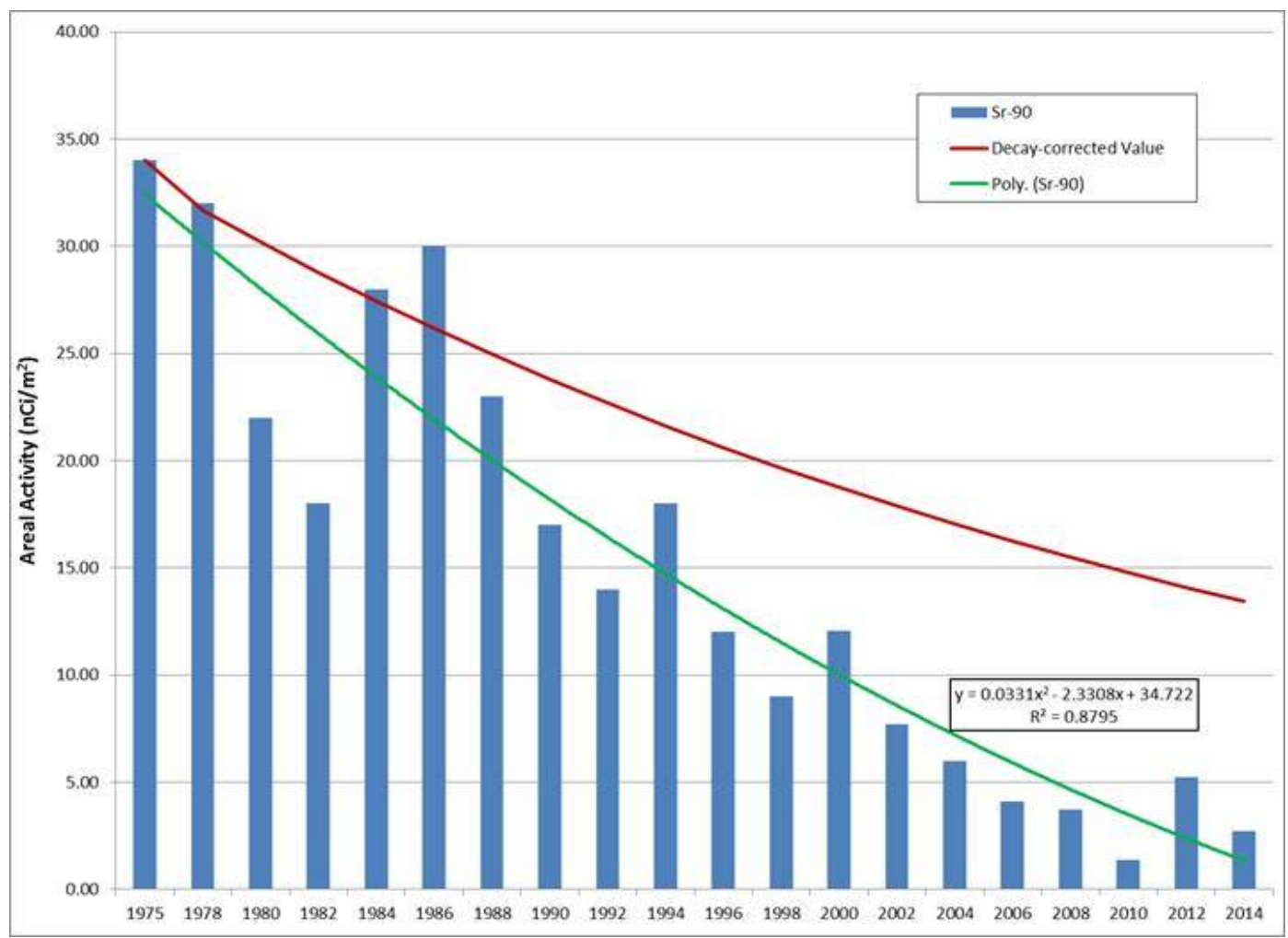

Figure A-3. Areal activity of Sr-90 in offsite surface soil sampled from 1975 through 2014. Each column represents the geometric average of all locations for that year.

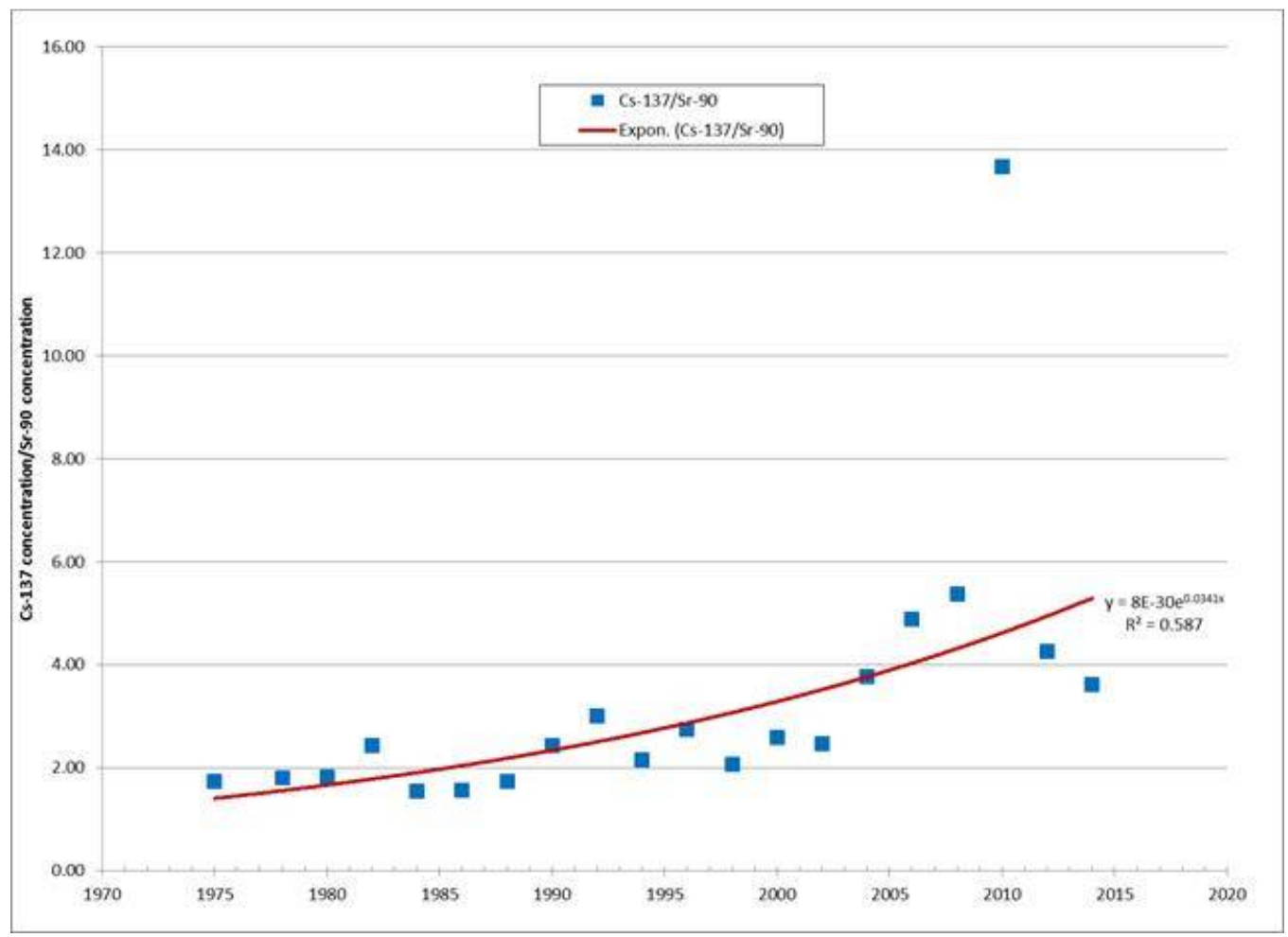

Figure A-4. Cs-137/Sr-90 ratios in offsite surface soil shown as a function of time. 


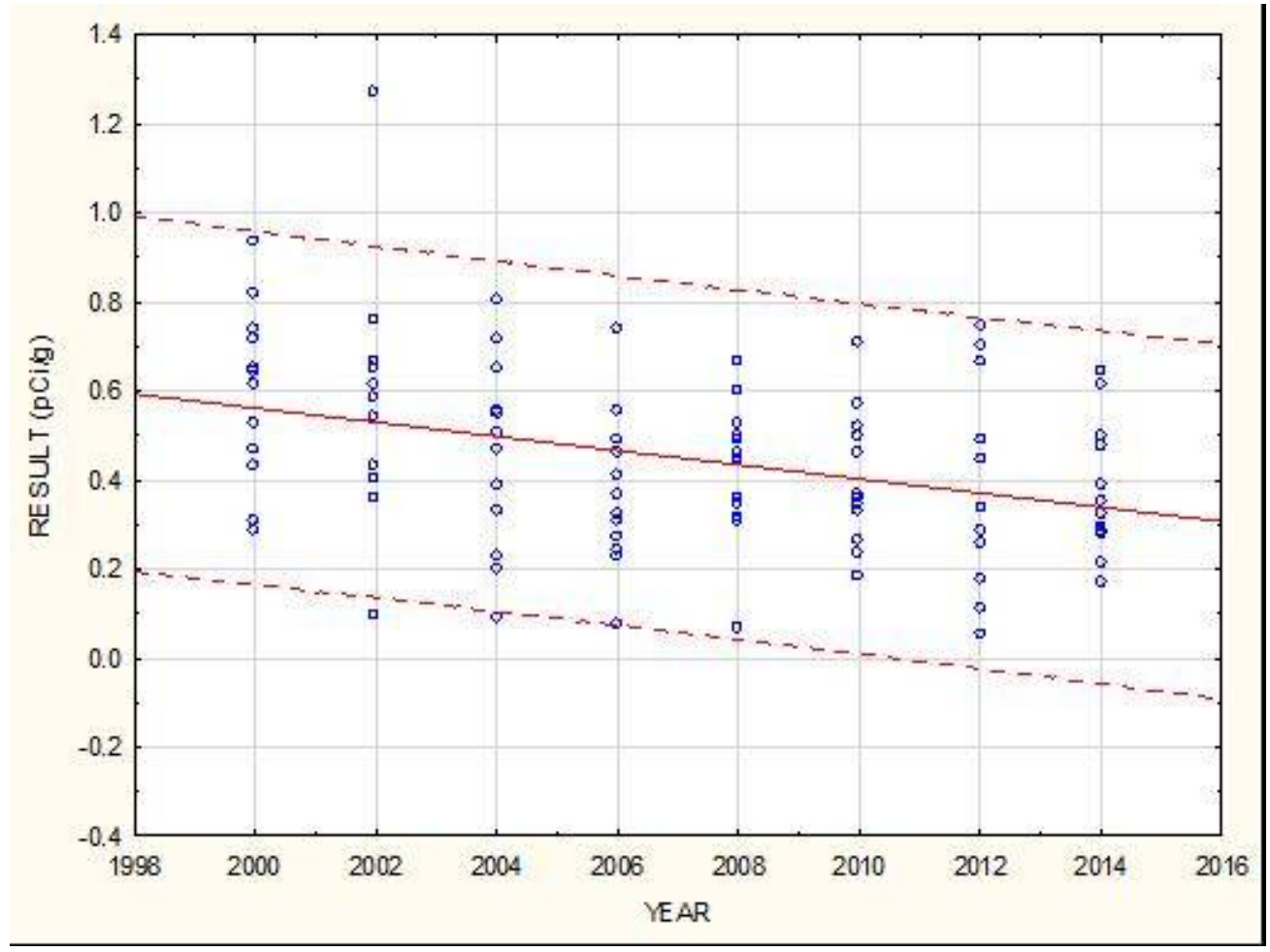

Figure A-5. Scatterplot of individual measurements of Cs-137 in surface soil samples collected at locations off the INL Site from 2000 to 2014. A trend line and 95\% confidence limits are shown.

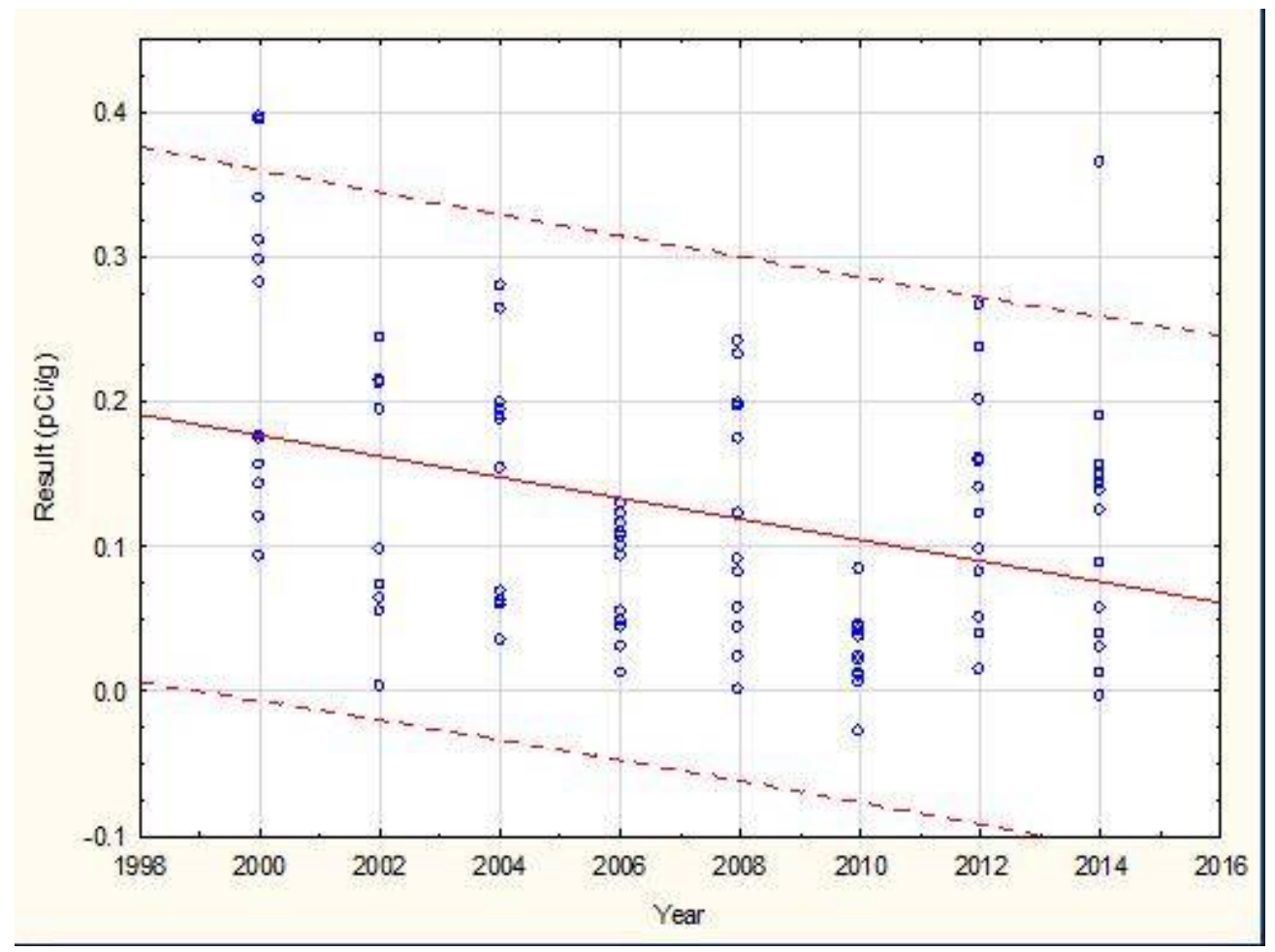

Figure A-6. Scatterplot of individual measurements of Sr-90 in surface soil samples collected at locations off the INL site from 2000 to 2014 . A trend line and 95\% confidence limits are shown. 
Cesium concentrations measured in surface $(0-5 \mathrm{~cm})$ and subsurface $(5-10 \mathrm{~cm})$ soil samples collected from 2000 to 2014 show a distinct difference between these two layers but a similarity in patterns (Figure A-7). The similarity suggests that differences from year to year not only reflect radioactive decay but sample area heterogeneity. In addition, radionuclide movement between soil layers is not currently indicated (i.e., the lower soil layer is not increasing in Cs-137 concentration when the upper layer is decreasing.)

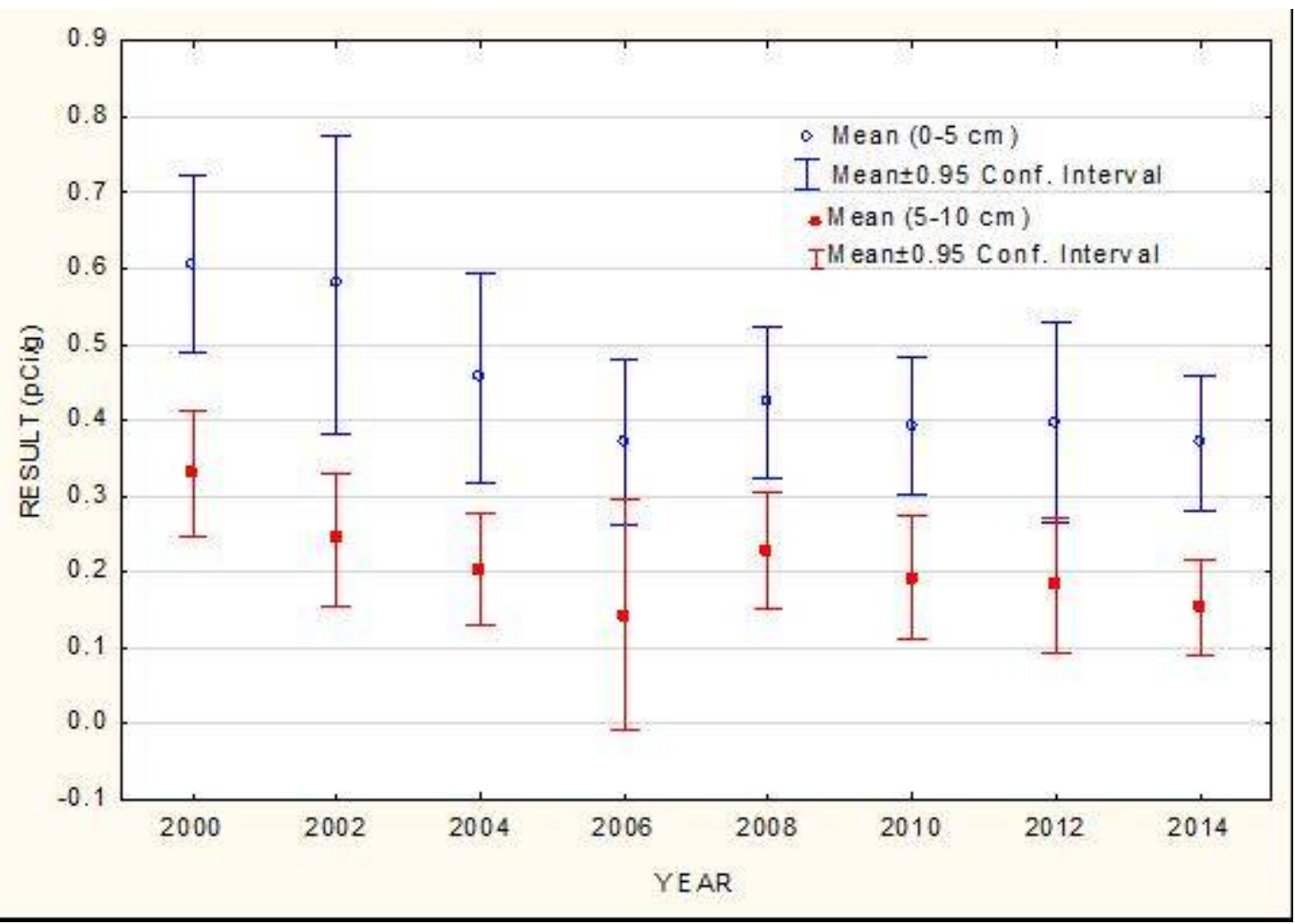

Figure A-7. Mean Cs-137 concentrations in upper and lower soil layers.

In summary, results of four decades of soil monitoring show that background concentrations of transuranics in undisturbed surface soils remain fairly constant over time. Cs-137 appears to be decreasing primarily from radioactive decay. Sr-90 is decreasing at a rate faster than radioactive decay indicating that other processes, such as uptake by plants, are involved. The majority of radionuclides appear to reside in the upper $5 \mathrm{~cm}$ of soil. There is no evidence of radionuclides being present in offsite soils as a result of INL Site releases. Rather it appears that Am-241, Cs-137, Pu-238, Pu-239/240, and Sr-90 were deposited as fallout from historical global nuclear weapons testing.

\section{A-2. MEASUREMENT OF RADIONUCLIDES IN INL SITE SOIL}

- Historical concentrations of radionuclides of interest in site soils:

- Soil sampling

- $\quad$ In situ sampling

- Trends.

- Locations of sites within the INL Site boundaries that have been historically monitored (initially by RESL) for contamination (Radioactive Waste Management Complex [RWMC] soils outside the RWMC fence, INTEC soils, others?)

- I-129 contamination outside of INTEC. 


\section{A-3. RADIONUCLIDE DEPOSITION AND DISPERSION PATTERNS}

Air dispersion modeling, using CALPUFF and INL Site meteorological data measured from 2006 through 2008, was performed to develop data quality objectives for radiological air surveillance for the INL Site using methodology documented in Rood and Sondrup (2014). The same methodology was used to discern deposition patterns. For deposition, it was assumed that the particulates released from the INL Site have a geometric mean (GM) diameter of 1 micron and a geometric standard deviation (GSD) of 1.5 microns. Based on historical NESHAP data, the major sources of Cs-137 and Sr-90 are the Advanced Test Reactor (ATR) Complex and INTEC facilities; the major source of I-129 is INTEC and the Materials and Fuels Complex (MFC); and the major source of Am-241 and isotopes of plutonium is RWMC. Figure A-8 shows the locations of these facilities. The deposition patterns resulting from these sources reflect the southwest/northeast wind patterns typical of the INL Site (e.g., Figures A-9 through A-12). The CALPUFF model also demonstrates that the maximum offsite deposition value is located between the southwest INL Site boundary and the Great Southern Butte for emissions from ATR, INTEC, and RWMC. The Highway26/20 rest stop is also a potential location of interest as the highway traverses the INL Site through higher regions of modeled deposition and is a location accessed by the public. The Mud Lake/Terreton area and Atomic City are an offsite areas primarily impacted by emissions from MFC. Howe is an offsite location of concern for deposition of particulates released from ATR.

Potential deposition of sources at individual air sampling stations was investigated further to determine where and how long it would take for radionuclide deposition to result in measureable concentrations. For this exercise three particle sizes were assumed:

1. $1 \mu \mathrm{m}$ (mitigated/filtered)

2. $10 \mu \mathrm{m}$ (unmitigated/unfiltered)

3. 30- $\mu \mathrm{m}$ (large particles, such as resuspended soil).

Particle sizes are important in terms of deposition rates and health consequences. In general, HEPA-filtered effluent results particles less than $1 \mu \mathrm{m}$, so deposition from filtered effluent is not expected to contribute appreciably to offsite soil contamination. This is significant because particles less than $3.5 \mu \mathrm{m}$ in diameter are respirable and can penetrate the deep respiratory tract (Sehmel 1984). Larger particle releases may result from unplanned releases or releases from fugitive dust sources. In these cases, deposition may be significant and measureable near the source. Particles in excess of $15 \mu \mathrm{m}$ are likely to be filtered out by nasal hair or impact only the nasopharyngeal surface (Sehmel 1984). Although largeparticle releases do not present an inhalation health risk because the particles are not inhalable, they may present a soil contamination issue depending on the magnitude of the release. The Environmental Protection Agency (EPA 2004) assumes for estimation of annual emissions of radionuclides from contaminated soils that erosion is highest for particles in the size range from 15 to $30 \mu \mathrm{m}$ and lower for smaller particle sizes.

The following sources were selected for further evaluation because they contribute at least $1 \%$ to the annual dose estimated for National Emission Standards for Hazardous Air Pollutants (NESHAP) compliance or because, in the case of MFC, they represent a geographical location of key operational activities on the INL Site:

1. ATR Complex
a. TRA-770 Stack
b. ATR Complex ground level release (Test Reactor Area [TRA] warm waste ponds)

2. INTEC ground level release (TMI-2 Independent Spent Storage Installation and INL Comprehensive Environmental Response, Compensation, and Liability Act (CERCLA) Disposal Facility (ICDF) 


\section{3. $\mathrm{MFC}$}

a. MFC-764 Stack

b. MFC ground level release

4. RWMC.

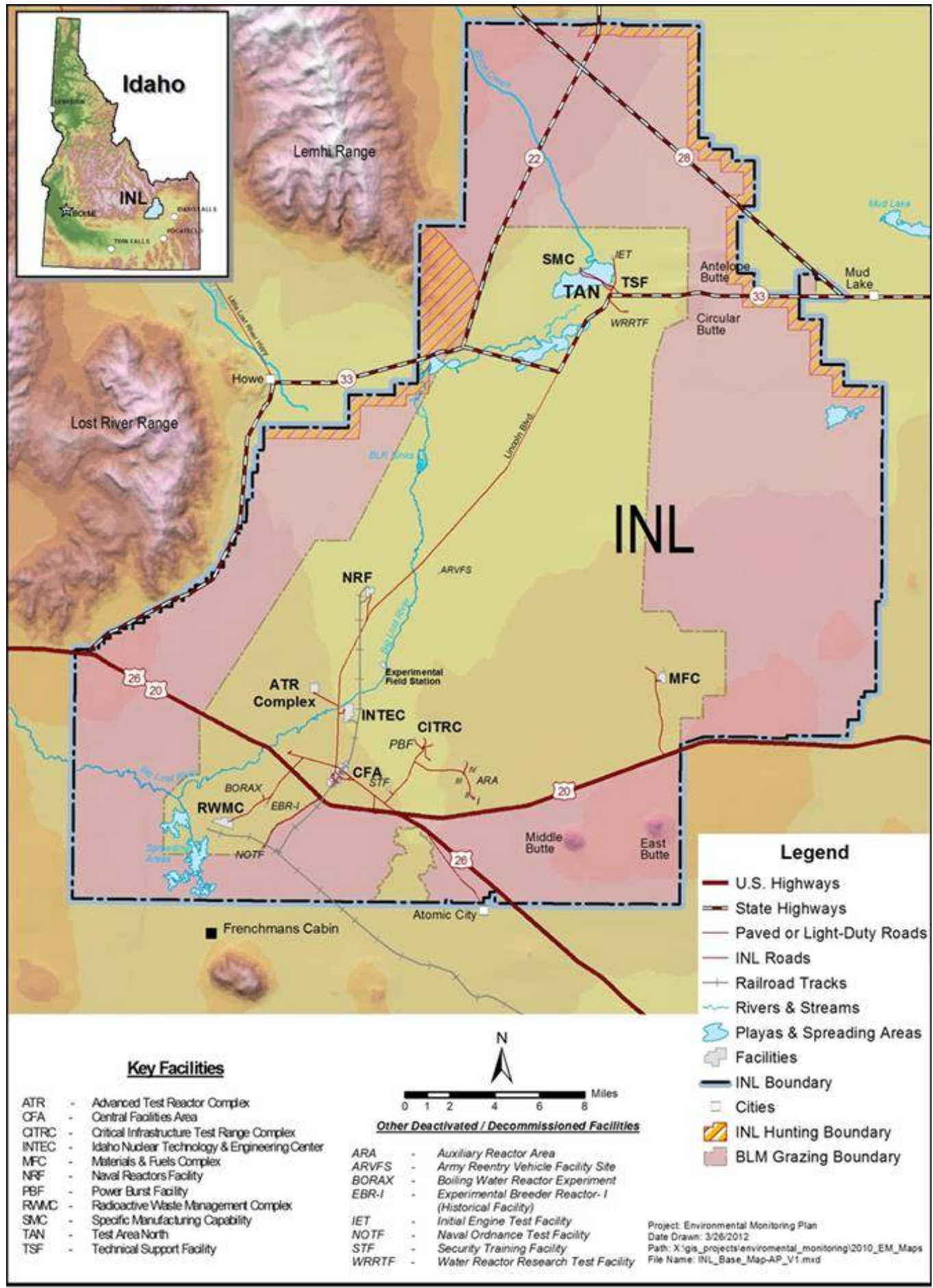

Figure A-8. Major facilities at the INL Site. 


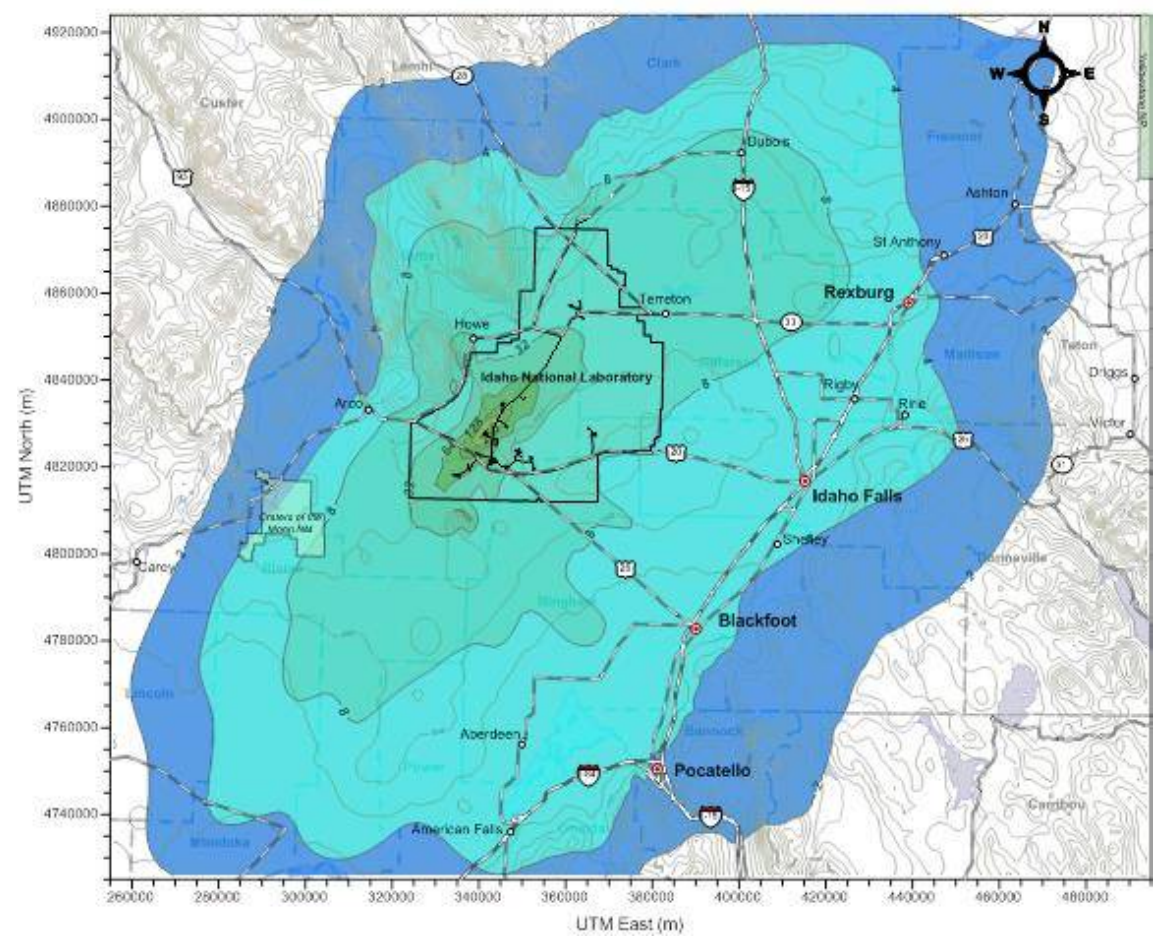

Figure A-9. Unit deposition values $\left(\mathrm{hr} \mathrm{m}^{-2} \times 10^{9}\right)$ for particles 1 micron in diameter released continuously from the ATR Complex TRA-770 stack for 1 year.

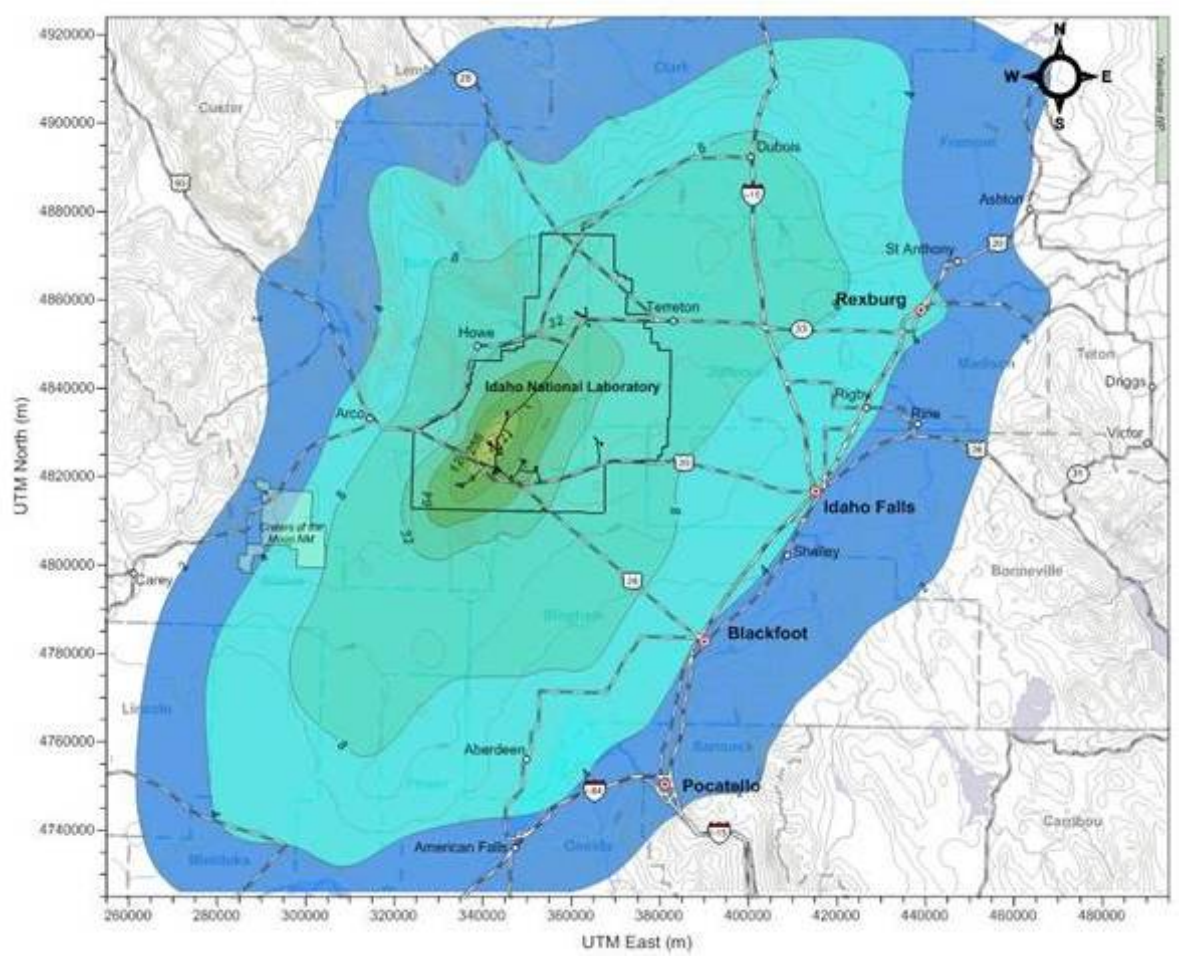

Figure A-10. Unit deposition values $\left(\mathrm{hr} \mathrm{m}^{-2} \times 10^{9}\right)$ for particles 1 micron in diameter released continuously at ground level from the ATR Complex for 1 year. 


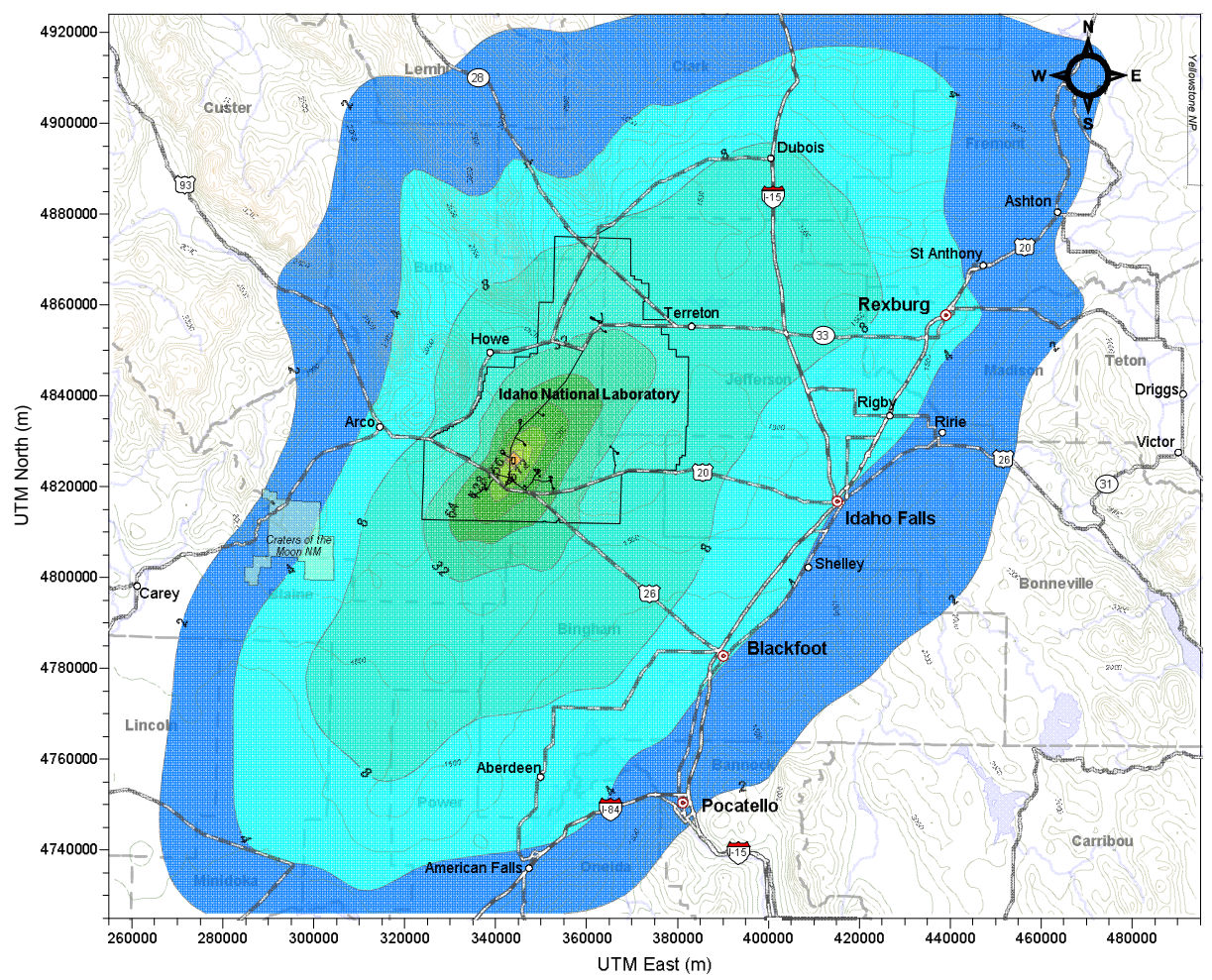

Figure A-11. Unit deposition values $\left(\mathrm{hr} \mathrm{m}^{-2} \times 10^{9}\right)$ for particles 1 micron in diameter released continuously at ground level from INTEC for 1 year.

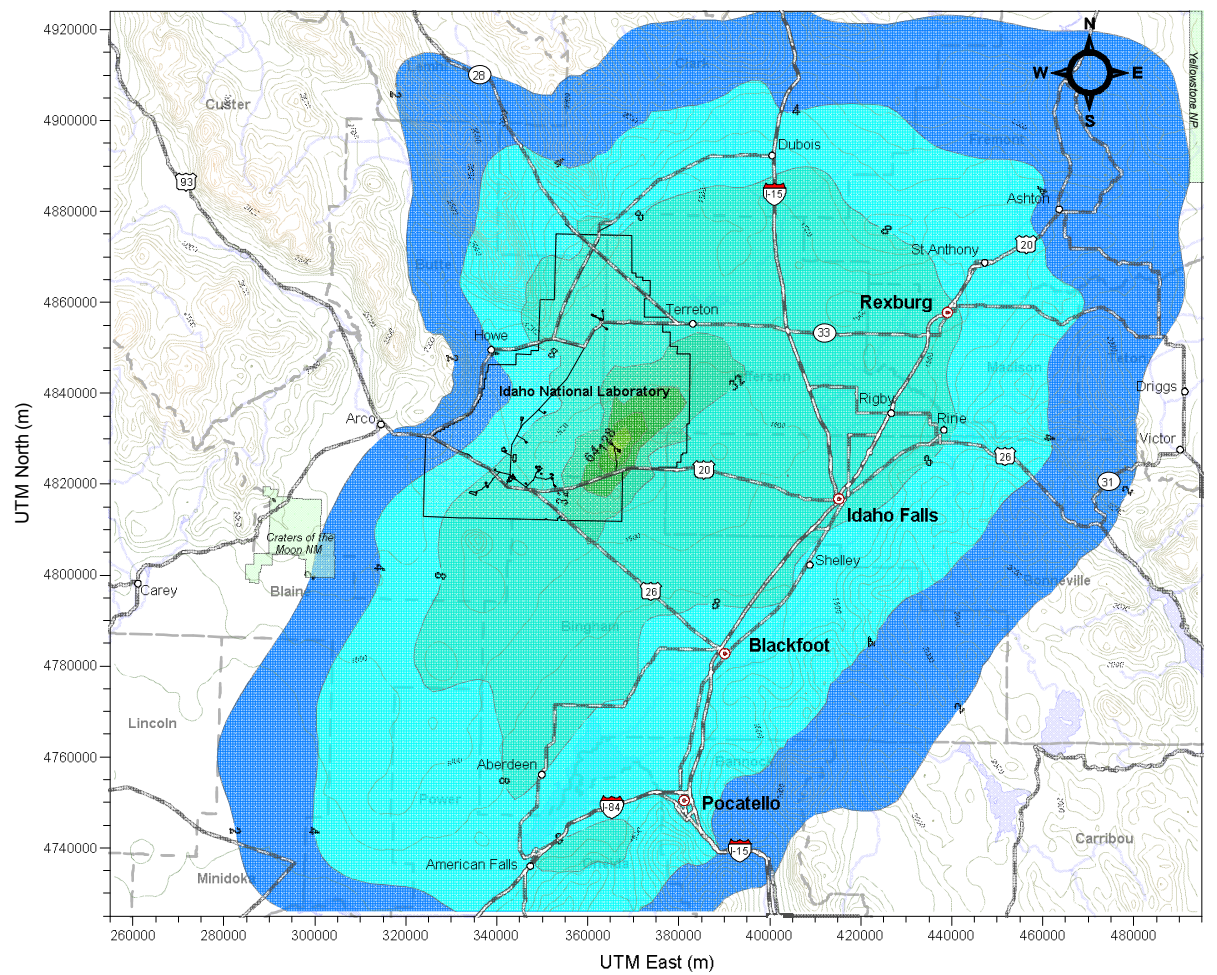

Figure A-12. Unit deposition values $\left(\mathrm{hr} \mathrm{m}^{-2} \times 10^{9}\right)$ for particles 1 micron in diameter released continuously from the MFC-764 stack for 1 year. 


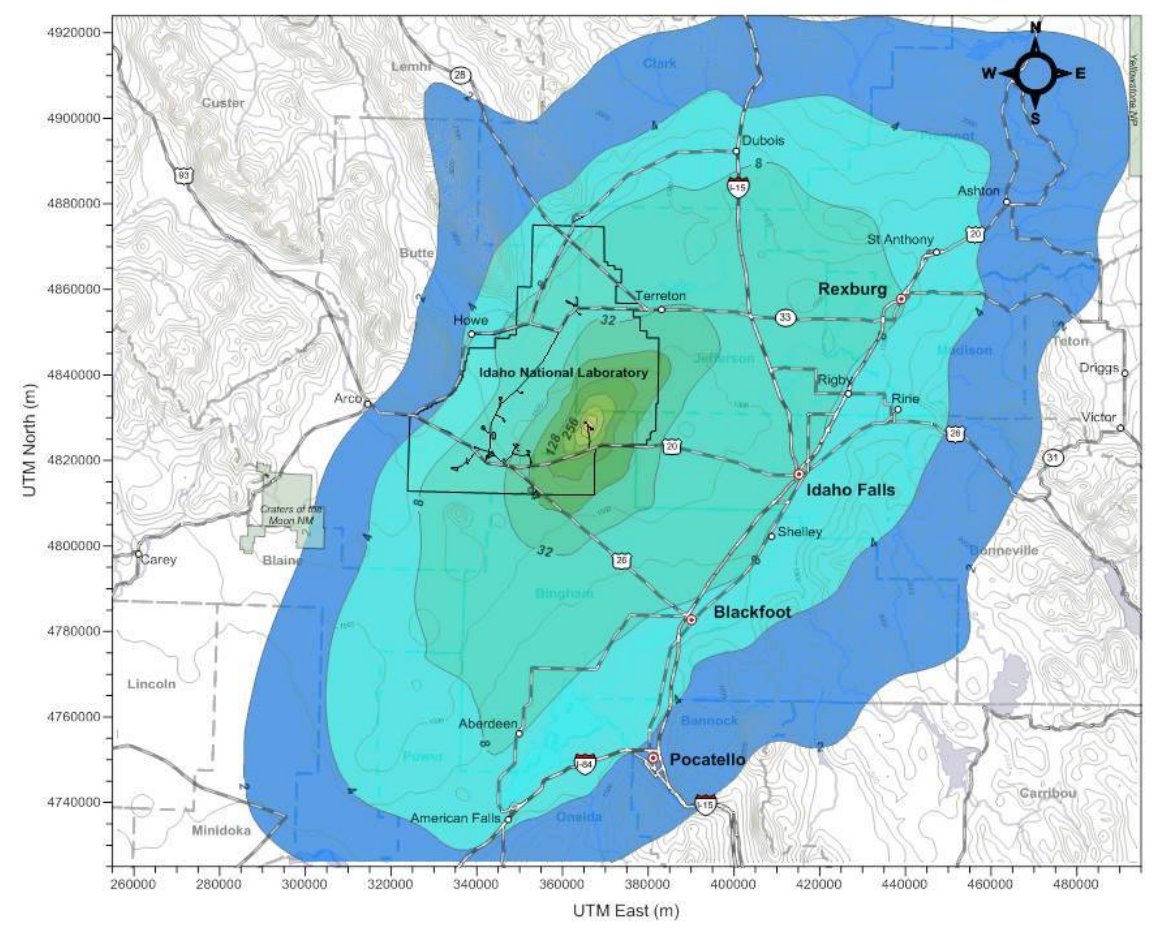

Figure A-13. Unit deposition values $\left(\mathrm{hr} \mathrm{m}^{-2} \times 10^{9}\right)$ for particles 1 micron in diameter released continuously at ground level from MFC for 1 year.

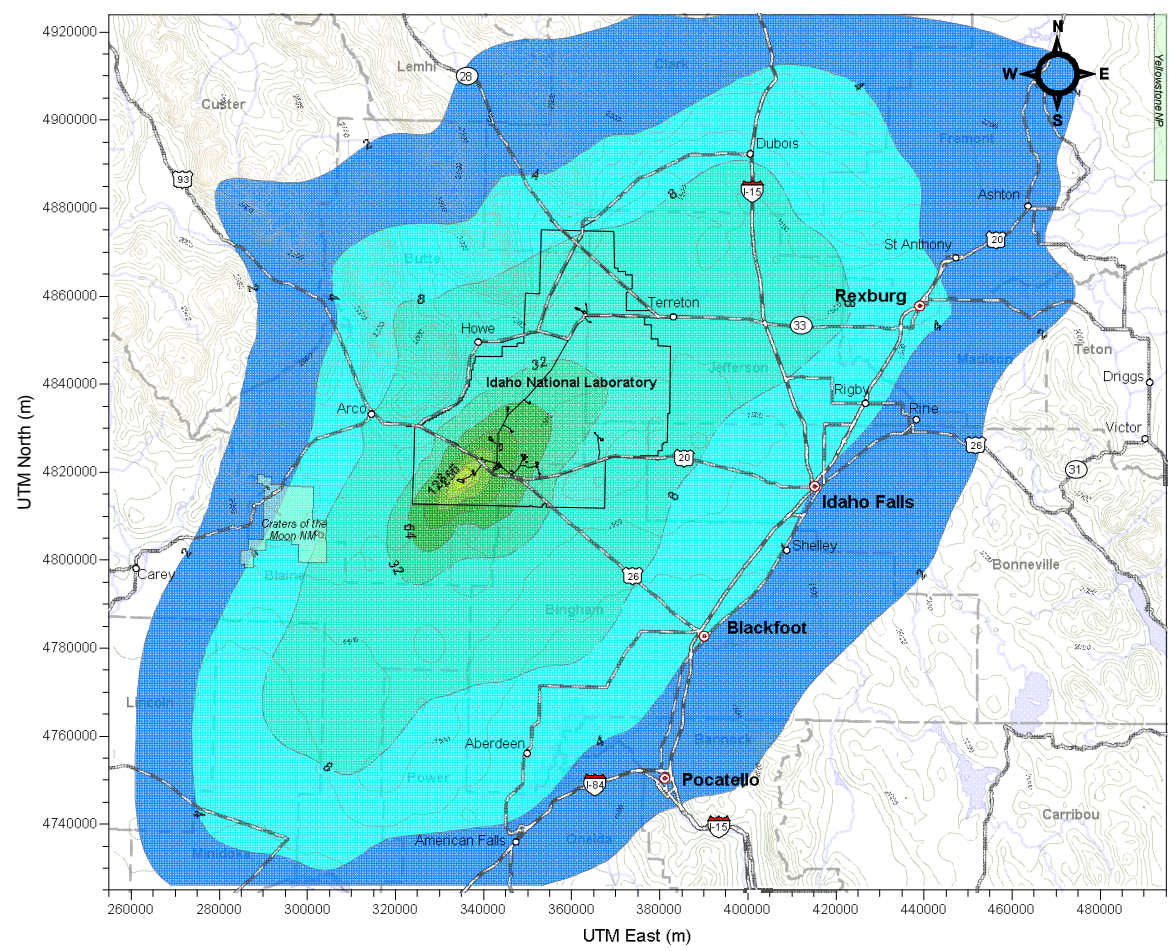

Figure A-14. Unit deposition values $\left(\mathrm{hr} \mathrm{m}^{-2} \times 10^{9}\right)$ for particles 1 micron in diameter released continuously at ground level from the RWMC for 1 year. 
Using the CALPUFF model, unit deposition factors $\left(\mathrm{nCi} / \mathrm{m}^{2}\right.$ per $1 \mathrm{Ci}$ released in air) were calculated for all INL Site air sampler locations and locations near a source were estimated for 1-, 10-, and $30-\mu \mathrm{m}$ particles. Details of the analytical exercise appear in Appendix C.

The annual amount of deposition $(\psi)$ that might occur from an annual release quantity can be estimated by

$\psi=\sum_{i=1}^{n} \frac{\psi_{u, i} Q_{i}}{Q_{u}}$

where $\psi_{u, i}=$ the annual deposition for a $1 \mathrm{Ci} / \mathrm{yr}$ release for particle size $i\left(\mathrm{Ci} / \mathrm{m}^{2}\right), \mathrm{Q}_{i}=$ the annual release for a radionuclide with particle size $i(\mathrm{Ci} / \mathrm{yr})$, and $\mathrm{Q}_{u}$ is the unit release quantity $(1 \mathrm{Ci} / \mathrm{yr})$. For this evaluation, it was simply assumed that each release was of a uniform particle size (i.e., $i=1-\mu \mathrm{m}, 10-\mu \mathrm{m}$, or $30 \mu \mathrm{m}$ ) so that $\psi=\psi_{u, i}$.

Assuming a constant release rate over time, the amount of activity that accumulates over time is the product of $\psi$ and the number of years of accumulation. The time necessary to accumulate enough activity such that it can be measured in surface soil above background is found by

$t=\frac{\Delta C}{\psi}$

where $\Delta \mathrm{C}$ is the amount of activity accumulated in surface soil that could be measured statistically above background. For this exercise, $\Delta \mathrm{C}$ was estimated by subtracting the mean value from the $95 \%$ upper confidence limit (UCL). It is assumed that the radionuclide can be detected if it is measured at that value above the background concentration. It is also assumed that $\Delta \mathrm{C}$ can be detected by the analytical laboratory (i.e., it is greater than the minimum detectable concentration [MDC]).

Means and 95\% UCLs of background soil concentrations for radionuclides of concern are presented in Table A-3, along with MDCs. Iodine-129 is not included in Table 3 and was not assessed in this exercise because it is not actually released to the environment. It is a hypothetical source term provided in the NESHAP report that is estimated as a fraction of TMI-2 dry fuel inventory in storage (Scott Lee, personal communication, April 22, 2015). The statistical descriptions of long-lived radionuclides (Am-241, Pu-238, and Pu-239/240) were based on data collected from 1975 through 2014. The earlier data collected by RESL from 1975 through 1992 and by the Environmental Science and Research Foundation from 1992 through 1996 were summarized in annual reports as geometric (lognormal) means and 95\% uncertainty limits. Results from 1998 through 2014 were analyzed for geometric means and UCLs The summary data were combined statistically to yield the results in Table A-3. In the cases of Sr-90 and Cs-137, the 2014 data were determined to be normally distributed; therefore, the arithmetic means and UCLs were accepted as the current statistics for these radionuclides.

It is important to note that the buildup calculation approach described above is very conservative in nature. That is, it only considers deposition on soil surface and does not include processes that act to deplete radionuclides in soil, such as radioactive decay and resuspension of contaminated particles via wind (see Figure A-11). Thus, the actual times to achieve measureable build-up would be longer. 
Table A-3. Key radionuclide background concentrations in offsite soil as of 2014. The means and upper confidence limits were calculated with all data regardless if they were above the detection level.

\begin{tabular}{|c|c|c|c|c|c|c|}
\hline \multirow[b]{2}{*}{ Radionuclide } & \multicolumn{2}{|c|}{2014} & \multicolumn{2}{|c|}{ 1975-2014 } & \multirow{2}{*}{$\begin{array}{c}\Delta \mathrm{C} \\
(\mathrm{UCL}-\mathrm{Mean}) \\
\left(\mathrm{nCi} / \mathrm{m}^{2}\right)\end{array}$} & \multirow[b]{2}{*}{$\begin{array}{c}\mathrm{MDC} \\
\left(\mathrm{nCi} / \mathrm{m}^{2}\right)\end{array}$} \\
\hline & $\begin{array}{c}\text { Mean } \\
\left(\mathrm{nCi} / \mathrm{m}^{2}\right)\end{array}$ & $\begin{array}{c}\mathrm{UCL} \\
\left(\mathrm{nCi} / \mathrm{m}^{2}\right)\end{array}$ & $\underset{\left(\mathrm{nCi} / \mathrm{m}^{2}\right)}{\text { Mean }}$ & $\begin{array}{c}\mathrm{UCL} \\
\left(\mathrm{nCi} / \mathrm{m}^{2}\right)\end{array}$ & & \\
\hline $\mathrm{Am}-241^{\mathrm{a}}$ & $\mathrm{NU}^{\mathrm{c}}$ & $\mathrm{NU}$ & 0.33 & 0.63 & 0.30 & 0.3 \\
\hline $\mathrm{Cs}-137^{\mathrm{b}}$ & 20.74 & 24.08 & $\mathrm{NU}$ & NU & 3.34 & 0.5 \\
\hline $\mathrm{Pu}-238^{\mathrm{a}}$ & $\mathrm{NU}$ & $\mathrm{NU}$ & 0.17 & 0.36 & 0.19 & 0.2 \\
\hline $\mathrm{Pu}-239^{\mathrm{a}}$ & NU & NU & 0.98 & 1.48 & 0.50 & 0.2 \\
\hline Sr- $90^{\mathrm{b}}$ & 6.36 & 8.80 & $\mathrm{NU}$ & $\mathrm{NU}$ & 2.44 & 2.2 \\
\hline
\end{tabular}

a. Combined data set derived from biennial results reported for offsite soils in annual reports from 1975-2014. All data were assumed to be lognormally distributed. Means are geometric averages.

b. Annual results for offsite soils collected in 2014. The results were determined to be normally distributed and arithmetic means and upper confidence limit.

c. $\mathrm{NU}=$ Not used

d. $\mathrm{MDC}=$ Minimum Detectable Concentration (based on lowest MDC reported by the current analytical laboratory for each radionuclide).

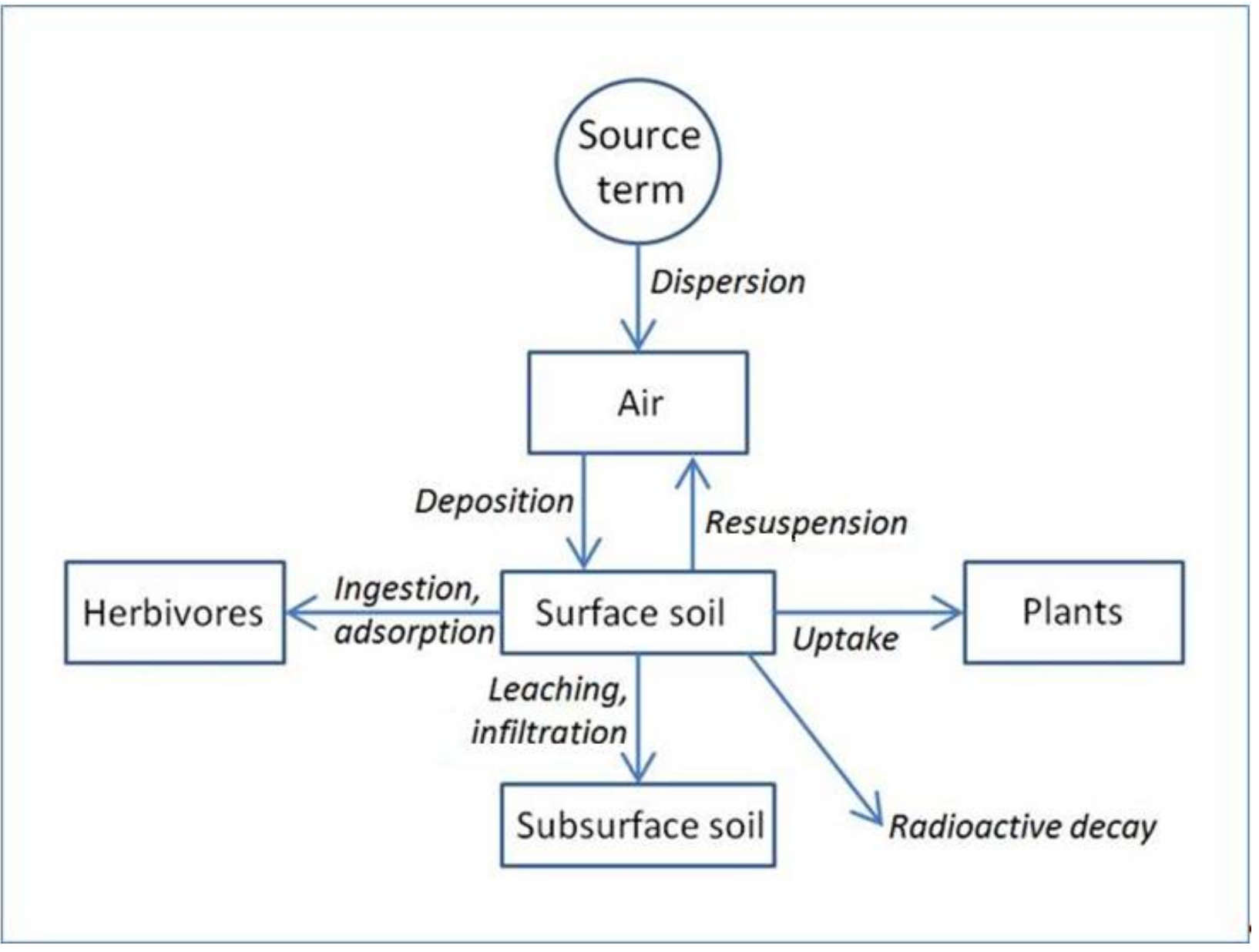

Figure A-15. Major process which affected radionuclide concentrations in surface soil. 


\section{A-3.1 Advanced Test Reactor Test Complex}

The ATR Complex (formerly known as the Reactor Technology Complex [RTC] and TRA) was established in the early 1950s for studying the effects of radiation on materials, fuels, and equipment. The ATR is currently the only operating reactor at the ATR Complex. The ATR tests materials for the nation's next generation of nuclear power plants. ATR is also used to manufacture a significant portion of the nation's medical nuclear isotopes. Radiological air emissions from the ATR Complex are primarily associated with operation of the ATR. These emissions are released through the TRA-770 stack and include noble gases, iodines, and other mixed fission and activation products. Other radiological air emissions are associated with sample analysis, site remediation, research and development activities, and decommissioning and demolition activities.

Table A-4 presents results of the time calculations at each air sampling location for a release from the TRA-770 stack. For this calculation it was assumed that the entire source term reported for the ATR Complex for the 2013 NESHAP report (DOE/ID 2014) is released through the stack. This assumes a filtered particle diameter of $1 \mu \mathrm{m}$. As can be seen in the table, the shortest time period needed to achieve the estimated build-up level is over 650 years for Sr-90 at air monitors located near the facility (Battelle Energy Alliance, LLC [BEA]-RTC and BEA-TRA) and over 1,300 years at the Highway 20/26 rest stop (a point of public access).

Table A-5 presents the results of calculations using a ground level release from the ATR Complex. The source terms used were based on fugitive emissions reported in the 2013 NESHAP report (DOE/ID 2014). Fugitive emissions consist of all air releases not released through a confined air stream and include diffuse sources (EPA 2004). Diffuse sources include soils, surface water/evaporation, buildings, tank venting, equipment venting, and releases from underground testing. The fugitive source terms estimated for Cs-137 and Sr-90 in the NESHAP report were based on a cumulative inventory in the TRA warm waste ponds. The emission rate from the ponds was derived by calculating the pond inventory for each radionuclide, in $\mathrm{Ci}$, by a resuspension rate of $1 \times 10^{-10} \mathrm{~s}^{-1}$ (DeWayne King, personal communication, April 29,2015). This calculation is highly unrealistic as contaminants in water most likely end up submerged in the pond sediments and are not available for resuspension. A more realistic approach assumes that at most $10 \%$ of the sediment along the pond edges is exposed to air, dried, and becomes available, as 30- $\mu \mathrm{m}$ particles, for erosion by wind. A resuspension rate factor of $1 \times 10^{-12} \mathrm{~s}^{-1}$ has been estimated for undisturbed soils at the INL Site using measured airborne particulate mass concentrations and particulate resuspension rate equations (EPA 2003) and was adopted for this exercise. The source terms used for generation of Table A-5 are thus three orders of magnitude smaller than those reported in DOE/ID (2014).

Table A-5 shows that Sr-90 attached to large particles would deposit close to the release site and would be detected at BEA-RTC and BEA-TRA after 160 years. It would take 50,000 years to be measureable above background at EFS and 54,000 years at the Highway 20/26 rest stop. 
Table A-4. Estimated time (years) to achieve build-up concentration in surface soil for $1 \mu \mathrm{m}$ particles released continuously for 1 year from a stack (TRA-770 stack) at the Advanced Test Reactor Complex. ${ }^{\mathrm{a}}$ The top five results are highlighted. Two of the sampler locations, Van Buren and EFS, have replicate samplers (BEA-VAN and ESER-VAN, BEA-EFS, and ESER-EFS).

\begin{tabular}{|c|c|c|c|c|c|c|}
\hline SOURCE & SAMPLER & Am-241 & Cs-137 & Pu-238 & Pu-239 & Sr-90 \\
\hline TRA-770 & BEA-TRA & $5.64 \mathrm{E}+04$ & $2.15 \mathrm{E}+03$ & $6.27 \mathrm{E}+13$ & $2.45 \mathrm{E}+05$ & $7.77 \mathrm{E}+02$ \\
\hline TRA-770 & BEA-СРP & $6.24 \mathrm{E}+05$ & $2.38 \mathrm{E}+04$ & $6.94 \mathrm{E}+14$ & $2.71 \mathrm{E}+06$ & $8.59 \mathrm{E}+03$ \\
\hline TRA-770 & BEA-RWMC & $2.76 \mathrm{E}+05$ & $1.05 \mathrm{E}+04$ & $3.07 \mathrm{E}+14$ & $1.20 \mathrm{E}+06$ & $3.80 \mathrm{E}+03$ \\
\hline TRA-770 & BEA-VAN & $2.48 \mathrm{E}+05$ & $9.43 \mathrm{E}+03$ & $2.75 \mathrm{E}+14$ & $1.08 \mathrm{E}+06$ & $3.41 \mathrm{E}+03$ \\
\hline TRA-770 & BEA-IRC & $6.42 \mathrm{E}+06$ & $2.44 \mathrm{E}+05$ & $7.13 \mathrm{E}+15$ & $2.79 \mathrm{E}+07$ & $8.83 \mathrm{E}+04$ \\
\hline TRA-770 & BEA-BLKFT & $5.74 \mathrm{E}+06$ & $2.18 \mathrm{E}+05$ & $6.37 \mathrm{E}+15$ & $2.49 \mathrm{E}+07$ & $7.90 \mathrm{E}+04$ \\
\hline TRA-770 & BEA-SMC & $1.37 \mathrm{E}+06$ & $5.21 \mathrm{E}+04$ & $1.52 \mathrm{E}+15$ & $5.94 \mathrm{E}+06$ & $1.88 \mathrm{E}+04$ \\
\hline TRA-770 & BEA-GATE & $9.15 \mathrm{E}+05$ & $3.48 \mathrm{E}+04$ & $1.02 \mathrm{E}+15$ & $3.97 \mathrm{E}+06$ & $1.26 \mathrm{E}+04$ \\
\hline TRA-770 & BEA-ARA & $1.94 \mathrm{E}+06$ & $7.38 \mathrm{E}+04$ & $2.15 \mathrm{E}+15$ & $8.42 \mathrm{E}+06$ & $2.67 \mathrm{E}+04$ \\
\hline TRA-770 & BEA-REST & $9.72 \mathrm{E}+04$ & $3.70 \mathrm{E}+03$ & $1.08 \mathrm{E}+14$ & $4.22 \mathrm{E}+05$ & $1.34 \mathrm{E}+03$ \\
\hline TRA-770 & BEA-IF & $6.49 \mathrm{E}+06$ & $2.47 \mathrm{E}+05$ & $7.21 \mathrm{E}+15$ & $2.82 \mathrm{E}+07$ & $8.94 \mathrm{E}+04$ \\
\hline TRA-770 & BEA-NRF & $3.33 \mathrm{E}+05$ & $1.27 \mathrm{E}+04$ & $3.70 \mathrm{E}+14$ & $1.45 \mathrm{E}+06$ & $4.58 \mathrm{E}+03$ \\
\hline TRA-770 & BEA-RTC & $4.76 \mathrm{E}+04$ & $1.81 \mathrm{E}+03$ & $5.29 \mathrm{E}+13$ & $2.07 \mathrm{E}+05$ & $6.55 \mathrm{E}+02$ \\
\hline TRA-770 & BEA-EBR & $2.93 \mathrm{E}+05$ & $1.12 \mathrm{E}+04$ & $3.25 \mathrm{E}+14$ & $1.27 \mathrm{E}+06$ & $4.03 \mathrm{E}+03$ \\
\hline TRA-770 & BEA-MFC & $2.54 \mathrm{E}+06$ & $9.68 \mathrm{E}+04$ & $2.83 \mathrm{E}+15$ & $1.11 \mathrm{E}+07$ & $3.50 \mathrm{E}+04$ \\
\hline TRA-770 & BEA-SUGAR & $8.75 \mathrm{E}+06$ & $3.33 \mathrm{E}+05$ & $9.72 \mathrm{E}+15$ & $3.80 \mathrm{E}+07$ & $1.20 \mathrm{E}+05$ \\
\hline TRA-770 & BEA-PBF & $1.31 \mathrm{E}+06$ & $4.99 \mathrm{E}+04$ & $1.46 \mathrm{E}+15$ & $5.70 \mathrm{E}+06$ & $1.81 \mathrm{E}+04$ \\
\hline TRA-770 & BEA-INTEC & $5.68 \mathrm{E}+05$ & $2.16 \mathrm{E}+04$ & $6.32 \mathrm{E}+14$ & $2.47 \mathrm{E}+06$ & $7.83 \mathrm{E}+03$ \\
\hline TRA-770 & BEA-CFA & $4.93 \mathrm{E}+05$ & $1.88 \mathrm{E}+04$ & $5.48 \mathrm{E}+14$ & $2.14 \mathrm{E}+06$ & $6.79 \mathrm{E}+03$ \\
\hline TRA-770 & BEA-EFS & $2.30 \mathrm{E}+05$ & $8.75 \mathrm{E}+03$ & $2.56 \mathrm{E}+14$ & $9.99 \mathrm{E}+05$ & $3.17 \mathrm{E}+03$ \\
\hline TRA-770 & BEA-CRATE & $5.44 \mathrm{E}+06$ & $2.07 \mathrm{E}+05$ & $6.05 \mathrm{E}+15$ & $2.37 \mathrm{E}+07$ & $7.49 \mathrm{E}+04$ \\
\hline TRA-770 & ESER-VAN & $2.48 \mathrm{E}+05$ & $9.44 \mathrm{E}+03$ & $2.75 \mathrm{E}+14$ & $1.08 \mathrm{E}+06$ & $3.41 \mathrm{E}+03$ \\
\hline TRA-770 & ESER-CRA & $5.44 \mathrm{E}+06$ & $2.07 \mathrm{E}+05$ & $6.05 \mathrm{E}+15$ & $2.37 \mathrm{E}+07$ & $7.49 \mathrm{E}+04$ \\
\hline TRA-770 & ESER-ARC & $4.52 \mathrm{E}+06$ & $1.72 \mathrm{E}+05$ & $5.02 \mathrm{E}+15$ & $1.97 \mathrm{E}+07$ & $6.22 \mathrm{E}+04$ \\
\hline TRA-770 & ESER-SUG & $8.75 \mathrm{E}+06$ & $3.33 \mathrm{E}+05$ & $9.72 \mathrm{E}+15$ & $3.80 \mathrm{E}+07$ & $1.20 \mathrm{E}+05$ \\
\hline TRA-770 & ESER-IDA & $6.49 \mathrm{E}+06$ & $2.47 \mathrm{E}+05$ & $7.21 \mathrm{E}+15$ & $2.82 \mathrm{E}+07$ & $8.94 \mathrm{E}+04$ \\
\hline TRA-770 & ESER-DUB & $3.61 \mathrm{E}+06$ & $1.37 \mathrm{E}+05$ & $4.01 \mathrm{E}+15$ & $1.57 \mathrm{E}+07$ & $4.97 \mathrm{E}+04$ \\
\hline
\end{tabular}


Table A-4. (continued).

\begin{tabular}{|c|c|c|c|c|c|c|}
\hline SOURCE & SAMPLER & Am-241 & Cs-137 & Pu-238 & Pu-239 & Sr-90 \\
\hline TRA-770 & ESER-MON & $2.92 \mathrm{E}+06$ & $1.11 \mathrm{E}+05$ & $3.25 \mathrm{E}+15$ & $1.27 \mathrm{E}+07$ & $4.02 \mathrm{E}+04$ \\
\hline TRA-770 & ESER-FAA & $6.21 \mathrm{E}+06$ & $2.36 \mathrm{E}+05$ & $6.90 \mathrm{E}+15$ & $2.70 \mathrm{E}+07$ & $8.55 \mathrm{E}+04$ \\
\hline TRA-770 & ESER-MAI & $1.28 \mathrm{E}+06$ & $4.87 \mathrm{E}+04$ & $1.42 \mathrm{E}+15$ & $5.56 \mathrm{E}+06$ & $1.76 \mathrm{E}+04$ \\
\hline TRA-770 & ESER-EFS & $2.30 \mathrm{E}+05$ & $8.75 \mathrm{E}+03$ & $2.55 \mathrm{E}+14$ & $9.99 \mathrm{E}+05$ & $3.16 \mathrm{E}+03$ \\
\hline TRA-770 & ESER-ATO & $2.41 \mathrm{E}+06$ & $9.19 \mathrm{E}+04$ & $2.68 \mathrm{E}+15$ & $1.05 \mathrm{E}+07$ & $3.32 \mathrm{E}+04$ \\
\hline TRA-770 & ESER-MOU & $5.74 \mathrm{E}+06$ & $2.18 \mathrm{E}+05$ & $6.37 \mathrm{E}+15$ & $2.49 \mathrm{E}+07$ & $7.90 \mathrm{E}+04$ \\
\hline TRA-770 & ESER-BLU & $5.25 \mathrm{E}+06$ & $2.00 \mathrm{E}+05$ & $5.83 \mathrm{E}+15$ & $2.28 \mathrm{E}+07$ & $7.22 \mathrm{E}+04$ \\
\hline TRA-770 & ESER-TER & $2.51 \mathrm{E}+06$ & $9.57 \mathrm{E}+04$ & $2.79 \mathrm{E}+15$ & $1.09 \mathrm{E}+07$ & $3.46 \mathrm{E}+04$ \\
\hline TRA-770 & ESER-HOW & $1.28 \mathrm{E}+06$ & $4.86 \mathrm{E}+04$ & $1.42 \mathrm{E}+15$ & $5.55 \mathrm{E}+06$ & $1.76 \mathrm{E}+04$ \\
\hline
\end{tabular}

Table A-5. Estimated time (years) to achieve build-up concentration in surface soil for $30 \mu \mathrm{m}$ particles released continuously for 1 year at ground level from the Advanced Test Reactor Complex. ${ }^{a}$ The shortest five time periods are highlighted in yellow. Two of the sampler locations, Van Buren and EFS, have replicate samplers (BEA-VAN and ESER-VAN, BEA-EFS, and ESER-EFS).

\begin{tabular}{|c|c|c|c|c|c|c|}
\hline SOURCE & SAMPLER & Am-241 & Cs-137 & $\mathrm{Pu}-238^{\mathrm{b}}$ & Pu-239 & Sr-90 \\
\hline ATR-GL & BEA-TRA & $1.21 \mathrm{E}+04$ & $4.59 \mathrm{E}+02$ & $1.34 \mathrm{E}+13$ & $5.24 \mathrm{E}+04$ & $1.66 \mathrm{E}+02$ \\
\hline ATR-GL & BEA-CPP & $4.31 \mathrm{E}+06$ & $1.64 \mathrm{E}+05$ & $4.79 \mathrm{E}+15$ & $1.87 \mathrm{E}+07$ & $5.93 \mathrm{E}+04$ \\
\hline ATR-GL & BEA-RWMC & $1.73 \mathrm{E}+07$ & $6.57 \mathrm{E}+05$ & $1.92 \mathrm{E}+16$ & $7.50 \mathrm{E}+07$ & $2.37 \mathrm{E}+05$ \\
\hline ATR-GL & BEA-VAN & $5.05 \mathrm{E}+06$ & $1.92 \mathrm{E}+05$ & $5.61 \mathrm{E}+15$ & $2.19 \mathrm{E}+07$ & $6.95 \mathrm{E}+04$ \\
\hline ATR-GL & BEA-IRC & $8.55 \mathrm{E}+09$ & $3.26 \mathrm{E}+08$ & $9.50 \mathrm{E}+18$ & $3.72 \mathrm{E}+10$ & $1.18 \mathrm{E}+08$ \\
\hline ATR-GL & BEA-BLKFT & $1.74 \mathrm{E}+10$ & $6.61 \mathrm{E}+08$ & $1.93 \mathrm{E}+19$ & $7.54 \mathrm{E}+10$ & $2.39 \mathrm{E}+08$ \\
\hline ATR-GL & BEA-SMC & $1.47 \mathrm{E}+08$ & $5.61 \mathrm{E}+06$ & $1.64 \mathrm{E}+17$ & $6.41 \mathrm{E}+08$ & $2.03 \mathrm{E}+06$ \\
\hline ATR-GL & BEA-GATE & $5.96 \mathrm{E}+07$ & $2.27 \mathrm{E}+06$ & $6.63 \mathrm{E}+16$ & $2.59 \mathrm{E}+08$ & $8.21 \mathrm{E}+05$ \\
\hline ATR-GL & BEA-ARA & $4.78 \mathrm{E}+08$ & $1.82 \mathrm{E}+07$ & $5.32 \mathrm{E}+17$ & $2.08 \mathrm{E}+09$ & $6.58 \mathrm{E}+06$ \\
\hline ATR-GL & BEA-REST & $3.90 \mathrm{E}+06$ & $1.49 \mathrm{E}+05$ & $4.33 \mathrm{E}+15$ & $1.70 \mathrm{E}+07$ & $5.37 \mathrm{E}+04$ \\
\hline ATR-GL & BEA-IF & $8.17 \mathrm{E}+09$ & $3.11 \mathrm{E}+08$ & $9.08 \mathrm{E}+18$ & $3.55 \mathrm{E}+10$ & $1.12 \mathrm{E}+08$ \\
\hline ATR-GL & BEA-NRF & $4.80 \mathrm{E}+06$ & $1.83 \mathrm{E}+05$ & $5.34 \mathrm{E}+15$ & $2.09 \mathrm{E}+07$ & $6.61 \mathrm{E}+04$ \\
\hline ATR-GL & BEA-RTC & $1.46 \mathrm{E}+04$ & $5.56 \mathrm{E}+02$ & $1.62 \mathrm{E}+13$ & $6.35 \mathrm{E}+04$ & $2.01 \mathrm{E}+02$ \\
\hline ATR-GL & BEA-EBR & $1.32 \mathrm{E}+07$ & $5.01 \mathrm{E}+05$ & $1.46 \mathrm{E}+16$ & $5.72 \mathrm{E}+07$ & $1.81 \mathrm{E}+05$ \\
\hline
\end{tabular}


Table A-5. (continued).

\begin{tabular}{|c|c|c|c|c|c|c|}
\hline SOURCE & SAMPLER & Am-241 & Cs-137 & $\mathrm{Pu}-238^{\mathrm{b}}$ & Pu-239 & Sr-90 \\
\hline ATR-GL & BEA-MFC & $6.19 \mathrm{E}+08$ & $2.36 \mathrm{E}+07$ & $6.87 \mathrm{E}+17$ & $2.69 \mathrm{E}+09$ & $8.52 \mathrm{E}+06$ \\
\hline ATR-GL & BEA-SUGAR & $4.84 \mathrm{E}+09$ & $1.84 \mathrm{E}+08$ & $5.38 \mathrm{E}+18$ & $2.10 \mathrm{E}+10$ & $6.67 \mathrm{E}+07$ \\
\hline ATR-GL & BEA-PBF & $1.55 \mathrm{E}+08$ & $5.90 \mathrm{E}+06$ & $1.72 \mathrm{E}+17$ & $6.73 \mathrm{E}+08$ & $2.13 E+06$ \\
\hline ATR-GL & BEA-INTEC & $4.32 \mathrm{E}+06$ & $1.65 \mathrm{E}+05$ & $4.80 \mathrm{E}+15$ & $1.88 \mathrm{E}+07$ & $5.95 \mathrm{E}+04$ \\
\hline ATR-GL & BEA-CFA & $1.92 \mathrm{E}+07$ & $7.32 \mathrm{E}+05$ & $2.14 \mathrm{E}+16$ & $8.36 \mathrm{E}+07$ & $2.65 \mathrm{E}+05$ \\
\hline ATR-GL & BEA-EFS & $3.63 \mathrm{E}+06$ & $1.38 \mathrm{E}+05$ & $4.04 \mathrm{E}+15$ & $1.58 \mathrm{E}+07$ & $5.00 \mathrm{E}+04$ \\
\hline ATR-GL & BEA-CRATE & $4.08 \mathrm{E}+09$ & $1.55 \mathrm{E}+08$ & $4.54 \mathrm{E}+18$ & $1.78 \mathrm{E}+10$ & $5.62 \mathrm{E}+07$ \\
\hline ATR-GL & ESER-VAN & $5.05 \mathrm{E}+06$ & $1.92 \mathrm{E}+05$ & $5.62 \mathrm{E}+15$ & $2.20 \mathrm{E}+07$ & $6.96 \mathrm{E}+04$ \\
\hline ATR-GL & ESER-CRA & $4.08 \mathrm{E}+09$ & $1.55 \mathrm{E}+08$ & $4.54 \mathrm{E}+18$ & $1.78 \mathrm{E}+10$ & $5.62 \mathrm{E}+07$ \\
\hline ATR-GL & ESER-ARC & $1.85 \mathrm{E}+09$ & $7.03 \mathrm{E}+07$ & $2.05 \mathrm{E}+18$ & $8.02 \mathrm{E}+09$ & $2.54 \mathrm{E}+07$ \\
\hline ATR-GL & ESER-SUG & $4.84 \mathrm{E}+09$ & $1.84 \mathrm{E}+08$ & $5.38 \mathrm{E}+18$ & $2.10 \mathrm{E}+10$ & $6.67 \mathrm{E}+07$ \\
\hline ATR-GL & ESER-IDA & $8.17 \mathrm{E}+09$ & $3.11 \mathrm{E}+08$ & $9.08 \mathrm{E}+18$ & $3.55 \mathrm{E}+10$ & $1.12 \mathrm{E}+08$ \\
\hline ATR-GL & ESER-DUB & $1.18 \mathrm{E}+09$ & $4.49 \mathrm{E}+07$ & $1.31 \mathrm{E}+18$ & $5.12 \mathrm{E}+09$ & $1.62 \mathrm{E}+07$ \\
\hline ATR-GL & ESER-MON & $3.41 \mathrm{E}+08$ & $1.30 \mathrm{E}+07$ & $3.79 \mathrm{E}+17$ & $1.48 \mathrm{E}+09$ & $4.69 \mathrm{E}+06$ \\
\hline ATR-GL & ESER-FAA & $1.67 \mathrm{E}+09$ & $6.34 \mathrm{E}+07$ & $1.85 \mathrm{E}+18$ & $7.24 \mathrm{E}+09$ & $2.29 \mathrm{E}+07$ \\
\hline ATR-GL & ESER-MAI & $2.10 \mathrm{E}+08$ & $8.00 \mathrm{E}+06$ & $2.34 \mathrm{E}+17$ & $9.13 \mathrm{E}+08$ & $2.89 \mathrm{E}+06$ \\
\hline ATR-GL & ESER-EFS & $3.65 \mathrm{E}+06$ & $1.39 \mathrm{E}+05$ & $4.06 \mathrm{E}+15$ & $1.59 \mathrm{E}+07$ & $5.03 \mathrm{E}+04$ \\
\hline ATR-GL & ESER-ATO & $1.39 \mathrm{E}+09$ & $5.29 \mathrm{E}+07$ & $1.54 \mathrm{E}+18$ & $6.04 \mathrm{E}+09$ & $1.91 \mathrm{E}+07$ \\
\hline ATR-GL & ESER-MOU & $1.74 \mathrm{E}+10$ & $6.61 \mathrm{E}+08$ & $1.93 \mathrm{E}+19$ & $7.55 \mathrm{E}+10$ & $2.39 \mathrm{E}+08$ \\
\hline ATR-GL & ESER-BLU & $1.69 \mathrm{E}+09$ & $6.42 \mathrm{E}+07$ & $1.88 \mathrm{E}+18$ & $7.33 \mathrm{E}+09$ & $2.32 \mathrm{E}+07$ \\
\hline ATR-GL & ESER-TER & $2.25 \mathrm{E}+08$ & $8.57 \mathrm{E}+06$ & $2.50 \mathrm{E}+17$ & $9.78 \mathrm{E}+08$ & $3.10 \mathrm{E}+06$ \\
\hline ATR-GL & ESER-HOW & $2.19 \mathrm{E}+08$ & $8.35 \mathrm{E}+06$ & $2.44 \mathrm{E}+17$ & $9.53 \mathrm{E}+08$ & $3.02 \mathrm{E}+06$ \\
\hline
\end{tabular}




\section{A-3.2 Idaho Nuclear Technology and Engineering Center}

Idaho Nuclear Technology and Engineering Center (INTEC) was established in the 1950s to recover usable uranium from spent nuclear fuel generated in government reactors and to store spent nuclear fuel. Radiological air emissions from INTEC sources are primarily associated with liquid-waste operations, including effluents from the Tank Farm Facility, Process Equipment Waste Evaporator, and Liquid Effluent Treatment and Disposal, which are exhausted through the main stack. These radioactive emissions include particulates and gaseous radionuclides. Additional radioactive emissions are associated with decommissioning and decontamination activities, wet-to-dry spent nuclear fuel transfers, environmental remediation, remote-handled transuranic waste management, radiological and hazardouswaste storage facilities, and contaminated-equipment maintenance.

In 2013, air doses from INTEC activities were estimated primarily from fugitive emissions from CPP-1774 (TMI-2 Independent Spent Storage Installation) and from ICDF. Most of the INTEC doses are due to hypothetical releases from the TMI-2 Independent Spent Storage Installation. Given the fact that the fuel is stored in casks inside of a vault, the calculated emissions are unrealistic. The ICDF pond and landfill could potentially release radionuclides through resuspension. However, the estimated release rates are orders of magnitude less than that calculated for the TMI-2 Independent Spent Storage Installation.

For this exercise, it was conservatively assumed that $10 \%$ of the total INTEC source term (not including stack releases) was actually available for release to air as unfiltered $10-\mu \mathrm{m}$ particles. The results are shown in Table A-6. According to the modeled results, it would take over 160 years for Cs-137 to be detected in soil at the nearest air samplers (BEA-CPP and BEA-INTEC) and over 1,000 years at EFS. 
Table A-6. Estimated time (years) to achieve build-up concentration in surface soil for 10- $\mu$ m particles released continuously for 1 year at ground level from Idaho Nuclear Technology and Engineering Complex. ${ }^{\mathrm{a}}$ The top five results are highlighted in yellow. Two of the sampler locations,

Van Buren and EFS, have replicate samplers (BEA-VAN and ESER-VAN, BEA-EFS, and ESER-EFS).

\begin{tabular}{|c|c|c|c|c|c|c|}
\hline SOURCE & SAMPLER & Am-241 & Cs-137 & Pu-238b & Pu-239 & Sr-90 \\
\hline INTEC-GL & BEA-TRA & $1.45 \mathrm{E}+08$ & $5.36 \mathrm{E}+03$ & $8.98 \mathrm{E}+04$ & $5.24 \mathrm{E}+04$ & $7.18 \mathrm{E}+03$ \\
\hline INTEC-GL & BEA-CPP & $4.04 \mathrm{E}+06$ & $1.49 \mathrm{E}+02$ & $2.50 \mathrm{E}+03$ & $1.46 \mathrm{E}+03$ & $2.00 \mathrm{E}+02$ \\
\hline INTEC-GL & BEA-RWMC & $2.81 \mathrm{E}+08$ & $1.04 \mathrm{E}+04$ & $1.74 \mathrm{E}+05$ & $1.01 \mathrm{E}+05$ & $1.39 \mathrm{E}+04$ \\
\hline INTEC-GL & BEA-VAN & $6.79 \mathrm{E}+07$ & $2.51 \mathrm{E}+03$ & $4.20 \mathrm{E}+04$ & $2.45 \mathrm{E}+04$ & $3.36 \mathrm{E}+03$ \\
\hline INTEC-GL & BEA-IRC & $2.12 \mathrm{E}+10$ & $7.81 \mathrm{E}+05$ & $1.31 \mathrm{E}+07$ & $7.64 \mathrm{E}+06$ & $1.05 \mathrm{E}+06$ \\
\hline INTEC-GL & BEA-BLKFT & $3.47 \mathrm{E}+10$ & $1.28 \mathrm{E}+06$ & $2.15 \mathrm{E}+07$ & $1.25 \mathrm{E}+07$ & $1.72 \mathrm{E}+06$ \\
\hline INTEC-GL & BEA-SMC & $1.80 \mathrm{E}+09$ & $6.64 \mathrm{E}+04$ & $1.11 \mathrm{E}+06$ & $6.50 \mathrm{E}+05$ & $8.90 \mathrm{E}+04$ \\
\hline INTEC-GL & BEA-GATE & $7.00 \mathrm{E}+08$ & $2.58 \mathrm{E}+04$ & $4.33 \mathrm{E}+05$ & $2.53 \mathrm{E}+05$ & $3.46 \mathrm{E}+04$ \\
\hline INTEC-GL & BEA-ARA & $6.27 \mathrm{E}+08$ & $2.31 \mathrm{E}+04$ & $3.88 \mathrm{E}+05$ & $2.26 \mathrm{E}+05$ & $3.10 \mathrm{E}+04$ \\
\hline INTEC-GL & BEA-REST & $2.72 \mathrm{E}+08$ & $1.00 \mathrm{E}+04$ & $1.68 \mathrm{E}+05$ & $9.81 \mathrm{E}+04$ & $1.34 \mathrm{E}+04$ \\
\hline INTEC-GL & BEA-IF & $2.03 \mathrm{E}+10$ & $7.50 \mathrm{E}+05$ & $1.26 \mathrm{E}+07$ & $7.33 \mathrm{E}+06$ & $1.00 \mathrm{E}+06$ \\
\hline INTEC-GL & BEA-NRF & $1.86 \mathrm{E}+08$ & $6.85 \mathrm{E}+03$ & $1.15 \mathrm{E}+05$ & $6.70 \mathrm{E}+04$ & $9.18 \mathrm{E}+03$ \\
\hline INTEC-GL & BEA-RTC & $1.16 \mathrm{E}+08$ & $4.29 \mathrm{E}+03$ & $7.18 \mathrm{E}+04$ & $4.19 \mathrm{E}+04$ & $5.74 \mathrm{E}+03$ \\
\hline INTEC-GL & BEA-EBR & $1.23 \mathrm{E}+08$ & $4.54 \mathrm{E}+03$ & $7.60 \mathrm{E}+04$ & $4.44 \mathrm{E}+04$ & $6.08 \mathrm{E}+03$ \\
\hline INTEC-GL & BEA-MFC & $2.33 \mathrm{E}+09$ & $8.58 \mathrm{E}+04$ & $1.44 \mathrm{E}+06$ & $8.39 \mathrm{E}+05$ & $1.15 \mathrm{E}+05$ \\
\hline INTEC-GL & BEA-SUGAR & $2.27 \mathrm{E}+10$ & $8.39 \mathrm{E}+05$ & $1.41 \mathrm{E}+07$ & $8.21 \mathrm{E}+06$ & $1.12 \mathrm{E}+06$ \\
\hline INTEC-GL & BEA-PBF & $1.66 \mathrm{E}+08$ & $6.12 \mathrm{E}+03$ & $1.03 \mathrm{E}+05$ & $5.99 \mathrm{E}+04$ & $8.20 \mathrm{E}+03$ \\
\hline INTEC-GL & BEA-INTEC & $3.82 \mathrm{E}+06$ & $1.41 \mathrm{E}+02$ & $2.37 \mathrm{E}+03$ & $1.38 \mathrm{E}+03$ & $1.89 \mathrm{E}+02$ \\
\hline INTEC-GL & BEA-CFA & $2.26 \mathrm{E}+07$ & $8.33 \mathrm{E}+02$ & $1.40 \mathrm{E}+04$ & $8.15 \mathrm{E}+03$ & $1.12 \mathrm{E}+03$ \\
\hline INTEC-GL & BEA-EFS & $2.96 \mathrm{E}+07$ & $1.09 \mathrm{E}+03$ & $1.83 \mathrm{E}+04$ & $1.07 \mathrm{E}+04$ & $1.47 \mathrm{E}+03$ \\
\hline INTEC-GL & BEA-CRATE & $3.40 \mathrm{E}+10$ & $1.26 \mathrm{E}+06$ & $2.10 \mathrm{E}+07$ & $1.23 \mathrm{E}+07$ & $1.68 \mathrm{E}+06$ \\
\hline INTEC-GL & ESER-VAN & $6.78 \mathrm{E}+07$ & $2.50 \mathrm{E}+03$ & $4.20 \mathrm{E}+04$ & $2.45 \mathrm{E}+04$ & $3.35 \mathrm{E}+03$ \\
\hline INTEC-GL & ESER-CRA & $3.40 \mathrm{E}+10$ & $1.26 \mathrm{E}+06$ & $2.10 \mathrm{E}+07$ & $1.23 \mathrm{E}+07$ & $1.68 \mathrm{E}+06$ \\
\hline INTEC-GL & ESER-ARC & $1.65 \mathrm{E}+10$ & $6.11 \mathrm{E}+05$ & $1.02 \mathrm{E}+07$ & $5.97 \mathrm{E}+06$ & $8.18 \mathrm{E}+05$ \\
\hline INTEC-GL & ESER-SUG & $2.27 \mathrm{E}+10$ & $8.39 \mathrm{E}+05$ & $1.41 \mathrm{E}+07$ & $8.21 \mathrm{E}+06$ & $1.12 \mathrm{E}+06$ \\
\hline INTEC-GL & ESER-IDA & $2.03 \mathrm{E}+10$ & $7.50 \mathrm{E}+05$ & $1.26 \mathrm{E}+07$ & $7.33 \mathrm{E}+06$ & $1.00 \mathrm{E}+06$ \\
\hline INTEC-GL & ESER-DUB & $8.91 E+09$ & $3.29 \mathrm{E}+05$ & $5.51 \mathrm{E}+06$ & $3.22 \mathrm{E}+06$ & $4.40 \mathrm{E}+05$ \\
\hline
\end{tabular}


Table A-6. (continued).

\begin{tabular}{|c|c|c|c|c|c|c|}
\hline SOURCE & SAMPLER & Am-241 & Cs-137 & Pu-238b & Pu-239 & Sr-90 \\
\hline INTEC-GL & ESER-MON & $2.21 \mathrm{E}+09$ & $8.15 \mathrm{E}+04$ & $1.36 \mathrm{E}+06$ & $7.97 \mathrm{E}+05$ & $1.09 \mathrm{E}+05$ \\
\hline INTEC-GL & ESER-FAA & $5.96 \mathrm{E}+09$ & $2.20 \mathrm{E}+05$ & $3.69 \mathrm{E}+06$ & $2.15 \mathrm{E}+06$ & $2.95 \mathrm{E}+05$ \\
\hline INTEC-GL & ESER-MAI & $1.83 \mathrm{E}+08$ & $6.76 \mathrm{E}+03$ & $1.13 \mathrm{E}+05$ & $6.61 \mathrm{E}+04$ & $9.05 \mathrm{E}+03$ \\
\hline INTEC-GL & ESER-EFS & $2.96 \mathrm{E}+07$ & $1.09 \mathrm{E}+03$ & $1.83 \mathrm{E}+04$ & $1.07 \mathrm{E}+04$ & $1.46 \mathrm{E}+03$ \\
\hline INTEC-GL & ESER-ATO & $1.38 \mathrm{E}+09$ & $5.08 \mathrm{E}+04$ & $8.51 \mathrm{E}+05$ & $4.97 \mathrm{E}+05$ & $6.80 \mathrm{E}+04$ \\
\hline INTEC-GL & ESER-MOU & $3.47 \mathrm{E}+10$ & $1.28 \mathrm{E}+06$ & $2.15 \mathrm{E}+07$ & $1.25 \mathrm{E}+07$ & $1.72 \mathrm{E}+06$ \\
\hline INTEC-GL & ESER-BLU & $1.13 \mathrm{E}+10$ & $4.18 \mathrm{E}+05$ & $7.01 \mathrm{E}+06$ & $4.09 \mathrm{E}+06$ & $5.60 \mathrm{E}+05$ \\
\hline INTEC-GL & ESER-TER & $1.55 \mathrm{E}+09$ & $5.72 \mathrm{E}+04$ & $9.59 \mathrm{E}+05$ & $5.60 \mathrm{E}+05$ & $7.66 \mathrm{E}+04$ \\
\hline INTEC-GL & ESER-HOW & $2.06 \mathrm{E}+09$ & $7.59 \mathrm{E}+04$ & $1.27 \mathrm{E}+06$ & $7.42 \mathrm{E}+05$ & $1.02 \mathrm{E}+05$ \\
\hline
\end{tabular}




\section{A-3.3 Materials and Fuels Complex}

Materials and Fuels Complex (MFC), originally called Argonne National Laboratory-West, was established in the 1950s to research and develop nuclear reactors and fuel. Four reactors have been constructed at MFC: Transient Reactor Test Facility, Experimental Breeder Reactor II (EBR-II), Zero Power Physics Reactor and Neutron Radiography Reactor (NRAD). Only one of these reactors, NRAD, is currently operating. Today, the MFC is the prime testing center in the U.S. for demonstration and proofof-concept of nuclear energy technologies. Research and development activities at MFC are focused on areas of national concern, including energy, nuclear safety, spent nuclear fuel treatment, nuclear material disposal, nonproliferation, decommissioning and decontamination technologies, projects to support space exploration, and homeland security.

Radiological air emissions are primarily associated with spent-fuel treatment at the Fuel Conditioning Facility (FCF) and waste characterization at the Hot Fuel Examination Facility (HFEF). These facilities are equipped with continuous emission monitoring (CEM) systems. On a monthly basis, the effluent streams from FCF, HFEF, and other non-CEM radiological facilities are sampled and analyzed for particulate radionuclides. Gaseous and particulate radionuclides may also be released from other MFC facilities during laboratory research activities, sample analysis, waste handling and storage, and maintenance operations. Both measured and estimated emissions from MFC sources are consolidated for NESHAPS reporting on an annual basis.

In 2013, none of the facilities at MFC contributed at least $1 \%$ of the total estimated dose to the MEI. However, given the isolated location of the MFC and the potential for new projects at that site, deposition calculations were made to demonstrate potential buildup patterns in surface soils surrounding the facility.

Tables A-7 and A-8 present results of build-up calculations made with source terms used for the 2013 NESHAPS report (DOE/ID 2014). In addition to the air sampling locations listed in previous tables, two new potential locations were added: the MFC parking lot and the MFC guard shack. These are the only locations downwind of the MFC that have access to power. Results show that, given the current source term, it would take millions of years for radionuclides to be detected in surface soils at current and proposed air sampling locations. 
Table A-7. Estimated time (years) to achieve build-up concentration in surface soil for $1 \mu \mathrm{m}$ particles released continuously for 1 year from a stack (MFC-764 stack) at the Materials and Fuel Complex. The top five results are highlighted. Two of the sampler locations, MFC Parking Lots and MFC Guard Shack, are proposed.

\begin{tabular}{|c|c|c|c|c|c|c|}
\hline SOURCE & SAMPLER & Am-241 & Cs-137 & Pu-238 & Pu-239 & Sr-90 \\
\hline MFC-764 & BEA-TRA & NA & NA & NA & $4.32 \mathrm{E}+10$ & $2.00 \mathrm{E}+10$ \\
\hline MFC-764 & BEA-CPP & NA & NA & NA & $3.14 \mathrm{E}+10$ & $1.46 \mathrm{E}+10$ \\
\hline MFC-764 & BEA-RWMC & NA & NA & NA & $3.73 \mathrm{E}+10$ & $1.73 \mathrm{E}+10$ \\
\hline MFC-764 & BEA-VAN & NA & NA & NA & $3.53 \mathrm{E}+10$ & $1.64 \mathrm{E}+10$ \\
\hline MFC-764 & BEA-IRC & NA & NA & NA & $6.15 \mathrm{E}+10$ & $2.86 \mathrm{E}+10$ \\
\hline MFC-764 & BEA-BLKFT & NA & NA & NA & $5.94 \mathrm{E}+10$ & $2.76 \mathrm{E}+10$ \\
\hline MFC-764 & BEA-SMC & NA & NA & NA & $3.52 \mathrm{E}+10$ & $1.63 \mathrm{E}+10$ \\
\hline MFC-764 & BEA-GATE & NA & NA & NA & $3.64 \mathrm{E}+10$ & $1.69 \mathrm{E}+10$ \\
\hline MFC-764 & BEA-ARA & NA & NA & NA & $1.52 \mathrm{E}+10$ & $7.05 \mathrm{E}+09$ \\
\hline MFC-764 & BEA-REST & NA & NA & NA & $4.41 \mathrm{E}+10$ & $2.05 \mathrm{E}+10$ \\
\hline MFC-764 & BEA-IF & NA & NA & NA & $6.08 \mathrm{E}+10$ & $2.82 \mathrm{E}+10$ \\
\hline MFC-764 & BEA-NRF & NA & NA & NA & $4.47 \mathrm{E}+10$ & $2.07 \mathrm{E}+10$ \\
\hline MFC-764 & BEA-RTC & NA & NA & NA & $4.36 \mathrm{E}+10$ & $2.02 \mathrm{E}+10$ \\
\hline MFC-764 & BEA-EBR & NA & NA & NA & $3.57 \mathrm{E}+10$ & $1.66 \mathrm{E}+10$ \\
\hline MFC-764 & BEA-MFC & NA & NA & NA & $3.78 \mathrm{E}+08$ & $1.76 \mathrm{E}+08$ \\
\hline MFC-764 & BEA-SUGAR & NA & NA & NA & $6.87 \mathrm{E}+10$ & $3.19 \mathrm{E}+10$ \\
\hline MFC-764 & BEA-PBF & NA & NA & NA & $1.97 \mathrm{E}+10$ & $9.14 \mathrm{E}+09$ \\
\hline MFC-764 & BEA-INTEC & NA & NA & NA & $3.11 \mathrm{E}+10$ & $1.45 \mathrm{E}+10$ \\
\hline MFC-764 & BEA-CFA & NA & NA & NA & $2.93 \mathrm{E}+10$ & $1.36 \mathrm{E}+10$ \\
\hline MFC-764 & BEA-EFS & NA & NA & NA & $3.08 \mathrm{E}+10$ & $1.43 \mathrm{E}+10$ \\
\hline MFC-764 & BEA-CRATE & NA & NA & NA & $2.16 \mathrm{E}+11$ & $1.00 \mathrm{E}+11$ \\
\hline MFC-764 & ESER-VAN & NA & NA & NA & $3.53 \mathrm{E}+10$ & $1.64 \mathrm{E}+10$ \\
\hline MFC-764 & ESER-CRA & NA & NA & NA & $2.16 \mathrm{E}+11$ & $1.00 \mathrm{E}+11$ \\
\hline MFC-764 & ESER-ARC & NA & NA & NA & $1.68 \mathrm{E}+11$ & $7.79 \mathrm{E}+10$ \\
\hline MFC-764 & ESER-SUG & NA & NA & NA & $6.87 \mathrm{E}+10$ & $3.19 \mathrm{E}+10$ \\
\hline MFC-764 & ESER-IDA & NA & NA & NA & $6.08 \mathrm{E}+10$ & $2.82 \mathrm{E}+10$ \\
\hline MFC-764 & ESER-DUB & NA & NA & NA & $5.58 \mathrm{E}+10$ & $2.59 \mathrm{E}+10$ \\
\hline
\end{tabular}


Table A-7. (continued).

\begin{tabular}{|c|c|c|c|c|c|c|}
\hline SOURCE & SAMPLER & Am-241 & Cs-137 & $\mathrm{Pu}-238$ & Pu-239 & Sr-90 \\
\hline MFC-764 & ESER-MON & NA & NA & NA & $3.21 \mathrm{E}+10$ & $1.49 \mathrm{E}+10$ \\
\hline MFC-764 & ESER-FAA & NA & NA & NA & $1.57 \mathrm{E}+10$ & $7.27 \mathrm{E}+09$ \\
\hline MFC-764 & ESER-MAI & NA & NA & NA & $2.42 \mathrm{E}+10$ & $1.12 \mathrm{E}+10$ \\
\hline MFC-764 & ESER-EFS & NA & NA & NA & $3.08 \mathrm{E}+10$ & $1.43 \mathrm{E}+10$ \\
\hline MFC-764 & ESER-ATO & NA & NA & NA & $1.17 \mathrm{E}+10$ & $5.43 \mathrm{E}+09$ \\
\hline MFC-764 & ESER-MOU & NA & NA & NA & $5.94 \mathrm{E}+10$ & $2.76 \mathrm{E}+10$ \\
\hline MFC-764 & ESER-BLU & NA & NA & NA & $6.21 \mathrm{E}+10$ & $2.89 \mathrm{E}+10$ \\
\hline MFC-764 & ESER-TER & NA & NA & $\mathrm{NA}$ & $1.59 \mathrm{E}+10$ & $7.37 \mathrm{E}+09$ \\
\hline MFC-764 & ESER-HOW & NA & NA & NA & $1.06 \mathrm{E}+11$ & $4.92 \mathrm{E}+10$ \\
\hline MFC-764 & MFC Parking Lot & NA & NA & NA & $6.22 \mathrm{E}+08$ & $2.89 \mathrm{E}+08$ \\
\hline MFC-764 & MFC Guard Shack & NA & NA & NA & $8.27 \mathrm{E}+08$ & $3.84 \mathrm{E}+08$ \\
\hline
\end{tabular}

Table A-8. Estimated time (years) to achieve build-up concentration in surface soil for $30 \mu \mathrm{m}$ particles released continuously for 1 year at ground level from the Materials and Fuels Complex. The shortest five time periods are highlighted in yellow. Two of the sampler locations, MFC Parking Lots and MFC Guard Shack, are proposed.

\begin{tabular}{|llllllc|}
\hline SOURCE & \multicolumn{1}{c}{ SAMPLER } & Am-241 & Cs-137 & Pu-238 & Pu-239 \\
\hline MFC-GL & BEA-TRA & $9.91 \mathrm{E}+12$ & $2.24 \mathrm{E}+08$ & $1.04 \mathrm{E}+19$ & $1.13 \mathrm{E}+09$ & $3.61 \mathrm{E}+08$ \\
\hline MFC-GL & BEA-CPP & $7.21 \mathrm{E}+12$ & $1.63 \mathrm{E}+08$ & $7.55 \mathrm{E}+18$ & $8.25 \mathrm{E}+08$ & $2.63 \mathrm{E}+08$ \\
\hline MFC-GL & BEA-RWMC & $8.56 \mathrm{E}+12$ & $1.93 \mathrm{E}+08$ & $8.96 \mathrm{E}+18$ & $9.80 \mathrm{E}+08$ & $3.12 \mathrm{E}+08$ \\
\hline MFC-GL & BEA-VAN & $8.10 \mathrm{E}+12$ & $1.83 \mathrm{E}+08$ & $8.48 \mathrm{E}+18$ & $9.28 \mathrm{E}+08$ & $2.95 \mathrm{E}+08$ \\
\hline MFC-GL & BEA-IRC & $1.41 \mathrm{E}+13$ & $3.19 \mathrm{E}+08$ & $1.48 \mathrm{E}+19$ & $1.62 \mathrm{E}+09$ & $5.15 \mathrm{E}+08$ \\
\hline MFC-GL & BEA-BLKFT & $1.36 \mathrm{E}+13$ & $3.08 \mathrm{E}+08$ & $1.43 \mathrm{E}+19$ & $1.56 \mathrm{E}+09$ & $4.97 \mathrm{E}+08$ \\
\hline MFC-GL & BEA-SMC & $8.08 \mathrm{E}+12$ & $1.82 \mathrm{E}+08$ & $8.46 \mathrm{E}+18$ & $9.25 \mathrm{E}+08$ & $2.94 \mathrm{E}+08$ \\
\hline MFC-GL & BEA-GATE & $8.36 \mathrm{E}+12$ & $1.89 \mathrm{E}+08$ & $8.75 \mathrm{E}+18$ & $9.57 \mathrm{E}+08$ & $3.05 \mathrm{E}+08$ \\
\hline MFC-GL & BEA-ARA & $3.48 \mathrm{E}+12$ & $7.86 \mathrm{E}+07$ & $3.65 \mathrm{E}+18$ & $3.99 \mathrm{E}+08$ & $1.27 \mathrm{E}+08$ \\
\hline MFC-GL & BEA-REST & $1.01 \mathrm{E}+13$ & $2.28 \mathrm{E}+08$ & $1.06 \mathrm{E}+19$ & $1.16 \mathrm{E}+09$ & $3.69 \mathrm{E}+08$ \\
\hline MFC-GL & BEA-IF & $1.40 \mathrm{E}+13$ & $3.15 \mathrm{E}+08$ & $1.46 \mathrm{E}+19$ & $1.60 \mathrm{E}+09$ & $1.17 \mathrm{E}+09$ \\
\hline MFC-GL & BEA-NRF & $1.03 \mathrm{E}+13$ & $2.31 \mathrm{E}+08$ & $1.07 \mathrm{E}+19$ & $3.08 \mathrm{E}+08$ \\
\hline
\end{tabular}


Table A-8. (continued).

\begin{tabular}{|c|c|c|c|c|c|c|}
\hline SOURCE & SAMPLER & Am-241 & Cs-137 & Pu-238 & Pu-239 & Sr-90 \\
\hline MFC-GL & BEA-RTC & $1.00 \mathrm{E}+13$ & $2.26 \mathrm{E}+08$ & $1.05 \mathrm{E}+19$ & $1.15 \mathrm{E}+09$ & $3.65 \mathrm{E}+08$ \\
\hline MFC-GL & BEA-EBR & $8.20 \mathrm{E}+12$ & $1.85 \mathrm{E}+08$ & $8.58 \mathrm{E}+18$ & $9.38 \mathrm{E}+08$ & $2.99 \mathrm{E}+08$ \\
\hline MFC-GL & BEA-MFC & $8.69 \mathrm{E}+10$ & $1.96 \mathrm{E}+06$ & $9.10 \mathrm{E}+16$ & $9.95 \mathrm{E}+06$ & $3.16 \mathrm{E}+06$ \\
\hline MFC-GL & BEA-SUGAR & $1.58 \mathrm{E}+13$ & $3.56 \mathrm{E}+08$ & $1.65 \mathrm{E}+19$ & $1.80 \mathrm{E}+09$ & $5.74 \mathrm{E}+08$ \\
\hline MFC-GL & BEA-PBF & $4.52 \mathrm{E}+12$ & $1.02 \mathrm{E}+08$ & $4.73 \mathrm{E}+18$ & $5.17 \mathrm{E}+08$ & $1.65 \mathrm{E}+08$ \\
\hline MFC-GL & BEA-INTEC & $7.15 \mathrm{E}+12$ & $1.61 \mathrm{E}+08$ & $7.49 \mathrm{E}+18$ & $8.19 \mathrm{E}+08$ & $2.60 \mathrm{E}+08$ \\
\hline MFC-GL & BEA-CFA & $6.72 \mathrm{E}+12$ & $1.52 \mathrm{E}+08$ & $7.03 \mathrm{E}+18$ & $7.69 \mathrm{E}+08$ & $2.45 \mathrm{E}+08$ \\
\hline MFC-GL & BEA-EFS & $7.07 \mathrm{E}+12$ & $1.59 \mathrm{E}+08$ & $7.40 \mathrm{E}+18$ & $8.09 \mathrm{E}+08$ & $2.58 \mathrm{E}+08$ \\
\hline MFC-GL & BEA-CRATE & $4.95 \mathrm{E}+13$ & $1.12 \mathrm{E}+09$ & $5.18 \mathrm{E}+19$ & $5.67 \mathrm{E}+09$ & $1.80 \mathrm{E}+09$ \\
\hline MFC-GL & ESER-VAN & $8.10 \mathrm{E}+12$ & $1.83 \mathrm{E}+08$ & $8.48 \mathrm{E}+18$ & $9.27 \mathrm{E}+08$ & $2.95 \mathrm{E}+08$ \\
\hline MFC-GL & ESER-CRA & $4.95 \mathrm{E}+13$ & $1.12 \mathrm{E}+09$ & $5.18 \mathrm{E}+19$ & $5.67 \mathrm{E}+09$ & $1.80 \mathrm{E}+09$ \\
\hline MFC-GL & ESER-ARC & $3.85 \mathrm{E}+13$ & $8.69 \mathrm{E}+08$ & $4.03 \mathrm{E}+19$ & $4.41 \mathrm{E}+09$ & $1.40 \mathrm{E}+09$ \\
\hline MFC-GL & ESER-SUG & $1.58 \mathrm{E}+13$ & $3.56 \mathrm{E}+08$ & $1.65 \mathrm{E}+19$ & $1.80 \mathrm{E}+09$ & $5.74 \mathrm{E}+08$ \\
\hline MFC-GL & ESER-IDA & $1.40 \mathrm{E}+13$ & $3.15 \mathrm{E}+08$ & $1.46 \mathrm{E}+19$ & $1.60 \mathrm{E}+09$ & $5.08 \mathrm{E}+08$ \\
\hline MFC-GL & ESER-DUB & $1.28 \mathrm{E}+13$ & $2.89 \mathrm{E}+08$ & $1.34 \mathrm{E}+19$ & $1.47 \mathrm{E}+09$ & $4.67 \mathrm{E}+08$ \\
\hline MFC-GL & ESER-MON & $7.37 \mathrm{E}+12$ & $1.66 \mathrm{E}+08$ & $7.72 \mathrm{E}+18$ & $8.44 \mathrm{E}+08$ & $2.69 \mathrm{E}+08$ \\
\hline MFC-GL & ESER-FAA & $3.59 \mathrm{E}+12$ & $8.11 \mathrm{E}+07$ & $3.76 \mathrm{E}+18$ & $4.12 \mathrm{E}+08$ & $1.31 \mathrm{E}+08$ \\
\hline MFC-GL & ESER-MAI & $5.55 \mathrm{E}+12$ & $1.25 \mathrm{E}+08$ & $5.81 \mathrm{E}+18$ & $6.35 \mathrm{E}+08$ & $2.02 \mathrm{E}+08$ \\
\hline MFC-GL & ESER-EFS & $7.07 \mathrm{E}+12$ & $1.59 \mathrm{E}+08$ & $7.40 \mathrm{E}+18$ & $8.09 \mathrm{E}+08$ & $2.57 \mathrm{E}+08$ \\
\hline MFC-GL & ESER-ATO & $2.68 \mathrm{E}+12$ & $6.05 \mathrm{E}+07$ & $2.81 \mathrm{E}+18$ & $3.07 \mathrm{E}+08$ & $9.77 \mathrm{E}+07$ \\
\hline MFC-GL & ESER-MOU & $1.36 \mathrm{E}+13$ & $3.08 \mathrm{E}+08$ & $1.43 \mathrm{E}+19$ & $1.56 \mathrm{E}+09$ & $4.97 \mathrm{E}+08$ \\
\hline MFC-GL & ESER-BLU & $1.43 \mathrm{E}+13$ & $3.22 \mathrm{E}+08$ & $1.49 \mathrm{E}+19$ & $1.63 \mathrm{E}+09$ & $5.20 \mathrm{E}+08$ \\
\hline MFC-GL & ESER-TER & $3.64 \mathrm{E}+12$ & $8.22 \mathrm{E}+07$ & $3.81 \mathrm{E}+18$ & $4.17 \mathrm{E}+08$ & $1.33 \mathrm{E}+08$ \\
\hline MFC-GL & ESER-HOW & $2.43 \mathrm{E}+13$ & $5.48 \mathrm{E}+08$ & $2.55 \mathrm{E}+19$ & $2.78 \mathrm{E}+09$ & $8.86 \mathrm{E}+08$ \\
\hline MFC-GL & MFC Parking Lot & $1.43 \mathrm{E}+11$ & $3.22 \mathrm{E}+06$ & $1.49 \mathrm{E}+17$ & $1.63 \mathrm{E}+07$ & $5.20 \mathrm{E}+06$ \\
\hline MFC-GL & MFC Guard Shack & $1.90 \mathrm{E}+11$ & $4.29 \mathrm{E}+06$ & $1.99 \mathrm{E}+17$ & $2.17 \mathrm{E}+07$ & $6.92 \mathrm{E}+06$ \\
\hline
\end{tabular}




\section{A-3.4 Radioactive Waste Management Complex}

The Radioactive Waste Management Complex (RWMC), located in the southwestern corner of the INL Site, is a controlled-access area with a primary mission to dispose of INL-generated low-level radioactive waste and to temporarily store contact-handled and remote-handled transuranic waste that will be shipped to other designated facilities for disposal. The Accelerated Retrieval Project (ARP), regulated under CERCLA, is removing targeted waste from the subsurface disposal area (SDA), disposing of transuranic waste at an offsite facility, and remediating and closing the SDA.

Current operations at the RWMC include the Advanced Mixed Waste Treatment Program (AMWTP). The AMWTP includes the retrieval of mixed transuranic waste from temporary storage, characterizing the waste, treating the waste to meet disposal criteria, and packaging the waste for shipment to the Waste Isolation Pilot Plant in Carlsbad, New Mexico. Radiological air emissions from the AMWTP may result from the retrieval, characterization, and treatment of transuranic waste, alpha-contaminated low-level mixed waste (alpha LLMW), and low-level mixed waste (LLMW).

In 2014, air emissions at the RWMC, which contributed at least $1 \%$ of the total estimated dose to the MEI, were primarily from activities associated with the ARP, the Drum Treatment Facility, and tritium releases from buried beryllium blocks. Table A-9 shows the results of calculations using the entire RWMC source term for 2013 (including the AMWTP) as a ground level release. According to these calculations, most of the fugitive releases would be deposited close to the RWMC and Am-241 could be detected within 7 years. The same radionuclide would be detectable at EBR-1 after 700 years. It is important to note that environmental surveillance is conducted by CWI as part of waste management operations within the RWMC and includes continual air sampling along the perimeter of the facility, as well as soil sampling within the waste disposal area on a biennial basis. These data are not discussed here. 
Table A-9. Estimated time (years) to achieve build-up concentration in surface soil for $30 \mu \mathrm{m}$ particles released continuously for 1 year at ground level from the Radioactive Waste Management Complex. The five shortest time periods are highlighted in yellow.

\begin{tabular}{|c|c|c|c|c|c|c|}
\hline SOURCE & SAMPLER & Am-241 & Cs-137 & $\mathbf{P u}-238$ & Pu-239 & Sr-90 \\
\hline RWMC-GL & BEA-TRA & $5.22 \mathrm{E}+03$ & $4.80 \mathrm{E}+07$ & $8.54 \mathrm{E}+04$ & $5.40 \mathrm{E}+03$ & $3.25 \mathrm{E}+07$ \\
\hline RWMC-GL & BEA-CPP & $4.52 \mathrm{E}+03$ & $4.15 \mathrm{E}+07$ & $7.39 \mathrm{E}+04$ & $4.67 \mathrm{E}+03$ & $2.82 \mathrm{E}+07$ \\
\hline RWMC-GL & BEA-RWMC & $6.58 \mathrm{E}+00$ & $6.04 \mathrm{E}+04$ & $1.08 \mathrm{E}+02$ & $6.80 \mathrm{E}+00$ & $4.10 \mathrm{E}+04$ \\
\hline RWMC-GL & BEA-VAN & $1.61 \mathrm{E}+03$ & $1.48 \mathrm{E}+07$ & $2.64 \mathrm{E}+04$ & $1.67 \mathrm{E}+03$ & $1.01 \mathrm{E}+07$ \\
\hline RWMC-GL & BEA-IRC & $4.68 \mathrm{E}+06$ & $4.30 \mathrm{E}+10$ & $7.65 \mathrm{E}+07$ & $4.84 \mathrm{E}+06$ & $2.92 \mathrm{E}+10$ \\
\hline RWMC-GL & BEA-BLKFT & $1.01 \mathrm{E}+07$ & $9.31 \mathrm{E}+10$ & $1.66 \mathrm{E}+08$ & $1.05 \mathrm{E}+07$ & $6.32 \mathrm{E}+10$ \\
\hline RWMC-GL & BEA-SMC & $1.97 \mathrm{E}+05$ & $1.81 \mathrm{E}+09$ & $3.22 \mathrm{E}+06$ & $2.04 \mathrm{E}+05$ & $1.23 \mathrm{E}+09$ \\
\hline RWMC-GL & BEA-GATE & $8.97 \mathrm{E}+04$ & $8.24 \mathrm{E}+08$ & $1.47 \mathrm{E}+06$ & $9.27 \mathrm{E}+04$ & $5.59 \mathrm{E}+08$ \\
\hline RWMC-GL & BEA-ARA & $1.10 \mathrm{E}+05$ & $1.01 \mathrm{E}+09$ & $1.80 \mathrm{E}+06$ & $1.14 \mathrm{E}+05$ & $6.88 \mathrm{E}+08$ \\
\hline RWMC-GL & BEA-REST & $1.66 \mathrm{E}+03$ & $1.52 \mathrm{E}+07$ & $2.71 \mathrm{E}+04$ & $1.71 \mathrm{E}+03$ & $1.03 \mathrm{E}+07$ \\
\hline RWMC-GL & BEA-IF & $4.37 \mathrm{E}+06$ & $4.02 \mathrm{E}+10$ & $7.15 \mathrm{E}+07$ & $4.52 \mathrm{E}+06$ & $2.73 \mathrm{E}+10$ \\
\hline RWMC-GL & BEA-NRF & $2.20 \mathrm{E}+04$ & $2.02 \mathrm{E}+08$ & $3.60 \mathrm{E}+05$ & $2.28 \mathrm{E}+04$ & $1.37 \mathrm{E}+08$ \\
\hline RWMC-GL & BEA-RTC & $6.25 \mathrm{E}+03$ & $5.74 \mathrm{E}+07$ & $1.02 \mathrm{E}+05$ & $6.45 \mathrm{E}+03$ & $3.89 \mathrm{E}+07$ \\
\hline RWMC-GL & BEA-EBR & $7.39 \mathrm{E}+02$ & $6.78 \mathrm{E}+06$ & $1.21 \mathrm{E}+04$ & $7.64 \mathrm{E}+02$ & $4.60 \mathrm{E}+06$ \\
\hline RWMC-GL & BEA-MFC & $1.91 \mathrm{E}+05$ & $1.75 \mathrm{E}+09$ & $3.11 \mathrm{E}+06$ & $1.97 \mathrm{E}+05$ & $1.19 \mathrm{E}+09$ \\
\hline RWMC-GL & BEA-SUGAR & $2.24 \mathrm{E}+06$ & $2.05 \mathrm{E}+10$ & $3.65 \mathrm{E}+07$ & $2.31 \mathrm{E}+06$ & $1.39 \mathrm{E}+10$ \\
\hline RWMC-GL & BEA-PBF & $2.87 \mathrm{E}+04$ & $2.63 \mathrm{E}+08$ & $4.69 \mathrm{E}+05$ & $2.96 \mathrm{E}+04$ & $1.79 \mathrm{E}+08$ \\
\hline RWMC-GL & BEA-INTEC & $5.27 \mathrm{E}+03$ & $4.84 \mathrm{E}+07$ & $8.62 \mathrm{E}+04$ & $5.45 \mathrm{E}+03$ & $3.29 \mathrm{E}+07$ \\
\hline RWMC-GL & BEA-CFA & $6.73 \mathrm{E}+03$ & $6.18 \mathrm{E}+07$ & $1.10 \mathrm{E}+05$ & $6.95 \mathrm{E}+03$ & $4.19 \mathrm{E}+07$ \\
\hline RWMC-GL & BEA-EFS & $9.14 \mathrm{E}+03$ & $8.39 \mathrm{E}+07$ & $1.49 \mathrm{E}+05$ & $9.44 \mathrm{E}+03$ & $5.69 \mathrm{E}+07$ \\
\hline RWMC-GL & BEA-CRATE & $2.39 \mathrm{E}+06$ & $2.20 \mathrm{E}+10$ & $3.91 \mathrm{E}+07$ & $2.47 \mathrm{E}+06$ & $1.49 \mathrm{E}+10$ \\
\hline RWMC-GL & ESER-VAN & $1.62 \mathrm{E}+03$ & $1.48 \mathrm{E}+07$ & $2.64 \mathrm{E}+04$ & $1.67 \mathrm{E}+03$ & $1.01 \mathrm{E}+07$ \\
\hline RWMC-GL & ESER-CRA & $2.39 \mathrm{E}+06$ & $2.19 \mathrm{E}+10$ & $3.91 \mathrm{E}+07$ & $2.47 \mathrm{E}+06$ & $1.49 \mathrm{E}+10$ \\
\hline RWMC-GL & ESER-ARC & $9.20 \mathrm{E}+05$ & $8.45 \mathrm{E}+09$ & $1.50 \mathrm{E}+07$ & $9.51 \mathrm{E}+05$ & $5.73 \mathrm{E}+09$ \\
\hline RWMC-GL & ESER-SUG & $2.24 \mathrm{E}+06$ & $2.05 \mathrm{E}+10$ & $3.65 \mathrm{E}+07$ & $2.31 \mathrm{E}+06$ & $1.39 \mathrm{E}+10$ \\
\hline RWMC-GL & ESER-IDA & $4.37 \mathrm{E}+06$ & $4.02 \mathrm{E}+10$ & $7.15 \mathrm{E}+07$ & $4.52 \mathrm{E}+06$ & $2.72 \mathrm{E}+10$ \\
\hline RWMC-GL & ESER-DUB & $1.02 \mathrm{E}+06$ & $9.38 \mathrm{E}+09$ & $1.67 \mathrm{E}+07$ & $1.06 \mathrm{E}+06$ & $6.36 \mathrm{E}+09$ \\
\hline RWMC-GL & ESER-MON & $3.63 \mathrm{E}+05$ & $3.34 \mathrm{E}+09$ & $5.94 \mathrm{E}+06$ & $3.75 \mathrm{E}+05$ & $2.26 \mathrm{E}+09$ \\
\hline
\end{tabular}


Table A-9. (continued).

\begin{tabular}{|c|c|c|c|c|c|c|}
\hline SOURCE & SAMPLER & Am-241 & Cs-137 & $\mathrm{Pu}-238$ & Pu-239 & Sr-90 \\
\hline RWMC-GL & ESER-FAA & $7.19 \mathrm{E}+05$ & $6.61 \mathrm{E}+09$ & $1.18 \mathrm{E}+07$ & $7.43 \mathrm{E}+05$ & $4.48 \mathrm{E}+09$ \\
\hline RWMC-GL & ESER-MAI & $4.93 \mathrm{E}+04$ & $4.52 \mathrm{E}+08$ & $8.05 \mathrm{E}+05$ & $5.09 \mathrm{E}+04$ & $3.07 \mathrm{E}+08$ \\
\hline RWMC-GL & ESER-EFS & $9.13 \mathrm{E}+03$ & $8.39 \mathrm{E}+07$ & $1.49 \mathrm{E}+05$ & $9.44 \mathrm{E}+03$ & $5.69 \mathrm{E}+07$ \\
\hline RWMC-GL & ESER-ATO & $5.08 \mathrm{E}+05$ & $4.66 \mathrm{E}+09$ & $8.30 \mathrm{E}+06$ & $5.25 \mathrm{E}+05$ & $3.16 \mathrm{E}+09$ \\
\hline RWMC-GL & ESER-MOU & $1.01 \mathrm{E}+07$ & $9.32 \mathrm{E}+10$ & $1.66 \mathrm{E}+08$ & $1.05 \mathrm{E}+07$ & $6.32 \mathrm{E}+10$ \\
\hline RWMC-GL & ESER-BLU & $1.62 \mathrm{E}+06$ & $1.49 \mathrm{E}+10$ & $2.65 \mathrm{E}+07$ & $1.68 \mathrm{E}+06$ & $1.01 \mathrm{E}+10$ \\
\hline RWMC-GL & ESER-TER & $1.91 \mathrm{E}+05$ & $1.76 \mathrm{E}+09$ & $3.13 \mathrm{E}+06$ & $1.98 \mathrm{E}+05$ & $1.19 \mathrm{E}+09$ \\
\hline RWMC-GL & ESER-HOW & $2.95 \mathrm{E}+05$ & $2.71 \mathrm{E}+09$ & $4.83 \mathrm{E}+06$ & $3.05 \mathrm{E}+05$ & $1.84 \mathrm{E}+09$ \\
\hline
\end{tabular}




\section{A-3.5 Summary of Deposition Modeling Results}

In most cases, detection of radionuclides of concern is not possible within INL Site boundaries for many, many years, assuming continuous releases from the facilities analyzed, with the exception of Am-241 in soil at the RWMC air monitor (BEA-RWMC). Releases from the ARP facilities could be detected within 7 years in the soil. INL Site releases are never detected at offsite locations. Based on the deposition modeling, it is apparent that soil sampling for routine monitoring of current air emissions from INL Site facilities is not necessary on an annual to 3-year basis, as recommended in DOE (2015). Radionuclides released from INL Site activities or accidental releases are much more likely to be observed in air monitor filters than in soil.

\section{A-4 SAMPLING TECHNIQUES}

Both ex-situ (physical soil sampling with laboratory analysis) and field measurements by in-situ gamma spectroscopy (ISGS) have been used at INL in the past and they have complementary roles in assessing radionuclide inventories in surface soils. The random analytical uncertainty in ISGS field methods can exceed that for ex situ measurements made in the controlled laboratory conditions. However, for ex-situ measurements, heterogeneous contamination can result in higher levels of random sampling uncertainty. At the same time, ex-situ techniques can detect radionuclides that ISGS cannot and ex-situ data can provide accurate depth profiles so that collocated ISGS data can be modelled with greater confidence. Additionally, because in situ measurements are substantially less costly, a greater number of measurements can be made potentially reducing the uncertainty on the mean over a larger area than is possible using ex situ methods alone.

Soil sampling with laboratory analysis has been conducted at locations outside the INL Site since the program was established in 1978 by RESL. The process described below is used to produce a relatively homogeneous sample from which gamma analysis and then a relatively small aliquot ( $10 \mathrm{~g})$ may be drawn for radiochemical analyses. A standard 10-m square soil sampling grid is used to collect composite samples at each location. A core sampler $10 \mathrm{~cm}$ in diameter and $5 \mathrm{~cm}$ deep is driven into the soil at each corner and at the center of the grid for a total of five sub-samples (Figure 11).The five sub-samples are combined to generate a single $0-5 \mathrm{~cm}$ composite sample. This process is repeated at the same collection points to obtain a 5-10 $\mathrm{cm}$ depth composite sample. The composited samples are thoroughly mixed and passed through a 35 mesh $(0.5 \mathrm{~mm})$ sieve. A $500-\mathrm{mL}$ Marinelli Beaker is filled with the sieved material for gamma analysis. Approximately 50-g aliquots are split from the composite samples and placed in pre-weighed $70-\mathrm{mL}$ sample containers. These samples are sent to the radiochemistry laboratory for analysis of Am-241, plutonium isotopes and Sr-90.

The soil monitoring program also employs ISGS to identify gamma-emitting radionuclides in soil. ISGS is a sensitive and selective method for detecting manmade gamma-emitting radionuclides, particularly Cs-137. The major advantage of this method is a large number of analyses can be carried out in a relatively short time compared to the effort required for soil sampling, preparation, and analyses (NCRP 1999). By measuring a larger volume of soil, ISGS results are commonly less affected by smallscale sampling variability introduced by physical soil sampling. ISGS is particularly useful because Cs-137 is the most prevalent manmade radionuclide in surface soil and it is widely used as an indicator species for general contamination trends; however, only gamma-emitting radionuclides like Cs-137can be detected, precluding radionuclides like Sr-90 (a beta-emitting radionuclide) and $\mathrm{Pu}-239 / 240$ (an alphaemitting radionuclide). In addition, the detector usually cannot distinguish the location of the radionuclides in the soil (i.e., the horizontal or vertical distribution). 
Because Cs-137 is the predominant anthropogenic radionuclide in the known radionuclide-contaminated soil areas at the INL Site, and because it is relatively easy to detect even at low concentrations using the in-situ instruments, ISGS soil surveillance focuses on Cs-137. NCRP (1999) states that the horizontal distribution can be assumed to be fairly uniformly distributed within the field of view and data from a limited number of soil samples can provide sufficient information of the depth profile to allow one to use ISGS as a primary means of charactering the concentration of soil in a contaminated site. Between 2006 and 2010, a series of studies were conducted to compare in-situ gamma analysis results with soil sampling and laboratory analysis at various onsite sampling locations. The methodology was that used by Walker (2000). For example, at 34 locations in 2007, 10 split-spoon samples were collected from a predetermined array centered at the in-situ location out to $30 \mathrm{ft}$. Each of the 10 split-spoon samples were removed and divided by 1-in. depth to form 12 composite samples. Each of these 1-in. composite samples from the 34 in-situ locations were then analyzed for gamma emitting radionuclides in the laboratory to determine the Cs-137 depth profiles for the in-situ measurement technique. These data are still used for today's in-situ measurements and data analysis.

ISGS technology is limited in the case of areas contaminated solely by transuranic (TRU) radionuclides or Sr-90. Transuranic radionuclides have very low-energy photon emissions so that low levels would be difficult to detect in the field, except in the case of unplanned releases with concentrations that are measureable with ISGS. Sr-90 cannot be detected with ISGS technology.

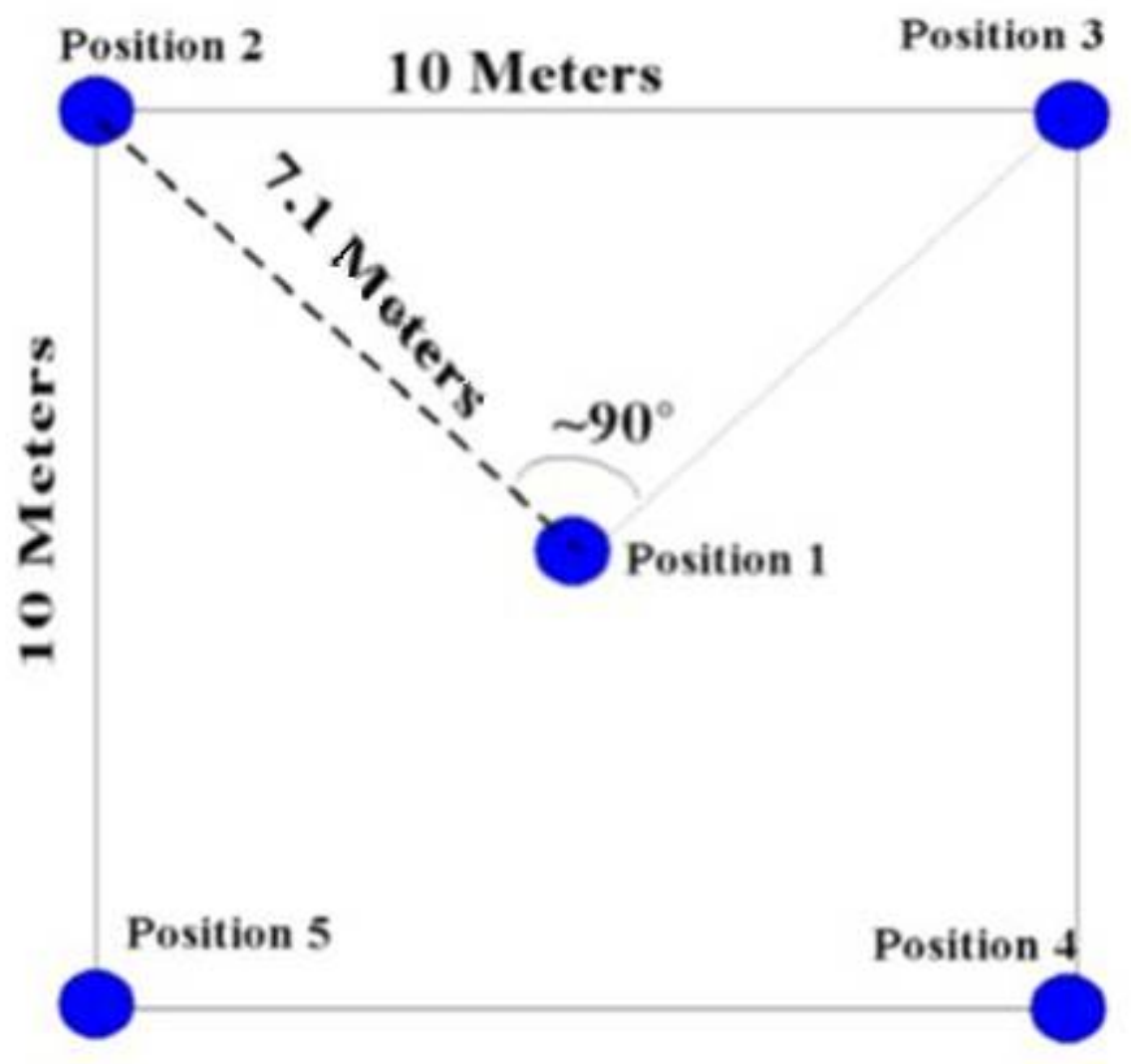

Figure A-16. Soil sampling grid. 


\section{Appendix B}

\section{Statistical Analysis Associated with Soil Monitoring Data Quality Objectives}

The Data Quality Objectives (DQOs) reference a great deal of statistical concepts and techniques because background levels used to establish radionuclide inventory in the soils were computed using statistical methods. This section provides a detailed explanation of the methods used to obtain the background levels, justification for their selection, and information on how to use and update them. The primary statistical method that is used in these DQOs is upper tolerance limits (UTLs) to compute background levels. The following sections discuss each of these methods separately.

\section{B-1. BACKGROUND LEVELS}

Background levels were computed for each of the near facility and distant areas that are sampled under the monitoring program. Area-specific background levels are necessary for this program because radionuclide concentrations can vary widely in each area due to variation in natural radiation and localized activities. Thus, historical data obtained from each of the areas was used to compute a UTL for each area that can be used as a background level.

\section{B-1.1 Use of Background Levels}

The background level is computed using a $99 \% / 95 \%$ UTL. The $99 \% / 95 \%$ UTL is a value such that $99 \%$ of the data are less than the UTL with $95 \%$ confidence. Thus, a UTL computed using background data is designed to cover all but a small percentage of the background population measurements (EPA 2009). It is an appropriate measure for providing an upper threshold on measurements obtained from an area and is often used to construct a background level for the area. Individual measurements are compared to the UTL. If the UTL is exceeded, that measurement may indicate an unusually high concentration for that area.

Approximately $1 \%$ of the doses are expected to be greater than the UTL when there is no actual increase in dose. Therefore, when a measured concentration exceeds the UTL it should not be directly assumed that radionuclide concentrations have increased. Rather, the concentration should be compared to other concentrations in the area and to concentrations previously obtained from that sampling location to provide context. The UTL provides a threshold that allows identification of concentrations that may alert the monitoring program to a potential release, but an exceedance does not always indicate an increase in radionuclide concentration for that area.

It is important that background levels remain relevant to the area in which they describe. Therefore, UTLs should be updated approximately every 5 years to ensure the background levels remain relevant to current area radionuclide concentrations. Data should be carefully examined to ensure that the data used to construct the UTL are indicative of current site conditions. 


\section{B-1.2 Computation of Background Levels}

UTLs were computed using ProUCL Version 5.0.0 (EPA 2013). The data were assessed to determine if they were normally distributed, gamma distributed, or did not have a discernable distribution (non-parametric). The appropriate UTL was selected based on the distribution of the data. The ProUCL Technical Guide (EPA 2013) contains the details on how UTLs are computed and selected. Data were also examined for outliers or other trends that may bias the UTL in a manner that is not indicative of the true background for the area. All data obtained from 1972 to 2014 were used to construct the UTLs unless data from those years are not available, or the data were deemed to be unusable due to quality issues. If the minimum detectable activity (MDA) for a sample was larger than the largest detected concentration it was removed from the data set. Data were obtained from RESL sampling activities (INEL 1994) and from other soil sampling results obtained under the soil monitoring program dating from 1972 to 2014 .

UTLs require a minimum of 8 data points to be computed. Thus, areas where fewer than 8 dose measurements are available did not have background levels computed. UTLs will need to be computed for those areas once a minimum of 8 data points are available.

The UTLs for each of the near facility areas are listed in Tables B-1 through B-6. The UTLs and summary statistics for the distant regions are listed in Tables B-7 through B-16. The tables also lists how many points were used to compute the background level for that area and summary statistics. If sufficient data were not available to compute a UTL, it is noted on the table along with the number of measurements currently available for that area. 
Table B-1. Summary statistics for data collected at ARA.

\begin{tabular}{|c|c|c|c|c|c|c|c|c|}
\hline Radionuclide & $\mathrm{N}$ & $\begin{array}{l}\text { Percent } \\
\text { Detected }\end{array}$ & $\begin{array}{c}\operatorname{Minimum}_{(\mathrm{pCi} / \mathrm{g})}^{\mathrm{a}} \\
\end{array}$ & $\begin{array}{l}\text { Maximum } \\
(\mathrm{pCi} / \mathrm{g})\end{array}$ & $\begin{array}{l}\text { Mean } \\
(\mathrm{pCi} / \mathrm{g})\end{array}$ & $\begin{array}{c}\text { Standard } \\
\text { Deviation } \\
\text { (pCi/g) }\end{array}$ & $\begin{array}{l}\text { Distribution } \\
\text { of the Data }\end{array}$ & $\begin{array}{c}\mathrm{UTL} \\
(\mathrm{pCi} / \mathrm{g})\end{array}$ \\
\hline Am-241 & 93 & 10 & $<0.003$ & 0.401 & $\mathrm{NA}^{\mathrm{b}}$ & $\mathrm{NA}^{\mathrm{b}}$ & $\mathrm{NA}^{\mathrm{b}}$ & $0.401 \mathrm{c}$ \\
\hline Co-60 & 157 & 62 & $<0.00081$ & 0.37 & 0.065 & 0.0604 & Gamma & 0.242 \\
\hline Cs-134 & 213 & 70 & $<0.0093$ & 1.687 & 0.0862 & 0.146 & Non-Parametric & 1.687 \\
\hline $\begin{array}{l}\text { Cs-134 (1 Outlier } \\
\text { Removed) }\end{array}$ & 212 & 70 & $<0.0093$ & 0.667 & 0.0754 & 0.0621 & Non-Parametric & 0.667 \\
\hline $\begin{array}{l}\text { Cs-134 (2 Outliers } \\
\text { Removed) }{ }^{\text {e }}\end{array}$ & 211 & 70 & $<0.0093$ & 0.306 & 0.0713 & 0.0383 & Non-Parametric & 0.306 \\
\hline Cs-137 & 432 & 95 & $<0.065$ & 373 & 5.879 & 25.37 & Non-Parametric & 133 \\
\hline Eu-152 & 92 & 50 & $<0.0402$ & 1.248 & 0.446 & 0.335 & Non-Parametric & 1.248 \\
\hline $\mathrm{Pu}-238$ & 14 & 36 & $<0.0018$ & 0.025 & $\mathrm{NA}^{\mathrm{b}}$ & $\mathrm{NA}^{\mathrm{b}}$ & $\mathrm{NA}^{\mathrm{b}}$ & $0.025^{\mathrm{c}}$ \\
\hline $\mathrm{Pu}-239 / 240$ & 13 & 100 & 0.009 & 0.047 & 0.0192 & 0.0105 & Normal & 0.0577 \\
\hline $\mathrm{Sb}-125$ & 93 & 49 & $<0.0435$ & 1.415 & 0.365 & 0.274 & Non-Parametric & 1.415 \\
\hline Sr-90 & 16 & 100 & 0.38 & 57 & 7.381 & 14.8 & Non-Parametric & 57 \\
\hline $\begin{array}{l}\text { Sr-90 (1 Outlier } \\
\text { Removed })^{\mathrm{f}}\end{array}$ & 15 & 100 & 0.38 & 22.3 & 4.073 & 6.853 & Non-Parametric & 22.3 \\
\hline U-234 & 192 & 46 & $<0.714$ & 201.4 & 69.17 & 54.57 & Non-Parametric & 201.4 \\
\hline U-235 & 270 & 53 & $<0.063$ & 3.7 & 0.934 & 0.895 & Non-Parametric & 3.677 \\
\hline U-238 & 246 & 47 & $<0.131$ & 238.5 & 27.39 & 43.6 & Non-Parametric & 216.9 \\
\hline \multicolumn{9}{|c|}{$\begin{array}{l}\text { a. Less than sign indicates that th } \\
\text { b. A minimum of } 8 \text { detects and } 30 \\
\text { c. Insufficient detected data are a } \\
\text { d. Outlier at ARA-37 in } 2007 \\
\text { e. Outlier at ARA-65 in } 2007 \\
\text { f. Outlier at } 0^{\circ} \mathrm{C} 500 \text { in } 1977\end{array}$} \\
\hline
\end{tabular}


Table B-2. Summary statistics for data collected at ATR.

\begin{tabular}{|c|c|c|c|c|c|c|c|c|}
\hline Radionuclide & $\mathrm{N}$ & $\begin{array}{l}\text { Percent } \\
\text { Detected }\end{array}$ & $\begin{array}{c}\operatorname{Minimum}^{\mathrm{a}} \\
(\mathrm{pCi} / \mathrm{g})\end{array}$ & $\begin{array}{l}\text { Maximum } \\
(\mathrm{pCi} / \mathrm{g})\end{array}$ & $\begin{array}{l}\text { Mean } \\
(\mathrm{pCi} / \mathrm{g})\end{array}$ & $\begin{array}{l}\text { Standard } \\
\text { Deviation } \\
(\mathrm{pCi} / \mathrm{g})\end{array}$ & $\begin{array}{l}\text { Distribution } \\
\text { of the Data }\end{array}$ & $\begin{array}{c}\mathrm{UTL} \\
(\mathrm{pCi} / \mathrm{g})\end{array}$ \\
\hline Am-241 & 41 & 22 & $<0.002$ & 0.49 & $\mathrm{NA}^{\mathrm{b}}$ & $\mathrm{NA}^{\mathrm{b}}$ & $\mathrm{NA}^{\mathrm{b}}$ & $0.49^{\mathrm{c}}$ \\
\hline Co-60 & 102 & 84 & $<0.00278$ & 66.4 & 1.806 & 8.523 & Non-Parametric & 66.4 \\
\hline $\begin{array}{l}\text { Co-60 (1 Outlier } \\
\text { Removed })^{\mathrm{d}}\end{array}$ & 101 & 84 & $<0.00278$ & 40.3 & 1.046 & 4.822 & Non-Parametric & 40.3 \\
\hline $\begin{array}{l}\text { Co-60 (2 Outliers } \\
\text { Removed) }\end{array}$ & 100 & 84 & $<0.00278$ & 18.2 & 0.578 & 2.179 & Non-Parametric & 18.2 \\
\hline Cs-134 & 63 & 60 & $<0.0117$ & 0.172 & 0.0572 & 0.0393 & Normal & 0.167 \\
\hline Cs-137 & 181 & 100 & 0.0759 & 223 & 3.318 & 21.85 & Non-Parametric & 223 \\
\hline Eu-152 & 31 & 6 & $<0.066$ & 0.775 & $\mathrm{NA}^{\mathrm{b}}$ & $\mathrm{NA}^{\mathrm{b}}$ & $\mathrm{NA}^{\mathrm{b}}$ & $0.775^{\mathrm{c}}$ \\
\hline $\mathrm{Pu}-238$ & 12 & 33 & $<0.003$ & 0.009 & 0.00424 & 0.00196 & Normal & 0.0116 \\
\hline $\mathrm{Pu}-239 / 240$ & 12 & 100 & 0.012 & 0.056 & 0.0261 & 0.0125 & Normal & 0.0728 \\
\hline Sb-125 & 33 & 12 & $<0.087$ & 0.725 & $\mathrm{NA}^{\mathrm{b}}$ & $\mathrm{NA}^{\mathrm{b}}$ & $\mathrm{NA}^{\mathrm{b}}$ & $0.725^{\mathrm{c}}$ \\
\hline $\begin{array}{l}\text { Sb-125 (1 Outlier } \\
\text { Removed) }\end{array}$ & 32 & 9 & $<0.087$ & 0.218 & $\mathrm{NA}^{\mathrm{b}}$ & $\mathrm{NA}^{\mathrm{b}}$ & $\mathrm{NA}^{\mathrm{b}}$ & $0.218^{\mathrm{c}}$ \\
\hline Sr-90 & 29 & 100 & 0.41 & 1.2 & 0.761 & 0.191 & Normal & 1.349 \\
\hline $\mathrm{U}-234$ & 43 & 70 & $<2.277$ & 195.3 & 108.5 & 53.74 & Non-Parametric & 195.3 \\
\hline U-235 & 90 & 34 & $<0.131$ & 2.65 & 0.733 & 0.631 & Gamma & 1.284 \\
\hline $\mathrm{U}-238$ & 82 & 27 & $<0.594$ & 64.21 & 18.74 & 13.43 & Gamma & 66.59 \\
\hline $\begin{array}{l}\text { U-238 (1 Outlier } \\
\text { Removed) }\end{array}$ & 81 & 26 & $<0.594$ & 34.68 & 5.409 & 8.339 & Normal & 28.13 \\
\hline \multicolumn{9}{|c|}{$\begin{array}{l}\text { a. Less than sign indicates } \\
\text { b. A minimum of } 8 \text { detects } \\
\text { c. Insufficient detected dat } \\
\text { d. Outlier at } 4.3 \text { in } 1983 \text {. } \\
\text { e. Outlier at } 4.2 \text { in } 1990 . \\
\text { f. Outlier at } 2.3 \text { in } 2011 . \\
\text { g. Outlier at } 8.2 \text { in } 2006 \text {. }\end{array}$} \\
\hline
\end{tabular}


Table B-3. Summary statistics for data collected at INTEC.

\begin{tabular}{|c|c|c|c|c|c|c|c|c|}
\hline Radionuclide & $\mathrm{N}$ & $\begin{array}{c}\text { Percent } \\
\text { Detected }\end{array}$ & $\begin{array}{c}\operatorname{Minimum}^{\mathrm{a}} \\
(\mathrm{pCi} / \mathrm{g})\end{array}$ & $\begin{array}{c}\text { Maximum } \\
(\mathrm{pCi} / \mathrm{g})\end{array}$ & $\begin{array}{c}\text { Mean } \\
(\mathrm{pCi} / \mathrm{g})\end{array}$ & $\begin{array}{c}\text { Standard } \\
\text { Deviation } \\
\text { (pCi } / \mathrm{g})\end{array}$ & $\begin{array}{l}\text { Distribution } \\
\text { of the Data }\end{array}$ & $\begin{array}{c}\mathrm{UTL} \\
(\mathrm{pCi} / \mathrm{g})\end{array}$ \\
\hline Am-241 & 204 & 12 & $<0.0041$ & 0.9 & $\mathrm{NA}^{\mathrm{b}}$ & $\mathrm{NA}^{\mathrm{b}}$ & $\mathrm{NA}^{\mathrm{b}}$ & $0.9^{\mathrm{c}}$ \\
\hline $\begin{array}{l}\text { Am-241 (1 Outlier } \\
\text { Removed) }\end{array}$ & 202 & 12 & $<0.0041$ & 0.67 & $\mathrm{NA}^{\mathrm{b}}$ & $\mathrm{NA}^{\mathrm{b}}$ & $\mathrm{NA}^{\mathrm{b}}$ & $0.67^{c}$ \\
\hline Co-60 & 268 & 53 & $<0.000847$ & 1.995 & 0.0619 & 0.191 & Non-Parametric & 1.1 \\
\hline $\begin{array}{l}\text { Co-60 (1 Outlier } \\
\text { Removed })^{\mathrm{e}}\end{array}$ & 267 & 53 & $<0.000847$ & 1.1 & 0.0482 & 0.099 & Non-Parametric & 0.4 \\
\hline $\begin{array}{l}\text { Co-60 }(2 \text { Outliers } \\
\text { Removed })^{\mathrm{f}}\end{array}$ & 266 & 53 & $<0.000847$ & 0.4 & 0.0407 & 0.0431 & Non-Parametric & 0.155 \\
\hline $\begin{array}{l}\text { Co-60 (3 Outliers } \\
\text { Removed })^{\mathrm{g}}\end{array}$ & 265 & 52 & $<0.000847$ & 0.155 & 0.0381 & 0.0304 & Non-Parametric & 0.149 \\
\hline Cs-134 & 219 & 68 & $<0.0128$ & 1.1 & 0.122 & 0.14 & Non-Parametric & 1.1 \\
\hline Cs-137 & 494 & 98 & $<0.00761$ & 52.7 & 3.544 & 6.646 & Non-Parametric & 40 \\
\hline $\mathrm{Eu}-152$ & 182 & 47 & $<0.0509$ & 2.096 & 0.422 & 0.345 & Non-Parametric & 2.096 \\
\hline $\mathrm{Pu}-238$ & 38 & 84 & $<0.0027$ & 0.387 & 0.0529 & 0.0835 & Non-Parametric & 0.387 \\
\hline $\begin{array}{l}\text { Pu-238 (1 Outlier } \\
\text { Removed) }{ }^{\text {h }}\end{array}$ & 37 & 84 & $<0.0027$ & 0.255 & 0.0421 & 0.058 & Non-Parametric & 0.255 \\
\hline $\mathrm{Pu}-239 / 240$ & 39 & 90 & $<0.003$ & 0.73 & 0.0505 & 0.132 & Non-Parametric & 0.73 \\
\hline $\begin{array}{l}\text { Pu-239/240 (1 } \\
\text { Outlier Removed })^{\mathrm{i}}\end{array}$ & 38 & 89 & $<0.003$ & 0.37 & 0.0306 & 0.0605 & Non-Parametric & 0.37 \\
\hline $\begin{array}{l}\text { Pu-239/240 (2 } \\
\text { Outliers Removed })^{j}\end{array}$ & 35 & 94 & $<0.003$ & 0.038 & 0.0194 & 0.00841 & Normal & 0.0445 \\
\hline $\mathrm{Sb}-125$ & 181 & 44 & $<0.0606$ & 1.492 & 0.373 & 0.222 & Non-Parametric & 1.492 \\
\hline $\begin{array}{l}\text { Sb-125 (1 Outlier } \\
\text { Removed) }\end{array}$ & 180 & 43 & $<0.0606$ & 1.075 & 0.359 & 0.183 & Gamma & 1.331 \\
\hline Sr-90 & 11 & 100 & 0.39 & 10.6 & 2.966 & 3.098 & Normal & 14.9 \\
\hline
\end{tabular}


Table B-3. (continued).

\begin{tabular}{|c|c|c|c|c|c|c|c|c|}
\hline Radionuclide & $\mathrm{N}$ & $\begin{array}{c}\text { Percent } \\
\text { Detected }\end{array}$ & $\begin{array}{l}\operatorname{Minimum}^{\mathrm{a}} \\
(\mathrm{pCi} / \mathrm{g})\end{array}$ & $\begin{array}{l}\text { Maximum } \\
(\mathrm{pCi} / \mathrm{g})\end{array}$ & $\begin{array}{l}\text { Mean } \\
(\mathrm{pCi} / \mathrm{g})\end{array}$ & $\begin{array}{c}\text { Standard } \\
\text { Deviation } \\
(\mathrm{pCi} / \mathrm{g})\end{array}$ & $\begin{array}{l}\text { Distribution } \\
\text { of the Data }\end{array}$ & $\begin{array}{c}\text { UTL } \\
(\mathrm{pCi} / \mathrm{g})\end{array}$ \\
\hline $\begin{array}{l}\text { Sr-90 (1 Outlier } \\
\text { Removed) }\end{array}$ & 10 & 100 & 0.39 & 5.7 & 2.203 & 1.883 & Normal & 9.698 \\
\hline U-234 & 203 & 14 & $<6.501$ & 669.5 & $\mathrm{NA}^{\mathrm{b}}$ & $\mathrm{NA}^{\mathrm{b}}$ & $\mathrm{NA}^{\mathrm{b}}$ & $669.5^{\mathrm{c}}$ \\
\hline $\begin{array}{l}\text { U-234 (1 Outlier } \\
\text { Removed) }{ }^{\mathrm{m}}\end{array}$ & 119 & 21 & $<6.501$ & 171 & $\mathrm{NA}^{\mathrm{b}}$ & $\mathrm{NA}^{\mathrm{b}}$ & $\mathrm{NA}^{\mathrm{b}}$ & $171^{\mathrm{c}}$ \\
\hline $\mathrm{U}-235$ & 311 & 43 & $<0.044$ & 3.598 & 0.69 & 0.637 & Non-Parametric & 3.213 \\
\hline U-238 & 299 & 59 & $<0.237$ & 256.3 & 15.62 & 37.22 & Non-Parametric & 227.9 \\
\hline \multicolumn{9}{|c|}{$\begin{array}{l}\text { ND }=\text { nondetect } \\
\text { a. Less than sign indicates that the actual minimum concentration is unknown, but it known to be less than a certain value. } \\
\text { b. A minimum of } 8 \text { detects and } 30 \% \text { of the data detected are needed to estimate a mean, standard deviation, assess the distributio } \\
\text { c. Insufficient detected data are available to compute a UTL. The maximum detected value was used as the UTL. } \\
\text { d. Outlier at B } 80 \text { in } 2007 \text {. An additional ND was removed because the MDA was higher than the next detected value. } \\
\text { e. Outlier at B69 in } 2007 . \\
\text { f. Outlier at B37 in } 1974 . \\
\text { g. Outlier at B38 in } 1974 . \\
\text { h. Outlier at B96 in } 1989 . \\
\text { i. Outlier at B96 in } 1989 \text {. } \\
\text { j. Outlier at A17 in } 1982 \text {. Two additional NDs were removed because the MDAs were higher than the next detected value. } \\
\text { k. Outlier at B69 in 2007. } \\
\text { 1. Outlier at A46 in } 1989 \text {. } \\
\text { m. Outlier at B36 in } 2007 \text {. Forty-six additional NDs were removed because the MDAs were higher than the next detected value. }\end{array}$} \\
\hline
\end{tabular}

Table B-4. Summary statistics for data collected at MFC.

\begin{tabular}{|l|c|c|c|c|c|c|c|c|}
\hline \multicolumn{1}{|c|}{ Radionuclide } & $\mathrm{N}$ & $\begin{array}{c}\text { Percent } \\
\text { Detected }\end{array}$ & $\begin{array}{c}\text { Minimum } \\
(\mathrm{pCi} / \mathrm{g})\end{array}$ & $\begin{array}{c}\text { Maximum } \\
(\mathrm{pCi} / \mathrm{g})\end{array}$ & $\begin{array}{c}\text { Mean } \\
(\mathrm{pCi} / \mathrm{g})\end{array}$ & $\begin{array}{c}\text { Standard } \\
\text { Deviation } \\
(\mathrm{pCi} / \mathrm{g})\end{array}$ & $\begin{array}{c}\text { Distribution } \\
\text { of the Data }\end{array}$ & $\begin{array}{c}\text { UTL } \\
(\mathrm{pCi} / \mathrm{g})\end{array}$ \\
\hline Am-241 & 9 & 44 & $<0.0042$ & 0.008 & $\mathrm{NA}^{\mathrm{b}}$ & $\mathrm{NA}^{\mathrm{b}}$ & $\mathrm{NA}^{\mathrm{b}}$ & $0.008^{\mathrm{c}}$ \\
\hline Co-60 & 51 & 31 & $<0.000939$ & 0.203 & 0.0323 & 0.0548 & Normal & 0.188 \\
\hline Cs-134 & 87 & 61 & $<0.00905$ & 0.131 & 0.0414 & 0.0275 & Normal & 0.116 \\
\hline Cs-137 & 156 & 98 & $<0.0523$ & 1.99 & 0.455 & 0.37 & Non-Parametric & 1.99 \\
\hline
\end{tabular}


Table B-4. (continued).

\begin{tabular}{|c|c|c|c|c|c|c|c|c|}
\hline Radionuclide & $\mathrm{N}$ & $\begin{array}{c}\text { Percent } \\
\text { Detected }\end{array}$ & $\begin{array}{l}\text { Minimum }^{\mathrm{a}} \\
(\mathrm{pCi} / \mathrm{g})\end{array}$ & $\begin{array}{l}\text { Maximum } \\
(\mathrm{pCi} / \mathrm{g})\end{array}$ & $\begin{array}{l}\text { Mean } \\
(\mathrm{pCi} / \mathrm{g})\end{array}$ & $\begin{array}{c}\text { Standard } \\
\text { Deviation } \\
(\mathrm{pCi} / \mathrm{g})\end{array}$ & $\begin{array}{l}\text { Distribution } \\
\text { of the Data }\end{array}$ & $\begin{array}{c}\text { UTL } \\
(\mathrm{pCi} / \mathrm{g})\end{array}$ \\
\hline Eu-152 & 38 & 8 & $<0.0449$ & 0.276 & $\mathrm{NA}^{\mathrm{b}}$ & $\mathrm{NA}^{\mathrm{b}}$ & $\mathrm{NA}^{\mathrm{b}}$ & $0.276^{\mathrm{c}}$ \\
\hline $\begin{array}{l}\text { Eu-152 (1 Outlier } \\
\text { Removed })^{d}\end{array}$ & 37 & 5 & $<0.0449$ & 0.145 & $\mathrm{NA}^{\mathrm{b}}$ & $\mathrm{NA}^{\mathrm{b}}$ & $\mathrm{NA}^{\mathrm{b}}$ & $0.145^{\mathrm{c}}$ \\
\hline $\mathrm{Pu}-238$ & 9 & 11 & $<0.0021$ & 0.01 & $\mathrm{NA}^{\mathrm{b}}$ & $\mathrm{NA}^{\mathrm{b}}$ & $\mathrm{NA}^{\mathrm{b}}$ & $0.01^{\mathrm{c}}$ \\
\hline $\mathrm{Pu}-239 / 240$ & 9 & 100 & 0.009 & 0.032 & 0.0198 & 0.00698 & Normal & 0.0487 \\
\hline $\mathrm{Sb}-125$ & 38 & 16 & $<0.0484$ & 0.393 & $\mathrm{NA}^{\mathrm{b}}$ & $\mathrm{NA}^{\mathrm{b}}$ & $\mathrm{NA}^{\mathrm{b}}$ & $0.393^{\mathrm{c}}$ \\
\hline $\begin{array}{l}\text { Sb-125 (1 Outlier } \\
\text { Removed) }\end{array}$ & 37 & 14 & $<0.0484$ & 0.226 & $\mathrm{NA}^{\mathrm{b}}$ & $\mathrm{NA}^{\mathrm{b}}$ & $\mathrm{NA}^{\mathrm{b}}$ & $0.226^{\mathrm{c}}$ \\
\hline Sr-90 & 9 & 100 & 0.45 & 0.74 & 0.578 & 0.0907 & Normal & 0.953 \\
\hline U-234 & 86 & 47 & $<6.782$ & 328.9 & 81.52 & 72.2 & Gamma & 498.1 \\
\hline $\begin{array}{l}\text { U-234 (1 Outlier } \\
\text { Removed) }{ }^{\mathrm{f}}\end{array}$ & 81 & 48 & $<6.782$ & 238.8 & 75.18 & 60.82 & Gamma & 411.8 \\
\hline U-235 & 101 & 38 & $<0.161$ & 2.289 & 0.749 & 0.796 & Non-Parametric & 3.29 \\
\hline U-238 & 95 & 25 & $<1.18$ & 164.5 & 29.58 & 34.12 & Gamma & 85.44 \\
\hline $\begin{array}{l}\text { U-238 (1 Outliers } \\
\text { Removed) }\end{array}$ & 92 & 25 & $<1.18$ & 87.7 & 7.381 & 13.44 & Normal & 43.64 \\
\hline $\begin{array}{l}\text { U-238 (3 Outliers } \\
\text { Removed) }{ }^{\mathrm{h}}\end{array}$ & 91 & 24 & $<1.18$ & 45.31 & 6.498 & 10.54 & Normal & 34.94 \\
\hline \multicolumn{9}{|c|}{$\begin{array}{l}\text { ND }=\text { nondetect } \\
\text { a. Less than sign indicates that the actual minimum concentration is unknown, but it known to be less than a certain value. } \\
\text { b. A minimum of } 8 \text { detects and } 30 \% \text { of the data detected are needed to estimate a mean, standard deviation, assess the distribution } \\
\text { c. Insufficient detected data are available to compute a UTL. The maximum detected value was used as the UTL. } \\
\text { d. Outlier at EBRII-10 in } 2013 \text {. } \\
\text { e. Outlier at EBRII-1 } 1 \text { in } 2013 \text {. } \\
\text { f. Outlier at TREAT- } 4 \text { in } 2007 \text {. Four additional NDs were removed because the MDAs were higher than the next detected value. } \\
\text { g. Outlier at TREAT-1 in } 2010 \text {. Two additional NDs were removed because the MDAs were higher than the next detected value. } \\
\text { h. Outlier at TREAT-3 in } 2010 \text {. Two additional NDs were removed because the MDAs were higher than the next detected value. }\end{array}$} \\
\hline
\end{tabular}


Table B-5. Summary statistics for data collected at RWMC.

\begin{tabular}{|c|c|c|c|c|c|c|c|c|}
\hline Radionuclide & $\mathrm{N}$ & $\begin{array}{c}\text { Percent } \\
\text { Detected }\end{array}$ & $\begin{array}{l}\operatorname{Minimum}^{\mathrm{a}} \\
(\mathrm{pCi} / \mathrm{g})\end{array}$ & $\begin{array}{l}\text { Maximum } \\
(\mathrm{pCi} / \mathrm{g})\end{array}$ & $\begin{array}{c}\text { Mean } \\
(\mathrm{pCi} / \mathrm{g})\end{array}$ & $\begin{array}{c}\text { Standard } \\
\text { Deviation } \\
(\mathrm{pCi} / \mathrm{g})\end{array}$ & $\begin{array}{l}\text { Distribution } \\
\text { of the Data }\end{array}$ & $\begin{array}{c}\text { UTL } \\
(\mathrm{pCi} / \mathrm{g})\end{array}$ \\
\hline Am-241 & 156 & 76 & $<0.00195$ & 15.27 & 0.435 & 1.658 & Non-Parametric & 15.27 \\
\hline $\begin{array}{l}\text { Am-241 (1 Outlier } \\
\text { Removed) })^{b}\end{array}$ & 155 & 76 & $<0.00195$ & 8.4 & 0.309 & 0.936 & Non-Parametric & 8.4 \\
\hline Co-60 & 165 & 16 & $<0.00242$ & 53 & $\mathrm{NA}^{\mathrm{c}}$ & $\mathrm{NA}^{\mathrm{c}}$ & $\mathrm{NA}^{\mathrm{c}}$ & $53^{\mathrm{d}}$ \\
\hline Cs-134 & 84 & 70 & $<0.0152$ & 0.142 & 0.0488 & 0.0247 & Normal & 0.116 \\
\hline Cs-137 & 286 & 83 & $<0.0525$ & 3.76 & 0.5 & 0.55 & Non-Parametric & 3.54 \\
\hline Eu-152 & 79 & 58 & $<0.0627$ & 1.403 & 0.37 & 0.273 & Non-Parametric & 1.403 \\
\hline $\mathrm{Pu}-238$ & 112 & 38 & $<0.0005$ & 0.058 & 0.0093 & 0.0116 & Non-Parametric & 0.058 \\
\hline $\begin{array}{l}\text { Pu-238 (2 Outliers } \\
\text { Removed) })^{\mathrm{e}}\end{array}$ & 109 & 38 & $<0.0005$ & 0.02 & 0.00693 & 0.0041 & Non-Parametric & 0.02 \\
\hline $\mathrm{Pu}-239 / 240$ & 112 & 96 & $<0.0027$ & 2.57 & 0.09 & 0.263 & Non-Parametric & 2.57 \\
\hline $\begin{array}{l}\mathrm{Pu}-239 / 240(1 \\
\text { Outlier Removed })^{\mathrm{f}}\end{array}$ & 111 & 96 & $<0.0027$ & 0.644 & 0.0668 & 0.106 & Non-Parametric & 0.644 \\
\hline $\mathrm{Sb}-125$ & 86 & 53 & $<0.0897$ & 0.672 & 0.2 & 0.133 & Normal & 0.561 \\
\hline Sr-90 & 20 & 100 & 0.36 & 2.52 & 0.861 & 0.488 & Normal & 2.47 \\
\hline $\begin{array}{l}\text { Sr-90 (1 Outlier } \\
\text { Removed })^{\mathrm{g}}\end{array}$ & 19 & 100 & 0.36 & 1.31 & 0.774 & 0.301 & Normal & 1.777 \\
\hline U-234 & 29 & 100 & 0.86 & 1.18 & 1.045 & 0.0671 & Normal & 1.252 \\
\hline $\mathrm{U}-235$ & 98 & 82 & $<3.03$ & 188.2 & 33.95 & 29.42 & Non-Parametric & 188.2 \\
\hline
\end{tabular}


Table B-5. (continued).

\begin{tabular}{|c|c|c|c|c|c|c|c|c|}
\hline Radionuclide & $\mathrm{N}$ & $\begin{array}{c}\text { Percent } \\
\text { Detected }\end{array}$ & $\begin{array}{l}\text { Minimum }^{\mathrm{a}} \\
(\mathrm{pCi} / \mathrm{g})\end{array}$ & $\begin{array}{l}\text { Maximum } \\
(\mathrm{pCi} / \mathrm{g})\end{array}$ & $\begin{array}{l}\text { Mean } \\
(\mathrm{pCi} / \mathrm{g})\end{array}$ & $\begin{array}{c}\text { Standard } \\
\text { Deviation } \\
(\mathrm{pCi} / \mathrm{g})\end{array}$ & $\begin{array}{l}\text { Distribution } \\
\text { of the Data }\end{array}$ & $\begin{array}{c}\text { UTL } \\
(\mathrm{pCi} / \mathrm{g})\end{array}$ \\
\hline U-238 & 216 & 81 & $<0.043$ & 11.9 & 0.656 & 1.154 & Non-Parametric & 11.9 \\
\hline $\begin{array}{l}\text { U-238 (1 Outlier } \\
\text { Removed })^{\mathrm{h}}\end{array}$ & 215 & 80 & $<0.043$ & 5.797 & 0.591 & 0.774 & Non-Parametric & 5.797 \\
\hline \multicolumn{9}{|c|}{$\begin{array}{l}\text { ND }=\text { nondetect } \\
\text { a. Less than sign indicates that } \\
\text { b. Outlier at 2-4 in } 2013 . \\
\text { c. A minimum of } 8 \text { detects and } \\
\text { d. Insufficient detected data are } \\
\text { e. Outlier at 5-1 in } 1973 \text { and } 7 \\
\text { f. Outlier at 5-1 in } 1973 . \\
\text { g. Outlier at 3-2 in } 1978 . \\
\text { h. Outlier at 5-4 in 2010. }\end{array}$} \\
\hline
\end{tabular}

Table B-6. Summary statistics for data collected at TAN.

\begin{tabular}{|c|c|c|c|c|c|c|c|c|}
\hline Radionuclide & $\mathrm{N}$ & $\begin{array}{l}\text { Percent } \\
\text { Detected }\end{array}$ & $\begin{array}{c}\operatorname{Minimum}^{\mathrm{a}} \\
\mathrm{pCi} / \mathrm{g})\end{array}$ & $\begin{array}{l}\text { Maximum } \\
(\mathrm{pCi} / \mathrm{g})\end{array}$ & $\begin{array}{l}\text { Mean } \\
(\mathrm{pCi} / \mathrm{g})\end{array}$ & $\begin{array}{c}\text { Standard } \\
\text { Deviation } \\
\text { (pCi } / \mathrm{g})\end{array}$ & $\begin{array}{l}\text { Distribution } \\
\text { of the Data }\end{array}$ & $\begin{array}{c}\text { UTL } \\
(\mathrm{pCi} / \mathrm{g})\end{array}$ \\
\hline Am-241 & 11 & 36 & $<0.003$ & 0.086 & $\mathrm{NA}^{\mathrm{b}}$ & $\mathrm{NA}^{\mathrm{b}}$ & $\mathrm{NA}^{\mathrm{b}}$ & $0.086^{\mathrm{c}}$ \\
\hline $\begin{array}{l}\text { Am-241 (1 Outlier } \\
\text { Removed) }{ }^{\mathrm{d}}\end{array}$ & 10 & 30 & $<0.003$ & 0.029 & $N A^{b}$ & $N A^{b}$ & $\mathrm{NA}^{\mathrm{b}}$ & $0.029^{c}$ \\
\hline Сo-60 & 47 & 28 & $<0.00306$ & 0.21 & $\mathrm{NA}^{\mathrm{b}}$ & $\mathrm{NA}^{\mathrm{b}}$ & $\mathrm{NA}^{\mathrm{b}}$ & $0.21^{\mathrm{c}}$ \\
\hline $\begin{array}{l}\text { Co-60 (1 Outlier } \\
\text { Removed })^{\mathrm{e}}\end{array}$ & 46 & 26 & $<0.00306$ & 0.131 & $\mathrm{NA}^{\mathrm{b}}$ & $\mathrm{NA}^{\mathrm{b}}$ & $\mathrm{NA}^{\mathrm{b}}$ & $0.131^{\mathrm{c}}$ \\
\hline Cs-134 & 62 & 65 & $<0.0144$ & 0.108 & 0.0461 & 0.0251 & Normal & 0.116 \\
\hline Cs-137 & 135 & 99 & $<0.0164$ & 23.8 & 0.879 & 2.443 & Non-Parametric & 23.8 \\
\hline $\begin{array}{l}\text { Cs-137 (1 Outlier } \\
\text { Removed) }\end{array}$ & 134 & 99 & $<0.0164$ & 12.7 & 0.707 & 1.415 & Non-Parametric & 12.7 \\
\hline Eu-152 & 28 & 7 & $<0.0483$ & 0.155 & $\mathrm{NA}^{\mathrm{b}}$ & $\mathrm{NA}^{\mathrm{b}}$ & $\mathrm{NA}^{\mathrm{b}}$ & $0.155^{\mathrm{c}}$ \\
\hline $\mathrm{Pu}-238$ & 11 & 18 & $<0.003$ & 0.014 & $\mathrm{NA}^{\mathrm{b}}$ & $\mathrm{NA}^{\mathrm{b}}$ & $\mathrm{NA}^{\mathrm{b}}$ & $0.014^{\mathrm{c}}$ \\
\hline
\end{tabular}


Table B-6. (continued).

\begin{tabular}{|c|c|c|c|c|c|c|c|c|}
\hline Radionuclide & $\mathrm{N}$ & $\begin{array}{l}\text { Percent } \\
\text { Detected }\end{array}$ & $\begin{array}{c}\left.\operatorname{Minimum}^{\mathrm{a}} \mathrm{pCi} / \mathrm{g}\right) \\
\end{array}$ & $\begin{array}{l}\text { Maximum } \\
(\mathrm{pCi} / \mathrm{g})\end{array}$ & $\begin{array}{c}\text { Mean } \\
(\mathrm{pCi} / \mathrm{g})\end{array}$ & $\begin{array}{l}\text { Standard } \\
\text { Deviation } \\
(\mathrm{pCi} / \mathrm{g})\end{array}$ & $\begin{array}{l}\text { Distribution } \\
\text { of the Data }\end{array}$ & $\begin{array}{c}\mathrm{UTL} \\
(\mathrm{pCi} / \mathrm{g})\end{array}$ \\
\hline $\mathrm{Pu}-239 / 240$ & 11 & 100 & 0.008 & 0.029 & 0.0155 & 0.00712 & Non-Parametric & 0.029 \\
\hline $\mathrm{Sb}-125$ & 28 & 11 & $<0.0747$ & 0.804 & $\mathrm{NA}^{\mathrm{b}}$ & $\mathrm{NA}^{\mathrm{b}}$ & $\mathrm{NA}^{\mathrm{b}}$ & $0.804^{\mathrm{c}}$ \\
\hline $\begin{array}{l}\text { Sb-125 (1 Outlier } \\
\text { Removed) }\end{array}$ & 27 & 7 & $<0.0747$ & 0.686 & $\mathrm{NA}^{\mathrm{b}}$ & $\mathrm{NA}^{\mathrm{b}}$ & $\mathrm{NA}^{\mathrm{b}}$ & $0.686^{\mathrm{c}}$ \\
\hline $\begin{array}{l}\text { Sb-125 (2 Outliers } \\
\text { Removed })^{\mathrm{h}}\end{array}$ & 26 & 4 & $<0.0747$ & 0.267 & $\mathrm{NA}^{\mathrm{b}}$ & $\mathrm{NA}^{\mathrm{b}}$ & $\mathrm{NA}^{\mathrm{b}}$ & $0.267^{\mathrm{c}}$ \\
\hline Sr-90 & 8 & 100 & 039 & 1.15 & 0.636 & 0.257 & Normal & 1.754 \\
\hline U-234 & 62 & 55 & $<5.891$ & 413.9 & 64.66 & 71.75 & Non-Parametric & 413.9 \\
\hline $\begin{array}{l}\text { U-234 (1 Outlier } \\
\text { Removed) }\end{array}$ & 36 & 92 & $<5.891$ & 111.3 & 54.07 & 37.17 & Non-Parametric & 111.3 \\
\hline U-235 & 83 & 40 & $<0.129$ & 3.193 & 0.845 & 0.829 & Non-Parametric & 3.193 \\
\hline $\mathrm{U}-238$ & 69 & 29 & $<1.368$ & 111.4 & $\mathrm{NA}^{\mathrm{b}}$ & $\mathrm{NA}^{\mathrm{b}}$ & $\mathrm{NA}^{\mathrm{b}}$ & $111.4^{\mathrm{c}}$ \\
\hline $\begin{array}{l}\text { U-238 (1 Outlier } \\
\text { Removed) }\end{array}$ & 68 & 28 & $<1.368$ & 86.8 & $\mathrm{NA}^{\mathrm{b}}$ & $\mathrm{NA}^{\mathrm{b}}$ & $\mathrm{NA}^{\mathrm{b}}$ & $86.8^{\mathrm{c}}$ \\
\hline \multicolumn{9}{|c|}{$\begin{array}{l}\text { ND }=\text { nondetect } \\
\text { a. Less than sign indicates that the actual minimum concentration is unknown, but it known to be less than a certain value. } \\
\text { b. A minimum of } 8 \text { detects and } 30 \% \text { of the data detected are needed to estimate a mean, standard deviation, assess the distribution of } \\
\text { c. Insufficient detected data are available to compute a UTL. The maximum detected value was used as the UTL. } \\
\text { d. Outlier at TSF-6 in } 1988 \text {. } \\
\text { e. Outlier at TSF-3 in } 1981 . \\
\text { f. Outlier at TSF-3 in } 2007 \text {. } \\
\text { g. Outlier at TSF-9 in } 2014 \text {. } \\
\text { h. Outlier at L2-76 in } 2011 \text {. } \\
\text { i. Outlier at TSF-8 in } 2007 \text {. Twenty-five additional NDs were removed because the MDAs were higher than the next detected value. } \\
\text { j. Outlier at IET-8 in } 2007 \text {. }\end{array}$} \\
\hline
\end{tabular}


Table B-7. Summary statistics for data collected at Atomic City.

\begin{tabular}{|c|c|c|c|c|c|c|c|c|}
\hline Radionuclide & $\mathrm{N}$ & $\begin{array}{c}\text { Percent } \\
\text { Detected }\end{array}$ & $\begin{array}{c}\text { Minimum }^{\mathrm{a}} \\
(\mathrm{pCi} / \mathrm{g})\end{array}$ & $\begin{array}{c}\text { Maximum } \\
(\mathrm{pCi} / \mathrm{g})\end{array}$ & $\begin{array}{c}\text { Mean } \\
(\mathrm{pCi} / \mathrm{g})\end{array}$ & $\begin{array}{c}\text { Standard } \\
\text { Deviation } \\
(\mathrm{pCi} / \mathrm{g})\end{array}$ & $\begin{array}{l}\text { Distribution } \\
\text { of the Data }\end{array}$ & $\begin{array}{c}\text { UTL } \\
\text { (pCi/g) }\end{array}$ \\
\hline Am-241 & 11 & 64 & $<0.00569$ & 0.02 & 0.00919 & 0.00484 & Normal & 0.0278 \\
\hline Cs-137 & 21 & 95 & $<0.0788$ & 0.81 & 0.349 & 0.203 & Normal & 1.012 \\
\hline $\mathrm{Pu}-238$ & 10 & 80 & $<0.00211$ & 0.014 & 0.00536 & 0.00406 & Normal & 0.0227 \\
\hline $\mathrm{Pu}-239 / 240$ & 11 & 91 & $<0.011$ & 0.041 & 0.0234 & 0.00881 & Normal & 0.0573 \\
\hline Sr-90 & 9 & 100 & 0.0453 & 0.42 & 0.225 & 0.123 & Normal & 0.734 \\
\hline
\end{tabular}

Table B-8. Summary statistics for data collected at Blackfoot.

\begin{tabular}{|c|c|c|c|c|c|c|c|c|}
\hline Radionuclide & $\mathrm{N}$ & $\begin{array}{c}\text { Percent } \\
\text { Detected }\end{array}$ & $\begin{array}{c}\text { Minimum }^{\mathrm{a}} \\
(\mathrm{pCi} / \mathrm{g})\end{array}$ & $\begin{array}{c}\text { Maximum } \\
(\mathrm{pCi} / \mathrm{g})\end{array}$ & $\begin{array}{c}\text { Mean } \\
(\mathrm{pCi} / \mathrm{g})\end{array}$ & $\begin{array}{c}\text { Standard } \\
\text { Deviation } \\
(\mathrm{pCi} / \mathrm{g})\end{array}$ & $\begin{array}{l}\text { Distribution } \\
\text { of the Data }\end{array}$ & $\begin{array}{c}\text { UTL } \\
(\mathrm{pCi} / \mathrm{g})\end{array}$ \\
\hline Am-241 & 11 & 91 & $<0.00423$ & 0.0209 & 0.00952 & 0.00498 & Gamma & 0.0405 \\
\hline Cs-137 & 20 & 100 & 0.0347 & 1.3 & 0.408 & 0.322 & Gamma & 2.697 \\
\hline $\mathrm{Pu}-238$ & 12 & 67 & $<0.002$ & 0.154 & 0.028 & 0.0512 & Non-Parametric & 0.154 \\
\hline $\mathrm{Pu}-239 / 240$ & 12 & 92 & $<0.0019$ & 0.0885 & 0.0228 & 0.0229 & Gamma & 0.239 \\
\hline Sr-90 & 11 & 91 & $<0.0115$ & 0.27 & 0.0964 & 0.0783 & Normal & 0.398 \\
\hline
\end{tabular}


Table B- 9. Summary statistics for data collected at Butte City.

\begin{tabular}{|l|c|c|c|c|c|c|c|c|}
\hline \multicolumn{1}{|c|}{ Radionuclide } & $\mathrm{N}$ & $\begin{array}{c}\text { Percent } \\
\text { Detected }\end{array}$ & $\begin{array}{c}\text { Minimum } \\
(\mathrm{pCi} / \mathrm{g})\end{array}$ & $\begin{array}{c}\text { Maximum } \\
(\mathrm{pCi} / \mathrm{g})\end{array}$ & $\begin{array}{c}\text { Mean } \\
(\mathrm{pCi} / \mathrm{g})\end{array}$ & $\begin{array}{c}\text { Standard } \\
\text { Deviation } \\
(\mathrm{pCi} / \mathrm{g})\end{array}$ & $\begin{array}{c}\text { Distribution } \\
\text { of the Data }\end{array}$ & $\begin{array}{c}\text { UTL } \\
(\mathrm{pCi} / \mathrm{g})\end{array}$ \\
\hline $\mathrm{Am}-241$ & 11 & 82 & $<0.002$ & 0.0942 & 0.0173 & 0.0291 & Non-Parametric & 0.0942 \\
\hline $\mathrm{Cs}-137$ & 21 & 100 & 0.0698 & 0.96 & 0.373 & 0.268 & Normal & 1.248 \\
\hline $\mathrm{Pu}-238$ & 11 & 73 & $<0.00166$ & 0.0192 & 0.00835 & 0.00657 & Normal & 0.0337 \\
\hline $\mathrm{Pu}-239 / 240$ & 10 & 80 & $<0.00261$ & 0.03 & 0.0179 & 0.00771 & Normal & 0.0487 \\
\hline Sr-90 & 9 & 100 & 0.0448 & 0.31 & 0.177 & 0.177 & Normal & 0.56 \\
\hline
\end{tabular}

Table B-10. Summary statistics for data collected at Carey.

\begin{tabular}{|l|c|c|c|c|c|c|c|c|}
\hline \multicolumn{1}{|c|}{ Radionuclide } & $\mathrm{N}$ & $\begin{array}{c}\text { Percent } \\
\text { Detected }\end{array}$ & $\begin{array}{c}\text { Minimum } \\
(\mathrm{pCi} / \mathrm{g})\end{array}$ & $\begin{array}{c}\text { Maximum } \\
(\mathrm{pCi} / \mathrm{g})\end{array}$ & $\begin{array}{c}\text { Mean } \\
(\mathrm{pCi} / \mathrm{g})\end{array}$ & $\begin{array}{c}\text { Standard } \\
\text { Deviation } \\
(\mathrm{pCi} / \mathrm{g})\end{array}$ & $\begin{array}{c}\text { Distribution } \\
\text { of the Data }\end{array}$ & $\begin{array}{c}\text { UTL } \\
(\mathrm{pCi} / \mathrm{g})\end{array}$ \\
\hline $\mathrm{Am}-241$ & 10 & 60 & $<0.00913$ & 0.0556 & $\mathrm{NA}^{\mathrm{b}}$ & $\mathrm{NA}^{\mathrm{b}}$ & $\mathrm{NA}^{\mathrm{b}}$ & $0.0556^{\mathrm{c}}$ \\
\hline $\mathrm{Cs}-137$ & 21 & 100 & 0.0876 & 0.759 & 0.347 & 0.189 & Normal & 0.963 \\
\hline $\mathrm{Pu}-238$ & 11 & 82 & $<0.002$ & 0.0336 & 0.0096 & 0.0091 & Normal & 0.0447 \\
\hline $\mathrm{Pu}-239 / 240$ & 11 & 91 & $<0.00867$ & 0.0442 & 0.0241 & 0.0112 & Normal & 0.0671 \\
\hline $\mathrm{Sr}-90$ & 11 & 82 & $<0.0447$ & 0.36 & 0.145 & 0.101 & Normal \\
\hline
\end{tabular}


Table B-11. Summary statistics for data collected at FAA.

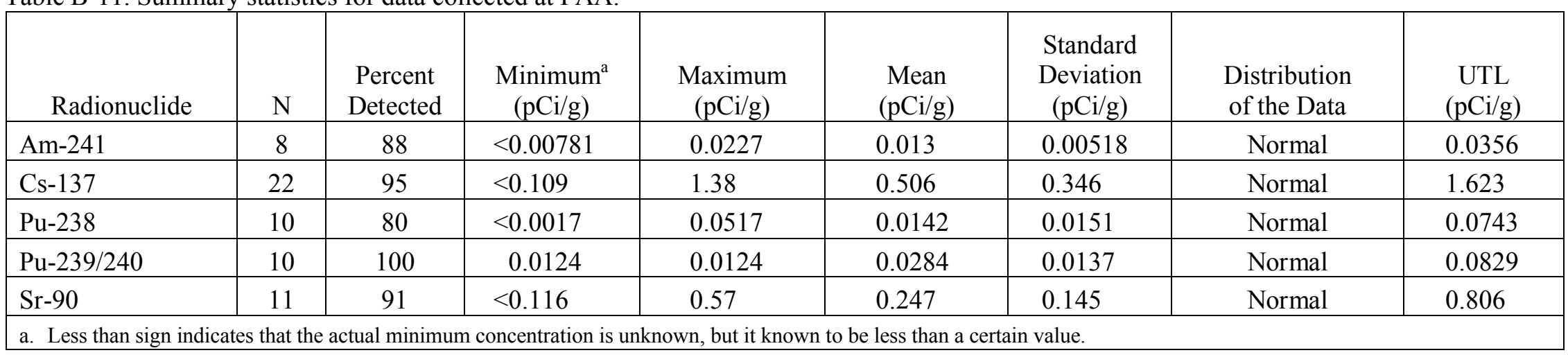

Table B-12. Summary statistics for data collected at Howe.

\begin{tabular}{|c|c|c|c|c|c|c|c|c|}
\hline Radionuclide & $\mathrm{N}$ & $\begin{array}{c}\text { Percent } \\
\text { Detected }\end{array}$ & $\begin{array}{c}\operatorname{Minimum}^{\mathrm{a}} \\
(\mathrm{pCi} / \mathrm{g})\end{array}$ & $\begin{array}{c}\text { Maximum } \\
(\mathrm{pCi} / \mathrm{g})\end{array}$ & $\begin{array}{c}\text { Mean } \\
(\mathrm{pCi} / \mathrm{g})\end{array}$ & $\begin{array}{c}\text { Standard } \\
\text { Deviation } \\
(\mathrm{pCi} / \mathrm{g})\end{array}$ & $\begin{array}{c}\text { Distribution } \\
\text { of the Data }\end{array}$ & $\begin{array}{c}\text { UTL } \\
(\mathrm{pCi} / \mathrm{g})\end{array}$ \\
\hline Am-241 & 9 & 33 & $<0.00424$ & 0.01 & $\mathrm{NA}^{\mathrm{b}}$ & $\mathrm{NA}^{\mathrm{b}}$ & $\mathrm{NA}^{\mathrm{b}}$ & $0.01^{\mathrm{c}}$ \\
\hline Cs-137 & 22 & 100 & 0.074 & 0.525 & 0.264 & 0.135 & Normal & 0.7 \\
\hline $\mathrm{Pu}-238$ & 9 & 44 & $<0.001$ & 0.0119 & $\mathrm{NA}^{\mathrm{b}}$ & $\mathrm{NA}^{\mathrm{b}}$ & $\mathrm{NA}^{\mathrm{b}}$ & $0.0119^{\mathrm{c}}$ \\
\hline $\mathrm{Pu}-239 / 240$ & 9 & 89 & $<0.00561$ & 0.02 & 0.0122 & 0.00559 & Normal & 0.0353 \\
\hline Sr-90 & 9 & 100 & 0.0204 & 0.35 & 0.157 & 0.124 & Normal & 0.67 \\
\hline
\end{tabular}


Table B-13. Summary statistics for data collected at Monteview.

\begin{tabular}{|c|c|c|c|c|c|c|c|c|}
\hline Radionuclide & $\mathrm{N}$ & $\begin{array}{l}\text { Percent } \\
\text { Detected }\end{array}$ & $\underset{(\mathrm{pCi} / \mathrm{g})}{\operatorname{Minimum}^{\mathrm{a}}}$ & $\begin{array}{l}\text { Maximum } \\
(\mathrm{pCi} / \mathrm{g})\end{array}$ & $\begin{array}{c}\text { Mean } \\
(\mathrm{pCi} / \mathrm{g})\end{array}$ & $\begin{array}{l}\text { Standard } \\
\text { Deviation } \\
\text { (pCi } / \mathrm{g})\end{array}$ & $\begin{array}{l}\text { Distribution } \\
\text { of the Data }\end{array}$ & $\begin{array}{c}\mathrm{UTL} \\
(\mathrm{pCi} / \mathrm{g})\end{array}$ \\
\hline $\mathrm{Am}-241$ & 10 & 60 & $<0.00335$ & 0.0194 & $\mathrm{NA}^{\mathrm{b}}$ & $\mathrm{NA}^{\mathrm{b}}$ & $\mathrm{NA}^{\mathrm{b}}$ & $0.0194^{\mathrm{c}}$ \\
\hline Cs-137 & 22 & 100 & 0.1 & 1.11 & 0.357 & 0.219 & Non-Parametric & 1.11 \\
\hline $\begin{array}{l}\text { Cs-137 (1 Outlier } \\
\text { Removed) }\end{array}$ & 21 & 100 & 0.1 & 0.82 & 0.321 & 0.144 & Gamma & 0.986 \\
\hline $\mathrm{Pu}-238$ & 10 & 80 & $<0.000619$ & 0.0227 & 0.00685 & 0.00706 & Normal & 0.035 \\
\hline $\mathrm{Pu}-239 / 240$ & 11 & 73 & $<0.000619$ & 0.03 & 0.0152 & 0.00844 & Normal & 0.0477 \\
\hline Sr-90 & 9 & 89 & $<0.0297$ & 0.17 & 0.0827 & 0.0447 & Normal & 0.268 \\
\hline
\end{tabular}

Table B-14. Summary statistics for data collected at Mud Lake.

\begin{tabular}{|l|c|c|c|c|c|c|c|c|}
\hline \multicolumn{1}{|c|}{ Radionuclide } & $\mathrm{N}$ & $\begin{array}{c}\text { Percent } \\
\text { Detected }\end{array}$ & $\begin{array}{c}\text { Minimum } \\
(\mathrm{pCi} / \mathrm{g})\end{array}$ & $\begin{array}{c}\text { Maximum } \\
(\mathrm{pCi} / \mathrm{g})\end{array}$ & $\begin{array}{c}\text { Mean } \\
(\mathrm{pCi} / \mathrm{g})\end{array}$ & $\begin{array}{c}\text { Standard } \\
\text { Deviation } \\
(\mathrm{pCi} / \mathrm{g})\end{array}$ & $\begin{array}{c}\text { Distribution } \\
\text { of the Data }\end{array}$ & $\begin{array}{c}\text { UTL } \\
(\mathrm{pCi} / \mathrm{g})\end{array}$ \\
\hline $\mathrm{Am}-241$ & 23 & 78 & $<0.00166$ & 0.0875 & 0.00989 & 0.0198 & Non-Parametric & 0.0875 \\
\hline $\mathrm{Cs}-137$ & 45 & 100 & $5.04 \mathrm{E}-02$ & 0.59 & 0.236 & 0.134 & Normal & 0.624 \\
\hline $\mathrm{Pu}-238$ & 21 & 57 & $<0.002$ & 0.044 & 0.0115 & 0.0115 & Gamma & 0.0514 \\
\hline $\mathrm{Pu}-239 / 240$ & 22 & 86 & $<0.00183$ & 0.05 & 0.0143 & 0.0136 & Gamma & 0.0892 \\
\hline Sr-90 & 17 & 88 & $<0.0243$ & 0.32 & 0.0872 & 0.0183 & Normal & 0.335 \\
\hline
\end{tabular}


Table B-15. Summary statistics for data collected at Blue Dome/Birch Creek Hydro.

\begin{tabular}{|c|c|c|c|c|c|c|c|c|}
\hline Radionuclide & $\mathrm{N}$ & $\begin{array}{l}\text { Percent } \\
\text { Detected }\end{array}$ & $\underset{(\mathrm{pCi} / \mathrm{g})}{\operatorname{Minimum}^{\mathrm{a}}}$ & $\begin{array}{l}\text { Maximum } \\
(\mathrm{pCi} / \mathrm{g})\end{array}$ & $\begin{array}{l}\text { Mean } \\
(\mathrm{pCi} / \mathrm{g})\end{array}$ & $\begin{array}{c}\text { Standard } \\
\text { Deviation } \\
\text { (pCi/g) }\end{array}$ & $\begin{array}{l}\text { Distribution } \\
\text { of the Data }\end{array}$ & $\begin{array}{c}\text { UTL } \\
(\mathrm{pCi} / \mathrm{g})\end{array}$ \\
\hline Am-241 & 10 & 100 & 0.007 & 0.0268 & 0.0126 & 0.00673 & Non-Parametric & 0.0268 \\
\hline Cs-137 & 17 & 100 & 0.0839 & 1.3 & 0.494 & 0.319 & Normal & 1.583 \\
\hline $\mathrm{Pu}-238$ & 11 & 45 & $<0.000617$ & 0.0144 & $\mathrm{NA}^{\mathrm{b}}$ & $\mathrm{NA}^{\mathrm{b}}$ & $\mathrm{NA}^{\mathrm{b}}$ & $0.0144^{\mathrm{c}}$ \\
\hline $\mathrm{Pu}-239 / 240$ & 10 & 80 & $<0.012$ & 0.043 & 0.025 & 0.0107 & Normal & 0.0677 \\
\hline Sr-90 & 9 & 100 & 0.023 & 0.52 & 0.266 & 0.156 & Normal & 0.911 \\
\hline
\end{tabular}

Table B-16. Summary statistics for data collected at St. Anthony.

\begin{tabular}{|l|c|c|c|c|c|c|c|c|}
\hline \multicolumn{1}{|c|}{ Radionuclide } & $\mathrm{N}$ & $\begin{array}{c}\text { Percent } \\
\text { Detected }\end{array}$ & $\begin{array}{c}\text { Minimum } \\
(\mathrm{pCi} / \mathrm{g})\end{array}$ & $\begin{array}{c}\text { Maximum } \\
(\mathrm{pCi} / \mathrm{g})\end{array}$ & $\begin{array}{c}\text { Mean } \\
(\mathrm{pCi} / \mathrm{g})\end{array}$ & $\begin{array}{c}\text { Standard } \\
\text { Deviation } \\
(\mathrm{pCi} / \mathrm{g})\end{array}$ & $\begin{array}{c}\text { Distribution } \\
\text { of the Data }\end{array}$ & $\begin{array}{c}\text { UTL } \\
(\mathrm{pCi} / \mathrm{g})\end{array}$ \\
\hline $\mathrm{Am}-241$ & 10 & 100 & 0.00543 & 0.0287 & 0.0125 & 0.00746 & Normal & 0.0422 \\
\hline $\mathrm{Cs}-137$ & 21 & 100 & 0.0752 & 1.27 & 0.657 & 0.337 & Normal & 1.758 \\
\hline $\mathrm{Pu}-238$ & 11 & 82 & $<0.00147$ & 0.0657 & 0.0149 & 0.0184 & Normal & 0.0857 \\
\hline $\mathrm{Pu}-239 / 240$ & 10 & 100 & 0.00147 & 0.06 & 0.0257 & 0.0175 & Normal & 0.0954 \\
\hline Sr-90 & 9 & 100 & 0.0432 & 0.56 & 0.242 & 0.17 & Normal & 0.948 \\
\hline
\end{tabular}




\section{Appendix C \\ Data Used for Baseline Inventory}

Tables C-1 and C-2 contain the radionuclide measurements that were used to compute background levels used to establish a baseline inventory for near facility and distant sites.

Table C-1. Radionuclides concentrations in surface soils associated with ARA.

\begin{tabular}{|c|c|c|c|c|c|c|}
\hline Radionuclide & $\begin{array}{c}\text { Sample } \\
\text { Location }\end{array}$ & Date & $\begin{array}{l}\text { Concentrations } \\
(\mathrm{pCi} / \mathrm{g})\end{array}$ & $\begin{array}{l}\text { Uncertainty } \\
(\mathrm{pCi} / \mathrm{g})\end{array}$ & $\begin{array}{l}\text { MDA } \\
(\mathrm{pCi} / \mathrm{g})\end{array}$ & Flag \\
\hline Am-241 & $0^{\circ} 250^{\prime}$ & 1977 & 0.01 & 0.002 & 0.006 & - \\
\hline Am-241 & $22.5^{\circ} 250^{\prime}$ & 1977 & 0.02 & 0.002 & 0.006 & - \\
\hline Am-241 & $225^{\circ} 500^{\prime}$ & 1977 & 0.002 & 0.005 & 0.015 & $\mathrm{U}$ \\
\hline Am-241 & $270^{\circ} 1500^{\prime}$ & 1977 & 0.003 & 0.001 & 0.003 & - \\
\hline Am-241 & $270^{\circ} 500^{\prime}$ & 1977 & 0.003 & 0.001 & 0.003 & - \\
\hline Am-241 & $45^{\circ} 1500^{\prime}$ & 1977 & 0.004 & 0.001 & 0.003 & - \\
\hline Am-241 & $45^{\circ} 250^{\prime}$ & 1977 & 0.005 & 0.003 & 0.009 & $\mathrm{U}$ \\
\hline Am-241 & $45^{\circ} 2500^{\prime}$ & 1977 & 0.005 & 0.001 & 0.003 & - \\
\hline Am-241 & $45^{\circ} 500^{\prime}$ & 1977 & 0.0054 & 0.0016 & 0.0048 & - \\
\hline Am-241 & $90^{\circ} 500^{\prime}$ & 1977 & 0.008 & 0.002 & 0.006 & - \\
\hline Am-241 & $0^{\circ} 1000^{\prime}$ & 1985 & 0.003 & 0.002 & 0.006 & $\mathrm{U}$ \\
\hline Am-241 & $22.5^{\circ} 2500^{\prime}$ & 1985 & 0.006 & 0.003 & 0.009 & $\mathrm{U}$ \\
\hline Am-241 & $67.5^{\circ} 2000^{\prime}$ & 1985 & 0.003 & 0.002 & 0.006 & $\mathrm{U}$ \\
\hline Am-241 & $67.5^{\circ} 500^{\prime}$ & 1985 & 0 & 0.002 & 0.006 & $\mathrm{U}$ \\
\hline Am-241 & ARA-0 & 2007 & 0.2213 & 0.0895 & 0.2685 & $\mathrm{U}$ \\
\hline Am-241 & ARA-11 & 2007 & 0.401 & 0.126 & 0.378 & - \\
\hline Am-241 & ARA-14 & 2007 & 0.1272 & 0.078 & 0.234 & $\mathrm{U}$ \\
\hline Am-241 & ARA-19 & 2007 & 0.07257 & 0.0535 & 0.1605 & $\mathrm{U}$ \\
\hline Am-241 & ARA-2 & 2007 & 0.1254 & 0.062 & 0.186 & $\mathrm{U}$ \\
\hline Am-241 & ARA-21 & 2007 & 0.1662 & 0.063 & 0.189 & $\mathrm{U}$ \\
\hline Am-241 & ARA-23 & 2007 & 0.2921 & 0.1105 & 0.3315 & $\mathrm{U}$ \\
\hline Am-241 & ARA-24 & 2007 & 0.05789 & 0.03215 & 0.09645 & $\mathrm{U}$ \\
\hline Am-241 & ARA-36 & 2007 & 0.2042 & 0.0885 & 0.2655 & $\mathrm{U}$ \\
\hline Am-241 & ARA-37 & 2007 & 0.1176 & 0.08 & 0.24 & $\mathrm{U}$ \\
\hline Am-241 & ARA-4 & 2007 & 0.1533 & 0.078 & 0.234 & $\mathrm{U}$ \\
\hline Am-241 & ARA-43 & 2007 & 0.02696 & 0.057 & 0.171 & $\mathrm{U}$ \\
\hline Am-241 & ARA-45 & 2007 & 0.07517 & 0.0525 & 0.1575 & $\mathrm{U}$ \\
\hline Am-241 & ARA-46 & 2007 & 0 & 0.04055 & 0.12165 & $\mathrm{U}$ \\
\hline Am-241 & ARA-47 & 2007 & 0.03163 & 0.0279 & 0.0837 & $\mathrm{U}$ \\
\hline Am-241 & ARA-48 & 2007 & 0.1157 & 0.0715 & 0.2145 & $\mathrm{U}$ \\
\hline Am-241 & ARA-49 & 2007 & 0.101 & 0.04885 & 0.14655 & $\mathrm{U}$ \\
\hline Am-241 & ARA-5 & 2007 & 0.1349 & 0.0725 & 0.2175 & $\mathrm{U}$ \\
\hline
\end{tabular}


Table C-1. (continued).

\begin{tabular}{|c|c|c|c|c|c|c|}
\hline Radionuclide & $\begin{array}{c}\text { Sample } \\
\text { Location }\end{array}$ & Date & $\begin{array}{c}\text { Concentrations } \\
(\mathrm{pCi} / \mathrm{g})\end{array}$ & $\begin{array}{l}\text { Uncertainty } \\
(\mathrm{pCi} / \mathrm{g})\end{array}$ & $\begin{array}{c}\text { MDA } \\
(\mathrm{pCi} / \mathrm{g})\end{array}$ & Flag \\
\hline Am-241 & ARA-51 & 2007 & -0.01711 & 0.063 & 0.189 & $\mathrm{U}$ \\
\hline Am-241 & ARA-56 & 2007 & 0.06139 & 0.0414 & 0.1242 & $\mathrm{U}$ \\
\hline Am-241 & ARA-57 & 2007 & 0.06861 & 0.056 & 0.168 & $\mathrm{U}$ \\
\hline Am-241 & ARA-58 & 2007 & 0.01031 & 0.059 & 0.177 & $\mathrm{U}$ \\
\hline Am-241 & ARA-59 & 2007 & 0.07199 & 0.0585 & 0.1755 & $\mathrm{U}$ \\
\hline Am-241 & ARA-6 & 2007 & 0.1231 & 0.071 & 0.213 & $\mathrm{U}$ \\
\hline Am-241 & ARA-60 & 2007 & 0.002381 & 0.04195 & 0.12585 & $\mathrm{U}$ \\
\hline Am-241 & ARA-65 & 2007 & 0.08867 & 0.05 & 0.15 & $\mathrm{U}$ \\
\hline Am-241 & ARA-7 & 2007 & 0.05277 & 0.05 & 0.15 & $\mathrm{U}$ \\
\hline Am-241 & ARA-73 & 2007 & 0.01521 & 0.081 & 0.243 & $\mathrm{U}$ \\
\hline Am-241 & ARA-74 & 2007 & 0.0003457 & 0.03885 & 0.11655 & $\mathrm{U}$ \\
\hline Am-241 & ARA-75 & 2007 & 0.113 & 0.072 & 0.216 & $\mathrm{U}$ \\
\hline Am-241 & ARA-77 & 2007 & 0.04561 & 0.03855 & 0.11565 & $\mathrm{U}$ \\
\hline Am-241 & ARA-1 & 2011 & 0.01294 & 0.071 & 0.213 & $\mathrm{U}$ \\
\hline Am-241 & ARA-14 & 2011 & 0.04576 & 0.0701 & 0.2103 & $\mathrm{U}$ \\
\hline Am-241 & ARA-2 & 2011 & -0.02348 & 0.0767 & 0.2301 & $\mathrm{U}$ \\
\hline Am-241 & ARA-24 & 2011 & 0.02742 & 0.0828 & 0.2484 & $\mathrm{U}$ \\
\hline Am-241 & ARA-28 & 2011 & 0.01409 & 0.0622 & 0.1866 & $\mathrm{U}$ \\
\hline Am-241 & ARA-29 & 2011 & -0.01569 & 0.0462 & 0.1386 & $\mathrm{U}$ \\
\hline Am-241 & ARA-31 & 2011 & -0.02227 & 0.0599 & 0.1797 & $\mathrm{U}$ \\
\hline Am-241 & ARA-32 & 2011 & 0.05305 & 0.0987 & 0.2961 & $\mathrm{U}$ \\
\hline Am-241 & ARA-34 & 2011 & 0.0472 & 0.093 & 0.279 & $\mathrm{U}$ \\
\hline Am-241 & ARA-38 & 2011 & 0.08759 & 0.0928 & 0.2784 & $\mathrm{U}$ \\
\hline Am-241 & ARA-4 & 2011 & -0.03804 & 0.0424 & 0.1272 & $\mathrm{U}$ \\
\hline Am-241 & ARA-42 & 2011 & 0.06746 & 0.0953 & 0.2859 & $\mathrm{U}$ \\
\hline Am-241 & ARA-43 & 2011 & -0.04122 & 0.0537 & 0.1611 & $\mathrm{U}$ \\
\hline Am-241 & ARA-47 & 2011 & -0.001312 & 0.0591 & 0.1773 & $\mathrm{U}$ \\
\hline Am-241 & ARA-50 & 2011 & -0.04888 & 0.0822 & 0.2466 & $\mathrm{U}$ \\
\hline Am-241 & ARA-51 & 2011 & -0.003077 & 0.0543 & 0.1629 & $\mathrm{U}$ \\
\hline Am-241 & ARA-62 & 2011 & 0.0171 & 0.0911 & 0.2733 & $\mathrm{U}$ \\
\hline Am-241 & ARA-65 & 2011 & 0.1064 & 0.0881 & 0.2643 & $\mathrm{U}$ \\
\hline Am-241 & ARA-74 & 2011 & 0.0043 & 0.0593 & 0.1779 & $\mathrm{U}$ \\
\hline Am-241 & ARA-75 & 2011 & 0.107 & 0.0883 & 0.2649 & $\mathrm{U}$ \\
\hline Am-241 & ARA-77 & 2011 & 0.06909 & 0.0569 & 0.1707 & $\mathrm{U}$ \\
\hline Am-241 & ARA-9 & 2011 & 0.01591 & 0.041 & 0.123 & $\mathrm{U}$ \\
\hline Am-241 & ARA-11 & 2012 & 0.07631 & 0.0821 & 0.2463 & $\mathrm{U}$ \\
\hline Am-241 & ARA-16 & 2012 & 0.1188 & 0.0936 & 0.2808 & $\mathrm{U}$ \\
\hline
\end{tabular}


Table C-1. (continued).

\begin{tabular}{|c|c|c|c|c|c|c|}
\hline Radionuclide & $\begin{array}{c}\text { Sample } \\
\text { Location }\end{array}$ & Date & $\begin{array}{c}\text { Concentrations } \\
(\mathrm{pCi} / \mathrm{g})\end{array}$ & $\begin{array}{l}\text { Uncertainty } \\
(\mathrm{pCi} / \mathrm{g})\end{array}$ & $\begin{array}{c}\text { MDA } \\
(\mathrm{pCi} / \mathrm{g})\end{array}$ & Flag \\
\hline Am-241 & ARA-31 & 2012 & 0.005208 & 0.0808 & 0.2424 & $\mathrm{U}$ \\
\hline Am-241 & ARA-32 & 2012 & -0.02262 & 0.0951 & 0.2853 & $\mathrm{U}$ \\
\hline Am-241 & ARA-48 & 2012 & 0.007558 & 0.0451 & 0.1353 & $\mathrm{U}$ \\
\hline Am-241 & ARA-53 & 2012 & 0.08028 & 0.0753 & 0.2259 & $\mathrm{U}$ \\
\hline Am-241 & ARA-57 & 2012 & 0.1233 & 0.0799 & 0.2397 & $\mathrm{U}$ \\
\hline Am-241 & ARA-58 & 2012 & 0.01109 & 0.0416 & 0.1248 & $\mathrm{U}$ \\
\hline Am-241 & ARA-59 & 2012 & 0.09303 & 0.0709 & 0.2127 & $\mathrm{U}$ \\
\hline Am-241 & ARA-60 & 2012 & 0.05766 & 0.0793 & 0.2379 & $\mathrm{U}$ \\
\hline Am-241 & ARA-62 & 2012 & -0.002745 & 0.0833 & 0.2499 & $\mathrm{U}$ \\
\hline Am-241 & ARA-65 & 2012 & -0.02205 & 0.045 & 0.135 & $\mathrm{U}$ \\
\hline Am-241 & ARA-73 & 2012 & 0.03367 & 0.044 & 0.132 & $\mathrm{U}$ \\
\hline Am-241 & ARA-31 & 2013 & -0.01316 & 0.073 & 0.219 & $\mathrm{U}$ \\
\hline Am-241 & ARA-32 & 2013 & 0.03202 & 0.058 & 0.174 & $\mathrm{U}$ \\
\hline Am-241 & ARA-33 & 2013 & 0.02818 & 0.074 & 0.223 & $\mathrm{U}$ \\
\hline Am-241 & ARA-40 & 2013 & 0.0345 & 0.065 & 0.196 & $\mathrm{U}$ \\
\hline Am-241 & ARA-48 & 2013 & 0.04875 & 0.058 & 0.175 & $\mathrm{U}$ \\
\hline Am-241 & ARA-65 & 2013 & 0.04136 & 0.057 & 0.17 & $\mathrm{U}$ \\
\hline Am-241 & $\begin{array}{c}2 \text { INCH AIR } \\
- \text { ARA }\end{array}$ & 2014 & 0.03432 & 0.116 & 0.348 & $\mathrm{U}$ \\
\hline Am-241 & ARA-1 & 2014 & -0.002003 & 0.0457 & 0.1371 & $\mathrm{U}$ \\
\hline Am-241 & ARA-24 & 2014 & -0.01891 & 0.0708 & 0.2124 & $\mathrm{U}$ \\
\hline Am-241 & ARA-31 & 2014 & 0.08178 & 0.112 & 0.336 & $\mathrm{U}$ \\
\hline Am-241 & ARA-32 & 2014 & 0.07562 & 0.0851 & 0.2553 & $\mathrm{U}$ \\
\hline Am-241 & ARA-65 & 2014 & 0.1799 & 0.07 & 0.21 & $\mathrm{U}$ \\
\hline Am-241 & ARA-71 & 2014 & 0.1069 & 0.0534 & 0.1602 & $\mathrm{U}$ \\
\hline Co-60 & $0^{\circ} 250^{\prime}$ & 1977 & 0.37 & 0.06 & 0.18 & - \\
\hline Co-60 & $135^{\circ} 250^{\prime}$ & 1977 & 0.043 & 0.014 & 0.042 & - \\
\hline Co-60 & $157^{\circ} 1000^{\prime}$ & 1977 & 0.025 & 0.013 & 0.039 & $\mathrm{U}$ \\
\hline Co-60 & $202^{\circ} 500^{\prime}$ & 1977 & 0.015 & 0.01 & 0.03 & $\mathrm{U}$ \\
\hline $\mathrm{Co}-60$ & $22^{\circ} 500^{\prime}$ & 1977 & 0.14 & 0.05 & 0.15 & $\mathrm{U}$ \\
\hline Co-60 & $225^{\circ} 500^{\prime}$ & 1977 & 0.06 & 0.009 & 0.027 & - \\
\hline Co-60 & $292^{\circ} 500^{\prime}$ & 1977 & 0.012 & 0.011 & 0.033 & $\mathrm{U}$ \\
\hline Co-60 & $337.5^{\circ} 250^{\prime}$ & 1977 & 0.08 & 0.01 & 0.03 & - \\
\hline Co-60 & $45^{\circ} 1000^{\prime}$ & 1977 & 0.021 & 0.011 & 0.033 & $\mathrm{U}$ \\
\hline Co-60 & $45^{\circ} 250^{\prime}$ & 1977 & 0.16 & 0.04 & 0.12 & - \\
\hline Co-60 & $45^{\circ} 5000^{\prime}$ & 1977 & 0.043 & 0.009 & 0.027 & - \\
\hline Co-60 & $67^{\circ} 500^{\prime}$ & 1977 & 0.083 & 0.013 & 0.039 & - \\
\hline
\end{tabular}


Table C-1. (continued).

\begin{tabular}{|c|c|c|c|c|c|c|}
\hline Radionuclide & $\begin{array}{c}\text { Sample } \\
\text { Location }\end{array}$ & Date & $\begin{array}{c}\text { Concentrations } \\
(\mathrm{pCi} / \mathrm{g})\end{array}$ & $\begin{array}{l}\text { Uncertainty } \\
(\mathrm{pCi} / \mathrm{g})\end{array}$ & $\begin{array}{c}\text { MDA } \\
(\mathrm{pCi} / \mathrm{g})\end{array}$ & Flag \\
\hline Co-60 & $90^{\circ} 250^{\prime}$ & 1977 & 0.3 & 0.015 & 0.045 & - \\
\hline Co-60 & ARA-0 & 2006 & 0.1672 & 0.0326 & 0.0978 & - \\
\hline Co-60 & ARA-1 & 2006 & 0.0186 & 0.003895 & 0.011685 & - \\
\hline Co-60 & ARA-10 & 2006 & 0.1053 & 0.02955 & 0.08865 & - \\
\hline Co-60 & ARA-13 & 2006 & 0.1696 & 0.0219 & 0.0657 & - \\
\hline Co-60 & ARA-18 & 2006 & 0.06164 & 0.0154 & 0.0462 & - \\
\hline Co-60 & ARA-2 & 2006 & 0.1849 & 0.0326 & 0.0978 & - \\
\hline Co-60 & ARA-20 & 2006 & 0.06734 & 0.01215 & 0.03645 & - \\
\hline Co-60 & ARA-26 & 2006 & 0.1102 & 0.02015 & 0.06045 & - \\
\hline Co-60 & ARA-3 & 2006 & 0.06975 & 0.01375 & 0.04125 & - \\
\hline Co-60 & ARA-30 & 2006 & 0.03297 & 0.00795 & 0.02385 & - \\
\hline Co-60 & ARA-31 & 2006 & 0.05475 & 0.0121 & 0.0363 & - \\
\hline Co-60 & ARA-32 & 2006 & 0.07927 & 0.01475 & 0.04425 & - \\
\hline Co-60 & ARA-33 & 2006 & 0.1431 & 0.03655 & 0.10965 & - \\
\hline Co-60 & ARA-34 & 2006 & 0.07441 & 0.0139 & 0.0417 & - \\
\hline Co-60 & ARA-39 & 2006 & 0.08151 & 0.0146 & 0.0438 & - \\
\hline Co-60 & ARA-4 & 2006 & 0.2281 & 0.0535 & 0.1605 & - \\
\hline Co-60 & ARA-40 & 2006 & 0.04222 & 0.0097 & 0.0291 & - \\
\hline Co-60 & ARA-41 & 2006 & 0.1021 & 0.0167 & 0.0501 & - \\
\hline Co-60 & ARA-42 & 2006 & 0.06697 & 0.01125 & 0.03375 & - \\
\hline Co-60 & ARA-43 & 2006 & 0.0596 & 0.0116 & 0.0348 & - \\
\hline Co-60 & ARA-44 & 2006 & 0.03293 & 0.00625 & 0.01875 & - \\
\hline Co-60 & ARA-48 & 2006 & 0.0596 & 0.0116 & 0.0348 & - \\
\hline Co-60 & ARA-49 & 2006 & 0.06872 & 0.01365 & 0.04095 & - \\
\hline Co-60 & ARA-5 & 2006 & 0.0784 & 0.0118 & 0.0354 & - \\
\hline Co-60 & ARA-50 & 2006 & 0.05144 & 0.00765 & 0.02295 & - \\
\hline Co-60 & ARA-51 & 2006 & 0.06972 & 0.01665 & 0.04995 & - \\
\hline Co-60 & ARA-53 & 2006 & 0.061 & 0.01025 & 0.03075 & - \\
\hline Co-60 & ARA-54 & 2006 & 0.0364 & 0.00645 & 0.01935 & - \\
\hline Co-60 & ARA-55 & 2006 & 0.07131 & 0.016 & 0.048 & - \\
\hline Co-60 & ARA-56 & 2006 & 0.07058 & 0.0133 & 0.0399 & - \\
\hline Co-60 & ARA-57 & 2006 & 0.1612 & 0.03065 & 0.09195 & - \\
\hline Co-60 & ARA-58 & 2006 & 0.04992 & 0.00885 & 0.02655 & - \\
\hline Co-60 & ARA-59 & 2006 & 0.04369 & 0.00855 & 0.02565 & - \\
\hline Co-60 & ARA-6 & 2006 & 0.1396 & 0.01855 & 0.05565 & - \\
\hline Co-60 & ARA-60 & 2006 & 0.04132 & 0.008 & 0.024 & - \\
\hline Co-60 & ARA-61 & 2006 & 0.08935 & 0.0148 & 0.0444 & - \\
\hline
\end{tabular}


Table C-1. (continued).

\begin{tabular}{|c|c|c|c|c|c|c|}
\hline Radionuclide & $\begin{array}{c}\text { Sample } \\
\text { Location }\end{array}$ & Date & $\begin{array}{c}\text { Concentrations } \\
(\mathrm{pCi} / \mathrm{g})\end{array}$ & $\begin{array}{l}\text { Uncertainty } \\
(\mathrm{pCi} / \mathrm{g})\end{array}$ & $\begin{array}{c}\text { MDA } \\
(\mathrm{pCi} / \mathrm{g})\end{array}$ & Flag \\
\hline Co-60 & ARA-62 & 2006 & 0.0859 & 0.01655 & 0.04965 & - \\
\hline Co-60 & ARA-63 & 2006 & 0.0007154 & 0.00032 & 0.00096 & $\mathrm{U}$ \\
\hline Co-60 & ARA-65 & 2006 & 0.06494 & 0.01095 & 0.03285 & - \\
\hline Co-60 & ARA-66 & 2006 & 0.08009 & 0.0167 & 0.0501 & - \\
\hline Co-60 & ARA-67 & 2006 & 0.05132 & 0.01075 & 0.03225 & - \\
\hline Co-60 & ARA-68 & 2006 & 0.1757 & 0.0311 & 0.0933 & - \\
\hline Co-60 & ARA-69 & 2006 & 0.07075 & 0.0103 & 0.0309 & - \\
\hline Co-60 & ARA-7 & 2006 & 0.0994 & 0.0223 & 0.0669 & - \\
\hline Co-60 & ARA-70 & 2006 & 0.1109 & 0.01965 & 0.05895 & - \\
\hline Co-60 & ARA-71 & 2006 & 0.03037 & 0.0097 & 0.0291 & - \\
\hline Co-60 & ARA-72 & 2006 & 0.1068 & 0.01985 & 0.05955 & - \\
\hline Co-60 & ARA-73 & 2006 & 0.03255 & 0.0075 & 0.0225 & - \\
\hline Co-60 & ARA-74 & 2006 & 0.07171 & 0.01275 & 0.03825 & - \\
\hline Co-60 & ARA-75 & 2006 & 0.06387 & 0.0103 & 0.0309 & - \\
\hline Co-60 & ARA-76 & 2006 & 0.05846 & 0.01445 & 0.04335 & - \\
\hline Co-60 & ARA-77 & 2006 & 0.02402 & 0.00625 & 0.01875 & - \\
\hline Co-60 & ARA-8 & 2006 & 0.08591 & 0.0156 & 0.0468 & - \\
\hline Co-60 & ARA-9 & 2006 & 0.1309 & 0.0139 & 0.0417 & - \\
\hline Co-60 & ARA-0 & 2007 & 0.02313 & 0.00505 & 0.01515 & - \\
\hline Co-60 & ARA-11 & 2007 & 0.01861 & 0.00472 & 0.01416 & - \\
\hline Co-60 & ARA-12 & 2007 & 0.05611 & 0.0124 & 0.0372 & - \\
\hline Co-60 & ARA-14 & 2007 & 0.009533 & 0.002725 & 0.008175 & - \\
\hline Co-60 & ARA-15 & 2007 & 0.0157 & 0.003815 & 0.011445 & - \\
\hline Co-60 & ARA-17 & 2007 & 0.01854 & 0.00469 & 0.01407 & - \\
\hline Co-60 & ARA-19 & 2007 & 0.03212 & 0.00675 & 0.02025 & - \\
\hline Co-60 & ARA-2 & 2007 & 0.01397 & 0.00367 & 0.01101 & - \\
\hline Co-60 & ARA-21 & 2007 & 0.01706 & 0.00407 & 0.01221 & - \\
\hline Co-60 & ARA-23 & 2007 & 0.0413 & 0.00835 & 0.02505 & - \\
\hline Co-60 & ARA-24 & 2007 & 0.01183 & 0.00292 & 0.00876 & - \\
\hline Co-60 & ARA-28 & 2007 & 0.04033 & 0.0074 & 0.0222 & - \\
\hline Co-60 & ARA-29 & 2007 & 0.009198 & 0.00312 & 0.00936 & $\mathrm{U}$ \\
\hline Co-60 & ARA-31 & 2007 & 0.03806 & 0.00865 & 0.02595 & - \\
\hline Co-60 & ARA-32 & 2007 & 0.03456 & 0.00695 & 0.02085 & - \\
\hline Co-60 & ARA-36 & 2007 & 0.0274 & 0.0062 & 0.0186 & - \\
\hline Co-60 & ARA-37 & 2007 & 0.02591 & 0.0058 & 0.0174 & - \\
\hline Co-60 & ARA-38 & 2007 & 0.006232 & 0.002495 & 0.007485 & $\mathrm{U}$ \\
\hline Co-60 & ARA-4 & 2007 & 0.03237 & 0.00655 & 0.01965 & - \\
\hline
\end{tabular}


Table C-1. (continued).

\begin{tabular}{|c|c|c|c|c|c|c|}
\hline Radionuclide & $\begin{array}{c}\text { Sample } \\
\text { Location }\end{array}$ & Date & $\begin{array}{c}\text { Concentrations } \\
(\mathrm{pCi} / \mathrm{g})\end{array}$ & $\begin{array}{c}\text { Uncertainty } \\
(\mathrm{pCi} / \mathrm{g})\end{array}$ & $\begin{array}{c}\text { MDA } \\
(\mathrm{pCi} / \mathrm{g})\end{array}$ & Flag \\
\hline Co-60 & ARA-42 & 2007 & 0.01697 & 0.004925 & 0.014775 & - \\
\hline Co-60 & ARA-43 & 2007 & 0.02278 & 0.00437 & 0.01311 & - \\
\hline Co-60 & ARA-45 & 2007 & 0.03343 & 0.00865 & 0.02595 & - \\
\hline Co-60 & ARA-46 & 2007 & 0.01031 & 0.003105 & 0.009315 & - \\
\hline Co-60 & ARA-47 & 2007 & 0.02455 & 0.0055 & 0.0165 & - \\
\hline Co-60 & ARA-48 & 2007 & 0.02453 & 0.00735 & 0.02205 & - \\
\hline Co-60 & ARA-49 & 2007 & 0.01348 & 0.00334 & 0.01002 & - \\
\hline Co-60 & ARA-5 & 2007 & 0.02601 & 0.0054 & 0.0162 & - \\
\hline Co-60 & ARA-50 & 2007 & 0.01078 & 0.002395 & 0.007185 & - \\
\hline Co-60 & ARA-51 & 2007 & 0.01801 & 0.00615 & 0.01845 & $\mathrm{U}$ \\
\hline Co-60 & ARA-52 & 2007 & 0.01407 & 0.00341 & 0.01023 & - \\
\hline Co-60 & ARA-56 & 2007 & 0.02097 & 0.00462 & 0.01386 & - \\
\hline Co-60 & ARA-57 & 2007 & 0.02345 & 0.006 & 0.018 & - \\
\hline Co-60 & ARA-58 & 2007 & 0.01702 & 0.003855 & 0.011565 & - \\
\hline Co-60 & ARA-6 & 2007 & 0.003777 & 0.002345 & 0.007035 & $\mathrm{U}$ \\
\hline Co-60 & ARA-60 & 2007 & 0.01447 & 0.004625 & 0.013875 & - \\
\hline Co-60 & ARA-65 & 2007 & 0.02401 & 0.00575 & 0.01725 & - \\
\hline Co-60 & ARA-7 & 2007 & 0.01757 & 0.003915 & 0.011745 & - \\
\hline Co-60 & ARA-73 & 2007 & 0.0173 & 0.00397 & 0.01191 & - \\
\hline Co-60 & ARA-74 & 2007 & 0.01117 & 0.004935 & 0.014805 & $\mathrm{U}$ \\
\hline Co-60 & ARA-75 & 2007 & 0.03548 & 0.0089 & 0.0267 & - \\
\hline Co-60 & ARA-77 & 2007 & 0.006336 & 0.002835 & 0.008505 & $\mathrm{U}$ \\
\hline Co-60 & ARA-1 & 2011 & 0.0002147 & 0.00236 & 0.00708 & $\mathrm{U}$ \\
\hline Co-60 & ARA-14 & 2011 & 0.0004696 & 0.00126 & 0.00378 & $\mathrm{U}$ \\
\hline Co-60 & ARA-16 & 2011 & 0.002014 & 0.00285 & 0.00855 & $\mathrm{U}$ \\
\hline Co-60 & ARA-2 & 2011 & -0.0003417 & 0.00229 & 0.00687 & $\mathrm{U}$ \\
\hline Co-60 & ARA-24 & 2011 & 0.002039 & 0.00266 & 0.00798 & $\mathrm{U}$ \\
\hline Co-60 & ARA-28 & 2011 & -0.0008401 & 0.00436 & 0.01308 & $\mathrm{U}$ \\
\hline Co-60 & ARA-29 & 2011 & -0.001381 & 0.00223 & 0.00669 & $\mathrm{U}$ \\
\hline Co-60 & ARA-31 & 2011 & -0.0008192 & 0.00386 & 0.01158 & $\mathrm{U}$ \\
\hline Co-60 & ARA-32 & 2011 & -0.003255 & 0.00545 & 0.01635 & $\mathrm{U}$ \\
\hline Co-60 & ARA-34 & 2011 & -0.001067 & 0.00403 & 0.01209 & $\mathrm{U}$ \\
\hline Co-60 & ARA-38 & 2011 & 0.002743 & 0.0045 & 0.0135 & $\mathrm{U}$ \\
\hline Co-60 & ARA-4 & 2011 & 0.001196 & 0.00192 & 0.00576 & $\mathrm{U}$ \\
\hline Co-60 & ARA-42 & 2011 & -0.003101 & 0.00472 & 0.01416 & $\mathrm{U}$ \\
\hline Co-60 & ARA-43 & 2011 & -0.00002657 & 0.0036 & 0.0108 & $\mathrm{U}$ \\
\hline Co-60 & ARA-47 & 2011 & 0.002008 & 0.00377 & 0.01131 & $\mathrm{U}$ \\
\hline
\end{tabular}


Table C-1. (continued).

\begin{tabular}{|c|c|c|c|c|c|c|}
\hline Radionuclide & $\begin{array}{c}\text { Sample } \\
\text { Location }\end{array}$ & Date & $\begin{array}{c}\text { Concentrations } \\
(\mathrm{pCi} / \mathrm{g})\end{array}$ & $\begin{array}{c}\text { Uncertainty } \\
(\mathrm{pCi} / \mathrm{g})\end{array}$ & $\begin{array}{c}\text { MDA } \\
(\mathrm{pCi} / \mathrm{g})\end{array}$ & Flag \\
\hline Co-60 & ARA-50 & 2011 & 0.0001911 & 0.00286 & 0.00858 & $\mathrm{U}$ \\
\hline Co-60 & ARA-51 & 2011 & 0.0001462 & 0.00502 & 0.01506 & $\mathrm{U}$ \\
\hline Co-60 & ARA-62 & 2011 & 0.0009998 & 0.00281 & 0.00843 & $\mathrm{U}$ \\
\hline Co-60 & ARA-65 & 2011 & 0.0007134 & 0.00221 & 0.00663 & $\mathrm{U}$ \\
\hline Co-60 & ARA-74 & 2011 & -0.002245 & 0.00415 & 0.01245 & $\mathrm{U}$ \\
\hline Co-60 & ARA-75 & 2011 & 0.0003345 & 0.00435 & 0.01305 & $\mathrm{U}$ \\
\hline Co-60 & ARA-77 & 2011 & 0.002575 & 0.00359 & 0.01077 & $\mathrm{U}$ \\
\hline Co-60 & ARA-9 & 2011 & 0.0009574 & 0.00184 & 0.00552 & $\mathrm{U}$ \\
\hline Co-60 & ARA-11 & 2012 & 0.0006428 & 0.00395 & 0.01185 & $\mathrm{U}$ \\
\hline Co-60 & ARA-16 & 2012 & -0.0002781 & 0.00544 & 0.01632 & $\mathrm{U}$ \\
\hline Co-60 & ARA-31 & 2012 & 0.002699 & 0.0039 & 0.0117 & $\mathrm{U}$ \\
\hline Co-60 & ARA-32 & 2012 & 0.0002451 & 0.00453 & 0.01359 & $\mathrm{U}$ \\
\hline Co-60 & ARA-48 & 2012 & 0.004188 & 0.00394 & 0.01182 & $\mathrm{U}$ \\
\hline Co-60 & ARA-53 & 2012 & 0.00116 & 0.00378 & 0.01134 & $\mathrm{U}$ \\
\hline Co-60 & ARA-57 & 2012 & 0.003762 & 0.0043 & 0.0129 & $\mathrm{U}$ \\
\hline Co-60 & ARA-58 & 2012 & 0.001196 & 0.00418 & 0.01254 & $\mathrm{U}$ \\
\hline Co-60 & ARA-59 & 2012 & -0.00008167 & 0.00332 & 0.00996 & $\mathrm{U}$ \\
\hline Co-60 & ARA-60 & 2012 & -0.002254 & 0.00407 & 0.01221 & $\mathrm{U}$ \\
\hline Co-60 & ARA-62 & 2012 & 0.001208 & 0.00464 & 0.01392 & $\mathrm{U}$ \\
\hline Co-60 & ARA-65 & 2012 & 0.001349 & 0.00356 & 0.01068 & $\mathrm{U}$ \\
\hline Co-60 & ARA-73 & 2012 & 0.0001252 & 0.00423 & 0.01269 & $\mathrm{U}$ \\
\hline Co-60 & ARA-31 & 2013 & 0.01054 & 0.0035 & 0.0105 & - \\
\hline Co-60 & ARA-32 & 2013 & 0.0002091 & 0.000318 & 0.000953 & $\mathrm{U}$ \\
\hline Co-60 & ARA-33 & 2013 & 0.003221 & 0.00074 & 0.00222 & - \\
\hline Co-60 & ARA-40 & 2013 & 0.006411 & 0.002453 & 0.00736 & $\mathrm{U}$ \\
\hline Co-60 & ARA-48 & 2013 & -0.0000275 & 0.000315 & 0.000944 & $\mathrm{U}$ \\
\hline Co-60 & ARA-65 & 2013 & 0.000277 & 0.00027 & 0.00081 & $\mathrm{U}$ \\
\hline Co-60 & $\begin{array}{c}2 \text { INCH AIR } \\
\text { - ARA }\end{array}$ & 2014 & -0.0005734 & 0.00179 & 0.00537 & $\mathrm{U}$ \\
\hline Co-60 & ARA-1 & 2014 & 0.001465 & 0.0014 & 0.0042 & $\mathrm{U}$ \\
\hline Co-60 & ARA-24 & 2014 & 0.00001018 & 0.00102 & 0.00306 & $\mathrm{U}$ \\
\hline Co-60 & ARA-31 & 2014 & 0.0001038 & 0.0014 & 0.0042 & $\mathrm{U}$ \\
\hline Co-60 & ARA-32 & 2014 & 0.0001427 & 0.00112 & 0.00336 & $\mathrm{U}$ \\
\hline Co-60 & ARA-65 & 2014 & -0.0004769 & 0.0014 & 0.0042 & $\mathrm{U}$ \\
\hline $\mathrm{Co}-60$ & ARA-71 & 2014 & -0.0005109 & 0.000661 & 0.001983 & $\mathrm{U}$ \\
\hline Cs-134 & $67^{\circ} 500^{\prime}$ & 1977 & 0.14 & 0.03 & 0.09 & - \\
\hline Cs-134 & $90^{\circ} 500^{\prime}$ & 1977 & 0.046 & 0.01 & 0.03 & - \\
\hline
\end{tabular}


Table C-1. (continued).

\begin{tabular}{|c|c|c|c|c|c|c|}
\hline Radionuclide & $\begin{array}{c}\text { Sample } \\
\text { Location }\end{array}$ & Date & $\begin{array}{c}\text { Concentrations } \\
(\mathrm{pCi} / \mathrm{g})\end{array}$ & $\begin{array}{l}\text { Uncertainty } \\
(\mathrm{pCi} / \mathrm{g})\end{array}$ & $\begin{array}{c}\text { MDA } \\
(\mathrm{pCi} / \mathrm{g})\end{array}$ & Flag \\
\hline Cs-134 & ARA-0 & 2007 & 0.1378 & 0.01505 & 0.04515 & - \\
\hline Cs-134 & ARA-1 & 2007 & 0.03485 & 0.0025 & 0.0075 & - \\
\hline Cs-134 & ARA-10 & 2007 & 0.05036 & 0.00835 & 0.02505 & - \\
\hline Cs-134 & ARA-11 & 2007 & 0.1367 & 0.01505 & 0.04515 & - \\
\hline Cs-134 & ARA-12 & 2007 & 0.04648 & 0.00525 & 0.01575 & - \\
\hline Cs-134 & ARA-13 & 2007 & 0.006443 & 0.00815 & 0.02445 & $\mathrm{U}$ \\
\hline Cs-134 & ARA-14 & 2007 & 0.1025 & 0.01115 & 0.03345 & - \\
\hline Cs-134 & ARA-15 & 2007 & 0.1182 & 0.0129 & 0.0387 & - \\
\hline Cs-134 & ARA-16 & 2007 & 0.1366 & 0.0204 & 0.0612 & - \\
\hline Cs-134 & ARA-17 & 2007 & 0.08374 & 0.01175 & 0.03525 & - \\
\hline Cs-134 & ARA-18 & 2007 & 0.0554 & 0.0101 & 0.0303 & - \\
\hline Cs-134 & ARA-19 & 2007 & 0.1808 & 0.02205 & 0.06615 & - \\
\hline Cs-134 & ARA-2 & 2007 & 0.1267 & 0.02355 & 0.07065 & - \\
\hline Cs-134 & ARA-20 & 2007 & 0.05433 & 0.0097 & 0.0291 & - \\
\hline Cs-134 & ARA-21 & 2007 & 0.09053 & 0.0123 & 0.0369 & - \\
\hline Cs-134 & ARA-22 & 2007 & 0.0619 & 0.00782 & 0.02346 & - \\
\hline Cs-134 & ARA-23 & 2007 & 0.09117 & 0.0108 & 0.0324 & - \\
\hline Cs-134 & ARA-24 & 2007 & 0.1004 & 0.018 & 0.054 & - \\
\hline Cs-134 & ARA-25 & 2007 & 0.07364 & 0.0121 & 0.0363 & - \\
\hline Cs-134 & ARA-26 & 2007 & 0.04116 & 0.00719 & 0.02157 & - \\
\hline Cs-134 & ARA-27 & 2007 & 0.0528 & 0.00754 & 0.02262 & - \\
\hline Cs-134 & ARA-28 & 2007 & 0.08601 & 0.01535 & 0.04605 & - \\
\hline Cs-134 & ARA-29 & 2007 & 0.1094 & 0.0109 & 0.0327 & - \\
\hline Cs-134 & ARA-3 & 2007 & 0.07118 & 0.0114 & 0.0342 & - \\
\hline Cs-134 & ARA-30 & 2007 & 0.04575 & 0.00618 & 0.01854 & - \\
\hline Cs-134 & ARA-31 & 2007 & 0.3059 & 0.03645 & 0.10935 & - \\
\hline Cs-134 & ARA-32 & 2007 & 0.1051 & 0.0109 & 0.0327 & - \\
\hline Cs-134 & ARA-33 & 2007 & 0.03109 & 0.00645 & 0.01935 & - \\
\hline Cs-134 & ARA-34 & 2007 & 0.0361 & 0.00567 & 0.01701 & - \\
\hline Cs-134 & ARA-35 & 2007 & 0.05728 & 0.016 & 0.048 & - \\
\hline Cs-134 & ARA-36 & 2007 & 0.0891 & 0.01365 & 0.04095 & - \\
\hline Cs-134 & ARA-37 & 2007 & 1.687 & 0.1565 & 0.4695 & - \\
\hline Cs-134 & ARA-38 & 2007 & 0.1271 & 0.015 & 0.045 & - \\
\hline Cs-134 & ARA-39 & 2007 & 0.04376 & 0.0093 & 0.0279 & - \\
\hline Cs-134 & ARA-4 & 2007 & 0.06495 & 0.0113 & 0.0339 & - \\
\hline Cs-134 & ARA-40 & 2007 & 0.0553 & 0.0108 & 0.0324 & 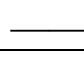 \\
\hline Cs-134 & ARA-41 & 2007 & 0.0558 & 0.00707 & 0.02121 & - \\
\hline
\end{tabular}


Table C-1. (continued).

\begin{tabular}{|c|c|c|c|c|c|c|}
\hline Radionuclide & $\begin{array}{c}\text { Sample } \\
\text { Location }\end{array}$ & Date & $\begin{array}{c}\text { Concentrations } \\
(\mathrm{pCi} / \mathrm{g})\end{array}$ & $\begin{array}{l}\text { Uncertainty } \\
(\mathrm{pCi} / \mathrm{g})\end{array}$ & $\begin{array}{c}\text { MDA } \\
(\mathrm{pCi} / \mathrm{g})\end{array}$ & Flag \\
\hline Cs-134 & ARA-42 & 2007 & 0.06382 & 0.00955 & 0.02865 & - \\
\hline Cs-134 & ARA-43 & 2007 & 0.09555 & 0.0121 & 0.0363 & - \\
\hline Cs-134 & ARA-44 & 2007 & 0.05398 & 0.00665 & 0.01995 & - \\
\hline Cs-134 & ARA-45 & 2007 & 0.1082 & 0.0151 & 0.0453 & - \\
\hline Cs-134 & ARA-46 & 2007 & 0.1337 & 0.01565 & 0.04695 & - \\
\hline Cs-134 & ARA-47 & 2007 & 0.1407 & 0.0217 & 0.0651 & - \\
\hline Cs-134 & ARA-48 & 2007 & 0.1028 & 0.0131 & 0.0393 & - \\
\hline Cs-134 & ARA-49 & 2007 & 0.1072 & 0.01145 & 0.03435 & - \\
\hline Cs-134 & ARA-5 & 2007 & 0.1125 & 0.014 & 0.042 & - \\
\hline Cs-134 & ARA-50 & 2007 & 0.05309 & 0.0072 & 0.0216 & - \\
\hline Cs-134 & ARA-51 & 2007 & 0.08249 & 0.01095 & 0.03285 & - \\
\hline Cs-134 & ARA-52 & 2007 & 0.0497 & 0.00825 & 0.02475 & - \\
\hline Cs-134 & ARA-53 & 2007 & 0.05168 & 0.0069 & 0.0207 & - \\
\hline Cs-134 & ARA-54 & 2007 & 0.07459 & 0.0122 & 0.0366 & - \\
\hline Cs-134 & ARA-55 & 2007 & 0.03102 & 0.0055 & 0.0165 & - \\
\hline Cs-134 & ARA-56 & 2007 & 0.1154 & 0.013 & 0.039 & - \\
\hline Cs-134 & ARA-57 & 2007 & 0.06439 & 0.0123 & 0.0369 & - \\
\hline Cs-134 & ARA-58 & 2007 & 0.1115 & 0.017 & 0.051 & - \\
\hline Cs-134 & ARA-59 & 2007 & 0.1103 & 0.01755 & 0.05265 & - \\
\hline Cs-134 & ARA-6 & 2007 & 0.06572 & 0.0099 & 0.0297 & - \\
\hline Cs-134 & ARA-60 & 2007 & 0.1354 & 0.0173 & 0.0519 & - \\
\hline Cs-134 & ARA-61 & 2007 & 0.03989 & 0.00933 & 0.02799 & - \\
\hline Cs-134 & ARA-62 & 2007 & 0.06257 & 0.00928 & 0.02784 & - \\
\hline Cs-134 & ARA-63 & 2007 & 0.07209 & 0.0128 & 0.0384 & - \\
\hline Cs-134 & ARA-65 & 2007 & 0.6668 & 0.121 & 0.363 & - \\
\hline Cs-134 & ARA-66 & 2007 & 0.04994 & 0.00855 & 0.02565 & - \\
\hline Cs-134 & ARA-67 & 2007 & 0.02802 & 0.00572 & 0.01716 & - \\
\hline Cs-134 & ARA-68 & 2007 & 0.03591 & 0.00677 & 0.02031 & - \\
\hline Cs-134 & ARA-69 & 2007 & 0.07437 & 0.0128 & 0.0384 & - \\
\hline Cs-134 & ARA-7 & 2007 & 0.05345 & 0.0058 & 0.0174 & - \\
\hline Cs-134 & ARA-70 & 2007 & 0.05034 & 0.00932 & 0.02796 & - \\
\hline Cs-134 & ARA-71 & 2007 & 0.03086 & 0.00867 & 0.02601 & - \\
\hline Cs-134 & ARA-72 & 2007 & 0.04523 & 0.00573 & 0.01719 & - \\
\hline Cs-134 & ARA-73 & 2007 & 0.1384 & 0.016 & 0.048 & - \\
\hline Cs-134 & ARA-74 & 2007 & 0.1283 & 0.015 & 0.045 & - \\
\hline Cs-134 & ARA-75 & 2007 & 0.135 & 0.01385 & 0.04155 & - \\
\hline Cs-134 & ARA-76 & 2007 & 0.04247 & 0.00829 & 0.02487 & - \\
\hline
\end{tabular}


Table C-1. (continued).

\begin{tabular}{|c|c|c|c|c|c|c|}
\hline Radionuclide & $\begin{array}{c}\text { Sample } \\
\text { Location }\end{array}$ & Date & $\begin{array}{l}\text { Concentrations } \\
(\mathrm{pCi} / \mathrm{g})\end{array}$ & $\begin{array}{l}\text { Uncertainty } \\
\text { (pCi/g) }\end{array}$ & $\begin{array}{l}\text { MDA } \\
(\mathrm{pCi} / \mathrm{g})\end{array}$ & Flag \\
\hline Cs-134 & ARA-77 & 2007 & 0.1068 & 0.01295 & 0.03885 & - \\
\hline Cs-134 & ARA-8 & 2007 & 0.06107 & 0.0105 & 0.0315 & 一 \\
\hline Cs-134 & ARA-9 & 2007 & 0.04735 & 0.00965 & 0.02895 & - \\
\hline Cs-134 & ARA-10 & 2009 & 0.02878 & 0.0099 & 0.0297 & $\mathrm{U}$ \\
\hline Cs-134 & ARA-13 & 2009 & 0.002409 & 0.01255 & 0.03765 & $\mathrm{U}$ \\
\hline Cs-134 & ARA-18 & 2009 & 0.02375 & 0.01045 & 0.03135 & $\mathrm{U}$ \\
\hline Cs-134 & ARA-20 & 2009 & 0.01748 & 0.0096 & 0.0288 & $\mathrm{U}$ \\
\hline Cs-134 & ARA-21 & 2009 & 0.01696 & 0.0114 & 0.0342 & $\mathrm{U}$ \\
\hline Cs-134 & ARA-25 & 2009 & 0.01217 & 0.0051 & 0.0153 & $\mathrm{U}$ \\
\hline Cs-134 & ARA-26 & 2009 & 0.008734 & 0.0109 & 0.0327 & $\mathrm{U}$ \\
\hline Cs-134 & ARA-27 & 2009 & 0.01828 & 0.00454 & 0.01362 & - \\
\hline Cs-134 & ARA-3 & 2009 & 0.02831 & 0.01675 & 0.05025 & $\mathrm{U}$ \\
\hline Cs-134 & ARA-30 & 2009 & 0.03252 & 0.0196 & 0.0588 & $\mathrm{U}$ \\
\hline Cs-134 & ARA-31 & 2009 & 0.01683 & 0.0087 & 0.0261 & $\mathrm{U}$ \\
\hline Cs-134 & ARA-35 & 2009 & 0.02519 & 0.0114 & 0.0342 & $\mathrm{U}$ \\
\hline Cs-134 & ARA-39 & 2009 & -0.008447 & 0.01105 & 0.03315 & $\mathrm{U}$ \\
\hline Cs-134 & ARA-40 & 2009 & 0.009578 & 0.00615 & 0.01845 & $\mathrm{U}$ \\
\hline Cs-134 & ARA-44 & 2009 & -0.02249 & 0.01325 & 0.03975 & $\mathrm{U}$ \\
\hline Cs-134 & ARA-47 & 2009 & 0.01035 & 0.00715 & 0.02145 & $\mathrm{U}$ \\
\hline Cs-134 & ARA-56 & 2009 & 0.003943 & 0.0031 & 0.0093 & $\mathrm{U}$ \\
\hline Cs-134 & ARA-58 & 2009 & 0.03603 & 0.00575 & 0.01725 & - \\
\hline Cs-134 & ARA-61 & 2009 & -0.02965 & 0.01215 & 0.03645 & $\mathrm{U}$ \\
\hline Cs-134 & ARA-62 & 2009 & 0.02241 & 0.0183 & 0.0549 & $\mathrm{U}$ \\
\hline Cs-134 & ARA-63 & 2009 & 0.03051 & 0.0176 & 0.0528 & $\mathrm{U}$ \\
\hline Cs-134 & ARA-67 & 2009 & 0.02668 & 0.0142 & 0.0426 & $\mathrm{U}$ \\
\hline Cs-134 & ARA-68 & 2009 & 0.01774 & 0.0077 & 0.0231 & $\mathrm{U}$ \\
\hline Cs-134 & ARA-69 & 2009 & 0.0007141 & 0.01255 & 0.03765 & $\mathrm{U}$ \\
\hline Cs-134 & ARA-70 & 2009 & 0.02026 & 0.0087 & 0.0261 & $\mathrm{U}$ \\
\hline Cs-134 & ARA-71 & 2009 & 0.0214 & 0.00875 & 0.02625 & $\mathrm{U}$ \\
\hline Cs-134 & ARA-72 & 2009 & 0.01757 & 0.01375 & 0.04125 & $\mathrm{U}$ \\
\hline Cs-134 & ARA-74 & 2009 & -0.00406 & 0.0134 & 0.0402 & $\mathrm{U}$ \\
\hline Cs-134 & ARA-75 & 2009 & -0.006927 & 0.0133 & 0.0399 & $\mathrm{U}$ \\
\hline Cs-134 & ARA-77 & 2009 & 0.02815 & 0.0054 & 0.0162 & - \\
\hline Cs-134 & ARA-8 & 2009 & 0.01254 & 0.0145 & 0.0435 & $\mathrm{U}$ \\
\hline Cs-134 & ARA-0 & 2010 & 0.003588 & 0.005 & 0.015 & $\mathrm{U}$ \\
\hline Cs-134 & ARA-1 & 2010 & 0.174 & 0.0249 & 0.0747 & - \\
\hline Cs-134 & ARA-11 & 2010 & 0.01054 & 0.00579 & 0.01737 & $\mathrm{U}$ \\
\hline
\end{tabular}


Table C-1. (continued).

\begin{tabular}{|c|c|c|c|c|c|c|}
\hline Radionuclide & $\begin{array}{c}\text { Sample } \\
\text { Location }\end{array}$ & Date & $\begin{array}{c}\text { Concentrations } \\
(\mathrm{pCi} / \mathrm{g})\end{array}$ & $\begin{array}{l}\text { Uncertainty } \\
(\mathrm{pCi} / \mathrm{g})\end{array}$ & $\begin{array}{c}\text { MDA } \\
(\mathrm{pCi} / \mathrm{g})\end{array}$ & Flag \\
\hline Cs-134 & ARA-12 & 2010 & 0.06383 & 0.00868 & 0.02604 & - \\
\hline Cs-134 & ARA-14 & 2010 & 0.05387 & 0.00768 & 0.02304 & - \\
\hline Cs-134 & ARA-15 & 2010 & 0.04957 & 0.00792 & 0.02376 & - \\
\hline Cs-134 & ARA-16 & 2010 & 0.07601 & 0.013 & 0.039 & - \\
\hline Cs-134 & ARA-17 & 2010 & 0.02711 & 0.00674 & 0.02022 & - \\
\hline Cs-134 & ARA-19 & 2010 & 0.08229 & 0.0125 & 0.0375 & - \\
\hline Cs-134 & ARA-2 & 2010 & 0.05579 & 0.00777 & 0.02331 & - \\
\hline Cs-134 & ARA-21 & 2010 & 0.0326 & 0.00821 & 0.02463 & - \\
\hline Cs-134 & ARA-22 & 2010 & 0.09117 & 0.0137 & 0.0411 & - \\
\hline Cs-134 & ARA-23 & 2010 & 0.03971 & 0.0065 & 0.0195 & - \\
\hline Cs-134 & ARA-24 & 2010 & 0.04705 & 0.00802 & 0.02406 & - \\
\hline Cs-134 & ARA-27 & 2010 & 0.04713 & 0.00523 & 0.01569 & - \\
\hline Cs-134 & ARA-28 & 2010 & 0.06926 & 0.0133 & 0.0399 & - \\
\hline Cs-134 & ARA-29 & 2010 & 0.04325 & 0.0057 & 0.0171 & - \\
\hline Cs-134 & ARA-31 & 2010 & 0.0734 & 0.0109 & 0.0327 & - \\
\hline Cs-134 & ARA-32 & 2010 & 0.04751 & 0.00554 & 0.01662 & - \\
\hline Cs-134 & ARA-34 & 2010 & 0.06969 & 0.0103 & 0.0309 & - \\
\hline Cs-134 & ARA-35 & 2010 & -0.004071 & 0.00523 & 0.01569 & $\mathrm{U}$ \\
\hline Cs-134 & ARA-36 & 2010 & 0.04468 & 0.0102 & 0.0306 & - \\
\hline Cs-134 & ARA-37 & 2010 & 0.06164 & 0.00971 & 0.02913 & - \\
\hline Cs-134 & ARA-38 & 2010 & 0.05337 & 0.00727 & 0.02181 & - \\
\hline Cs-134 & ARA-4 & 2010 & 0.0462 & 0.00922 & 0.02766 & - \\
\hline Cs-134 & ARA-40 & 2010 & 0.04406 & 0.00696 & 0.02088 & - \\
\hline Cs-134 & ARA-41 & 2010 & 0.05824 & 0.0328 & 0.0984 & $\mathrm{U}$ \\
\hline Cs-134 & ARA-42 & 2010 & 0.05133 & 0.0062 & 0.0186 & - \\
\hline Cs-134 & ARA-43 & 2010 & 0.009824 & 0.00275 & 0.00825 & - \\
\hline Cs-134 & ARA-44 & 2010 & 0.05967 & 0.0103 & 0.0309 & - \\
\hline Cs-134 & ARA-45 & 2010 & 0.1443 & 0.0249 & 0.0747 & - \\
\hline Cs-134 & ARA-46 & 2010 & 0.04361 & 0.00692 & 0.02076 & - \\
\hline Cs-134 & ARA-47 & 2010 & 0.04244 & 0.00559 & 0.01677 & - \\
\hline Cs-134 & ARA-48 & 2010 & 0.04532 & 0.00641 & 0.01923 & - \\
\hline Cs-134 & ARA-5 & 2010 & 0.04748 & 0.0069 & 0.0207 & - \\
\hline Cs-134 & ARA-50 & 2010 & 0.03549 & 0.00544 & 0.01632 & - \\
\hline Cs-134 & ARA-51 & 2010 & 0.1 & 0.0133 & 0.0399 & - \\
\hline Cs-134 & ARA-52 & 2010 & 0.08762 & 0.0152 & 0.0456 & - \\
\hline Cs-134 & ARA-53 & 2010 & 0.04774 & 0.00744 & 0.02232 & - \\
\hline Cs-134 & ARA-54 & 2010 & 0.05725 & 0.00917 & 0.02751 & - \\
\hline
\end{tabular}


Table C-1. (continued).

\begin{tabular}{|c|c|c|c|c|c|c|}
\hline Radionuclide & $\begin{array}{c}\text { Sample } \\
\text { Location }\end{array}$ & Date & $\begin{array}{c}\text { Concentrations } \\
(\mathrm{pCi} / \mathrm{g})\end{array}$ & $\begin{array}{l}\text { Uncertainty } \\
(\mathrm{pCi} / \mathrm{g})\end{array}$ & $\begin{array}{c}\text { MDA } \\
(\mathrm{pCi} / \mathrm{g})\end{array}$ & Flag \\
\hline Cs-134 & ARA-55 & 2010 & 0.06347 & 0.0121 & 0.0363 & - \\
\hline Cs-134 & ARA-56 & 2010 & 0.06608 & 0.00899 & 0.02697 & - \\
\hline Cs-134 & ARA-57 & 2010 & 0.01827 & 0.00553 & 0.01659 & - \\
\hline Cs-134 & ARA-58 & 2010 & 0.08448 & 0.0153 & 0.0459 & - \\
\hline Cs-134 & ARA-59 & 2010 & 0.0368 & 0.00674 & 0.02022 & - \\
\hline Cs-134 & ARA-6 & 2010 & 0.05869 & 0.0107 & 0.0321 & - \\
\hline Cs-134 & ARA-60 & 2010 & 0.05732 & 0.00952 & 0.02856 & - \\
\hline Cs-134 & ARA-65 & 2010 & 0.07861 & 0.0112 & 0.0336 & - \\
\hline Cs-134 & ARA-7 & 2010 & 0.0629 & 0.0173 & 0.0519 & \\
\hline Cs-134 & ARA-73 & 2010 & 0.05227 & 0.00797 & 0.02391 & - \\
\hline Cs-134 & ARA-74 & 2010 & 0.07505 & 0.0221 & 0.0663 & - \\
\hline Cs-134 & ARA-75 & 2010 & 0.06441 & 0.00997 & 0.02991 & - \\
\hline Cs-134 & ARA-77 & 2010 & 0.1059 & 0.0167 & 0.0501 & - \\
\hline Cs-134 & ARA-9 & 2010 & 0.05986 & 0.0092 & 0.0276 & - \\
\hline Cs-134 & ARA-1 & 2011 & -0.003997 & 0.0165 & 0.0495 & $\mathrm{U}$ \\
\hline Cs-134 & ARA-14 & 2011 & -0.00205 & 0.0105 & 0.0315 & $\mathrm{U}$ \\
\hline Cs-134 & ARA-16 & 2011 & 0.00297 & 0.016 & 0.048 & $\mathrm{U}$ \\
\hline Cs-134 & ARA-2 & 2011 & 0.004703 & 0.0128 & 0.0384 & $\mathrm{U}$ \\
\hline Cs-134 & ARA-24 & 2011 & -0.003188 & 0.00525 & 0.01575 & $\mathrm{U}$ \\
\hline Cs-134 & ARA-28 & 2011 & -0.0746 & 0.0108 & 0.0324 & $\mathrm{U}$ \\
\hline Cs-134 & ARA-29 & 2011 & -0.004136 & 0.0122 & 0.0366 & $\mathrm{U}$ \\
\hline Cs-134 & ARA-31 & 2011 & -0.01086 & 0.0154 & 0.0462 & $\mathrm{U}$ \\
\hline Cs-134 & ARA-32 & 2011 & -0.01427 & 0.0248 & 0.0744 & $\mathrm{U}$ \\
\hline Cs-134 & ARA-34 & 2011 & -0.002829 & 0.0177 & 0.0531 & $\mathrm{U}$ \\
\hline Cs-134 & ARA-38 & 2011 & 0.00105 & 0.0225 & 0.0675 & $\mathrm{U}$ \\
\hline Cs-134 & ARA-4 & 2011 & -0.008226 & 0.0117 & 0.0351 & $\mathrm{U}$ \\
\hline Cs-134 & ARA-42 & 2011 & -0.01544 & 0.0118 & 0.0354 & $\mathrm{U}$ \\
\hline Cs-134 & ARA-43 & 2011 & -0.006347 & 0.02 & 0.06 & $\mathrm{U}$ \\
\hline Cs-134 & ARA-47 & 2011 & -0.00499 & 0.0214 & 0.0642 & $\mathrm{U}$ \\
\hline Cs-134 & ARA-50 & 2011 & -0.02878 & 0.0112 & 0.0336 & $\mathrm{U}$ \\
\hline Cs-134 & ARA-51 & 2011 & -0.01321 & 0.0137 & 0.0411 & $\mathrm{U}$ \\
\hline Cs-134 & ARA-62 & 2011 & -0.004405 & 0.0187 & 0.0561 & $\mathrm{U}$ \\
\hline Cs-134 & ARA-65 & 2011 & 0.001224 & 0.0155 & 0.0465 & $\mathrm{U}$ \\
\hline Cs-134 & ARA-74 & 2011 & -0.08548 & 0.0102 & 0.0306 & $\mathrm{U}$ \\
\hline Cs-134 & ARA-75 & 2011 & -0.0007343 & 0.017 & 0.051 & $\mathrm{U}$ \\
\hline Cs-134 & ARA-77 & 2011 & -0.0444 & 0.0104 & 0.0312 & $\mathrm{U}$ \\
\hline Cs-134 & ARA-9 & 2011 & 0.002184 & 0.00522 & 0.01566 & $\mathrm{U}$ \\
\hline
\end{tabular}


Table C-1. (continued).

\begin{tabular}{|c|c|c|c|c|c|c|}
\hline Radionuclide & $\begin{array}{c}\text { Sample } \\
\text { Location }\end{array}$ & Date & $\begin{array}{c}\text { Concentrations } \\
(\mathrm{pCi} / \mathrm{g})\end{array}$ & $\begin{array}{l}\text { Uncertainty } \\
(\mathrm{pCi} / \mathrm{g})\end{array}$ & $\begin{array}{c}\text { MDA } \\
(\mathrm{pCi} / \mathrm{g})\end{array}$ & Flag \\
\hline Cs-134 & ARA-11 & 2012 & 0.05365 & 0.00886 & 0.02658 & - \\
\hline Cs-134 & ARA-16 & 2012 & 0.06425 & 0.0107 & 0.0321 & - \\
\hline Cs-134 & ARA-31 & 2012 & 0.07447 & 0.0079 & 0.0237 & - \\
\hline Cs-134 & ARA-32 & 2012 & 0.07669 & 0.00938 & 0.02814 & - \\
\hline Cs-134 & ARA-48 & 2012 & 0.05646 & 0.00834 & 0.02502 & - \\
\hline Cs-134 & ARA-53 & 2012 & 0.06261 & 0.00768 & 0.02304 & - \\
\hline Cs-134 & ARA-57 & 2012 & 0.0554 & 0.00867 & 0.02601 & - \\
\hline Cs-134 & ARA-58 & 2012 & 0.06722 & 0.00848 & 0.02544 & - \\
\hline Cs-134 & ARA-59 & 2012 & 0.06266 & 0.00732 & 0.02196 & - \\
\hline Cs-134 & ARA-60 & 2012 & 0.072 & 0.00801 & 0.02403 & - \\
\hline Cs-134 & ARA-62 & 2012 & 0.06219 & 0.00913 & 0.02739 & - \\
\hline Cs-134 & ARA-65 & 2012 & 0.04952 & 0.00755 & 0.02265 & - \\
\hline Cs-134 & ARA-73 & 2012 & 0.01761 & 0.00747 & 0.02241 & $\mathrm{U}$ \\
\hline Cs-134 & ARA-31 & 2013 & 0.07174 & 0.0168 & 0.0504 & - \\
\hline Cs-134 & ARA-32 & 2013 & 0.0007087 & 0.00997 & 0.0299 & $\mathrm{U}$ \\
\hline Cs-134 & ARA-33 & 2013 & 0.02681 & 0.0096 & 0.0288 & $\mathrm{U}$ \\
\hline Cs-134 & ARA-40 & 2013 & 0.04153 & 0.0105 & 0.0316 & - \\
\hline Cs-134 & ARA-48 & 2013 & -0.03367 & 0.00663 & 0.0199 & $\mathrm{U}$ \\
\hline Cs-134 & ARA-65 & 2013 & 0.0009309 & 0.0056 & 0.0168 & $\mathrm{U}$ \\
\hline Cs-134 & $\begin{array}{c}2 \text { INCH AIR } \\
\text { - ARA }\end{array}$ & 2014 & 0.01788 & 0.0167 & 0.0501 & $\mathrm{U}$ \\
\hline Cs-134 & ARA-1 & 2014 & 0.05923 & 0.0125 & 0.0375 & - \\
\hline Cs-134 & ARA-24 & 2014 & 0.04786 & 0.0108 & 0.0324 & - \\
\hline Cs-134 & ARA-31 & 2014 & 0.02944 & 0.0145 & 0.0435 & $\mathrm{U}$ \\
\hline Cs-134 & ARA-32 & 2014 & 0.04228 & 0.0102 & 0.0306 & \\
\hline Cs-134 & ARA-65 & 2014 & 0.03176 & 0.0135 & 0.0405 & $\mathrm{U}$ \\
\hline Cs-134 & ARA-71 & 2014 & 0.02232 & 0.00637 & 0.01911 & - \\
\hline Cs-137 & $0^{\circ} 1000^{\prime}$ & 1977 & 25.6 & 0.8 & 2.4 & - \\
\hline Cs-137 & $0^{\circ} 1500^{\prime}$ & 1977 & 5.5 & 0.2 & 0.6 & - \\
\hline Cs-137 & $0^{\circ} 250^{\prime}$ & 1977 & 71 & 2 & 6 & - \\
\hline Cs-137 & $0^{\circ} 2500^{\prime}$ & 1977 & 2.88 & 0.09 & 0.27 & - \\
\hline Cs-137 & $0^{\circ} 500^{\prime}$ & 1977 & 373 & 11 & 33 & - \\
\hline Cs-137 & $112.5^{\circ} 250^{\prime}$ & 1977 & 4.21 & 0.1 & 0.3 & - \\
\hline Cs-137 & $112^{\circ} 1000^{\prime}$ & 1977 & 1.97 & 0.06 & 0.18 & - \\
\hline Cs-137 & $112^{\circ} 2000^{\prime}$ & 1977 & 0.76 & 0.07 & 0.21 & - \\
\hline Cs-137 & $135^{\circ} 1000^{\prime}$ & 1977 & 2.91 & 0.09 & 0.27 & - \\
\hline Cs-137 & $135^{\circ} 250^{\prime}$ & 1977 & 67 & 1.5 & 4.5 & - \\
\hline
\end{tabular}


Table C-1. (continued).

\begin{tabular}{|c|c|c|c|c|c|c|}
\hline Radionuclide & $\begin{array}{c}\text { Sample } \\
\text { Location }\end{array}$ & Date & $\begin{array}{c}\text { Concentrations } \\
(\mathrm{pCi} / \mathrm{g})\end{array}$ & $\begin{array}{c}\text { Uncertainty } \\
(\mathrm{pCi} / \mathrm{g})\end{array}$ & $\begin{array}{c}\mathrm{MDA} \\
(\mathrm{pCi} / \mathrm{g})\end{array}$ & Flag \\
\hline Cs-137 & $135^{\circ} 2500^{\prime}$ & 1977 & 0.81 & 0.03 & 0.09 & - \\
\hline Cs-137 & $157^{\circ} 1000^{\prime}$ & 1977 & 8.5 & 0.3 & 0.9 & - \\
\hline Cs-137 & $157^{\circ} 2000^{\prime}$ & 1977 & 1.6 & 0.1 & 0.3 & - \\
\hline Cs-137 & $157^{\circ} 500^{\prime}$ & 1977 & 38 & 1 & 3 & - \\
\hline Cs-137 & $180^{\circ} 1000^{\prime}$ & 1977 & 8.6 & 0.3 & 0.9 & - \\
\hline Cs-137 & $180^{\circ} 1500^{\prime}$ & 1977 & 3.87 & 0.17 & 0.51 & - \\
\hline Cs-137 & $180^{\circ} 2500^{\prime}$ & 1977 & 1.1 & 0.09 & 0.27 & - \\
\hline Cs-137 & $180^{\circ} 500^{\prime}$ & 1977 & 30.4 & 0.9 & 2.7 & - \\
\hline Cs-137 & $202^{\circ} 2000^{\prime}$ & 1977 & 5.46 & 0.17 & 0.51 & - \\
\hline Cs-137 & $202^{\circ} 500^{\prime}$ & 1977 & 26.3 & 0.8 & 2.4 & - \\
\hline Cs-137 & $22.5^{\circ} 250^{\prime}$ & 1977 & 35 & 1 & 3 & - \\
\hline Cs-137 & $22^{\circ} 1000^{\prime}$ & 1977 & 11.6 & 0.4 & 1.2 & - \\
\hline Cs-137 & $22^{\circ} 2000^{\prime}$ & 1977 & 2.98 & 0.09 & 0.27 & - \\
\hline Cs-137 & $22^{\circ} 2500^{\prime}$ & 1977 & 2.16 & 0.07 & 0.21 & - \\
\hline Cs-137 & $22^{\circ} 500^{\prime}$ & 1977 & 76 & 2 & 6 & - \\
\hline Cs-137 & $225^{\circ} 1000^{\prime}$ & 1977 & 13.3 & 0.4 & 1.2 & - \\
\hline Cs-137 & $225^{\circ} 2500^{\prime}$ & 1977 & 3.74 & 0.17 & 0.51 & - \\
\hline Cs-137 & $225^{\circ} 500^{\prime}$ & 1977 & 56.6 & 1.7 & 5.1 & - \\
\hline Cs-137 & $247^{\circ} 2000^{\prime}$ & 1977 & 1.77 & 0.06 & 0.18 & - \\
\hline Cs-137 & $247^{\circ} 500^{\prime}$ & 1977 & 21.8 & 0.7 & 2.1 & - \\
\hline Cs-137 & $270^{\circ} 1000^{\prime}$ & 1977 & 1.24 & 0.04 & 0.12 & - \\
\hline Cs-137 & $270^{\circ} 1500^{\prime}$ & 1977 & 1.63 & 0.06 & 0.18 & - \\
\hline Cs-137 & $270^{\circ} 2500^{\prime}$ & 1977 & 0.56 & 0.07 & 0.21 & - \\
\hline Cs-137 & $270^{\circ} 500^{\prime}$ & 1977 & 26.2 & 0.8 & 2.4 & - \\
\hline Cs-137 & $292^{\circ} 2000^{\prime}$ & 1977 & 7.5 & 0.3 & 0.9 & - \\
\hline Cs-137 & $292^{\circ} 500^{\prime}$ & 1977 & 2.66 & 0.08 & 0.24 & - \\
\hline Cs-137 & $315^{\circ} 1000^{\prime}$ & 1977 & 1.94 & 0.06 & 0.18 & - \\
\hline Cs-137 & $315^{\circ} 1500^{\prime}$ & 1977 & 0.76 & 0.08 & 0.24 & - \\
\hline Cs-137 & $315^{\circ} 250^{\prime}$ & 1977 & 1.64 & 0.06 & 0.18 & - \\
\hline Cs-137 & $315^{\circ} 2500^{\prime}$ & 1977 & 0.91 & 0.08 & 0.24 & - \\
\hline Cs-137 & $315^{\circ} 500^{\prime}$ & 1977 & 8.2 & 0.2 & 0.6 & - \\
\hline Cs-137 & $337.5^{\circ} 250^{\prime}$ & 1977 & 29.6 & 0.9 & 2.7 & - \\
\hline Cs-137 & $337^{\circ} 1000^{\prime}$ & 1977 & 44.1 & 1.3 & 3.9 & - \\
\hline Cs-137 & $337^{\circ} 2000^{\prime}$ & 1977 & 1.49 & 0.05 & 0.15 & - \\
\hline Cs-137 & $45^{\circ} 1000^{\prime}$ & 1977 & 16.7 & 0.5 & 1.5 & - \\
\hline Cs-137 & $45^{\circ} 1500^{\prime}$ & 1977 & 17.6 & 0.5 & 1.5 & - \\
\hline Cs-137 & $45^{\circ} 250^{\prime}$ & 1977 & 1.46 & 0.19 & 0.57 & - \\
\hline
\end{tabular}


Table C-1. (continued).

\begin{tabular}{|c|c|c|c|c|c|c|}
\hline Radionuclide & $\begin{array}{c}\text { Sample } \\
\text { Location }\end{array}$ & Date & $\begin{array}{c}\text { Concentrations } \\
(\mathrm{pCi} / \mathrm{g})\end{array}$ & $\begin{array}{l}\text { Uncertainty } \\
(\mathrm{pCi} / \mathrm{g})\end{array}$ & $\begin{array}{c}\text { MDA } \\
(\mathrm{pCi} / \mathrm{g})\end{array}$ & Flag \\
\hline Cs-137 & $45^{\circ} 2500^{\prime}$ & 1977 & 9.8 & 0.3 & 0.9 & - \\
\hline Cs-137 & $45^{\circ} 500^{\prime}$ & 1977 & 109 & 3 & 9 & - \\
\hline Cs-137 & $45^{\circ} 5000^{\prime}$ & 1977 & 2.13 & 0.07 & 0.21 & - \\
\hline Cs-137 & $67^{\circ} 1000^{\prime}$ & 1977 & 121 & 4 & 12 & - \\
\hline Cs-137 & $67^{\circ} 2000^{\prime}$ & 1977 & 30.5 & 0.9 & 2.7 & - \\
\hline Cs-137 & $67^{\circ} 2500^{\prime}$ & 1977 & 5.95 & 0.19 & 0.57 & - \\
\hline Cs-137 & $67^{\circ} 500^{\prime}$ & 1977 & 241 & 7 & 21 & - \\
\hline Cs-137 & $90^{\circ} 1000^{\prime}$ & 1977 & 3.2 & 0.09 & 0.27 & - \\
\hline Cs-137 & $90^{\circ} 1500^{\prime}$ & 1977 & 1.18 & 0.09 & 0.27 & - \\
\hline Cs-137 & $90^{\circ} 250^{\prime}$ & 1977 & 9.3 & 0.2 & 0.6 & - \\
\hline Cs-137 & $90^{\circ} 2500^{\prime}$ & 1977 & 0.99 & 0.08 & 0.24 & - \\
\hline Cs-137 & $90^{\circ} 500^{\prime}$ & 1977 & 17.7 & 0.5 & 1.5 & - \\
\hline Cs-137 & $0^{\circ} 1000^{\prime}$ & 1985 & 7.3 & 0.3 & 0.9 & - \\
\hline Cs-137 & $0^{\circ} 2500^{\prime}$ & 1985 & 2.29 & 0.13 & 0.39 & - \\
\hline Cs-137 & $112.5^{\circ} 1500^{\prime}$ & 1985 & 1.1 & 0.08 & 0.24 & - \\
\hline Cs-137 & $135^{\circ} 2500^{\prime}$ & 1985 & 3.49 & 0.18 & 0.54 & - \\
\hline Cs-137 & $135^{\circ} 2500^{\prime}$ & 1985 & 1.11 & 0.09 & 0.27 & - \\
\hline Cs-137 & $157.5^{\circ} 2000^{\prime}$ & 1985 & 1.97 & 0.12 & 0.36 & - \\
\hline Cs-137 & $180^{\circ} 1500^{\prime}$ & 1985 & 3.04 & 0.16 & 0.48 & - \\
\hline Cs-137 & $180^{\circ} 2500^{\prime}$ & 1985 & 1.08 & 0.09 & 0.27 & - \\
\hline Cs-137 & $202.5^{\circ} 1000^{\prime}$ & 1985 & 11.9 & 0.5 & 1.5 & - \\
\hline Cs-137 & $202.5^{\circ} 2000^{\prime}$ & 1985 & 2.8 & 0.15 & 0.45 & - \\
\hline Cs-137 & $202.5^{\circ} 3500^{\prime}$ & 1985 & 2.5 & 0.14 & 0.42 & - \\
\hline Cs-137 & $22.5^{\circ} 1500^{\prime}$ & 1985 & 2.98 & 0.16 & 0.48 & - \\
\hline Cs-137 & $22.5^{\circ} 2500^{\prime}$ & 1985 & 2.27 & 0.13 & 0.39 & - \\
\hline Cs-137 & $225^{\circ} 2500^{\prime}$ & 1985 & 1.67 & 0.11 & 0.33 & - \\
\hline Cs-137 & $225^{\circ} 500^{\prime}$ & 1985 & 46.8 & 1.8 & 5.4 & - \\
\hline Cs-137 & $247.5^{\circ} 1500^{\prime}$ & 1985 & 2.04 & 0.12 & 0.36 & - \\
\hline Cs-137 & $270^{\circ} 1000^{\prime}$ & 1985 & 2.7 & 0.15 & 0.45 & - \\
\hline Cs-137 & $270^{\circ} 2000^{\prime}$ & 1985 & 0.76 & 0.07 & 0.21 & - \\
\hline Cs-137 & $292.5^{\circ} 2000^{\prime}$ & 1985 & 7 & 0.3 & 0.9 & - \\
\hline Cs-137 & $292.5^{\circ} 500^{\prime}$ & 1985 & 2.16 & 0.13 & 0.39 & - \\
\hline Cs-137 & $315^{\circ} 2000^{\prime}$ & 1985 & 0.78 & 0.07 & 0.21 & - \\
\hline Cs-137 & $33.75^{\circ} 2000^{\prime}$ & 1985 & 3.91 & 0.2 & 0.6 & - \\
\hline Cs-137 & $\begin{array}{c}337 . .5^{\circ} \\
1500^{\prime}\end{array}$ & 1985 & 1.43 & 0.1 & 0.3 & - \\
\hline Cs-137 & $337.5^{\circ} 2500^{\prime}$ & 1985 & 0.67 & 0.06 & 0.18 & - \\
\hline
\end{tabular}


Table C-1. (continued).

\begin{tabular}{|c|c|c|c|c|c|c|}
\hline Radionuclide & $\begin{array}{c}\text { Sample } \\
\text { Location }\end{array}$ & Date & $\begin{array}{c}\text { Concentrations } \\
(\mathrm{pCi} / \mathrm{g})\end{array}$ & $\begin{array}{l}\text { Uncertainty } \\
(\mathrm{pCi} / \mathrm{g})\end{array}$ & $\begin{array}{l}\text { MDA } \\
(\mathrm{pCi} / \mathrm{g})\end{array}$ & Flag \\
\hline Cs-137 & $45^{\circ} 1000^{\prime}$ & 1985 & 12.1 & 0.5 & 1.5 & - \\
\hline Cs-137 & $45^{\circ} 2500^{\prime}$ & 1985 & 9.5 & 0.4 & 1.2 & - \\
\hline Cs-137 & $45^{\circ} 3250^{\prime}$ & 1985 & 1.71 & 0.1 & 0.3 & 一 \\
\hline Cs-137 & $56.25^{\circ} 2000^{\prime}$ & 1985 & 16.8 & 0.7 & 2.1 & - \\
\hline Cs-137 & $67.5^{\circ} 2000^{\prime}$ & 1985 & 14.8 & 0.6 & 1.8 & - \\
\hline Cs-137 & $67.5^{\circ} 500^{\prime}$ & 1985 & 133 & 5 & 15 & - \\
\hline Cs-137 & $90^{\circ} 1000^{\prime}$ & 1985 & 2.75 & 0.15 & 0.45 & 一 \\
\hline Cs-137 & $90^{\circ} 2000^{\prime}$ & 1985 & 1.16 & 0.09 & 0.27 & 一 \\
\hline Cs-137 & $112.5^{\circ} 2000^{\prime}$ & 1991 & 0.95 & 0.07 & 0.21 & 一 \\
\hline Cs-137 & $135^{\circ} 2500^{\prime}$ & 1991 & 1.02 & 0.07 & 0.21 & - \\
\hline Cs-137 & $157.5^{\circ} 1500^{\prime}$ & 1991 & 2.3 & 0.11 & 0.33 & - \\
\hline Cs-137 & $157.5^{\circ} 500^{\prime}$ & 1991 & 31.4 & 1.2 & 3.6 & 一 \\
\hline Cs-137 & $270^{\circ} 1000^{\prime}$ & 1991 & 1.54 & 0.09 & 0.27 & 一 \\
\hline Cs-137 & $315^{\circ} 2500^{\prime}$ & 1991 & 0.67 & 0.06 & 0.18 & - \\
\hline Cs-137 & $337.5^{\circ} 1500^{\prime}$ & 1991 & 1.09 & 0.07 & 0.21 & - \\
\hline Cs-137 & $90^{\circ} 2500^{\prime}$ & 1991 & 1.23 & 0.08 & 0.24 & - \\
\hline Cs-137 & ARA-0 & 2006 & 7.334 & 0.1085 & 0.3255 & - \\
\hline Cs-137 & ARA-1 & 2006 & 0.1086 & 0.00645 & 0.01935 & - \\
\hline Cs-137 & ARA-10 & 2006 & 0.6276 & 0.0361 & 0.1083 & - \\
\hline Cs-137 & ARA-11 & 2006 & 0.2194 & 23.45 & 70.35 & $\mathrm{U}$ \\
\hline Cs-137 & ARA-12 & 2006 & 0.3869 & 23.2 & 69.6 & $\mathrm{U}$ \\
\hline Cs-137 & ARA-13 & 2006 & 7.38 & 0.064 & 0.192 & - \\
\hline Cs-137 & ARA-14 & 2006 & 0.1468 & 4.9 & 14.7 & $\mathrm{U}$ \\
\hline Cs-137 & ARA-15 & 2006 & 0.8889 & 4 & 12 & $\mathrm{U}$ \\
\hline Cs-137 & ARA-16 & 2006 & 0.4734 & 9.5 & 28.5 & $\mathrm{U}$ \\
\hline Cs-137 & ARA-17 & 2006 & 1.647 & 2.1 & 6.3 & $\mathrm{U}$ \\
\hline Cs-137 & ARA-18 & 2006 & 0.7294 & 0.0655 & 0.1965 & - \\
\hline Cs-137 & ARA-19 & 2006 & 1.182 & 2.4 & 7.2 & $\mathrm{U}$ \\
\hline Cs-137 & ARA-2 & 2006 & 2.24 & 0.056 & 0.168 & - \\
\hline Cs-137 & ARA-20 & 2006 & 2.25 & 0.04005 & 0.12015 & - \\
\hline Cs-137 & ARA-21 & 2006 & 4.441 & 1.25 & 3.75 & - \\
\hline Cs-137 & ARA-22 & 2006 & 2.432 & 1.8 & 5.4 & $\mathrm{U}$ \\
\hline Cs-137 & ARA-23 & 2006 & 0.06294 & 1.35 & 4.05 & $\mathrm{U}$ \\
\hline Cs-137 & ARA-24 & 2006 & 0.9544 & 5.7 & 17.1 & $\mathrm{U}$ \\
\hline Cs-137 & ARA-26 & 2006 & 8.426 & 0.092 & 0.276 & - \\
\hline Cs-137 & ARA-27 & 2006 & 0.4941 & 3.4 & 10.2 & $\mathrm{U}$ \\
\hline Cs-137 & ARA-28 & 2006 & 0.3807 & 2.8 & 8.4 & $\mathrm{U}$ \\
\hline
\end{tabular}


Table C-1. (continued).

\begin{tabular}{|c|c|c|c|c|c|c|}
\hline Radionuclide & $\begin{array}{c}\text { Sample } \\
\text { Location }\end{array}$ & Date & $\begin{array}{c}\text { Concentrations } \\
(\mathrm{pCi} / \mathrm{g})\end{array}$ & $\begin{array}{l}\text { Uncertainty } \\
(\mathrm{pCi} / \mathrm{g})\end{array}$ & $\begin{array}{c}\text { MDA } \\
(\mathrm{pCi} / \mathrm{g})\end{array}$ & Flag \\
\hline Cs-137 & ARA-29 & 2006 & 0.2388 & 8.95 & 26.85 & $\mathrm{U}$ \\
\hline Cs-137 & ARA-3 & 2006 & 7.044 & 0.056 & 0.168 & - \\
\hline Cs-137 & ARA-30 & 2006 & 7.678 & 0.0665 & 0.1995 & - \\
\hline Cs-137 & ARA-31 & 2006 & 0.5219 & 0.04295 & 0.12885 & - \\
\hline Cs-137 & ARA-32 & 2006 & 0.4751 & 0.0685 & 0.2055 & 一 \\
\hline Cs-137 & ARA-33 & 2006 & 4.831 & 0.053 & 0.159 & - \\
\hline Cs-137 & ARA-34 & 2006 & 0.3508 & 0.0855 & 0.2565 & - \\
\hline Cs-137 & ARA-35 & 2006 & 0.9907 & 1.35 & 4.05 & $\mathrm{U}$ \\
\hline Cs-137 & ARA-36 & 2006 & 0.3587 & 2.75 & 8.25 & $\mathrm{U}$ \\
\hline Cs-137 & ARA-37 & 2006 & 0.2708 & 5.5 & 16.5 & $\mathrm{U}$ \\
\hline Cs-137 & ARA-38 & 2006 & 0.1846 & 7.2 & 21.6 & $\mathrm{U}$ \\
\hline Cs-137 & ARA-39 & 2006 & 4.301 & 0.0715 & 0.2145 & - \\
\hline Cs-137 & ARA-4 & 2006 & 0.2372 & 0.02535 & 0.07605 & - \\
\hline Cs-137 & ARA-40 & 2006 & 1.043 & 0.072 & 0.216 & - \\
\hline Cs-137 & ARA-41 & 2006 & 0.4455 & 0.098 & 0.294 & - \\
\hline Cs-137 & ARA-42 & 2006 & 0.2916 & 0.0645 & 0.1935 & - \\
\hline Cs-137 & ARA-43 & 2006 & 1.299 & 0.0675 & 0.2025 & - \\
\hline Cs-137 & ARA-44 & 2006 & 0.7157 & 0.0995 & 0.2985 & - \\
\hline Cs-137 & ARA-45 & 2006 & 0.1394 & 7.9 & 23.7 & $\mathrm{U}$ \\
\hline Cs-137 & ARA-46 & 2006 & 0.1102 & 7.55 & 22.65 & $\mathrm{U}$ \\
\hline Cs-137 & ARA-47 & 2006 & 0.09029 & 9 & 27 & $\mathrm{U}$ \\
\hline Cs-137 & ARA-48 & 2006 & 1.299 & 0.0675 & 0.2025 & - \\
\hline Cs-137 & ARA-49 & 2006 & 0.4117 & 0.078 & 0.234 & - \\
\hline Cs-137 & ARA-5 & 2006 & 1.475 & 0.0555 & 0.1665 & - \\
\hline Cs-137 & ARA-50 & 2006 & 0.3242 & 0.0635 & 0.1905 & - \\
\hline Cs-137 & ARA-51 & 2006 & 1.27 & 0.102 & 0.306 & - \\
\hline Cs-137 & ARA-52 & 2006 & 0.08828 & 12.45 & 37.35 & $\mathrm{U}$ \\
\hline Cs-137 & ARA-53 & 2006 & 0.179 & 0.051 & 0.153 & - \\
\hline Cs-137 & ARA-54 & 2006 & 0.3886 & 0.0525 & 0.1575 & - \\
\hline Cs-137 & ARA-55 & 2006 & 0.2129 & 0.0685 & 0.2055 & - \\
\hline Cs-137 & ARA-56 & 2006 & 0.3101 & 0.09 & 0.27 & - \\
\hline Cs-137 & ARA-57 & 2006 & 0.5972 & 0.0595 & 0.1785 & - \\
\hline Cs-137 & ARA-58 & 2006 & 0.1957 & 0.065 & 0.195 & - \\
\hline Cs-137 & ARA-59 & 2006 & 0.2239 & 0.059 & 0.177 & - \\
\hline Cs-137 & ARA-6 & 2006 & 0.8341 & 0.04115 & 0.12345 & - \\
\hline Cs-137 & ARA-60 & 2006 & 0.4089 & 0.093 & 0.279 & - \\
\hline Cs-137 & ARA-61 & 2006 & 10.38 & 0.087 & 0.261 & - \\
\hline
\end{tabular}


Table C-1. (continued).

\begin{tabular}{|c|c|c|c|c|c|c|}
\hline Radionuclide & $\begin{array}{c}\text { Sample } \\
\text { Location }\end{array}$ & Date & $\begin{array}{c}\text { Concentrations } \\
(\mathrm{pCi} / \mathrm{g})\end{array}$ & $\begin{array}{c}\text { Uncertainty } \\
(\mathrm{pCi} / \mathrm{g})\end{array}$ & $\begin{array}{c}\text { MDA } \\
(\mathrm{pCi} / \mathrm{g})\end{array}$ & Flag \\
\hline Cs-137 & ARA-62 & 2006 & 8.113 & 0.071 & 0.213 & - \\
\hline Cs-137 & ARA-63 & 2006 & 0.172 & 0.0076 & 0.0228 & - \\
\hline Cs-137 & ARA-65 & 2006 & 1.009 & 0.0625 & 0.1875 & - \\
\hline Cs-137 & ARA-66 & 2006 & 4.356 & 0.0895 & 0.2685 & - \\
\hline Cs-137 & ARA-67 & 2006 & 2.878 & 0.069 & 0.207 & - \\
\hline Cs-137 & ARA-68 & 2006 & 0.5706 & 0.0114 & 0.0342 & - \\
\hline Cs-137 & ARA-69 & 2006 & 15.44 & 0.1075 & 0.3225 & - \\
\hline Cs-137 & ARA-7 & 2006 & 0.8425 & 0.053 & 0.159 & - \\
\hline Cs-137 & ARA-70 & 2006 & 5.905 & 0.0575 & 0.1725 & - \\
\hline Cs-137 & ARA-71 & 2006 & 0.9692 & 0.0985 & 0.2955 & - \\
\hline Cs-137 & ARA-72 & 2006 & 2.448 & 0.0805 & 0.2415 & - \\
\hline Cs-137 & ARA-73 & 2006 & 0.5155 & 0.0905 & 0.2715 & - \\
\hline Cs-137 & ARA-74 & 2006 & 0.3194 & 0.0925 & 0.2775 & - \\
\hline Cs-137 & ARA-75 & 2006 & 0.4479 & 0.1025 & 0.3075 & - \\
\hline Cs-137 & ARA-76 & 2006 & 17.31 & 0.1055 & 0.3165 & - \\
\hline Cs-137 & ARA-77 & 2006 & 0.4814 & 0.119 & 0.357 & - \\
\hline Cs-137 & ARA-8 & 2006 & 12.9 & 0.0945 & 0.2835 & - \\
\hline Cs-137 & ARA-9 & 2006 & 0.7335 & 0.04925 & 0.14775 & - \\
\hline Cs-137 & ARA-0 & 2007 & 2.756 & 0.01715 & 0.05145 & - \\
\hline Cs-137 & ARA-1 & 2007 & 1.243 & 0.00271 & 0.00813 & - \\
\hline Cs-137 & ARA-10 & 2007 & 0.2615 & 0.0105 & 0.0315 & - \\
\hline Cs-137 & ARA-11 & 2007 & 0.2572 & 0.00456 & 0.01368 & - \\
\hline Cs-137 & ARA-12 & 2007 & 0.3087 & 0.0082 & 0.0246 & - \\
\hline Cs-137 & ARA-13 & 2007 & 4.453 & 0.0168 & 0.0504 & - \\
\hline Cs-137 & ARA-14 & 2007 & 0.2814 & 0.0091 & 0.0273 & - \\
\hline Cs-137 & ARA-15 & 2007 & 1.992 & 0.02045 & 0.06135 & - \\
\hline Cs-137 & ARA-16 & 2007 & 0.649 & 0.00959 & 0.02877 & - \\
\hline Cs-137 & ARA-17 & 2007 & 0.6889 & 0.01285 & 0.03855 & - \\
\hline Cs-137 & ARA-18 & 2007 & 5.061 & 0.0298 & 0.0894 & - \\
\hline Cs-137 & ARA-19 & 2007 & 0.4658 & 0.0196 & 0.0588 & - \\
\hline Cs-137 & ARA-2 & 2007 & 0.8085 & 0.0222 & 0.0666 & - \\
\hline Cs-137 & ARA-20 & 2007 & 1.032 & 0.0147 & 0.0441 & - \\
\hline Cs-137 & ARA-21 & 2007 & 1.858 & 0.0167 & 0.0501 & - \\
\hline Cs-137 & ARA-22 & 2007 & 1.029 & 0.0162 & 0.0486 & - \\
\hline Cs-137 & ARA-23 & 2007 & 0.5455 & 0.0121 & 0.0363 & - \\
\hline Cs-137 & ARA-24 & 2007 & 0.303 & 0.00489 & 0.01467 & - \\
\hline Cs-137 & ARA-25 & 2007 & 2.48 & 0.0202 & 0.0606 & - \\
\hline
\end{tabular}


Table C-1. (continued).

\begin{tabular}{|c|c|c|c|c|c|c|}
\hline Radionuclide & $\begin{array}{c}\text { Sample } \\
\text { Location }\end{array}$ & Date & $\begin{array}{c}\text { Concentrations } \\
(\mathrm{pCi} / \mathrm{g})\end{array}$ & $\begin{array}{l}\text { Uncertainty } \\
(\mathrm{pCi} / \mathrm{g})\end{array}$ & $\begin{array}{c}\text { MDA } \\
(\mathrm{pCi} / \mathrm{g})\end{array}$ & Flag \\
\hline Cs-137 & ARA-26 & 2007 & 3.513 & 0.0222 & 0.0666 & - \\
\hline Cs-137 & ARA-27 & 2007 & 1.943 & 0.0136 & 0.0408 & - \\
\hline Cs-137 & ARA-28 & 2007 & 1.348 & 0.0096 & 0.0288 & - \\
\hline Cs-137 & ARA-29 & 2007 & 0.7172 & 0.01205 & 0.03615 & - \\
\hline Cs-137 & ARA-3 & 2007 & 3.337 & 0.0206 & 0.0618 & 一 \\
\hline Cs-137 & ARA-30 & 2007 & 2.676 & 0.0169 & 0.0507 & - \\
\hline Cs-137 & ARA-31 & 2007 & 0.9774 & 0.0218 & 0.0654 & - \\
\hline Cs-137 & ARA-32 & 2007 & 0.7291 & 0.0202 & 0.0606 & - \\
\hline Cs-137 & ARA-33 & 2007 & 1.798 & 0.0138 & 0.0414 & - \\
\hline Cs-137 & ARA-34 & 2007 & 0.439 & 0.00659 & 0.01977 & - \\
\hline Cs-137 & ARA-35 & 2007 & 2.687 & 0.0151 & 0.0453 & - \\
\hline Cs-137 & ARA-36 & 2007 & 1.237 & 0.0135 & 0.0405 & - \\
\hline Cs-137 & ARA-37 & 2007 & 0.6494 & 0.0137 & 0.0411 & - \\
\hline Cs-137 & ARA-38 & 2007 & 0.6048 & 0.01175 & 0.03525 & - \\
\hline Cs-137 & ARA-39 & 2007 & 1.218 & 0.0158 & 0.0474 & - \\
\hline Cs-137 & ARA-4 & 2007 & 0.7505 & 0.0127 & 0.0381 & - \\
\hline Cs-137 & ARA-40 & 2007 & 0.9159 & 0.0148 & 0.0444 & - \\
\hline Cs-137 & ARA-41 & 2007 & 0.4337 & 0.00834 & 0.02502 & - \\
\hline Cs-137 & ARA-42 & 2007 & 0.4162 & 0.0096 & 0.0288 & - \\
\hline Cs-137 & ARA-43 & 2007 & 0.3697 & 0.00945 & 0.02835 & - \\
\hline Cs-137 & ARA-44 & 2007 & 0.6785 & 0.00657 & 0.01971 & - \\
\hline Cs-137 & ARA-45 & 2007 & 0.4444 & 0.0188 & 0.0564 & - \\
\hline Cs-137 & ARA-46 & 2007 & 0.3773 & 0.01285 & 0.03855 & - \\
\hline Cs-137 & ARA-47 & 2007 & 0.2996 & 0.0134 & 0.0402 & - \\
\hline Cs-137 & ARA-48 & 2007 & 2.34 & 0.0259 & 0.0777 & - \\
\hline Cs-137 & ARA-49 & 2007 & 0.2932 & 0.009 & 0.027 & - \\
\hline Cs-137 & ARA-5 & 2007 & 0.5303 & 0.0131 & 0.0393 & - \\
\hline Cs-137 & ARA-50 & 2007 & 0.2465 & 0.01555 & 0.04665 & - \\
\hline Cs-137 & ARA-51 & 2007 & 1.686 & 0.02065 & 0.06195 & - \\
\hline Cs-137 & ARA-52 & 2007 & 0.2448 & 0.01685 & 0.05055 & - \\
\hline Cs-137 & ARA-53 & 2007 & 0.2443 & 0.0101 & 0.0303 & - \\
\hline Cs-137 & ARA-54 & 2007 & 0.2271 & 0.0108 & 0.0324 & - \\
\hline Cs-137 & ARA-55 & 2007 & 0.1676 & 0.0137 & 0.0411 & - \\
\hline Cs-137 & ARA-56 & 2007 & 0.2312 & 0.008 & 0.024 & - \\
\hline Cs-137 & ARA-57 & 2007 & 0.2256 & 0.0151 & 0.0453 & - \\
\hline Cs-137 & ARA-58 & 2007 & 0.3719 & 0.01135 & 0.03405 & - \\
\hline Cs-137 & ARA-59 & 2007 & 0.2553 & 0.013 & 0.039 & - \\
\hline
\end{tabular}


Table C-1. (continued).

\begin{tabular}{|c|c|c|c|c|c|c|}
\hline Radionuclide & $\begin{array}{c}\text { Sample } \\
\text { Location }\end{array}$ & Date & $\begin{array}{c}\text { Concentrations } \\
(\mathrm{pCi} / \mathrm{g})\end{array}$ & $\begin{array}{l}\text { Uncertainty } \\
(\mathrm{pCi} / \mathrm{g})\end{array}$ & $\begin{array}{c}\text { MDA } \\
(\mathrm{pCi} / \mathrm{g})\end{array}$ & Flag \\
\hline Cs-137 & ARA-6 & 2007 & 0.3158 & 0.01 & 0.03 & - \\
\hline Cs-137 & ARA-60 & 2007 & 0.3157 & 0.00965 & 0.02895 & - \\
\hline Cs-137 & ARA-61 & 2007 & 3.367 & 0.0233 & 0.0699 & - \\
\hline Cs-137 & ARA-62 & 2007 & 3.339 & 0.0207 & 0.0621 & - \\
\hline Cs-137 & ARA-63 & 2007 & 3.795 & 0.0206 & 0.0618 & - \\
\hline Cs-137 & ARA-65 & 2007 & 1.868 & 0.0165 & 0.0495 & - \\
\hline Cs-137 & ARA-66 & 2007 & 0.6005 & 0.00818 & 0.02454 & - \\
\hline Cs-137 & ARA-67 & 2007 & 2.494 & 0.016 & 0.048 & - \\
\hline Cs-137 & ARA-68 & 2007 & 1.693 & 0.0147 & 0.0441 & - \\
\hline Cs-137 & ARA-69 & 2007 & 5.872 & 0.0205 & 0.0615 & - \\
\hline Cs-137 & ARA-7 & 2007 & 0.2316 & 0.0071 & 0.0213 & - \\
\hline Cs-137 & ARA-70 & 2007 & 2.523 & 0.0188 & 0.0564 & - \\
\hline Cs-137 & ARA-71 & 2007 & 3.827 & 0.0186 & 0.0558 & - \\
\hline Cs-137 & ARA-72 & 2007 & 0.9004 & 0.0138 & 0.0414 & - \\
\hline Cs-137 & ARA-73 & 2007 & 0.8435 & 0.01345 & 0.04035 & - \\
\hline Cs-137 & ARA-74 & 2007 & 0.434 & 0.01305 & 0.03915 & - \\
\hline Cs-137 & ARA-75 & 2007 & 0.3964 & 0.0118 & 0.0354 & - \\
\hline Cs-137 & ARA-76 & 2007 & 9.189 & 0.0308 & 0.0924 & - \\
\hline Cs-137 & ARA-77 & 2007 & 0.2793 & 0.0104 & 0.0312 & - \\
\hline Cs-137 & ARA-8 & 2007 & 5.04 & 0.0231 & 0.0693 & - \\
\hline Cs-137 & ARA-9 & 2007 & 0.1999 & 0.00772 & 0.02316 & - \\
\hline Cs-137 & ARA-0 & 2009 & 1.87 & 0.009 & 0.027 & - \\
\hline Cs-137 & ARA-1 & 2009 & 0.8197 & 0.00635 & 0.01905 & - \\
\hline Cs-137 & ARA-10 & 2009 & 0.2822 & 0.00945 & 0.02835 & - \\
\hline Cs-137 & ARA-11 & 2009 & 0.2658 & 0.008 & 0.024 & - \\
\hline Cs-137 & ARA-12 & 2009 & 0.296 & 0.0081 & 0.0243 & - \\
\hline Cs-137 & ARA-13 & 2009 & 4.5 & 0.02855 & 0.08565 & - \\
\hline Cs-137 & ARA-14 & 2009 & 0.2366 & 0.00595 & 0.01785 & - \\
\hline Cs-137 & ARA-15 & 2009 & 1.533 & 0.0122 & 0.0366 & - \\
\hline Cs-137 & ARA-16 & 2009 & 0.6973 & 0.0098 & 0.0294 & - \\
\hline Cs-137 & ARA-17 & 2009 & 0.5257 & 0.0073 & 0.0219 & - \\
\hline Cs-137 & ARA-18 & 2009 & 0.2576 & 0.01105 & 0.03315 & - \\
\hline Cs-137 & ARA-19 & 2009 & 0.3758 & 0.0089 & 0.0267 & - \\
\hline Cs-137 & ARA-2 & 2009 & 0.6175 & 0.00575 & 0.01725 & - \\
\hline Cs-137 & ARA-20 & 2009 & 0.9695 & 0.01505 & 0.04515 & - \\
\hline Cs-137 & ARA-21 & 2009 & 1.44 & 0.01175 & 0.03525 & - \\
\hline Cs-137 & ARA-22 & 2009 & 0.6917 & 0.0072 & 0.0216 & - \\
\hline
\end{tabular}


Table C-1. (continued).

\begin{tabular}{|c|c|c|c|c|c|c|}
\hline Radionuclide & $\begin{array}{c}\text { Sample } \\
\text { Location }\end{array}$ & Date & $\begin{array}{c}\text { Concentrations } \\
(\mathrm{pCi} / \mathrm{g})\end{array}$ & $\begin{array}{l}\text { Uncertainty } \\
(\mathrm{pCi} / \mathrm{g})\end{array}$ & $\begin{array}{c}\text { MDA } \\
(\mathrm{pCi} / \mathrm{g})\end{array}$ & Flag \\
\hline Cs-137 & ARA-23 & 2009 & 0.4066 & 0.00845 & 0.02535 & - \\
\hline Cs-137 & ARA-24 & 2009 & 0.06502 & 0.0063 & 0.0189 & - \\
\hline Cs-137 & ARA-25 & 2009 & 1.995 & 0.0129 & 0.0387 & - \\
\hline Cs-137 & ARA-26 & 2009 & 3.135 & 0.02285 & 0.06855 & - \\
\hline Cs-137 & ARA-27 & 2009 & 1.378 & 0.007 & 0.021 & - \\
\hline Cs-137 & ARA-28 & 2009 & 1.022 & 0.01065 & 0.03195 & - \\
\hline Cs-137 & ARA-29 & 2009 & 0.5661 & 0.00845 & 0.02535 & - \\
\hline Cs-137 & ARA-3 & 2009 & 2.931 & 0.024 & 0.072 & - \\
\hline Cs-137 & ARA-30 & 2009 & 2.221 & 0.0192 & 0.0576 & - \\
\hline Cs-137 & ARA-31 & 2009 & 0.8786 & 0.0102 & 0.0306 & - \\
\hline Cs-137 & ARA-33 & 2009 & 2.463 & 0.0145 & 0.0435 & - \\
\hline Cs-137 & ARA-34 & 2009 & 0.5281 & 0.00915 & 0.02745 & - \\
\hline Cs-137 & ARA-35 & 2009 & 2.725 & 0.01505 & 0.04515 & - \\
\hline Cs-137 & ARA-36 & 2009 & 0.9555 & 0.01035 & 0.03105 & - \\
\hline Cs-137 & ARA-37 & 2009 & 0.5692 & 0.0051 & 0.0153 & - \\
\hline Cs-137 & ARA-38 & 2009 & 0.4468 & 0.00785 & 0.02355 & - \\
\hline Cs-137 & ARA-39 & 2009 & 1.199 & 0.01605 & 0.04815 & - \\
\hline Cs-137 & ARA-4 & 2009 & 0.5201 & 0.00865 & 0.02595 & - \\
\hline Cs-137 & ARA-40 & 2009 & 1.22 & 0.0109 & 0.0327 & - \\
\hline Cs-137 & ARA-41 & 2009 & 0.7215 & 0.0098 & 0.0294 & - \\
\hline Cs-137 & ARA-42 & 2009 & 0.4231 & 0.00845 & 0.02535 & - \\
\hline Cs-137 & ARA-43 & 2009 & 0.2998 & 0.00615 & 0.01845 & - \\
\hline Cs-137 & ARA-44 & 2009 & 0.7318 & 0.0093 & 0.0279 & - \\
\hline Cs-137 & ARA-45 & 2009 & 0.3411 & 0.0045 & 0.0135 & - \\
\hline Cs-137 & ARA-46 & 2009 & 0.2988 & 0.00245 & 0.00735 & - \\
\hline Cs-137 & ARA-47 & 2009 & 0.2549 & 0.00915 & 0.02745 & - \\
\hline Cs-137 & ARA-48 & 2009 & 1.786 & 0.01315 & 0.03945 & - \\
\hline Cs-137 & ARA-49 & 2009 & 0.3155 & 0.00765 & 0.02295 & - \\
\hline Cs-137 & ARA-5 & 2009 & 0.4513 & 0.00885 & 0.02655 & - \\
\hline Cs-137 & ARA-50 & 2009 & 0.2252 & 0.004415 & 0.013245 & - \\
\hline Cs-137 & ARA-51 & 2009 & 1.409 & 0.01345 & 0.04035 & - \\
\hline Cs-137 & ARA-52 & 2009 & 0.1775 & 0.0069 & 0.0207 & - \\
\hline Cs-137 & ARA-53 & 2009 & 0.2647 & 0.00795 & 0.02385 & - \\
\hline Cs-137 & ARA-54 & 2009 & 0.07963 & 0.00313 & 0.00939 & - \\
\hline Cs-137 & ARA-55 & 2009 & 0.1548 & 0.00505 & 0.01515 & - \\
\hline Cs-137 & ARA-56 & 2009 & 0.1845 & 0.004765 & 0.014295 & - \\
\hline Cs-137 & ARA-57 & 2009 & 0.06779 & 0.00411 & 0.01233 & - \\
\hline
\end{tabular}


Table C-1. (continued).

\begin{tabular}{|c|c|c|c|c|c|c|}
\hline Radionuclide & $\begin{array}{c}\text { Sample } \\
\text { Location }\end{array}$ & Date & $\begin{array}{c}\text { Concentrations } \\
(\mathrm{pCi} / \mathrm{g})\end{array}$ & $\begin{array}{l}\text { Uncertainty } \\
(\mathrm{pCi} / \mathrm{g})\end{array}$ & $\begin{array}{c}\text { MDA } \\
(\mathrm{pCi} / \mathrm{g})\end{array}$ & Flag \\
\hline Cs-137 & ARA-58 & 2009 & 0.2515 & 0.0064 & 0.0192 & - \\
\hline Cs-137 & ARA-59 & 2009 & 0.2446 & 0.00486 & 0.01458 & - \\
\hline Cs-137 & ARA-6 & 2009 & 0.2679 & 0.00775 & 0.02325 & - \\
\hline Cs-137 & ARA-60 & 2009 & 0.2603 & 0.00835 & 0.02505 & - \\
\hline Cs-137 & ARA-61 & 2009 & 2.919 & 0.0197 & 0.0591 & - \\
\hline Cs-137 & ARA-62 & 2009 & 2.404 & 0.0193 & 0.0579 & - \\
\hline Cs-137 & ARA-63 & 2009 & 3.792 & 0.0261 & 0.0783 & - \\
\hline Cs-137 & ARA-65 & 2009 & 1.918 & 0.01265 & 0.03795 & - \\
\hline Cs-137 & ARA-66 & 2009 & 0.6254 & 0.0095 & 0.0285 & - \\
\hline Cs-137 & ARA-67 & 2009 & 2.348 & 0.0144 & 0.0432 & - \\
\hline Cs-137 & ARA-68 & 2009 & 1.487 & 0.0114 & 0.0342 & - \\
\hline Cs-137 & ARA-69 & 2009 & 5.458 & 0.03135 & 0.09405 & - \\
\hline Cs-137 & ARA-7 & 2009 & 0.2192 & 0.0061 & 0.0183 & - \\
\hline Cs-137 & ARA-70 & 2009 & 2.46 & 0.01895 & 0.05685 & - \\
\hline Cs-137 & ARA-71 & 2009 & 3.578 & 0.0176 & 0.0528 & - \\
\hline Cs-137 & ARA-72 & 2009 & 0.7575 & 0.0094 & 0.0282 & - \\
\hline Cs-137 & ARA-73 & 2009 & 0.6822 & 0.00995 & 0.02985 & - \\
\hline Cs-137 & ARA-74 & 2009 & 0.3511 & 0.00865 & 0.02595 & - \\
\hline Cs-137 & ARA-75 & 2009 & 0.3299 & 0.0095 & 0.0285 & - \\
\hline Cs-137 & ARA-76 & 2009 & 7.991 & 0.02455 & 0.07365 & - \\
\hline Cs-137 & ARA-77 & 2009 & 0.2383 & 0.00785 & 0.02355 & - \\
\hline Cs-137 & ARA-8 & 2009 & 4.877 & 0.0195 & 0.0585 & - \\
\hline Cs-137 & ARA-9 & 2009 & 0.186 & 0.00715 & 0.02145 & - \\
\hline Cs-137 & ARA-0 & 2010 & 2.271 & 0.0109 & 0.0327 & - \\
\hline Cs-137 & ARA-1 & 2010 & 1.222 & 0.0145 & 0.0435 & - \\
\hline Cs-137 & ARA-11 & 2010 & 0.254 & 0.00482 & 0.01446 & - \\
\hline Cs-137 & ARA-12 & 2010 & 0.2568 & 0.00805 & 0.02415 & - \\
\hline Cs-137 & ARA-14 & 2010 & 0.2518 & 0.00907 & 0.02721 & - \\
\hline Cs-137 & ARA-15 & 2010 & 1.334 & 0.0153 & 0.0459 & - \\
\hline Cs-137 & ARA-16 & 2010 & 0.6574 & 0.0141 & 0.0423 & - \\
\hline Cs-137 & ARA-17 & 2010 & 0.5511 & 0.00959 & 0.02877 & - \\
\hline Cs-137 & ARA-19 & 2010 & 0.4321 & 0.01 & 0.03 & - \\
\hline Cs-137 & ARA-2 & 2010 & 0.3311 & 0.00304 & 0.00912 & - \\
\hline Cs-137 & ARA-21 & 2010 & 1.38 & 0.00956 & 0.02868 & - \\
\hline Cs-137 & ARA-22 & 2010 & 0.8731 & 0.0108 & 0.0324 & - \\
\hline Cs-137 & ARA-23 & 2010 & 0.4366 & 0.00716 & 0.02148 & - \\
\hline Cs-137 & ARA-24 & 2010 & 0.278 & 0.00816 & 0.02448 & - \\
\hline
\end{tabular}


Table C-1. (continued).

\begin{tabular}{|c|c|c|c|c|c|c|}
\hline Radionuclide & $\begin{array}{c}\text { Sample } \\
\text { Location }\end{array}$ & Date & $\begin{array}{l}\text { Concentrations } \\
(\mathrm{pCi} / \mathrm{g})\end{array}$ & $\begin{array}{l}\text { Uncertainty } \\
\text { (pCi/g) }\end{array}$ & $\begin{array}{l}\text { MDA } \\
(\mathrm{pCi} / \mathrm{g})\end{array}$ & Flag \\
\hline Cs-137 & ARA-27 & 2010 & 0.8604 & 0.00653 & 0.01959 & - \\
\hline Cs-137 & ARA-28 & 2010 & 1.22 & 0.0152 & 0.0456 & 一 \\
\hline Cs-137 & ARA-29 & 2010 & 0.5318 & 0.0108 & 0.0324 & - \\
\hline Cs-137 & ARA-31 & 2010 & 0.9442 & 0.0177 & 0.0531 & - \\
\hline Cs-137 & ARA-32 & 2010 & 0.651 & 0.0067 & 0.0201 & - \\
\hline Cs-137 & ARA-34 & 2010 & 0.532 & 0.00984 & 0.02952 & - \\
\hline Cs-137 & ARA-35 & 2010 & 2.862 & 0.0115 & 0.0345 & - \\
\hline Cs-137 & ARA-36 & 2010 & 0.99 & 0.0126 & 0.0378 & - \\
\hline Cs-137 & ARA-37 & 2010 & 0.5538 & 0.00898 & 0.02694 & - \\
\hline Cs-137 & ARA-38 & 2010 & 0.4045 & 0.00926 & 0.02778 & - \\
\hline Cs-137 & ARA-4 & 2010 & 0.6033 & 0.0103 & 0.0309 & - \\
\hline Cs-137 & ARA-40 & 2010 & 1.34 & 0.0115 & 0.0345 & - \\
\hline Cs-137 & ARA-41 & 2010 & 0.6976 & 0.0118 & 0.0354 & - \\
\hline Cs-137 & ARA-42 & 2010 & 0.3141 & 0.00948 & 0.02844 & - \\
\hline Cs-137 & ARA-43 & 2010 & 0.2279 & 0.00348 & 0.01044 & - \\
\hline Cs-137 & ARA-44 & 2010 & 0.7383 & 0.0122 & 0.0366 & - \\
\hline Cs-137 & ARA-45 & 2010 & 0.418 & 0.0115 & 0.0345 & - \\
\hline Cs-137 & ARA-46 & 2010 & 0.3035 & 0.014 & 0.042 & - \\
\hline Cs-137 & ARA-47 & 2010 & 0.2303 & 0.00723 & 0.02169 & - \\
\hline Cs-137 & ARA-48 & 2010 & 0.9946 & 0.00568 & 0.01704 & - \\
\hline Cs-137 & ARA-5 & 2010 & 0.4013 & 0.0113 & 0.0339 & 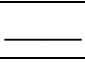 \\
\hline Cs-137 & ARA-50 & 2010 & 0.3074 & 0.00896 & 0.02688 & - \\
\hline Cs-137 & ARA-51 & 2010 & 1.303 & 0.00901 & 0.02703 & - \\
\hline Cs-137 & ARA-52 & 2010 & 0.2343 & 0.00945 & 0.02835 & - \\
\hline Cs-137 & ARA-53 & 2010 & 0.2854 & 0.0115 & 0.0345 & - \\
\hline Cs-137 & ARA-54 & 2010 & 0.2258 & 0.00851 & 0.02553 & - \\
\hline Cs-137 & ARA-55 & 2010 & 0.2692 & 0.00842 & 0.02526 & - \\
\hline Cs-137 & ARA-56 & 2010 & 0.2242 & 0.00948 & 0.02844 & - \\
\hline Cs-137 & ARA-57 & 2010 & 0.2016 & 0.00898 & 0.02694 & - \\
\hline Cs-137 & ARA-58 & 2010 & 0.3116 & 0.00894 & 0.02682 & - \\
\hline Cs-137 & ARA-59 & 2010 & 0.2957 & 0.00842 & 0.02526 & - \\
\hline Cs-137 & ARA-6 & 2010 & 0.3249 & 0.0101 & 0.0303 & - \\
\hline Cs-137 & ARA-60 & 2010 & 0.2701 & 0.00941 & 0.02823 & - \\
\hline Cs-137 & ARA-65 & 2010 & 1.805 & 0.0148 & 0.0444 & - \\
\hline Cs-137 & ARA-7 & 2010 & 0.2017 & 0.00455 & 0.01365 & - \\
\hline Cs-137 & ARA-73 & 2010 & 0.7083 & 0.0123 & 0.0369 & - \\
\hline Cs-137 & ARA-74 & 2010 & 0.3772 & 0.00843 & 0.02529 & - \\
\hline
\end{tabular}


Table C-1. (continued).

\begin{tabular}{|c|c|c|c|c|c|c|}
\hline Radionuclide & $\begin{array}{c}\text { Sample } \\
\text { Location }\end{array}$ & Date & $\begin{array}{c}\text { Concentrations } \\
(\mathrm{pCi} / \mathrm{g})\end{array}$ & $\begin{array}{l}\text { Uncertainty } \\
(\mathrm{pCi} / \mathrm{g})\end{array}$ & $\begin{array}{c}\text { MDA } \\
(\mathrm{pCi} / \mathrm{g})\end{array}$ & Flag \\
\hline Cs-137 & ARA-75 & 2010 & 0.3813 & 0.011 & 0.033 & - \\
\hline Cs-137 & ARA-77 & 2010 & 0.2462 & 0.0127 & 0.0381 & - \\
\hline Cs-137 & ARA-9 & 2010 & 0.2106 & 0.0105 & 0.0315 & - \\
\hline Cs-137 & ARA-1 & 2011 & 2.558 & 0.0203 & 0.0609 & - \\
\hline Cs-137 & ARA-14 & 2011 & 0.297 & 0.00718 & 0.02154 & - \\
\hline Cs-137 & ARA-16 & 2011 & 1.369 & 0.0148 & 0.0444 & - \\
\hline Cs-137 & ARA-2 & 2011 & 1.054 & 0.0137 & 0.0411 & - \\
\hline Cs-137 & ARA-24 & 2011 & 0.8482 & 0.0159 & 0.0477 & - \\
\hline Cs-137 & ARA-28 & 2011 & 2.441 & 0.0218 & 0.0654 & - \\
\hline Cs-137 & ARA-29 & 2011 & 0.6844 & 0.0087 & 0.0261 & - \\
\hline Cs-137 & ARA-31 & 2011 & 2.064 & 0.0207 & 0.0621 & - \\
\hline Cs-137 & ARA-32 & 2011 & 1.812 & 0.0257 & 0.0771 & - \\
\hline Cs-137 & ARA-34 & 2011 & 1.546 & 0.0247 & 0.0741 & - \\
\hline Cs-137 & ARA-38 & 2011 & 1.325 & 0.0242 & 0.0726 & - \\
\hline Cs-137 & ARA-4 & 2011 & 0.6981 & 0.00939 & 0.02817 & - \\
\hline Cs-137 & ARA-42 & 2011 & 1.061 & 0.0234 & 0.0702 & - \\
\hline Cs-137 & ARA-43 & 2011 & 0.6363 & 0.0135 & 0.0405 & - \\
\hline Cs-137 & ARA-47 & 2011 & 0.5158 & 0.0131 & 0.0393 & - \\
\hline Cs-137 & ARA-50 & 2011 & 0.651 & 0.0145 & 0.0435 & - \\
\hline Cs-137 & ARA-51 & 2011 & 3.622 & 0.0301 & 0.0903 & - \\
\hline Cs-137 & ARA-62 & 2011 & 0.49 & 0.0127 & 0.0381 & - \\
\hline Cs-137 & ARA-65 & 2011 & 4.678 & 0.0252 & 0.0756 & - \\
\hline Cs-137 & ARA-71 & 2011 & 9.583 & 0.5 & 1.5 & - \\
\hline Cs-137 & ARA-74 & 2011 & 0.7906 & 0.0147 & 0.0441 & - \\
\hline Cs-137 & ARA-75 & 2011 & 0.9302 & 0.0163 & 0.0489 & - \\
\hline Cs-137 & ARA-77 & 2011 & 0.4888 & 0.013 & 0.039 & - \\
\hline Cs-137 & ARA-9 & 2011 & 0.2335 & 0.00679 & 0.02037 & - \\
\hline Cs-137 & ARA-11 & 2012 & 0.2424 & 0.00986 & 0.02958 & - \\
\hline Cs-137 & ARA-16 & 2012 & 0.4904 & 0.0112 & 0.0336 & - \\
\hline Cs-137 & ARA-31 & 2012 & 1.253 & 0.0122 & 0.0366 & - \\
\hline Cs-137 & ARA-32 & 2012 & 0.5818 & 0.00838 & 0.02514 & - \\
\hline Cs-137 & ARA-48 & 2012 & 1.328 & 0.01 & 0.03 & - \\
\hline Cs-137 & ARA-53 & 2012 & 0.2027 & 0.00516 & 0.01548 & - \\
\hline Cs-137 & ARA-57 & 2012 & 0.08622 & 0.0062 & 0.0186 & - \\
\hline Cs-137 & ARA-58 & 2012 & 0.3202 & 0.00784 & 0.02352 & - \\
\hline Cs-137 & ARA-59 & 2012 & 0.2819 & 0.00809 & 0.02427 & - \\
\hline Cs-137 & ARA-60 & 2012 & 0.2291 & 0.00805 & 0.02415 & - \\
\hline
\end{tabular}


Table C-1. (continued).

\begin{tabular}{|c|c|c|c|c|c|c|}
\hline Radionuclide & $\begin{array}{c}\text { Sample } \\
\text { Location }\end{array}$ & Date & $\begin{array}{c}\text { Concentrations } \\
(\mathrm{pCi} / \mathrm{g})\end{array}$ & $\begin{array}{l}\text { Uncertainty } \\
(\mathrm{pCi} / \mathrm{g})\end{array}$ & $\begin{array}{c}\text { MDA } \\
(\mathrm{pCi} / \mathrm{g})\end{array}$ & Flag \\
\hline Cs-137 & ARA-62 & 2012 & 0.1267 & 0.00941 & 0.02823 & - \\
\hline Cs-137 & ARA-65 & 2012 & 1.618 & 0.015 & 0.045 & - \\
\hline Cs-137 & ARA-73 & 2012 & 0.5814 & 0.00702 & 0.02106 & - \\
\hline Cs-137 & ARA-31 & 2013 & 0.8052 & 0.0119 & 0.0357 & - \\
\hline Cs-137 & ARA-32 & 2013 & 0.2114 & 0.0038 & 0.0114 & - \\
\hline Cs-137 & ARA-33 & 2013 & 0.2264 & 0.012166667 & 0.0365 & - \\
\hline Cs-137 & ARA-40 & 2013 & 1.152 & 0.0156 & 0.0468 & - \\
\hline Cs-137 & ARA-48 & 2013 & 0.542 & 0.0059 & 0.0177 & - \\
\hline Cs-137 & ARA-65 & 2013 & 0.577 & 0.004633333 & 0.0139 & - \\
\hline Cs-137 & $\begin{array}{c}2 \text { INCH AIR } \\
\text { - ARA }\end{array}$ & 2014 & 0.3787 & 0.0146 & 0.0438 & - \\
\hline Cs-137 & ARA-1 & 2014 & 0.8636 & 0.00883 & 0.02649 & - \\
\hline Cs-137 & ARA-24 & 2014 & 0.31 & 0.012 & 0.036 & - \\
\hline Cs-137 & ARA-31 & 2014 & 0.862 & 0.0152 & 0.0456 & - \\
\hline Cs-137 & ARA-32 & 2014 & 0.1298 & 0.0065 & 0.0195 & - \\
\hline Cs-137 & ARA-65 & 2014 & 1.639 & 0.0146 & 0.0438 & - \\
\hline Cs-137 & ARA-71 & 2014 & 1.549 & 0.00767 & 0.02301 & - \\
\hline Eu-152 & $56.25^{\circ} 2000^{\prime}$ & 1985 & 0.6 & 0.05 & 0.15 & - \\
\hline Eu-152 & ARA-0 & 2007 & 0.1984 & 0.03685 & 0.11055 & - \\
\hline Eu-152 & ARA-11 & 2007 & 1.017 & 0.08 & 0.24 & - \\
\hline Eu-152 & ARA-12 & 2007 & 1.013 & 0.0675 & 0.2025 & - \\
\hline Eu-152 & ARA-14 & 2007 & 0.2634 & 0.02895 & 0.08685 & - \\
\hline Eu-152 & ARA-15 & 2007 & 0.3424 & 0.04625 & 0.13875 & - \\
\hline Eu-152 & ARA-17 & 2007 & 0.418 & 0.04415 & 0.13245 & - \\
\hline Eu-152 & ARA-19 & 2007 & 1.248 & 0.082 & 0.246 & - \\
\hline Eu-152 & ARA-2 & 2007 & 0.8257 & 0.055 & 0.165 & - \\
\hline Eu-152 & ARA-21 & 2007 & 0.2399 & 0.0279 & 0.0837 & - \\
\hline Eu-152 & ARA-23 & 2007 & 0.2833 & 0.0344 & 0.1032 & - \\
\hline $\mathrm{Eu}-152$ & ARA-24 & 2007 & 0.2276 & 0.02705 & 0.08115 & - \\
\hline Eu-152 & ARA-28 & 2007 & 0.3814 & 0.03805 & 0.11415 & - \\
\hline Eu-152 & ARA-29 & 2007 & 0.2089 & 0.02915 & 0.08745 & - \\
\hline Eu-152 & ARA-31 & 2007 & 0.622 & 0.059 & 0.177 & - \\
\hline Eu-152 & ARA-32 & 2007 & 0.7504 & 0.052 & 0.156 & - \\
\hline Eu-152 & ARA-36 & 2007 & 0.1301 & 0.02055 & 0.06165 & - \\
\hline Eu-152 & ARA-37 & 2007 & 1.187 & 0.093 & 0.279 & - \\
\hline Eu-152 & ARA-38 & 2007 & 0.2351 & 0.02745 & 0.08235 & - \\
\hline Eu-152 & ARA-4 & 2007 & 0.2935 & 0.03015 & 0.09045 & - \\
\hline
\end{tabular}


Table C-1. (continued).

\begin{tabular}{|c|c|c|c|c|c|c|}
\hline Radionuclide & $\begin{array}{c}\text { Sample } \\
\text { Location }\end{array}$ & Date & $\begin{array}{c}\text { Concentrations } \\
(\mathrm{pCi} / \mathrm{g})\end{array}$ & $\begin{array}{l}\text { Uncertainty } \\
(\mathrm{pCi} / \mathrm{g})\end{array}$ & $\begin{array}{c}\text { MDA } \\
(\mathrm{pCi} / \mathrm{g})\end{array}$ & Flag \\
\hline Eu-152 & ARA-42 & 2007 & 0.4242 & 0.051 & 0.153 & - \\
\hline Eu-152 & ARA-43 & 2007 & 0.221 & 0.02935 & 0.08805 & - \\
\hline Eu-152 & ARA-45 & 2007 & 1.077 & 0.059 & 0.177 & - \\
\hline Eu-152 & ARA-46 & 2007 & 0.2391 & 0.03305 & 0.09915 & - \\
\hline Eu-152 & ARA-47 & 2007 & 0.3586 & 0.036 & 0.108 & - \\
\hline Eu-152 & ARA-48 & 2007 & 0.1298 & 0.0341 & 0.1023 & - \\
\hline Eu-152 & ARA-49 & 2007 & 0.184 & 0.0246 & 0.0738 & - \\
\hline Eu-152 & ARA-5 & 2007 & 0.3412 & 0.04355 & 0.13065 & - \\
\hline Eu-152 & ARA-50 & 2007 & 0.7454 & 0.068 & 0.204 & - \\
\hline Eu-152 & ARA-51 & 2007 & 0.353 & 0.0404 & 0.1212 & - \\
\hline Eu-152 & ARA-52 & 2007 & 0.3845 & 0.04515 & 0.13545 & - \\
\hline Eu-152 & ARA-56 & 2007 & 0.2021 & 0.0259 & 0.0777 & - \\
\hline Eu-152 & ARA-57 & 2007 & 1.217 & 0.076 & 0.228 & - \\
\hline Eu-152 & ARA-58 & 2007 & 0.2532 & 0.0374 & 0.1122 & - \\
\hline Eu-152 & ARA-59 & 2007 & 0.3171 & 0.03585 & 0.10755 & - \\
\hline Eu-152 & ARA-6 & 2007 & 0.2392 & 0.02975 & 0.08925 & - \\
\hline Eu-152 & ARA-60 & 2007 & 0.2893 & 0.03755 & 0.11265 & - \\
\hline Eu-152 & ARA-65 & 2007 & 1.159 & 0.078 & 0.234 & - \\
\hline Eu-152 & ARA-7 & 2007 & 0.2856 & 0.03405 & 0.10215 & - \\
\hline Eu-152 & ARA-73 & 2007 & 0.209 & 0.02915 & 0.08745 & - \\
\hline Eu-152 & ARA-74 & 2007 & 0.2633 & 0.03135 & 0.09405 & - \\
\hline Eu-152 & ARA-75 & 2007 & 0.367 & 0.067 & 0.201 & - \\
\hline Eu-152 & ARA-77 & 2007 & 0.2755 & 0.03 & 0.09 & - \\
\hline Eu-152 & ARA-1 & 2011 & -0.01441 & 0.0217 & 0.0651 & $\mathrm{U}$ \\
\hline Eu-152 & ARA-14 & 2011 & 0.007048 & 0.0173 & 0.0519 & $\mathrm{U}$ \\
\hline Eu-152 & ARA-16 & 2011 & -0.002974 & 0.0315 & 0.0945 & $\mathrm{U}$ \\
\hline Eu-152 & ARA-2 & 2011 & 0.0102 & 0.0202 & 0.0606 & $\mathrm{U}$ \\
\hline Eu-152 & ARA-24 & 2011 & 0.0006984 & 0.0216 & 0.0648 & $\mathrm{U}$ \\
\hline Eu-152 & ARA-28 & 2011 & 0.02575 & 0.0272 & 0.0816 & $\mathrm{U}$ \\
\hline Eu-152 & ARA-29 & 2011 & 0.01154 & 0.017 & 0.051 & $\mathrm{U}$ \\
\hline Eu-152 & ARA-31 & 2011 & 0.008125 & 0.0266 & 0.0798 & $\mathrm{U}$ \\
\hline Eu-152 & ARA-32 & 2011 & 0.01698 & 0.0315 & 0.0945 & $\mathrm{U}$ \\
\hline Eu-152 & ARA-34 & 2011 & -0.0002467 & 0.0289 & 0.0867 & $\mathrm{U}$ \\
\hline Eu-152 & ARA-38 & 2011 & 0.02879 & 0.0291 & 0.0873 & $\mathrm{U}$ \\
\hline $\mathrm{Eu}-152$ & ARA-4 & 2011 & 0.002729 & 0.0163 & 0.0489 & $\mathrm{U}$ \\
\hline Eu-152 & ARA-42 & 2011 & 0.02726 & 0.0286 & 0.0858 & $\mathrm{U}$ \\
\hline Eu-152 & ARA-43 & 2011 & 0.009126 & 0.0206 & 0.0618 & $\mathrm{U}$ \\
\hline
\end{tabular}


Table C-1. (continued).

\begin{tabular}{|c|c|c|c|c|c|c|}
\hline Radionuclide & $\begin{array}{c}\text { Sample } \\
\text { Location }\end{array}$ & Date & $\begin{array}{c}\text { Concentrations } \\
(\mathrm{pCi} / \mathrm{g})\end{array}$ & $\begin{array}{l}\text { Uncertainty } \\
(\mathrm{pCi} / \mathrm{g})\end{array}$ & $\begin{array}{c}\text { MDA } \\
(\mathrm{pCi} / \mathrm{g})\end{array}$ & Flag \\
\hline Eu-152 & ARA-47 & 2011 & -0.01508 & 0.0257 & 0.0771 & $\mathrm{U}$ \\
\hline Eu-152 & ARA-50 & 2011 & 0.01953 & 0.0245 & 0.0735 & $\mathrm{U}$ \\
\hline Eu-152 & ARA-51 & 2011 & 0.01477 & 0.0288 & 0.0864 & $\mathrm{U}$ \\
\hline $\mathrm{Eu}-152$ & ARA-62 & 2011 & 0.01772 & 0.0231 & 0.0693 & $\mathrm{U}$ \\
\hline Eu-152 & ARA-65 & 2011 & -0.02214 & 0.0225 & 0.0675 & $\mathrm{U}$ \\
\hline Eu-152 & ARA-74 & 2011 & 0.02667 & 0.0256 & 0.0768 & $\mathrm{U}$ \\
\hline Eu-152 & ARA-75 & 2011 & 0.01495 & 0.0263 & 0.0789 & $\mathrm{U}$ \\
\hline Eu-152 & ARA-77 & 2011 & -0.005068 & 0.0252 & 0.0756 & $\mathrm{U}$ \\
\hline Eu-152 & ARA-9 & 2011 & 0.0249 & 0.0143 & 0.0429 & $\mathrm{U}$ \\
\hline Eu-152 & ARA-11 & 2012 & 0.009189 & 0.0258 & 0.0774 & $\mathrm{U}$ \\
\hline Eu-152 & ARA-16 & 2012 & -0.003108 & 0.0317 & 0.0951 & $\mathrm{U}$ \\
\hline Eu-152 & ARA-31 & 2012 & -0.008595 & 0.0247 & 0.0741 & $\mathrm{U}$ \\
\hline Eu-152 & ARA-32 & 2012 & -0.004131 & 0.0278 & 0.0834 & $\mathrm{U}$ \\
\hline Eu-152 & ARA-48 & 2012 & 0.04203 & 0.0233 & 0.0699 & $\mathrm{U}$ \\
\hline $\mathrm{Eu}-152$ & ARA-53 & 2012 & 0.005979 & 0.023 & 0.069 & $\mathrm{U}$ \\
\hline Eu-152 & ARA-57 & 2012 & 0.01051 & 0.0258 & 0.0774 & $\mathrm{U}$ \\
\hline Eu-152 & ARA-58 & 2012 & 0.03904 & 0.0219 & 0.0657 & $\mathrm{U}$ \\
\hline Eu-152 & ARA-59 & 2012 & 0.03 & 0.0222 & 0.0666 & $\mathrm{U}$ \\
\hline Eu-152 & ARA-60 & 2012 & 0.01173 & 0.0244 & 0.0732 & $\mathrm{U}$ \\
\hline Eu-152 & ARA-62 & 2012 & 0.01668 & 0.0269 & 0.0807 & $\mathrm{U}$ \\
\hline Eu-152 & ARA-65 & 2012 & 0.0246 & 0.0217 & 0.0651 & $\mathrm{U}$ \\
\hline Eu-152 & ARA-73 & 2012 & 0.03736 & 0.0224 & 0.0672 & $\mathrm{U}$ \\
\hline Eu-152 & ARA-31 & 2013 & 0.1388 & 0.0311 & 0.0934 & - \\
\hline Eu-152 & ARA-32 & 2013 & 0.01422 & 0.0156 & 0.0468 & $\mathrm{U}$ \\
\hline Eu-152 & ARA-33 & 2013 & 0.1558 & 0.0251 & 0.0753 & - \\
\hline Eu-152 & ARA-40 & 2013 & 0.1809 & 0.0357 & 0.107 & - \\
\hline Eu-152 & ARA-48 & 2013 & -0.001267 & 0.0161 & 0.0482 & $\mathrm{U}$ \\
\hline Eu-152 & ARA-65 & 2013 & 0.008385 & 0.0148 & 0.0445 & $\mathrm{U}$ \\
\hline Eu-152 & $\begin{array}{c}2 \text { INCH AIR } \\
\text { - ARA }\end{array}$ & 2014 & 0.03905 & 0.0347 & 0.1041 & $\mathrm{U}$ \\
\hline Eu-152 & ARA-1 & 2014 & 0.01375 & 0.0225 & 0.0675 & $\mathrm{U}$ \\
\hline Eu-152 & ARA-24 & 2014 & 0.001474 & 0.0194 & 0.0582 & $\mathrm{U}$ \\
\hline Eu-152 & ARA-31 & 2014 & -0.01088 & 0.0317 & 0.0951 & $\mathrm{U}$ \\
\hline Eu-152 & ARA-32 & 2014 & 0.02117 & 0.024 & 0.072 & $\mathrm{U}$ \\
\hline Eu-152 & ARA-65 & 2014 & 0.0046 & 0.0307 & 0.0921 & $\mathrm{U}$ \\
\hline Eu-152 & ARA-71 & 2014 & 0.01143 & 0.0134 & 0.0402 & $\mathrm{U}$ \\
\hline $\mathrm{Pu}-238$ & $0^{\circ} 250^{\prime}$ & 1977 & 0.024 & 0.003 & 0.009 & - \\
\hline
\end{tabular}


Table C-1. (continued).

\begin{tabular}{|c|c|c|c|c|c|c|}
\hline Radionuclide & $\begin{array}{l}\text { Sample } \\
\text { Location }\end{array}$ & Date & $\begin{array}{c}\text { Concentrations } \\
(\mathrm{pCi} / \mathrm{g})\end{array}$ & $\begin{array}{l}\text { Uncertainty } \\
(\mathrm{pCi} / \mathrm{g})\end{array}$ & $\begin{array}{c}\text { MDA } \\
(\mathrm{pCi} / \mathrm{g})\end{array}$ & Flag \\
\hline $\mathrm{Pu}-238$ & $22.5^{\circ} 250^{\prime}$ & 1977 & 0.002 & 0.002 & 0.006 & $\mathrm{U}$ \\
\hline $\mathrm{Pu}-238$ & $225^{\circ} 500^{\prime}$ & 1977 & 0.004 & 0.001 & 0.003 & - \\
\hline $\mathrm{Pu}-238$ & $270^{\circ} 1500^{\prime}$ & 1977 & 0.0011 & 0.0006 & 0.0018 & $\mathrm{U}$ \\
\hline $\mathrm{Pu}-238$ & $270^{\circ} 500^{\prime}$ & 1977 & 0.0011 & 0.0006 & 0.0018 & $\mathrm{U}$ \\
\hline $\mathrm{Pu}-238$ & $45^{\circ} 1500^{\prime}$ & 1977 & 0.003 & 0.001 & 0.003 & - \\
\hline $\mathrm{Pu}-238$ & $45^{\circ} 250^{\prime}$ & 1977 & 0.004 & 0.001 & 0.003 & - \\
\hline $\mathrm{Pu}-238$ & $45^{\circ} 2500^{\prime}$ & 1977 & 0 & 0.001 & 0.003 & $\mathrm{U}$ \\
\hline $\mathrm{Pu}-238$ & $45^{\circ} 500^{\prime}$ & 1977 & 0.025 & 0.003 & 0.009 & - \\
\hline $\mathrm{Pu}-238$ & $90^{\circ} 500^{\prime}$ & 1977 & 0.001 & 0.001 & 0.003 & $\mathrm{U}$ \\
\hline $\mathrm{Pu}-238$ & $0^{\circ} 1000^{\prime}$ & 1985 & 0.0012 & 0.0013 & 0.0039 & $\mathrm{U}$ \\
\hline $\mathrm{Pu}-238$ & $22.5^{\circ} 2500^{\prime}$ & 1985 & 0.003 & 0.002 & 0.006 & $\mathrm{U}$ \\
\hline $\mathrm{Pu}-238$ & $67.5^{\circ} 2000^{\prime}$ & 1985 & 0.001 & 0.0013 & 0.0039 & $\mathrm{U}$ \\
\hline $\mathrm{Pu}-238$ & $67.5^{\circ} 500^{\prime}$ & 1985 & 0.002 & 0.002 & 0.006 & $\mathrm{U}$ \\
\hline $\mathrm{Pu}-239 / 240$ & $0^{\circ} 250^{\prime}$ & 1977 & 0.047 & 0.004 & 0.012 & - \\
\hline $\mathrm{Pu}-239 / 240$ & $22.5^{\circ} 250^{\prime}$ & 1977 & 0.022 & 0.003 & 0.009 & - \\
\hline $\mathrm{Pu}-239 / 240$ & $225^{\circ} 500^{\prime}$ & 1977 & 0.011 & 0.002 & 0.006 & - \\
\hline $\mathrm{Pu}-239 / 240$ & $270^{\circ} 500^{\prime}$ & 1977 & 0.012 & 0.002 & 0.006 & - \\
\hline $\mathrm{Pu}-239 / 240$ & $45^{\circ} 1500^{\prime}$ & 1977 & 0.015 & 0.002 & 0.006 & - \\
\hline $\mathrm{Pu}-239 / 240$ & $45^{\circ} 250^{\prime}$ & 1977 & 0.016 & 0.002 & 0.006 & - \\
\hline $\mathrm{Pu}-239 / 240$ & $45^{\circ} 2500^{\prime}$ & 1977 & 0.018 & 0.002 & 0.006 & - \\
\hline $\mathrm{Pu}-239 / 240$ & $45^{\circ} 500^{\prime}$ & 1977 & 0.033 & 0.003 & 0.009 & - \\
\hline $\mathrm{Pu}-239 / 240$ & $90^{\circ} 500^{\prime}$ & 1977 & 0.021 & 0.003 & 0.009 & - \\
\hline $\mathrm{Pu}-239 / 240$ & $0^{\circ} 1000^{\prime}$ & 1985 & 0.014 & 0.002 & 0.006 & - \\
\hline $\mathrm{Pu}-239 / 240$ & $22.5^{\circ} 2500^{\prime}$ & 1985 & 0.021 & 0.003 & 0.009 & - \\
\hline $\mathrm{Pu}-239 / 240$ & $67.5^{\circ} 2000^{\prime}$ & 1985 & 0.011 & 0.002 & 0.006 & - \\
\hline $\mathrm{Pu}-239 / 240$ & $67.5^{\circ} 500^{\prime}$ & 1985 & 0.009 & 0.002 & 0.006 & - \\
\hline $\mathrm{Sb}-125$ & $270^{\circ} 1000^{\prime}$ & 1977 & 0.1 & 0.04 & 0.12 & $\mathrm{U}$ \\
\hline $\mathrm{Sb}-125$ & $315^{\circ} 500^{\prime}$ & 1977 & 0.17 & 0.06 & 0.18 & $\mathrm{U}$ \\
\hline $\mathrm{Sb}-125$ & ARA-0 & 2007 & 0.4902 & 0.0655 & 0.1965 & - \\
\hline Sb-125 & ARA-11 & 2007 & 1.01 & 0.1945 & 0.5835 & - \\
\hline Sb-125 & ARA-12 & 2007 & 0.586 & 0.125 & 0.375 & - \\
\hline $\mathrm{Sb}-125$ & ARA-14 & 2007 & 0.1637 & 0.04075 & 0.12225 & - \\
\hline $\mathrm{Sb}-125$ & ARA-15 & 2007 & 0.226 & 0.03935 & 0.11805 & - \\
\hline Sb-125 & ARA-17 & 2007 & 0.3805 & 0.06 & 0.18 & - \\
\hline $\mathrm{Sb}-125$ & ARA-19 & 2007 & 0.3985 & 0.1325 & 0.3975 & - \\
\hline $\mathrm{Sb}-125$ & ARA-2 & 2007 & 1.415 & 0.265 & 0.795 & - \\
\hline $\mathrm{Sb}-125$ & ARA-21 & 2007 & 0.1101 & 0.0437 & 0.1311 & $\mathrm{U}$ \\
\hline
\end{tabular}


Table C-1. (continued).

\begin{tabular}{|c|c|c|c|c|c|c|}
\hline Radionuclide & $\begin{array}{c}\text { Sample } \\
\text { Location }\end{array}$ & Date & $\begin{array}{c}\text { Concentrations } \\
(\mathrm{pCi} / \mathrm{g})\end{array}$ & $\begin{array}{l}\text { Uncertainty } \\
(\mathrm{pCi} / \mathrm{g})\end{array}$ & $\begin{array}{c}\text { MDA } \\
(\mathrm{pCi} / \mathrm{g})\end{array}$ & Flag \\
\hline $\mathrm{Sb}-125$ & ARA-23 & 2007 & 0.2591 & 0.0635 & 0.1905 & - \\
\hline $\mathrm{Sb}-125$ & ARA-24 & 2007 & 0.2465 & 0.03725 & 0.11175 & - \\
\hline $\mathrm{Sb}-125$ & ARA-28 & 2007 & 0.3409 & 0.065 & 0.195 & - \\
\hline $\mathrm{Sb}-125$ & ARA-29 & 2007 & 0.2129 & 0.04375 & 0.13125 & - \\
\hline $\mathrm{Sb}-125$ & ARA-31 & 2007 & 0.5463 & 0.112 & 0.336 & - \\
\hline $\mathrm{Sb}-125$ & ARA-32 & 2007 & 0.6003 & 0.089 & 0.267 & - \\
\hline $\mathrm{Sb}-125$ & ARA-36 & 2007 & 0.2576 & 0.0474 & 0.1422 & - \\
\hline $\mathrm{Sb}-125$ & ARA-37 & 2007 & 0.6505 & 0.103 & 0.309 & - \\
\hline $\mathrm{Sb}-125$ & ARA-38 & 2007 & 0.3246 & 0.0455 & 0.1365 & - \\
\hline $\mathrm{Sb}-125$ & ARA-4 & 2007 & 0.2618 & 0.051 & 0.153 & - \\
\hline $\mathrm{Sb}-125$ & ARA-42 & 2007 & 0.3039 & 0.0481 & 0.1443 & - \\
\hline $\mathrm{Sb}-125$ & ARA-43 & 2007 & 0.192 & 0.0505 & 0.1515 & - \\
\hline $\mathrm{Sb}-125$ & ARA-45 & 2007 & 1.152 & 0.2085 & 0.6255 & - \\
\hline $\mathrm{Sb}-125$ & ARA-46 & 2007 & 0.2414 & 0.04565 & 0.13695 & - \\
\hline Sb-125 & ARA-47 & 2007 & 0.3426 & 0.058 & 0.174 & - \\
\hline $\mathrm{Sb}-125$ & ARA-48 & 2007 & 0.2379 & 0.0464 & 0.1392 & - \\
\hline $\mathrm{Sb}-125$ & ARA-49 & 2007 & 0.2245 & 0.0424 & 0.1272 & - \\
\hline $\mathrm{Sb}-125$ & ARA-5 & 2007 & 0.2235 & 0.059 & 0.177 & - \\
\hline Sb-125 & ARA-50 & 2007 & 0.531 & 0.093 & 0.279 & - \\
\hline $\mathrm{Sb}-125$ & ARA-51 & 2007 & 0.4702 & 0.062 & 0.186 & - \\
\hline Sb-125 & ARA-52 & 2007 & 0.177 & 0.02645 & 0.07935 & - \\
\hline Sb-125 & ARA-56 & 2007 & 0.1579 & 0.0341 & 0.1023 & - \\
\hline $\mathrm{Sb}-125$ & ARA-57 & 2007 & 0.6054 & 0.113 & 0.339 & - \\
\hline $\mathrm{Sb}-125$ & ARA-58 & 2007 & 0.3235 & 0.057 & 0.171 & - \\
\hline $\mathrm{Sb}-125$ & ARA-59 & 2007 & 0.3663 & 0.0745 & 0.2235 & - \\
\hline $\mathrm{Sb}-125$ & ARA-6 & 2007 & 0.2458 & 0.0332 & 0.0996 & - \\
\hline Sb-125 & ARA-60 & 2007 & 0.1564 & 0.04535 & 0.13605 & - \\
\hline $\mathrm{Sb}-125$ & ARA-65 & 2007 & 0.5521 & 0.113 & 0.339 & - \\
\hline $\mathrm{Sb}-125$ & ARA-7 & 2007 & 0.2512 & 0.03995 & 0.11985 & - \\
\hline Sb-125 & ARA-73 & 2007 & 0.2343 & 0.04715 & 0.14145 & - \\
\hline Sb-125 & ARA-74 & 2007 & 0.1883 & 0.0487 & 0.1461 & - \\
\hline Sb-125 & ARA-75 & 2007 & 0.0789 & 0.0309 & 0.0927 & $\mathrm{U}$ \\
\hline Sb-125 & ARA-77 & 2007 & 0.2113 & 0.0351 & 0.1053 & - \\
\hline Sb-125 & ARA-1 & 2011 & 0.0719 & 0.0295 & 0.0885 & $\mathrm{U}$ \\
\hline $\mathrm{Sb}-125$ & ARA-14 & 2011 & -0.01036 & 0.0224 & 0.0672 & $\mathrm{U}$ \\
\hline $\mathrm{Sb}-125$ & ARA-16 & 2011 & 0.05636 & 0.0371 & 0.1113 & $\mathrm{U}$ \\
\hline $\mathrm{Sb}-125$ & ARA-2 & 2011 & 0.01497 & 0.0258 & 0.0774 & $\mathrm{U}$ \\
\hline
\end{tabular}


Table C-1. (continued).

\begin{tabular}{|c|c|c|c|c|c|c|}
\hline Radionuclide & $\begin{array}{c}\text { Sample } \\
\text { Location }\end{array}$ & Date & $\begin{array}{c}\text { Concentrations } \\
(\mathrm{pCi} / \mathrm{g})\end{array}$ & $\begin{array}{l}\text { Uncertainty } \\
(\mathrm{pCi} / \mathrm{g})\end{array}$ & $\begin{array}{c}\text { MDA } \\
(\mathrm{pCi} / \mathrm{g})\end{array}$ & Flag \\
\hline $\mathrm{Sb}-125$ & ARA-24 & 2011 & 0.07952 & 0.0263 & 0.0789 & - \\
\hline $\mathrm{Sb}-125$ & ARA-28 & 2011 & 0.05066 & 0.0359 & 0.1077 & $\mathrm{U}$ \\
\hline $\mathrm{Sb}-125$ & ARA-29 & 2011 & 0.0232 & 0.0224 & 0.0672 & $\mathrm{U}$ \\
\hline $\mathrm{Sb}-125$ & ARA-31 & 2011 & 0.03403 & 0.0395 & 0.1185 & $\mathrm{U}$ \\
\hline $\mathrm{Sb}-125$ & ARA-32 & 2011 & 0.11 & 0.0422 & 0.1266 & $\mathrm{U}$ \\
\hline $\mathrm{Sb}-125$ & ARA-34 & 2011 & -0.02466 & 0.0385 & 0.1155 & $\mathrm{U}$ \\
\hline $\mathrm{Sb}-125$ & ARA-38 & 2011 & 0.08144 & 0.04 & 0.12 & $\mathrm{U}$ \\
\hline $\mathrm{Sb}-125$ & ARA-4 & 2011 & 0.03117 & 0.022 & 0.066 & $\mathrm{U}$ \\
\hline $\mathrm{Sb}-125$ & ARA-42 & 2011 & 0.005976 & 0.0405 & 0.1215 & $\mathrm{U}$ \\
\hline $\mathrm{Sb}-125$ & ARA-43 & 2011 & 0.08471 & 0.0341 & 0.1023 & $\mathrm{U}$ \\
\hline $\mathrm{Sb}-125$ & ARA-47 & 2011 & 0.02753 & 0.0343 & 0.1029 & $\mathrm{U}$ \\
\hline $\mathrm{Sb}-125$ & ARA-50 & 2011 & 0.002701 & 0.0333 & 0.0999 & $\mathrm{U}$ \\
\hline $\mathrm{Sb}-125$ & ARA-51 & 2011 & 0.09535 & 0.0426 & 0.1278 & $\mathrm{U}$ \\
\hline $\mathrm{Sb}-125$ & ARA-62 & 2011 & 0.03584 & 0.0268 & 0.0804 & $\mathrm{U}$ \\
\hline Sb-125 & ARA-65 & 2011 & 0.5495 & 0.031 & 0.093 & - \\
\hline $\mathrm{Sb}-125$ & ARA-74 & 2011 & 0.01884 & 0.0375 & 0.1125 & $\mathrm{U}$ \\
\hline $\mathrm{Sb}-125$ & ARA-75 & 2011 & -0.003259 & 0.0385 & 0.1155 & $\mathrm{U}$ \\
\hline $\mathrm{Sb}-125$ & ARA-77 & 2011 & 0.03254 & 0.0352 & 0.1056 & $\mathrm{U}$ \\
\hline Sb-125 & ARA-9 & 2011 & 0.1444 & 0.0253 & 0.0759 & - \\
\hline $\mathrm{Sb}-125$ & ARA-11 & 2012 & 0.01697 & 0.0317 & 0.0951 & $\mathrm{U}$ \\
\hline Sb-125 & ARA-16 & 2012 & 0.02102 & 0.0433 & 0.1299 & $\mathrm{U}$ \\
\hline Sb-125 & ARA-31 & 2012 & -0.001805 & 0.0359 & 0.1077 & $\mathrm{U}$ \\
\hline $\mathrm{Sb}-125$ & ARA-32 & 2012 & 0.003851 & 0.0346 & 0.1038 & $\mathrm{U}$ \\
\hline $\mathrm{Sb}-125$ & ARA-48 & 2012 & 0.004137 & 0.0412 & 0.1236 & $\mathrm{U}$ \\
\hline $\mathrm{Sb}-125$ & ARA-53 & 2012 & 0.01073 & 0.0306 & 0.0918 & $\mathrm{U}$ \\
\hline $\mathrm{Sb}-125$ & ARA-57 & 2012 & 0.0003073 & 0.0343 & 0.1029 & $\mathrm{U}$ \\
\hline Sb-125 & ARA-58 & 2012 & 0.002329 & 0.0309 & 0.0927 & $\mathrm{U}$ \\
\hline $\mathrm{Sb}-125$ & ARA-59 & 2012 & 0.01127 & 0.0295 & 0.0885 & $\mathrm{U}$ \\
\hline $\mathrm{Sb}-125$ & ARA-60 & 2012 & 0.03591 & 0.032 & 0.096 & $\mathrm{U}$ \\
\hline Sb-125 & ARA-62 & 2012 & 0.01059 & 0.0255 & 0.0765 & $\mathrm{U}$ \\
\hline Sb-125 & ARA-65 & 2012 & 0.0032 & 0.0384 & 0.1152 & $\mathrm{U}$ \\
\hline $\mathrm{Sb}-125$ & ARA-73 & 2012 & -0.0146 & 0.034 & 0.102 & $\mathrm{U}$ \\
\hline Sb-125 & ARA-31 & 2013 & 0.02805 & 0.039 & 0.117 & $\mathrm{U}$ \\
\hline Sb-125 & ARA-32 & 2013 & 0.04352 & 0.0141 & 0.0424 & - \\
\hline $\mathrm{Sb}-125$ & ARA-33 & 2013 & 0.07421 & 0.0193 & 0.0578 & - \\
\hline $\mathrm{Sb}-125$ & ARA-40 & 2013 & 0.1355 & 0.0303 & 0.0908 & - \\
\hline $\mathrm{Sb}-125$ & ARA-48 & 2013 & 0.04925 & 0.0166 & 0.0497 & $\mathrm{U}$ \\
\hline
\end{tabular}


Table C-1. (continued).

\begin{tabular}{|c|c|c|c|c|c|c|}
\hline Radionuclide & $\begin{array}{c}\text { Sample } \\
\text { Location }\end{array}$ & Date & $\begin{array}{c}\text { Concentrations } \\
(\mathrm{pCi} / \mathrm{g})\end{array}$ & $\begin{array}{l}\text { Uncertainty } \\
(\mathrm{pCi} / \mathrm{g})\end{array}$ & $\begin{array}{c}\text { MDA } \\
(\mathrm{pCi} / \mathrm{g})\end{array}$ & Flag \\
\hline $\mathrm{Sb}-125$ & ARA-65 & 2013 & 0.03836 & 0.0150 & 0.0451 & $\mathrm{U}$ \\
\hline Sb-125 & $\begin{array}{c}2 \text { INCH AIR } \\
\text { - ARA }\end{array}$ & 2014 & -0.03332 & 0.0586 & 0.1758 & $\mathrm{U}$ \\
\hline $\mathrm{Sb}-125$ & ARA-1 & 2014 & -0.1056 & 0.0436 & 0.1308 & $\mathrm{U}$ \\
\hline $\mathrm{Sb}-125$ & ARA-24 & 2014 & 0.02015 & 0.0456 & 0.1368 & $\mathrm{U}$ \\
\hline $\mathrm{Sb}-125$ & ARA-31 & 2014 & -0.01094 & 0.0504 & 0.1512 & $\mathrm{U}$ \\
\hline $\mathrm{Sb}-125$ & ARA-32 & 2014 & -0.1258 & 0.0397 & 0.1191 & $\mathrm{U}$ \\
\hline $\mathrm{Sb}-125$ & ARA-65 & 2014 & -0.007183 & 0.0515 & 0.1545 & $\mathrm{U}$ \\
\hline $\mathrm{Sb}-125$ & ARA-71 & 2014 & 0.005412 & 0.0311 & 0.0933 & $\mathrm{U}$ \\
\hline Sr-90 & $0^{\circ} 500^{\prime}$ & 1977 & 57 & 2 & 6 & - \\
\hline Sr-90 & $270^{\circ} 500^{\prime}$ & 1977 & 0.41 & 0.05 & 0.15 & - \\
\hline Sr-90 & $0^{\circ} 1000^{\prime}$ & 1985 & 2.23 & 0.11 & 0.33 & - \\
\hline Sr-90 & $135^{\circ} 2500^{\prime}$ & 1985 & 0.62 & 0.06 & 0.18 & - \\
\hline Sr-90 & $135^{\circ} 2500^{\prime}$ & 1985 & 0.45 & 0.06 & 0.18 & - \\
\hline Sr-90 & $180^{\circ} 2500^{\prime}$ & 1985 & 0.46 & 0.06 & 0.18 & - \\
\hline Sr-90 & $202.5^{\circ} 2000^{\prime}$ & 1985 & 0.93 & 0.07 & 0.21 & - \\
\hline Sr-90 & $22.5^{\circ} 2500^{\prime}$ & 1985 & 0.74 & 0.07 & 0.21 & - \\
\hline Sr-90 & $225^{\circ} 2500^{\prime}$ & 1985 & 0.68 & 0.07 & 0.21 & - \\
\hline Sr-90 & $225^{\circ} 500^{\prime}$ & 1985 & 9.5 & 0.3 & 0.9 & - \\
\hline Sr-90 & $270^{\circ} 1000^{\prime}$ & 1985 & 0.5 & 0.06 & 0.18 & - \\
\hline Sr-90 & $337.5^{\circ} 2500^{\prime}$ & 1985 & 0.38 & 0.06 & 0.18 & - \\
\hline Sr-90 & $45^{\circ} 1000^{\prime}$ & 1985 & 2.86 & 0.13 & 0.39 & - \\
\hline Sr-90 & $45^{\circ} 2500^{\prime}$ & 1985 & 1.73 & 0.09 & 0.27 & 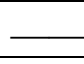 \\
\hline Sr-90 & $67.5^{\circ} 2000^{\prime}$ & 1985 & 17.3 & 0.5 & 1.5 & - \\
\hline Sr-90 & $67.5^{\circ} 500^{\prime}$ & 1985 & 22.3 & 0.7 & 2.1 & - \\
\hline U-233/234 & $0^{\circ} 1000^{\prime}$ & 1985 & 1.01 & 0.04 & 0.12 & - \\
\hline U-233/234 & $22.5^{\circ} 2500^{\prime}$ & 1985 & 0.94 & 0.03 & 0.09 & - \\
\hline U-233/234 & $67.5^{\circ} 2000^{\prime}$ & 1985 & 0.91 & 0.03 & 0.09 & - \\
\hline U-233/234 & $67.5^{\circ} 500^{\prime}$ & 1985 & 0.8 & 0.03 & 0.09 & - \\
\hline U-234 & ARA-0 & 2007 & 75.62 & 26.5 & 79.5 & $\mathrm{U}$ \\
\hline U-234 & ARA-1 & 2007 & 35.67 & 0.796 & 2.388 & - \\
\hline U-234 & ARA-10 & 2007 & 77.86 & 2.79 & 8.37 & - \\
\hline U-234 & ARA-11 & 2007 & 183.9 & 52.5 & 157.5 & - \\
\hline U-234 & ARA-13 & 2007 & 13.61 & 3.05 & 9.15 & - \\
\hline U-234 & ARA-14 & 2007 & 9.297 & 11.9 & 35.7 & $\mathrm{U}$ \\
\hline U-234 & ARA-15 & 2007 & 71.52 & 19.15 & 57.45 & - \\
\hline U-234 & ARA-16 & 2007 & 65.55 & 3.2 & 9.6 & - \\
\hline
\end{tabular}


Table C-1. (continued).

\begin{tabular}{|c|c|c|c|c|c|c|}
\hline Radionuclide & $\begin{array}{c}\text { Sample } \\
\text { Location }\end{array}$ & Date & $\begin{array}{c}\text { Concentrations } \\
(\mathrm{pCi} / \mathrm{g})\end{array}$ & $\begin{array}{l}\text { Uncertainty } \\
(\mathrm{pCi} / \mathrm{g})\end{array}$ & $\begin{array}{c}\text { MDA } \\
(\mathrm{pCi} / \mathrm{g})\end{array}$ & Flag \\
\hline U-234 & ARA-17 & 2007 & 39.43 & 33.25 & 99.75 & $\mathrm{U}$ \\
\hline U-234 & ARA-18 & 2007 & 124.7 & 6.53 & 19.59 & - \\
\hline U-234 & ARA-2 & 2007 & 31.83 & 12.2 & 36.6 & $\mathrm{U}$ \\
\hline U-234 & ARA-20 & 2007 & 137.5 & 4.3 & 12.9 & - \\
\hline U-234 & ARA-21 & 2007 & 32.59 & 21.4 & 64.2 & $\mathrm{U}$ \\
\hline U-234 & ARA-22 & 2007 & 35.86 & 2.57 & 7.71 & - \\
\hline U-234 & ARA-23 & 2007 & 67.37 & 31.85 & 95.55 & $\mathrm{U}$ \\
\hline $\mathrm{U}-234$ & ARA-24 & 2007 & 22.79 & 12.85 & 38.55 & $\mathrm{U}$ \\
\hline U-234 & ARA-25 & 2007 & 151.3 & 4.55 & 13.65 & - \\
\hline U-234 & ARA-26 & 2007 & 156 & 4.52 & 13.56 & - \\
\hline U-234 & ARA-27 & 2007 & 43.75 & 2.6 & 7.8 & - \\
\hline U-234 & ARA-28 & 2007 & 32.77 & 20.65 & 61.95 & $\mathrm{U}$ \\
\hline $\mathrm{U}-234$ & ARA-29 & 2007 & 62.55 & 41.05 & 123.15 & $\mathrm{U}$ \\
\hline $\mathrm{U}-234$ & ARA-3 & 2007 & 141.1 & 4.26 & 12.78 & - \\
\hline U-234 & ARA-30 & 2007 & 140.9 & 4.21 & 12.63 & - \\
\hline U-234 & ARA-31 & 2007 & 50.71 & 17.95 & 53.85 & $\mathrm{U}$ \\
\hline $\mathrm{U}-234$ & ARA-32 & 2007 & 74.43 & 28.5 & 85.5 & $\mathrm{U}$ \\
\hline U-234 & ARA-33 & 2007 & 124.7 & 3.98 & 11.94 & - \\
\hline U-234 & ARA-34 & 2007 & 114 & 3.72 & 11.16 & - \\
\hline U-234 & ARA-35 & 2007 & 118.2 & 4.02 & 12.06 & - \\
\hline U-234 & ARA-36 & 2007 & 13.21 & 18.05 & 54.15 & $\mathrm{U}$ \\
\hline U-234 & ARA-37 & 2007 & 71.51 & 26.45 & 79.35 & $\mathrm{U}$ \\
\hline U-234 & ARA-38 & 2007 & 29.17 & 25.25 & 75.75 & $\mathrm{U}$ \\
\hline U-234 & ARA-39 & 2007 & 95.54 & 3.03 & 9.09 & - \\
\hline U-234 & ARA-4 & 2007 & 18.64 & 20.9 & 62.7 & $\mathrm{U}$ \\
\hline U-234 & ARA-40 & 2007 & 138 & 3.83 & 11.49 & - \\
\hline U-234 & ARA-41 & 2007 & 134.3 & 3.88 & 11.64 & - \\
\hline U-234 & ARA-42 & 2007 & 24.23 & 17.65 & 52.95 & $\mathrm{U}$ \\
\hline U-234 & ARA-43 & 2007 & 39.56 & 17.3 & 51.9 & $\mathrm{U}$ \\
\hline U-234 & ARA-44 & 2007 & 91.35 & 4.11 & 12.33 & - \\
\hline U-234 & ARA-45 & 2007 & 40.48 & 12.8 & 38.4 & - \\
\hline U-234 & ARA-46 & 2007 & 34.6 & 16.05 & 48.15 & $\mathrm{U}$ \\
\hline U-234 & ARA-47 & 2007 & 37.31 & 11.1 & 33.3 & - \\
\hline U-234 & ARA-48 & 2007 & 46.73 & 23.05 & 69.15 & $\mathrm{U}$ \\
\hline U-234 & ARA-49 & 2007 & 25.42 & 12.4 & 37.2 & $\mathrm{U}$ \\
\hline U-234 & ARA-5 & 2007 & 31.24 & 19.75 & 59.25 & $\mathrm{U}$ \\
\hline U-234 & ARA-50 & 2007 & 13.69 & 8.2 & 24.6 & $\mathrm{U}$ \\
\hline
\end{tabular}


Table C-1. (continued).

\begin{tabular}{|c|c|c|c|c|c|c|}
\hline Radionuclide & $\begin{array}{c}\text { Sample } \\
\text { Location }\end{array}$ & Date & $\begin{array}{c}\text { Concentrations } \\
(\mathrm{pCi} / \mathrm{g})\end{array}$ & $\begin{array}{l}\text { Uncertainty } \\
(\mathrm{pCi} / \mathrm{g})\end{array}$ & $\begin{array}{c}\text { MDA } \\
(\mathrm{pCi} / \mathrm{g})\end{array}$ & Flag \\
\hline U-234 & ARA-51 & 2007 & 25.38 & 17.05 & 51.15 & $\mathrm{U}$ \\
\hline U-234 & ARA-53 & 2007 & 76.61 & 3.91 & 11.73 & - \\
\hline U-234 & ARA-54 & 2007 & 101.8 & 3.49 & 10.47 & - \\
\hline U-234 & ARA-55 & 2007 & 98.67 & 3.52 & 10.56 & - \\
\hline U-234 & ARA-56 & 2007 & 0.1117 & 12.85 & 38.55 & $\mathrm{U}$ \\
\hline U-234 & ARA-57 & 2007 & 26.67 & 14.6 & 43.8 & $\mathrm{U}$ \\
\hline U-234 & ARA-58 & 2007 & -1.273 & 20.85 & 62.55 & $\mathrm{U}$ \\
\hline $\mathrm{U}-234$ & ARA-59 & 2007 & 21.51 & 10.55 & 31.65 & $\mathrm{U}$ \\
\hline U-234 & ARA-6 & 2007 & 9.411 & 9.25 & 27.75 & $\mathrm{U}$ \\
\hline U-234 & ARA-60 & 2007 & 1.539 & 11.25 & 33.75 & $\mathrm{U}$ \\
\hline U-234 & ARA-61 & 2007 & 125.1 & 4.09 & 12.27 & - \\
\hline U-234 & ARA-62 & 2007 & 149.2 & 4.36 & 13.08 & - \\
\hline $\mathrm{U}-234$ & ARA-63 & 2007 & 152.2 & 4.31 & 12.93 & - \\
\hline $\mathrm{U}-234$ & ARA-65 & 2007 & 86.97 & 26.5 & 79.5 & - \\
\hline U-234 & ARA-66 & 2007 & 122.5 & 4.16 & 12.48 & - \\
\hline U-234 & ARA-67 & 2007 & 121.9 & 3.89 & 11.67 & - \\
\hline $\mathrm{U}-234$ & ARA-68 & 2007 & 131 & 3.96 & 11.88 & - \\
\hline U-234 & ARA-69 & 2007 & 155.2 & 4.4 & 13.2 & - \\
\hline U-234 & ARA-7 & 2007 & 53.79 & 23.05 & 69.15 & $\mathrm{U}$ \\
\hline U-234 & ARA-70 & 2007 & 143.6 & 4.2 & 12.6 & - \\
\hline U-234 & ARA-71 & 2007 & 152.8 & 4.3 & 12.9 & - \\
\hline U-234 & ARA-72 & 2007 & 99.44 & 4.15 & 12.45 & - \\
\hline U-234 & ARA-73 & 2007 & 37 & 18.15 & 54.45 & $\mathrm{U}$ \\
\hline U-234 & ARA-74 & 2007 & 59.06 & 22.5 & 67.5 & $\mathrm{U}$ \\
\hline U-234 & ARA-75 & 2007 & 10.52 & 12.15 & 36.45 & $\mathrm{U}$ \\
\hline U-234 & ARA-76 & 2007 & 201.4 & 5.18 & 15.54 & - \\
\hline U-234 & ARA-77 & 2007 & 43.89 & 22.35 & 67.05 & $\mathrm{U}$ \\
\hline U-234 & ARA-8 & 2007 & 186.6 & 4.8 & 14.4 & - \\
\hline U-234 & ARA-9 & 2007 & 86.7 & 3.31 & 9.93 & - \\
\hline U-234 & ARA-10 & 2009 & 1.289 & 4.275 & 12.825 & $\mathrm{U}$ \\
\hline U-234 & ARA-13 & 2009 & -1.396 & 4.65 & 13.95 & $\mathrm{U}$ \\
\hline U-234 & ARA-18 & 2009 & -0.8736 & 4.48 & 13.44 & $\mathrm{U}$ \\
\hline U-234 & ARA-20 & 2009 & 3.315 & 5.35 & 16.05 & $\mathrm{U}$ \\
\hline U-234 & ARA-21 & 2009 & 0 & 0.238 & 0.714 & $\mathrm{U}$ \\
\hline U-234 & ARA-26 & 2009 & -0.02759 & 5.9 & 17.7 & $\mathrm{U}$ \\
\hline U-234 & ARA-27 & 2009 & 8.558 & 3.415 & 10.245 & $\mathrm{U}$ \\
\hline U-234 & ARA-3 & 2009 & 29.3 & 17.6 & 52.8 & $\mathrm{U}$ \\
\hline
\end{tabular}


Table C-1. (continued).

\begin{tabular}{|c|c|c|c|c|c|c|}
\hline Radionuclide & $\begin{array}{c}\text { Sample } \\
\text { Location }\end{array}$ & Date & $\begin{array}{c}\text { Concentrations } \\
(\mathrm{pCi} / \mathrm{g})\end{array}$ & $\begin{array}{c}\text { Uncertainty } \\
(\mathrm{pCi} / \mathrm{g})\end{array}$ & $\begin{array}{c}\mathrm{MDA} \\
(\mathrm{pCi} / \mathrm{g})\end{array}$ & Flag \\
\hline U-234 & ARA-30 & 2009 & -1.539 & 9.05 & 27.15 & $\mathrm{U}$ \\
\hline U-234 & ARA-35 & 2009 & -2.809 & 6.45 & 19.35 & $\mathrm{U}$ \\
\hline U-234 & ARA-36 & 2009 & 3.278 & 2.105 & 6.315 & $\mathrm{U}$ \\
\hline U-234 & ARA-39 & 2009 & 0.3392 & 8.75 & 26.25 & $\mathrm{U}$ \\
\hline U-234 & ARA-40 & 2009 & 2.467 & 1.885 & 5.655 & $\mathrm{U}$ \\
\hline U-234 & ARA-43 & 2009 & 27.74 & 26.85 & 80.55 & $\mathrm{U}$ \\
\hline U-234 & ARA-44 & 2009 & -2.54 & 6.2 & 18.6 & $\mathrm{U}$ \\
\hline $\mathrm{U}-234$ & ARA-47 & 2009 & -9.892 & 6.85 & 20.55 & $\mathrm{U}$ \\
\hline U-234 & ARA-61 & 2009 & -0.07304 & 0.301 & 0.903 & $\mathrm{U}$ \\
\hline U-234 & ARA-62 & 2009 & 0.06726 & 0.447 & 1.341 & $\mathrm{U}$ \\
\hline U-234 & ARA-63 & 2009 & -1.779 & 12.05 & 36.15 & $\mathrm{U}$ \\
\hline U-234 & ARA-65 & 2009 & 4.008 & 2.07 & 6.21 & $\mathrm{U}$ \\
\hline $\mathrm{U}-234$ & ARA-67 & 2009 & -0.5938 & 4.59 & 13.77 & $\mathrm{U}$ \\
\hline $\mathrm{U}-234$ & ARA-68 & 2009 & -4.266 & 6.55 & 19.65 & $\mathrm{U}$ \\
\hline U-234 & ARA-69 & 2009 & -11.66 & 13.25 & 39.75 & $\mathrm{U}$ \\
\hline U-234 & ARA-70 & 2009 & 90.58 & 12.5 & 37.5 & - \\
\hline $\mathrm{U}-234$ & ARA-71 & 2009 & 2.427 & 6.2 & 18.6 & $\mathrm{U}$ \\
\hline U-234 & ARA-72 & 2009 & 4.814 & 4.34 & 13.02 & $\mathrm{U}$ \\
\hline U-234 & ARA-74 & 2009 & -3.189 & 6.5 & 19.5 & $\mathrm{U}$ \\
\hline U-234 & ARA-75 & 2009 & -1.672 & 5.9 & 17.7 & $\mathrm{U}$ \\
\hline U-234 & ARA-76 & 2009 & 18.4 & 5.85 & 17.55 & - \\
\hline U-234 & ARA-77 & 2009 & 0.5842 & 4.805 & 14.415 & $\mathrm{U}$ \\
\hline U-234 & ARA-8 & 2009 & 0.6721 & 5.25 & 15.75 & $\mathrm{U}$ \\
\hline U-234 & ARA-0 & 2010 & 2.561 & 2.86 & 8.58 & $\mathrm{U}$ \\
\hline U-234 & ARA-1 & 2010 & 18.31 & 1.75 & 5.25 & - \\
\hline U-234 & ARA-11 & 2010 & 22.03 & 1.64 & 4.92 & - \\
\hline U-234 & ARA-12 & 2010 & 109.8 & 3.74 & 11.22 & - \\
\hline U-234 & ARA-14 & 2010 & 21.88 & 2.06 & 6.18 & - \\
\hline U-234 & ARA-15 & 2010 & 114.8 & 3.85 & 11.55 & - \\
\hline U-234 & ARA-16 & 2010 & 115.2 & 3.83 & 11.49 & - \\
\hline U-234 & ARA-17 & 2010 & 15.34 & 1.69 & 5.07 & - \\
\hline U-234 & ARA-19 & 2010 & 20.29 & 2.12 & 6.36 & - \\
\hline U-234 & ARA-2 & 2010 & 7.034 & 2.22 & 6.66 & - \\
\hline U-234 & ARA-21 & 2010 & 5.738 & 2.18 & 6.54 & $\mathrm{U}$ \\
\hline U-234 & ARA-22 & 2010 & 21.8 & 2.12 & 6.36 & - \\
\hline U-234 & ARA-23 & 2010 & 8.55 & 1.98 & 5.94 & - \\
\hline U-234 & ARA-24 & 2010 & 109.6 & 3.67 & 11.01 & - \\
\hline
\end{tabular}


Table C-1. (continued).

\begin{tabular}{|c|c|c|c|c|c|c|}
\hline Radionuclide & $\begin{array}{c}\text { Sample } \\
\text { Location }\end{array}$ & Date & $\begin{array}{c}\text { Concentrations } \\
(\mathrm{pCi} / \mathrm{g})\end{array}$ & $\begin{array}{c}\text { Uncertainty } \\
(\mathrm{pCi} / \mathrm{g})\end{array}$ & $\begin{array}{c}\mathrm{MDA} \\
(\mathrm{pCi} / \mathrm{g})\end{array}$ & Flag \\
\hline U-234 & ARA-27 & 2010 & 9.462 & 2.16 & 6.48 & - \\
\hline U-234 & ARA-28 & 2010 & 29.52 & 2.15 & 6.45 & - \\
\hline U-234 & ARA-29 & 2010 & 17.53 & 1.64 & 4.92 & - \\
\hline U-234 & ARA-31 & 2010 & 39.7 & 2.33 & 6.99 & - \\
\hline U-234 & ARA-32 & 2010 & 27.13 & 2.08 & 6.24 & - \\
\hline U-234 & ARA-34 & 2010 & 37.58 & 2.26 & 6.78 & - \\
\hline U-234 & ARA-35 & 2010 & 2.896 & 2.89 & 8.67 & $\mathrm{U}$ \\
\hline $\mathrm{U}-234$ & ARA-36 & 2010 & 29.62 & 2.05 & 6.15 & - \\
\hline U-234 & ARA-37 & 2010 & 100 & 3.56 & 10.68 & - \\
\hline U-234 & ARA-38 & 2010 & 97.28 & 3.56 & 10.68 & - \\
\hline U-234 & ARA-4 & 2010 & 16.94 & 1.86 & 5.58 & - \\
\hline U-234 & ARA-40 & 2010 & 30.01 & 2 & 6 & - \\
\hline $\mathrm{U}-234$ & ARA-41 & 2010 & 20.44 & 1.7 & 5.1 & - \\
\hline $\mathrm{U}-234$ & ARA-42 & 2010 & 4.18 & 1.95 & 5.85 & $\mathrm{U}$ \\
\hline U-234 & ARA-43 & 2010 & 6.191 & 1.91 & 5.73 & - \\
\hline U-234 & ARA-44 & 2010 & 19.39 & 1.63 & 4.89 & - \\
\hline $\mathrm{U}-234$ & ARA-46 & 2010 & 14.64 & 1.99 & 5.97 & - \\
\hline U-234 & ARA-47 & 2010 & 2.664 & 1.97 & 5.91 & $\mathrm{U}$ \\
\hline U-234 & ARA-48 & 2010 & 6.129 & 2.21 & 6.63 & $\mathrm{U}$ \\
\hline U-234 & ARA-5 & 2010 & 107.1 & 3.78 & 11.34 & - \\
\hline U-234 & ARA-50 & 2010 & 11.52 & 1.66 & 4.98 & - \\
\hline U-234 & ARA-51 & 2010 & 9.755 & 2.13 & 6.39 & - \\
\hline U-234 & ARA-52 & 2010 & 30.26 & 1.96 & 5.88 & - \\
\hline U-234 & ARA-53 & 2010 & 26.65 & 2.1 & 6.3 & - \\
\hline U-234 & ARA-54 & 2010 & 14.7 & 1.48 & 4.44 & - \\
\hline U-234 & ARA-55 & 2010 & 22.38 & 1.89 & 5.67 & - \\
\hline U-234 & ARA-56 & 2010 & 14.95 & 1.61 & 4.83 & - \\
\hline U-234 & ARA-57 & 2010 & 19.68 & 2.03 & 6.09 & - \\
\hline U-234 & ARA-58 & 2010 & 15.51 & 1.65 & 4.95 & - \\
\hline U-234 & ARA-59 & 2010 & 16.85 & 1.47 & 4.41 & - \\
\hline U-234 & ARA-6 & 2010 & 12.99 & 1.53 & 4.59 & - \\
\hline U-234 & ARA-60 & 2010 & 29.57 & 2 & 6 & - \\
\hline U-234 & ARA-65 & 2010 & 19.14 & 1.71 & 5.13 & - \\
\hline U-234 & ARA-7 & 2010 & 28.13 & 1.97 & 5.91 & - \\
\hline U-234 & ARA-73 & 2010 & 20.88 & 1.66 & 4.98 & - \\
\hline U-234 & ARA-74 & 2010 & 20.4 & 1.68 & 5.04 & - \\
\hline U-234 & ARA-75 & 2010 & 39.47 & 2.26 & 6.78 & - \\
\hline
\end{tabular}


Table C-1. (continued).

\begin{tabular}{|c|c|c|c|c|c|c|}
\hline Radionuclide & $\begin{array}{c}\text { Sample } \\
\text { Location }\end{array}$ & Date & $\begin{array}{c}\text { Concentrations } \\
(\mathrm{pCi} / \mathrm{g})\end{array}$ & $\begin{array}{l}\text { Uncertainty } \\
(\mathrm{pCi} / \mathrm{g})\end{array}$ & $\begin{array}{c}\text { MDA } \\
(\mathrm{pCi} / \mathrm{g})\end{array}$ & Flag \\
\hline U-234 & ARA-77 & 2010 & 26.85 & 1.99 & 5.97 & - \\
\hline U-234 & ARA-9 & 2010 & 15.43 & 1.53 & 4.59 & - \\
\hline U-234 & ARA-1 & 2011 & -41.01 & 65.5 & 196.5 & $\mathrm{U}$ \\
\hline U-234 & ARA-14 & 2011 & 15.15 & 50.7 & 152.1 & $\mathrm{U}$ \\
\hline U-234 & ARA-2 & 2011 & -43.79 & 53.2 & 159.6 & $\mathrm{U}$ \\
\hline U-234 & ARA-24 & 2011 & -3.921 & 61.3 & 183.9 & $\mathrm{U}$ \\
\hline U-234 & ARA-29 & 2011 & 29.97 & 45.7 & 137.1 & $\mathrm{U}$ \\
\hline $\mathrm{U}-234$ & ARA-4 & 2011 & 20.76 & 37.8 & 113.4 & $\mathrm{U}$ \\
\hline U-234 & ARA-43 & 2011 & -49.67 & 59.5 & 178.5 & $\mathrm{U}$ \\
\hline U-234 & ARA-47 & 2011 & 17.68 & 63.3 & 189.9 & $\mathrm{U}$ \\
\hline U-234 & ARA-65 & 2011 & -11.2 & 60.9 & 182.7 & $\mathrm{U}$ \\
\hline U-234 & ARA-77 & 2011 & 8.652 & 61.8 & 185.4 & $\mathrm{U}$ \\
\hline $\mathrm{U}-234$ & ARA-9 & 2011 & -1.56 & 37.6 & 112.8 & $\mathrm{U}$ \\
\hline $\mathrm{U}-234$ & ARA-11 & 2012 & 16.78 & 24.5 & 73.5 & $\mathrm{U}$ \\
\hline U-234 & ARA-16 & 2012 & 5.204 & 30.1 & 90.3 & $\mathrm{U}$ \\
\hline U-234 & ARA-31 & 2012 & 7.898 & 24.5 & 73.5 & $\mathrm{U}$ \\
\hline $\mathrm{U}-234$ & ARA-32 & 2012 & 6.701 & 26.7 & 80.1 & $\mathrm{U}$ \\
\hline U-234 & ARA-48 & 2012 & 4.741 & 16.7 & 50.1 & $\mathrm{U}$ \\
\hline U-234 & ARA-53 & 2012 & 18.51 & 21.8 & 65.4 & $\mathrm{U}$ \\
\hline U-234 & ARA-57 & 2012 & 12.66 & 24.6 & 73.8 & $\mathrm{U}$ \\
\hline U-234 & ARA-58 & 2012 & -2.3 & 15.2 & 45.6 & $\mathrm{U}$ \\
\hline U-234 & ARA-59 & 2012 & 1.838 & 22.1 & 66.3 & $\mathrm{U}$ \\
\hline U-234 & ARA-60 & 2012 & 4.865 & 23.1 & 69.3 & $\mathrm{U}$ \\
\hline U-234 & ARA-62 & 2012 & 3.379 & 26.3 & 78.9 & $\mathrm{U}$ \\
\hline U-234 & ARA-65 & 2012 & -7.62 & 16 & 48 & $\mathrm{U}$ \\
\hline U-234 & ARA-73 & 2012 & -7.701 & 15.3 & 45.9 & $\mathrm{U}$ \\
\hline U-234 & ARA-31 & 2013 & 0.8604 & 32.0 & 96.1 & $\mathrm{U}$ \\
\hline U-234 & ARA-32 & 2013 & -19.62 & 44.3 & 133 & $\mathrm{U}$ \\
\hline U-234 & ARA-33 & 2013 & 22.64 & 26.0 & 77.9 & $\mathrm{U}$ \\
\hline U-234 & ARA-40 & 2013 & 46.54 & 41.3 & 124 & $\mathrm{U}$ \\
\hline U-234 & ARA-48 & 2013 & 1.623 & 46 & 138 & $\mathrm{U}$ \\
\hline U-234 & ARA-65 & 2013 & 20.68 & 39.7 & 119 & $\mathrm{U}$ \\
\hline U-234 & ARA-1 & 2014 & -3.863 & 47.4 & 142.2 & $\mathrm{U}$ \\
\hline U-234 & ARA-24 & 2014 & -60.49 & 60.5 & 181.5 & $\mathrm{U}$ \\
\hline U-234 & ARA-32 & 2014 & 10.13 & 54.9 & 164.7 & $\mathrm{U}$ \\
\hline U-234 & ARA-71 & 2014 & -5.107 & 43.3 & 129.9 & $\mathrm{U}$ \\
\hline U-235 & $0^{\circ} 1000^{\prime}$ & 1985 & 0.05 & 0.025 & 0.075 & $\mathrm{U}$ \\
\hline
\end{tabular}


Table C-1. (continued).

\begin{tabular}{|c|c|c|c|c|c|c|}
\hline Radionuclide & $\begin{array}{c}\text { Sample } \\
\text { Location }\end{array}$ & Date & $\begin{array}{c}\text { Concentrations } \\
(\mathrm{pCi} / \mathrm{g})\end{array}$ & $\begin{array}{l}\text { Uncertainty } \\
(\mathrm{pCi} / \mathrm{g})\end{array}$ & $\begin{array}{c}\text { MDA } \\
(\mathrm{pCi} / \mathrm{g})\end{array}$ & Flag \\
\hline U-235 & $22.5^{\circ} 2500^{\prime}$ & 1985 & 0.053 & 0.027 & 0.081 & $\mathrm{U}$ \\
\hline U-235 & $67.5^{\circ} 2000^{\prime}$ & 1985 & 0.053 & 0.027 & 0.081 & $\mathrm{U}$ \\
\hline U-235 & $67.5^{\circ} 500^{\prime}$ & 1985 & 0.043 & 0.021 & 0.063 & $\mathrm{U}$ \\
\hline U-235 & ARA-0 & 2006 & 3.7 & 1.12 & 3.36 & - \\
\hline U-235 & ARA-1 & 2006 & 0.3044 & 0.096 & 0.288 & - \\
\hline U-235 & ARA-10 & 2006 & 1.017 & 0.43 & 1.29 & $\mathrm{U}$ \\
\hline U-235 & ARA-13 & 2006 & 3.223 & 1.225 & 3.675 & $\mathrm{U}$ \\
\hline $\mathrm{U}-235$ & ARA-18 & 2006 & 1.328 & 0.685 & 2.055 & $\mathrm{U}$ \\
\hline $\mathrm{U}-235$ & ARA-2 & 2006 & 3.517 & 0.845 & 2.535 & - \\
\hline U-235 & ARA-20 & 2006 & -0.1246 & 0.935 & 2.805 & $\mathrm{U}$ \\
\hline $\mathrm{U}-235$ & ARA-26 & 2006 & 2.827 & 1.13 & 3.39 & $\mathrm{U}$ \\
\hline $\mathrm{U}-235$ & ARA-3 & 2006 & 3.541 & 1.11 & 3.33 & - \\
\hline $\mathrm{U}-235$ & ARA-30 & 2006 & 2.784 & 0.84 & 2.52 & - \\
\hline $\mathrm{U}-235$ & ARA-31 & 2006 & 1.998 & 0.4885 & 1.4655 & - \\
\hline $\mathrm{U}-235$ & ARA-32 & 2006 & 2.123 & 0.51 & 1.53 & - \\
\hline $\mathrm{U}-235$ & ARA-33 & 2006 & 0.8142 & 0.465 & 1.395 & $\mathrm{U}$ \\
\hline $\mathrm{U}-235$ & ARA-34 & 2006 & 1.415 & 0.4145 & 1.2435 & - \\
\hline U-235 & ARA-39 & 2006 & 1.892 & 0.95 & 2.85 & $\mathrm{U}$ \\
\hline U-235 & ARA-4 & 2006 & 2.419 & 0.69 & 2.07 & - \\
\hline U-235 & ARA-40 & 2006 & 1.421 & 0.4345 & 1.3035 & - \\
\hline U-235 & ARA-41 & 2006 & 0.5711 & 0.455 & 1.365 & $\mathrm{U}$ \\
\hline U-235 & ARA-42 & 2006 & 0.846 & 0.455 & 1.365 & $\mathrm{U}$ \\
\hline U-235 & ARA-43 & 2006 & 0.9046 & 0.3625 & 1.0875 & $\mathrm{U}$ \\
\hline U-235 & ARA-44 & 2006 & 1.194 & 0.4615 & 1.3845 & $\mathrm{U}$ \\
\hline U-235 & ARA-48 & 2006 & 0.9046 & 0.3625 & 1.0875 & $\mathrm{U}$ \\
\hline U-235 & ARA-49 & 2006 & 1.285 & 0.6 & 1.8 & $\mathrm{U}$ \\
\hline U-235 & ARA-5 & 2006 & 1.039 & 0.54 & 1.62 & $\mathrm{U}$ \\
\hline U-235 & ARA-50 & 2006 & 0.4406 & 0.206 & 0.618 & $\mathrm{U}$ \\
\hline U-235 & ARA-51 & 2006 & 2.011 & 0.61 & 1.83 & - \\
\hline U-235 & ARA-53 & 2006 & 1.104 & 0.545 & 1.635 & $\mathrm{U}$ \\
\hline U-235 & ARA-54 & 2006 & 1.273 & 0.3825 & 1.1475 & - \\
\hline U-235 & ARA-55 & 2006 & 1.437 & 0.5 & 1.5 & $\mathrm{U}$ \\
\hline U-235 & ARA-56 & 2006 & 1.176 & 0.4505 & 1.3515 & $\mathrm{U}$ \\
\hline U-235 & ARA-57 & 2006 & 1.292 & 0.4235 & 1.2705 & - \\
\hline U-235 & ARA-58 & 2006 & 0.2976 & 0.1785 & 0.5355 & $\mathrm{U}$ \\
\hline U-235 & ARA-59 & 2006 & 1.672 & 0.4435 & 1.3305 & - \\
\hline U-235 & ARA-6 & 2006 & 0.8321 & 0.52 & 1.56 & $\mathrm{U}$ \\
\hline
\end{tabular}


Table C-1. (continued).

\begin{tabular}{|c|c|c|c|c|c|c|}
\hline Radionuclide & $\begin{array}{c}\text { Sample } \\
\text { Location }\end{array}$ & Date & $\begin{array}{c}\text { Concentrations } \\
(\mathrm{pCi} / \mathrm{g})\end{array}$ & $\begin{array}{c}\text { Uncertainty } \\
(\mathrm{pCi} / \mathrm{g})\end{array}$ & $\begin{array}{c}\mathrm{MDA} \\
(\mathrm{pCi} / \mathrm{g})\end{array}$ & Flag \\
\hline U-235 & ARA-60 & 2006 & 0.4217 & 0.395 & 1.185 & $\mathrm{U}$ \\
\hline U-235 & ARA-61 & 2006 & 3.677 & 0.975 & 2.925 & - \\
\hline U-235 & ARA-62 & 2006 & 0.8498 & 0.655 & 1.965 & $\mathrm{U}$ \\
\hline U-235 & ARA-63 & 2006 & 0.8444 & 0.1465 & 0.4395 & - \\
\hline U-235 & ARA-65 & 2006 & 1.871 & 0.635 & 1.905 & $\mathrm{U}$ \\
\hline U-235 & ARA-66 & 2006 & 1.354 & 0.735 & 2.205 & $\mathrm{U}$ \\
\hline U-235 & ARA-67 & 2006 & 2.579 & 0.81 & 2.43 & - \\
\hline $\mathrm{U}-235$ & ARA-68 & 2006 & 2.453 & 0.96 & 2.88 & $\mathrm{U}$ \\
\hline $\mathrm{U}-235$ & ARA-69 & 2006 & 2.06 & 1.04 & 3.12 & $\mathrm{U}$ \\
\hline U-235 & ARA-7 & 2006 & 1.304 & 0.3495 & 1.0485 & - \\
\hline $\mathrm{U}-235$ & ARA-70 & 2006 & 3.591 & 0.815 & 2.445 & - \\
\hline $\mathrm{U}-235$ & ARA-71 & 2006 & 1.25 & 0.4865 & 1.4595 & $\mathrm{U}$ \\
\hline $\mathrm{U}-235$ & ARA-72 & 2006 & 1.468 & 0.78 & 2.34 & $\mathrm{U}$ \\
\hline $\mathrm{U}-235$ & ARA-73 & 2006 & 1.193 & 0.3595 & 1.0785 & - \\
\hline $\mathrm{U}-235$ & ARA-74 & 2006 & 0.8312 & 0.349 & 1.047 & $\mathrm{U}$ \\
\hline $\mathrm{U}-235$ & ARA-75 & 2006 & 1.903 & 0.44 & 1.32 & - \\
\hline $\mathrm{U}-235$ & ARA-76 & 2006 & 3.451 & 0.87 & 2.61 & - \\
\hline U-235 & ARA-77 & 2006 & 0.2374 & 0.555 & 1.665 & $\mathrm{U}$ \\
\hline U-235 & ARA-8 & 2006 & 2.402 & 1.08 & 3.24 & $\mathrm{U}$ \\
\hline U-235 & ARA-9 & 2006 & 1.728 & 0.545 & 1.635 & - \\
\hline U-235 & ARA-0 & 2007 & 0.264 & 0.0635 & 0.1905 & - \\
\hline U-235 & ARA-1 & 2007 & 0.225 & 0.0234 & 0.0702 & - \\
\hline U-235 & ARA-10 & 2007 & 0.09379 & 0.0498 & 0.1494 & $\mathrm{U}$ \\
\hline U-235 & ARA-11 & 2007 & 0.4989 & 0.0955 & 0.2865 & - \\
\hline U-235 & ARA-12 & 2007 & 0.6814 & 0.1085 & 0.3255 & - \\
\hline U-235 & ARA-13 & 2007 & 0.3049 & 0.0768 & 0.2304 & - \\
\hline U-235 & ARA-14 & 2007 & 0.3245 & 0.0765 & 0.2295 & - \\
\hline U-235 & ARA-15 & 2007 & 0.3804 & 0.078 & 0.234 & - \\
\hline U-235 & ARA-16 & 2007 & 0.9037 & 0.16 & 0.48 & - \\
\hline U-235 & ARA-17 & 2007 & 0.3794 & 0.0595 & 0.1785 & \\
\hline U-235 & ARA-18 & 2007 & 0.06535 & 0.102 & 0.306 & $\mathrm{U}$ \\
\hline U-235 & ARA-19 & 2007 & 0.2998 & 0.0865 & 0.2595 & - \\
\hline U-235 & ARA-2 & 2007 & 0.4862 & 0.111 & 0.333 & - \\
\hline U-235 & ARA-20 & 2007 & 0.1057 & 0.0854 & 0.2562 & $\mathrm{U}$ \\
\hline U-235 & ARA-21 & 2007 & 0.409 & 0.083 & 0.249 & - \\
\hline U-235 & ARA-22 & 2007 & 0.2483 & 0.0755 & 0.2265 & - \\
\hline U-235 & ARA-23 & 2007 & 0.3451 & 0.0565 & 0.1695 & - \\
\hline
\end{tabular}


Table C-1. (continued).

\begin{tabular}{|c|c|c|c|c|c|c|}
\hline Radionuclide & $\begin{array}{c}\text { Sample } \\
\text { Location }\end{array}$ & Date & $\begin{array}{c}\text { Concentrations } \\
(\mathrm{pCi} / \mathrm{g})\end{array}$ & $\begin{array}{l}\text { Uncertainty } \\
(\mathrm{pCi} / \mathrm{g})\end{array}$ & $\begin{array}{c}\text { MDA } \\
(\mathrm{pCi} / \mathrm{g})\end{array}$ & Flag \\
\hline U-235 & ARA-24 & 2007 & 0.2707 & 0.068 & 0.204 & - \\
\hline U-235 & ARA-25 & 2007 & 0.3237 & 0.0965 & 0.2895 & - \\
\hline U-235 & ARA-26 & 2007 & 0.1726 & 0.0844 & 0.2532 & $\mathrm{U}$ \\
\hline U-235 & ARA-27 & 2007 & 0.1971 & 0.0798 & 0.2394 & $\mathrm{U}$ \\
\hline U-235 & ARA-28 & 2007 & 0.4091 & 0.061 & 0.183 & - \\
\hline U-235 & ARA-29 & 2007 & 0.307 & 0.065 & 0.195 & - \\
\hline U-235 & ARA-3 & 2007 & 0.1224 & 0.0884 & 0.2652 & $\mathrm{U}$ \\
\hline $\mathrm{U}-235$ & ARA-30 & 2007 & 0.2025 & 0.0832 & 0.2496 & $\mathrm{U}$ \\
\hline $\mathrm{U}-235$ & ARA-31 & 2007 & 0.8034 & 0.1395 & 0.4185 & - \\
\hline U-235 & ARA-32 & 2007 & 0.7664 & 0.1295 & 0.3885 & - \\
\hline $\mathrm{U}-235$ & ARA-33 & 2007 & 0.09769 & 0.0632 & 0.1896 & $\mathrm{U}$ \\
\hline $\mathrm{U}-235$ & ARA-34 & 2007 & 0.1316 & 0.0761 & 0.2283 & $\mathrm{U}$ \\
\hline $\mathrm{U}-235$ & ARA-35 & 2007 & 0.3291 & 0.0858 & 0.2574 & - \\
\hline $\mathrm{U}-235$ & ARA-36 & 2007 & 0.3953 & 0.084 & 0.252 & - \\
\hline $\mathrm{U}-235$ & ARA-37 & 2007 & 0.6191 & 0.1165 & 0.3495 & - \\
\hline $\mathrm{U}-235$ & ARA-38 & 2007 & 0.4962 & 0.087 & 0.261 & - \\
\hline $\mathrm{U}-235$ & ARA-39 & 2007 & 0.1526 & 0.057 & 0.171 & $\mathrm{U}$ \\
\hline U-235 & ARA-4 & 2007 & 0.3477 & 0.088 & 0.264 & - \\
\hline U-235 & ARA-40 & 2007 & 2.492 & 0.449 & 1.347 & - \\
\hline U-235 & ARA-41 & 2007 & 1.568 & 0.323 & 0.969 & - \\
\hline U-235 & ARA-42 & 2007 & 0.4177 & 0.063 & 0.189 & - \\
\hline U-235 & ARA-43 & 2007 & 0.3537 & 0.07 & 0.21 & - \\
\hline U-235 & ARA-44 & 2007 & 0.165 & 0.0775 & 0.2325 & $\mathrm{U}$ \\
\hline U-235 & ARA-45 & 2007 & 0.4657 & 0.112 & 0.336 & - \\
\hline U-235 & ARA-46 & 2007 & 0.3408 & 0.0785 & 0.2355 & - \\
\hline U-235 & ARA-47 & 2007 & 0.3041 & 0.094 & 0.282 & - \\
\hline U-235 & ARA-48 & 2007 & 0.3878 & 0.1135 & 0.3405 & - \\
\hline U-235 & ARA-49 & 2007 & 0.589 & 0.1055 & 0.3165 & - \\
\hline U-235 & ARA-5 & 2007 & 0.2645 & 0.0685 & 0.2055 & - \\
\hline U-235 & ARA-50 & 2007 & 0.5514 & 0.0925 & 0.2775 & - \\
\hline U-235 & ARA-51 & 2007 & 0.3657 & 0.0655 & 0.1965 & - \\
\hline U-235 & ARA-52 & 2007 & 0.2532 & 0.04315 & 0.12945 & - \\
\hline U-235 & ARA-53 & 2007 & 0.1365 & 0.0769 & 0.2307 & $\mathrm{U}$ \\
\hline U-235 & ARA-54 & 2007 & 0.1679 & 0.0721 & 0.2163 & $\mathrm{U}$ \\
\hline U-235 & ARA-55 & 2007 & 0.218 & 0.0633 & 0.1899 & - \\
\hline U-235 & ARA-56 & 2007 & 0.3278 & 0.066 & 0.198 & - \\
\hline U-235 & ARA-57 & 2007 & 2.472 & 0.54 & 1.62 & - \\
\hline
\end{tabular}


Table C-1. (continued).

\begin{tabular}{|c|c|c|c|c|c|c|}
\hline Radionuclide & $\begin{array}{c}\text { Sample } \\
\text { Location }\end{array}$ & Date & $\begin{array}{c}\text { Concentrations } \\
(\mathrm{pCi} / \mathrm{g})\end{array}$ & $\begin{array}{c}\text { Uncertainty } \\
(\mathrm{pCi} / \mathrm{g})\end{array}$ & $\begin{array}{c}\text { MDA } \\
(\mathrm{pCi} / \mathrm{g})\end{array}$ & Flag \\
\hline U-235 & ARA-58 & 2007 & 0.3358 & 0.0605 & 0.1815 & - \\
\hline U-235 & ARA-59 & 2007 & 0.3217 & 0.0545 & 0.1635 & - \\
\hline U-235 & ARA-6 & 2007 & 0.3015 & 0.0615 & 0.1845 & - \\
\hline U-235 & ARA-60 & 2007 & 0.633 & 0.0885 & 0.2655 & - \\
\hline U-235 & ARA-61 & 2007 & 0.08486 & 0.0697 & 0.2091 & $\mathrm{U}$ \\
\hline U-235 & ARA-62 & 2007 & 0.194 & 0.0862 & 0.2586 & $\mathrm{U}$ \\
\hline U-235 & ARA-63 & 2007 & 0.2113 & 0.0814 & 0.2442 & $\mathrm{U}$ \\
\hline $\mathrm{U}-235$ & ARA-65 & 2007 & 0.6562 & 0.1425 & 0.4275 & - \\
\hline $\mathrm{U}-235$ & ARA-66 & 2007 & 0.1626 & 0.0589 & 0.1767 & $\mathrm{U}$ \\
\hline U-235 & ARA-67 & 2007 & 0.1892 & 0.0637 & 0.1911 & $\mathrm{U}$ \\
\hline $\mathrm{U}-235$ & ARA-68 & 2007 & 0.1867 & 0.0712 & 0.2136 & $\mathrm{U}$ \\
\hline $\mathrm{U}-235$ & ARA-69 & 2007 & 0.4761 & 0.0815 & 0.2445 & - \\
\hline $\mathrm{U}-235$ & ARA-7 & 2007 & 0.2918 & 0.057 & 0.171 & - \\
\hline $\mathrm{U}-235$ & ARA-70 & 2007 & 0.1675 & 0.0871 & 0.2613 & $\mathrm{U}$ \\
\hline $\mathrm{U}-235$ & ARA-71 & 2007 & 0.1547 & 0.0853 & 0.2559 & $\mathrm{U}$ \\
\hline $\mathrm{U}-235$ & ARA-72 & 2007 & 0.1596 & 0.0559 & 0.1677 & $\mathrm{U}$ \\
\hline $\mathrm{U}-235$ & ARA-73 & 2007 & 0.2323 & 0.0494 & 0.1482 & - \\
\hline U-235 & ARA-74 & 2007 & 0.353 & 0.055 & 0.165 & - \\
\hline U-235 & ARA-75 & 2007 & 0.4135 & 0.0665 & 0.1995 & - \\
\hline U-235 & ARA-76 & 2007 & 0.2846 & 0.079 & 0.237 & - \\
\hline U-235 & ARA-77 & 2007 & 0.5109 & 0.072 & 0.216 & - \\
\hline U-235 & ARA-8 & 2007 & 0.4685 & 0.0824 & 0.2472 & - \\
\hline U-235 & ARA-9 & 2007 & 0.2578 & 0.0599 & 0.1797 & - \\
\hline U-235 & ARA-10 & 2009 & 0.2566 & 0.0845 & 0.2535 & - \\
\hline U-235 & ARA-11 & 2009 & 0.1449 & 0.03525 & 0.10575 & - \\
\hline U-235 & ARA-13 & 2009 & 0.09463 & 0.1055 & 0.3165 & $\mathrm{U}$ \\
\hline U-235 & ARA-18 & 2009 & 0.25 & 0.083 & 0.249 & - \\
\hline U-235 & ARA-19 & 2009 & 0.2339 & 0.0525 & 0.1575 & - \\
\hline U-235 & ARA-2 & 2009 & 0.2225 & 0.0299 & 0.0897 & - \\
\hline U-235 & ARA-20 & 2009 & 0.1642 & 0.091 & 0.273 & $\mathrm{U}$ \\
\hline U-235 & ARA-21 & 2009 & 0.1169 & 0.0665 & 0.1995 & $\mathrm{U}$ \\
\hline U-235 & ARA-25 & 2009 & 0.2908 & 0.0575 & 0.1725 & - \\
\hline U-235 & ARA-26 & 2009 & 0.3346 & 0.102 & 0.306 & - \\
\hline U-235 & ARA-27 & 2009 & 0.2253 & 0.057 & 0.171 & - \\
\hline U-235 & ARA-29 & 2009 & 0.1577 & 0.149 & 0.447 & $\mathrm{U}$ \\
\hline U-235 & ARA-3 & 2009 & 0.2331 & 0.1065 & 0.3195 & $\mathrm{U}$ \\
\hline U-235 & ARA-30 & 2009 & 0.04459 & 0.087 & 0.261 & $\mathrm{U}$ \\
\hline
\end{tabular}


Table C-1. (continued).

\begin{tabular}{|c|c|c|c|c|c|c|}
\hline Radionuclide & $\begin{array}{c}\text { Sample } \\
\text { Location }\end{array}$ & Date & $\begin{array}{c}\text { Concentrations } \\
(\mathrm{pCi} / \mathrm{g})\end{array}$ & $\begin{array}{c}\text { Uncertainty } \\
(\mathrm{pCi} / \mathrm{g})\end{array}$ & $\begin{array}{c}\text { MDA } \\
(\mathrm{pCi} / \mathrm{g})\end{array}$ & Flag \\
\hline U-235 & ARA-35 & 2009 & 0.2065 & 0.07 & 0.21 & $\mathrm{U}$ \\
\hline U-235 & ARA-39 & 2009 & 0.08117 & 0.097 & 0.291 & $\mathrm{U}$ \\
\hline U-235 & ARA-44 & 2009 & 0.1364 & 0.0665 & 0.1995 & $\mathrm{U}$ \\
\hline U-235 & ARA-45 & 2009 & 0.1492 & 0.02775 & 0.08325 & - \\
\hline U-235 & ARA-47 & 2009 & 0.1605 & 0.0655 & 0.1965 & $\mathrm{U}$ \\
\hline U-235 & ARA-61 & 2009 & 0.1312 & 0.094 & 0.282 & $\mathrm{U}$ \\
\hline U-235 & ARA-62 & 2009 & 0.1536 & 0.092 & 0.276 & $\mathrm{U}$ \\
\hline $\mathrm{U}-235$ & ARA-63 & 2009 & 0.1097 & 0.111 & 0.333 & $\mathrm{U}$ \\
\hline $\mathrm{U}-235$ & ARA-67 & 2009 & 0.2288 & 0.0865 & 0.2595 & $\mathrm{U}$ \\
\hline U-235 & ARA-68 & 2009 & 0.2738 & 0.0925 & 0.2775 & $\mathrm{U}$ \\
\hline $\mathrm{U}-235$ & ARA-69 & 2009 & 0.1834 & 0.119 & 0.357 & $\mathrm{U}$ \\
\hline $\mathrm{U}-235$ & ARA-70 & 2009 & 0.02346 & 0.0895 & 0.2685 & $\mathrm{U}$ \\
\hline $\mathrm{U}-235$ & ARA-71 & 2009 & 0.4508 & 0.107 & 0.321 & - \\
\hline $\mathrm{U}-235$ & ARA-72 & 2009 & 0.215 & 0.0845 & 0.2535 & $\mathrm{U}$ \\
\hline $\mathrm{U}-235$ & ARA-74 & 2009 & 0.163 & 0.0625 & 0.1875 & $\mathrm{U}$ \\
\hline $\mathrm{U}-235$ & ARA-75 & 2009 & 0.2248 & 0.064 & 0.192 & - \\
\hline $\mathrm{U}-235$ & ARA-77 & 2009 & 0.1379 & 0.0625 & 0.1875 & $\mathrm{U}$ \\
\hline U-235 & ARA-8 & 2009 & 0.2027 & 0.104 & 0.312 & $\mathrm{U}$ \\
\hline U-235 & ARA-0 & 2010 & 0.3073 & 0.0572 & 0.1716 & - \\
\hline U-235 & ARA-1 & 2010 & 2.567 & 0.457 & 1.371 & - \\
\hline U-235 & ARA-11 & 2010 & 2.113 & 0.322 & 0.966 & - \\
\hline U-235 & ARA-12 & 2010 & 1.267 & 0.244 & 0.732 & - \\
\hline U-235 & ARA-14 & 2010 & 1.081 & 0.252 & 0.756 & - \\
\hline U-235 & ARA-15 & 2010 & 1.109 & 0.274 & 0.822 & - \\
\hline U-235 & ARA-16 & 2010 & 0.9979 & 0.275 & 0.825 & - \\
\hline U-235 & ARA-17 & 2010 & 2.902 & 0.614 & 1.842 & - \\
\hline U-235 & ARA-19 & 2010 & 1.496 & 0.284 & 0.852 & - \\
\hline U-235 & ARA-2 & 2010 & 0.9653 & 0.185 & 0.555 & - \\
\hline U-235 & ARA-21 & 2010 & 1.45 & 0.652 & 1.956 & $\mathrm{U}$ \\
\hline U-235 & ARA-22 & 2010 & 0.8477 & 0.213 & 0.639 & - \\
\hline U-235 & ARA-23 & 2010 & 0.6273 & 0.142 & 0.426 & - \\
\hline U-235 & ARA-24 & 2010 & 1.411 & 0.249 & 0.747 & - \\
\hline U-235 & ARA-27 & 2010 & 1.105 & 0.203 & 0.609 & - \\
\hline U-235 & ARA-28 & 2010 & 0.699 & 0.23 & 0.69 & - \\
\hline U-235 & ARA-29 & 2010 & 0.2251 & 0.0435 & 0.1305 & - \\
\hline U-235 & ARA-31 & 2010 & 0.6395 & 0.198 & 0.594 & - \\
\hline U-235 & ARA-32 & 2010 & 2.552 & 0.454 & 1.362 & - \\
\hline
\end{tabular}


Table C-1. (continued).

\begin{tabular}{|c|c|c|c|c|c|c|}
\hline Radionuclide & $\begin{array}{c}\text { Sample } \\
\text { Location }\end{array}$ & Date & $\begin{array}{c}\text { Concentrations } \\
(\mathrm{pCi} / \mathrm{g})\end{array}$ & $\begin{array}{l}\text { Uncertainty } \\
(\mathrm{pCi} / \mathrm{g})\end{array}$ & $\begin{array}{c}\text { MDA } \\
(\mathrm{pCi} / \mathrm{g})\end{array}$ & Flag \\
\hline U-235 & ARA-34 & 2010 & 0.9072 & 0.266 & 0.798 & - \\
\hline U-235 & ARA-35 & 2010 & 0.2218 & 0.0571 & 0.1713 & \\
\hline U-235 & ARA-36 & 2010 & 0.4253 & 0.162 & 0.486 & $\mathrm{U}$ \\
\hline U-235 & ARA-37 & 2010 & 1.003 & 0.208 & 0.624 & - \\
\hline U-235 & ARA-38 & 2010 & 0.8838 & 0.182 & 0.546 & - \\
\hline U-235 & ARA-4 & 2010 & 0.3338 & 0.0473 & 0.1419 & - \\
\hline U-235 & ARA-40 & 2010 & 0.7294 & 0.187 & 0.561 & - \\
\hline $\mathrm{U}-235$ & ARA-41 & 2010 & 1.75 & 0.32 & 0.96 & - \\
\hline $\mathrm{U}-235$ & ARA-42 & 2010 & 3.265 & 0.531 & 1.593 & - \\
\hline U-235 & ARA-43 & 2010 & 1.009 & 0.188 & 0.564 & - \\
\hline $\mathrm{U}-235$ & ARA-44 & 2010 & 0.1952 & 0.0469 & 0.1407 & - \\
\hline $\mathrm{U}-235$ & ARA-45 & 2010 & 0.8618 & 0.23 & 0.69 & - \\
\hline $\mathrm{U}-235$ & ARA-46 & 2010 & 0.2827 & 0.0583 & 0.1749 & - \\
\hline $\mathrm{U}-235$ & ARA-47 & 2010 & 0.6409 & 0.169 & 0.507 & - \\
\hline $\mathrm{U}-235$ & ARA-48 & 2010 & 1.207 & 0.203 & 0.609 & - \\
\hline $\mathrm{U}-235$ & ARA-5 & 2010 & 1.146 & 0.233 & 0.699 & - \\
\hline $\mathrm{U}-235$ & ARA-50 & 2010 & 1.238 & 0.22 & 0.66 & - \\
\hline $\mathrm{U}-235$ & ARA-51 & 2010 & 0.7514 & 0.664 & 1.992 & $\mathrm{U}$ \\
\hline U-235 & ARA-52 & 2010 & 0.7545 & 0.218 & 0.654 & - \\
\hline U-235 & ARA-53 & 2010 & 2.734 & 0.495 & 1.485 & - \\
\hline U-235 & ARA-54 & 2010 & 0.3296 & 0.0545 & 0.1635 & - \\
\hline U-235 & ARA-55 & 2010 & 1.479 & 0.222 & 0.666 & - \\
\hline U-235 & ARA-56 & 2010 & 1.359 & 0.266 & 0.798 & - \\
\hline U-235 & ARA-57 & 2010 & 0.2923 & 0.0585 & 0.1755 & - \\
\hline U-235 & ARA-58 & 2010 & 0.9526 & 0.198 & 0.594 & - \\
\hline U-235 & ARA-59 & 2010 & 0.8003 & 0.178 & 0.534 & - \\
\hline U-235 & ARA-6 & 2010 & 0.572 & 0.17 & 0.51 & - \\
\hline U-235 & ARA-60 & 2010 & 0.2824 & 0.0592 & 0.1776 & 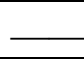 \\
\hline U-235 & ARA-65 & 2010 & 0.3153 & 0.0595 & 0.1785 & - \\
\hline U-235 & ARA-7 & 2010 & 0.6565 & 0.164 & 0.492 & - \\
\hline U-235 & ARA-73 & 2010 & 0.2906 & 0.0529 & 0.1587 & - \\
\hline U-235 & ARA-74 & 2010 & 1.65 & 0.354 & 1.062 & - \\
\hline U-235 & ARA-75 & 2010 & 0.2425 & 0.0432 & 0.1296 & - \\
\hline U-235 & ARA-77 & 2010 & 0.3201 & 0.0528 & 0.1584 & - \\
\hline U-235 & ARA-9 & 2010 & 0.3077 & 0.0528 & 0.1584 & - \\
\hline U-235 & ARA-1 & 2011 & 0.1014 & 0.14 & 0.42 & $\mathrm{U}$ \\
\hline U-235 & ARA-14 & 2011 & -0.06507 & 0.101 & 0.303 & $\mathrm{U}$ \\
\hline
\end{tabular}


Table C-1. (continued).

\begin{tabular}{|c|c|c|c|c|c|c|}
\hline Radionuclide & $\begin{array}{c}\text { Sample } \\
\text { Location }\end{array}$ & Date & $\begin{array}{c}\text { Concentrations } \\
(\mathrm{pCi} / \mathrm{g})\end{array}$ & $\begin{array}{l}\text { Uncertainty } \\
(\mathrm{pCi} / \mathrm{g})\end{array}$ & $\begin{array}{c}\text { MDA } \\
(\mathrm{pCi} / \mathrm{g})\end{array}$ & Flag \\
\hline U-235 & ARA-16 & 2011 & -0.01462 & 0.208 & 0.624 & $\mathrm{U}$ \\
\hline U-235 & ARA-2 & 2011 & -0.004737 & 0.111 & 0.333 & $\mathrm{U}$ \\
\hline U-235 & ARA-24 & 2011 & 0.06232 & 0.139 & 0.417 & $\mathrm{U}$ \\
\hline U-235 & ARA-28 & 2011 & 0.1959 & 0.174 & 0.522 & $\mathrm{U}$ \\
\hline U-235 & ARA-29 & 2011 & -0.004242 & 0.113 & 0.339 & $\mathrm{U}$ \\
\hline U-235 & ARA-31 & 2011 & 0.07835 & 0.18 & 0.54 & $\mathrm{U}$ \\
\hline U-235 & ARA-32 & 2011 & -0.09388 & 0.195 & 0.585 & $\mathrm{U}$ \\
\hline $\mathrm{U}-235$ & ARA-34 & 2011 & 0.1824 & 0.176 & 0.528 & $\mathrm{U}$ \\
\hline $\mathrm{U}-235$ & ARA-38 & 2011 & -0.01501 & 0.167 & 0.501 & $\mathrm{U}$ \\
\hline U-235 & ARA-4 & 2011 & -0.03097 & 0.106 & 0.318 & $\mathrm{U}$ \\
\hline $\mathrm{U}-235$ & ARA-42 & 2011 & 0.04697 & 0.18 & 0.54 & $\mathrm{U}$ \\
\hline $\mathrm{U}-235$ & ARA-43 & 2011 & -0.1353 & 0.144 & 0.432 & $\mathrm{U}$ \\
\hline $\mathrm{U}-235$ & ARA-47 & 2011 & 0.1152 & 0.16 & 0.48 & $\mathrm{U}$ \\
\hline $\mathrm{U}-235$ & ARA-50 & 2011 & 0.02772 & 0.13 & 0.39 & $\mathrm{U}$ \\
\hline $\mathrm{U}-235$ & ARA-51 & 2011 & -0.1392 & 0.184 & 0.552 & $\mathrm{U}$ \\
\hline $\mathrm{U}-235$ & ARA-62 & 2011 & 0.08036 & 0.151 & 0.453 & $\mathrm{U}$ \\
\hline $\mathrm{U}-235$ & ARA-65 & 2011 & -0.0565 & 0.134 & 0.402 & $\mathrm{U}$ \\
\hline U-235 & ARA-74 & 2011 & 0.1772 & 0.167 & 0.501 & $\mathrm{U}$ \\
\hline U-235 & ARA-75 & 2011 & 0.1721 & 0.18 & 0.54 & $\mathrm{U}$ \\
\hline U-235 & ARA-77 & 2011 & 0.04868 & 0.168 & 0.504 & $\mathrm{U}$ \\
\hline U-235 & ARA-9 & 2011 & 0.08159 & 0.102 & 0.306 & $\mathrm{U}$ \\
\hline U-235 & ARA-11 & 2012 & 0.03717 & 0.134 & 0.402 & $\mathrm{U}$ \\
\hline U-235 & ARA-16 & 2012 & 0.1813 & 0.202 & 0.606 & $\mathrm{U}$ \\
\hline U-235 & ARA-31 & 2012 & -0.02503 & 0.146 & 0.438 & $\mathrm{U}$ \\
\hline U-235 & ARA-32 & 2012 & -0.0347 & 0.173 & 0.519 & $\mathrm{U}$ \\
\hline U-235 & ARA-48 & 2012 & 0.04045 & 0.157 & 0.471 & $\mathrm{U}$ \\
\hline U-235 & ARA-53 & 2012 & 0.01952 & 0.139 & 0.417 & $\mathrm{U}$ \\
\hline U-235 & ARA-57 & 2012 & 0.06745 & 0.165 & 0.495 & $\mathrm{U}$ \\
\hline U-235 & ARA-58 & 2012 & 0.06569 & 0.138 & 0.414 & $\mathrm{U}$ \\
\hline U-235 & ARA-59 & 2012 & -0.0213 & 0.139 & 0.417 & $\mathrm{U}$ \\
\hline U-235 & ARA-60 & 2012 & -0.01049 & 0.146 & 0.438 & $\mathrm{U}$ \\
\hline U-235 & ARA-62 & 2012 & -0.01719 & 0.167 & 0.501 & $\mathrm{U}$ \\
\hline U-235 & ARA-65 & 2012 & 0.1006 & 0.137 & 0.411 & $\mathrm{U}$ \\
\hline U-235 & ARA-73 & 2012 & 0.05427 & 0.14 & 0.42 & $\mathrm{U}$ \\
\hline U-235 & ARA-31 & 2013 & 0.2439 & 0.0753 & 0.226 & - \\
\hline U-235 & ARA-32 & 2013 & 0.04475 & 0.0967 & 0.29 & $\mathrm{U}$ \\
\hline U-235 & ARA-33 & 2013 & 0.2517 & 0.0727 & 0.218 & - \\
\hline
\end{tabular}


Table C-1. (continued).

\begin{tabular}{|c|c|c|c|c|c|c|}
\hline Radionuclide & $\begin{array}{c}\text { Sample } \\
\text { Location }\end{array}$ & Date & $\begin{array}{c}\text { Concentrations } \\
(\mathrm{pCi} / \mathrm{g})\end{array}$ & $\begin{array}{l}\text { Uncertainty } \\
(\mathrm{pCi} / \mathrm{g})\end{array}$ & $\begin{array}{c}\text { MDA } \\
(\mathrm{pCi} / \mathrm{g})\end{array}$ & Flag \\
\hline U-235 & ARA-40 & 2013 & 0.2229 & 0.0887 & 0.266 & $\mathrm{U}$ \\
\hline U-235 & ARA-48 & 2013 & -0.01813 & 0.0917 & 0.275 & $\mathrm{U}$ \\
\hline U-235 & ARA-65 & 2013 & 0.05423 & 0.099 & 0.297 & $\mathrm{U}$ \\
\hline U-235 & $\begin{array}{c}2 \text { INCH AIR } \\
\text { - ARA }\end{array}$ & 2014 & 0.157 & 0.221 & 0.663 & $\mathrm{U}$ \\
\hline $\mathrm{U}-235$ & ARA-1 & 2014 & 0.1201 & 0.147 & 0.441 & $\mathrm{U}$ \\
\hline $\mathrm{U}-235$ & ARA-24 & 2014 & -0.07724 & 0.132 & 0.396 & $\mathrm{U}$ \\
\hline U-235 & ARA-31 & 2014 & -0.2694 & 0.201 & 0.603 & $\mathrm{U}$ \\
\hline U-235 & ARA-32 & 2014 & -0.05136 & 0.149 & 0.447 & $\mathrm{U}$ \\
\hline $\mathrm{U}-235$ & ARA-65 & 2014 & -0.1635 & 0.197 & 0.591 & $\mathrm{U}$ \\
\hline $\mathrm{U}-235$ & ARA-71 & 2014 & 0.01738 & 0.113 & 0.339 & $\mathrm{U}$ \\
\hline $\mathrm{U}-238$ & $0^{\circ} 1000^{\prime}$ & 1985 & 1.04 & 0.04 & 0.12 & - \\
\hline U-238 & $22.5^{\circ} 2500^{\prime}$ & 1985 & 1.06 & 0.04 & 0.12 & - \\
\hline U-238 & $67.5^{\circ} 2000^{\prime}$ & 1985 & 1.01 & 0.04 & 0.12 & - \\
\hline $\mathrm{U}-238$ & $67.5^{\circ} 500^{\prime}$ & 1985 & 0.84 & 0.03 & 0.09 & - \\
\hline $\mathrm{U}-238$ & ARA-0 & 2006 & 74.69 & 13.05 & 39.15 & - \\
\hline U-238 & ARA-1 & 2006 & 4.104 & 0.575 & 1.725 & - \\
\hline U-238 & ARA-10 & 2006 & 40.84 & 7.25 & 21.75 & - \\
\hline U-238 & ARA-11 & 2006 & 11.41 & 13.6 & 40.8 & $\mathrm{U}$ \\
\hline $\mathrm{U}-238$ & ARA-12 & 2006 & 123.2 & 14.6 & 43.8 & - \\
\hline U-238 & ARA-13 & 2006 & 13.34 & 2.87 & 8.61 & - \\
\hline U-238 & ARA-14 & 2006 & 4.105 & 27.9 & 83.7 & $\mathrm{U}$ \\
\hline U-238 & ARA-15 & 2006 & 14.17 & 17.2 & 51.6 & $\mathrm{U}$ \\
\hline $\mathrm{U}-238$ & ARA-16 & 2006 & 11.68 & 16.45 & 49.35 & $\mathrm{U}$ \\
\hline U-238 & ARA-17 & 2006 & 36.31 & 16.45 & 49.35 & $\mathrm{U}$ \\
\hline U-238 & ARA-18 & 2006 & 17.56 & 2.725 & 8.175 & - \\
\hline U-238 & ARA-19 & 2006 & 39.49 & 13.45 & 40.35 & $\mathrm{U}$ \\
\hline U-238 & ARA-2 & 2006 & 21.73 & 5.9 & 17.7 & - \\
\hline U-238 & ARA-20 & 2006 & 34.8 & 5.3 & 15.9 & - \\
\hline U-238 & ARA-21 & 2006 & 72.06 & 18.9 & 56.7 & - \\
\hline U-238 & ARA-22 & 2006 & 32.15 & 21.1 & 63.3 & $\mathrm{U}$ \\
\hline $\mathrm{U}-238$ & ARA-23 & 2006 & 2.708 & 15.5 & 46.5 & $\mathrm{U}$ \\
\hline U-238 & ARA-24 & 2006 & 39.64 & 34.45 & 103.35 & $\mathrm{U}$ \\
\hline U-238 & ARA-26 & 2006 & 19.06 & 4.525 & 13.575 & - \\
\hline U-238 & ARA-27 & 2006 & 56.39 & 24.35 & 73.05 & $\mathrm{U}$ \\
\hline U-238 & ARA-28 & 2006 & 13.61 & 22.8 & 68.4 & $\mathrm{U}$ \\
\hline U-238 & ARA-29 & 2006 & 11.51 & 38.85 & 116.55 & $\mathrm{U}$ \\
\hline
\end{tabular}


Table C-1. (continued).

\begin{tabular}{|c|c|c|c|c|c|c|}
\hline Radionuclide & $\begin{array}{c}\text { Sample } \\
\text { Location }\end{array}$ & Date & $\begin{array}{c}\text { Concentrations } \\
(\mathrm{pCi} / \mathrm{g})\end{array}$ & $\begin{array}{c}\text { Uncertainty } \\
(\mathrm{pCi} / \mathrm{g})\end{array}$ & $\begin{array}{c}\mathrm{MDA} \\
(\mathrm{pCi} / \mathrm{g})\end{array}$ & Flag \\
\hline U-238 & ARA-3 & 2006 & 29.33 & 5.1 & 15.3 & - \\
\hline U-238 & ARA-30 & 2006 & 37.61 & 4.6 & 13.8 & - \\
\hline $\mathrm{U}-238$ & ARA-31 & 2006 & 13.02 & 2.335 & 7.005 & - \\
\hline $\mathrm{U}-238$ & ARA-32 & 2006 & 18.77 & 3.065 & 9.195 & - \\
\hline U-238 & ARA-33 & 2006 & 17.44 & 2.805 & 8.415 & - \\
\hline U-238 & ARA-34 & 2006 & 25.77 & 3.25 & 9.75 & - \\
\hline $\mathrm{U}-238$ & ARA-35 & 2006 & 21.31 & 19.25 & 57.75 & $\mathrm{U}$ \\
\hline $\mathrm{U}-238$ & ARA-36 & 2006 & 26.73 & 18.4 & 55.2 & $\mathrm{U}$ \\
\hline $\mathrm{U}-238$ & ARA-37 & 2006 & 9.371 & 34.1 & 102.3 & $\mathrm{U}$ \\
\hline $\mathrm{U}-238$ & ARA-38 & 2006 & 6.389 & 33.65 & 100.95 & $\mathrm{U}$ \\
\hline $\mathrm{U}-238$ & ARA-39 & 2006 & 23.42 & 3.44 & 10.32 & - \\
\hline $\mathrm{U}-238$ & ARA-4 & 2006 & 9.425 & 2.08 & 6.24 & - \\
\hline $\mathrm{U}-238$ & ARA-40 & 2006 & 7.211 & 1.495 & 4.485 & - \\
\hline $\mathrm{U}-238$ & ARA-41 & 2006 & 22.73 & 2.905 & 8.715 & - \\
\hline $\mathrm{U}-238$ & ARA-42 & 2006 & 9.002 & 3.27 & 9.81 & $\mathrm{U}$ \\
\hline $\mathrm{U}-238$ & ARA-43 & 2006 & 10.25 & 2.165 & 6.495 & - \\
\hline $\mathrm{U}-238$ & ARA-44 & 2006 & 15.43 & 3.625 & 10.875 & - \\
\hline $\mathrm{U}-238$ & ARA-45 & 2006 & 20.77 & 20.25 & 60.75 & $\mathrm{U}$ \\
\hline U-238 & ARA-46 & 2006 & 33.98 & 19.85 & 59.55 & $\mathrm{U}$ \\
\hline U-238 & ARA-48 & 2006 & 10.25 & 2.165 & 6.495 & - \\
\hline U-238 & ARA-49 & 2006 & 18.84 & 2.385 & 7.155 & - \\
\hline U-238 & ARA-5 & 2006 & 7.416 & 2.165 & 6.495 & - \\
\hline U-238 & ARA-50 & 2006 & 11.74 & 1.525 & 4.575 & - \\
\hline $\mathrm{U}-238$ & ARA-51 & 2006 & 14.23 & 3.575 & 10.725 & - \\
\hline U-238 & ARA-52 & 2006 & 46.64 & 20.75 & 62.25 & $\mathrm{U}$ \\
\hline U-238 & ARA-53 & 2006 & 24.3 & 2.755 & 8.265 & - \\
\hline $\mathrm{U}-238$ & ARA-54 & 2006 & 10.72 & 2.15 & 6.45 & - \\
\hline U-238 & ARA-55 & 2006 & 15.45 & 2.37 & 7.11 & - \\
\hline U-238 & ARA-56 & 2006 & 11.72 & 1.755 & 5.265 & - \\
\hline U-238 & ARA-57 & 2006 & 6.709 & 2.11 & 6.33 & - \\
\hline U-238 & ARA-58 & 2006 & 14.65 & 2.835 & 8.505 & - \\
\hline U-238 & ARA-59 & 2006 & 12.94 & 2.02 & 6.06 & - \\
\hline U-238 & ARA-6 & 2006 & 56.14 & 8.75 & 26.25 & - \\
\hline U-238 & ARA-60 & 2006 & 5.323 & 1.385 & 4.155 & - \\
\hline $\mathrm{U}-238$ & ARA-61 & 2006 & 26.73 & 3.08 & 9.24 & - \\
\hline U-238 & ARA-62 & 2006 & 28.64 & 4.34 & 13.02 & - \\
\hline U-238 & ARA-63 & 2006 & 0.05039 & 0.0437 & 0.1311 & $\mathrm{U}$ \\
\hline
\end{tabular}


Table C-1. (continued).

\begin{tabular}{|c|c|c|c|c|c|c|}
\hline Radionuclide & $\begin{array}{c}\text { Sample } \\
\text { Location }\end{array}$ & Date & $\begin{array}{c}\text { Concentrations } \\
(\mathrm{pCi} / \mathrm{g})\end{array}$ & $\begin{array}{c}\text { Uncertainty } \\
(\mathrm{pCi} / \mathrm{g})\end{array}$ & $\begin{array}{c}\mathrm{MDA} \\
(\mathrm{pCi} / \mathrm{g})\end{array}$ & Flag \\
\hline U-238 & ARA-65 & 2006 & 7.193 & 1.645 & 4.935 & - \\
\hline U-238 & ARA-66 & 2006 & 33.41 & 4.5 & 13.5 & - \\
\hline $\mathrm{U}-238$ & ARA-67 & 2006 & 40.85 & 4.6 & 13.8 & - \\
\hline $\mathrm{U}-238$ & ARA-68 & 2006 & 2.177 & 0.84 & 2.52 & $\mathrm{U}$ \\
\hline U-238 & ARA-69 & 2006 & 27 & 4.485 & 13.455 & - \\
\hline U-238 & ARA-7 & 2006 & 101.8 & 17.15 & 51.45 & - \\
\hline $\mathrm{U}-238$ & ARA-70 & 2006 & 23.29 & 3.005 & 9.015 & - \\
\hline $\mathrm{U}-238$ & ARA-71 & 2006 & 15.56 & 3.88 & 11.64 & - \\
\hline $\mathrm{U}-238$ & ARA-72 & 2006 & 13.13 & 3.53 & 10.59 & - \\
\hline $\mathrm{U}-238$ & ARA-73 & 2006 & 13.17 & 2.285 & 6.855 & - \\
\hline $\mathrm{U}-238$ & ARA-74 & 2006 & 10.37 & 2.495 & 7.485 & - \\
\hline $\mathrm{U}-238$ & ARA-75 & 2006 & 5.368 & 0.91 & 2.73 & - \\
\hline $\mathrm{U}-238$ & ARA-76 & 2006 & 20.51 & 3.22 & 9.66 & - \\
\hline $\mathrm{U}-238$ & ARA-77 & 2006 & 22.84 & 3.17 & 9.51 & - \\
\hline $\mathrm{U}-238$ & ARA-8 & 2006 & 22.26 & 3.09 & 9.27 & - \\
\hline $\mathrm{U}-238$ & ARA-9 & 2006 & 47.79 & 6.35 & 19.05 & - \\
\hline $\mathrm{U}-238$ & ARA-0 & 2007 & 1.962 & 0.4145 & 1.2435 & - \\
\hline $\mathrm{U}-238$ & ARA-1 & 2007 & 0 & 1.11 & 3.33 & $\mathrm{U}$ \\
\hline U-238 & ARA-10 & 2007 & 0.0531 & 4.34 & 13.02 & $\mathrm{U}$ \\
\hline U-238 & ARA-11 & 2007 & 10.18 & 1.405 & 4.215 & - \\
\hline U-238 & ARA-12 & 2007 & 10.22 & 1.595 & 4.785 & - \\
\hline U-238 & ARA-13 & 2007 & 0.5049 & 4.21 & 12.63 & $\mathrm{U}$ \\
\hline U-238 & ARA-14 & 2007 & 0.664 & 0.414 & 1.242 & $\mathrm{U}$ \\
\hline $\mathrm{U}-238$ & ARA-15 & 2007 & 1.756 & 0.2715 & 0.8145 & - \\
\hline U-238 & ARA-17 & 2007 & 3.929 & 0.54 & 1.62 & - \\
\hline U-238 & ARA-18 & 2007 & 238.5 & 8.75 & 26.25 & - \\
\hline $\mathrm{U}-238$ & ARA-19 & 2007 & 4.543 & 0.635 & 1.905 & - \\
\hline U-238 & ARA-2 & 2007 & 12.07 & 1.695 & 5.085 & - \\
\hline U-238 & ARA-20 & 2007 & 0.2986 & 6.72 & 20.16 & $\mathrm{U}$ \\
\hline U-238 & ARA-21 & 2007 & 2.87 & 0.54 & 1.62 & - \\
\hline U-238 & ARA-22 & 2007 & 0 & 3.66 & 10.98 & $\mathrm{U}$ \\
\hline U-238 & ARA-23 & 2007 & 3.48 & 0.535 & 1.605 & - \\
\hline U-238 & ARA-24 & 2007 & 4.007 & 0.715 & 2.145 & - \\
\hline U-238 & ARA-25 & 2007 & 0.09051 & 7.07 & 21.21 & $\mathrm{U}$ \\
\hline $\mathrm{U}-238$ & ARA-26 & 2007 & 0 & 7.04 & 21.12 & $\mathrm{U}$ \\
\hline U-238 & ARA-27 & 2007 & 0 & 3.84 & 11.52 & $\mathrm{U}$ \\
\hline U-238 & ARA-28 & 2007 & 4.035 & 0.585 & 1.755 & - \\
\hline
\end{tabular}


Table C-1. (continued).

\begin{tabular}{|c|c|c|c|c|c|c|}
\hline Radionuclide & $\begin{array}{c}\text { Sample } \\
\text { Location }\end{array}$ & Date & $\begin{array}{c}\text { Concentrations } \\
(\mathrm{pCi} / \mathrm{g})\end{array}$ & $\begin{array}{l}\text { Uncertainty } \\
(\mathrm{pCi} / \mathrm{g})\end{array}$ & $\begin{array}{c}\text { MDA } \\
(\mathrm{pCi} / \mathrm{g})\end{array}$ & Flag \\
\hline U-238 & ARA-29 & 2007 & 3.417 & 0.4735 & 1.4205 & - \\
\hline U-238 & ARA-3 & 2007 & 0 & 6.28 & 18.84 & $\mathrm{U}$ \\
\hline $\mathrm{U}-238$ & ARA-30 & 2007 & 0 & 6.21 & 18.63 & $\mathrm{U}$ \\
\hline $\mathrm{U}-238$ & ARA-31 & 2007 & 3.395 & 0.525 & 1.575 & - \\
\hline U-238 & ARA-32 & 2007 & 5.96 & 0.795 & 2.385 & - \\
\hline U-238 & ARA-33 & 2007 & 0.1691 & 5.88 & 17.64 & $\mathrm{U}$ \\
\hline $\mathrm{U}-238$ & ARA-34 & 2007 & 0 & 5.5 & 16.5 & $\mathrm{U}$ \\
\hline $\mathrm{U}-238$ & ARA-35 & 2007 & 0 & 6.26 & 18.78 & $\mathrm{U}$ \\
\hline $\mathrm{U}-238$ & ARA-36 & 2007 & 1.273 & 0.3605 & 1.0815 & - \\
\hline $\mathrm{U}-238$ & ARA-37 & 2007 & 4.651 & 0.605 & 1.815 & - \\
\hline $\mathrm{U}-238$ & ARA-38 & 2007 & 3.992 & 0.4955 & 1.4865 & - \\
\hline $\mathrm{U}-238$ & ARA-39 & 2007 & 0 & 4.74 & 14.22 & $\mathrm{U}$ \\
\hline $\mathrm{U}-238$ & ARA-4 & 2007 & 2.174 & 0.4565 & 1.3695 & - \\
\hline $\mathrm{U}-238$ & ARA-41 & 2007 & 43.04 & 22.1 & 66.3 & $\mathrm{U}$ \\
\hline $\mathrm{U}-238$ & ARA-42 & 2007 & 1.948 & 0.515 & 1.545 & - \\
\hline $\mathrm{U}-238$ & ARA-43 & 2007 & 1.258 & 0.3735 & 1.1205 & - \\
\hline $\mathrm{U}-238$ & ARA-44 & 2007 & 156.7 & 5.89 & 17.67 & - \\
\hline $\mathrm{U}-238$ & ARA-45 & 2007 & 25.49 & 3.84 & 11.52 & - \\
\hline U-238 & ARA-46 & 2007 & 1.92 & 0.423 & 1.269 & - \\
\hline U-238 & ARA-47 & 2007 & 3.111 & 0.4335 & 1.3005 & - \\
\hline U-238 & ARA-48 & 2007 & 1.035 & 0.452 & 1.356 & $\mathrm{U}$ \\
\hline U-238 & ARA-49 & 2007 & 2.849 & 0.4265 & 1.2795 & - \\
\hline U-238 & ARA-5 & 2007 & 2.898 & 0.535 & 1.605 & - \\
\hline $\mathrm{U}-238$ & ARA-50 & 2007 & 6.735 & 1.32 & 3.96 & - \\
\hline U-238 & ARA-51 & 2007 & 2.745 & 0.775 & 2.325 & - \\
\hline U-238 & ARA-52 & 2007 & 4.756 & 0.6 & 1.8 & - \\
\hline $\mathrm{U}-238$ & ARA-53 & 2007 & 137.5 & 5.57 & 16.71 & - \\
\hline U-238 & ARA-54 & 2007 & 0.0795 & 5.16 & 15.48 & $\mathrm{U}$ \\
\hline U-238 & ARA-55 & 2007 & 0 & 5.2 & 15.6 & $\mathrm{U}$ \\
\hline U-238 & ARA-56 & 2007 & 2.821 & 0.4385 & 1.3155 & - \\
\hline U-238 & ARA-57 & 2007 & 47.28 & 7.85 & 23.55 & - \\
\hline U-238 & ARA-58 & 2007 & 1.568 & 0.371 & 1.113 & - \\
\hline U-238 & ARA-59 & 2007 & 1.205 & 0.4585 & 1.3755 & $\mathrm{U}$ \\
\hline U-238 & ARA-6 & 2007 & 2.371 & 0.414 & 1.242 & - \\
\hline $\mathrm{U}-238$ & ARA-60 & 2007 & 3.038 & 0.66 & 1.98 & - \\
\hline U-238 & ARA-61 & 2007 & 0.01376 & 6.01 & 18.03 & $\mathrm{U}$ \\
\hline U-238 & ARA-62 & 2007 & 0 & 6.85 & 20.55 & $\mathrm{U}$ \\
\hline
\end{tabular}


Table C-1. (continued).

\begin{tabular}{|c|c|c|c|c|c|c|}
\hline Radionuclide & $\begin{array}{c}\text { Sample } \\
\text { Location }\end{array}$ & Date & $\begin{array}{c}\text { Concentrations } \\
(\mathrm{pCi} / \mathrm{g})\end{array}$ & $\begin{array}{l}\text { Uncertainty } \\
(\mathrm{pCi} / \mathrm{g})\end{array}$ & $\begin{array}{c}\text { MDA } \\
(\mathrm{pCi} / \mathrm{g})\end{array}$ & Flag \\
\hline U-238 & ARA-63 & 2007 & 0.142 & 6.36 & 19.08 & $\mathrm{U}$ \\
\hline U-238 & ARA-65 & 2007 & 9.811 & 1.655 & 4.965 & - \\
\hline $\mathrm{U}-238$ & ARA-66 & 2007 & 0 & 6.43 & 19.29 & $\mathrm{U}$ \\
\hline $\mathrm{U}-238$ & ARA-67 & 2007 & 0.03178 & 5.77 & 17.31 & $\mathrm{U}$ \\
\hline U-238 & ARA-68 & 2007 & 0 & 5.86 & 17.58 & $\mathrm{U}$ \\
\hline U-238 & ARA-69 & 2007 & 0.9461 & 6.51 & 19.53 & $\mathrm{U}$ \\
\hline $\mathrm{U}-238$ & ARA-7 & 2007 & 6.417 & 0.825 & 2.475 & - \\
\hline $\mathrm{U}-238$ & ARA-70 & 2007 & 0.08092 & 6.21 & 18.63 & $\mathrm{U}$ \\
\hline $\mathrm{U}-238$ & ARA-71 & 2007 & 0.08097 & 6.34 & 19.02 & $\mathrm{U}$ \\
\hline $\mathrm{U}-238$ & ARA-72 & 2007 & 148.5 & 5.99 & 17.97 & - \\
\hline $\mathrm{U}-238$ & ARA-73 & 2007 & 2.594 & 0.555 & 1.665 & - \\
\hline $\mathrm{U}-238$ & ARA-74 & 2007 & 3.007 & 0.466 & 1.398 & - \\
\hline $\mathrm{U}-238$ & ARA-75 & 2007 & 1.596 & 0.4005 & 1.2015 & - \\
\hline $\mathrm{U}-238$ & ARA-76 & 2007 & 0.1228 & 8.1 & 24.3 & $\mathrm{U}$ \\
\hline $\mathrm{U}-238$ & ARA-77 & 2007 & 1.499 & 0.3855 & 1.1565 & - \\
\hline $\mathrm{U}-238$ & ARA-8 & 2007 & 0 & 7.08 & 21.24 & $\mathrm{U}$ \\
\hline $\mathrm{U}-238$ & ARA-9 & 2007 & 0.1887 & 4.88 & 14.64 & $\mathrm{U}$ \\
\hline $\mathrm{U}-238$ & ARA-10 & 2009 & -5.131 & 5.85 & 17.55 & $\mathrm{U}$ \\
\hline $\mathrm{U}-238$ & ARA-12 & 2009 & 141.2 & 48.3 & 144.9 & $\mathrm{U}$ \\
\hline U-238 & ARA-13 & 2009 & -5.523 & 7.2 & 21.6 & $\mathrm{U}$ \\
\hline U-238 & ARA-18 & 2009 & 3.633 & 6 & 18 & $\mathrm{U}$ \\
\hline U-238 & ARA-20 & 2009 & 3.621 & 7.35 & 22.05 & $\mathrm{U}$ \\
\hline U-238 & ARA-21 & 2009 & -10.28 & 8.45 & 25.35 & $\mathrm{U}$ \\
\hline $\mathrm{U}-238$ & ARA-26 & 2009 & 0.5363 & 7.6 & 22.8 & $\mathrm{U}$ \\
\hline $\mathrm{U}-238$ & ARA-27 & 2009 & 5.46 & 4.045 & 12.135 & $\mathrm{U}$ \\
\hline $\mathrm{U}-238$ & ARA-3 & 2009 & -0.1902 & 24.6 & 73.8 & $\mathrm{U}$ \\
\hline $\mathrm{U}-238$ & ARA-30 & 2009 & 216.9 & 8.5 & 25.5 & - \\
\hline U-238 & ARA-35 & 2009 & -1.5 & 8.8 & 26.4 & $\mathrm{U}$ \\
\hline U-238 & ARA-39 & 2009 & 3.96 & 13.8 & 41.4 & $\mathrm{U}$ \\
\hline $\mathrm{U}-238$ & ARA-44 & 2009 & -0.00009768 & 8.95 & 26.85 & $\mathrm{U}$ \\
\hline U-238 & ARA-47 & 2009 & 91.14 & 7.25 & 21.75 & - \\
\hline U-238 & ARA-61 & 2009 & 189.6 & 8.35 & 25.05 & - \\
\hline U-238 & ARA-62 & 2009 & -33.6 & 12.45 & 37.35 & $\mathrm{U}$ \\
\hline U-238 & ARA-63 & 2009 & -2.881 & 15.6 & 46.8 & $\mathrm{U}$ \\
\hline $\mathrm{U}-238$ & ARA-67 & 2009 & -3.669 & 5.9 & 17.7 & $\mathrm{U}$ \\
\hline U-238 & ARA-68 & 2009 & -8.346 & 9.3 & 27.9 & $\mathrm{U}$ \\
\hline U-238 & ARA-69 & 2009 & 3.409 & 16.6 & 49.8 & $\mathrm{U}$ \\
\hline
\end{tabular}


Table C-1. (continued).

\begin{tabular}{|c|c|c|c|c|c|c|}
\hline Radionuclide & $\begin{array}{c}\text { Sample } \\
\text { Location }\end{array}$ & Date & $\begin{array}{c}\text { Concentrations } \\
(\mathrm{pCi} / \mathrm{g})\end{array}$ & $\begin{array}{c}\text { Uncertainty } \\
(\mathrm{pCi} / \mathrm{g})\end{array}$ & $\begin{array}{c}\mathrm{MDA} \\
(\mathrm{pCi} / \mathrm{g})\end{array}$ & Flag \\
\hline U-238 & ARA-70 & 2009 & 76.38 & 7.75 & 23.25 & - \\
\hline U-238 & ARA-71 & 2009 & -8.285 & 9.65 & 28.95 & $\mathrm{U}$ \\
\hline $\mathrm{U}-238$ & ARA-72 & 2009 & 5.764 & 6.05 & 18.15 & $\mathrm{U}$ \\
\hline U-238 & ARA-74 & 2009 & -4.158 & 9.15 & 27.45 & $\mathrm{U}$ \\
\hline U-238 & ARA-75 & 2009 & -0.2417 & 7.9 & 23.7 & $\mathrm{U}$ \\
\hline U-238 & ARA-77 & 2009 & -12.23 & 8.25 & 24.75 & $\mathrm{U}$ \\
\hline $\mathrm{U}-238$ & ARA-8 & 2009 & -0.6352 & 7.5 & 22.5 & $\mathrm{U}$ \\
\hline $\mathrm{U}-238$ & ARA-0 & 2010 & 4.936 & 4.09 & 12.27 & $\mathrm{U}$ \\
\hline $\mathrm{U}-238$ & ARA-11 & 2010 & 36.14 & 23.2 & 69.6 & $\mathrm{U}$ \\
\hline $\mathrm{U}-238$ & ARA-12 & 2010 & 34.4 & 21.8 & 65.4 & $\mathrm{U}$ \\
\hline $\mathrm{U}-238$ & ARA-14 & 2010 & 40.22 & 2.78 & 8.34 & - \\
\hline $\mathrm{U}-238$ & ARA-16 & 2010 & 43.17 & 22.7 & 68.1 & $\mathrm{U}$ \\
\hline $\mathrm{U}-238$ & ARA-19 & 2010 & 41.49 & 2.86 & 8.58 & - \\
\hline $\mathrm{U}-238$ & ARA-2 & 2010 & 4.877 & 3.03 & 9.09 & $\mathrm{U}$ \\
\hline $\mathrm{U}-238$ & ARA-22 & 2010 & 41.46 & 2.85 & 8.55 & - \\
\hline $\mathrm{U}-238$ & ARA-27 & 2010 & 25.69 & 21.4 & 64.2 & $\mathrm{U}$ \\
\hline $\mathrm{U}-238$ & ARA-28 & 2010 & 48.29 & 3.07 & 9.21 & - \\
\hline $\mathrm{U}-238$ & ARA-35 & 2010 & 3.533 & 4.13 & 12.39 & $\mathrm{U}$ \\
\hline U-238 & ARA-50 & 2010 & 92.45 & 24.4 & 73.2 & - \\
\hline U-238 & ARA-52 & 2010 & 17.83 & 13 & 39 & $\mathrm{U}$ \\
\hline U-238 & ARA-53 & 2010 & 45.55 & 2.83 & 8.49 & \\
\hline U-238 & ARA-7 & 2010 & 29.5 & 15.1 & 45.3 & $\mathrm{U}$ \\
\hline U-238 & ARA-1 & 2011 & 0.9351 & 0.535 & 1.605 & $\mathrm{U}$ \\
\hline $\mathrm{U}-238$ & ARA-14 & 2011 & 0.5789 & 0.401 & 1.203 & $\mathrm{U}$ \\
\hline U-238 & ARA-16 & 2011 & 0.8651 & 0.571 & 1.713 & $\mathrm{U}$ \\
\hline U-238 & ARA-2 & 2011 & 0.852 & 0.453 & 1.359 & $\mathrm{U}$ \\
\hline $\mathrm{U}-238$ & ARA-24 & 2011 & 0.9776 & 0.509 & 1.527 & $\mathrm{U}$ \\
\hline U-238 & ARA-28 & 2011 & 0.928 & 0.791 & 2.373 & $\mathrm{U}$ \\
\hline U-238 & ARA-29 & 2011 & 0.5056 & 0.413 & 1.239 & $\mathrm{U}$ \\
\hline U-238 & ARA-31 & 2011 & 1.228 & 0.791 & 2.373 & $\mathrm{U}$ \\
\hline U-238 & ARA-32 & 2011 & 4.21 & 1.04 & 3.12 & - \\
\hline U-238 & ARA-34 & 2011 & 1.262 & 0.822 & 2.466 & $\mathrm{U}$ \\
\hline U-238 & ARA-38 & 2011 & 1.084 & 0.901 & 2.703 & $\mathrm{U}$ \\
\hline U-238 & ARA-4 & 2011 & 0.4905 & 0.39 & 1.17 & $\mathrm{U}$ \\
\hline $\mathrm{U}-238$ & ARA-42 & 2011 & 0.8891 & 0.592 & 1.776 & $\mathrm{U}$ \\
\hline U-238 & ARA-43 & 2011 & 1.018 & 0.714 & 2.142 & $\mathrm{U}$ \\
\hline U-238 & ARA-47 & 2011 & 1.006 & 0.796 & 2.388 & $\mathrm{U}$ \\
\hline
\end{tabular}


Table C-1. (continued).

\begin{tabular}{|c|c|c|c|c|c|c|}
\hline Radionuclide & $\begin{array}{c}\text { Sample } \\
\text { Location }\end{array}$ & Date & $\begin{array}{c}\text { Concentrations } \\
(\mathrm{pCi} / \mathrm{g})\end{array}$ & $\begin{array}{l}\text { Uncertainty } \\
(\mathrm{pCi} / \mathrm{g})\end{array}$ & $\begin{array}{c}\text { MDA } \\
(\mathrm{pCi} / \mathrm{g})\end{array}$ & Flag \\
\hline U-238 & ARA-50 & 2011 & 1.086 & 0.686 & 2.058 & $\mathrm{U}$ \\
\hline U-238 & ARA-51 & 2011 & 1.494 & 0.969 & 2.907 & $\mathrm{U}$ \\
\hline $\mathrm{U}-238$ & ARA-62 & 2011 & 0.9015 & 0.536 & 1.608 & $\mathrm{U}$ \\
\hline U-238 & ARA-65 & 2011 & 3.237 & 0.692 & 2.076 & - \\
\hline U-238 & ARA-71 & 2011 & 1.43 & 56.9 & 170.7 & $\mathrm{U}$ \\
\hline U-238 & ARA-74 & 2011 & 1.595 & 0.758 & 2.274 & $\mathrm{U}$ \\
\hline $\mathrm{U}-238$ & ARA-75 & 2011 & 1.352 & 0.843 & 2.529 & $\mathrm{U}$ \\
\hline $\mathrm{U}-238$ & ARA-77 & 2011 & 1.086 & 0.757 & 2.271 & $\mathrm{U}$ \\
\hline $\mathrm{U}-238$ & ARA-9 & 2011 & 0.1793 & 0.384 & 1.152 & $\mathrm{U}$ \\
\hline $\mathrm{U}-238$ & ARA-11 & 2012 & 0.7769 & 0.798 & 2.394 & $\mathrm{U}$ \\
\hline $\mathrm{U}-238$ & ARA-16 & 2012 & 5.471 & 1.46 & 4.38 & - \\
\hline $\mathrm{U}-238$ & ARA-31 & 2012 & 0.7178 & 0.734 & 2.202 & $\mathrm{U}$ \\
\hline U-238 & ARA-32 & 2012 & 1.679 & 0.866 & 2.598 & $\mathrm{U}$ \\
\hline $\mathrm{U}-238$ & ARA-48 & 2012 & 1.512 & 0.823 & 2.469 & $\mathrm{U}$ \\
\hline $\mathrm{U}-238$ & ARA-53 & 2012 & 0.9301 & 0.69 & 2.07 & $\mathrm{U}$ \\
\hline $\mathrm{U}-238$ & ARA-57 & 2012 & 1.477 & 0.803 & 2.409 & $\mathrm{U}$ \\
\hline $\mathrm{U}-238$ & ARA-58 & 2012 & 1.003 & 0.799 & 2.397 & $\mathrm{U}$ \\
\hline $\mathrm{U}-238$ & ARA-59 & 2012 & 0.00496 & 0.682 & 2.046 & $\mathrm{U}$ \\
\hline $\mathrm{U}-238$ & ARA-60 & 2012 & 0.7222 & 0.758 & 2.274 & $\mathrm{U}$ \\
\hline U-238 & ARA-62 & 2012 & 0.9615 & 0.803 & 2.409 & $\mathrm{U}$ \\
\hline U-238 & ARA-65 & 2012 & 1.128 & 0.694 & 2.082 & $\mathrm{U}$ \\
\hline U-238 & ARA-73 & 2012 & 0.5955 & 0.739 & 2.217 & $\mathrm{U}$ \\
\hline U-238 & ARA-31 & 2013 & 3.114 & 1.323 & 3.97 & $\mathrm{U}$ \\
\hline U-238 & ARA-32 & 2013 & 0.813 & 0.387 & 1.16 & $\mathrm{U}$ \\
\hline $\mathrm{U}-238$ & ARA-33 & 2013 & 0.2643 & 1.15 & 3.45 & $\mathrm{U}$ \\
\hline $\mathrm{U}-238$ & ARA-40 & 2013 & 14.64 & 2.27 & 6.81 & - \\
\hline $\mathrm{U}-238$ & ARA-48 & 2013 & 0.6165 & 0.377 & 1.13 & $\mathrm{U}$ \\
\hline U-238 & ARA-65 & 2013 & 0.4007 & 0.332 & 0.995 & $\mathrm{U}$ \\
\hline U-238 & $\begin{array}{c}2 \text { INCH AIR } \\
\text { - ARA }\end{array}$ & 2014 & 0.8957 & 1.03 & 3.09 & $\mathrm{U}$ \\
\hline $\mathrm{U}-238$ & ARA-1 & 2014 & 1.213 & 0.767 & 2.301 & $\mathrm{U}$ \\
\hline $\mathrm{U}-238$ & ARA-24 & 2014 & 0.5667 & 0.672 & 2.016 & $\mathrm{U}$ \\
\hline U-238 & ARA-31 & 2014 & 0.8805 & 0.893 & 2.679 & $\mathrm{U}$ \\
\hline U-238 & ARA-32 & 2014 & 1.35 & 0.692 & 2.076 & $\mathrm{U}$ \\
\hline U-238 & ARA-65 & 2014 & 1.703 & 0.791 & 2.373 & $\mathrm{U}$ \\
\hline $\mathrm{U}-238$ & ARA-71 & 2014 & 1.92 & 0.576 & 1.728 & - \\
\hline
\end{tabular}


Table C-2. Radionuclides concentrations in surface soils associated with ATR.

\begin{tabular}{|c|c|c|c|c|c|c|}
\hline Radionuclide & $\begin{array}{c}\text { Sample } \\
\text { Location }\end{array}$ & Date & $\begin{array}{c}\text { Concentrations } \\
(\mathrm{pCi} / \mathrm{g})\end{array}$ & $\begin{array}{c}\text { Uncertainty } \\
(\mathrm{pCi} / \mathrm{g})\end{array}$ & $\begin{array}{c}\text { MDA } \\
(\mathrm{pCi} / \mathrm{g})\end{array}$ & Flag \\
\hline Am-241 & LP 0.3 & 1976 & 0.007 & 0.002 & 0.006 & - \\
\hline Am-241 & LP -1.2 & 1976 & 0.0047 & 0.0015 & 0.0045 & - \\
\hline Am-241 & LP 2.2 & 1976 & 0.0089 & 0.0017 & 0.0051 & - \\
\hline Am-241 & LP 3.1 & 1976 & 0.002 & 0.0001 & 0.0003 & - \\
\hline Am-241 & LP 3.2 & 1976 & 0.006 & 0.002 & 0.006 & - \\
\hline Am-241 & LP 3.3 & 1976 & 0.0029 & 0.0012 & 0.0036 & $\mathrm{U}$ \\
\hline Am-241 & LP 5.3 & 1976 & 0.0035 & 0.0013 & 0.0039 & $\mathrm{U}$ \\
\hline Am-241 & LP 7.2 & 1976 & 0.011 & 0.002 & 0.006 & - \\
\hline Am-241 & LP 8.2 & 1976 & 0.004 & 0.002 & 0.006 & $\mathrm{U}$ \\
\hline Am-241 & A3.4 & 1977 & 0.007 & 0.002 & 0.006 & - \\
\hline Am-241 & LP -2.2 & 1977 & 0.003 & 0.003 & 0.009 & $\mathrm{U}$ \\
\hline Am-241 & LP 3.4 & 1977 & 0.004 & 0.002 & 0.006 & $\mathrm{U}$ \\
\hline Am-241 & RTC.3 & 2011 & -0.03042 & 0.0912 & 0.2736 & $\mathrm{U}$ \\
\hline Am-241 & RTC-0.1 & 2011 & 0.06534 & 0.0633 & 0.1899 & $\mathrm{U}$ \\
\hline Am-241 & RTC-1.1 & 2011 & 0.07889 & 0.0969 & 0.2907 & $\mathrm{U}$ \\
\hline Am-241 & RTC-1.2 & 2011 & 0.4901 & 0.147 & 0.441 & - \\
\hline Am-241 & RTC1.3 & 2011 & 0.02979 & 0.0997 & 0.2991 & $\mathrm{U}$ \\
\hline Am-241 & RTC1.4 & 2011 & 0.05194 & 0.0597 & 0.1791 & $\mathrm{U}$ \\
\hline Am-241 & RTC-2.2 & 2011 & 0.005518 & 0.0643 & 0.1929 & $\mathrm{U}$ \\
\hline Am-241 & RTC2.3 & 2011 & 0.01608 & 0.0914 & 0.2742 & $\mathrm{U}$ \\
\hline Am-241 & RTC-6.2 & 2011 & 0.03812 & 0.114 & 0.342 & $\mathrm{U}$ \\
\hline Am-241 & RTC-6.4 & 2011 & 0.1187 & 0.101 & 0.303 & $\mathrm{U}$ \\
\hline Am-241 & RTC-8.2 & 2011 & -0.05202 & 0.0625 & 0.1875 & $\mathrm{U}$ \\
\hline Am-241 & RTC-A1.2 & 2011 & -0.002923 & 0.052 & 0.156 & $\mathrm{U}$ \\
\hline Am-241 & RTC-A1.3 & 2011 & -0.04303 & 0.0634 & 0.1902 & $\mathrm{U}$ \\
\hline Am-241 & RTC-A2.2 & 2011 & 0.003935 & 0.0637 & 0.1911 & $\mathrm{U}$ \\
\hline Am-241 & RTC-A2.3 & 2011 & -0.01334 & 0.112 & 0.336 & $\mathrm{U}$ \\
\hline Am-241 & RTC-A3.2 & 2011 & -0.01533 & 0.0655 & 0.1965 & $\mathrm{U}$ \\
\hline Am-241 & RTC-A3.4 & 2011 & 0.1223 & 0.118 & 0.354 & $\mathrm{U}$ \\
\hline Am-241 & RTC-A3.5 & 2011 & -0.00117 & 0.0685 & 0.2055 & $\mathrm{U}$ \\
\hline Am-241 & RTC-A4.5 & 2011 & 0.07064 & 0.143 & 0.429 & $\mathrm{U}$ \\
\hline Am-241 & RTC.3 & 2012 & 0.05038 & 0.0439 & 0.1317 & $\mathrm{U}$ \\
\hline Am-241 & RTC-1.2 & 2012 & 0.1933 & 0.147 & 0.441 & $\mathrm{U}$ \\
\hline Am-241 & RTC-2.2 & 2012 & 0.1131 & 0.1 & 0.3 & $\mathrm{U}$ \\
\hline Am-241 & RTC-6.2 & 2012 & 0.3973 & 0.115 & 0.345 & - \\
\hline Am-241 & RTC-6.4 & 2012 & -0.0009055 & 0.0365 & 0.1095 & $\mathrm{U}$ \\
\hline Am-241 & RTC-A2.2 & 2012 & 0.006511 & 0.0743 & 0.2229 & $\mathrm{U}$ \\
\hline
\end{tabular}


Table C-2. (continued).

\begin{tabular}{|c|c|c|c|c|c|c|}
\hline Radionuclide & $\begin{array}{c}\text { Sample } \\
\text { Location }\end{array}$ & Date & $\begin{array}{c}\text { Concentrations } \\
(\mathrm{pCi} / \mathrm{g})\end{array}$ & $\begin{array}{l}\text { Uncertainty } \\
(\mathrm{pCi} / \mathrm{g})\end{array}$ & $\begin{array}{c}\mathrm{MDA} \\
(\mathrm{pCi} / \mathrm{g})\end{array}$ & Flag \\
\hline Am-241 & RTC-A2.3 & 2012 & 0.01548 & 0.0824 & 0.2472 & $\mathrm{U}$ \\
\hline Am-241 & RTC-A3.2 & 2012 & -0.02406 & 0.0431 & 0.1293 & $\mathrm{U}$ \\
\hline Am-241 & RTC-A3.4 & 2012 & 0.0006616 & 0.0517 & 0.1551 & $\mathrm{U}$ \\
\hline Am-241 & RTC-A3.5 & 2012 & 0.04509 & 0.0906 & 0.2718 & $\mathrm{U}$ \\
\hline Co-60 & LP 0.1 & 1976 & 0.19 & 0.011 & 0.033 & - \\
\hline Co-60 & LP 0.3 & 1976 & 0.033 & 0.009 & 0.027 & - \\
\hline Co-60 & LP 1.1 & 1976 & 0.02 & 0.008 & 0.024 & $\mathrm{U}$ \\
\hline Co-60 & LP 1.2 & 1976 & 0.34 & 0.01 & 0.03 & - \\
\hline Co-60 & LP -1.2 & 1976 & 0.09 & 0.01 & 0.03 & - \\
\hline Co-60 & LP 2.1 & 1976 & 0.029 & 0.009 & 0.027 & - \\
\hline $\mathrm{Co}-60$ & LP 2.2 & 1976 & 0.48 & 0.02 & 0.06 & - \\
\hline Co-60 & LP 2.3 & 1976 & 0.136 & 0.009 & 0.027 & - \\
\hline Co-60 & LP 3.1 & 1976 & 0.17 & 0.01 & 0.03 & - \\
\hline Co-60 & LP 3.2 & 1976 & 1.11 & 0.04 & 0.12 & - \\
\hline Co-60 & LP 3.3 & 1976 & 0.27 & 0.002 & 0.006 & - \\
\hline Co-60 & LP 4.1 & 1976 & 0.19 & 0.01 & 0.03 & - \\
\hline $\mathrm{Co}-60$ & LP 4.2 & 1976 & 1.1 & 0.04 & 0.12 & - \\
\hline $\mathrm{Co}-60$ & LP 4.3 & 1976 & 0.054 & 0.009 & 0.027 & - \\
\hline Co-60 & LP 4.4 & 1976 & 0.026 & 0.009 & 0.027 & $\mathrm{U}$ \\
\hline Co-60 & LP 5.2 & 1976 & 1.49 & 0.05 & 0.15 & - \\
\hline Co-60 & LP 5.3 & 1976 & 0.2 & 0.01 & 0.03 & - \\
\hline Co-60 & LP 6.2 & 1976 & 0.006 & 0.001 & 0.003 & - \\
\hline Co-60 & LP 6.3 & 1976 & 0.1 & 0.01 & 0.03 & - \\
\hline Co-60 & LP 7.2 & 1976 & 0.067 & 0.008 & 0.024 & - \\
\hline Co-60 & LP1-2 & 1976 & 0.34 & 0.01 & 0.03 & - \\
\hline Co-60 & LP2-2 & 1976 & 0.48 & 0.02 & 0.06 & - \\
\hline Co-60 & LP3-2 & 1976 & 1.11 & 0.04 & 0.12 & - \\
\hline Co-60 & LP4-2 & 1976 & 18.2 & 0.5 & 1.5 & - \\
\hline Co-60 & LP5-2 & 1976 & 4 & 0.1 & 0.3 & - \\
\hline Co-60 & LP6-2 & 1976 & 0.093 & 0.011 & 0.033 & - \\
\hline Co-60 & A1.3 & 1977 & 0.056 & 0.011 & 0.033 & - \\
\hline Co-60 & $\mathrm{A} 2.2$ & 1977 & 0.0297 & 0.01 & 0.03 & $\mathrm{U}$ \\
\hline Co-60 & A 3.2 & 1977 & 0.121 & 0.011 & 0.033 & - \\
\hline Co-60 & A3.3 & 1977 & 0.0813 & 0.0088 & 0.0264 & - \\
\hline Co-60 & A 3.4 & 1977 & 0.14 & 0.012 & 0.036 & - \\
\hline Co-60 & LP 1.4 & 1977 & 0.48 & 0.009 & 0.027 & - \\
\hline Co-60 & LP -1.4 & 1977 & 0.027 & 0.008 & 0.024 & - \\
\hline
\end{tabular}


Table C-2. (continued).

\begin{tabular}{|c|c|c|c|c|c|c|}
\hline Radionuclide & $\begin{array}{c}\text { Sample } \\
\text { Location }\end{array}$ & Date & $\begin{array}{l}\text { Concentrations } \\
(\mathrm{pCi} / \mathrm{g})\end{array}$ & $\begin{array}{l}\text { Uncertainty } \\
(\mathrm{pCi} / \mathrm{g})\end{array}$ & $\begin{array}{c}\text { MDA } \\
(\mathrm{pCi} / \mathrm{g})\end{array}$ & Flag \\
\hline Co-60 & LP 6.4 & 1977 & 0.45 & 0.009 & 0.027 & - \\
\hline Co-60 & 2.2 & 1983 & 1.32 & 0.11 & 0.33 & - \\
\hline Co-60 & 3.1 & 1983 & 0.13 & 0.04 & 0.12 & 一 \\
\hline $\mathrm{Co}-60$ & 3.3 & 1983 & 0.26 & 0.06 & 0.18 & - \\
\hline Co-60 & 4.2 & 1983 & 66.4 & 2.6 & 7.8 & 一 \\
\hline Co-60 & 0.2 & 1990 & 0.31 & 0.05 & 0.15 & - \\
\hline Co-60 & 1.1 & 1990 & 0.31 & 0.04 & 0.12 & - \\
\hline Co-60 & 2.2 & 1990 & 1.96 & 0.11 & 0.33 & - \\
\hline $\mathrm{Co}-60$ & 3.3 & 1990 & 0.23 & 0.05 & 0.15 & - \\
\hline Co-60 & 4.2 & 1990 & 40.3 & 1.5 & 4.5 & - \\
\hline Co-60 & 5.2 & 1990 & 7.8 & 0.3 & 0.9 & - \\
\hline Co-60 & 5.3 & 1990 & 0.3 & 0.05 & 0.15 & - \\
\hline Co-60 & 6.2 & 1990 & 0.48 & 0.05 & 0.15 & 一 \\
\hline Co-60 & A1.3 & 1990 & 0.28 & 0.04 & 0.12 & - \\
\hline Co-60 & RTC.3 & 2006 & 0.06351 & 0.01075 & 0.03225 & - \\
\hline Co-60 & RTC-0.1 & 2006 & 0.1513 & 0.0126 & 0.0378 & - \\
\hline $\mathrm{Co}-60$ & RTC-1.1 & 2006 & 0.1416 & 0.013 & 0.039 & - \\
\hline Co-60 & RTC-1.2 & 2006 & 0.2364 & 0.0406 & 0.1218 & - \\
\hline Co-60 & RTC1.3 & 2006 & 0.1377 & 0.02185 & 0.06555 & - \\
\hline Co-60 & RTC-1.3 & 2006 & 0.1158 & 0.025 & 0.07635 & - \\
\hline Co-60 & RTC1.4 & 2006 & 0.1357 & 0.02375 & 0.07125 & - \\
\hline Co-60 & RTC-2.2 & 2006 & 0.1095 & 0.0126 & 0.0378 & - \\
\hline Co-60 & RTC2.3 & 2006 & 0.042 & 0.00725 & 0.02175 & - \\
\hline Co-60 & RTC-2.3 & 2006 & 0.1387 & 0.02515 & 0.07545 & - \\
\hline Co-60 & RTC-3.4 & 2006 & 0.04854 & 0.0103 & 0.0309 & - \\
\hline Co-60 & RTC-6.2 & 2006 & 0.2049 & 0.0183 & 0.0549 & - \\
\hline Co-60 & RTC-6.4 & 2006 & 0.05395 & 0.0091 & 0.0273 & - \\
\hline $\mathrm{Co}-60$ & RTC-8.2 & 2006 & 0.134 & 0.01935 & 0.05805 & - \\
\hline $\mathrm{Co}-60$ & RTC-A1.2 & 2006 & 0.03471 & 0.00675 & 0.02025 & - \\
\hline Co-60 & RTC-A1.3 & 2006 & 0.095 & 0.01515 & 0.04545 & - \\
\hline Co-60 & RTC-A2.2 & 2006 & 0.1169 & 0.020 & 0.06135 & - \\
\hline $\mathrm{Co}-60$ & RTC-A2.3 & 2006 & 0.06067 & 0.00955 & 0.02865 & - \\
\hline Co-60 & RTC-A2.4 & 2006 & 0.04949 & 0.0071 & 0.0213 & - \\
\hline Co-60 & RTC-A3.2 & 2006 & 0.0866 & 0.01815 & 0.05445 & - \\
\hline Co-60 & RTC-A3.3 & 2006 & 0.04218 & 0.00705 & 0.02115 & - \\
\hline Co-60 & RTC-A3.4 & 2006 & 0.1147 & 0.021 & 0.0621 & - \\
\hline Co-60 & RTC-A3.5 & 2006 & 0.1094 & 0.015 & 0.045 & - \\
\hline
\end{tabular}


Table C-2. (continued).

\begin{tabular}{|c|c|c|c|c|c|c|}
\hline Radionuclide & $\begin{array}{l}\text { Sample } \\
\text { Location }\end{array}$ & Date & $\begin{array}{l}\text { Concentrations } \\
(\mathrm{pCi} / \mathrm{g})\end{array}$ & $\begin{array}{l}\text { Uncertainty } \\
(\mathrm{pCi} / \mathrm{g})\end{array}$ & $\begin{array}{c}\mathrm{MDA} \\
(\mathrm{pCi} / \mathrm{g})\end{array}$ & Flag \\
\hline Co-60 & RTC-A4.5 & 2006 & 0.1897 & 0.01555 & 0.04665 & - \\
\hline Co-60 & RTC.3 & 2011 & 0.005069 & 0.00263 & 0.00789 & $\mathrm{U}$ \\
\hline $\mathrm{Co}-60$ & RTC-0.1 & 2011 & 0.09775 & 0.00789 & 0.02367 & - \\
\hline $\mathrm{Co}-60$ & RTC-1.1 & 2011 & 0.04789 & 0.00398 & 0.01194 & - \\
\hline $\mathrm{Co}-60$ & RTC-1.2 & 2011 & 0.06151 & 0.00703 & 0.02109 & - \\
\hline $\mathrm{Co}-60$ & RTC1.3 & 2011 & 0.01258 & 0.00499 & 0.01497 & $\mathrm{U}$ \\
\hline $\mathrm{Co}-60$ & RTC1.4 & 2011 & 0.01008 & 0.00499 & 0.01497 & $\mathrm{U}$ \\
\hline $\mathrm{Co}-60$ & RTC-2.2 & 2011 & 0.0371 & 0.00578 & 0.01734 & - \\
\hline $\mathrm{Co}-60$ & RTC2.3 & 2011 & 0.04756 & 0.00476 & 0.01428 & - \\
\hline $\mathrm{Co}-60$ & RTC-6.2 & 2011 & 0.09073 & 0.00769 & 0.02307 & - \\
\hline Co-60 & RTC-6.4 & 2011 & 0.01139 & 0.0051 & 0.0153 & $\mathrm{U}$ \\
\hline Co-60 & RTC-8.2 & 2011 & 0.04147 & 0.00765 & 0.02295 & - \\
\hline $\mathrm{Co}-60$ & RTC-A1.2 & 2011 & 0.008498 & 0.00332 & 0.00996 & $\mathrm{U}$ \\
\hline $\mathrm{Co}-60$ & RTC-A1.3 & 2011 & 0.02644 & 0.00519 & 0.01557 & - \\
\hline $\mathrm{Co}-60$ & RTC-A2.2 & 2011 & 0.002649 & 0.00442 & 0.01326 & $\mathrm{U}$ \\
\hline $\mathrm{Co}-60$ & RTC-A2.3 & 2011 & 0.007284 & 0.00522 & 0.01566 & $\mathrm{U}$ \\
\hline $\mathrm{Co}-60$ & RTC-A2.4 & 2011 & 0.005536 & 0.00255 & 0.00765 & $\mathrm{U}$ \\
\hline Co-60 & RTC-A3.2 & 2011 & 0.006172 & 0.00442 & 0.01326 & $\mathrm{U}$ \\
\hline Co-60 & RTC-A3.4 & 2011 & 0.0534 & 0.00685 & 0.02055 & - \\
\hline Co-60 & RTC-A3.5 & 2011 & 0.02541 & 0.00489 & 0.01467 & - \\
\hline Co-60 & RTC-A4.5 & 2011 & 0.06475 & 0.00757 & 0.02271 & - \\
\hline $\mathrm{Co}-60$ & RTC. 3 & 2012 & 0.02559 & 0.00537 & 0.01611 & - \\
\hline Co-60 & RTC-1.2 & 2012 & 0.04464 & 0.00809 & 0.02427 & - \\
\hline Co-60 & RTC-2.2 & 2012 & 0.05277 & 0.00671 & 0.02013 & - \\
\hline $\mathrm{Co}-60$ & RTC-6.2 & 2012 & 0.1079 & 0.00879 & 0.02637 & - \\
\hline $\mathrm{Co}-60$ & RTC-6.4 & 2012 & 0.009474 & 0.00469 & 0.01407 & $\mathrm{U}$ \\
\hline $\mathrm{Co}-60$ & RTC-A2.2 & 2012 & 0.007701 & 0.00456 & 0.01368 & $\mathrm{U}$ \\
\hline $\mathrm{Co}-60$ & RTC-A2.3 & 2012 & 0.08023 & 0.00908 & 0.02724 & - \\
\hline Co-60 & RTC-A3.2 & 2012 & 0.01536 & 0.0048 & 0.0144 & - \\
\hline Co-60 & RTC-A3.4 & 2012 & 0.01319 & 0.00484 & 0.01452 & $\mathrm{U}$ \\
\hline Co-60 & RTC-A3.5 & 2012 & 0.03358 & 0.00531 & 0.01593 & - \\
\hline Co-60 & $\begin{array}{l}2 \text { INCH AIR } \\
\text { - TRA }\end{array}$ & 2014 & 0.0002374 & 0.000928 & 0.002784 & $\mathrm{U}$ \\
\hline Cs-134 & RTC. 3 & 2007 & 0.1118 & 0.0158 & 0.0474 & - \\
\hline Cs-134 & RTC-0.1 & 2007 & 0.01776 & 0.00507 & 0.01521 & - \\
\hline Cs-134 & RTC-1.1 & 2007 & 0.01866 & 0.00841 & 0.02523 & $\mathrm{U}$ \\
\hline Cs-134 & RTC-1.2 & 2007 & 0.1154 & 0.0146 & 0.0438 & - \\
\hline
\end{tabular}


Table C-2. (continued).

\begin{tabular}{|c|c|c|c|c|c|c|}
\hline Radionuclide & $\begin{array}{c}\text { Sample } \\
\text { Location }\end{array}$ & Date & $\begin{array}{l}\text { Concentrations } \\
(\mathrm{pCi} / \mathrm{g})\end{array}$ & $\begin{array}{l}\text { Uncertainty } \\
(\mathrm{pCi} / \mathrm{g})\end{array}$ & $\begin{array}{c}\text { MDA } \\
(\mathrm{pCi} / \mathrm{g})\end{array}$ & Flag \\
\hline Cs-134 & RTC1.3 & 2007 & 0.002544 & 0.0043 & 0.0129 & $\mathrm{U}$ \\
\hline Cs-134 & RTC-1.3 & 2007 & 0.13 & 0.0208 & 0.0624 & - \\
\hline Cs-134 & RTC1.4 & 2007 & 0.08755 & 0.0164 & 0.0492 & - \\
\hline Cs-134 & RTC-2.2 & 2007 & 0.08189 & 0.0111 & 0.0333 & - \\
\hline Cs-134 & RTC2.3 & 2007 & 0.07935 & 0.0136 & 0.0408 & - \\
\hline Cs-134 & RTC-2.3 & 2007 & 0.1661 & 0.0207 & 0.0621 & - \\
\hline Cs-134 & RTC-3.4 & 2007 & 0.04885 & 0.0087 & 0.0261 & - \\
\hline Cs-134 & RTC-6.2 & 2007 & 0.06382 & 0.0157 & 0.0471 & - \\
\hline Cs-134 & RTC-6.4 & 2007 & 0.09783 & 0.0176 & 0.0528 & - \\
\hline Cs-134 & RTC-8.2 & 2007 & 0.1179 & 0.0145 & 0.0435 & - \\
\hline Cs-134 & RTC-A1.2 & 2007 & 0.02392 & 0.00696 & 0.02088 & - \\
\hline Cs-134 & RTC-A1.3 & 2007 & 0.06297 & 0.0126 & 0.0378 & - \\
\hline Cs-134 & RTC-A2.2 & 2007 & 0.007519 & 0.0191 & 0.0573 & $\mathrm{U}$ \\
\hline Cs-134 & RTC-A2.3 & 2007 & 0.1089 & 0.0168 & 0.0504 & - \\
\hline Cs-134 & RTC-A2.4 & 2007 & 0.05306 & 0.00957 & 0.02871 & - \\
\hline Cs-134 & RTC-A3.2 & 2007 & 0.1716 & 0.0219 & 0.0657 & - \\
\hline Cs-134 & RTC-A3.3 & 2007 & 0.1249 & 0.0171 & 0.0513 & - \\
\hline Cs-134 & RTC-A3.4 & 2007 & 0.1249 & 0.0134 & 0.0402 & - \\
\hline Cs-134 & RTC-A3.5 & 2007 & 0.03875 & 0.0282 & 0.0846 & $\mathrm{U}$ \\
\hline Cs-134 & RTC-A4.5 & 2007 & 0.03781 & 0.00768 & 0.02304 & - \\
\hline Cs-134 & RTC-3.4 & 2008 & 0.1001 & 0.0132 & 0.0396 & - \\
\hline Cs-134 & RTC-A3.3 & 2008 & 0.08253 & 0.0141 & 0.0423 & - \\
\hline Cs-134 & RTC-A3.4 & 2008 & 0.02916 & 0.00773 & 0.02319 & - \\
\hline Cs-134 & RTC1.4 & 2009 & 0.008943 & 0.003895 & 0.011685 & $\mathrm{U}$ \\
\hline Cs-134 & RTC-A3.2 & 2009 & 0.04535 & 0.0068 & 0.0204 & - \\
\hline Cs-134 & RTC-A3.5 & 2009 & 0.006815 & 0.004725 & 0.014175 & $\mathrm{U}$ \\
\hline Cs-134 & RTC-8.2 & 2010 & 0.05283 & 0.00692 & 0.02076 & 一 \\
\hline Cs-134 & RTC-A4.5 & 2010 & 0.0153 & 0.00322 & 0.00966 & - \\
\hline Cs-134 & RTC.3 & 2011 & -0.1121 & 0.00795 & 0.02385 & $\mathrm{U}$ \\
\hline Cs-134 & RTC-0.1 & 2011 & 0.005431 & 0.0145 & 0.0435 & $\mathrm{U}$ \\
\hline Cs-134 & RTC-1.1 & 2011 & 0.00798 & 0.0192 & 0.0576 & $\mathrm{U}$ \\
\hline Cs-134 & RTC-1.2 & 2011 & -0.001626 & 0.0258 & 0.0774 & $\mathrm{U}$ \\
\hline Cs-134 & RTC1.3 & 2011 & -0.003505 & 0.0253 & 0.0759 & $\mathrm{U}$ \\
\hline Cs-134 & RTC1.4 & 2011 & 0.001093 & 0.0132 & 0.0396 & $\mathrm{U}$ \\
\hline Cs-134 & RTC-2.2 & 2011 & -0.007413 & 0.0236 & 0.0708 & $\mathrm{U}$ \\
\hline Cs-134 & RTC2.3 & 2011 & 0.01064 & 0.0178 & 0.0534 & $\mathrm{U}$ \\
\hline Cs-134 & RTC-6.2 & 2011 & -0.004116 & 0.0241 & 0.0723 & $\mathrm{U}$ \\
\hline
\end{tabular}


Table C-2. (continued).

\begin{tabular}{|c|c|c|c|c|c|c|}
\hline Radionuclide & $\begin{array}{l}\text { Sample } \\
\text { Location }\end{array}$ & Date & $\begin{array}{l}\text { Concentrations } \\
(\mathrm{pCi} / \mathrm{g})\end{array}$ & $\begin{array}{l}\text { Uncertainty } \\
(\mathrm{pCi} / \mathrm{g})\end{array}$ & $\begin{array}{c}\mathrm{MDA} \\
(\mathrm{pCi} / \mathrm{g})\end{array}$ & Flag \\
\hline Cs-134 & RTC-6.4 & 2011 & -0.01676 & 0.025 & 0.075 & $\mathrm{U}$ \\
\hline Cs-134 & RTC-8.2 & 2011 & -0.006582 & 0.0145 & 0.0435 & $\mathrm{U}$ \\
\hline Cs-134 & RTC-A1.2 & 2011 & -0.001184 & 0.0213 & 0.0639 & $\mathrm{U}$ \\
\hline Cs-134 & RTC-A1.3 & 2011 & -0.002305 & 0.0226 & 0.0678 & $\mathrm{U}$ \\
\hline Cs-134 & RTC-A2.2 & 2011 & -0.01363 & 0.024 & 0.072 & $\mathrm{U}$ \\
\hline Cs-134 & RTC-A2.3 & 2011 & -0.01804 & 0.0332 & 0.0996 & $\mathrm{U}$ \\
\hline Cs-134 & RTC-A2.4 & 2011 & -0.001358 & 0.0126 & 0.0378 & $\mathrm{U}$ \\
\hline Cs-134 & RTC-A3.2 & 2011 & 0.0021 & 0.0235 & 0.0705 & $\mathrm{U}$ \\
\hline Cs-134 & RTC-A3.4 & 2011 & 0.0086 & 0.0248 & 0.0744 & $\mathrm{U}$ \\
\hline Cs-134 & RTC-A3.5 & 2011 & 0.05303 & 0.0109 & 0.0327 & - \\
\hline Cs-134 & RTC-A4.5 & 2011 & 0.002122 & 0.0245 & 0.0735 & $\mathrm{U}$ \\
\hline Cs-134 & RTC.3 & 2012 & 0.05988 & 0.00899 & 0.02697 & - \\
\hline Cs-134 & RTC-1.2 & 2012 & 0.07708 & 0.0131 & 0.0393 & - \\
\hline Cs-134 & RTC-2.2 & 2012 & 0.0667 & 0.00998 & 0.02994 & - \\
\hline Cs-134 & RTC-6.2 & 2012 & 0.02404 & 0.00704 & 0.02112 & - \\
\hline Cs-134 & RTC-6.4 & 2012 & 0.08045 & 0.00898 & 0.02694 & - \\
\hline Cs-134 & RTC-A2.2 & 2012 & 0.06831 & 0.00872 & 0.02616 & - \\
\hline Cs-134 & RTC-A2.3 & 2012 & 0.05793 & 0.00993 & 0.02979 & - \\
\hline Cs-134 & RTC-A3.2 & 2012 & 0.07143 & 0.00937 & 0.02811 & - \\
\hline Cs-134 & RTC-A3.4 & 2012 & 0.07192 & 0.00995 & 0.02985 & - \\
\hline Cs-134 & RTC-A3.5 & 2012 & 0.06206 & 0.00816 & 0.02448 & 一 \\
\hline Cs-134 & $\begin{array}{l}2 \text { INCH AIR } \\
\text { - TRA }\end{array}$ & 2014 & 0.03925 & 0.00944 & 0.02832 & - \\
\hline Cs-137 & LP 0.1 & 1976 & 0.99 & 0.04 & 0.12 & - \\
\hline Cs-137 & LP 0.3 & 1976 & 1.84 & 0.06 & 0.18 & - \\
\hline Cs-137 & LP 1.1 & 1976 & 0.82 & 0.03 & 0.09 & 一 \\
\hline Cs-137 & LP -1.1 & 1976 & 0.95 & 0.04 & 0.12 & - \\
\hline Cs-137 & LP 1.2 & 1976 & 2.37 & 0.08 & 0.24 & - \\
\hline Cs-137 & LP -1.2 & 1976 & 1.84 & 0.06 & 0.18 & - \\
\hline Cs-137 & LP 1.3 & 1976 & 0.85 & 0.003 & 0.009 & - \\
\hline Cs-137 & LP -1.3 & 1976 & 0.55 & 0.02 & 0.06 & - \\
\hline Cs-137 & LP 2.1 & 1976 & 0.81 & 0.03 & 0.09 & - \\
\hline Cs-137 & LP 2.2 & 1976 & 3 & 0.1 & 0.3 & - \\
\hline Cs-137 & LP 2.3 & 1976 & 1.29 & 0.05 & 0.15 & - \\
\hline Cs-137 & LP 3.1 & 1976 & 1.04 & 0.04 & 0.12 & - \\
\hline Cs-137 & LP 3.2 & 1976 & 3.1 & 0.1 & 0.3 & - \\
\hline Cs-137 & LP 3.3 & 1976 & 1.22 & 0.02 & 0.06 & - \\
\hline
\end{tabular}


Table C-2. (continued).

\begin{tabular}{|c|c|c|c|c|c|c|}
\hline Radionuclide & $\begin{array}{l}\text { Sample } \\
\text { Location }\end{array}$ & Date & $\begin{array}{l}\text { Concentrations } \\
(\mathrm{pCi} / \mathrm{g})\end{array}$ & $\begin{array}{l}\text { Uncertainty } \\
(\mathrm{pCi} / \mathrm{g})\end{array}$ & $\begin{array}{c}\text { MDA } \\
(\mathrm{pCi} / \mathrm{g})\end{array}$ & Flag \\
\hline Cs-137 & LP 4.1 & 1976 & 0.28 & 0.02 & 0.06 & - \\
\hline Cs-137 & LP 4.2 & 1976 & 1.36 & 0.05 & 0.15 & 一 \\
\hline Cs-137 & LP 4.3 & 1976 & 0.26 & 0.02 & 0.06 & - \\
\hline Cs-137 & LP 4.4 & 1976 & 0.73 & 0.003 & 0.009 & - \\
\hline Cs-137 & LP 5.2 & 1976 & 3.4 & 0.1 & 0.3 & - \\
\hline Cs-137 & LP 5.3 & 1976 & 1.48 & 0.050 & 0.15 & - \\
\hline Cs-137 & LP 6.2 & 1976 & 0.76 & 0.03 & 0.09 & - \\
\hline Cs-137 & LP 6.3 & 1976 & 1.6 & 0.06 & 0.18 & - \\
\hline Cs-137 & LP 7.2 & 1976 & 1.5 & 0.05 & 0.15 & - \\
\hline Cs-137 & LP 7.3 & 1976 & 0.28 & 0.02 & 0.06 & - \\
\hline Cs-137 & LP 8.2 & 1976 & 0.99 & 0.04 & 0.12 & - \\
\hline Cs-137 & A 1.3 & 1977 & 1.23 & 0.045 & 0.135 & - \\
\hline Cs-137 & $\mathrm{A} 2.2$ & 1977 & 1.16 & 0.043 & 0.129 & - \\
\hline Cs-137 & $\mathrm{A} 2.3$ & 1977 & 0.216 & 0.013 & 0.039 & - \\
\hline Cs-137 & A2.4 & 1977 & 1.48 & 0.05 & 0.15 & - \\
\hline Cs-137 & $\mathrm{A} 2.5$ & 1977 & 1.52 & 0.03 & 0.09 & - \\
\hline Cs-137 & A 3.2 & 1977 & 2.14 & 0.071 & 0.213 & - \\
\hline Cs-137 & A 3.3 & 1977 & 1.02 & 0.04 & 0.12 & - \\
\hline Cs-137 & A3.4 & 1977 & 1.79 & 0.058 & 0.174 & - \\
\hline Cs-137 & A 3.5 & 1977 & 1.43 & 0.052 & 0.156 & - \\
\hline Cs-137 & A4.5 & 1977 & 1.51 & 0.048 & 0.144 & - \\
\hline Cs-137 & A5.5 & 1977 & 0.857 & 0.031 & 0.093 & - \\
\hline Cs-137 & LP -1.3 & 1977 & 0.55 & 0.02 & 0.06 & - \\
\hline Cs-137 & LP 1.4 & 1977 & 1.34 & 0.05 & 0.15 & - \\
\hline Cs-137 & LP -1.4 & 1977 & 1.25 & 0.04 & 0.12 & - \\
\hline Cs-137 & LP -2.2 & 1977 & 1.09 & 0.04 & 0.12 & - \\
\hline Cs-137 & LP -2.3 & 1977 & 1.29 & 0.04 & 0.12 & - \\
\hline Cs-137 & LP 3.4 & 1977 & 1.52 & 0.05 & 0.15 & - \\
\hline Cs-137 & LP 6.4 & 1977 & 1.58 & 0.05 & 0.15 & 一 \\
\hline Cs-137 & 1.1 & 1983 & 1.5 & 0.010 & 0.03 & 一 \\
\hline Cs-137 & -1.1 & 1983 & 1.15 & 0.08 & 0.24 & - \\
\hline Cs-137 & -1.2 & 1983 & 1.26 & 0.09 & 0.27 & - \\
\hline Cs-137 & 2.2 & 1983 & 5.47 & 0.25 & 0.75 & - \\
\hline Cs-137 & 3.1 & 1983 & 0.86 & 0.08 & 0.24 & - \\
\hline Cs-137 & 3.3 & 1983 & 1.65 & 0.1 & 0.3 & - \\
\hline Cs-137 & 4.2 & 1983 & 192 & 7 & 21 & - \\
\hline Cs-137 & 6.4 & 1983 & 0.71 & 0.07 & 0.21 & - \\
\hline
\end{tabular}


Table C-2. (continued).

\begin{tabular}{|c|c|c|c|c|c|c|}
\hline Radionuclide & $\begin{array}{l}\text { Sample } \\
\text { Location }\end{array}$ & Date & $\begin{array}{l}\text { Concentrations } \\
(\mathrm{pCi} / \mathrm{g})\end{array}$ & $\begin{array}{l}\text { Uncertainty } \\
(\mathrm{pCi} / \mathrm{g})\end{array}$ & $\begin{array}{c}\text { MDA } \\
(\mathrm{pCi} / \mathrm{g})\end{array}$ & Flag \\
\hline Cs-137 & 8.2 & 1983 & 0.75 & 0.07 & 0.21 & - \\
\hline Cs-137 & A1.3 & 1984 & 0.81 & 0.07 & 0.21 & 一 \\
\hline Cs-137 & $\mathrm{A} 2.2$ & 1984 & 1.05 & 0.08 & 0.24 & - \\
\hline Cs-137 & $\mathrm{A} 2.5$ & 1984 & 0.7 & 0.07 & 0.21 & - \\
\hline Cs-137 & A 3.4 & 1984 & 1.02 & 0.08 & 0.24 & - \\
\hline Cs-137 & A4.5 & 1984 & 0.67 & 0.07 & 0.21 & - \\
\hline Cs-137 & 0.2 & 1990 & 1.89 & 0.1 & 0.3 & - \\
\hline Cs-137 & 1.1 & 1990 & 1.85 & 0.1 & 0.3 & - \\
\hline Cs-137 & 1.3 & 1990 & 1.62 & 0.09 & 0.27 & - \\
\hline Cs-137 & -1.3 & 1990 & 1.64 & 0.09 & 0.27 & - \\
\hline Cs-137 & 1.4 & 1990 & 0.84 & 0.07 & 0.21 & - \\
\hline Cs-137 & 2.2 & 1990 & 9.1 & 0.4 & 1.2 & - \\
\hline Cs-137 & -2.2 & 1990 & 1.07 & 0.07 & 0.21 & - \\
\hline Cs-137 & 3.3 & 1990 & 1.75 & 0.1 & 0.3 & - \\
\hline Cs-137 & 3.4 & 1990 & 1.29 & 0.08 & 0.24 & - \\
\hline Cs-137 & 4.2 & 1990 & 223 & 8 & 24 & - \\
\hline Cs-137 & 5.2 & 1990 & 31.8 & 1.2 & 3.6 & - \\
\hline Cs-137 & 5.3 & 1990 & 3.11 & 0.15 & 0.45 & - \\
\hline Cs-137 & 6.2 & 1990 & 2.18 & 0.11 & 0.33 & - \\
\hline Cs-137 & 6.4 & 1990 & 1.29 & 0.09 & 0.27 & - \\
\hline Cs-137 & A 1.3 & 1990 & 2.1 & 0.11 & 0.33 & - \\
\hline Cs-137 & $\mathrm{A} 2.2$ & 1990 & 0.86 & 0.07 & 0.21 & - \\
\hline Cs-137 & $\mathrm{A} 2.5$ & 1990 & 0.55 & 0.05 & 0.15 & - \\
\hline Cs-137 & A 3.3 & 1990 & 0.63 & 0.06 & 0.18 & - \\
\hline Cs-137 & A3.4 & 1990 & 0.54 & 0.05 & 0.15 & - \\
\hline Cs-137 & A4.5 & 1990 & 0.6 & 0.06 & 0.18 & 一 \\
\hline Cs-137 & RTC. 3 & 2006 & 0.1834 & 0.00635 & 0.01905 & 一 \\
\hline Cs-137 & RTC-0.1 & 2006 & 0.4068 & 0.0072 & 0.0216 & - \\
\hline Cs-137 & RTC-1.1 & 2006 & 0.3454 & 0.00775 & 0.02325 & 一 \\
\hline Cs-137 & RTC-1.2 & 2006 & 0.3943 & 0.003985 & 0.011955 & 一 \\
\hline Cs-137 & RTC-1.3 & 2006 & 0.1406 & 0.0117 & 0.0351 & - \\
\hline Cs-137 & RTC1.4 & 2006 & 0.1322 & 0.0061 & 0.0183 & - \\
\hline Cs- 137 & RTC-2.2 & 2006 & 0.3159 & 0.0099 & 0.0297 & - \\
\hline Cs-137 & RTC2.3 & 2006 & 0.131 & 0.00401 & 0.01203 & - \\
\hline Cs-137 & RTC-2.3 & 2006 & 0.2906 & 0.0068 & 0.0204 & - \\
\hline Cs-137 & RTC-3.4 & 2006 & 0.2047 & 0.0084 & 0.0252 & - \\
\hline Cs-137 & RTC-6.2 & 2006 & 0.3759 & 0.00895 & 0.02685 & - \\
\hline
\end{tabular}


Table C-2. (continued).

\begin{tabular}{|c|c|c|c|c|c|c|}
\hline Radionuclide & $\begin{array}{l}\text { Sample } \\
\text { Location }\end{array}$ & Date & $\begin{array}{l}\text { Concentrations } \\
(\mathrm{pCi} / \mathrm{g})\end{array}$ & $\begin{array}{l}\text { Uncertainty } \\
(\mathrm{pCi} / \mathrm{g})\end{array}$ & $\begin{array}{c}\text { MDA } \\
(\mathrm{pCi} / \mathrm{g})\end{array}$ & Flag \\
\hline Cs-137 & RTC-6.4 & 2006 & 0.186 & 0.00875 & 0.02625 & 一 \\
\hline Cs-137 & RTC-8.2 & 2006 & 0.1657 & 0.00795 & 0.02385 & 一 \\
\hline Cs-137 & RTC-A1.2 & 2006 & 0.126 & 0.00411 & 0.01233 & - \\
\hline Cs-137 & RTC-A1.3 & 2006 & 0.2044 & 0.00615 & 0.01845 & - \\
\hline Cs-137 & RTC-A2.2 & 2006 & 0.1342 & 0.0054 & 0.0162 & - \\
\hline Cs-137 & RTC-A2.3 & 2006 & 0.1321 & 0.0084 & 0.0252 & - \\
\hline Cs-137 & RTC-A2.4 & 2006 & 0.07823 & 0.00338 & 0.01014 & - \\
\hline Cs-137 & RTC-A3.2 & 2006 & 0.1438 & 0.00264 & 0.00792 & - \\
\hline Cs-137 & RTC-A3.3 & 2006 & 0.1499 & 0.00735 & 0.02205 & - \\
\hline Cs-137 & RTC-A3.4 & 2006 & 0.155 & 0.00855 & 0.02565 & - \\
\hline Cs-137 & RTC-A3.5 & 2006 & 0.1149 & 0.0059 & 0.0177 & - \\
\hline Cs-137 & RTC-A4.5 & 2006 & 0.1648 & 0.0126 & 0.0378 & - \\
\hline Cs-137 & RTC.3 & 2007 & 0.6213 & 0.0244 & 0.0732 & - \\
\hline Cs-137 & RTC-0.1 & 2007 & 0.7973 & 0.00887 & 0.02661 & - \\
\hline Cs-137 & RTC-1.1 & 2007 & 0.8395 & 0.0262 & 0.0786 & - \\
\hline Cs-137 & RTC-1.2 & 2007 & 1.047 & 0.0281 & 0.0843 & - \\
\hline Cs-137 & RTC1.3 & 2007 & 0.421 & 0.0104 & 0.0312 & - \\
\hline Cs-137 & RTC-1.3 & 2007 & 0.4638 & 0.0204 & 0.0612 & - \\
\hline Cs-137 & RTC1.4 & 2007 & 0.4739 & 0.0226 & 0.0678 & - \\
\hline Cs-137 & RTC-2.2 & 2007 & 0.747 & 0.0228 & 0.0684 & - \\
\hline Cs-137 & RTC2.3 & 2007 & 0.7481 & 0.0212 & 0.0636 & - \\
\hline Cs-137 & RTC-2.3 & 2007 & 0.542 & 0.0126 & 0.0378 & - \\
\hline Cs-137 & RTC-3.4 & 2007 & 0.4543 & 0.0241 & 0.0723 & - \\
\hline Cs-137 & RTC-6.2 & 2007 & 1.304 & 0.0246 & 0.0738 & - \\
\hline Cs-137 & RTC-6.4 & 2007 & 0.4443 & 0.014 & 0.042 & 一 \\
\hline Cs-137 & RTC-8.2 & 2007 & 0.3192 & 0.0129 & 0.0387 & 一 \\
\hline Cs-137 & RTC-A1.2 & 2007 & 0.3767 & 0.0128 & 0.0384 & 一 \\
\hline Cs-137 & RTC-A1.3 & 2007 & 0.4828 & 0.0254 & 0.0762 & - \\
\hline Cs-137 & RTC-A2.2 & 2007 & 0.3406 & 0.00693 & 0.02079 & 一 \\
\hline Cs-137 & RTC-A2.3 & 2007 & 0.222 & 0.00541 & 0.01623 & 一 \\
\hline Cs-137 & RTC-A2.4 & 2007 & 0.2153 & 0.0191 & 0.0573 & - \\
\hline Cs-137 & RTC-A3.2 & 2007 & 0.4117 & 0.0536 & 0.1608 & - \\
\hline Cs-137 & RTC-A3.3 & 2007 & 0.3568 & 0.0183 & 0.0549 & - \\
\hline Cs-137 & RTC-A3.4 & 2007 & 0.3845 & 0.0193 & 0.0579 & - \\
\hline Cs-137 & RTC-A3.5 & 2007 & 0.308 & 0.025 & 0.075 & - \\
\hline Cs-137 & RTC-A4.5 & 2007 & 0.3767 & 0.0252 & 0.0756 & - \\
\hline Cs-137 & RTC-3.4 & 2008 & 0.3312 & 0.0119 & 0.0357 & - \\
\hline
\end{tabular}


Table C-2. (continued).

\begin{tabular}{|c|c|c|c|c|c|c|}
\hline Radionuclide & $\begin{array}{c}\text { Sample } \\
\text { Location }\end{array}$ & Date & $\begin{array}{l}\text { Concentrations } \\
(\mathrm{pCi} / \mathrm{g})\end{array}$ & $\begin{array}{l}\text { Uncertainty } \\
(\mathrm{pCi} / \mathrm{g})\end{array}$ & $\begin{array}{c}\text { MDA } \\
(\mathrm{pCi} / \mathrm{g})\end{array}$ & Flag \\
\hline Cs-137 & RTC-A3.3 & 2008 & 0.2621 & 0.0139 & 0.0417 & 一 \\
\hline Cs-137 & RTC-A3.4 & 2008 & 0.2235 & 0.00707 & 0.02121 & 一 \\
\hline Cs-137 & RTC.3 & 2009 & 0.325 & 0.001805 & 0.005415 & - \\
\hline Cs-137 & RTC-0.1 & 2009 & 0.6821 & 0.00885 & 0.02655 & - \\
\hline Cs-137 & RTC-1.1 & 2009 & 0.5534 & 0.006 & 0.018 & - \\
\hline Cs-137 & RTC-1.2 & 2009 & 0.5852 & 0.00935 & 0.02805 & - \\
\hline Cs-137 & RTC1.3 & 2009 & 0.3254 & 0.0082 & 0.0246 & - \\
\hline Cs-137 & RTC1.4 & 2009 & 0.288 & 0.00515 & 0.01545 & - \\
\hline Cs-137 & RTC-2.2 & 2009 & 0.4329 & 0.00815 & 0.02445 & - \\
\hline Cs-137 & RTC2.3 & 2009 & 0.543 & 0.00895 & 0.02685 & - \\
\hline Cs-137 & RTC-2.3 & 2009 & 0.3139 & 0.00865 & 0.02595 & - \\
\hline Cs-137 & RTC-3.4 & 2009 & 0.1997 & 0.00429 & 0.01287 & - \\
\hline Cs-137 & RTC-6.2 & 2009 & 0.6389 & 0.0066 & 0.0198 & - \\
\hline Cs-137 & RTC-6.4 & 2009 & 0.2483 & 0.00625 & 0.01875 & - \\
\hline Cs-137 & RTC-8.2 & 2009 & 0.2058 & 0.00705 & 0.02115 & - \\
\hline Cs-137 & RTC-A1.2 & 2009 & 0.2034 & 0.00436 & 0.01308 & - \\
\hline Cs-137 & RTC-A1.3 & 2009 & 0.3257 & 0.0051 & 0.0153 & - \\
\hline Cs-137 & RTC-A2.2 & 2009 & 0.1971 & 0.00665 & 0.01995 & - \\
\hline Cs-137 & RTC-A2.3 & 2009 & 0.1746 & 0.00675 & 0.02025 & - \\
\hline Cs-137 & RTC-A3.2 & 2009 & 0.2387 & 0.00765 & 0.02295 & - \\
\hline Cs-137 & RTC-A3.3 & 2009 & 0.1699 & 0.00515 & 0.01545 & - \\
\hline Cs-137 & RTC-A3.5 & 2009 & 0.1765 & 0.00815 & 0.02445 & - \\
\hline Cs-137 & RTC-A4.5 & 2009 & 0.1734 & 0.00795 & 0.02385 & - \\
\hline Cs-137 & RTC-8.2 & 2010 & 0.2398 & 0.016 & 0.048 & - \\
\hline Cs-137 & RTC-A4.5 & 2010 & 0.1364 & 0.00148 & 0.00444 & 一 \\
\hline Cs-137 & RTC. 3 & 2011 & 0.8786 & 0.0144 & 0.0432 & 一 \\
\hline Cs-137 & RTC-0.1 & 2011 & 1.878 & 0.023 & 0.069 & 一 \\
\hline Cs-137 & RTC-1.1 & 2011 & 1.501 & 0.0194 & 0.0582 & - \\
\hline Cs-137 & RTC-1.2 & 2011 & 1.751 & 0.0257 & 0.0771 & - \\
\hline Cs-137 & RTC1.3 & 2011 & 0.8372 & 0.0212 & 0.0636 & 一 \\
\hline Cs-137 & RTC1.4 & 2011 & 0.8556 & 0.0175 & 0.0525 & - \\
\hline Cs-137 & RTC-2.2 & 2011 & 1.032 & 0.0161 & 0.0483 & - \\
\hline Cs-137 & RTC2.3 & 2011 & 1.403 & 0.019 & 0.057 & - \\
\hline Cs-137 & RTC-6.2 & 2011 & 1.738 & 0.0249 & 0.0747 & - \\
\hline Cs-137 & RTC-6.4 & 2011 & 0.8259 & 0.0205 & 0.0615 & - \\
\hline Cs-137 & RTC-8.2 & 2011 & 0.6268 & 0.0167 & 0.0501 & - \\
\hline Cs-137 & RTC-A1.2 & 2011 & 0.4954 & 0.0118 & 0.0354 & - \\
\hline
\end{tabular}


Table C-2. (continued).

\begin{tabular}{|c|c|c|c|c|c|c|}
\hline Radionuclide & $\begin{array}{l}\text { Sample } \\
\text { Location }\end{array}$ & Date & $\begin{array}{l}\text { Concentrations } \\
(\mathrm{pCi} / \mathrm{g})\end{array}$ & $\begin{array}{l}\text { Uncertainty } \\
(\mathrm{pCi} / \mathrm{g})\end{array}$ & $\begin{array}{c}\text { MDA } \\
(\mathrm{pCi} / \mathrm{g})\end{array}$ & Flag \\
\hline Cs-137 & RTC-A1.3 & 2011 & 0.7331 & 0.0124 & 0.0372 & 一 \\
\hline Cs-137 & RTC-A2.2 & 2011 & 0.479 & 0.0134 & 0.0402 & 一 \\
\hline Cs-137 & RTC-A2.3 & 2011 & 0.4508 & 0.0188 & 0.0564 & - \\
\hline Cs-137 & RTC-A2.4 & 2011 & 0.155 & 0.00859 & 0.02577 & - \\
\hline Cs-137 & RTC-A3.2 & 2011 & 0.5849 & 0.0141 & 0.0423 & - \\
\hline Cs-137 & RTC-A3.4 & 2011 & 0.6499 & 0.0268 & 0.0804 & - \\
\hline Cs-137 & RTC-A3.5 & 2011 & 0.3683 & 0.0134 & 0.0402 & - \\
\hline Cs-137 & RTC-A4.5 & 2011 & 0.5039 & 0.0248 & 0.0744 & - \\
\hline Cs-137 & RTC.3 & 2012 & 0.2552 & 0.00529 & 0.01587 & - \\
\hline Cs-137 & RTC-1.2 & 2012 & 0.4285 & 0.0114 & 0.0342 & - \\
\hline Cs-137 & RTC-2.2 & 2012 & 0.4863 & 0.00869 & 0.02607 & - \\
\hline Cs-137 & RTC-6.2 & 2012 & 0.8307 & 0.00925 & 0.02775 & - \\
\hline Cs-137 & RTC-6.4 & 2012 & 0.2566 & 0.00534 & 0.01602 & - \\
\hline Cs-137 & RTC-A2.2 & 2012 & 0.2071 & 0.00771 & 0.02313 & - \\
\hline Cs-137 & RTC-A2.3 & 2012 & 0.6036 & 0.00946 & 0.02838 & - \\
\hline Cs-137 & RTC-A3.2 & 2012 & 0.1568 & 0.00633 & 0.01899 & - \\
\hline Cs-137 & RTC-A3.4 & 2012 & 0.232 & 0.0091 & 0.0273 & - \\
\hline Cs-137 & RTC-A3.5 & 2012 & 0.07696 & 0.00759 & 0.02277 & - \\
\hline Cs-137 & $\begin{array}{l}2 \text { INCH AIR } \\
\text { - TRA }\end{array}$ & 2014 & 0.07592 & 0.00754 & 0.02262 & 一 \\
\hline Eu-152 & RTC. 3 & 2011 & 0.01541 & 0.0242 & 0.0726 & $\mathrm{U}$ \\
\hline Eu-152 & RTC-0.1 & 2011 & 0.0121 & 0.0275 & 0.0825 & $\mathrm{U}$ \\
\hline Eu-152 & RTC-1.1 & 2011 & 0.02707 & 0.0245 & 0.0735 & $\mathrm{U}$ \\
\hline Eu-152 & RTC-1.2 & 2011 & 0.03889 & 0.0316 & 0.0948 & $\mathrm{U}$ \\
\hline Eu-152 & RTC1.3 & 2011 & 0.02213 & 0.0321 & 0.0963 & $\mathrm{U}$ \\
\hline Eu-152 & RTC1.4 & 2011 & 0.01046 & 0.0261 & 0.0783 & $\mathrm{U}$ \\
\hline Eu-152 & RTC-2.2 & 2011 & 0.0009363 & 0.028 & 0.084 & $\mathrm{U}$ \\
\hline Eu-152 & RTC2.3 & 2011 & 0.002431 & 0.0237 & 0.0711 & $\mathrm{U}$ \\
\hline Eu-152 & RTC-6.2 & 2011 & 0.01159 & 0.0331 & 0.0993 & $\mathrm{U}$ \\
\hline Eu-152 & RTC-6.4 & 2011 & 0.04785 & 0.0264 & 0.0792 & $\mathrm{U}$ \\
\hline Eu-152 & RTC-8.2 & 2011 & 0.0555 & 0.0287 & 0.0861 & $\mathrm{U}$ \\
\hline Eu-152 & RTC-A1.2 & 2011 & 0.01452 & 0.0227 & 0.0681 & $\mathrm{U}$ \\
\hline Eu-152 & RTC-A1.3 & 2011 & 0.03154 & 0.0267 & 0.0801 & $\mathrm{U}$ \\
\hline Eu-152 & RTC-A2.2 & 2011 & 0.03046 & 0.0279 & 0.0837 & $\mathrm{U}$ \\
\hline Eu-152 & RTC-A2.3 & 2011 & 0.02941 & 0.0332 & 0.0996 & $\mathrm{U}$ \\
\hline Eu-152 & RTC-A2.4 & 2011 & -0.006851 & 0.0371 & 0.1113 & $\mathrm{U}$ \\
\hline Eu-152 & RTC-A3.2 & 2011 & 0.009706 & 0.0262 & 0.0786 & $\mathrm{U}$ \\
\hline
\end{tabular}


Table C-2. (continued).

\begin{tabular}{|c|c|c|c|c|c|c|}
\hline Radionuclide & $\begin{array}{l}\text { Sample } \\
\text { Location }\end{array}$ & Date & $\begin{array}{l}\text { Concentrations } \\
(\mathrm{pCi} / \mathrm{g})\end{array}$ & $\begin{array}{l}\text { Uncertainty } \\
\text { (pCi } / \mathrm{g})\end{array}$ & $\begin{array}{c}\text { MDA } \\
(\mathrm{pCi} / \mathrm{g})\end{array}$ & Flag \\
\hline Eu-152 & RTC-A3.4 & 2011 & 0.627 & 0.0426 & 0.1278 & - \\
\hline Eu-152 & RTC-A3.5 & 2011 & 0.06371 & 0.0271 & 0.0813 & $\mathrm{U}$ \\
\hline Eu-152 & RTC-A4.5 & 2011 & 0.07326 & 0.0377 & 0.1131 & $\mathrm{U}$ \\
\hline Eu-152 & RTC. 3 & 2012 & 0.01806 & 0.0236 & 0.0708 & $\mathrm{U}$ \\
\hline Eu-152 & RTC-1.2 & 2012 & 0.03193 & 0.0389 & 0.1167 & $\mathrm{U}$ \\
\hline Eu-152 & RTC-2.2 & 2012 & 0.02902 & 0.0294 & 0.0882 & $\mathrm{U}$ \\
\hline Eu-152 & RTC-6.2 & 2012 & 0.01269 & 0.025 & 0.075 & $\mathrm{U}$ \\
\hline Eu-152 & RTC-6.4 & 2012 & 0.00002383 & 0.023 & 0.069 & $\mathrm{U}$ \\
\hline Eu-152 & RTC-A2.2 & 2012 & 0.04105 & 0.0256 & 0.0768 & $\mathrm{U}$ \\
\hline Eu-152 & RTC-A2.3 & 2012 & 0.01393 & 0.0281 & 0.0843 & $\mathrm{U}$ \\
\hline Eu-152 & RTC-A3.2 & 2012 & 0.04328 & 0.0242 & 0.0726 & $\mathrm{U}$ \\
\hline Eu-152 & RTC-A3.4 & 2012 & 0.02296 & 0.0243 & 0.0729 & $\mathrm{U}$ \\
\hline Eu-152 & RTC-A3.5 & 2012 & 0.7753 & 0.0318 & 0.0954 & - \\
\hline Eu-152 & $\begin{array}{l}2 \text { INCH AIR } \\
\text { - TRA }\end{array}$ & 2014 & 0.001068 & 0.022 & 0.066 & $\mathrm{U}$ \\
\hline $\mathrm{Pu}-238$ & LP 0.3 & 1976 & 0.009 & 0.002 & 0.006 & - \\
\hline $\mathrm{Pu}-238$ & LP -1.2 & 1976 & 0.0069 & 0.0015 & 0.0045 & - \\
\hline $\mathrm{Pu}-238$ & LP 2.2 & 1976 & 0.0066 & 0.0014 & 0.0042 & - \\
\hline $\mathrm{Pu}-238$ & LP 3.1 & 1976 & 0.0033 & 0.0011 & 0.0033 & - \\
\hline $\mathrm{Pu}-238$ & LP 3.2 & 1976 & 0.003 & 0.002 & 0.006 & $\mathrm{U}$ \\
\hline $\mathrm{Pu}-238$ & LP 3.3 & 1976 & 0.004 & 0.002 & 0.006 & $\mathrm{U}$ \\
\hline $\mathrm{Pu}-238$ & LP 5.3 & 1976 & 0.005 & 0.002 & 0.006 & $\mathrm{U}$ \\
\hline $\mathrm{Pu}-238$ & LP 7.2 & 1976 & 0.0032 & 0.0014 & 0.0042 & $\mathrm{U}$ \\
\hline $\mathrm{Pu}-238$ & LP 8.2 & 1976 & 0.002 & 0.002 & 0.006 & $\mathrm{U}$ \\
\hline $\mathrm{Pu}-238$ & A 3.4 & 1977 & 0.0026 & 0.0014 & 0.0042 & $\mathrm{U}$ \\
\hline $\mathrm{Pu}-238$ & LP -2.2 & 1977 & 0 & 0.001 & 0.003 & $\mathrm{U}$ \\
\hline $\mathrm{Pu}-238$ & LP 3.4 & 1977 & 0.0026 & 0.0012 & 0.0036 & $\mathrm{U}$ \\
\hline $\mathrm{Pu}-239 / 240$ & LP 0.3 & 1976 & 0.031 & 0.003 & 0.009 & - \\
\hline $\mathrm{Pu}-239 / 240$ & LP -1.2 & 1976 & 0.034 & 0.003 & 0.009 & - \\
\hline $\mathrm{Pu}-239 / 240$ & LP 2.2 & 1976 & 0.056 & 0.004 & 0.012 & - \\
\hline $\mathrm{Pu}-239 / 240$ & LP 3.1 & 1976 & 0.018 & 0.002 & 0.006 & - \\
\hline $\mathrm{Pu}-239 / 240$ & LP 3.2 & 1976 & 0.039 & 0.003 & 0.009 & - \\
\hline $\mathrm{Pu}-239 / 240$ & LP 3.3 & 1976 & 0.012 & 0.002 & 0.006 & - \\
\hline $\mathrm{Pu}-239 / 240$ & LP 5.3 & 1976 & 0.019 & 0.003 & 0.009 & - \\
\hline $\mathrm{Pu}-239 / 240$ & LP 7.2 & 1976 & 0.022 & 0.003 & 0.009 & - \\
\hline $\mathrm{Pu}-239 / 240$ & LP 8.2 & 1976 & 0.015 & 0.002 & 0.006 & - \\
\hline $\mathrm{Pu}-239 / 240$ & A 3.4 & 1977 & 0.029 & 0.003 & 0.009 & - \\
\hline
\end{tabular}


Table C-2. (continued).

\begin{tabular}{|c|c|c|c|c|c|c|}
\hline Radionuclide & $\begin{array}{c}\text { Sample } \\
\text { Location }\end{array}$ & Date & $\begin{array}{c}\text { Concentrations } \\
(\mathrm{pCi} / \mathrm{g})\end{array}$ & $\begin{array}{l}\text { Uncertainty } \\
(\mathrm{pCi} / \mathrm{g})\end{array}$ & $\begin{array}{l}\text { MDA } \\
(\mathrm{pCi} / \mathrm{g})\end{array}$ & Flag \\
\hline $\mathrm{Pu}-239 / 240$ & LP -2.2 & 1977 & 0.018 & 0.002 & 0.006 & - \\
\hline $\mathrm{Pu}-239 / 240$ & LP 3.4 & 1977 & 0.02 & 0.002 & 0.006 & - \\
\hline $\mathrm{Sb}-125$ & A 3.2 & 1977 & 0.0869 & 0.034 & 0.102 & $\mathrm{U}$ \\
\hline $\mathrm{Sb}-125$ & A 3.5 & 1977 & 0.218 & 0.048 & 0.144 & - \\
\hline $\mathrm{Sb}-125$ & RTC.3 & 2011 & 0.09469 & 0.0301 & 0.0903 & - \\
\hline $\mathrm{Sb}-125$ & RTC-0.1 & 2011 & -0.06318 & 0.0445 & 0.1335 & $\mathrm{U}$ \\
\hline $\mathrm{Sb}-125$ & RTC-1.1 & 2011 & 0.07594 & 0.03200 & 0.096 & $\mathrm{U}$ \\
\hline $\mathrm{Sb}-125$ & RTC-1.2 & 2011 & 0.04583 & 0.0407 & 0.1221 & $\mathrm{U}$ \\
\hline $\mathrm{Sb}-125$ & RTC1.3 & 2011 & 0.08626 & 0.0403 & 0.1209 & $\mathrm{U}$ \\
\hline $\mathrm{Sb}-125$ & RTC1.4 & 2011 & 0.1805 & 0.0395 & 0.1185 & - \\
\hline $\mathrm{Sb}-125$ & RTC-2.2 & 2011 & 0.0267 & 0.0393 & 0.1179 & $\mathrm{U}$ \\
\hline $\mathrm{Sb}-125$ & RTC2.3 & 2011 & 0.7251 & 0.0316 & 0.0948 & - \\
\hline $\mathrm{Sb}-125$ & RTC-6.2 & 2011 & 0.08155 & 0.0461 & 0.1383 & $\mathrm{U}$ \\
\hline $\mathrm{Sb}-125$ & RTC-6.4 & 2011 & 0.09308 & 0.0384 & 0.1152 & $\mathrm{U}$ \\
\hline $\mathrm{Sb}-125$ & RTC-8.2 & 2011 & -0.06156 & 0.0462 & 0.1386 & $\mathrm{U}$ \\
\hline $\mathrm{Sb}-125$ & RTC-A1.2 & 2011 & 0.03776 & 0.029 & 0.087 & $\mathrm{U}$ \\
\hline $\mathrm{Sb}-125$ & RTC-A1.3 & 2011 & 0.05835 & 0.03860 & 0.1158 & $\mathrm{U}$ \\
\hline $\mathrm{Sb}-125$ & RTC-A2.2 & 2011 & 0.04631 & 0.0379 & 0.1137 & $\mathrm{U}$ \\
\hline $\mathrm{Sb}-125$ & RTC-A2.3 & 2011 & 0.07528 & 0.0453 & 0.1359 & $\mathrm{U}$ \\
\hline Sb-125 & RTC-A2.4 & 2011 & -0.01462 & 0.0337 & 0.1011 & $\mathrm{U}$ \\
\hline $\mathrm{Sb}-125$ & RTC-A3.2 & 2011 & 0.06654 & 0.0397 & 0.1191 & $\mathrm{U}$ \\
\hline $\mathrm{Sb}-125$ & RTC-A3.4 & 2011 & 0.002491 & 0.0483 & 0.1449 & $\mathrm{U}$ \\
\hline $\mathrm{Sb}-125$ & RTC-A3.5 & 2011 & 0.03213 & 0.0408 & 0.1224 & $\mathrm{U}$ \\
\hline $\mathrm{Sb}-125$ & RTC-A4.5 & 2011 & 0.09604 & 0.0482 & 0.1446 & $\mathrm{U}$ \\
\hline $\mathrm{Sb}-125$ & RTC.3 & 2012 & 0.005981 & 0.0349 & 0.1047 & $\mathrm{U}$ \\
\hline $\mathrm{Sb}-125$ & RTC-1.2 & 2012 & -0.01076 & 0.0537 & 0.1611 & $\mathrm{U}$ \\
\hline $\mathrm{Sb}-125$ & RTC-2.2 & 2012 & 0.0217 & 0.0393 & 0.1179 & $\mathrm{U}$ \\
\hline Sb-125 & RTC-6.2 & 2012 & -0.004582 & 0.0361 & 0.1083 & $\mathrm{U}$ \\
\hline $\mathrm{Sb}-125$ & RTC-6.4 & 2012 & 0.006869 & 0.0371 & 0.1113 & $\mathrm{U}$ \\
\hline $\mathrm{Sb}-125$ & RTC-A2.2 & 2012 & -0.008378 & 0.0319 & 0.0957 & $\mathrm{U}$ \\
\hline $\mathrm{Sb}-125$ & RTC-A2.3 & 2012 & -0.005957 & 0.0391 & 0.1173 & $\mathrm{U}$ \\
\hline $\mathrm{Sb}-125$ & RTC-A3.2 & 2012 & 0.0008879 & 0.0345 & 0.1035 & $\mathrm{U}$ \\
\hline $\mathrm{Sb}-125$ & RTC-A3.4 & 2012 & 0.001198 & 0.0351 & 0.1053 & $\mathrm{U}$ \\
\hline $\mathrm{Sb}-125$ & RTC-A3.5 & 2012 & -0.003376 & 0.0325 & 0.0975 & $\mathrm{U}$ \\
\hline $\mathrm{Sb}-125$ & $\begin{array}{l}2 \text { INCH AIR } \\
\text { - TRA }\end{array}$ & 2014 & 0.004974 & 0.0449 & 0.1347 & $\mathrm{U}$ \\
\hline Sr-90 & LP 0.1 & 1976 & 0.62 & 0.06 & 0.18 & - \\
\hline
\end{tabular}


Table C-2. (continued).

\begin{tabular}{|c|c|c|c|c|c|c|}
\hline Radionuclide & $\begin{array}{c}\text { Sample } \\
\text { Location }\end{array}$ & Date & $\begin{array}{c}\text { Concentrations } \\
(\mathrm{pCi} / \mathrm{g})\end{array}$ & $\begin{array}{l}\text { Uncertainty } \\
(\mathrm{pCi} / \mathrm{g})\end{array}$ & $\begin{array}{c}\mathrm{MDA} \\
(\mathrm{pCi} / \mathrm{g})\end{array}$ & Flag \\
\hline Sr-90 & LP -1.1 & 1976 & 0.62 & 0.06 & 0.18 & 一 \\
\hline Sr-90 & LP 1.2 & 1976 & 1.01 & 0.06 & 0.18 & 一 \\
\hline Sr-90 & LP -1.2 & 1976 & 0.9 & 0.06 & 0.18 & - \\
\hline Sr-90 & LP 1.3 & 1976 & 0.5 & 0.05 & 0.15 & - \\
\hline Sr-90 & LP 2.1 & 1976 & 0.64 & 0.05 & 0.15 & - \\
\hline Sr-90 & LP 2.2 & 1976 & 0.97 & 0.06 & 0.18 & - \\
\hline Sr-90 & LP 2.3 & 1976 & 0.87 & 0.06 & 0.18 & - \\
\hline Sr-90 & LP 3.1 & 1976 & 0.58 & 0.05 & 0.15 & - \\
\hline Sr-90 & LP 3.2 & 1976 & 0.98 & 0.06 & 0.18 & - \\
\hline Sr-90 & LP 3.3 & 1976 & 0.67 & 0.07 & 0.21 & - \\
\hline Sr-90 & LP 4.4 & 1976 & 0.6 & 0.01 & 0.03 & - \\
\hline Sr-90 & LP 5.3 & 1976 & 0.69 & 0.05 & 0.15 & - \\
\hline Sr-90 & LP 6.3 & 1976 & 0.66 & 0.05 & 0.15 & - \\
\hline Sr-90 & LP 7.2 & 1976 & 0.71 & 0.05 & 0.15 & - \\
\hline Sr-90 & LP 8.2 & 1976 & 0.41 & 0.04 & 0.12 & - \\
\hline Sr-90 & $\mathrm{A} 1.2$ & 1977 & 0.68 & 0.05 & 0.15 & - \\
\hline Sr-90 & A1.3 & 1977 & 0.63 & 0.05 & 0.15 & - \\
\hline Sr-90 & $\mathrm{A} 2.5$ & 1977 & 0.81 & 0.06 & 0.18 & - \\
\hline Sr-90 & A 3.2 & 1977 & 0.93 & 0.06 & 0.18 & - \\
\hline Sr-90 & A3.3 & 1977 & 0.7 & 0.05 & 0.15 & - \\
\hline Sr-90 & A4.5 & 1977 & 0.86 & 0.06 & 0.18 & - \\
\hline Sr-90 & LP 0.2 & 1977 & 0.61 & 0.05 & 0.15 & - \\
\hline Sr-90 & LP 1.4 & 1977 & 0.89 & 0.06 & 0.18 & - \\
\hline Sr-90 & LP -2.2 & 1977 & 0.84 & 0.06 & 0.18 & - \\
\hline Sr-90 & LP -2.3 & 1977 & 0.72 & 0.05 & 0.15 & - \\
\hline Sr-90 & LP 3.4 & 1977 & 1.17 & 0.07 & 0.21 & 一 \\
\hline Sr-90 & LP 6.4 & 1977 & 1.2 & 0.01 & 0.03 & 一 \\
\hline Sr-90 & 8.2 & 1983 & 0.59 & 0.06 & 0.18 & - \\
\hline U-234 & RTC. 3 & 2007 & 45.15 & 2.65 & 7.95 & - \\
\hline U-234 & RTC-0.1 & 2007 & 153 & 4.05 & 12.15 & 一 \\
\hline U-234 & RTC-1.1 & 2007 & 143.5 & 4.02 & 12.06 & - \\
\hline U-234 & RTC-1.2 & 2007 & 122.8 & 3.78 & 11.34 & - \\
\hline U-234 & RTC1.3 & 2007 & 112.5 & 3 & 9 & - \\
\hline U-234 & RTC-1.3 & 2007 & 147.9 & 4.02 & 12.06 & - \\
\hline U-234 & RTC1.4 & 2007 & 149.2 & 4.06 & 12.18 & - \\
\hline U-234 & RTC-2.2 & 2007 & 144 & 4.16 & 12.48 & - \\
\hline U-234 & RTC2.3 & 2007 & 195.3 & 4.85 & 14.55 & - \\
\hline
\end{tabular}


Table C-2. (continued).

\begin{tabular}{|c|c|c|c|c|c|c|}
\hline Radionuclide & $\begin{array}{c}\text { Sample } \\
\text { Location }\end{array}$ & Date & $\begin{array}{c}\text { Concentrations } \\
(\mathrm{pCi} / \mathrm{g})\end{array}$ & $\begin{array}{l}\text { Uncertainty } \\
(\mathrm{pCi} / \mathrm{g})\end{array}$ & $\begin{array}{c}\text { MDA } \\
(\mathrm{pCi} / \mathrm{g})\end{array}$ & Flag \\
\hline U-234 & RTC-2.3 & 2007 & 47.72 & 2.64 & 7.92 & 一 \\
\hline U-234 & RTC-3.4 & 2007 & 128.1 & 3.28 & 9.84 & 一 \\
\hline U-234 & RTC-6.2 & 2007 & 43.22 & 2.82 & 8.46 & - \\
\hline U-234 & RTC-6.4 & 2007 & 146.7 & 3.96 & 11.88 & - \\
\hline U-234 & RTC-8.2 & 2007 & 157.2 & 4.02 & 12.06 & - \\
\hline U-234 & RTC-A1.2 & 2007 & 29.53 & 2.29 & 6.87 & - \\
\hline U-234 & RTC-A1.3 & 2007 & 146 & 3.97 & 11.91 & - \\
\hline U-234 & RTC-A2.2 & 2007 & 114.3 & 3.71 & 11.13 & - \\
\hline U-234 & RTC-A2.3 & 2007 & 145.9 & 3.97 & 11.91 & - \\
\hline U-234 & RTC-A2.4 & 2007 & 68.37 & 2.42 & 7.26 & - \\
\hline U-234 & RTC-A3.2 & 2007 & 154 & 4.24 & 12.72 & - \\
\hline U-234 & RTC-A3.3 & 2007 & 157.5 & 4.09 & 12.27 & - \\
\hline U-234 & RTC-A3.4 & 2007 & 123 & 3.82 & 11.46 & - \\
\hline U-234 & RTC-A3.5 & 2007 & 152 & 4.07 & 12.21 & - \\
\hline U-234 & RTC-A4.5 & 2007 & 139.1 & 4.02 & 12.06 & - \\
\hline U-234 & RTC-3.4 & 2008 & 32.29 & 1.98 & 5.94 & - \\
\hline U-234 & RTC-A3.3 & 2008 & 38.23 & 2.11 & 6.33 & - \\
\hline U-234 & RTC-A3.4 & 2008 & 48.32 & 2.36 & 7.08 & - \\
\hline U-234 & RTC. 3 & 2009 & 2.277 & 0.73 & 2.19 & - \\
\hline U-234 & RTC-A1.3 & 2009 & 6.974 & 3.99 & 11.97 & $\mathrm{U}$ \\
\hline $\mathrm{U}-234$ & RTC-8.2 & 2010 & 143.4 & 4.36 & 13.08 & - \\
\hline U-234 & RTC-A4.5 & 2010 & 24.4 & 0.674 & 2.022 & - \\
\hline U-234 & RTC-1.1 & 2011 & 13.39 & 52.7 & 158.1 & $\mathrm{U}$ \\
\hline U-234 & RTC-A1.2 & 2011 & -34.7 & 61.5 & 184.5 & $\mathrm{U}$ \\
\hline U-234 & RTC.3 & 2012 & -2.746 & 13.7 & 41.1 & U \\
\hline U-234 & RTC-1.2 & 2012 & 9.593 & 37.1 & 111.3 & U \\
\hline U-234 & RTC-2.2 & 2012 & 12.63 & 27.6 & 82.8 & $\mathrm{U}$ \\
\hline U-234 & RTC-6.2 & 2012 & 19.64 & 24.2 & 72.6 & $\mathrm{U}$ \\
\hline U-234 & RTC-6.4 & 2012 & 10.47 & 16.3 & 48.9 & $\mathrm{U}$ \\
\hline U-234 & RTC-A2.2 & 2012 & -3.014 & 25.4 & 76.2 & $\mathrm{U}$ \\
\hline U-234 & RTC-A2.3 & 2012 & 7.015 & 27.8 & 83.4 & $\mathrm{U}$ \\
\hline U-234 & RTC-A3.2 & 2012 & -8.367 & 15.5 & 46.5 & $\mathrm{U}$ \\
\hline U-234 & RTC-A3.4 & 2012 & 3.115 & 18 & 54 & $\mathrm{U}$ \\
\hline U-234 & RTC-A3.5 & 2012 & -35.05 & 26 & 78 & $\mathrm{U}$ \\
\hline U-235 & A 2.2 & 1977 & 0.195 & 0.092 & 0.276 & $\mathrm{U}$ \\
\hline U-235 & $\mathrm{A} 2.3$ & 1977 & 0.131 & 0.015 & 0.045 & 一 \\
\hline U-235 & RTC. 3 & 2006 & 0.9055 & 0.291 & 0.873 & - \\
\hline
\end{tabular}


Table C-2. (continued).

\begin{tabular}{|c|c|c|c|c|c|c|}
\hline Radionuclide & $\begin{array}{c}\text { Sample } \\
\text { Location }\end{array}$ & Date & $\begin{array}{l}\text { Concentrations } \\
(\mathrm{pCi} / \mathrm{g})\end{array}$ & $\begin{array}{l}\text { Uncertainty } \\
(\mathrm{pCi} / \mathrm{g})\end{array}$ & $\begin{array}{c}\text { MDA } \\
(\mathrm{pCi} / \mathrm{g})\end{array}$ & Flag \\
\hline U-235 & RTC-0.1 & 2006 & 0.9713 & 0.4495 & 1.3485 & $\mathrm{U}$ \\
\hline U-235 & RTC-1.1 & 2006 & 0.9688 & 0.3925 & 1.1775 & $\mathrm{U}$ \\
\hline U-235 & RTC-1.2 & 2006 & 2.65 & 0.65 & 1.95 & - \\
\hline U-235 & RTC1.3 & 2006 & 1.444 & 0.62 & 1.86 & $\mathrm{U}$ \\
\hline U-235 & RTC1.4 & 2006 & 1.927 & 0.8 & 2.4 & $\mathrm{U}$ \\
\hline U-235 & RTC-2.2 & 2006 & 1.223 & 0.409 & 1.227 & $\mathrm{U}$ \\
\hline U-235 & RTC2.3 & 2006 & 0.9813 & 0.4375 & 1.3125 & $\mathrm{U}$ \\
\hline $\mathrm{U}-235$ & RTC-2.3 & 2006 & 1.269 & 0.459 & 1.377 & $\mathrm{U}$ \\
\hline $\mathrm{U}-235$ & RTC-3.4 & 2006 & 2.369 & 0.4695 & 1.4085 & - \\
\hline U-235 & RTC-6.2 & 2006 & 1.406 & 0.4315 & 1.2945 & - \\
\hline U-235 & RTC-6.4 & 2006 & 1.248 & 0.429 & 1.287 & $\mathrm{U}$ \\
\hline U-235 & RTC-8.2 & 2006 & 1.358 & 0.4365 & 1.3095 & - \\
\hline U-235 & RTC-A1.2 & 2006 & 0.4553 & 0.1425 & 0.4275 & - \\
\hline U-235 & RTC-A1.3 & 2006 & 0.7162 & 0.316 & 0.948 & $\mathrm{U}$ \\
\hline U-235 & RTC-A2.2 & 2006 & 0.9894 & 0.59 & 1.77 & $\mathrm{U}$ \\
\hline U-235 & RTC-A2.3 & 2006 & 1.373 & 0.387 & 1.161 & - \\
\hline U-235 & RTC-A2.4 & 2006 & 1.567 & 0.665 & 1.995 & $\mathrm{U}$ \\
\hline U-235 & RTC-A3.2 & 2006 & 1.456 & 0.555 & 1.665 & $\mathrm{U}$ \\
\hline U-235 & RTC-A3.3 & 2006 & 0.9995 & 0.35 & 1.05 & $\mathrm{U}$ \\
\hline U-235 & RTC-A3.4 & 2006 & 1.762 & 0.54 & 1.62 & - \\
\hline U-235 & RTC-A3.5 & 2006 & 0.4339 & 0.3785 & 1.1355 & $\mathrm{U}$ \\
\hline U-235 & RTC-A4.5 & 2006 & 2.217 & 0.77 & 2.31 & $\mathrm{U}$ \\
\hline U-235 & RTC. 3 & 2007 & 0.9139 & 0.127 & 0.381 & - \\
\hline U-235 & RTC-0.1 & 2007 & 0.2985 & 0.1 & 0.3 & $\mathrm{U}$ \\
\hline U-235 & RTC-1.1 & 2007 & 0.1771 & 0.0663 & 0.1989 & $\mathrm{U}$ \\
\hline U-235 & RTC-1.2 & 2007 & 0.6334 & 0.11 & 0.33 & - \\
\hline U-235 & RTC1.3 & 2007 & 0.4977 & 0.0934 & 0.2802 & - \\
\hline U-235 & RTC-1.3 & 2007 & 0.1917 & 0.0878 & 0.2634 & $\mathrm{U}$ \\
\hline U-235 & RTC1.4 & 2007 & 0.1173 & 0.0757 & 0.2271 & $\mathrm{U}$ \\
\hline U-235 & RTC-2.2 & 2007 & 0.6459 & 0.127 & 0.381 & - \\
\hline U-235 & RTC2.3 & 2007 & 0.3693 & 0.102 & 0.306 & - \\
\hline U-235 & RTC-2.3 & 2007 & 0.5734 & 0.116 & 0.348 & - \\
\hline U-235 & RTC-3.4 & 2007 & 0.1748 & 0.089 & 0.267 & $\mathrm{U}$ \\
\hline U-235 & RTC-6.2 & 2007 & 0.3796 & 0.0978 & 0.2934 & - \\
\hline U-235 & RTC-6.4 & 2007 & 0.2794 & 0.0842 & 0.2526 & - \\
\hline U-235 & RTC-8.2 & 2007 & 0.677 & 0.116 & 0.348 & - \\
\hline U-235 & RTC-A1.2 & 2007 & 0.1597 & 0.079 & 0.237 & $\mathrm{U}$ \\
\hline
\end{tabular}


Table C-2. (continued).

\begin{tabular}{|c|c|c|c|c|c|c|}
\hline Radionuclide & $\begin{array}{c}\text { Sample } \\
\text { Location }\end{array}$ & Date & $\begin{array}{c}\text { Concentrations } \\
(\mathrm{pCi} / \mathrm{g})\end{array}$ & $\begin{array}{l}\text { Uncertainty } \\
(\mathrm{pCi} / \mathrm{g})\end{array}$ & $\begin{array}{c}\text { MDA } \\
(\mathrm{pCi} / \mathrm{g})\end{array}$ & Flag \\
\hline U-235 & RTC-A1.3 & 2007 & 0.6291 & 0.115 & 0.345 & - \\
\hline U-235 & RTC-A2.2 & 2007 & 0.1401 & 0.0881 & 0.2643 & $\mathrm{U}$ \\
\hline U-235 & RTC-A2.3 & 2007 & 0.2112 & 0.095 & 0.285 & $\mathrm{U}$ \\
\hline $\mathrm{U}-235$ & RTC-A2.4 & 2007 & 0.2275 & 0.0721 & 0.2163 & - \\
\hline U-235 & RTC-A3.2 & 2007 & 0.4716 & 0.114 & 0.342 & - \\
\hline U-235 & RTC-A3.3 & 2007 & 0.7601 & 0.0982 & 0.2946 & - \\
\hline U-235 & RTC-A3.4 & 2007 & 0.3305 & 0.0999 & 0.2997 & - \\
\hline $\mathrm{U}-235$ & RTC-A3.5 & 2007 & 0.2081 & 0.0728 & 0.2184 & $\mathrm{U}$ \\
\hline $\mathrm{U}-235$ & RTC-A4.5 & 2007 & 0.4503 & 0.134 & 0.402 & - \\
\hline U-235 & RTC-3.4 & 2008 & 0.1737 & 0.0716 & 0.2148 & $\mathrm{U}$ \\
\hline U-235 & RTC-A3.3 & 2008 & 0.2766 & 0.0923 & 0.2769 & $\mathrm{U}$ \\
\hline U-235 & RTC-A3.4 & 2008 & 0.1181 & 0.0816 & 0.2448 & $\mathrm{U}$ \\
\hline U-235 & RTC.3 & 2009 & 0.1932 & 0.056 & 0.168 & - \\
\hline $\mathrm{U}-235$ & RTC-1.1 & 2009 & 0.221 & 0.0296 & 0.0888 & - \\
\hline U-235 & RTC-1.2 & 2009 & 0.2174 & 0.055 & 0.165 & - \\
\hline $\mathrm{U}-235$ & RTC-8.2 & 2009 & 0.2465 & 0.0575 & 0.1725 & - \\
\hline U-235 & RTC-A2.2 & 2009 & 0.2696 & 0.058 & 0.174 & - \\
\hline U-235 & RTC-8.2 & 2010 & 1.103 & 0.226 & 0.678 & - \\
\hline U-235 & RTC-A4.5 & 2010 & 0.2353 & 0.0192 & 0.0576 & - \\
\hline U-235 & RTC.3 & 2011 & -0.09157 & 0.154 & 0.462 & $\mathrm{U}$ \\
\hline U-235 & RTC-0.1 & 2011 & 0.1115 & 0.185 & 0.555 & $\mathrm{U}$ \\
\hline U-235 & RTC-1.1 & 2011 & 0.0441 & 0.151 & 0.453 & $\mathrm{U}$ \\
\hline U-235 & RTC-1.2 & 2011 & -0.0257 & 0.191 & 0.573 & $\mathrm{U}$ \\
\hline U-235 & RTC1.3 & 2011 & 0.008094 & 0.201 & 0.603 & $\mathrm{U}$ \\
\hline U-235 & RTC1.4 & 2011 & 0.1213 & 0.167 & 0.501 & $\mathrm{U}$ \\
\hline U-235 & RTC-2.2 & 2011 & 0.1275 & 0.178 & 0.534 & $\mathrm{U}$ \\
\hline U-235 & RTC2.3 & 2011 & -0.01914 & 0.149 & 0.447 & $\mathrm{U}$ \\
\hline U-235 & RTC-6.2 & 2011 & 0.2562 & 0.199 & 0.597 & $\mathrm{U}$ \\
\hline $\mathrm{U}-235$ & RTC-6.4 & 2011 & -0.2428 & 0.18 & 0.54 & $\mathrm{U}$ \\
\hline U-235 & RTC-8.2 & 2011 & 0.1096 & 0.196 & 0.588 & $\mathrm{U}$ \\
\hline U-235 & RTC-A1.2 & 2011 & 0.1473 & 0.151 & 0.453 & $\mathrm{U}$ \\
\hline U-235 & RTC-A1.3 & 2011 & -0.01972 & 0.158 & 0.474 & $\mathrm{U}$ \\
\hline U-235 & RTC-A2.2 & 2011 & 0.0798 & 0.175 & 0.525 & $\mathrm{U}$ \\
\hline U-235 & RTC-A2.3 & 2011 & 0.1007 & 0.207 & 0.621 & $\mathrm{U}$ \\
\hline U-235 & RTC-A2.4 & 2011 & -0.07842 & 0.215 & 0.645 & $\mathrm{U}$ \\
\hline U-235 & RTC-A3.2 & 2011 & -0.08751 & 0.17 & 0.51 & $\mathrm{U}$ \\
\hline U-235 & RTC-A3.4 & 2011 & 0.09186 & 0.195 & 0.585 & $\mathrm{U}$ \\
\hline
\end{tabular}


Table C-2. (continued).

\begin{tabular}{|c|c|c|c|c|c|c|}
\hline Radionuclide & $\begin{array}{c}\text { Sample } \\
\text { Location }\end{array}$ & Date & $\begin{array}{c}\text { Concentrations } \\
(\mathrm{pCi} / \mathrm{g})\end{array}$ & $\begin{array}{l}\text { Uncertainty } \\
(\mathrm{pCi} / \mathrm{g})\end{array}$ & $\begin{array}{c}\mathrm{MDA} \\
(\mathrm{pCi} / \mathrm{g})\end{array}$ & Flag \\
\hline $\mathrm{U}-235$ & RTC-A3.5 & 2011 & -0.01959 & 0.194 & 0.582 & $\mathrm{U}$ \\
\hline U-235 & RTC-A4.5 & 2011 & 0.07577 & 0.227 & 0.681 & $\mathrm{U}$ \\
\hline U-235 & RTC. 3 & 2012 & 0.02181 & 0.134 & 0.402 & $\mathrm{U}$ \\
\hline U-235 & RTC-1.2 & 2012 & 0.02846 & 0.247 & 0.741 & $\mathrm{U}$ \\
\hline U-235 & RTC-2.2 & 2012 & 0.08543 & 0.18 & 0.54 & $\mathrm{U}$ \\
\hline U-235 & RTC-6.2 & 2012 & -0.03779 & 0.154 & 0.462 & $\mathrm{U}$ \\
\hline U-235 & RTC-6.4 & 2012 & 0.03336 & 0.136 & 0.408 & $\mathrm{U}$ \\
\hline U-235 & RTC-A2.2 & 2012 & -0.03647 & 0.162 & 0.486 & $\mathrm{U}$ \\
\hline U-235 & RTC-A2.3 & 2012 & 0.1996 & 0.183 & 0.549 & $\mathrm{U}$ \\
\hline U-235 & RTC-A3.2 & 2012 & 0.0567 & 0.15 & 0.45 & $\mathrm{U}$ \\
\hline $\mathrm{U}-235$ & RTC-A3.4 & 2012 & 0.08665 & 0.167 & 0.501 & $\mathrm{U}$ \\
\hline U-235 & RTC-A3.5 & 2012 & 0.1422 & 0.156 & 0.468 & $\mathrm{U}$ \\
\hline U-235 & $\begin{array}{l}2 \text { INCH AIR } \\
\text { - TRA }\end{array}$ & 2014 & -0.07184 & 0.143 & 0.429 & $\mathrm{U}$ \\
\hline U-238 & RTC.3 & 2006 & 12.52 & 1.365 & 4.095 & - \\
\hline U-238 & RTC-0.1 & 2006 & 19.59 & 2.14 & 6.42 & - \\
\hline U-238 & RTC-1.1 & 2006 & 18.75 & 3.195 & 9.585 & - \\
\hline U-238 & RTC-1.2 & 2006 & 5.185 & 3.955 & 11.865 & $\mathrm{U}$ \\
\hline U-238 & RTC1.3 & 2006 & 30.55 & 3.355 & 10.065 & - \\
\hline U-238 & RTC-1.3 & 2006 & 24.53 & 4.48 & 13.44 & - \\
\hline U-238 & RTC1.4 & 2006 & 23.39 & 2.94 & 8.82 & - \\
\hline U-238 & RTC-2.2 & 2006 & 34.68 & 3.63 & 10.89 & - \\
\hline U-238 & RTC2.3 & 2006 & 7.216 & 2.085 & 6.255 & - \\
\hline U-238 & RTC-2.3 & 2006 & 4.501 & 1.39 & 4.17 & - \\
\hline U-238 & RTC-3.4 & 2006 & 5.708 & 2.265 & 6.795 & $\mathrm{U}$ \\
\hline U-238 & RTC-6.2 & 2006 & 22.9 & 2.465 & 7.395 & 一 \\
\hline U-238 & RTC-6.4 & 2006 & 24.8 & 2.95 & 8.85 & - \\
\hline U-238 & RTC-8.2 & 2006 & 64.21 & 12.25 & 36.75 & - \\
\hline U-238 & RTC-A1.2 & 2006 & 7.786 & 2.165 & 6.495 & - \\
\hline U-238 & RTC-A1.3 & 2006 & 16.09 & 2.065 & 6.195 & - \\
\hline U-238 & RTC-A2.2 & 2006 & 13.78 & 1.66 & 4.98 & - \\
\hline U-238 & RTC-A2.3 & 2006 & 16.77 & 2.195 & 6.585 & - \\
\hline U-238 & RTC-A2.4 & 2006 & 5.576 & 1.04 & 3.12 & - \\
\hline U-238 & RTC-A3.2 & 2006 & 5.006 & 1.215 & 3.645 & 一 \\
\hline U-238 & RTC-A3.3 & 2006 & 19.66 & 2.165 & 6.495 & - \\
\hline U-238 & RTC-A3.4 & 2006 & 23.14 & 2.875 & 8.625 & 一 \\
\hline U-238 & RTC-A3.5 & 2006 & 15.18 & 2.455 & 7.365 & 一 \\
\hline
\end{tabular}


Table C-2. (continued).

\begin{tabular}{|c|c|c|c|c|c|c|}
\hline Radionuclide & $\begin{array}{c}\text { Sample } \\
\text { Location }\end{array}$ & Date & $\begin{array}{l}\text { Concentrations } \\
(\mathrm{pCi} / \mathrm{g})\end{array}$ & $\begin{array}{l}\text { Uncertainty } \\
(\mathrm{pCi} / \mathrm{g})\end{array}$ & $\begin{array}{c}\text { MDA } \\
(\mathrm{pCi} / \mathrm{g})\end{array}$ & Flag \\
\hline U-238 & RTC-A4.5 & 2006 & 12.6 & 5.7 & 17.1 & $\mathrm{U}$ \\
\hline U-238 & RTC. 3 & 2007 & 0 & 3.92 & 11.76 & $\mathrm{U}$ \\
\hline U-238 & RTC-0.1 & 2007 & 0.3659 & 6.04 & 18.12 & $\mathrm{U}$ \\
\hline $\mathrm{U}-238$ & RTC-1.1 & 2007 & 0.2946 & 6 & 18 & $\mathrm{U}$ \\
\hline $\mathrm{U}-238$ & RTC-1.2 & 2007 & 0 & 5.99 & 17.97 & $\mathrm{U}$ \\
\hline U-238 & RTC1.3 & 2007 & 0.1908 & 4.78 & 14.34 & $\mathrm{U}$ \\
\hline $\mathrm{U}-238$ & RTC-1.3 & 2007 & 0.2574 & 5.98 & 17.94 & $\mathrm{U}$ \\
\hline $\mathrm{U}-238$ & RTC1.4 & 2007 & 0.2194 & 6.04 & 18.12 & $\mathrm{U}$ \\
\hline $\mathrm{U}-238$ & RTC-2.2 & 2007 & 0.02662 & 6.57 & 19.71 & $\mathrm{U}$ \\
\hline U-238 & RTC2.3 & 2007 & 0.2639 & 7.62 & 22.86 & $\mathrm{U}$ \\
\hline U-238 & RTC-2.3 & 2007 & 0 & 3.93 & 11.79 & $\mathrm{U}$ \\
\hline U-238 & RTC-3.4 & 2007 & 0.05795 & 5.21 & 15.63 & $\mathrm{U}$ \\
\hline $\mathrm{U}-238$ & RTC-6.2 & 2007 & 0.1963 & 4.03 & 12.09 & $\mathrm{U}$ \\
\hline $\mathrm{U}-238$ & RTC-6.4 & 2007 & 0.2015 & 5.91 & 17.73 & $\mathrm{U}$ \\
\hline U-238 & RTC-8.2 & 2007 & 0.1292 & 6.02 & 18.06 & $\mathrm{U}$ \\
\hline U-238 & RTC-A1.2 & 2007 & 0 & 3.37 & 10.11 & $\mathrm{U}$ \\
\hline $\mathrm{U}-238$ & RTC-A1.3 & 2007 & 0.089 & 5.93 & 17.79 & $\mathrm{U}$ \\
\hline U-238 & RTC-A2.2 & 2007 & 0 & 5.88 & 17.64 & $\mathrm{U}$ \\
\hline U-238 & RTC-A2.3 & 2007 & 0 & 5.92 & 17.76 & $\mathrm{U}$ \\
\hline U-238 & RTC-A2.4 & 2007 & 0.09197 & 3.85 & 11.55 & $\mathrm{U}$ \\
\hline U-238 & RTC-A3.2 & 2007 & 0 & 6.7 & 20.1 & $\mathrm{U}$ \\
\hline U-238 & RTC-A3.3 & 2007 & 0.1634 & 6.09 & 18.27 & $\mathrm{U}$ \\
\hline U-238 & RTC-A3.4 & 2007 & 0 & 6.03 & 18.09 & $\mathrm{U}$ \\
\hline U-238 & RTC-A3.5 & 2007 & 0.09305 & 6.07 & 18.21 & $\mathrm{U}$ \\
\hline U-238 & RTC-A4.5 & 2007 & 0 & 6.31 & 18.93 & $\mathrm{U}$ \\
\hline U-238 & RTC-3.4 & 2008 & 0.559 & 0.278 & 0.834 & $\mathrm{U}$ \\
\hline U-238 & RTC-A3.3 & 2008 & 0.4099 & 0.277 & 0.831 & $\mathrm{U}$ \\
\hline U-238 & RTC-A3.4 & 2008 & 1.683 & 0.341 & 1.023 & - \\
\hline U-238 & RTC.3 & 2011 & 0.9252 & 0.6 & 1.8 & $\mathrm{U}$ \\
\hline U-238 & RTC-0.1 & 2011 & 1.351 & 1.02 & 3.06 & $\mathrm{U}$ \\
\hline U-238 & RTC-1.1 & 2011 & 1.259 & 0.607 & 1.821 & $\mathrm{U}$ \\
\hline U-238 & RTC-1.2 & 2011 & 0.7186 & 0.973 & 2.919 & $\mathrm{U}$ \\
\hline U-238 & RTC1.3 & 2011 & 1.876 & 0.978 & 2.934 & $\mathrm{U}$ \\
\hline U-238 & RTC1.4 & 2011 & 1.503 & 0.925 & 2.775 & $\mathrm{U}$ \\
\hline U-238 & RTC-2.2 & 2011 & 1.573 & 0.826 & 2.478 & $\mathrm{U}$ \\
\hline U-238 & RTC2.3 & 2011 & 0.9657 & 0.58 & 1.74 & $\mathrm{U}$ \\
\hline U-238 & RTC-6.2 & 2011 & -0.04248 & 0.979 & 2.937 & $\mathrm{U}$ \\
\hline
\end{tabular}


Table C-2. (continued).

\begin{tabular}{|c|c|c|c|c|c|c|}
\hline Radionuclide & $\begin{array}{l}\text { Sample } \\
\text { Location }\end{array}$ & Date & $\begin{array}{l}\text { Concentrations } \\
(\mathrm{pCi} / \mathrm{g})\end{array}$ & $\begin{array}{l}\text { Uncertainty } \\
\text { (pCi } / \mathrm{g})\end{array}$ & $\begin{array}{c}\mathrm{MDA} \\
(\mathrm{pCi} / \mathrm{g})\end{array}$ & Flag \\
\hline U-238 & RTC-6.4 & 2011 & 1.022 & 0.98 & 2.94 & $\mathrm{U}$ \\
\hline U-238 & RTC-8.2 & 2011 & 1.705 & 1.04 & 3.12 & $\mathrm{U}$ \\
\hline $\mathrm{U}-238$ & RTC-A1.2 & 2011 & 0.9353 & 0.652 & 1.956 & $\mathrm{U}$ \\
\hline $\mathrm{U}-238$ & RTC-A1.3 & 2011 & 1.328 & 0.798 & 2.394 & $\mathrm{U}$ \\
\hline $\mathrm{U}-238$ & RTC-A2.2 & 2011 & 1.13 & 0.843 & 2.529 & $\mathrm{U}$ \\
\hline U-238 & RTC-A2.3 & 2011 & 1.431 & 1.04 & 3.12 & $\mathrm{U}$ \\
\hline $\mathrm{U}-238$ & RTC-A2.4 & 2011 & 1.149 & 0.494 & 1.482 & $\mathrm{U}$ \\
\hline $\mathrm{U}-238$ & RTC-A3.2 & 2011 & 1.414 & 0.826 & 2.478 & $\mathrm{U}$ \\
\hline $\mathrm{U}-238$ & RTC-A3.4 & 2011 & 2.902 & 1.07 & 3.21 & $\mathrm{U}$ \\
\hline $\mathrm{U}-238$ & RTC-A3.5 & 2011 & 1.13 & 0.651 & 1.953 & $\mathrm{U}$ \\
\hline $\mathrm{U}-238$ & RTC-A4.5 & 2011 & 1.508 & 1.07 & 3.21 & $\mathrm{U}$ \\
\hline U-238 & RTC.3 & 2012 & 2.095 & 0.816 & 2.448 & $\mathrm{U}$ \\
\hline $\mathrm{U}-238$ & RTC-1.2 & 2012 & 0.9104 & 1.19 & 3.57 & $\mathrm{U}$ \\
\hline $\mathrm{U}-238$ & RTC-2.2 & 2012 & 1.964 & 0.904 & 2.712 & $\mathrm{U}$ \\
\hline U-238 & RTC-6.2 & 2012 & 0.7613 & 0.774 & 2.322 & $\mathrm{U}$ \\
\hline U-238 & RTC-6.4 & 2012 & 0.9675 & 0.828 & 2.484 & $\mathrm{U}$ \\
\hline U-238 & RTC-A2.2 & 2012 & 1.347 & 0.805 & 2.415 & $\mathrm{U}$ \\
\hline U-238 & RTC-A2.3 & 2012 & 0.5758 & 0.887 & 2.661 & $\mathrm{U}$ \\
\hline U-238 & RTC-A3.2 & 2012 & 0 & 0.198 & 0.594 & $\mathrm{U}$ \\
\hline U-238 & RTC-A3.4 & 2012 & 1.145 & 0.877 & 2.631 & $\mathrm{U}$ \\
\hline U-238 & RTC-A3.5 & 2012 & 0.1262 & 0.712 & 2.136 & $\mathrm{U}$ \\
\hline U-238 & $\begin{array}{l}2 \text { INCH AIR } \\
\text { - TRA }\end{array}$ & 2014 & 1.428 & 0.551 & 1.653 & $\mathrm{U}$ \\
\hline
\end{tabular}

Table C-3. Radionuclides concentrations in surface soils associated with INTEC.

\begin{tabular}{|c|c|c|c|c|c|c|}
\hline Radionuclide & $\begin{array}{c}\text { Sample } \\
\text { Location }\end{array}$ & Date & $\begin{array}{c}\text { Concentrations } \\
(\mathrm{pCi} / \mathrm{g})\end{array}$ & $\begin{array}{c}\text { Uncertainty } \\
(\mathrm{pCi} / \mathrm{g})\end{array}$ & $\begin{array}{c}\text { MDA } \\
(\mathrm{pCi} / \mathrm{g})\end{array}$ & Flag \\
\hline Am-241 & A118 & 1974 & 0.0036 & 0.0022 & 0.0066 & $\mathrm{U}$ \\
\hline Am-241 & A120 & 1974 & -0.0009 & 0.0009 & 0.0027 & $\mathrm{U}$ \\
\hline Am-241 & A52 & 1974 & 0.0036 & 0.00135 & 0.00405 & $\mathrm{U}$ \\
\hline Am-241 & A71 & 1974 & 0.0059 & 0.0013 & 0.0039 & - \\
\hline Am-241 & A87 & 1974 & 0.005 & 0.0018 & 0.0054 & $\mathrm{U}$ \\
\hline Am-241 & A91 & 1974 & 0.0018 & 0.0013 & 0.0039 & $\mathrm{U}$ \\
\hline Am-241 & B117 & 1974 & 0.0054 & 0.0013 & 0.0039 & - \\
\hline Am-241 & B120 & 1974 & 0.0045 & 0.004 & 0.012 & $\mathrm{U}$ \\
\hline Am-241 & B5 & 1974 & 0.0068 & 0.0018 & 0.0054 & - \\
\hline Am-241 & B61 & 1974 & 0.009 & 0.0032 & 0.0096 & $\mathrm{U}$ \\
\hline
\end{tabular}


Table C-3. (continued).

\begin{tabular}{|c|c|c|c|c|c|c|}
\hline Radionuclide & $\begin{array}{l}\text { Sample } \\
\text { Location }\end{array}$ & Date & $\begin{array}{c}\text { Concentrations } \\
(\mathrm{pCi} / \mathrm{g})\end{array}$ & $\begin{array}{l}\text { Uncertainty } \\
(\mathrm{pCi} / \mathrm{g})\end{array}$ & $\begin{array}{l}\text { MDA } \\
(\mathrm{pCi} / \mathrm{g})\end{array}$ & Flag \\
\hline Am-241 & B79 & 1974 & 0.016 & 0.002 & 0.006 & - \\
\hline Am-241 & B86 & 1974 & -0.1 & 0 & 0 & $\mathrm{U}$ \\
\hline Am-241 & B90 & 1974 & 0.0041 & 0.0022 & 0.0066 & $\mathrm{U}$ \\
\hline Am-241 & $\mathrm{C} 36$ & 1974 & 0.006 & 0.002 & 0.006 & - \\
\hline Am-241 & $\mathrm{C} 46$ & 1974 & 0.0023 & 0.0016 & 0.0048 & $\mathrm{U}$ \\
\hline Am-241 & D47 & 1974 & 0.0041 & 0.0013 & 0.0039 & 一 \\
\hline Am-241 & A-110 & 1982 & 0.015 & 0.003 & 0.009 & - \\
\hline Am-241 & A-120 & 1982 & 0.012 & 0.003 & 0.009 & - \\
\hline Am-241 & B-120 & 1982 & 0.014 & 0.003 & 0.009 & - \\
\hline Am-241 & B-25 & 1982 & 0.011 & 0.002 & 0.006 & - \\
\hline Am-241 & B-69 & 1982 & 0.016 & 0.003 & 0.009 & - \\
\hline Am-241 & C-21 & 1982 & 0.007 & 0.003 & 0.009 & $\mathrm{U}$ \\
\hline Am-241 & D-114 & 1982 & 0.026 & 0.003 & 0.009 & - \\
\hline Am-241 & D-96 & 1982 & 0.007 & 0.002 & 0.006 & 一 \\
\hline Am-241 & A108 & 1989 & 0.029 & 0.004 & 0.012 & 一 \\
\hline Am-241 & A17 & 1989 & 0.007 & 0.002 & 0.006 & 一 \\
\hline Am-241 & A66 & 1989 & 0.01 & 0.002 & 0.006 & - \\
\hline Am-241 & $\mathrm{B} 25$ & 1989 & 0.008 & 0.002 & 0.006 & - \\
\hline Am-241 & B90 & 1989 & 0.016 & 0.003 & 0.009 & - \\
\hline Am-241 & B96 & 1989 & 0.035 & 0.004 & 0.012 & 一 \\
\hline Am-241 & $\mathrm{C} 48$ & 1989 & 0.0026 & 0.0016 & 0.0048 & $\mathrm{U}$ \\
\hline Am-241 & C79 & 1989 & 0.004 & 0.002 & 0.006 & $\mathrm{U}$ \\
\hline Am-241 & D30 & 1989 & 0.0037 & 0.0013 & 0.0039 & $\mathrm{U}$ \\
\hline Am-241 & D96 & 1989 & 0.0014 & 0.0016 & 0.0048 & $\mathrm{U}$ \\
\hline Am-241 & A15 & 2007 & 0.06804 & 0.15 & 0.45 & $\mathrm{U}$ \\
\hline Am-241 & A17 & 2007 & -0.0643 & 0.102 & 0.306 & $\mathrm{U}$ \\
\hline Am-241 & A27 & 2007 & 0.1325 & 0.0585 & 0.1755 & $\mathrm{U}$ \\
\hline Am-241 & A28 & 2007 & 0.2065 & 0.1075 & 0.3225 & $\mathrm{U}$ \\
\hline Am-241 & A38 & 2007 & 0.1352 & 0.0745 & 0.2235 & $\mathrm{U}$ \\
\hline Am-241 & A4 & 2007 & -0.06878 & 0.1045 & 0.3135 & $\mathrm{U}$ \\
\hline Am-241 & A44 & 2007 & 0.1063 & 0.084 & 0.252 & $\mathrm{U}$ \\
\hline Am-241 & A45 & 2007 & 0.06609 & 0.082 & 0.2445 & $\mathrm{U}$ \\
\hline Am-241 & A47 & 2007 & 0.1804 & 0.1075 & 0.3225 & $\mathrm{U}$ \\
\hline Am-241 & A48 & 2007 & 0.09976 & 0.0835 & 0.2505 & $\mathrm{U}$ \\
\hline Am-241 & A49 & 2007 & 0.06879 & 0.0795 & 0.2385 & $\mathrm{U}$ \\
\hline Am-241 & A5 & 2007 & 0.05341 & 0.02825 & 0.08475 & $\mathrm{U}$ \\
\hline Am-241 & A50 & 2007 & 0.1743 & 0.076 & 0.228 & $\mathrm{U}$ \\
\hline
\end{tabular}


Table C-3. (continued).

\begin{tabular}{|c|c|c|c|c|c|c|}
\hline Radionuclide & $\begin{array}{l}\text { Sample } \\
\text { Location }\end{array}$ & Date & $\begin{array}{c}\text { Concentrations } \\
(\mathrm{pCi} / \mathrm{g})\end{array}$ & $\begin{array}{l}\text { Uncertainty } \\
(\mathrm{pCi} / \mathrm{g})\end{array}$ & $\begin{array}{l}\text { MDA } \\
(\mathrm{pCi} / \mathrm{g})\end{array}$ & Flag \\
\hline Am-241 & A58 & 2007 & 0.04673 & 0.0496 & 0.1488 & $\mathrm{U}$ \\
\hline Am-241 & A59 & 2007 & 0.01033 & 0.04625 & 0.13875 & $\mathrm{U}$ \\
\hline Am-241 & A6 & 2007 & 0.1427 & 0.065 & 0.195 & $\mathrm{U}$ \\
\hline Am-241 & A60 & 2007 & 0.1114 & 0.1055 & 0.3165 & $\mathrm{U}$ \\
\hline Am-241 & A61 & 2007 & 0.1187 & 0.074 & 0.222 & $\mathrm{U}$ \\
\hline Am-241 & A67 & 2007 & 0.1141 & 0.0795 & 0.2385 & $\mathrm{U}$ \\
\hline Am-241 & A69 & 2007 & 0.3781 & 0.1695 & 0.5085 & $\mathrm{U}$ \\
\hline Am-241 & A70 & 2007 & 0.07273 & 0.0463 & 0.1389 & $\mathrm{U}$ \\
\hline Am-241 & A71 & 2007 & 0.1941 & 0.04685 & 0.14055 & - \\
\hline Am-241 & A72 & 2007 & 0.09614 & 0.0715 & 0.2145 & $\mathrm{U}$ \\
\hline Am-241 & B101 & 2007 & 0.1143 & 0.072 & 0.216 & $\mathrm{U}$ \\
\hline Am-241 & B102 & 2007 & 0.1264 & 0.07 & 0.21 & $\mathrm{U}$ \\
\hline Am-241 & $\mathrm{B} 103$ & 2007 & 0.1088 & 0.066 & 0.198 & $\mathrm{U}$ \\
\hline Am-241 & B104 & 2007 & 0.01965 & 0.039 & 0.11745 & $\mathrm{U}$ \\
\hline Am-241 & B105 & 2007 & 0.2345 & 0.087 & 0.261 & $\mathrm{U}$ \\
\hline Am-241 & $\mathrm{B} 14$ & 2007 & 0.02149 & 0.0311 & 0.0933 & $\mathrm{U}$ \\
\hline Am-241 & $\mathrm{B} 17$ & 2007 & 0.1611 & 0.0835 & 0.2505 & $\mathrm{U}$ \\
\hline Am-241 & $\mathrm{B} 25$ & 2007 & 0.03616 & 0.056 & 0.168 & $\mathrm{U}$ \\
\hline Am-241 & B26 & 2007 & 0.1273 & 0.104 & 0.312 & $\mathrm{U}$ \\
\hline Am-241 & B28 & 2007 & -0.01002 & 0.0785 & 0.2355 & $\mathrm{U}$ \\
\hline Am-241 & B3 & 2007 & 0.08054 & 0.089 & 0.267 & $\mathrm{U}$ \\
\hline Am-241 & B40 & 2007 & -0.1091 & 0.166 & 0.498 & $\mathrm{U}$ \\
\hline Am-241 & B47 & 2007 & 0.1002 & 0.195 & 0.585 & $\mathrm{U}$ \\
\hline Am-241 & B48 & 2007 & 0.1517 & 0.087 & 0.261 & $\mathrm{U}$ \\
\hline Am-241 & B50 & 2007 & 0.1383 & 0.095 & 0.285 & $\mathrm{U}$ \\
\hline Am-241 & B51 & 2007 & 0.03342 & 0.1275 & 0.3825 & $\mathrm{U}$ \\
\hline Am-241 & B52 & 2007 & 0.06706 & 0.0685 & 0.2055 & $\mathrm{U}$ \\
\hline Am-241 & B59 & 2007 & 0.6702 & 0.1725 & 0.5175 & - \\
\hline Am-241 & B60 & 2007 & 0.2608 & 0.1525 & 0.4575 & $\mathrm{U}$ \\
\hline Am-241 & B61 & 2007 & 0.132 & 0.0605 & 0.1815 & $\mathrm{U}$ \\
\hline Am-241 & B62 & 2007 & 0.05238 & 0.04515 & 0.13545 & $\mathrm{U}$ \\
\hline Am-241 & B71 & 2007 & 0.4585 & 0.1475 & 0.4425 & - \\
\hline Am-241 & B73 & 2007 & 0.02904 & 0.0815 & 0.2445 & $\mathrm{U}$ \\
\hline Am-241 & B80 & 2007 & 0.9001 & 0.1985 & 0.5955 & - \\
\hline Am-241 & B81 & 2007 & 0.1479 & 0.1185 & 0.3555 & $\mathrm{U}$ \\
\hline Am-241 & B82 & 2007 & 0.1931 & 0.1405 & 0.4215 & $\mathrm{U}$ \\
\hline Am-241 & B83 & 2007 & 0.05148 & 0.0474 & 0.1422 & $\mathrm{U}$ \\
\hline
\end{tabular}


Table C-3. (continued).

\begin{tabular}{|c|c|c|c|c|c|c|}
\hline Radionuclide & $\begin{array}{l}\text { Sample } \\
\text { Location }\end{array}$ & Date & $\begin{array}{c}\text { Concentrations } \\
(\mathrm{pCi} / \mathrm{g})\end{array}$ & $\begin{array}{l}\text { Uncertainty } \\
(\mathrm{pCi} / \mathrm{g})\end{array}$ & $\begin{array}{l}\text { MDA } \\
(\mathrm{pCi} / \mathrm{g})\end{array}$ & Flag \\
\hline Am-241 & B88 & 2007 & 0.15 & 0.0785 & 0.2355 & $\mathrm{U}$ \\
\hline Am-241 & B90 & 2007 & -0.04847 & 0.094 & 0.282 & $\mathrm{U}$ \\
\hline Am-241 & B93 & 2007 & 0.2399 & 0.0635 & 0.1905 & - \\
\hline Am-241 & B94 & 2007 & 0.08205 & 0.093 & 0.279 & $\mathrm{U}$ \\
\hline Am-241 & B95 & 2007 & 0.06123 & 0.1045 & 0.3135 & $\mathrm{U}$ \\
\hline Am-241 & B99 & 2007 & 0.1238 & 0.09 & 0.27 & $\mathrm{U}$ \\
\hline Am-241 & $\mathrm{C} 14$ & 2007 & 0.2261 & 0.0955 & 0.2865 & $\mathrm{U}$ \\
\hline Am-241 & $\mathrm{C} 15$ & 2007 & 0.2863 & 0.111 & 0.333 & $\mathrm{U}$ \\
\hline Am-241 & C37 & 2007 & 0.05351 & 0.1285 & 0.3855 & $\mathrm{U}$ \\
\hline Am-241 & $\mathrm{C} 46$ & 2007 & 0.0476 & 0.03275 & 0.09825 & $\mathrm{U}$ \\
\hline Am-241 & $\mathrm{C} 48$ & 2007 & -0.07859 & 0.078 & 0.234 & $\mathrm{U}$ \\
\hline Am-241 & C56 & 2007 & 0.1334 & 0.0765 & 0.2295 & $\mathrm{U}$ \\
\hline Am-241 & C57 & 2007 & 0.1398 & 0.0595 & 0.1785 & $\mathrm{U}$ \\
\hline Am-241 & D58 & 2007 & 0.1833 & 0.082 & 0.246 & $\mathrm{U}$ \\
\hline Am-241 & D68 & 2007 & 0.159 & 0.102 & 0.306 & $\mathrm{U}$ \\
\hline Am-241 & D69 & 2007 & 0.0446 & 0.04495 & 0.13485 & $\mathrm{U}$ \\
\hline Am-241 & D80 & 2007 & 0.05021 & 0.103 & 0.309 & $\mathrm{U}$ \\
\hline Am-241 & D89 & 2007 & 0.1919 & 0.0805 & 0.2415 & $\mathrm{U}$ \\
\hline Am-241 & D90 & 2007 & 0.2088 & 0.0605 & 0.1815 & - \\
\hline Am-241 & D91 & 2007 & 0.2014 & 0.085 & 0.255 & $\mathrm{U}$ \\
\hline Am-241 & A15 & 2011 & 0.01596 & 0.0785 & 0.2355 & $\mathrm{U}$ \\
\hline Am-241 & A16 & 2011 & 0.04304 & 0.0976 & 0.2928 & $\mathrm{U}$ \\
\hline Am-241 & A17 & 2011 & -0.002077 & 0.0579 & 0.1737 & $\mathrm{U}$ \\
\hline Am-241 & A27 & 2011 & -0.01936 & 0.107 & 0.321 & $\mathrm{U}$ \\
\hline Am-241 & A28 & 2011 & 0.03814 & 0.0693 & 0.2079 & $\mathrm{U}$ \\
\hline Am-241 & A38 & 2011 & 0.0784 & 0.104 & 0.312 & $\mathrm{U}$ \\
\hline Am-241 & A39 & 2011 & 0.03364 & 0.102 & 0.306 & $\mathrm{U}$ \\
\hline Am-241 & A4 & 2011 & -0.01331 & 0.106 & 0.318 & $\mathrm{U}$ \\
\hline Am-241 & A44 & 2011 & 0.0781 & 0.163 & 0.489 & $\mathrm{U}$ \\
\hline Am-241 & A47 & 2011 & -0.006958 & 0.118 & 0.354 & $\mathrm{U}$ \\
\hline Am-241 & A49 & 2011 & 0.1063 & 0.122 & 0.366 & $\mathrm{U}$ \\
\hline Am-241 & A50 & 2011 & -0.01074 & 0.108 & 0.324 & $\mathrm{U}$ \\
\hline Am-241 & A55 & 2011 & 0.1464 & 0.118 & 0.354 & $\mathrm{U}$ \\
\hline Am-241 & A58 & 2011 & 0.05153 & 0.12 & 0.36 & $\mathrm{U}$ \\
\hline Am-241 & A59 & 2011 & 0.01319 & 0.106 & 0.318 & $\mathrm{U}$ \\
\hline Am-241 & A6 & 2011 & -0.004171 & 0.062 & 0.186 & $\mathrm{U}$ \\
\hline Am-241 & A61 & 2011 & 0.02828 & 0.115 & 0.345 & $\mathrm{U}$ \\
\hline
\end{tabular}


Table C-3. (continued).

\begin{tabular}{|c|c|c|c|c|c|c|}
\hline Radionuclide & $\begin{array}{l}\text { Sample } \\
\text { Location }\end{array}$ & Date & $\begin{array}{c}\text { Concentrations } \\
(\mathrm{pCi} / \mathrm{g})\end{array}$ & $\begin{array}{l}\text { Uncertainty } \\
(\mathrm{pCi} / \mathrm{g})\end{array}$ & $\begin{array}{c}\mathrm{MDA} \\
(\mathrm{pCi} / \mathrm{g})\end{array}$ & Flag \\
\hline Am-241 & A66 & 2011 & -0.03881 & 0.0644 & 0.1932 & $\mathrm{U}$ \\
\hline Am-241 & A69 & 2011 & 0.0275 & 0.12 & 0.36 & $\mathrm{U}$ \\
\hline Am-241 & A70 & 2011 & 0.05056 & 0.102 & 0.306 & $\mathrm{U}$ \\
\hline Am-241 & A72 & 2011 & 0.08474 & 0.11 & 0.33 & $\mathrm{U}$ \\
\hline Am-241 & $\mathrm{B} 101$ & 2011 & -0.002668 & 0.061 & 0.183 & $\mathrm{U}$ \\
\hline Am-241 & $\mathrm{B} 102$ & 2011 & 0.06281 & 0.121 & 0.363 & $\mathrm{U}$ \\
\hline Am-241 & B103 & 2011 & -0.04662 & 0.118 & 0.354 & $\mathrm{U}$ \\
\hline Am-241 & B104 & 2011 & 0.07609 & 0.0999 & 0.2997 & $\mathrm{U}$ \\
\hline Am-241 & B106 & 2011 & -0.00133 & 0.0706 & 0.2118 & $\mathrm{U}$ \\
\hline Am-241 & B14 & 2011 & 0.02834 & 0.0756 & 0.2268 & $\mathrm{U}$ \\
\hline Am-241 & B15 & 2011 & -0.05507 & 0.0999 & 0.2997 & $\mathrm{U}$ \\
\hline Am-241 & B17 & 2011 & -0.001653 & 0.134 & 0.402 & $\mathrm{U}$ \\
\hline Am-241 & $\mathrm{B} 25$ & 2011 & -0.005495 & 0.0975 & 0.2925 & $\mathrm{U}$ \\
\hline Am-241 & $\mathrm{B} 28$ & 2011 & -0.001728 & 0.0971 & 0.2913 & $\mathrm{U}$ \\
\hline Am-241 & B29 & 2011 & 0.1035 & 0.103 & 0.309 & $\mathrm{U}$ \\
\hline Am-241 & B3 & 2011 & 0.01172 & 0.105 & 0.315 & $\mathrm{U}$ \\
\hline Am-241 & B39 & 2011 & 0.02529 & 0.136 & 0.408 & $\mathrm{U}$ \\
\hline Am-241 & B4 & 2011 & 0.07723 & 0.0692 & 0.2076 & $\mathrm{U}$ \\
\hline Am-241 & B40 & 2011 & -0.09726 & 0.127 & 0.381 & $\mathrm{U}$ \\
\hline Am-241 & B47 & 2011 & -0.03913 & 0.109 & 0.327 & $\mathrm{U}$ \\
\hline Am-241 & B48 & 2011 & -0.06353 & 0.131 & 0.393 & $\mathrm{U}$ \\
\hline Am-241 & B50 & 2011 & 0.02622 & 0.097 & 0.291 & $\mathrm{U}$ \\
\hline Am-241 & B52 & 2011 & -0.005631 & 0.089 & 0.267 & $\mathrm{U}$ \\
\hline Am-241 & B61 & 2011 & -0.03311 & 0.0718 & 0.2154 & $\mathrm{U}$ \\
\hline Am-241 & B62 & 2011 & -0.05575 & 0.067 & 0.201 & $\mathrm{U}$ \\
\hline Am-241 & B71 & 2011 & -0.002432 & 0.195 & 0.585 & $\mathrm{U}$ \\
\hline Am-241 & B73 & 2011 & -0.02071 & 0.0687 & 0.2061 & $\mathrm{U}$ \\
\hline Am-241 & B83 & 2011 & 0.04614 & 0.059 & 0.177 & $\mathrm{U}$ \\
\hline Am-241 & B84 & 2011 & -0.04101 & 0.0703 & 0.2109 & $\mathrm{U}$ \\
\hline Am-241 & B89 & 2011 & 0.09178 & 0.129 & 0.387 & $\mathrm{U}$ \\
\hline Am-241 & B90 & 2011 & -0.009398 & 0.0779 & 0.2337 & $\mathrm{U}$ \\
\hline Am-241 & B91 & 2011 & -0.08238 & 0.151 & 0.453 & $\mathrm{U}$ \\
\hline Am-241 & B92 & 2011 & -0.03261 & 0.163 & 0.489 & $\mathrm{U}$ \\
\hline Am-241 & B93 & 2011 & 0.1489 & 0.0984 & 0.2952 & $\mathrm{U}$ \\
\hline Am-241 & B94 & 2011 & -0.05024 & 0.0952 & 0.2856 & $\mathrm{U}$ \\
\hline Am-241 & B99 & 2011 & 0.004382 & 0.0602 & 0.1806 & $\mathrm{U}$ \\
\hline Am-241 & $\mathrm{C} 15$ & 2011 & 0.006492 & 0.0557 & 0.1671 & $\mathrm{U}$ \\
\hline
\end{tabular}


Table C-3. (continued).

\begin{tabular}{|c|c|c|c|c|c|c|}
\hline Radionuclide & $\begin{array}{l}\text { Sample } \\
\text { Location }\end{array}$ & Date & $\begin{array}{c}\text { Concentrations } \\
(\mathrm{pCi} / \mathrm{g})\end{array}$ & $\begin{array}{l}\text { Uncertainty } \\
(\mathrm{pCi} / \mathrm{g})\end{array}$ & $\begin{array}{l}\text { MDA } \\
(\mathrm{pCi} / \mathrm{g})\end{array}$ & Flag \\
\hline Am-241 & $\mathrm{C} 25$ & 2011 & -0.04044 & 0.115 & 0.345 & $\mathrm{U}$ \\
\hline Am-241 & C37 & 2011 & 0.02541 & 0.0671 & 0.2013 & $\mathrm{U}$ \\
\hline Am-241 & $\mathrm{C} 46$ & 2011 & 0.01323 & 0.0696 & 0.2088 & $\mathrm{U}$ \\
\hline Am-241 & $\mathrm{C} 48$ & 2011 & -0.0001466 & 0.0874 & 0.2622 & $\mathrm{U}$ \\
\hline Am-241 & C56 & 2011 & 0.0575 & 0.067 & 0.201 & $\mathrm{U}$ \\
\hline Am-241 & C57 & 2011 & -0.008812 & 0.0665 & 0.1995 & $\mathrm{U}$ \\
\hline Am-241 & D58 & 2011 & 0.00009982 & 0.0897 & 0.2691 & $\mathrm{U}$ \\
\hline Am-241 & D68 & 2011 & 0.01191 & 0.0875 & 0.2625 & $\mathrm{U}$ \\
\hline Am-241 & D89 & 2011 & 0.03372 & 0.105 & 0.315 & $\mathrm{U}$ \\
\hline Am-241 & D91 & 2011 & -0.08838 & 0.0602 & 0.1806 & $\mathrm{U}$ \\
\hline Am-241 & A15 & 2012 & 0.005052 & 0.0875 & 0.2625 & $\mathrm{U}$ \\
\hline Am-241 & A38 & 2012 & 0.05491 & 0.107 & 0.321 & $\mathrm{U}$ \\
\hline Am-241 & A44 & 2012 & 0.05469 & 0.127 & 0.381 & $\mathrm{U}$ \\
\hline Am-241 & A47 & 2012 & 0.1363 & 0.115 & 0.345 & $\mathrm{U}$ \\
\hline Am-241 & A59 & 2012 & 0.08795 & 0.0935 & 0.2805 & $\mathrm{U}$ \\
\hline Am-241 & A6 & 2012 & 0.1148 & 0.104 & 0.312 & $\mathrm{U}$ \\
\hline Am-241 & A69 & 2012 & 0.3527 & 0.135 & 0.405 & $\mathrm{U}$ \\
\hline Am-241 & A70 & 2012 & -0.02921 & 0.0802 & 0.2406 & $\mathrm{U}$ \\
\hline Am-241 & A72 & 2012 & -0.03679 & 0.0553 & 0.1659 & $\mathrm{U}$ \\
\hline Am-241 & B14 & 2012 & 0.01437 & 0.0571 & 0.1713 & $\mathrm{U}$ \\
\hline Am-241 & B48 & 2012 & 0.007055 & 0.133 & 0.399 & $\mathrm{U}$ \\
\hline Am-241 & B83 & 2012 & 0.002165 & 0.0933 & 0.2799 & $\mathrm{U}$ \\
\hline Am-241 & $\mathrm{C} 46$ & 2012 & 0.01585 & 0.111 & 0.333 & $\mathrm{U}$ \\
\hline Am-241 & C57 & 2012 & -0.009905 & 0.1 & 0.3 & $\mathrm{U}$ \\
\hline Am-241 & $\begin{array}{l}2 \text { INCH AIR } \\
\text { - INTEC }\end{array}$ & 2013 & 0.01194 & 0.0803 & 0.241 & $\mathrm{U}$ \\
\hline Am-241 & A47 & 2013 & -0.05179 & 0.0917 & 0.275 & $\mathrm{U}$ \\
\hline Am-241 & A49 & 2013 & 0.008678 & 0.0653 & 0.196 & $\mathrm{U}$ \\
\hline Am-241 & A59 & 2013 & -0.0009546 & 0.0657 & 0.197 & $\mathrm{U}$ \\
\hline Am-241 & A71 & 2013 & 0.02736 & 0.0787 & 0.236 & $\mathrm{U}$ \\
\hline Am-241 & A72 & 2013 & 0.03325 & 0.0637 & 0.191 & $\mathrm{U}$ \\
\hline Am-241 & $\begin{array}{c}2 \text { INCH AIR } \\
- \text { CPP }\end{array}$ & 2014 & -0.01026 & 0.129 & 0.387 & $\mathrm{U}$ \\
\hline Am-241 & $\begin{array}{l}2 \text { INCH AIR } \\
\text { - INTEC }\end{array}$ & 2014 & 0.01929 & 0.0544 & 0.1632 & $\mathrm{U}$ \\
\hline Am-241 & A38 & 2014 & 0.01513 & 0.0507 & 0.1521 & $\mathrm{U}$ \\
\hline Am-241 & A47 & 2014 & 0.05181 & 0.104 & 0.312 & $\mathrm{U}$ \\
\hline Am-241 & A58 & 2014 & 0.04227 & 0.0538 & 0.1614 & $\mathrm{U}$ \\
\hline
\end{tabular}


Table C-3. (continued).

\begin{tabular}{|c|c|c|c|c|c|c|}
\hline Radionuclide & $\begin{array}{c}\text { Sample } \\
\text { Location }\end{array}$ & Date & $\begin{array}{c}\text { Concentrations } \\
(\mathrm{pCi} / \mathrm{g})\end{array}$ & $\begin{array}{l}\text { Uncertainty } \\
(\mathrm{pCi} / \mathrm{g})\end{array}$ & $\begin{array}{c}\text { MDA } \\
(\mathrm{pCi} / \mathrm{g})\end{array}$ & Flag \\
\hline Am-241 & A59 & 2014 & 0.06005 & 0.0941 & 0.2823 & $\mathrm{U}$ \\
\hline Am-241 & B15 & 2014 & 0.1693 & 0.153 & 0.459 & $\mathrm{U}$ \\
\hline Am-241 & $\mathrm{B} 28$ & 2014 & 0.0915 & 0.139 & 0.417 & $\mathrm{U}$ \\
\hline Am-241 & B29 & 2014 & 0.4678 & 0.199 & 0.597 & $\mathrm{U}$ \\
\hline Am-241 & B3 & 2014 & 0.09989 & 0.157 & 0.471 & $\mathrm{U}$ \\
\hline Am-241 & B3 & 2014 & -0.03795 & 0.0865 & 0.2595 & $\mathrm{U}$ \\
\hline Am-241 & B4 & 2014 & 0.06915 & 0.0784 & 0.2352 & $\mathrm{U}$ \\
\hline Am-241 & B40 & 2014 & 0.07699 & 0.0753 & 0.2259 & $\mathrm{U}$ \\
\hline Am-241 & B50 & 2014 & 0.7642 & 0.28 & 0.84 & $\mathrm{U}$ \\
\hline Am-241 & B52 & 2014 & 0.02817 & 0.106 & 0.318 & $\mathrm{U}$ \\
\hline Am-241 & B52 & 2014 & 0.002643 & 0.124 & 0.372 & $\mathrm{U}$ \\
\hline Co-60 & B37 & 1974 & 1.1 & 0.2 & 0.6 & - \\
\hline Co-60 & B38 & 1974 & 0.4 & 0.1 & 0.3 & - \\
\hline Co-60 & A16 & 2006 & 0.02893 & 0.0083 & 0.0249 & - \\
\hline Co-60 & A17 & 2006 & 0.04218 & 0.01315 & 0.03945 & - \\
\hline Co-60 & $\mathrm{A} 27$ & 2006 & 0.03882 & 0.00895 & 0.02685 & - \\
\hline Co-60 & A28 & 2006 & 0.02672 & 0.0067 & 0.0201 & - \\
\hline Co-60 & A38 & 2006 & 0.08766 & 0.01795 & 0.05385 & - \\
\hline Co-60 & A39 & 2006 & 0.02192 & 0.00535 & 0.01605 & - \\
\hline Co-60 & A4 & 2006 & 0.02008 & 0.004995 & 0.014985 & - \\
\hline Co-60 & A44 & 2006 & 0.04218 & 0.01255 & 0.03765 & - \\
\hline Co-60 & A45 & 2006 & 0.05951 & 0.01075 & 0.03225 & - \\
\hline Co-60 & A48 & 2006 & 0.02178 & 0.0068 & 0.0204 & - \\
\hline Co-60 & A49 & 2006 & 0.03222 & 0.00565 & 0.01695 & - \\
\hline Co-60 & A5 & 2006 & 0.05834 & 0.01225 & 0.03675 & - \\
\hline Co-60 & A50 & 2006 & 0.0359 & 0.01025 & 0.03075 & - \\
\hline Co-60 & A55 & 2006 & 0.01498 & 0.00382 & 0.01146 & - \\
\hline Co-60 & A58 & 2006 & 0.1275 & 0.02285 & 0.06855 & - \\
\hline Co-60 & A59 & 2006 & 0.07712 & 0.01425 & 0.04275 & - \\
\hline Co-60 & A6 & 2006 & 0.1554 & 0.02655 & 0.07965 & - \\
\hline Co-60 & A61 & 2006 & 0.0447 & 0.0088 & 0.0264 & - \\
\hline Co-60 & A66 & 2006 & 0.102 & 0.0228 & 0.0684 & - \\
\hline Co-60 & A67 & 2006 & 0.02089 & 0.0063 & 0.0189 & - \\
\hline Co-60 & A68 & 2006 & 0.06231 & 0.02015 & 0.06045 & - \\
\hline Co-60 & A69 & 2006 & 0.04014 & 0.01215 & 0.03645 & - \\
\hline Co-60 & A71 & 2006 & 0.03184 & 0.0088 & 0.0264 & - \\
\hline Co-60 & A72 & 2006 & 0.1299 & 0.01925 & 0.05775 & - \\
\hline
\end{tabular}


Table C-3. (continued).

\begin{tabular}{|c|c|c|c|c|c|c|}
\hline Radionuclide & $\begin{array}{c}\text { Sample } \\
\text { Location }\end{array}$ & Date & $\begin{array}{c}\text { Concentrations } \\
(\mathrm{pCi} / \mathrm{g})\end{array}$ & $\begin{array}{l}\text { Uncertainty } \\
(\mathrm{pCi} / \mathrm{g})\end{array}$ & $\begin{array}{c}\text { MDA } \\
(\mathrm{pCi} / \mathrm{g})\end{array}$ & Flag \\
\hline Co-60 & $\mathrm{B} 100$ & 2006 & 0.02708 & 0.0096 & 0.0288 & $\mathrm{U}$ \\
\hline Co-60 & $\mathrm{B} 101$ & 2006 & 0.0291 & 0.00595 & 0.01785 & 一 \\
\hline Co-60 & $\mathrm{B} 102$ & 2006 & 0.0379 & 0.00885 & 0.02655 & 一 \\
\hline Co-60 & B103 & 2006 & 0.02392 & 0.00625 & 0.01875 & - \\
\hline Co-60 & B104 & 2006 & 0.0926 & 0.018 & 0.054 & - \\
\hline Co-60 & $\mathrm{B} 105$ & 2006 & 0.08389 & 0.01135 & 0.03405 & - \\
\hline Co-60 & B106 & 2006 & 0.1136 & 0.01635 & 0.04905 & - \\
\hline Co-60 & B14 & 2006 & 0.03625 & 0.01125 & 0.03375 & - \\
\hline Co-60 & $\mathrm{B} 15$ & 2006 & 0.02796 & 0.00605 & 0.01815 & - \\
\hline Co-60 & B17 & 2006 & 0.02607 & 0.00725 & 0.02175 & - \\
\hline Co-60 & $\mathrm{B} 25$ & 2006 & 0.02563 & 0.00575 & 0.01725 & - \\
\hline Co-60 & B26 & 2006 & 0.00941 & 0.0035 & 0.0105 & $\mathrm{U}$ \\
\hline Co-60 & B28 & 2006 & 0.01984 & 0.00555 & 0.01665 & - \\
\hline Co-60 & B29 & 2006 & 0.03217 & 0.0074 & 0.0222 & - \\
\hline Co-60 & B3 & 2006 & 0.03025 & 0.0083 & 0.0249 & - \\
\hline Co-60 & B36 & 2006 & 0.1341 & 0.0248 & 0.0744 & - \\
\hline Co-60 & B37 & 2006 & 0.01897 & 0.00515 & 0.01545 & - \\
\hline Co-60 & B39 & 2006 & 0.07522 & 0.014 & 0.042 & - \\
\hline Co-60 & $\mathrm{B} 4$ & 2006 & 0.01442 & 0.00493 & 0.01479 & $\mathrm{U}$ \\
\hline Co-60 & B40 & 2006 & 0.04728 & 0.009 & 0.02835 & - \\
\hline Co-60 & B47 & 2006 & 0.05599 & 0.0069 & 0.0207 & - \\
\hline Co-60 & B48 & 2006 & 0.03795 & 0.00615 & 0.01845 & - \\
\hline Co-60 & B50 & 2006 & 0.02768 & 0.0074 & 0.0222 & - \\
\hline Co-60 & B51 & 2006 & 0.1485 & 0.02625 & 0.07875 & - \\
\hline Co-60 & B52 & 2006 & 0.02748 & 0.00995 & 0.02985 & $\mathrm{U}$ \\
\hline Co-60 & B59 & 2006 & 0.1131 & 0.01565 & 0.04695 & - \\
\hline Co-60 & B60 & 2006 & 0.05645 & 0.0103 & 0.0309 & - \\
\hline Co-60 & B61 & 2006 & 0.02188 & 0.00725 & 0.02175 & - \\
\hline Co-60 & B62 & 2006 & 0.07585 & 0.0212 & 0.0636 & - \\
\hline Co-60 & B68 & 2006 & 0.02498 & 0.0073 & 0.0219 & - \\
\hline Co-60 & B71 & 2006 & 0.01469 & 0.00505 & 0.01515 & $\mathrm{U}$ \\
\hline Co-60 & B73 & 2006 & 0.03224 & 0.00835 & 0.02505 & - \\
\hline Co-60 & $\mathrm{B} 80$ & 2006 & 0.05981 & 0.01575 & 0.04725 & - \\
\hline Co-60 & B81 & 2006 & 0.03946 & 0.00625 & 0.01875 & - \\
\hline Co-60 & B82 & 2006 & 0.02824 & 0.0059 & 0.0177 & - \\
\hline Co-60 & B83 & 2006 & 0.01124 & 0.003935 & 0.011805 & $\mathrm{U}$ \\
\hline Co-60 & B84 & 2006 & 0.05375 & 0.01345 & 0.04035 & - \\
\hline
\end{tabular}


Table C-3. (continued).

\begin{tabular}{|c|c|c|c|c|c|c|}
\hline Radionuclide & $\begin{array}{c}\text { Sample } \\
\text { Location }\end{array}$ & Date & $\begin{array}{c}\text { Concentrations } \\
(\mathrm{pCi} / \mathrm{g})\end{array}$ & $\begin{array}{l}\text { Uncertainty } \\
(\mathrm{pCi} / \mathrm{g})\end{array}$ & $\begin{array}{c}\mathrm{MDA} \\
(\mathrm{pCi} / \mathrm{g})\end{array}$ & Flag \\
\hline Co-60 & B88 & 2006 & 0.02135 & 0.00525 & 0.01575 & - \\
\hline Co-60 & B90 & 2006 & 0.00752 & 0.00213 & 0.00639 & 一 \\
\hline Co-60 & B91 & 2006 & 0.03287 & 0.0082 & 0.0246 & 一 \\
\hline Co-60 & B92 & 2006 & 0.02446 & 0.00715 & 0.02145 & - \\
\hline Co-60 & B93 & 2006 & 0.08499 & 0.0164 & 0.0492 & - \\
\hline Co-60 & B94 & 2006 & 0.1307 & 0.0278 & 0.0834 & 一 \\
\hline Co-60 & B95 & 2006 & 0.02725 & 0.0126 & 0.0378 & $\mathrm{U}$ \\
\hline Co-60 & B99 & 2006 & 0.0709 & 0.01395 & 0.04185 & 一 \\
\hline Co-60 & $\mathrm{C} 14$ & 2006 & 0.08567 & 0.01385 & 0.04155 & - \\
\hline Co-60 & $\mathrm{C} 15$ & 2006 & 0.01426 & 0.0057 & 0.0171 & $\mathrm{U}$ \\
\hline Co-60 & $\mathrm{C} 25$ & 2006 & 0.03824 & 0.0077 & 0.0231 & - \\
\hline Co-60 & C36 & 2006 & 0.03566 & 0.00945 & 0.02835 & - \\
\hline Co-60 & C37 & 2006 & 0.02251 & 0.00815 & 0.02445 & $\mathrm{U}$ \\
\hline Co-60 & $\mathrm{C} 46$ & 2006 & 0.02261 & 0.0066 & 0.0198 & - \\
\hline Co-60 & $\mathrm{C} 47$ & 2006 & 0.06422 & 0.013 & 0.039 & - \\
\hline Co-60 & $\mathrm{C} 48$ & 2006 & 0.03878 & 0.011 & 0.0327 & - \\
\hline Co-60 & $\mathrm{C} 56$ & 2006 & 0.02848 & 0.0093 & 0.0279 & - \\
\hline Co-60 & C57 & 2006 & 0.04876 & 0.0105 & 0.0315 & - \\
\hline Co-60 & D57 & 2006 & 0.0265 & 0.0074 & 0.0222 & - \\
\hline Co-60 & D58 & 2006 & 0.01512 & 0.004675 & 0.014025 & - \\
\hline Co-60 & D68 & 2006 & 0.005999 & 0.002845 & 0.008535 & $\mathrm{U}$ \\
\hline Сo-60 & D79 & 2006 & 0.01831 & 0.0062 & 0.0186 & $\mathrm{U}$ \\
\hline Co-60 & D89 & 2006 & 0.02331 & 0.00655 & 0.01965 & - \\
\hline Co-60 & A15 & 2007 & 0.02607 & 0.0061 & 0.0183 & - \\
\hline Co-60 & A16 & 2007 & 0.02211 & 0.0065 & 0.0195 & 一 \\
\hline Co-60 & A17 & 2007 & 0.01353 & 0.00315 & 0.00945 & - \\
\hline Co-60 & A27 & 2007 & 0.01145 & 0.004425 & 0.013275 & $\mathrm{U}$ \\
\hline Co-60 & A28 & 2007 & 0.01403 & 0.00349 & 0.01047 & - \\
\hline Co-60 & A37 & 2007 & 0.009977 & 0.003135 & 0.009405 & - \\
\hline Co-60 & A38 & 2007 & 0.009675 & 0.0034 & 0.0102 & $\mathrm{U}$ \\
\hline Co-60 & $\mathrm{A} 4$ & 2007 & 0.01267 & 0.00244 & 0.00732 & - \\
\hline Co-60 & A44 & 2007 & 0.01054 & 0.00305 & 0.00915 & - \\
\hline Co-60 & A45 & 2007 & 0.01604 & 0.0038 & 0.0114 & - \\
\hline Co-60 & A47 & 2007 & 0.003268 & 0.00326 & 0.00978 & $\mathrm{U}$ \\
\hline Co-60 & A48 & 2007 & 0.01565 & 0.00404 & 0.01212 & 一 \\
\hline Co-60 & A49 & 2007 & 0.01944 & 0.00465 & 0.01395 & - \\
\hline Co-60 & A5 & 2007 & 0.02082 & 0.00565 & 0.01695 & - \\
\hline
\end{tabular}


Table C-3. (continued).

\begin{tabular}{|c|c|c|c|c|c|c|}
\hline Radionuclide & $\begin{array}{c}\text { Sample } \\
\text { Location }\end{array}$ & Date & $\begin{array}{c}\text { Concentrations } \\
(\mathrm{pCi} / \mathrm{g})\end{array}$ & $\begin{array}{l}\text { Uncertainty } \\
(\mathrm{pCi} / \mathrm{g})\end{array}$ & $\begin{array}{c}\mathrm{MDA} \\
(\mathrm{pCi} / \mathrm{g})\end{array}$ & Flag \\
\hline Co-60 & $\mathrm{A} 50$ & 2007 & 0.0146 & 0.003055 & 0.009165 & 一 \\
\hline Co-60 & A55 & 2007 & 0.02679 & 0.00955 & 0.02865 & $\mathrm{U}$ \\
\hline Co-60 & A58 & 2007 & 0.0137 & 0.00392 & 0.01176 & 一 \\
\hline Co-60 & A59 & 2007 & 0.01218 & 0.00392 & 0.01176 & - \\
\hline Co-60 & A6 & 2007 & 0.02109 & 0.0059 & 0.0177 & - \\
\hline Co-60 & A60 & 2007 & 0.02003 & 0.004165 & 0.012495 & - \\
\hline Co-60 & A61 & 2007 & 0.01074 & 0.00459 & 0.01377 & $\mathrm{U}$ \\
\hline Co-60 & A67 & 2007 & 0.01897 & 0.0059 & 0.0177 & - \\
\hline Co-60 & A69 & 2007 & 0.03645 & 0.00685 & 0.02055 & - \\
\hline Co-60 & A70 & 2007 & 0.03195 & 0.009 & 0.027 & - \\
\hline Co-60 & A71 & 2007 & 0.01033 & 0.00296 & 0.00888 & - \\
\hline Co-60 & A72 & 2007 & 0.007218 & 0.0047 & 0.0141 & $\mathrm{U}$ \\
\hline Co-60 & $\mathrm{B} 101$ & 2007 & 0.02231 & 0.00535 & 0.01605 & - \\
\hline Co-60 & $\mathrm{B} 102$ & 2007 & 0.005028 & 0.004545 & 0.013635 & $\mathrm{U}$ \\
\hline Co-60 & B103 & 2007 & 0.01485 & 0.003715 & 0.011145 & 一 \\
\hline Co-60 & B104 & 2007 & 0.01459 & 0.00474 & 0.01422 & 一 \\
\hline Co-60 & $\mathrm{B} 105$ & 2007 & 0.007294 & 0.003105 & 0.009315 & $\mathrm{U}$ \\
\hline Co-60 & $\mathrm{B} 14$ & 2007 & 0.009919 & 0.00322 & 0.00966 & - \\
\hline Co-60 & B17 & 2007 & 0.008609 & 0.003215 & 0.009645 & $\mathrm{U}$ \\
\hline Co-60 & $\mathrm{B} 25$ & 2007 & 0.01355 & 0.004445 & 0.013335 & - \\
\hline Co-60 & B26 & 2007 & 0.01748 & 0.003695 & 0.011085 & 一 \\
\hline Co-60 & B28 & 2007 & 0.01878 & 0.004575 & 0.013725 & - \\
\hline Co-60 & B29 & 2007 & 0.007089 & 0.003385 & 0.010155 & $\mathrm{U}$ \\
\hline Co-60 & $\mathrm{B} 3$ & 2007 & 0.01592 & 0.00515 & 0.01545 & - \\
\hline Co-60 & B4 & 2007 & 0.05333 & 0.0132 & 0.0396 & - \\
\hline Co-60 & B40 & 2007 & 0.04053 & 0.01105 & 0.03315 & - \\
\hline Co-60 & B47 & 2007 & 0.03652 & 0.0054 & 0.0162 & - \\
\hline Co-60 & B48 & 2007 & 0.02455 & 0.004415 & 0.013245 & - \\
\hline Co-60 & B50 & 2007 & 0.04717 & 0.00915 & 0.02745 & - \\
\hline Co-60 & B51 & 2007 & 0.01839 & 0.00565 & 0.01695 & - \\
\hline Co-60 & B52 & 2007 & 0.03245 & 0.00815 & 0.02445 & - \\
\hline Co-60 & B59 & 2007 & 0.0355 & 0.00885 & 0.02655 & - \\
\hline Co-60 & B60 & 2007 & 0.05791 & 0.0096 & 0.0288 & - \\
\hline Co-60 & B61 & 2007 & 0.01402 & 0.00351 & 0.01053 & - \\
\hline Co-60 & B62 & 2007 & 0.01194 & 0.003365 & 0.010095 & - \\
\hline Co-60 & B69 & 2007 & 1.995 & 0.0192 & 0.0576 & - \\
\hline Co-60 & B71 & 2007 & 0.05099 & 0.01115 & 0.03345 & - \\
\hline
\end{tabular}


Table C-3. (continued).

\begin{tabular}{|c|c|c|c|c|c|c|}
\hline Radionuclide & $\begin{array}{c}\text { Sample } \\
\text { Location }\end{array}$ & Date & $\begin{array}{c}\text { Concentrations } \\
(\mathrm{pCi} / \mathrm{g})\end{array}$ & $\begin{array}{l}\text { Uncertainty } \\
(\mathrm{pCi} / \mathrm{g})\end{array}$ & $\begin{array}{c}\text { MDA } \\
(\mathrm{pCi} / \mathrm{g})\end{array}$ & Flag \\
\hline Co-60 & B73 & 2007 & 0.01735 & 0.00386 & 0.01158 & - \\
\hline Co-60 & $\mathrm{B} 80$ & 2007 & 0.01352 & 0.0056 & 0.0168 & $\mathrm{U}$ \\
\hline Co-60 & B81 & 2007 & 0.04813 & 0.01005 & 0.03015 & 一 \\
\hline Co-60 & B82 & 2007 & 0.01342 & 0.00378 & 0.01134 & - \\
\hline Co-60 & B83 & 2007 & 0.03325 & 0.007 & 0.021 & - \\
\hline Co-60 & B88 & 2007 & 0.01325 & 0.00344 & 0.01032 & - \\
\hline Co-60 & B89 & 2007 & 0.01348 & 0.003845 & 0.011535 & 一 \\
\hline Co-60 & B90 & 2007 & 0.005214 & 0.003255 & 0.009765 & $\mathrm{U}$ \\
\hline Co-60 & B92 & 2007 & 0.01441 & 0.00458 & 0.01374 & - \\
\hline Co-60 & B93 & 2007 & 0.01193 & 0.003515 & 0.010545 & - \\
\hline Co-60 & B94 & 2007 & 0.0005117 & 0.00665 & 0.01995 & $\mathrm{U}$ \\
\hline $\mathrm{Co}-60$ & B95 & 2007 & 0.009196 & 0.00186 & 0.00558 & - \\
\hline Co-60 & B99 & 2007 & 0.03985 & 0.00735 & 0.02205 & - \\
\hline Co-60 & $\mathrm{C} 14$ & 2007 & 0.02674 & 0.00695 & 0.02085 & 一 \\
\hline Co-60 & $\mathrm{C} 15$ & 2007 & 0.02005 & 0.00805 & 0.02415 & $\mathrm{U}$ \\
\hline Co-60 & $\mathrm{C} 36$ & 2007 & 0.02428 & 0.00585 & 0.01755 & 一 \\
\hline Co-60 & $\mathrm{C} 37$ & 2007 & 0.03644 & 0.00805 & 0.02415 & - \\
\hline Co-60 & $\mathrm{C} 46$ & 2007 & 0.02468 & 0.00585 & 0.01755 & - \\
\hline Co-60 & $\mathrm{C} 47$ & 2007 & 0.0124 & 0.00423 & 0.01269 & $\mathrm{U}$ \\
\hline Co-60 & $\mathrm{C} 48$ & 2007 & 0.02523 & 0.0062 & 0.0186 & - \\
\hline Co-60 & C56 & 2007 & 0.02294 & 0.00505 & 0.01515 & 一 \\
\hline Co-60 & C57 & 2007 & 0.0159 & 0.00445 & 0.01335 & - \\
\hline Co-60 & D58 & 2007 & 0.01733 & 0.00383 & 0.01149 & - \\
\hline Co-60 & D68 & 2007 & 0.02769 & 0.0077 & 0.0231 & - \\
\hline Co-60 & D69 & 2007 & 0.02689 & 0.0063 & 0.0189 & 一 \\
\hline Co-60 & D79 & 2007 & 0.0216 & 0.007 & 0.021 & 一 \\
\hline Co-60 & D80 & 2007 & 0.006289 & 0.0024 & 0.0072 & $\mathrm{U}$ \\
\hline $\mathrm{Co}-60$ & D89 & 2007 & 0.01056 & 0.00333 & 0.00999 & - \\
\hline Co-60 & D90 & 2007 & 0.0165 & 0.00397 & 0.01191 & - \\
\hline Co-60 & D91 & 2007 & 0.009077 & 0.00415 & 0.01245 & $\mathrm{U}$ \\
\hline Co-60 & A15 & 2011 & 0.002487 & 0.00274 & 0.00822 & $\mathrm{U}$ \\
\hline Co-60 & A16 & 2011 & -0.002467 & 0.00436 & 0.01308 & $\mathrm{U}$ \\
\hline Co-60 & A17 & 2011 & 0.003452 & 0.00455 & 0.01365 & $\mathrm{U}$ \\
\hline Co-60 & A27 & 2011 & 0.002232 & 0.0043 & 0.0129 & $\mathrm{U}$ \\
\hline Co-60 & A28 & 2011 & 0.00366 & 0.0046 & 0.0138 & $\mathrm{U}$ \\
\hline Co-60 & A38 & 2011 & 0.003989 & 0.00377 & 0.01131 & $\mathrm{U}$ \\
\hline Co-60 & A39 & 2011 & 0.001858 & 0.00378 & 0.01134 & $\mathrm{U}$ \\
\hline
\end{tabular}


Table C-3. (continued).

\begin{tabular}{|c|c|c|c|c|c|c|}
\hline Radionuclide & $\begin{array}{l}\text { Sample } \\
\text { Location }\end{array}$ & Date & $\begin{array}{c}\text { Concentrations } \\
(\mathrm{pCi} / \mathrm{g})\end{array}$ & $\begin{array}{l}\text { Uncertainty } \\
(\mathrm{pCi} / \mathrm{g})\end{array}$ & $\begin{array}{l}\text { MDA } \\
(\mathrm{pCi} / \mathrm{g})\end{array}$ & Flag \\
\hline Co-60 & $\mathrm{A} 4$ & 2011 & 0.001971 & 0.00474 & 0.01422 & $\mathrm{U}$ \\
\hline Co-60 & A44 & 2011 & 0.03294 & 0.00573 & 0.01719 & - \\
\hline Co-60 & A47 & 2011 & 0.00781 & 0.0052 & 0.0156 & $\mathrm{U}$ \\
\hline Co-60 & A49 & 2011 & 0.001225 & 0.00514 & 0.01542 & $\mathrm{U}$ \\
\hline Co-60 & A50 & 2011 & 0.002241 & 0.00534 & 0.01602 & $\mathrm{U}$ \\
\hline Co-60 & A55 & 2011 & 0.001262 & 0.0043 & 0.0129 & $\mathrm{U}$ \\
\hline Co-60 & A58 & 2011 & 0.003362 & 0.00345 & 0.01035 & $\mathrm{U}$ \\
\hline Co-60 & A59 & 2011 & -0.00008337 & 0.00352 & 0.01056 & $\mathrm{U}$ \\
\hline Co-60 & A6 & 2011 & 0.0002505 & 0.00589 & 0.01767 & $\mathrm{U}$ \\
\hline Co-60 & A61 & 2011 & 0.002682 & 0.00309 & 0.00927 & $\mathrm{U}$ \\
\hline Co-60 & A66 & 2011 & 0.0008304 & 0.00533 & 0.01599 & $\mathrm{U}$ \\
\hline Co-60 & A69 & 2011 & 0.005979 & 0.00474 & 0.01422 & $\mathrm{U}$ \\
\hline Co-60 & A70 & 2011 & -0.002624 & 0.00378 & 0.01134 & $\mathrm{U}$ \\
\hline Co-60 & A72 & 2011 & 0.002688 & 0.00349 & 0.01047 & $\mathrm{U}$ \\
\hline Co-60 & B101 & 2011 & 0.001451 & 0.00444 & 0.01332 & $\mathrm{U}$ \\
\hline Co-60 & B102 & 2011 & 0.01897 & 0.00671 & 0.02013 & $\mathrm{U}$ \\
\hline Co-60 & $\mathrm{B} 103$ & 2011 & 0.005821 & 0.00495 & 0.01485 & $\mathrm{U}$ \\
\hline Co-60 & B104 & 2011 & 0.001895 & 0.00375 & 0.01125 & $\mathrm{U}$ \\
\hline Co-60 & B106 & 2011 & -0.001054 & 0.00436 & 0.01308 & $\mathrm{U}$ \\
\hline Co-60 & $\mathrm{B} 14$ & 2011 & 0.003939 & 0.00552 & 0.01656 & $\mathrm{U}$ \\
\hline Co-60 & B15 & 2011 & -0.00109 & 0.00372 & 0.01116 & $\mathrm{U}$ \\
\hline Co-60 & $\mathrm{B} 17$ & 2011 & 0.0006075 & 0.00159 & 0.00477 & $\mathrm{U}$ \\
\hline Co-60 & $\mathrm{B} 25$ & 2011 & 0.003908 & 0.00526 & 0.01578 & $\mathrm{U}$ \\
\hline Co-60 & B28 & 2011 & 0.001297 & 0.00364 & 0.01092 & $\mathrm{U}$ \\
\hline Co-60 & B29 & 2011 & -0.0002173 & 0.00495 & 0.01485 & $\mathrm{U}$ \\
\hline Co-60 & B3 & 2011 & -0.002371 & 0.00394 & 0.01182 & $\mathrm{U}$ \\
\hline Co-60 & B39 & 2011 & -0.0002005 & 0.00144 & 0.00432 & $\mathrm{U}$ \\
\hline Co-60 & $\mathrm{B} 4$ & 2011 & -0.001915 & 0.00449 & 0.01347 & $\mathrm{U}$ \\
\hline Co-60 & B40 & 2011 & 0.0003215 & 0.00161 & 0.00483 & $\mathrm{U}$ \\
\hline Co-60 & B47 & 2011 & 0.05543 & 0.00711 & 0.02133 & - \\
\hline Co-60 & B48 & 2011 & 0.003104 & 0.00418 & 0.01254 & $\mathrm{U}$ \\
\hline Co-60 & B50 & 2011 & 0.001956 & 0.00393 & 0.01179 & $\mathrm{U}$ \\
\hline Co-60 & B52 & 2011 & 0.002192 & 0.00385 & 0.01155 & $\mathrm{U}$ \\
\hline Co-60 & B61 & 2011 & 0.00137 & 0.00516 & 0.01548 & $\mathrm{U}$ \\
\hline Co-60 & B62 & 2011 & -0.0006257 & 0.00459 & 0.01377 & $\mathrm{U}$ \\
\hline Co-60 & B71 & 2011 & -0.000434 & 0.00351 & 0.01053 & $\mathrm{U}$ \\
\hline Co-60 & B73 & 2011 & 0.0004195 & 0.00557 & 0.01671 & $\mathrm{U}$ \\
\hline
\end{tabular}


Table C-3. (continued).

\begin{tabular}{|c|c|c|c|c|c|c|}
\hline Radionuclide & $\begin{array}{c}\text { Sample } \\
\text { Location }\end{array}$ & Date & $\begin{array}{c}\text { Concentrations } \\
(\mathrm{pCi} / \mathrm{g})\end{array}$ & $\begin{array}{l}\text { Uncertainty } \\
(\mathrm{pCi} / \mathrm{g})\end{array}$ & $\begin{array}{c}\mathrm{MDA} \\
(\mathrm{pCi} / \mathrm{g})\end{array}$ & Flag \\
\hline Co-60 & B80 & 2011 & 0.01242 & 0.00442 & 0.01326 & $\mathrm{U}$ \\
\hline Co-60 & B83 & 2011 & 0.00207 & 0.00405 & 0.01215 & $\mathrm{U}$ \\
\hline Co-60 & B84 & 2011 & 0.003326 & 0.00555 & 0.01665 & $\mathrm{U}$ \\
\hline Co-60 & B89 & 2011 & -0.003933 & 0.00493 & 0.01479 & $\mathrm{U}$ \\
\hline Co-60 & B90 & 2011 & 0.0006691 & 0.00441 & 0.01323 & $\mathrm{U}$ \\
\hline Co-60 & B91 & 2011 & 0.001037 & 0.0042 & 0.0126 & $\mathrm{U}$ \\
\hline Co-60 & B92 & 2011 & 0.002843 & 0.00339 & 0.01017 & $\mathrm{U}$ \\
\hline Co-60 & B93 & 2011 & 0.003059 & 0.00351 & 0.01053 & $\mathrm{U}$ \\
\hline Co-60 & B94 & 2011 & 0.00006901 & 0.00384 & 0.01152 & $\mathrm{U}$ \\
\hline Co-60 & B99 & 2011 & 0.003785 & 0.00423 & 0.01269 & $\mathrm{U}$ \\
\hline Co-60 & $\mathrm{C} 15$ & 2011 & 0.0004493 & 0.00459 & 0.01377 & $\mathrm{U}$ \\
\hline Co-60 & $\mathrm{C} 25$ & 2011 & -0.001734 & 0.00337 & 0.01011 & $\mathrm{U}$ \\
\hline Co-60 & C37 & 2011 & 0.004336 & 0.00402 & 0.01206 & $\mathrm{U}$ \\
\hline Co-60 & $\mathrm{C} 46$ & 2011 & -0.001172 & 0.0040 & 0.01212 & $\mathrm{U}$ \\
\hline Co-60 & $\mathrm{C} 48$ & 2011 & -0.002664 & 0.00346 & 0.01038 & $\mathrm{U}$ \\
\hline Co-60 & C56 & 2011 & 0.00602 & 0.00529 & 0.01587 & $\mathrm{U}$ \\
\hline Co-60 & C57 & 2011 & 0.000615 & 0.00426 & 0.01278 & $\mathrm{U}$ \\
\hline Co-60 & D58 & 2011 & 0.001286 & 0.00363 & 0.01089 & $\mathrm{U}$ \\
\hline Co-60 & D68 & 2011 & 0.001274 & 0.00379 & 0.01137 & $\mathrm{U}$ \\
\hline Co-60 & D89 & 2011 & 0.002224 & 0.00414 & 0.01242 & $\mathrm{U}$ \\
\hline Co-60 & D91 & 2011 & 0.0001192 & 0.00514 & 0.01542 & $\mathrm{U}$ \\
\hline $\mathrm{Co}-60$ & A15 & 2012 & 0.001077 & 0.00413 & 0.01239 & $\mathrm{U}$ \\
\hline Co-60 & A38 & 2012 & 0.002167 & 0.00461 & 0.01383 & $\mathrm{U}$ \\
\hline Co-60 & A44 & 2012 & 0.0331 & 0.00534 & 0.01602 & - \\
\hline Co-60 & A47 & 2012 & 0.01051 & 0.0045 & 0.0135 & $\mathrm{U}$ \\
\hline Co-60 & A59 & 2012 & 0.001315 & 0.00422 & 0.01266 & $\mathrm{U}$ \\
\hline Co-60 & A6 & 2012 & 0.0008794 & 0.00533 & 0.01599 & $\mathrm{U}$ \\
\hline Co-60 & A69 & 2012 & 0.006386 & 0.00508 & 0.01524 & $\mathrm{U}$ \\
\hline Co-60 & A70 & 2012 & -0.001215 & 0.0041 & 0.0123 & $\mathrm{U}$ \\
\hline Co-60 & A72 & 2012 & -0.002314 & 0.00523 & 0.01569 & $\mathrm{U}$ \\
\hline Co-60 & B14 & 2012 & 0.005959 & 0.00468 & 0.01404 & $\mathrm{U}$ \\
\hline Co-60 & B48 & 2012 & 0.01007 & 0.00486 & 0.01458 & $\mathrm{U}$ \\
\hline Co-60 & B83 & 2012 & 0.002842 & 0.00399 & 0.01197 & $\mathrm{U}$ \\
\hline Co-60 & $\mathrm{C} 46$ & 2012 & -0.00108 & 0.00483 & 0.01449 & $\mathrm{U}$ \\
\hline Co-60 & $\mathrm{C} 57$ & 2012 & -0.002414 & 0.00479 & 0.01437 & $\mathrm{U}$ \\
\hline Co-60 & $\begin{array}{l}2 \text { INCH AIR } \\
\text { - INTEC }\end{array}$ & 2013 & 0.009973 & 0.0033 & 0.01 & $\mathrm{U}$ \\
\hline
\end{tabular}


Table C-3. (continued).

\begin{tabular}{|c|c|c|c|c|c|c|}
\hline Radionuclide & $\begin{array}{l}\text { Sample } \\
\text { Location }\end{array}$ & Date & $\begin{array}{c}\text { Concentrations } \\
(\mathrm{pCi} / \mathrm{g})\end{array}$ & $\begin{array}{l}\text { Uncertainty } \\
(\mathrm{pCi} / \mathrm{g})\end{array}$ & $\begin{array}{l}\text { MDA } \\
(\mathrm{pCi} / \mathrm{g})\end{array}$ & Flag \\
\hline Co-60 & A47 & 2013 & -0.001489 & 0.00306 & 0.00918 & $\mathrm{U}$ \\
\hline Co-60 & A49 & 2013 & 0.0002302 & 0.00028 & 0.000847 & $\mathrm{U}$ \\
\hline Co-60 & A59 & 2013 & 0.000358 & 0.00309 & 0.00927 & $\mathrm{U}$ \\
\hline Co-60 & A71 & 2013 & 0.00776 & 0.00297 & 0.00892 & $\mathrm{U}$ \\
\hline Co-60 & A72 & 2013 & 0.00004954 & 0.00036 & 0.00107 & $\mathrm{U}$ \\
\hline Co-60 & $\begin{array}{c}2 \text { INCH AIR } \\
- \text { CPP }\end{array}$ & 2014 & 0.0006881 & 0.00121 & 0.00363 & $\mathrm{U}$ \\
\hline Co-60 & $\begin{array}{l}2 \text { INCH AIR } \\
\text { - INTEC }\end{array}$ & 2014 & 0.00107 & 0.00126 & 0.00378 & $\mathrm{U}$ \\
\hline Co-60 & A38 & 2014 & -0.0004377 & 0.0014 & 0.0042 & $\mathrm{U}$ \\
\hline Co-60 & A47 & 2014 & -0.0001923 & 0.00129 & 0.00387 & $\mathrm{U}$ \\
\hline Co-60 & A58 & 2014 & 0.000498 & 0.00136 & 0.00408 & $\mathrm{U}$ \\
\hline Co-60 & A59 & 2014 & -0.0009825 & 0.00151 & 0.00453 & $\mathrm{U}$ \\
\hline Co-60 & $\mathrm{B} 15$ & 2014 & 0.001186 & 0.00246 & 0.00738 & $\mathrm{U}$ \\
\hline Co-60 & B28 & 2014 & 0.0005723 & 0.0012 & 0.0036 & $\mathrm{U}$ \\
\hline Co-60 & B29 & 2014 & 0.001195 & 0.00106 & 0.00318 & $\mathrm{U}$ \\
\hline Co-60 & B3 & 2014 & 0.0002284 & 0.000668 & 0.002004 & $\mathrm{U}$ \\
\hline $\mathrm{Co}-60$ & B3 & 2014 & -0.0002563 & 0.00209 & 0.00627 & $\mathrm{U}$ \\
\hline Co-60 & B4 & 2014 & -0.0005574 & 0.000806 & 0.002418 & $\mathrm{U}$ \\
\hline $\mathrm{Co}-60$ & B40 & 2014 & 0.00005314 & 0.000887 & 0.002661 & $\mathrm{U}$ \\
\hline $\mathrm{Co}-60$ & B50 & 2014 & 0.00001053 & 0.00109 & 0.00327 & $\mathrm{U}$ \\
\hline Co-60 & B52 & 2014 & 0.001077 & 0.00175 & 0.00525 & $\mathrm{U}$ \\
\hline Co-60 & B52 & 2014 & 0.0006405 & 0.00113 & 0.00339 & $\mathrm{U}$ \\
\hline Cs-134 & A36 & 1974 & 1.1 & 0.2 & 0.6 & 一 \\
\hline Cs-134 & A37 & 1974 & 0.6 & 0.2 & 0.6 & 一 \\
\hline Cs-134 & A46 & 1974 & 0.6 & 0.1 & 0.3 & - \\
\hline Cs-134 & A47 & 1974 & 0.3 & 0.1 & 0.3 & - \\
\hline Cs-134 & A48 & 1974 & 0.5 & 0.1 & 0.3 & - \\
\hline Cs-134 & $\mathrm{B} 25$ & 1974 & 0.6 & 0.2 & 0.6 & - \\
\hline Cs-134 & B37 & 1974 & 0.4 & 0.1 & 0.3 & - \\
\hline Cs-134 & B5 & 1974 & 0.2 & 0.06 & 0.18 & - \\
\hline Cs-134 & B76 & 1974 & 0.8 & 0.2 & 0.6 & 一 \\
\hline Cs-134 & B-120 & 1982 & 0.1 & 0.06 & 0.18 & $\mathrm{U}$ \\
\hline Cs-134 & A15 & 2007 & 0.07047 & 0.01395 & 0.04185 & 一 \\
\hline Cs-134 & A16 & 2007 & 0.1 & 0.01035 & 0.03105 & - \\
\hline Cs-134 & A17 & 2007 & 0.1059 & 0.015 & 0.045 & - \\
\hline Cs-134 & A27 & 2007 & 0.08698 & 0.01035 & 0.03105 & - \\
\hline
\end{tabular}


Table C-3. (continued).

\begin{tabular}{|c|c|c|c|c|c|c|}
\hline Radionuclide & $\begin{array}{c}\text { Sample } \\
\text { Location }\end{array}$ & Date & $\begin{array}{c}\text { Concentrations } \\
(\mathrm{pCi} / \mathrm{g})\end{array}$ & $\begin{array}{l}\text { Uncertainty } \\
(\mathrm{pCi} / \mathrm{g})\end{array}$ & $\begin{array}{c}\mathrm{MDA} \\
(\mathrm{pCi} / \mathrm{g})\end{array}$ & Flag \\
\hline Cs-134 & A28 & 2007 & 0.1338 & 0.01735 & 0.05205 & - \\
\hline Cs-134 & A37 & 2007 & 0.1068 & 0.0163 & 0.0489 & 一 \\
\hline Cs-134 & A38 & 2007 & 0.12 & 0.0196 & 0.0588 & 一 \\
\hline Cs-134 & A39 & 2007 & 0.1096 & 0.0183 & 0.0549 & - \\
\hline Cs-134 & $\mathrm{A} 4$ & 2007 & 0.07081 & 0.00865 & 0.02595 & - \\
\hline Cs-134 & A44 & 2007 & 0.04801 & 0.0058 & 0.0174 & - \\
\hline Cs-134 & A45 & 2007 & 0.1188 & 0.0185 & 0.0555 & - \\
\hline Cs-134 & A47 & 2007 & 0.09184 & 0.01045 & 0.03135 & - \\
\hline Cs-134 & A48 & 2007 & 0.1335 & 0.01685 & 0.05055 & - \\
\hline Cs-134 & A49 & 2007 & 0.1548 & 0.0198 & 0.0594 & - \\
\hline Cs-134 & A5 & 2007 & 0.142 & 0.0182 & 0.0546 & - \\
\hline Cs-134 & A50 & 2007 & 0.1235 & 0.0144 & 0.0432 & - \\
\hline Cs-134 & A55 & 2007 & 0.08063 & 0.012 & 0.036 & - \\
\hline Cs-134 & A56 & 2007 & 0.05898 & 0.0108 & 0.0324 & - \\
\hline Cs-134 & A58 & 2007 & 0.1013 & 0.0129 & 0.0387 & - \\
\hline Cs-134 & A59 & 2007 & 0.09886 & 0.01 & 0.03 & - \\
\hline Cs-134 & A6 & 2007 & 0.08253 & 0.0104 & 0.0312 & - \\
\hline Cs-134 & A60 & 2007 & 0.07276 & 0.0106 & 0.0318 & - \\
\hline Cs-134 & A61 & 2007 & 0.1156 & 0.01565 & 0.04695 & - \\
\hline Cs-134 & A66 & 2007 & 0.1298 & 0.0172 & 0.0516 & 一 \\
\hline Cs-134 & A67 & 2007 & 0.0916 & 0.0122 & 0.0366 & - \\
\hline Cs-134 & A68 & 2007 & 0.06147 & 0.00881 & 0.02643 & - \\
\hline Cs-134 & A69 & 2007 & 0.1458 & 0.0198 & 0.0594 & - \\
\hline Cs-134 & A70 & 2007 & 0.1187 & 0.01385 & 0.04155 & - \\
\hline Cs-134 & A71 & 2007 & 0.1357 & 0.01585 & 0.04755 & - \\
\hline Cs-134 & A72 & 2007 & 0.08464 & 0.0152 & 0.0456 & - \\
\hline Cs-134 & $\mathrm{B} 100$ & 2007 & 0.1397 & 0.0166 & 0.0498 & - \\
\hline Cs-134 & B101 & 2007 & 0.1017 & 0.0122 & 0.0366 & - \\
\hline Cs-134 & B102 & 2007 & 0.08446 & 0.0118 & 0.0354 & - \\
\hline Cs-134 & B103 & 2007 & 0.0769 & 0.0086 & 0.0258 & - \\
\hline Cs-134 & B104 & 2007 & 0.1444 & 0.0158 & 0.0474 & - \\
\hline Cs-134 & B105 & 2007 & 0.09322 & 0.01105 & 0.03315 & - \\
\hline Cs-134 & B106 & 2007 & 0.03704 & 0.00895 & 0.02685 & - \\
\hline Cs-134 & B14 & 2007 & 0.125 & 0.0157 & 0.0471 & - \\
\hline Cs-134 & B15 & 2007 & 0.07921 & 0.00888 & 0.02664 & - \\
\hline Cs-134 & $\mathrm{B} 17$ & 2007 & 0.1427 & 0.01715 & 0.05145 & - \\
\hline Cs-134 & $\mathrm{B} 25$ & 2007 & 0.1699 & 0.01815 & 0.05445 & - \\
\hline
\end{tabular}


Table C-3. (continued).

\begin{tabular}{|c|c|c|c|c|c|c|}
\hline Radionuclide & $\begin{array}{c}\text { Sample } \\
\text { Location }\end{array}$ & Date & $\begin{array}{c}\text { Concentrations } \\
(\mathrm{pCi} / \mathrm{g})\end{array}$ & $\begin{array}{l}\text { Uncertainty } \\
(\mathrm{pCi} / \mathrm{g})\end{array}$ & $\begin{array}{c}\mathrm{MDA} \\
(\mathrm{pCi} / \mathrm{g})\end{array}$ & Flag \\
\hline Cs-134 & $\mathrm{B} 26$ & 2007 & 0.08375 & 0.0114 & 0.0342 & - \\
\hline Cs-134 & B28 & 2007 & 0.1313 & 0.0157 & 0.0471 & 一 \\
\hline Cs-134 & B29 & 2007 & 0.3521 & 0.0615 & 0.1845 & 一 \\
\hline Cs-134 & B3 & 2007 & 0.1229 & 0.0172 & 0.0516 & - \\
\hline Cs-134 & B36 & 2007 & 0.06584 & 0.0126 & 0.0378 & - \\
\hline Cs-134 & B37 & 2007 & 0.05998 & 0.00803 & 0.02409 & - \\
\hline Cs-134 & B39 & 2007 & 0.09937 & 0.0134 & 0.0402 & 一 \\
\hline Cs-134 & B4 & 2007 & 0.0873 & 0.01005 & 0.03015 & - \\
\hline Cs-134 & B40 & 2007 & 0.1497 & 0.01565 & 0.04695 & - \\
\hline Cs-134 & B47 & 2007 & 0.09062 & 0.0149 & 0.0447 & - \\
\hline Cs-134 & B48 & 2007 & 0.0575 & 0.01125 & 0.03375 & - \\
\hline Cs-134 & B50 & 2007 & 0.1034 & 0.01815 & 0.05445 & - \\
\hline Cs-134 & B51 & 2007 & 0.07774 & 0.018 & 0.054 & - \\
\hline Cs-134 & B52 & 2007 & 0.0876 & 0.0098 & 0.0294 & - \\
\hline Cs-134 & B59 & 2007 & 0.2955 & 0.02915 & 0.08745 & - \\
\hline Cs-134 & B60 & 2007 & 0.3795 & 0.0291 & 0.0873 & - \\
\hline Cs-134 & B61 & 2007 & 0.0664 & 0.01315 & 0.03945 & - \\
\hline Cs-134 & B62 & 2007 & 0.07682 & 0.01225 & 0.03675 & - \\
\hline Cs-134 & B69 & 2007 & 0.2775 & 0.0765 & 0.2295 & - \\
\hline Cs-134 & B71 & 2007 & 0.2276 & 0.02455 & 0.07365 & - \\
\hline Cs-134 & B73 & 2007 & 0.1635 & 0.0173 & 0.0519 & - \\
\hline Cs-134 & $\mathrm{B} 80$ & 2007 & 0.1449 & 0.01985 & 0.05955 & - \\
\hline Cs-134 & B81 & 2007 & 0.1199 & 0.01745 & 0.05235 & - \\
\hline Cs-134 & B82 & 2007 & 0.07056 & 0.0144 & 0.0432 & - \\
\hline Cs-134 & B83 & 2007 & 0.1141 & 0.0139 & 0.0417 & - \\
\hline Cs-134 & B84 & 2007 & 0.0923 & 0.0143 & 0.0429 & - \\
\hline Cs-134 & B88 & 2007 & 0.1663 & 0.01635 & 0.04905 & - \\
\hline Cs-134 & B89 & 2007 & 0.09183 & 0.01255 & 0.03765 & - \\
\hline Cs-134 & B90 & 2007 & 0.1074 & 0.01665 & 0.04995 & - \\
\hline Cs-134 & B91 & 2007 & 0.0481 & 0.0069 & 0.0207 & - \\
\hline Cs-134 & B92 & 2007 & 0.102 & 0.0118 & 0.0354 & - \\
\hline Cs-134 & B93 & 2007 & 0.07183 & 0.00955 & 0.02865 & - \\
\hline Cs-134 & B94 & 2007 & 0.08766 & 0.0096 & 0.0288 & - \\
\hline Cs-134 & B95 & 2007 & 0.04935 & 0.00965 & 0.02895 & - \\
\hline Cs-134 & B99 & 2007 & 0.09587 & 0.01305 & 0.03915 & - \\
\hline Cs-134 & $\mathrm{C} 14$ & 2007 & 0.07217 & 0.0127 & 0.0381 & - \\
\hline Cs-134 & $\mathrm{C} 15$ & 2007 & 0.07853 & 0.00935 & 0.02805 & - \\
\hline
\end{tabular}


Table C-3. (continued).

\begin{tabular}{|c|c|c|c|c|c|c|}
\hline Radionuclide & $\begin{array}{c}\text { Sample } \\
\text { Location }\end{array}$ & Date & $\begin{array}{c}\text { Concentrations } \\
(\mathrm{pCi} / \mathrm{g})\end{array}$ & $\begin{array}{l}\text { Uncertainty } \\
(\mathrm{pCi} / \mathrm{g})\end{array}$ & $\begin{array}{c}\mathrm{MDA} \\
(\mathrm{pCi} / \mathrm{g})\end{array}$ & Flag \\
\hline Cs-134 & $\mathrm{C} 25$ & 2007 & 0.04077 & 0.00741 & 0.02223 & - \\
\hline Cs-134 & $\mathrm{C} 36$ & 2007 & 0.08481 & 0.0114 & 0.0342 & 一 \\
\hline Cs-134 & C37 & 2007 & 0.1669 & 0.01875 & 0.05625 & 一 \\
\hline Cs-134 & $\mathrm{C} 46$ & 2007 & 0.07452 & 0.0096 & 0.0288 & - \\
\hline Cs-134 & $\mathrm{C} 47$ & 2007 & 0.1065 & 0.01305 & 0.03915 & - \\
\hline Cs-134 & $\mathrm{C} 48$ & 2007 & 0.13 & 0.0141 & 0.0423 & - \\
\hline Cs-134 & C56 & 2007 & 0.1021 & 0.0111 & 0.0333 & - \\
\hline Cs-134 & $\mathrm{C} 57$ & 2007 & 0.07234 & 0.01395 & 0.04185 & - \\
\hline Cs-134 & D57 & 2007 & 0.05067 & 0.00754 & 0.02262 & - \\
\hline Cs-134 & D58 & 2007 & 0.1409 & 0.01465 & 0.04395 & - \\
\hline Cs-134 & D68 & 2007 & 0.1293 & 0.01895 & 0.05685 & - \\
\hline Cs-134 & D69 & 2007 & 0.1068 & 0.01355 & 0.04065 & - \\
\hline Cs-134 & D79 & 2007 & 0.0981 & 0.0148 & 0.0444 & - \\
\hline Cs-134 & D80 & 2007 & 0.08197 & 0.01015 & 0.03045 & - \\
\hline Cs-134 & D89 & 2007 & 0.1001 & 0.01055 & 0.03165 & - \\
\hline Cs-134 & D90 & 2007 & 0.1101 & 0.0139 & 0.0417 & - \\
\hline Cs-134 & D91 & 2007 & 0.1006 & 0.0125 & 0.0375 & - \\
\hline Cs-134 & B104 & 2008 & 0.03616 & 0.00588 & 0.01764 & - \\
\hline Cs-134 & A50 & 2009 & 0.007494 & 0.00428 & 0.01284 & $\mathrm{U}$ \\
\hline Cs-134 & $\mathrm{A} 27$ & 2010 & 0.0526 & 0.0105 & 0.0315 & - \\
\hline Cs-134 & A28 & 2010 & 0.03638 & 0.0118 & 0.0354 & - \\
\hline Cs-134 & A38 & 2010 & 0.06152 & 0.00959 & 0.02877 & - \\
\hline Cs-134 & A39 & 2010 & 0.04226 & 0.00415 & 0.01245 & - \\
\hline Cs-134 & A49 & 2010 & 0.05003 & 0.0076 & 0.0228 & - \\
\hline Cs-134 & A50 & 2010 & 0.03721 & 0.0107 & 0.0321 & - \\
\hline Cs-134 & A59 & 2010 & 0.05724 & 0.0139 & 0.0417 & - \\
\hline Cs-134 & A60 & 2010 & 0.03871 & 0.00781 & 0.02343 & - \\
\hline Cs-134 & A61 & 2010 & 0.05064 & 0.0063 & 0.0189 & - \\
\hline Cs-134 & A71 & 2010 & 0.03527 & 0.0105 & 0.0315 & - \\
\hline Cs-134 & A72 & 2010 & 0.0814 & 0.0113 & 0.0339 & - \\
\hline Cs-134 & A 15 & 2011 & -0.01394 & 0.0112 & 0.0336 & $\mathrm{U}$ \\
\hline Cs-134 & A16 & 2011 & 0.007038 & 0.0131 & 0.0393 & $\mathrm{U}$ \\
\hline Cs-134 & A17 & 2011 & 0.01711 & 0.0167 & 0.0501 & $\mathrm{U}$ \\
\hline Cs-134 & A27 & 2011 & -0.001129 & 0.0107 & 0.0321 & $\mathrm{U}$ \\
\hline Cs-134 & A28 & 2011 & 0.0006597 & 0.0248 & 0.0744 & $\mathrm{U}$ \\
\hline Cs-134 & A38 & 2011 & -0.005745 & 0.0173 & 0.0519 & $\mathrm{U}$ \\
\hline Cs-134 & A39 & 2011 & -0.0005636 & 0.0139 & 0.0417 & $\mathrm{U}$ \\
\hline
\end{tabular}


Table C-3. (continued).

\begin{tabular}{|c|c|c|c|c|c|c|}
\hline Radionuclide & $\begin{array}{c}\text { Sample } \\
\text { Location }\end{array}$ & Date & $\begin{array}{c}\text { Concentrations } \\
(\mathrm{pCi} / \mathrm{g})\end{array}$ & $\begin{array}{l}\text { Uncertainty } \\
(\mathrm{pCi} / \mathrm{g})\end{array}$ & $\begin{array}{c}\mathrm{MDA} \\
(\mathrm{pCi} / \mathrm{g})\end{array}$ & Flag \\
\hline Cs-134 & A4 & 2011 & -0.007031 & 0.0141 & 0.0423 & $\mathrm{U}$ \\
\hline Cs-134 & A44 & 2011 & -0.001563 & 0.024 & 0.072 & $\mathrm{U}$ \\
\hline Cs-134 & A47 & 2011 & -0.003504 & 0.0251 & 0.0753 & $\mathrm{U}$ \\
\hline Cs-134 & A49 & 2011 & 0.002134 & 0.0257 & 0.0771 & $\mathrm{U}$ \\
\hline Cs-134 & A50 & 2011 & -0.0114 & 0.0263 & 0.0789 & $\mathrm{U}$ \\
\hline Cs-134 & A55 & 2011 & -0.003762 & 0.014 & 0.042 & $\mathrm{U}$ \\
\hline Cs-134 & A58 & 2011 & -0.0084 & 0.0145 & 0.0435 & $\mathrm{U}$ \\
\hline Cs-134 & A59 & 2011 & 0.004225 & 0.0255 & 0.0765 & $\mathrm{U}$ \\
\hline Cs-134 & A6 & 2011 & -0.02617 & 0.0154 & 0.0462 & $\mathrm{U}$ \\
\hline Cs-134 & A61 & 2011 & -0.01099 & 0.0118 & 0.0354 & $\mathrm{U}$ \\
\hline Cs-134 & A66 & 2011 & -0.01043 & 0.0147 & 0.0441 & $\mathrm{U}$ \\
\hline Cs-134 & A69 & 2011 & -0.005644 & 0.0226 & 0.0678 & $\mathrm{U}$ \\
\hline Cs-134 & A70 & 2011 & 0.002402 & 0.0245 & 0.0735 & $\mathrm{U}$ \\
\hline Cs-134 & A72 & 2011 & 0.002162 & 0.0117 & 0.0351 & $\mathrm{U}$ \\
\hline Cs-134 & B101 & 2011 & 0.003715 & 0.0238 & 0.0714 & $\mathrm{U}$ \\
\hline Cs-134 & B102 & 2011 & -0.009781 & 0.0236 & 0.0708 & $\mathrm{U}$ \\
\hline Cs-134 & $\mathrm{B} 103$ & 2011 & -0.006791 & 0.0237 & 0.0711 & $\mathrm{U}$ \\
\hline Cs-134 & B104 & 2011 & -0.018 & 0.0131 & 0.0393 & $\mathrm{U}$ \\
\hline Cs-134 & B106 & 2011 & 0.001978 & 0.0264 & 0.0792 & $\mathrm{U}$ \\
\hline Cs-134 & $\mathrm{B} 14$ & 2011 & -0.02135 & 0.0159 & 0.0477 & $\mathrm{U}$ \\
\hline Cs-134 & B15 & 2011 & -0.0002064 & 0.0121 & 0.0363 & $\mathrm{U}$ \\
\hline Cs-134 & $\mathrm{B} 17$ & 2011 & -0.02687 & 0.00962 & 0.02886 & $\mathrm{U}$ \\
\hline Cs-134 & $\mathrm{B} 25$ & 2011 & -0.02834 & 0.0191 & 0.0573 & $\mathrm{U}$ \\
\hline Cs-134 & B28 & 2011 & -0.01044 & 0.0119 & 0.0357 & $\mathrm{U}$ \\
\hline Cs-134 & B29 & 2011 & -0.003382 & 0.0136 & 0.0408 & $\mathrm{U}$ \\
\hline Cs-134 & B3 & 2011 & -0.01778 & 0.0135 & 0.0405 & $\mathrm{U}$ \\
\hline Cs-134 & B39 & 2011 & -0.009049 & 0.00947 & 0.02841 & $\mathrm{U}$ \\
\hline Cs-134 & B4 & 2011 & -0.07655 & 0.0151 & 0.0453 & $\mathrm{U}$ \\
\hline Cs-134 & B40 & 2011 & -0.001086 & 0.00998 & 0.02994 & $\mathrm{U}$ \\
\hline Cs-134 & B47 & 2011 & -0.009866 & 0.0186 & 0.0558 & $\mathrm{U}$ \\
\hline Cs-134 & B48 & 2011 & -0.003563 & 0.0144 & 0.0432 & $\mathrm{U}$ \\
\hline Cs-134 & B50 & 2011 & -0.01777 & 0.0133 & 0.0399 & $\mathrm{U}$ \\
\hline Cs-134 & B52 & 2011 & -0.001545 & 0.0127 & 0.0381 & $\mathrm{U}$ \\
\hline Cs-134 & B61 & 2011 & 0.0006328 & 0.0171 & 0.0513 & $\mathrm{U}$ \\
\hline Cs-134 & B62 & 2011 & 0.01328 & 0.025 & 0.075 & $\mathrm{U}$ \\
\hline Cs-134 & B71 & 2011 & -0.009664 & 0.0202 & 0.0606 & $\mathrm{U}$ \\
\hline Cs-134 & B73 & 2011 & 0.01321 & 0.0114 & 0.0342 & $\mathrm{U}$ \\
\hline
\end{tabular}


Table C-3. (continued).

\begin{tabular}{|c|c|c|c|c|c|c|}
\hline Radionuclide & $\begin{array}{c}\text { Sample } \\
\text { Location }\end{array}$ & Date & $\begin{array}{c}\text { Concentrations } \\
(\mathrm{pCi} / \mathrm{g})\end{array}$ & $\begin{array}{c}\text { Uncertainty } \\
(\mathrm{pCi} / \mathrm{g})\end{array}$ & $\begin{array}{c}\text { MDA } \\
(\mathrm{pCi} / \mathrm{g})\end{array}$ & Flag \\
\hline Cs-134 & B80 & 2011 & -0.005139 & 0.0205 & 0.0615 & $\mathrm{U}$ \\
\hline Cs-134 & B83 & 2011 & 0.009197 & 0.0253 & 0.0759 & $\mathrm{U}$ \\
\hline Cs-134 & B84 & 2011 & -0.01913 & 0.0145 & 0.0435 & $\mathrm{U}$ \\
\hline Cs-134 & B89 & 2011 & 0.0491 & 0.0139 & 0.0417 & - \\
\hline Cs-134 & B90 & 2011 & -0.02797 & 0.016 & 0.048 & $\mathrm{U}$ \\
\hline Cs-134 & B91 & 2011 & 0.00005192 & 0.0194 & 0.0582 & $\mathrm{U}$ \\
\hline Cs-134 & B92 & 2011 & -0.04093 & 0.0121 & 0.0363 & $\mathrm{U}$ \\
\hline Cs-134 & B93 & 2011 & -0.000725 & 0.0115 & 0.0345 & $\mathrm{U}$ \\
\hline Cs-134 & B94 & 2011 & -0.02201 & 0.0114 & 0.0342 & $\mathrm{U}$ \\
\hline Cs-134 & B99 & 2011 & -0.0007449 & 0.0235 & 0.0705 & $\mathrm{U}$ \\
\hline Cs-134 & $\mathrm{C} 15$ & 2011 & 0.0007147 & 0.0132 & 0.0396 & $\mathrm{U}$ \\
\hline Cs-134 & $\mathrm{C} 25$ & 2011 & -0.03956 & 0.00914 & 0.02742 & $\mathrm{U}$ \\
\hline Cs-134 & C37 & 2011 & -0.0171 & 0.0256 & 0.0768 & $\mathrm{U}$ \\
\hline Cs-134 & $\mathrm{C} 46$ & 2011 & -0.2347 & 0.0169 & 0.0507 & $\mathrm{U}$ \\
\hline Cs-134 & $\mathrm{C} 48$ & 2011 & -0.002039 & 0.0208 & 0.0624 & $\mathrm{U}$ \\
\hline Cs-134 & C56 & 2011 & -0.01419 & 0.014 & 0.042 & $\mathrm{U}$ \\
\hline Cs-134 & C57 & 2011 & -0.01414 & 0.0105 & 0.0315 & $\mathrm{U}$ \\
\hline Cs-134 & D58 & 2011 & -0.008843 & 0.0117 & 0.0351 & $\mathrm{U}$ \\
\hline Cs-134 & D68 & 2011 & -0.01207 & 0.0239 & 0.0717 & $\mathrm{U}$ \\
\hline Cs-134 & D89 & 2011 & -0.00247 & 0.0189 & 0.0567 & $\mathrm{U}$ \\
\hline Cs-134 & D91 & 2011 & -0.009981 & 0.0135 & 0.0405 & $\mathrm{U}$ \\
\hline Cs-134 & A 15 & 2012 & 0.07316 & 0.00847 & 0.02541 & - \\
\hline Cs-134 & A38 & 2012 & 0.06792 & 0.00974 & 0.02922 & - \\
\hline Cs-134 & A44 & 2012 & 0.0706 & 0.00897 & 0.02691 & - \\
\hline Cs-134 & A47 & 2012 & 0.05849 & 0.00883 & 0.02649 & - \\
\hline Cs-134 & A59 & 2012 & 0.07781 & 0.00835 & 0.02505 & - \\
\hline Cs-134 & A6 & 2012 & 0.07395 & 0.0101 & 0.0303 & - \\
\hline Cs-134 & A69 & 2012 & 0.06467 & 0.01 & 0.03 & - \\
\hline Cs-134 & A70 & 2012 & 0.07268 & 0.0081 & 0.0243 & - \\
\hline Cs-134 & A72 & 2012 & 0.07116 & 0.01 & 0.03 & - \\
\hline Cs-134 & B14 & 2012 & 0.01813 & 0.00747 & 0.02241 & $\mathrm{U}$ \\
\hline Cs-134 & B48 & 2012 & 0.06781 & 0.00999 & 0.02997 & - \\
\hline Cs-134 & B83 & 2012 & 0.06728 & 0.00803 & 0.02409 & - \\
\hline Cs-134 & $\mathrm{C} 46$ & 2012 & 0.05312 & 0.00965 & 0.02895 & - \\
\hline Cs-134 & $\mathrm{C} 57$ & 2012 & 0.07259 & 0.0092 & 0.0276 & - \\
\hline Cs-134 & $\begin{array}{l}2 \text { INCH AIR } \\
\text { - INTEC }\end{array}$ & 2013 & 0.05303 & 0.0125 & 0.0374 & - \\
\hline
\end{tabular}


Table C-3. (continued).

\begin{tabular}{|c|c|c|c|c|c|c|}
\hline Radionuclide & $\begin{array}{c}\text { Sample } \\
\text { Location }\end{array}$ & Date & $\begin{array}{l}\text { Concentrations } \\
(\mathrm{pCi} / \mathrm{g})\end{array}$ & $\begin{array}{l}\text { Uncertainty } \\
(\mathrm{pCi} / \mathrm{g})\end{array}$ & $\begin{array}{c}\text { MDA } \\
(\mathrm{pCi} / \mathrm{g})\end{array}$ & Flag \\
\hline Cs-134 & A47 & 2013 & 0.06732 & 0.0129 & 0.0387 & - \\
\hline Cs-134 & A49 & 2013 & -0.006587 & 0.0113 & 0.0339 & $\mathrm{U}$ \\
\hline Cs-134 & A59 & 2013 & 0.08837 & 0.0167 & 0.0502 & - \\
\hline Cs-134 & A71 & 2013 & 0.05974 & 0.013 & 0.039 & 一 \\
\hline Cs-134 & A72 & 2013 & 0.01927 & 0.0059 & 0.0178 & - \\
\hline Cs-134 & $\begin{array}{c}2 \text { INCH AIR } \\
- \text { CPP }\end{array}$ & 2014 & 0.04829 & 0.0116 & 0.0348 & 一 \\
\hline Cs-134 & $\begin{array}{l}2 \text { INCH AIR } \\
\text { - INTEC }\end{array}$ & 2014 & 0.04165 & 0.0124 & 0.0372 & - \\
\hline Cs-134 & A38 & 2014 & 0.05365 & 0.0138 & 0.0414 & - \\
\hline Cs-134 & $\mathrm{A} 47$ & 2014 & 0.04106 & 0.0133 & 0.0399 & 一 \\
\hline Cs-134 & A58 & 2014 & 0.05459 & 0.0139 & 0.0417 & 一 \\
\hline Cs-134 & A59 & 2014 & 0.07062 & 0.0121 & 0.0363 & - \\
\hline Cs-134 & $\mathrm{B} 15$ & 2014 & 0.03674 & 0.0242 & 0.0726 & $\mathrm{U}$ \\
\hline Cs-134 & B28 & 2014 & 0.04318 & 0.0118 & 0.0354 & - \\
\hline Cs-134 & B29 & 2014 & 0.04242 & 0.012 & 0.036 & - \\
\hline Cs-134 & $\mathrm{B} 3$ & 2014 & 0.0376 & 0.0211 & 0.0633 & $\mathrm{U}$ \\
\hline Cs-134 & B3 & 2014 & 0.03359 & 0.00787 & 0.02361 & - \\
\hline Cs-134 & $\mathrm{B} 4$ & 2014 & 0.04311 & 0.00629 & 0.01887 & - \\
\hline Cs-134 & B40 & 2014 & 0.03528 & 0.00802 & 0.02406 & - \\
\hline Cs-134 & $\mathrm{B} 50$ & 2014 & 0.04071 & 0.013 & 0.039 & - \\
\hline Cs-134 & B52 & 2014 & 0.07505 & 0.015 & 0.045 & - \\
\hline Cs-134 & B52 & 2014 & 0.01826 & 0.0151 & 0.0453 & $\mathrm{U}$ \\
\hline Cs-137 & A105 & 1974 & 6.8 & 0.3 & 0.9 & - \\
\hline Cs-137 & A116 & 1974 & 1.32 & 0.5 & 1.5 & $\mathrm{U}$ \\
\hline Cs-137 & A36 & 1974 & 32.6 & 0.6 & 1.8 & - \\
\hline Cs-137 & A 37 & 1974 & 26.6 & 0.5 & 1.5 & - \\
\hline Cs-137 & A45 & 1974 & 14.7 & 0.4 & 1.2 & - \\
\hline Cs-137 & A46 & 1974 & 31.1 & 0.5 & 1.5 & - \\
\hline Cs-137 & A47 & 1974 & 17.6 & 0.4 & 1.2 & 一 \\
\hline Cs-137 & A 48 & 1974 & 22.3 & 0.5 & 1.5 & 一 \\
\hline Cs-137 & A5 & 1974 & 9.8 & 0.4 & 1.2 & - \\
\hline Cs-137 & $\mathrm{B} 115$ & 1974 & 5.3 & 0.3 & 0.9 & - \\
\hline Cs-137 & B120 & 1974 & 1.3 & 0.1 & 0.3 & - \\
\hline Cs-137 & B14 & 1974 & 18 & 4 & 12 & - \\
\hline Cs-137 & $\mathrm{B} 25$ & 1974 & 31.9 & 0.6 & 1.8 & - \\
\hline Cs-137 & B26 & 1974 & 11.2 & 0.4 & 1.2 & - \\
\hline
\end{tabular}


Table C-3. (continued).

\begin{tabular}{|c|c|c|c|c|c|c|}
\hline Radionuclide & $\begin{array}{c}\text { Sample } \\
\text { Location }\end{array}$ & Date & $\begin{array}{c}\text { Concentrations } \\
(\mathrm{pCi} / \mathrm{g})\end{array}$ & $\begin{array}{l}\text { Uncertainty } \\
(\mathrm{pCi} / \mathrm{g})\end{array}$ & $\begin{array}{c}\mathrm{MDA} \\
(\mathrm{pCi} / \mathrm{g})\end{array}$ & Flag \\
\hline Cs-137 & B37 & 1974 & 21.5 & 0.5 & 1.5 & - \\
\hline Cs-137 & B38 & 1974 & 12.7 & 0.4 & 1.2 & 一 \\
\hline Cs-137 & B47 & 1974 & 31.3 & 0.6 & 1.8 & 一 \\
\hline Cs-137 & B49 & 1974 & 19.8 & 0.5 & 1.5 & - \\
\hline Cs-137 & B5 & 1974 & 3.8 & 0.1 & 0.3 & - \\
\hline Cs-137 & B59 & 1974 & 40 & 0.6 & 1.8 & - \\
\hline Cs-137 & B60 & 1974 & 18.6 & 0.4 & 1.2 & - \\
\hline Cs-137 & B66 & 1974 & 52.7 & 0.5 & 1.5 & - \\
\hline Cs-137 & B67 & 1974 & 11.3 & 0.3 & 0.9 & - \\
\hline Cs-137 & B70 & 1974 & 17.7 & 0.5 & 1.5 & - \\
\hline Cs-137 & B72 & 1974 & 9.4 & 0.3 & 0.9 & - \\
\hline Cs-137 & B76 & 1974 & 2.7 & 0.2 & 0.6 & - \\
\hline Cs-137 & B78 & 1974 & 34.1 & 0.6 & 1.8 & - \\
\hline Cs-137 & $\mathrm{B} 80$ & 1974 & 16.1 & 0.4 & 1.2 & - \\
\hline Cs-137 & B82 & 1974 & 19.7 & 0.5 & 1.5 & - \\
\hline Cs-137 & B89 & 1974 & 12.5 & 0.4 & 1.2 & - \\
\hline Cs-137 & B90 & 1974 & 11 & 0.3 & 0.9 & - \\
\hline Cs-137 & B92 & 1974 & 23.3 & 0.5 & 1.5 & - \\
\hline Cs-137 & B94 & 1974 & 16.1 & 0.4 & 1.2 & - \\
\hline Cs-137 & $\mathrm{C} 100$ & 1974 & 1.8 & 0.27 & 0.81 & - \\
\hline Cs-137 & C117 & 1974 & 1.1 & 0.2 & 0.6 & - \\
\hline Cs-137 & $\mathrm{C} 12 \mathrm{a}$ & 1974 & 12.4 & 0.4 & 1.2 & - \\
\hline Cs-137 & $\mathrm{C} 14$ & 1974 & 2.7 & 0.2 & 0.6 & - \\
\hline Cs-137 & $\mathrm{C} 25$ & 1974 & 4.3 & 0.2 & 0.6 & - \\
\hline Cs-137 & $\mathrm{C} 26$ & 1974 & 2.5 & 0.2 & 0.6 & - \\
\hline Cs-137 & C31 & 1974 & 2.3 & 0.2 & 0.6 & - \\
\hline Cs-137 & $\mathrm{C} 45$ & 1974 & 4 & 0.2 & 0.6 & - \\
\hline Cs-137 & C49 & 1974 & 3.7 & 0.2 & 0.6 & - \\
\hline Cs-137 & D40 & 1974 & 1.2 & 0.1 & 0.3 & - \\
\hline Cs-137 & D58 & 1974 & 2.6 & 0.2 & 0.6 & - \\
\hline Cs-137 & A-110 & 1982 & 2.54 & 0.12 & 0.36 & - \\
\hline Cs- 137 & A-115 & 1982 & 7.5 & 0.13 & 0.39 & - \\
\hline Cs-137 & A-120 & 1982 & 3.71 & 0.16 & 0.48 & - \\
\hline Cs-137 & A-17 & 1982 & 7.9 & 0.29 & 0.87 & - \\
\hline Cs-137 & A-44 & 1982 & 7.6 & 0.3 & 0.9 & - \\
\hline Cs-137 & A-46 & 1982 & 27.4 & 0.9 & 2.7 & - \\
\hline Cs-137 & A-48 & 1982 & 21.7 & 0.7 & 2.1 & - \\
\hline
\end{tabular}


Table C-3. (continued).

\begin{tabular}{|c|c|c|c|c|c|c|}
\hline Radionuclide & $\begin{array}{l}\text { Sample } \\
\text { Location }\end{array}$ & Date & $\begin{array}{c}\text { Concentrations } \\
(\mathrm{pCi} / \mathrm{g})\end{array}$ & $\begin{array}{l}\text { Uncertainty } \\
(\mathrm{pCi} / \mathrm{g})\end{array}$ & $\begin{array}{c}\mathrm{MDA} \\
(\mathrm{pCi} / \mathrm{g})\end{array}$ & Flag \\
\hline Cs-137 & A-72 & 1982 & 8.78 & 0.32 & 0.96 & - \\
\hline Cs-137 & A-90 & 1982 & 5.76 & 0.22 & 0.66 & 一 \\
\hline Cs-137 & B-115 & 1982 & 8.3 & 0.3 & 0.9 & - \\
\hline Cs-137 & B-120 & 1982 & 1.89 & 0.11 & 0.33 & - \\
\hline Cs-137 & B-25 & 1982 & 37.1 & 1.2 & 3.6 & - \\
\hline Cs-137 & B-27 & 1982 & 4.14 & 0.18 & 0.54 & - \\
\hline Cs-137 & B-4 & 1982 & 4.6 & 0.2 & 0.6 & - \\
\hline Cs-137 & B-48 & 1982 & 24.3 & 0.82 & 2.46 & - \\
\hline Cs-137 & B-52 & 1982 & 4.7 & 0.2 & 0.6 & - \\
\hline Cs-137 & B-69 & 1982 & 19.6 & 0.07 & 0.21 & - \\
\hline Cs-137 & B-83 & 1982 & 13.7 & 0.5 & 1.5 & - \\
\hline Cs-137 & B-89 & 1982 & 5.8 & 0.2 & 0.6 & - \\
\hline Cs-137 & $\mathrm{C}-114$ & 1982 & 1.71 & 0.1 & 0.3 & - \\
\hline Cs-137 & $\mathrm{C}-14$ & 1982 & 4.4 & 0.2 & 0.6 & - \\
\hline Cs-137 & $\mathrm{C}-21$ & 1982 & 1.7 & 0.1 & 0.3 & - \\
\hline Cs-137 & C-48 & 1982 & 2.35 & 0.12 & 0.36 & - \\
\hline Cs-137 & $\mathrm{C}-85$ & 1982 & 1.1 & 0.09 & 0.27 & - \\
\hline Cs-137 & D-114 & 1982 & 3.01 & 0.14 & 0.42 & - \\
\hline Cs-137 & D-49 & 1982 & 1.83 & 0.1 & 0.3 & - \\
\hline Cs-137 & D-57 & 1982 & 4.18 & 0.18 & 0.54 & - \\
\hline Cs-137 & D-78 & 1982 & 6.7 & 0.3 & 0.9 & - \\
\hline Cs-137 & D-96 & 1982 & 0.88 & 0.07 & 0.21 & - \\
\hline Cs-137 & A108 & 1989 & 6.2 & 0.3 & 0.9 & - \\
\hline Cs-137 & A115 & 1989 & 9.9 & 0.4 & 1.2 & - \\
\hline Cs-137 & A17 & 1989 & 4.53 & 0.21 & 0.63 & - \\
\hline Cs-137 & A36 & 1989 & 37.8 & 1.4 & 4.2 & - \\
\hline Cs- 137 & A46 & 1989 & 14.9 & 0.6 & 1.8 & - \\
\hline Cs-137 & A52 & 1989 & 5.9 & 0.3 & 0.9 & - \\
\hline Cs-137 & A66 & 1989 & 3.31 & 0.15 & 0.45 & - \\
\hline Cs-137 & A72 & 1989 & 9.9 & 0.4 & 1.2 & - \\
\hline Cs-137 & A8 & 1989 & 1.89 & 0.11 & 0.33 & - \\
\hline Cs-137 & A90 & 1989 & 3.26 & 0.16 & 0.48 & - \\
\hline Cs-137 & B115 & 1989 & 4.14 & 0.16 & 0.48 & - \\
\hline Cs-137 & B119 & 1989 & 6.8 & 0.3 & 0.9 & - \\
\hline Cs-137 & B18 & 1989 & 2.71 & 0.15 & 0.45 & - \\
\hline Cs-137 & B25 & 1989 & 22.2 & 0.9 & 2.7 & - \\
\hline Cs-137 & B5 & 1989 & 4.61 & 0.2 & 0.6 & - \\
\hline
\end{tabular}


Table C-3. (continued).

\begin{tabular}{|c|c|c|c|c|c|c|}
\hline Radionuclide & $\begin{array}{c}\text { Sample } \\
\text { Location }\end{array}$ & Date & $\begin{array}{c}\text { Concentrations } \\
(\mathrm{pCi} / \mathrm{g})\end{array}$ & $\begin{array}{l}\text { Uncertainty } \\
(\mathrm{pCi} / \mathrm{g})\end{array}$ & $\begin{array}{c}\mathrm{MDA} \\
(\mathrm{pCi} / \mathrm{g})\end{array}$ & Flag \\
\hline Cs-137 & B52 & 1989 & 2.52 & 0.12 & 0.36 & - \\
\hline Cs-137 & B70 & 1989 & 7.9 & 0.3 & 0.9 & 一 \\
\hline Cs-137 & B73 & 1989 & 4.88 & 0.21 & 0.63 & 一 \\
\hline Cs-137 & B90 & 1989 & 5.9 & 0.3 & 0.9 & - \\
\hline Cs-137 & B96 & 1989 & 9.2 & 0.2 & 0.6 & - \\
\hline Cs-137 & $\mathrm{C} 114$ & 1989 & 2.02 & 0.12 & 0.36 & - \\
\hline Cs-137 & C40 & 1989 & 0.85 & 0.08 & 0.24 & - \\
\hline Cs-137 & $\mathrm{C} 48$ & 1989 & 1.55 & 0.09 & 0.27 & - \\
\hline Cs-137 & C79 & 1989 & 2.11 & 0.12 & 0.36 & - \\
\hline Cs-137 & C96 & 1989 & 1.75 & 1.1 & 3.3 & $\mathrm{U}$ \\
\hline Cs-137 & D30 & 1989 & 1.69 & 0.09 & 0.27 & - \\
\hline Cs-137 & D37 & 1989 & 2.84 & 0.14 & 0.42 & - \\
\hline Cs-137 & D63 & 1989 & 1.06 & 0.09 & 0.27 & - \\
\hline Cs-137 & D92 & 1989 & 1.91 & 0.12 & 0.36 & - \\
\hline Cs-137 & D96 & 1989 & 0.89 & 0.07 & 0.21 & - \\
\hline Cs-137 & A 15 & 2006 & 1.162 & 0.0106 & 0.0318 & - \\
\hline Cs-137 & A16 & 2006 & 0.8737 & 0.00805 & 0.02415 & - \\
\hline Cs-137 & A17 & 2006 & 0.6244 & 0.0067 & 0.0201 & - \\
\hline Cs-137 & A27 & 2006 & 1.02 & 0.01105 & 0.03315 & - \\
\hline Cs-137 & A28 & 2006 & 0.4925 & 0.0063 & 0.0189 & - \\
\hline Cs-137 & A38 & 2006 & 0.962 & 0.01195 & 0.03585 & - \\
\hline Cs-137 & A39 & 2006 & 1.143 & 0.0074 & 0.0222 & - \\
\hline Cs-137 & $\mathrm{A} 4$ & 2006 & 0.6364 & 0.0068 & 0.0204 & - \\
\hline Cs-137 & A44 & 2006 & 0.5795 & 0.0062 & 0.0186 & - \\
\hline Cs-137 & A45 & 2006 & 1.47 & 0.0102 & 0.0306 & - \\
\hline Cs-137 & A47 & 2006 & 2.289 & 0.01075 & 0.03225 & - \\
\hline Cs-137 & A48 & 2006 & 2.666 & 0.0109 & 0.0327 & - \\
\hline Cs-137 & A49 & 2006 & 1.901 & 0.00815 & 0.02445 & - \\
\hline Cs-137 & A5 & 2006 & 0.2011 & 0.00795 & 0.02385 & - \\
\hline Cs-137 & A50 & 2006 & 2.064 & 0.01205 & 0.03615 & - \\
\hline Cs-137 & A55 & 2006 & 0.629 & 0.0052 & 0.0156 & - \\
\hline Cs-137 & A56 & 2006 & 0.2694 & 0.00453 & 0.01359 & - \\
\hline Cs-137 & A58 & 2006 & 1.743 & 0.0127 & 0.0381 & - \\
\hline Cs-137 & A59 & 2006 & 1.639 & 0.0122 & 0.0366 & - \\
\hline Cs-137 & A6 & 2006 & 0.2498 & 0.00605 & 0.01815 & - \\
\hline Cs-137 & A61 & 2006 & 1.46 & 0.0089 & 0.0267 & - \\
\hline Cs-137 & A66 & 2006 & 0.6147 & 0.01025 & 0.03075 & - \\
\hline
\end{tabular}


Table C-3. (continued).

\begin{tabular}{|c|c|c|c|c|c|c|}
\hline Radionuclide & $\begin{array}{c}\text { Sample } \\
\text { Location }\end{array}$ & Date & $\begin{array}{c}\text { Concentrations } \\
(\mathrm{pCi} / \mathrm{g})\end{array}$ & $\begin{array}{l}\text { Uncertainty } \\
(\mathrm{pCi} / \mathrm{g})\end{array}$ & $\begin{array}{c}\mathrm{MDA} \\
(\mathrm{pCi} / \mathrm{g})\end{array}$ & Flag \\
\hline Cs-137 & A67 & 2006 & 0.3901 & 0.0105 & 0.0315 & - \\
\hline Cs-137 & A68 & 2006 & 0.5643 & 0.0077 & 0.0231 & 一 \\
\hline Cs-137 & A69 & 2006 & 0.9628 & 0.0084 & 0.0252 & 一 \\
\hline Cs-137 & A70 & 2006 & 1.144 & 0.00645 & 0.01935 & - \\
\hline Cs-137 & A71 & 2006 & 1.158 & 0.0081 & 0.0243 & - \\
\hline Cs-137 & A72 & 2006 & 1.397 & 0.0171 & 0.0513 & - \\
\hline Cs-137 & B100 & 2006 & 0.3913 & 0.0081 & 0.0243 & - \\
\hline Cs-137 & B101 & 2006 & 0.4751 & 0.004785 & 0.014355 & - \\
\hline Cs-137 & B102 & 2006 & 0.2786 & 0.003345 & 0.010035 & - \\
\hline Cs-137 & B103 & 2006 & 0.02748 & 0.003385 & 0.010155 & - \\
\hline Cs-137 & B104 & 2006 & 0.784 & 0.0098 & 0.0294 & - \\
\hline Cs-137 & B105 & 2006 & 0.6936 & 0.00965 & 0.02895 & - \\
\hline Cs-137 & B106 & 2006 & 0.6116 & 0.0083 & 0.0249 & - \\
\hline Cs-137 & B14 & 2006 & 1.435 & 0.0103 & 0.0309 & - \\
\hline Cs-137 & B15 & 2006 & 0.5403 & 0.00545 & 0.01635 & - \\
\hline Cs-137 & $\mathrm{B} 17$ & 2006 & 0.3234 & 0.00387 & 0.01161 & - \\
\hline Cs-137 & $\mathrm{B} 25$ & 2006 & 2.659 & 0.01225 & 0.03675 & - \\
\hline Cs-137 & B26 & 2006 & 0.7709 & 0.0062 & 0.0186 & - \\
\hline Cs-137 & $\mathrm{B} 28$ & 2006 & 0.3476 & 0.00412 & 0.01236 & - \\
\hline Cs-137 & B29 & 2006 & 0.2993 & 0.00349 & 0.01047 & - \\
\hline Cs-137 & B3 & 2006 & 1.076 & 0.01075 & 0.03225 & - \\
\hline Cs-137 & B36 & 2006 & 2.301 & 0.01345 & 0.04035 & - \\
\hline Cs-137 & B37 & 2006 & 0.8525 & 0.008 & 0.024 & - \\
\hline Cs-137 & B39 & 2006 & 0.5066 & 0.01075 & 0.03225 & - \\
\hline Cs-137 & B4 & 2006 & 0.4512 & 0.0037 & 0.0111 & - \\
\hline Cs-137 & B40 & 2006 & 0.3954 & 0.00575 & 0.01725 & - \\
\hline Cs-137 & B47 & 2006 & 1.747 & 0.01 & 0.03 & - \\
\hline Cs-137 & B48 & 2006 & 1.373 & 0.00695 & 0.02085 & - \\
\hline Cs-137 & B50 & 2006 & 0.4518 & 0.004335 & 0.013005 & - \\
\hline Cs-137 & B51 & 2006 & 0.3459 & 0.003335 & 0.010005 & - \\
\hline Cs-137 & B52 & 2006 & 0.3007 & 0.00515 & 0.01545 & - \\
\hline Cs-137 & B59 & 2006 & 0.1425 & 0.00595 & 0.01785 & - \\
\hline Cs-137 & B60 & 2006 & 0.136 & 0.00665 & 0.01995 & - \\
\hline Cs-137 & B61 & 2006 & 0.6369 & 0.0054 & 0.0162 & - \\
\hline Cs-137 & B62 & 2006 & 0.4835 & 0.0069 & 0.0207 & - \\
\hline Cs-137 & B71 & 2006 & 0.6154 & 0.0062 & 0.0186 & - \\
\hline Cs-137 & B73 & 2006 & 0.499 & 0.0063 & 0.0189 & - \\
\hline
\end{tabular}


Table C-3. (continued).

\begin{tabular}{|c|c|c|c|c|c|c|}
\hline Radionuclide & $\begin{array}{l}\text { Sample } \\
\text { Location }\end{array}$ & Date & $\begin{array}{c}\text { Concentrations } \\
(\mathrm{pCi} / \mathrm{g})\end{array}$ & $\begin{array}{l}\text { Uncertainty } \\
(\mathrm{pCi} / \mathrm{g})\end{array}$ & $\begin{array}{l}\text { MDA } \\
(\mathrm{pCi} / \mathrm{g})\end{array}$ & Flag \\
\hline Cs-137 & $\mathrm{B} 80$ & 2006 & 0.6458 & 0.00775 & 0.02325 & 一 \\
\hline Cs-137 & B81 & 2006 & 0.5445 & 0.0057 & 0.0171 & 一 \\
\hline Cs-137 & B82 & 2006 & 0.188 & 0.00444 & 0.01332 & 一 \\
\hline Cs-137 & B83 & 2006 & 0.9389 & 0.0067 & 0.0201 & - \\
\hline Cs-137 & B84 & 2006 & 0.4988 & 0.00805 & 0.02415 & - \\
\hline Cs-137 & B88 & 2006 & 0.462 & 0.0065 & 0.0195 & - \\
\hline Cs-137 & B89 & 2006 & 0.4841 & 0.00491 & 0.01473 & - \\
\hline Cs-137 & B90 & 2006 & 0.5428 & 0.0056 & 0.0168 & - \\
\hline Cs-137 & B91 & 2006 & 0.6455 & 0.00525 & 0.01575 & - \\
\hline Cs-137 & B92 & 2006 & 0.7188 & 0.00455 & 0.01365 & - \\
\hline Cs-137 & B93 & 2006 & 0.8962 & 0.01165 & 0.03495 & - \\
\hline Cs-137 & B94 & 2006 & 0.7178 & 0.0082 & 0.0246 & - \\
\hline Cs-137 & B95 & 2006 & 0.7867 & 0.00955 & 0.02865 & - \\
\hline Cs-137 & B99 & 2006 & 0.3959 & 0.01125 & 0.03375 & - \\
\hline Cs-137 & $\mathrm{C} 14$ & 2006 & 0.8078 & 0.01245 & 0.03735 & - \\
\hline Cs-137 & $\mathrm{C} 15$ & 2006 & 0.3282 & 0.00459 & 0.01377 & - \\
\hline Cs-137 & $\mathrm{C} 25$ & 2006 & 0.4583 & 0.00535 & 0.01605 & - \\
\hline Cs-137 & $\mathrm{C} 36$ & 2006 & 0.3184 & 0.00318 & 0.00954 & - \\
\hline Cs-137 & $\mathrm{C} 37$ & 2006 & 0.3376 & 0.00735 & 0.02205 & - \\
\hline Cs-137 & $\mathrm{C} 46$ & 2006 & 0.5585 & 0.00585 & 0.01755 & - \\
\hline Cs-137 & $\mathrm{C} 47$ & 2006 & 0.4458 & 0.0065 & 0.0195 & - \\
\hline Cs-137 & $\mathrm{C} 48$ & 2006 & 0.2746 & 0.009 & 0.027 & - \\
\hline Cs-137 & C56 & 2006 & 0.5547 & 0.00605 & 0.01815 & - \\
\hline Cs-137 & C57 & 2006 & 0.3477 & 0.0064 & 0.0192 & - \\
\hline Cs-137 & D57 & 2006 & 0.4091 & 0.00765 & 0.02295 & - \\
\hline Cs-137 & D58 & 2006 & 0.3591 & 0.004755 & 0.014265 & - \\
\hline Cs-137 & D68 & 2006 & 0.3745 & 0.00565 & 0.01695 & - \\
\hline Cs-137 & D69 & 2006 & 0.2646 & 2.45 & 7.35 & $\mathrm{U}$ \\
\hline Cs-137 & D79 & 2006 & 0.4763 & 0.00625 & 0.01875 & - \\
\hline Cs-137 & D80 & 2006 & 0.2384 & 1.7 & 5.1 & $\mathrm{U}$ \\
\hline Cs-137 & D89 & 2006 & 0.5785 & 0.00625 & 0.01875 & - \\
\hline Cs-137 & D90 & 2006 & 0.4577 & 1.35 & 4.05 & $\mathrm{U}$ \\
\hline Cs-137 & D91 & 2006 & 0.2763 & 1.65 & 4.95 & $\mathrm{U}$ \\
\hline Cs-137 & A 15 & 2007 & 2.165 & 0.0155 & 0.0465 & - \\
\hline Cs-137 & A16 & 2007 & 1.203 & 0.0115 & 0.0345 & - \\
\hline Cs-137 & A17 & 2007 & 1.025 & 0.01115 & 0.03345 & - \\
\hline Cs-137 & $\mathrm{A} 27$ & 2007 & 1.375 & 0.0123 & 0.0369 & - \\
\hline
\end{tabular}


Table C-3. (continued).

\begin{tabular}{|c|c|c|c|c|c|c|}
\hline Radionuclide & $\begin{array}{c}\text { Sample } \\
\text { Location }\end{array}$ & Date & $\begin{array}{c}\text { Concentrations } \\
(\mathrm{pCi} / \mathrm{g})\end{array}$ & $\begin{array}{l}\text { Uncertainty } \\
(\mathrm{pCi} / \mathrm{g})\end{array}$ & $\begin{array}{c}\mathrm{MDA} \\
(\mathrm{pCi} / \mathrm{g})\end{array}$ & Flag \\
\hline Cs-137 & A28 & 2007 & 1.423 & 0.0176 & 0.0528 & - \\
\hline Cs-137 & A37 & 2007 & 4.497 & 0.0178 & 0.0534 & 一 \\
\hline Cs-137 & A38 & 2007 & 1.349 & 0.01295 & 0.03885 & 一 \\
\hline Cs-137 & A39 & 2007 & 1.458 & 0.0124 & 0.0372 & - \\
\hline Cs-137 & $\mathrm{A} 4$ & 2007 & 1.101 & 0.00735 & 0.02205 & - \\
\hline Cs-137 & A44 & 2007 & 1.747 & 0.01385 & 0.04155 & - \\
\hline Cs-137 & A45 & 2007 & 2.387 & 0.01495 & 0.04485 & - \\
\hline Cs-137 & A47 & 2007 & 3.833 & 0.0129 & 0.0387 & - \\
\hline Cs-137 & A48 & 2007 & 4.826 & 0.02115 & 0.06345 & - \\
\hline Cs-137 & A49 & 2007 & 3.398 & 0.01715 & 0.05145 & - \\
\hline Cs-137 & A5 & 2007 & 0.5418 & 0.00865 & 0.02595 & - \\
\hline Cs-137 & A50 & 2007 & 1.914 & 0.0241 & 0.0723 & - \\
\hline Cs-137 & A55 & 2007 & 1.127 & 0.01125 & 0.03375 & - \\
\hline Cs-137 & A56 & 2007 & 0.6982 & 0.0079 & 0.0237 & - \\
\hline Cs-137 & A58 & 2007 & 2.829 & 0.01945 & 0.05835 & - \\
\hline Cs-137 & A59 & 2007 & 2.849 & 0.01125 & 0.03375 & - \\
\hline Cs-137 & A6 & 2007 & 0.6309 & 0.01095 & 0.03285 & - \\
\hline Cs-137 & A60 & 2007 & 2.349 & 0.0156 & 0.0468 & - \\
\hline Cs-137 & A61 & 2007 & 2.288 & 0.01435 & 0.04305 & - \\
\hline Cs-137 & A66 & 2007 & 0.9702 & 0.0202 & 0.0606 & - \\
\hline Cs-137 & A67 & 2007 & 1.565 & 0.0159 & 0.0477 & - \\
\hline Cs-137 & A68 & 2007 & 1.405 & 0.0203 & 0.0609 & - \\
\hline Cs-137 & A69 & 2007 & 2.406 & 0.0179 & 0.0537 & - \\
\hline Cs-137 & A70 & 2007 & 2.304 & 0.01795 & 0.05385 & - \\
\hline Cs-137 & A71 & 2007 & 1.918 & 0.0143 & 0.0429 & - \\
\hline Cs-137 & A72 & 2007 & 2.176 & 0.01495 & 0.04485 & - \\
\hline Cs-137 & $\mathrm{B} 100$ & 2007 & 0.7727 & 0.0119 & 0.0357 & - \\
\hline Cs-137 & B101 & 2007 & 0.8 & 0.01455 & 0.04365 & - \\
\hline Cs-137 & B102 & 2007 & 0.6156 & 0.0121 & 0.0363 & - \\
\hline Cs-137 & B103 & 2007 & 0.0531 & 0.006 & 0.018 & - \\
\hline Cs-137 & B104 & 2007 & 1.35 & 0.013 & 0.039 & - \\
\hline Cs-137 & B105 & 2007 & 1.249 & 0.01715 & 0.05145 & - \\
\hline Cs-137 & B106 & 2007 & 1.263 & 0.0168 & 0.0504 & - \\
\hline Cs-137 & B14 & 2007 & 2.306 & 0.01645 & 0.04935 & - \\
\hline Cs-137 & B15 & 2007 & 0.9534 & 0.011 & 0.033 & - \\
\hline Cs-137 & $\mathrm{B} 17$ & 2007 & 0.7011 & 0.0132 & 0.0396 & - \\
\hline Cs-137 & $\mathrm{B} 25$ & 2007 & 4.33 & 0.0211 & 0.0633 & - \\
\hline
\end{tabular}


Table C-3. (continued).

\begin{tabular}{|c|c|c|c|c|c|c|}
\hline Radionuclide & $\begin{array}{c}\text { Sample } \\
\text { Location }\end{array}$ & Date & $\begin{array}{c}\text { Concentrations } \\
(\mathrm{pCi} / \mathrm{g})\end{array}$ & $\begin{array}{l}\text { Uncertainty } \\
(\mathrm{pCi} / \mathrm{g})\end{array}$ & $\begin{array}{c}\mathrm{MDA} \\
(\mathrm{pCi} / \mathrm{g})\end{array}$ & Flag \\
\hline Cs-137 & $\mathrm{B} 26$ & 2007 & 1.401 & 0.01295 & 0.03885 & - \\
\hline Cs-137 & B28 & 2007 & 0.6992 & 0.0097 & 0.0291 & 一 \\
\hline Cs-137 & B29 & 2007 & 0.4985 & 0.01135 & 0.03405 & 一 \\
\hline Cs-137 & B3 & 2007 & 1.749 & 0.01325 & 0.03975 & - \\
\hline Cs-137 & B36 & 2007 & 4.74 & 0.0248 & 0.0744 & - \\
\hline Cs-137 & B37 & 2007 & 1.543 & 0.0223 & 0.0669 & - \\
\hline Cs-137 & B39 & 2007 & 0.6256 & 0.00774 & 0.02322 & - \\
\hline Cs-137 & B4 & 2007 & 0.859 & 0.01295 & 0.03885 & - \\
\hline Cs-137 & B40 & 2007 & 0.6985 & 0.01045 & 0.03135 & - \\
\hline Cs-137 & B47 & 2007 & 3.383 & 0.0171 & 0.0513 & - \\
\hline Cs-137 & B48 & 2007 & 2.074 & 0.01025 & 0.03075 & - \\
\hline Cs-137 & B50 & 2007 & 1.098 & 0.012 & 0.036 & - \\
\hline Cs-137 & B51 & 2007 & 0.8647 & 0.0164 & 0.0492 & - \\
\hline Cs-137 & B52 & 2007 & 0.478 & 0.0101 & 0.0303 & - \\
\hline Cs-137 & B59 & 2007 & 0.01488 & 0.00367 & 0.01101 & - \\
\hline Cs-137 & B60 & 2007 & 0.1857 & 0.01045 & 0.03135 & - \\
\hline Cs-137 & B61 & 2007 & 1.186 & 0.0121 & 0.0363 & - \\
\hline Cs-137 & B62 & 2007 & 0.8465 & 0.01345 & 0.04035 & - \\
\hline Cs-137 & B69 & 2007 & 50.3 & 0.092 & 0.276 & - \\
\hline Cs-137 & B71 & 2007 & 0.9012 & 0.0215 & 0.0645 & - \\
\hline Cs-137 & B73 & 2007 & 0.9943 & 0.0141 & 0.0423 & - \\
\hline Cs-137 & $\mathrm{B} 80$ & 2007 & 0.08106 & 0.00895 & 0.02685 & - \\
\hline Cs-137 & B81 & 2007 & 0.7352 & 0.0152 & 0.0456 & - \\
\hline Cs-137 & B82 & 2007 & 0.5103 & 0.02685 & 0.08055 & - \\
\hline Cs-137 & B83 & 2007 & 1.762 & 0.01345 & 0.04035 & - \\
\hline Cs-137 & B84 & 2007 & 1.148 & 0.0123 & 0.0369 & - \\
\hline Cs-137 & B88 & 2007 & 0.8258 & 0.0103 & 0.0309 & - \\
\hline Cs-137 & B89 & 2007 & 0.8762 & 0.0128 & 0.0384 & - \\
\hline Cs-137 & B90 & 2007 & 1.139 & 0.013 & 0.039 & - \\
\hline Cs-137 & B91 & 2007 & 1.156 & 0.0132 & 0.0396 & - \\
\hline Cs-137 & B92 & 2007 & 1.301 & 0.0207 & 0.0621 & - \\
\hline Cs-137 & B93 & 2007 & 1.702 & 0.00915 & 0.02745 & - \\
\hline Cs-137 & B94 & 2007 & 1.22 & 0.0085 & 0.0255 & - \\
\hline Cs-137 & B95 & 2007 & 1.368 & 0.0096 & 0.0288 & - \\
\hline Cs-137 & B99 & 2007 & 0.5841 & 0.0073 & 0.0219 & - \\
\hline Cs-137 & $\mathrm{C} 14$ & 2007 & 1.289 & 0.0159 & 0.0477 & - \\
\hline Cs-137 & $\mathrm{C} 15$ & 2007 & 0.587 & 0.01185 & 0.03555 & - \\
\hline
\end{tabular}


Table C-3. (continued).

\begin{tabular}{|c|c|c|c|c|c|c|}
\hline Radionuclide & $\begin{array}{c}\text { Sample } \\
\text { Location }\end{array}$ & Date & $\begin{array}{c}\text { Concentrations } \\
(\mathrm{pCi} / \mathrm{g})\end{array}$ & $\begin{array}{l}\text { Uncertainty } \\
(\mathrm{pCi} / \mathrm{g})\end{array}$ & $\begin{array}{c}\mathrm{MDA} \\
(\mathrm{pCi} / \mathrm{g})\end{array}$ & Flag \\
\hline Cs-137 & $\mathrm{C} 25$ & 2007 & 0.564 & 0.00762 & 0.02286 & - \\
\hline Cs-137 & $\mathrm{C} 36$ & 2007 & 0.557 & 0.0076 & 0.0228 & 一 \\
\hline Cs-137 & C37 & 2007 & 0.6352 & 0.01455 & 0.04365 & 一 \\
\hline Cs-137 & $\mathrm{C} 46$ & 2007 & 0.9699 & 0.01415 & 0.04245 & - \\
\hline Cs-137 & $\mathrm{C} 47$ & 2007 & 0.6083 & 0.00965 & 0.02895 & - \\
\hline Cs-137 & $\mathrm{C} 48$ & 2007 & 0.4108 & 0.01125 & 0.03375 & - \\
\hline Cs-137 & C56 & 2007 & 0.9796 & 0.0098 & 0.0294 & - \\
\hline Cs-137 & $\mathrm{C} 57$ & 2007 & 0.5979 & 0.01395 & 0.04185 & - \\
\hline Cs-137 & D57 & 2007 & 0.6723 & 0.0125 & 0.0375 & - \\
\hline Cs-137 & D58 & 2007 & 0.5828 & 0.0115 & 0.0345 & - \\
\hline Cs-137 & D68 & 2007 & 0.5814 & 0.0083 & 0.0249 & - \\
\hline Cs-137 & D69 & 2007 & 0.4874 & 0.0134 & 0.0402 & - \\
\hline Cs-137 & D79 & 2007 & 0.7343 & 0.0127 & 0.0381 & - \\
\hline Cs-137 & D80 & 2007 & 0.4145 & 0.0077 & 0.0231 & - \\
\hline Cs-137 & D89 & 2007 & 0.8544 & 0.01075 & 0.03225 & - \\
\hline Cs-137 & D90 & 2007 & 0.7343 & 0.0122 & 0.0366 & - \\
\hline Cs-137 & D91 & 2007 & 0.5111 & 0.01085 & 0.03255 & - \\
\hline Cs-137 & B104 & 2008 & 1.18 & 0.00824 & 0.02472 & - \\
\hline Cs-137 & A15 & 2009 & 1.784 & 0.01225 & 0.03675 & - \\
\hline Cs-137 & A16 & 2009 & 1.048 & 0.00975 & 0.02925 & - \\
\hline Cs-137 & A17 & 2009 & 0.8989 & 0.0102 & 0.0306 & - \\
\hline Cs-137 & $\mathrm{A} 27$ & 2009 & 1.268 & 0.0109 & 0.0327 & - \\
\hline Cs-137 & A28 & 2009 & 1.329 & 0.01165 & 0.03495 & - \\
\hline Cs-137 & A38 & 2009 & 1.496 & 0.01065 & 0.03195 & - \\
\hline Cs-137 & A39 & 2009 & 1.645 & 0.01155 & 0.03465 & - \\
\hline Cs-137 & $\mathrm{A} 4$ & 2009 & 0.7845 & 0.0116 & 0.0348 & - \\
\hline Cs-137 & A44 & 2009 & 1.544 & 0.0079 & 0.0237 & - \\
\hline Cs-137 & A45 & 2009 & 1.935 & 0.0085 & 0.0255 & - \\
\hline Cs-137 & A47 & 2009 & 3.471 & 0.01545 & 0.04635 & - \\
\hline Cs-137 & A48 & 2009 & 3.763 & 0.01145 & 0.03435 & - \\
\hline Cs-137 & A49 & 2009 & 2.91 & 0.0138 & 0.0414 & - \\
\hline Cs-137 & A5 & 2009 & 0.457 & 0.00745 & 0.02235 & - \\
\hline Cs-137 & A50 & 2009 & 1.832 & 0.00835 & 0.02505 & - \\
\hline Cs-137 & A55 & 2009 & 0.9924 & 0.0069 & 0.0207 & - \\
\hline Cs-137 & A56 & 2009 & 0.3895 & 0.0061 & 0.0183 & - \\
\hline Cs-137 & A58 & 2009 & 2.078 & 0.01175 & 0.03525 & - \\
\hline Cs-137 & A59 & 2009 & 2.358 & 0.00945 & 0.02835 & - \\
\hline
\end{tabular}


Table C-3. (continued).

\begin{tabular}{|c|c|c|c|c|c|c|}
\hline Radionuclide & $\begin{array}{c}\text { Sample } \\
\text { Location }\end{array}$ & Date & $\begin{array}{c}\text { Concentrations } \\
(\mathrm{pCi} / \mathrm{g})\end{array}$ & $\begin{array}{l}\text { Uncertainty } \\
(\mathrm{pCi} / \mathrm{g})\end{array}$ & $\begin{array}{c}\mathrm{MDA} \\
(\mathrm{pCi} / \mathrm{g})\end{array}$ & Flag \\
\hline Cs-137 & A6 & 2009 & 0.5842 & 0.0083 & 0.0249 & - \\
\hline Cs-137 & A60 & 2009 & 1.809 & 0.00865 & 0.02595 & 一 \\
\hline Cs-137 & A61 & 2009 & 2.174 & 0.0092 & 0.0276 & 一 \\
\hline Cs-137 & A66 & 2009 & 0.1531 & 0.004885 & 0.014655 & - \\
\hline Cs-137 & A67 & 2009 & 1.273 & 0.00725 & 0.02175 & - \\
\hline Cs-137 & A68 & 2009 & 0.7407 & 0.0088 & 0.0264 & - \\
\hline Cs-137 & A69 & 2009 & 1.107 & 0.00935 & 0.02805 & - \\
\hline Cs-137 & A70 & 2009 & 1.704 & 0.01065 & 0.03195 & - \\
\hline Cs-137 & A71 & 2009 & 1.54 & 0.00785 & 0.02355 & - \\
\hline Cs-137 & A72 & 2009 & 2.032 & 0.009 & 0.027 & - \\
\hline Cs-137 & $\mathrm{B} 100$ & 2009 & 0.6571 & 0.009 & 0.027 & - \\
\hline Cs-137 & B101 & 2009 & 0.6523 & 0.00745 & 0.02235 & - \\
\hline Cs-137 & B102 & 2009 & 0.3548 & 0.0076 & 0.0228 & - \\
\hline Cs-137 & B103 & 2009 & 0.06502 & 0.0063 & 0.0189 & - \\
\hline Cs-137 & B104 & 2009 & 0.9738 & 0.0088 & 0.0264 & - \\
\hline Cs-137 & B105 & 2009 & 1.061 & 0.0089 & 0.0267 & - \\
\hline Cs-137 & B106 & 2009 & 0.7588 & 0.00855 & 0.02565 & - \\
\hline Cs-137 & B14 & 2009 & 2.004 & 0.009 & 0.027 & - \\
\hline Cs-137 & $\mathrm{B} 15$ & 2009 & 0.9596 & 0.00995 & 0.02985 & - \\
\hline Cs-137 & $\mathrm{B} 25$ & 2009 & 3.462 & 0.01155 & 0.03465 & - \\
\hline Cs-137 & B26 & 2009 & 1.243 & 0.01025 & 0.03075 & - \\
\hline Cs-137 & $\mathrm{B} 28$ & 2009 & 0.6255 & 0.00885 & 0.02655 & - \\
\hline Cs-137 & B29 & 2009 & 0.4637 & 0.0062 & 0.0186 & - \\
\hline Cs-137 & B3 & 2009 & 1.283 & 0.00945 & 0.02835 & - \\
\hline Cs-137 & B36 & 2009 & 3.129 & 0.0155 & 0.0465 & - \\
\hline Cs-137 & B37 & 2009 & 1.276 & 0.01055 & 0.03165 & - \\
\hline Cs-137 & B39 & 2009 & 0.6985 & 0.00915 & 0.02745 & - \\
\hline Cs-137 & B4 & 2009 & 0.8977 & 0.01015 & 0.03045 & - \\
\hline Cs-137 & B40 & 2009 & 0.4846 & 0.00565 & 0.01695 & - \\
\hline Cs-137 & B47 & 2009 & 2.751 & 0.01455 & 0.04365 & - \\
\hline Cs-137 & B48 & 2009 & 1.957 & 0.0123 & 0.0369 & - \\
\hline Cs-137 & B50 & 2009 & 0.7595 & 0.00905 & 0.02715 & - \\
\hline Cs-137 & B51 & 2009 & 0.761 & 0.00985 & 0.02955 & - \\
\hline Cs-137 & B52 & 2009 & 0.3804 & 0.0064 & 0.0192 & - \\
\hline Cs-137 & B59 & 2009 & 0.04894 & 0.00347 & 0.01041 & - \\
\hline Cs-137 & B60 & 2009 & 0.07562 & 0.003995 & 0.011985 & - \\
\hline Cs-137 & B61 & 2009 & 1 & 0.0092 & 0.0276 & - \\
\hline
\end{tabular}


Table C-3. (continued).

\begin{tabular}{|c|c|c|c|c|c|c|}
\hline Radionuclide & $\begin{array}{c}\text { Sample } \\
\text { Location }\end{array}$ & Date & $\begin{array}{l}\text { Concentrations } \\
(\mathrm{pCi} / \mathrm{g})\end{array}$ & $\begin{array}{l}\text { Uncertainty } \\
(\mathrm{pCi} / \mathrm{g})\end{array}$ & $\begin{array}{c}\mathrm{MDA} \\
(\mathrm{pCi} / \mathrm{g})\end{array}$ & Flag \\
\hline Cs-137 & B62 & 2009 & 0.6813 & 0.0066 & 0.0198 & - \\
\hline Cs-137 & B71 & 2009 & 0.6848 & 0.00665 & 0.01995 & - \\
\hline Cs-137 & B73 & 2009 & 0.433 & 0.00775 & 0.02325 & - \\
\hline Cs-137 & B80 & 2009 & 1.531 & 0.0082 & 0.0246 & - \\
\hline Cs-137 & B81 & 2009 & 1.035 & 0.0103 & 0.0309 & - \\
\hline Cs-137 & B82 & 2009 & 0.3318 & 0.0071 & 0.0213 & - \\
\hline Cs-137 & B83 & 2009 & 1.478 & 0.00775 & 0.02325 & - \\
\hline Cs-137 & B84 & 2009 & 0.8224 & 0.00915 & 0.02745 & - \\
\hline Cs-137 & B88 & 2009 & 0.007609 & 0.000665 & 0.001995 & - \\
\hline Cs-137 & B89 & 2009 & 0.733 & 0.00895 & 0.02685 & - \\
\hline Cs-137 & B90 & 2009 & 0.9998 & 0.00995 & 0.02985 & 一 \\
\hline Cs-137 & B91 & 2009 & 1.115 & 0.0071 & 0.0213 & - \\
\hline Cs-137 & B92 & 2009 & 1.303 & 0.01015 & 0.03045 & - \\
\hline Cs-137 & B93 & 2009 & 1.496 & 0.008 & 0.024 & - \\
\hline Cs-137 & B94 & 2009 & 1.025 & 0.00745 & 0.02235 & - \\
\hline Cs-137 & B95 & 2009 & 1.026 & 0.0091 & 0.0273 & - \\
\hline Cs-137 & B99 & 2009 & 0.6179 & 0.00695 & 0.02085 & - \\
\hline Cs-137 & $\mathrm{C} 14$ & 2009 & 1.018 & 0.00685 & 0.02055 & - \\
\hline Cs-137 & $\mathrm{C} 15$ & 2009 & 0.5243 & 0.0061 & 0.0183 & - \\
\hline Cs-137 & $\mathrm{C} 25$ & 2009 & 0.5976 & 0.0092 & 0.0276 & - \\
\hline Cs-137 & $\mathrm{C} 36$ & 2009 & 0.598 & 0.0078 & 0.0234 & - \\
\hline Cs-137 & $\mathrm{C} 37$ & 2009 & 0.4815 & 0.00835 & 0.02505 & - \\
\hline Cs-137 & $\mathrm{C} 46$ & 2009 & 0.9883 & 0.0071 & 0.0213 & - \\
\hline Cs-137 & $\mathrm{C} 47$ & 2009 & 0.6329 & 0.00795 & 0.02385 & - \\
\hline Cs-137 & $\mathrm{C} 48$ & 2009 & 0.2824 & 0.00595 & 0.01785 & 一 \\
\hline Cs-137 & C56 & 2009 & 0.8222 & 0.0092 & 0.0276 & 一 \\
\hline Cs-137 & $\mathrm{C} 57$ & 2009 & 0.4852 & 0.003825 & 0.011475 & - \\
\hline Cs-137 & D58 & 2009 & 0.443 & 0.00725 & 0.02175 & - \\
\hline Cs-137 & D68 & 2009 & 0.5744 & 0.00825 & 0.02475 & - \\
\hline Cs-137 & D69 & 2009 & 0.42 & 0.00785 & 0.02355 & - \\
\hline Cs-137 & D79 & 2009 & 0.6882 & 0.0063 & 0.0189 & - \\
\hline Cs-137 & D80 & 2009 & 0.3584 & 0.0056 & 0.0168 & - \\
\hline Cs-137 & D89 & 2009 & 0.7213 & 0.009 & 0.027 & - \\
\hline Cs-137 & D91 & 2009 & 0.3931 & 0.0051 & 0.0153 & 一 \\
\hline Cs-137 & $\mathrm{A} 27$ & 2010 & 1.364 & 0.0104 & 0.0312 & 一 \\
\hline Cs-137 & A28 & 2010 & 1.076 & 0.00562 & 0.01686 & - \\
\hline Cs-137 & A 38 & 2010 & 1.439 & 0.0121 & 0.0363 & - \\
\hline
\end{tabular}


Table C-3. (continued).

\begin{tabular}{|c|c|c|c|c|c|c|}
\hline Radionuclide & $\begin{array}{c}\text { Sample } \\
\text { Location }\end{array}$ & Date & $\begin{array}{c}\text { Concentrations } \\
(\mathrm{pCi} / \mathrm{g})\end{array}$ & $\begin{array}{l}\text { Uncertainty } \\
(\mathrm{pCi} / \mathrm{g})\end{array}$ & $\begin{array}{l}\text { MDA } \\
(\mathrm{pCi} / \mathrm{g})\end{array}$ & Flag \\
\hline Cs-137 & A39 & 2010 & 1.499 & 0.00969 & 0.02907 & 一 \\
\hline Cs-137 & A49 & 2010 & 2.281 & 0.0127 & 0.0381 & 一 \\
\hline Cs-137 & A50 & 2010 & 1.727 & 0.0131 & 0.0393 & 一 \\
\hline Cs-137 & A59 & 2010 & 2.16 & 0.0143 & 0.0429 & - \\
\hline Cs-137 & A60 & 2010 & 1.588 & 0.0116 & 0.0348 & - \\
\hline Cs-137 & A61 & 2010 & 2.171 & 0.0134 & 0.0402 & - \\
\hline Cs-137 & A71 & 2010 & 1.293 & 0.0171 & 0.0513 & - \\
\hline Cs-137 & A72 & 2010 & 2.002 & 0.0142 & 0.0426 & - \\
\hline Cs-137 & A 15 & 2011 & 2.215 & 0.0222 & 0.0666 & - \\
\hline Cs-137 & A16 & 2011 & 3.202 & 0.0267 & 0.0801 & - \\
\hline Cs-137 & A17 & 2011 & 2.471 & 0.0223 & 0.0669 & - \\
\hline Cs-137 & $\mathrm{A} 27$ & 2011 & 4.5 & 0.0341 & 0.1023 & - \\
\hline Cs-137 & A28 & 2011 & 3.261 & 0.0251 & 0.0753 & - \\
\hline Cs-137 & A38 & 2011 & 4.313 & 0.0278 & 0.0834 & - \\
\hline Cs-137 & A39 & 2011 & 5.001 & 0.0352 & 0.1056 & - \\
\hline Cs-137 & $\mathrm{A} 4$ & 2011 & 2.405 & 0.0273 & 0.0819 & - \\
\hline Cs-137 & A44 & 2011 & 5.507 & 0.0312 & 0.0936 & - \\
\hline Cs-137 & A47 & 2011 & 10.97 & 0.0499 & 0.1497 & - \\
\hline Cs-137 & A49 & 2011 & 9.795 & 0.0481 & 0.1443 & - \\
\hline Cs-137 & A50 & 2011 & 6.151 & 0.041 & 0.123 & - \\
\hline Cs-137 & A55 & 2011 & 3.778 & 0.0293 & 0.0879 & - \\
\hline Cs-137 & A58 & 2011 & 8.052 & 0.0409 & 0.1227 & - \\
\hline Cs-137 & A59 & 2011 & 7.374 & 0.0353 & 0.1059 & - \\
\hline Cs-137 & A6 & 2011 & 1.925 & 0.0235 & 0.0705 & - \\
\hline Cs-137 & A61 & 2011 & 7.384 & 0.0322 & 0.0966 & - \\
\hline Cs-137 & A66 & 2011 & 2.324 & 0.0253 & 0.0759 & - \\
\hline Cs-137 & A69 & 2011 & 4.196 & 0.0324 & 0.0972 & - \\
\hline Cs-137 & A70 & 2011 & 5.512 & 0.0309 & 0.0927 & - \\
\hline Cs-137 & A72 & 2011 & 6.859 & 0.0295 & 0.0885 & - \\
\hline Cs-137 & B101 & 2011 & 2.016 & 0.0206 & 0.0618 & - \\
\hline Cs-137 & B102 & 2011 & 0.251 & 0.0123 & 0.0369 & - \\
\hline Cs-137 & B103 & 2011 & 0.2188 & 0.0186 & 0.0558 & - \\
\hline Cs-137 & B104 & 2011 & 3.312 & 0.0249 & 0.0747 & - \\
\hline Cs-137 & B106 & 2011 & 2.43 & 0.0224 & 0.0672 & - \\
\hline Cs-137 & B14 & 2011 & 6.787 & 0.0398 & 0.1194 & - \\
\hline Cs-137 & $\mathrm{B} 15$ & 2011 & 2.644 & 0.0227 & 0.0681 & 一 \\
\hline Cs-137 & B17 & 2011 & 0.006682 & 0.006 & 0.018 & $\mathrm{U}$ \\
\hline
\end{tabular}


Table C-3. (continued).

\begin{tabular}{|c|c|c|c|c|c|c|}
\hline Radionuclide & $\begin{array}{c}\text { Sample } \\
\text { Location }\end{array}$ & Date & $\begin{array}{c}\text { Concentrations } \\
(\mathrm{pCi} / \mathrm{g})\end{array}$ & $\begin{array}{l}\text { Uncertainty } \\
(\mathrm{pCi} / \mathrm{g})\end{array}$ & $\begin{array}{c}\mathrm{MDA} \\
(\mathrm{pCi} / \mathrm{g})\end{array}$ & Flag \\
\hline Cs-137 & $\mathrm{B} 25$ & 2011 & 12.68 & 0.0534 & 0.1602 & - \\
\hline Cs-137 & B28 & 2011 & 1.878 & 0.0198 & 0.0594 & 一 \\
\hline Cs-137 & B29 & 2011 & 1.569 & 0.0257 & 0.0771 & 一 \\
\hline Cs-137 & B3 & 2011 & 4.241 & 0.028 & 0.084 & - \\
\hline Cs-137 & B39 & 2011 & 0.0004929 & 0.00568 & 0.01704 & $\mathrm{U}$ \\
\hline Cs-137 & B4 & 2011 & 2.352 & 0.0207 & 0.0621 & - \\
\hline Cs-137 & B40 & 2011 & 0.0004997 & 0.00623 & 0.01869 & $\mathrm{U}$ \\
\hline Cs-137 & B47 & 2011 & 8.087 & 0.0436 & 0.1308 & 一 \\
\hline Cs-137 & B48 & 2011 & 6.631 & 0.0393 & 0.1179 & - \\
\hline Cs-137 & $\mathrm{B} 50$ & 2011 & 2.643 & 0.0254 & 0.0762 & - \\
\hline Cs-137 & B52 & 2011 & 1.264 & 0.0173 & 0.0519 & - \\
\hline Cs-137 & B61 & 2011 & 3.992 & 0.0314 & 0.0942 & - \\
\hline Cs-137 & B62 & 2011 & 1.954 & 0.0205 & 0.0615 & - \\
\hline Cs-137 & B71 & 2011 & 1.709 & 0.02 & 0.06 & - \\
\hline Cs-137 & B73 & 2011 & 2.898 & 0.0276 & 0.0828 & - \\
\hline Cs-137 & B80 & 2011 & 0.2095 & 0.0199 & 0.0597 & - \\
\hline Cs-137 & B83 & 2011 & 4.241 & 0.0277 & 0.0831 & - \\
\hline Cs-137 & B84 & 2011 & 2.639 & 0.0268 & 0.0804 & - \\
\hline Cs-137 & B89 & 2011 & 2.681 & 0.0312 & 0.0936 & - \\
\hline Cs-137 & B90 & 2011 & 3.343 & 0.0315 & 0.0945 & - \\
\hline Cs-137 & B91 & 2011 & 3.896 & 0.0308 & 0.0924 & - \\
\hline Cs-137 & B92 & 2011 & 4.218 & 0.0237 & 0.0711 & - \\
\hline Cs-137 & B93 & 2011 & 4.949 & 0.0274 & 0.0822 & - \\
\hline Cs-137 & B94 & 2011 & 3.304 & 0.0249 & 0.0747 & - \\
\hline Cs-137 & B99 & 2011 & 1.642 & 0.0191 & 0.0573 & - \\
\hline Cs-137 & $\mathrm{C} 15$ & 2011 & 1.498 & 0.019 & 0.057 & - \\
\hline Cs-137 & $\mathrm{C} 25$ & 2011 & 1.87 & 0.0181 & 0.0543 & - \\
\hline Cs-137 & C37 & 2011 & 1.041 & 0.0145 & 0.0435 & - \\
\hline Cs-137 & $\mathrm{C} 46$ & 2011 & 2.944 & 0.0238 & 0.0714 & - \\
\hline Cs-137 & $\mathrm{C} 48$ & 2011 & 1.197 & 0.015 & 0.045 & - \\
\hline Cs-137 & C56 & 2011 & 3.446 & 0.0295 & 0.0885 & - \\
\hline Cs-137 & $\mathrm{C} 57$ & 2011 & 1.599 & 0.0189 & 0.0567 & - \\
\hline Cs-137 & D58 & 2011 & 1.46 & 0.0181 & 0.0543 & - \\
\hline Cs-137 & D68 & 2011 & 0.01088 & 0.00996 & 0.02988 & $\mathrm{U}$ \\
\hline Cs-137 & D89 & 2011 & 2.933 & 0.03 & 0.09 & 一 \\
\hline Cs-137 & D91 & 2011 & 1.375 & 0.0207 & 0.0621 & - \\
\hline Cs-137 & A 15 & 2012 & 2.087 & 0.0132 & 0.0396 & - \\
\hline
\end{tabular}


Table C-3. (continued).

\begin{tabular}{|c|c|c|c|c|c|c|}
\hline Radionuclide & $\begin{array}{l}\text { Sample } \\
\text { Location }\end{array}$ & Date & $\begin{array}{c}\text { Concentrations } \\
(\mathrm{pCi} / \mathrm{g})\end{array}$ & $\begin{array}{l}\text { Uncertainty } \\
(\mathrm{pCi} / \mathrm{g})\end{array}$ & $\begin{array}{l}\text { MDA } \\
(\mathrm{pCi} / \mathrm{g})\end{array}$ & Flag \\
\hline Cs-137 & A38 & 2012 & 1.238 & 0.00842 & 0.02526 & - \\
\hline Cs-137 & A44 & 2012 & 1.769 & 0.0132 & 0.0396 & - \\
\hline Cs-137 & A47 & 2012 & 2.724 & 0.0136 & 0.0408 & 一 \\
\hline Cs-137 & A59 & 2012 & 2.522 & 0.0136 & 0.0408 & - \\
\hline Cs-137 & A6 & 2012 & 0.6699 & 0.0106 & 0.0318 & - \\
\hline Cs-137 & A69 & 2012 & 0.9396 & 0.011 & 0.033 & - \\
\hline Cs-137 & A70 & 2012 & 1.989 & 0.012 & 0.036 & 一 \\
\hline Cs-137 & A72 & 2012 & 1.439 & 0.0108 & 0.0324 & 一 \\
\hline Cs-137 & B14 & 2012 & 1.925 & 0.0115 & 0.0345 & - \\
\hline Cs-137 & B48 & 2012 & 2.088 & 0.0131 & 0.0393 & - \\
\hline Cs-137 & B83 & 2012 & 1.366 & 0.0107 & 0.0321 & 一 \\
\hline Cs-137 & $\mathrm{C} 46$ & 2012 & 0.6768 & 0.0098 & 0.0294 & 一 \\
\hline Cs-137 & $\mathrm{C} 57$ & 2012 & 0.5333 & 0.00807 & 0.02421 & - \\
\hline Cs-137 & D68 & 2012 & 0.5367 & 1.3 & 3.9 & $\mathrm{U}$ \\
\hline Cs-137 & $\begin{array}{l}2 \text { INCH AIR } \\
\text { - INTEC }\end{array}$ & 2013 & 0.2726 & 0.0133 & 0.0398 & 一 \\
\hline Cs-137 & A47 & 2013 & 3.721 & 0.0189 & 0.0566 & - \\
\hline Cs-137 & A49 & 2013 & 0.9745 & 0.0050 & 0.0149 & - \\
\hline Cs-137 & A59 & 2013 & 2.624 & 0.018 & 0.054 & - \\
\hline Cs-137 & A71 & 2013 & 1.681 & 0.016 & 0.048 & - \\
\hline Cs-137 & A72 & 2013 & 0.7715 & 0.0049 & 0.0146 & - \\
\hline Cs-137 & $\begin{array}{c}2 \text { INCH AIR } \\
- \text { CPP }\end{array}$ & 2014 & 0.9737 & 0.00974 & 0.02922 & 一 \\
\hline Cs-137 & $\begin{array}{l}2 \text { INCH AIR } \\
\text { - INTEC }\end{array}$ & 2014 & 0.1601 & 0.00466 & 0.01398 & - \\
\hline Cs-137 & A38 & 2014 & 1.28 & 0.00976 & 0.02928 & - \\
\hline Cs-137 & A47 & 2014 & 3.536 & 0.0225 & 0.0675 & - \\
\hline Cs-137 & A58 & 2014 & 2.298 & 0.0127 & 0.0381 & - \\
\hline Cs-137 & A59 & 2014 & 3.19 & 0.0193 & 0.0579 & - \\
\hline Cs-137 & B15 & 2014 & 0.7498 & 0.0137 & 0.0411 & - \\
\hline Cs-137 & B28 & 2014 & 0.6065 & 0.0152 & 0.0456 & - \\
\hline Cs-137 & B29 & 2014 & 0.4371 & 0.0136 & 0.0408 & - \\
\hline Cs-137 & B3 & 2014 & 1.447 & 0.0278 & 0.0834 & - \\
\hline Cs-137 & B3 & 2014 & 1.23 & 0.00602 & 0.01806 & - \\
\hline Cs-137 & B4 & 2014 & 0.6929 & 0.00947 & 0.02841 & - \\
\hline Cs-137 & B40 & 2014 & 0.5079 & 0.00884 & 0.02652 & - \\
\hline Cs-137 & $\mathrm{B} 50$ & 2014 & 0.8812 & 0.0161 & 0.0483 & - \\
\hline Cs-137 & B52 & 2014 & 0.7312 & 0.0195 & 0.0585 & - \\
\hline
\end{tabular}


Table C-3. (continued).

\begin{tabular}{|c|c|c|c|c|c|c|}
\hline Radionuclide & $\begin{array}{c}\text { Sample } \\
\text { Location }\end{array}$ & Date & $\begin{array}{c}\text { Concentrations } \\
(\mathrm{pCi} / \mathrm{g})\end{array}$ & $\begin{array}{l}\text { Uncertainty } \\
(\mathrm{pCi} / \mathrm{g})\end{array}$ & $\begin{array}{c}\mathrm{MDA} \\
(\mathrm{pCi} / \mathrm{g})\end{array}$ & Flag \\
\hline Cs-137 & B52 & 2014 & 0.4379 & 0.0172 & 0.0516 & - \\
\hline Eu-152 & A15 & 2007 & 0.6259 & 0.0489 & 0.1467 & 一 \\
\hline Eu-152 & A16 & 2007 & 0.3066 & 0.0358 & 0.1074 & 一 \\
\hline Eu-152 & A17 & 2007 & 0.2771 & 0.0311 & 0.0933 & - \\
\hline Eu-152 & A27 & 2007 & 0.4524 & 0.0665 & 0.1995 & - \\
\hline Eu-152 & A28 & 2007 & 0.3364 & 0.03685 & 0.11055 & - \\
\hline Eu-152 & A37 & 2007 & 0.2955 & 0.052 & 0.156 & - \\
\hline Eu-152 & A38 & 2007 & 0.2886 & 0.0355 & 0.1065 & - \\
\hline Eu-152 & A4 & 2007 & 0.1603 & 0.0225 & 0.0675 & - \\
\hline Eu-152 & A44 & 2007 & 0.3065 & 0.04385 & 0.13155 & - \\
\hline Eu-152 & A45 & 2007 & 0.4514 & 0.05 & 0.15 & - \\
\hline Eu-152 & A47 & 2007 & 0.2437 & 0.0295 & 0.0885 & - \\
\hline Eu-152 & A48 & 2007 & 0.4937 & 0.051 & 0.153 & - \\
\hline Eu-152 & A49 & 2007 & 0.2854 & 0.051 & 0.153 & - \\
\hline Eu-152 & A5 & 2007 & 0.2967 & 0.0399 & 0.1197 & - \\
\hline Eu-152 & A50 & 2007 & 1.482 & 0.111 & 0.333 & - \\
\hline Eu-152 & A55 & 2007 & 0.5748 & 0.052 & 0.156 & - \\
\hline Eu-152 & A58 & 2007 & 0.3638 & 0.03815 & 0.11445 & - \\
\hline Eu-152 & A59 & 2007 & 0.1887 & 0.0271 & 0.0813 & - \\
\hline Eu-152 & A6 & 2007 & 0.3102 & 0.03365 & 0.10095 & - \\
\hline Eu-152 & A60 & 2007 & 0.3034 & 0.0515 & 0.1545 & - \\
\hline Eu-152 & A61 & 2007 & 0.2991 & 0.0333 & 0.0999 & - \\
\hline Eu-152 & A67 & 2007 & 0.2509 & 0.0333 & 0.0999 & - \\
\hline Eu-152 & A69 & 2007 & 0.1944 & 0.0374 & 0.1122 & - \\
\hline Eu-152 & A70 & 2007 & 0.4256 & 0.0494 & 0.1482 & - \\
\hline Eu-152 & A71 & 2007 & 0.2254 & 0.0446 & 0.1338 & - \\
\hline Eu-152 & A72 & 2007 & 0.5999 & 0.059 & 0.177 & - \\
\hline Eu-152 & B101 & 2007 & 0.3486 & 0.04235 & 0.12705 & - \\
\hline Eu-152 & B102 & 2007 & 0.4176 & 0.0401 & 0.1203 & - \\
\hline Eu-152 & B103 & 2007 & 0.3564 & 0.0347 & 0.1041 & - \\
\hline Eu-152 & B104 & 2007 & 0.2406 & 0.03105 & 0.09315 & - \\
\hline Eu-152 & B105 & 2007 & 0.3637 & 0.03875 & 0.11625 & - \\
\hline Eu-152 & B14 & 2007 & 0.2535 & 0.0352 & 0.1056 & - \\
\hline Eu-152 & B17 & 2007 & 0.2643 & 0.02965 & 0.08895 & - \\
\hline Eu-152 & B25 & 2007 & 0.3228 & 0.0535 & 0.1605 & - \\
\hline Eu-152 & B26 & 2007 & 0.3901 & 0.0414 & 0.1242 & - \\
\hline Eu-152 & $\mathrm{B} 28$ & 2007 & 0.1499 & 0.03455 & 0.10365 & - \\
\hline
\end{tabular}


Table C-3. (continued).

\begin{tabular}{|c|c|c|c|c|c|c|}
\hline Radionuclide & $\begin{array}{c}\text { Sample } \\
\text { Location }\end{array}$ & Date & $\begin{array}{c}\text { Concentrations } \\
(\mathrm{pCi} / \mathrm{g})\end{array}$ & $\begin{array}{l}\text { Uncertainty } \\
(\mathrm{pCi} / \mathrm{g})\end{array}$ & $\begin{array}{c}\mathrm{MDA} \\
(\mathrm{pCi} / \mathrm{g})\end{array}$ & Flag \\
\hline Eu-152 & B29 & 2007 & 0.3365 & 0.0392 & 0.1176 & - \\
\hline Eu-152 & B3 & 2007 & 0.2449 & 0.0368 & 0.1104 & 一 \\
\hline Eu-152 & $\mathrm{B} 4$ & 2007 & 0.5645 & 0.0665 & 0.1995 & 一 \\
\hline Eu-152 & B40 & 2007 & 0.3171 & 0.0477 & 0.1431 & - \\
\hline Eu-152 & B47 & 2007 & 0.3746 & 0.0575 & 0.1725 & - \\
\hline Eu-152 & B48 & 2007 & 0.5597 & 0.0725 & 0.2175 & - \\
\hline Eu-152 & $\mathrm{B} 50$ & 2007 & 0.3408 & 0.0448 & 0.1344 & - \\
\hline Eu-152 & B51 & 2007 & 0.2556 & 0.0339 & 0.1017 & - \\
\hline Eu-152 & B52 & 2007 & 0.322 & 0.03645 & 0.10935 & - \\
\hline Eu-152 & B59 & 2007 & 1.283 & 0.1075 & 0.3225 & - \\
\hline Eu-152 & B60 & 2007 & 1.719 & 0.12 & 0.36 & - \\
\hline Eu-152 & B61 & 2007 & 0.6259 & 0.0765 & 0.2295 & - \\
\hline Eu-152 & B62 & 2007 & 0.3486 & 0.04235 & 0.12705 & - \\
\hline Eu-152 & B69 & 2007 & 2.096 & 0.263 & 0.789 & - \\
\hline Eu-152 & B71 & 2007 & 1.688 & 0.113 & 0.339 & - \\
\hline Eu-152 & B73 & 2007 & 0.292 & 0.03715 & 0.11145 & - \\
\hline Eu-152 & $\mathrm{B} 80$ & 2007 & 0.5678 & 0.079 & 0.237 & - \\
\hline Eu-152 & B81 & 2007 & 0.845 & 0.0965 & 0.2895 & - \\
\hline Eu-152 & B82 & 2007 & 0.3791 & 0.0488 & 0.1464 & - \\
\hline Eu-152 & B83 & 2007 & 0.2684 & 0.0351 & 0.1053 & - \\
\hline Eu-152 & B88 & 2007 & 0.3422 & 0.0408 & 0.1224 & - \\
\hline Eu-152 & B89 & 2007 & 0.2397 & 0.0312 & 0.0936 & - \\
\hline Eu-152 & B90 & 2007 & 0.3043 & 0.035 & 0.105 & - \\
\hline Eu-152 & B92 & 2007 & 0.5616 & 0.0655 & 0.1965 & - \\
\hline Eu-152 & B93 & 2007 & 0.2397 & 0.0311 & 0.0933 & - \\
\hline Eu-152 & B94 & 2007 & 0.2374 & 0.0283 & 0.0849 & - \\
\hline Eu-152 & B95 & 2007 & 0.1966 & 0.0227 & 0.0681 & - \\
\hline Eu-152 & B99 & 2007 & 0.2398 & 0.0417 & 0.1251 & - \\
\hline Eu-152 & $\mathrm{C} 14$ & 2007 & 0.2399 & 0.04015 & 0.12045 & - \\
\hline Eu-152 & $\mathrm{C} 15$ & 2007 & 0.3611 & 0.051 & 0.153 & - \\
\hline Eu-152 & $\mathrm{C} 36$ & 2007 & 0.3994 & 0.04845 & 0.14535 & - \\
\hline Eu-152 & C37 & 2007 & 0.9008 & 0.069 & 0.207 & - \\
\hline Eu-152 & $\mathrm{C} 46$ & 2007 & 0.3345 & 0.04845 & 0.14535 & - \\
\hline Eu-152 & $\mathrm{C} 47$ & 2007 & 0.3839 & 0.04705 & 0.14115 & - \\
\hline Eu-152 & $\mathrm{C} 48$ & 2007 & 0.1481 & 0.02705 & 0.08115 & - \\
\hline Eu-152 & C56 & 2007 & 0.5915 & 0.0855 & 0.2565 & - \\
\hline Eu-152 & C57 & 2007 & 0.3849 & 0.0635 & 0.1905 & - \\
\hline
\end{tabular}


Table C-3. (continued).

\begin{tabular}{|c|c|c|c|c|c|c|}
\hline Radionuclide & $\begin{array}{l}\text { Sample } \\
\text { Location }\end{array}$ & Date & $\begin{array}{c}\text { Concentrations } \\
(\mathrm{pCi} / \mathrm{g})\end{array}$ & $\begin{array}{l}\text { Uncertainty } \\
(\mathrm{pCi} / \mathrm{g})\end{array}$ & $\begin{array}{l}\text { MDA } \\
(\mathrm{pCi} / \mathrm{g})\end{array}$ & Flag \\
\hline Eu-152 & D58 & 2007 & 0.2344 & 0.02945 & 0.08835 & - \\
\hline Eu-152 & D68 & 2007 & 0.3918 & 0.0445 & 0.1335 & 一 \\
\hline Eu-152 & D69 & 2007 & 0.2658 & 0.0371 & 0.1113 & 一 \\
\hline Eu-152 & D79 & 2007 & 0.314 & 0.0385 & 0.1155 & - \\
\hline Eu-152 & D80 & 2007 & 0.2023 & 0.0296 & 0.0888 & - \\
\hline Eu-152 & D89 & 2007 & 0.3309 & 0.03905 & 0.11715 & - \\
\hline Eu-152 & D90 & 2007 & 0.5425 & 0.0705 & 0.2115 & - \\
\hline Eu-152 & D91 & 2007 & 0.3087 & 0.03455 & 0.10365 & - \\
\hline Eu-152 & A 15 & 2011 & 0.003547 & 0.0243 & 0.0729 & $\mathrm{U}$ \\
\hline Eu-152 & A16 & 2011 & 0.03537 & 0.0318 & 0.0954 & $\mathrm{U}$ \\
\hline Eu-152 & A17 & 2011 & 0.0373 & 0.0289 & 0.0867 & $\mathrm{U}$ \\
\hline $\mathrm{Eu}-152$ & A27 & 2011 & 0.05231 & 0.0319 & 0.0957 & $\mathrm{U}$ \\
\hline Eu-152 & A28 & 2011 & -0.003045 & 0.0288 & 0.0864 & $\mathrm{U}$ \\
\hline Eu-152 & A38 & 2011 & 0.04439 & 0.0312 & 0.0936 & $\mathrm{U}$ \\
\hline Eu-152 & A39 & 2011 & 0.02731 & 0.0343 & 0.1029 & $\mathrm{U}$ \\
\hline $\mathrm{Eu}-152$ & $\mathrm{~A} 4$ & 2011 & 0.004367 & 0.0319 & 0.0957 & $\mathrm{U}$ \\
\hline Eu-152 & A44 & 2011 & 0.0495 & 0.0357 & 0.1071 & $\mathrm{U}$ \\
\hline Eu-152 & A47 & 2011 & 0.02045 & 0.0399 & 0.1197 & $\mathrm{U}$ \\
\hline Eu-152 & A49 & 2011 & 0.008224 & 0.0363 & 0.1089 & $\mathrm{U}$ \\
\hline Eu-152 & A50 & 2011 & 0.01526 & 0.0368 & 0.1104 & $\mathrm{U}$ \\
\hline Eu-152 & A55 & 2011 & 0.02868 & 0.031 & 0.093 & $\mathrm{U}$ \\
\hline Eu-152 & A58 & 2011 & 0.001434 & 0.0343 & 0.1029 & $\mathrm{U}$ \\
\hline Eu-152 & A59 & 2011 & 0.005663 & 0.0322 & 0.0966 & $\mathrm{U}$ \\
\hline Eu-152 & A6 & 2011 & 0.03772 & 0.0265 & 0.0795 & $\mathrm{U}$ \\
\hline Eu-152 & A61 & 2011 & 0.006908 & 0.031 & 0.093 & $\mathrm{U}$ \\
\hline Eu-152 & A66 & 2011 & 0.09174 & 0.0297 & 0.0891 & - \\
\hline Eu-152 & A69 & 2011 & -0.01187 & 0.034 & 0.102 & $\mathrm{U}$ \\
\hline Eu-152 & A70 & 2011 & -0.01581 & 0.0294 & 0.0882 & $\mathrm{U}$ \\
\hline Eu-152 & A72 & 2011 & -0.05117 & 0.0298 & 0.0894 & $\mathrm{U}$ \\
\hline Eu-152 & B101 & 2011 & 0.0162 & 0.0297 & 0.0891 & $\mathrm{U}$ \\
\hline Eu-152 & B102 & 2011 & -0.01858 & 0.0332 & 0.0996 & $\mathrm{U}$ \\
\hline Eu-152 & B103 & 2011 & 0.01613 & 0.0317 & 0.0951 & $\mathrm{U}$ \\
\hline Eu-152 & B104 & 2011 & -0.03272 & 0.0318 & 0.0954 & $\mathrm{U}$ \\
\hline Eu-152 & B106 & 2011 & 0.003659 & 0.0304 & 0.0912 & $\mathrm{U}$ \\
\hline Eu-152 & B14 & 2011 & 0.05833 & 0.0339 & 0.1017 & $\mathrm{U}$ \\
\hline Eu-152 & $\mathrm{B} 15$ & 2011 & -0.01682 & 0.0331 & 0.0993 & $\mathrm{U}$ \\
\hline Eu-152 & $\mathrm{B} 17$ & 2011 & -0.07519 & 0.0217 & 0.0651 & $\mathrm{U}$ \\
\hline
\end{tabular}


Table C-3. (continued).

\begin{tabular}{|c|c|c|c|c|c|c|}
\hline Radionuclide & $\begin{array}{l}\text { Sample } \\
\text { Location }\end{array}$ & Date & $\begin{array}{c}\text { Concentrations } \\
(\mathrm{pCi} / \mathrm{g})\end{array}$ & $\begin{array}{l}\text { Uncertainty } \\
(\mathrm{pCi} / \mathrm{g})\end{array}$ & $\begin{array}{l}\text { MDA } \\
(\mathrm{pCi} / \mathrm{g})\end{array}$ & Flag \\
\hline Eu-152 & $\mathrm{B} 25$ & 2011 & 0.03906 & 0.0403 & 0.1209 & $\mathrm{U}$ \\
\hline Eu-152 & B28 & 2011 & 0.007721 & 0.0316 & 0.0948 & $\mathrm{U}$ \\
\hline Eu-152 & B29 & 2011 & 0.03066 & 0.0315 & 0.0945 & $\mathrm{U}$ \\
\hline Eu-152 & B3 & 2011 & 0.001218 & 0.034 & 0.102 & $\mathrm{U}$ \\
\hline Eu-152 & B39 & 2011 & -0.08723 & 0.0219 & 0.0657 & $\mathrm{U}$ \\
\hline Eu-152 & B4 & 2011 & 0.003829 & 0.0289 & 0.0867 & $\mathrm{U}$ \\
\hline Eu-152 & B40 & 2011 & -0.0762 & 0.0209 & 0.0627 & $\mathrm{U}$ \\
\hline Eu-152 & B47 & 2011 & 0.03013 & 0.046 & 0.138 & $\mathrm{U}$ \\
\hline Eu-152 & B48 & 2011 & 0.002985 & 0.0411 & 0.1233 & $\mathrm{U}$ \\
\hline Eu-152 & B50 & 2011 & 0.01985 & 0.0309 & 0.0927 & $\mathrm{U}$ \\
\hline Eu-152 & B52 & 2011 & -0.03506 & 0.0318 & 0.0954 & $\mathrm{U}$ \\
\hline $\mathrm{Eu}-152$ & B61 & 2011 & 0.04174 & 0.0323 & 0.0969 & $\mathrm{U}$ \\
\hline Eu-152 & B62 & 2011 & 0.004623 & 0.0288 & 0.0864 & $\mathrm{U}$ \\
\hline Eu-152 & B71 & 2011 & 0.01483 & 0.0407 & 0.1221 & $\mathrm{U}$ \\
\hline Eu-152 & B73 & 2011 & 0.05174 & 0.0317 & 0.0951 & $\mathrm{U}$ \\
\hline $\mathrm{Eu}-152$ & $\mathrm{~B} 80$ & 2011 & 0.02425 & 0.0698 & 0.2094 & $\mathrm{U}$ \\
\hline Eu-152 & B83 & 2011 & 0.03608 & 0.0312 & 0.0936 & $\mathrm{U}$ \\
\hline Eu-152 & B84 & 2011 & 0.005122 & 0.0326 & 0.0978 & $\mathrm{U}$ \\
\hline Eu-152 & B89 & 2011 & 0.001161 & 0.0338 & 0.1014 & $\mathrm{U}$ \\
\hline Eu-152 & B90 & 2011 & 0.04061 & 0.0328 & 0.0984 & $\mathrm{U}$ \\
\hline Eu-152 & B91 & 2011 & 0.01448 & 0.0401 & 0.1203 & $\mathrm{U}$ \\
\hline Eu-152 & B92 & 2011 & 0.03623 & 0.0374 & 0.1122 & $\mathrm{U}$ \\
\hline Eu-152 & B93 & 2011 & 0.05885 & 0.0304 & 0.0912 & $\mathrm{U}$ \\
\hline Eu-152 & B94 & 2011 & -0.001849 & 0.0319 & 0.0957 & $\mathrm{U}$ \\
\hline Eu-152 & B99 & 2011 & 0.02519 & 0.0288 & 0.0864 & $\mathrm{U}$ \\
\hline Eu-152 & $\mathrm{C} 15$ & 2011 & 0.01417 & 0.0289 & 0.0867 & $\mathrm{U}$ \\
\hline Eu-152 & $\mathrm{C} 25$ & 2011 & 0.04829 & 0.0291 & 0.0873 & $\mathrm{U}$ \\
\hline Eu-152 & $\mathrm{C} 37$ & 2011 & 0.004199 & 0.0289 & 0.0867 & $\mathrm{U}$ \\
\hline Eu-152 & $\mathrm{C} 46$ & 2011 & 0.02195 & 0.0236 & 0.0708 & $\mathrm{U}$ \\
\hline Eu-152 & $\mathrm{C} 48$ & 2011 & 0.005796 & 0.0282 & 0.0846 & $\mathrm{U}$ \\
\hline Eu-152 & C56 & 2011 & 0.01381 & 0.0308 & 0.0924 & $\mathrm{U}$ \\
\hline Eu-152 & C57 & 2011 & -0.01231 & 0.0274 & 0.0822 & $\mathrm{U}$ \\
\hline Eu-152 & D58 & 2011 & 0.009544 & 0.0267 & 0.0801 & $\mathrm{U}$ \\
\hline Eu-152 & D68 & 2011 & -0.03775 & 0.0329 & 0.0987 & $\mathrm{U}$ \\
\hline Eu-152 & D89 & 2011 & 0.02699 & 0.0315 & 0.0945 & $\mathrm{U}$ \\
\hline Eu-152 & D91 & 2011 & 0.01728 & 0.0255 & 0.0765 & $\mathrm{U}$ \\
\hline Eu-152 & A15 & 2012 & 0.05855 & 0.0291 & 0.0873 & $\mathrm{U}$ \\
\hline
\end{tabular}


Table C-3. (continued).

\begin{tabular}{|c|c|c|c|c|c|c|}
\hline Radionuclide & $\begin{array}{l}\text { Sample } \\
\text { Location }\end{array}$ & Date & $\begin{array}{c}\text { Concentrations } \\
(\mathrm{pCi} / \mathrm{g})\end{array}$ & $\begin{array}{l}\text { Uncertainty } \\
(\mathrm{pCi} / \mathrm{g})\end{array}$ & $\begin{array}{l}\text { MDA } \\
(\mathrm{pCi} / \mathrm{g})\end{array}$ & Flag \\
\hline Eu-152 & A38 & 2012 & 0.01167 & 0.0283 & 0.0849 & $\mathrm{U}$ \\
\hline $\mathrm{Eu}-152$ & A44 & 2012 & 0.03056 & 0.0333 & 0.0999 & $\mathrm{U}$ \\
\hline Eu-152 & A47 & 2012 & 0.02021 & 0.0329 & 0.0987 & $\mathrm{U}$ \\
\hline Eu-152 & A59 & 2012 & -0.009103 & 0.0267 & 0.0801 & $\mathrm{U}$ \\
\hline Eu-152 & A6 & 2012 & 0.03136 & 0.0286 & 0.0858 & $\mathrm{U}$ \\
\hline Eu-152 & A69 & 2012 & 0.03053 & 0.0304 & 0.0912 & $\mathrm{U}$ \\
\hline Eu-152 & A70 & 2012 & 0.006673 & 0.0268 & 0.0804 & $\mathrm{U}$ \\
\hline Eu-152 & A72 & 2012 & 0.02897 & 0.028 & 0.084 & $\mathrm{U}$ \\
\hline Eu-152 & B14 & 2012 & 0.02654 & 0.0275 & 0.0825 & $\mathrm{U}$ \\
\hline Eu-152 & B48 & 2012 & 0.007149 & 0.0345 & 0.1035 & $\mathrm{U}$ \\
\hline Eu-152 & B83 & 2012 & 0.02991 & 0.0268 & 0.0804 & $\mathrm{U}$ \\
\hline Eu-152 & $\mathrm{C} 46$ & 2012 & 0.01685 & 0.0302 & 0.0906 & $\mathrm{U}$ \\
\hline $\mathrm{Eu}-152$ & $\mathrm{C} 57$ & 2012 & 0.01068 & 0.0291 & 0.0873 & $\mathrm{U}$ \\
\hline Eu-152 & $\begin{array}{c}2 \text { INCH AIR } \\
\text { - INTEC }\end{array}$ & 2013 & 0.1429 & 0.0327 & 0.098 & - \\
\hline Eu-152 & A47 & 2013 & 0.3031 & 0.042 & 0.126 & - \\
\hline Eu-152 & A49 & 2013 & 0.005708 & 0.0176 & 0.0527 & $\mathrm{U}$ \\
\hline Eu-152 & A59 & 2013 & 0.229 & 0.0393 & 0.118 & - \\
\hline Eu-152 & A71 & 2013 & 0.2423 & 0.041 & 0.123 & - \\
\hline Eu-152 & A72 & 2013 & 0.0001521 & 0.0170 & 0.0509 & $\mathrm{U}$ \\
\hline Eu-152 & $\begin{array}{c}2 \text { INCH AIR } \\
- \text { CPP }\end{array}$ & 2014 & -0.02874 & 0.0325 & 0.0975 & $\mathrm{U}$ \\
\hline Eu-152 & $\begin{array}{l}2 \text { INCH AIR } \\
\text { - INTEC }\end{array}$ & 2014 & 0.01944 & 0.0249 & 0.0747 & $\mathrm{U}$ \\
\hline Eu-152 & A38 & 2014 & 0.02179 & 0.0263 & 0.0789 & $\mathrm{U}$ \\
\hline Eu-152 & A47 & 2014 & -0.01113 & 0.0303 & 0.0909 & $\mathrm{U}$ \\
\hline Eu-152 & A58 & 2014 & 0.02631 & 0.0262 & 0.0786 & $\mathrm{U}$ \\
\hline Eu-152 & A59 & 2014 & 0.004343 & 0.0277 & 0.0831 & $\mathrm{U}$ \\
\hline Eu-152 & B15 & 2014 & 0.008766 & 0.0465 & 0.1395 & $\mathrm{U}$ \\
\hline Eu-152 & B28 & 2014 & 0.004446 & 0.0291 & 0.0873 & $\mathrm{U}$ \\
\hline Eu-152 & B29 & 2014 & 0.001111 & 0.0277 & 0.0831 & $\mathrm{U}$ \\
\hline Eu-152 & B3 & 2014 & 0.06007 & 0.023 & 0.069 & $\mathrm{U}$ \\
\hline Eu-152 & B3 & 2014 & 0.01607 & 0.0498 & 0.1494 & $\mathrm{U}$ \\
\hline Eu-152 & B4 & 2014 & 0.009103 & 0.0187 & 0.0561 & $\mathrm{U}$ \\
\hline Eu-152 & B40 & 2014 & 0.04558 & 0.0204 & 0.0612 & $\mathrm{U}$ \\
\hline Eu-152 & B50 & 2014 & 0.009252 & 0.0284 & 0.0852 & $\mathrm{U}$ \\
\hline Eu-152 & B52 & 2014 & -0.01747 & 0.036 & 0.108 & $\mathrm{U}$ \\
\hline Eu-152 & B52 & 2014 & -0.02322 & 0.0298 & 0.0894 & $\mathrm{U}$ \\
\hline
\end{tabular}


Table C-3. (continued).

\begin{tabular}{|c|c|c|c|c|c|c|}
\hline Radionuclide & $\begin{array}{c}\text { Sample } \\
\text { Location }\end{array}$ & Date & $\begin{array}{c}\text { Concentrations } \\
(\mathrm{pCi} / \mathrm{g})\end{array}$ & $\begin{array}{l}\text { Uncertainty } \\
(\mathrm{pCi} / \mathrm{g})\end{array}$ & $\begin{array}{c}\mathrm{MDA} \\
(\mathrm{pCi} / \mathrm{g})\end{array}$ & Flag \\
\hline $\mathrm{Pu}-238$ & A118 & 1974 & 0.013 & 0.0018 & 0.0054 & 一 \\
\hline $\mathrm{Pu}-238$ & A120 & 1974 & 0.0072 & 0.0027 & 0.0081 & $\mathrm{U}$ \\
\hline $\mathrm{Pu}-238$ & A52 & 1974 & 0.019 & 0.0009 & 0.0027 & 一 \\
\hline $\mathrm{Pu}-238$ & A71 & 1974 & 0.034 & 0.003 & 0.009 & - \\
\hline $\mathrm{Pu}-238$ & A87 & 1974 & 0.009 & 0.0022 & 0.0066 & - \\
\hline $\mathrm{Pu}-238$ & A91 & 1974 & 0.0068 & 0.0009 & 0.0027 & - \\
\hline $\mathrm{Pu}-238$ & B117 & 1974 & 0.025 & 0.0013 & 0.0039 & - \\
\hline $\mathrm{Pu}-238$ & B120 & 1974 & 0.018 & 0.0018 & 0.0054 & - \\
\hline $\mathrm{Pu}-238$ & B5 & 1974 & 0.0095 & 0.0014 & 0.0042 & - \\
\hline $\mathrm{Pu}-238$ & B61 & 1974 & 0.024 & 0.0018 & 0.0054 & - \\
\hline $\mathrm{Pu}-238$ & B79 & 1974 & 0.185 & 0.005 & 0.015 & 一 \\
\hline $\mathrm{Pu}-238$ & B86 & 1974 & 0.0041 & 0.004 & 0.012 & $\mathrm{U}$ \\
\hline $\mathrm{Pu}-238$ & B90 & 1974 & 0.032 & 0.0014 & 0.0042 & - \\
\hline $\mathrm{Pu}-238$ & $\mathrm{C} 36$ & 1974 & 0.0063 & 0.0027 & 0.0081 & $\mathrm{U}$ \\
\hline $\mathrm{Pu}-238$ & $\mathrm{C} 46$ & 1974 & 0.007 & 0.0022 & 0.0066 & - \\
\hline $\mathrm{Pu}-238$ & D47 & 1974 & 0.015 & 0.004 & 0.012 & - \\
\hline $\mathrm{Pu}-238$ & A-110 & 1982 & 0.008 & 0.002 & 0.006 & - \\
\hline $\mathrm{Pu}-238$ & A-120 & 1982 & 0.017 & 0.002 & 0.006 & - \\
\hline $\mathrm{Pu}-238$ & A-17 & 1982 & 0.043 & 0.004 & 0.012 & - \\
\hline $\mathrm{Pu}-238$ & A-48 & 1982 & 0.112 & 0.007 & 0.021 & - \\
\hline $\mathrm{Pu}-238$ & B-120 & 1982 & 0.0043 & 0.0012 & 0.0036 & - \\
\hline $\mathrm{Pu}-238$ & B-27 & 1982 & 0.015 & 0.003 & 0.009 & - \\
\hline $\mathrm{Pu}-238$ & B-48 & 1982 & 0.092 & 0.006 & 0.018 & - \\
\hline $\mathrm{Pu}-238$ & B-52 & 1982 & 0.014 & 0.002 & 0.006 & - \\
\hline $\mathrm{Pu}-238$ & B-69 & 1982 & 0.135 & 0.007 & 0.021 & - \\
\hline $\mathrm{Pu}-238$ & C-21 & 1982 & 0.011 & 0.002 & 0.006 & - \\
\hline $\mathrm{Pu}-238$ & D-114 & 1982 & 0.255 & 0.011 & 0.033 & - \\
\hline $\mathrm{Pu}-238$ & D-57 & 1982 & 0.019 & 0.004 & 0.012 & - \\
\hline $\mathrm{Pu}-238$ & D-96 & 1982 & 0.0011 & 0.0009 & 0.0027 & $\mathrm{U}$ \\
\hline $\mathrm{Pu}-238$ & A108 & 1989 & 0.074 & 0.004 & 0.012 & - \\
\hline $\mathrm{Pu}-238$ & A66 & 1989 & 0.01 & 0.002 & 0.006 & - \\
\hline $\mathrm{Pu}-238$ & B25 & 1989 & 0.056 & 0.003 & 0.009 & - \\
\hline $\mathrm{Pu}-238$ & B90 & 1989 & 0.029 & 0.003 & 0.009 & - \\
\hline $\mathrm{Pu}-238$ & B96 & 1989 & 0.387 & 0.017 & 0.051 & - \\
\hline $\mathrm{Pu}-238$ & $\mathrm{C} 48$ & 1989 & 0.0037 & 0.0013 & 0.0039 & $\mathrm{U}$ \\
\hline $\mathrm{Pu}-238$ & C79 & 1989 & 0.01 & 0.002 & 0.006 & - \\
\hline $\mathrm{Pu}-238$ & D30 & 1989 & 0.0045 & 0.0015 & 0.0045 & - \\
\hline
\end{tabular}


Table C-3. (continued).

\begin{tabular}{|c|c|c|c|c|c|c|}
\hline Radionuclide & $\begin{array}{c}\text { Sample } \\
\text { Location }\end{array}$ & Date & $\begin{array}{c}\text { Concentrations } \\
(\mathrm{pCi} / \mathrm{g})\end{array}$ & $\begin{array}{l}\text { Uncertainty } \\
(\mathrm{pCi} / \mathrm{g})\end{array}$ & $\begin{array}{c}\mathrm{MDA} \\
(\mathrm{pCi} / \mathrm{g})\end{array}$ & Flag \\
\hline $\mathrm{Pu}-238$ & D96 & 1989 & 0.0011 & 0.001 & 0.003 & $\mathrm{U}$ \\
\hline $\mathrm{Pu}-239 / 240$ & A118 & 1974 & 0.011 & 0.0018 & 0.0054 & 一 \\
\hline $\mathrm{Pu}-239 / 240$ & A120 & 1974 & 0.0086 & 0.0018 & 0.0054 & 一 \\
\hline $\mathrm{Pu}-239 / 240$ & A52 & 1974 & 0.026 & 0.0014 & 0.0042 & - \\
\hline $\mathrm{Pu}-239 / 240$ & A71 & 1974 & 0.024 & 0.0023 & 0.0069 & - \\
\hline $\mathrm{Pu}-239 / 240$ & A87 & 1974 & 0.0099 & 0.0036 & 0.0108 & $\mathrm{U}$ \\
\hline $\mathrm{Pu}-239 / 240$ & A91 & 1974 & 0.0135 & 0.0009 & 0.0027 & - \\
\hline $\mathrm{Pu}-239 / 240$ & B117 & 1974 & 0.018 & 0.0009 & 0.0027 & - \\
\hline $\mathrm{Pu}-239 / 240$ & B120 & 1974 & 0.016 & 0.0018 & 0.0054 & - \\
\hline $\mathrm{Pu}-239 / 240$ & B5 & 1974 & 0.021 & 0.0023 & 0.0069 & - \\
\hline $\mathrm{Pu}-239 / 240$ & B61 & 1974 & 0.026 & 0.0018 & 0.0054 & - \\
\hline $\mathrm{Pu}-239 / 240$ & B79 & 1974 & 0.049 & 0.03 & 0.09 & $\mathrm{U}$ \\
\hline $\mathrm{Pu}-239 / 240$ & B86 & 1974 & 0.023 & 0.0045 & 0.0135 & - \\
\hline $\mathrm{Pu}-239 / 240$ & B90 & 1974 & 0.035 & 0.0014 & 0.0042 & - \\
\hline $\mathrm{Pu}-239 / 240$ & C36 & 1974 & 0.016 & 0.0036 & 0.0108 & - \\
\hline $\mathrm{Pu}-239 / 240$ & $\mathrm{C} 46$ & 1974 & 0.016 & 0.003 & 0.009 & - \\
\hline $\mathrm{Pu}-239 / 240$ & D47 & 1974 & 0.023 & 0.004 & 0.012 & - \\
\hline $\mathrm{Pu}-239 / 240$ & A-110 & 1982 & 0.02 & 0.002 & 0.006 & - \\
\hline $\mathrm{Pu}-239 / 240$ & A-120 & 1982 & 0.017 & 0.002 & 0.006 & - \\
\hline $\mathrm{Pu}-239 / 240$ & A-17 & 1982 & 0.37 & 0.004 & 0.012 & - \\
\hline $\mathrm{Pu}-239 / 240$ & A-48 & 1982 & 0.022 & 0.044 & 0.132 & $\mathrm{U}$ \\
\hline $\mathrm{Pu}-239 / 240$ & B-120 & 1982 & 0.0017 & 0.001 & 0.003 & $\mathrm{U}$ \\
\hline $\mathrm{Pu}-239 / 240$ & B-27 & 1982 & 0.009 & 0.003 & 0.009 & - \\
\hline $\mathrm{Pu}-239 / 240$ & B-48 & 1982 & 0.014 & 0.004 & 0.012 & - \\
\hline $\mathrm{Pu}-239 / 240$ & B-52 & 1982 & 0.032 & 0.003 & 0.009 & 一 \\
\hline $\mathrm{Pu}-239 / 240$ & B-69 & 1982 & 0.037 & 0.004 & 0.012 & 一 \\
\hline $\mathrm{Pu}-239 / 240$ & $\mathrm{C}-21$ & 1982 & 0.026 & 0.003 & 0.009 & - \\
\hline $\mathrm{Pu}-239 / 240$ & D-114 & 1982 & 0.038 & 0.003 & 0.009 & - \\
\hline $\mathrm{Pu}-239 / 240$ & D-57 & 1982 & 0.009 & 0.003 & 0.009 & - \\
\hline $\mathrm{Pu}-239 / 240$ & D-96 & 1982 & 0.013 & 0.002 & 0.006 & - \\
\hline $\mathrm{Pu}-239 / 240$ & A108 & 1989 & 0.025 & 0.002 & 0.006 & - \\
\hline $\mathrm{Pu}-239 / 240$ & A17 & 1989 & 0.018 & 0.003 & 0.009 & - \\
\hline $\mathrm{Pu}-239 / 240$ & A66 & 1989 & 0.017 & 0.002 & 0.006 & - \\
\hline $\mathrm{Pu}-239 / 240$ & B25 & 1989 & 0.03 & 0.003 & 0.009 & - \\
\hline $\mathrm{Pu}-239 / 240$ & B90 & 1989 & 0.023 & 0.003 & 0.009 & - \\
\hline $\mathrm{Pu}-239 / 240$ & B96 & 1989 & 0.73 & 0.005 & 0.015 & - \\
\hline $\mathrm{Pu}-239 / 240$ & $\mathrm{C} 48$ & 1989 & 0.0099 & 0.0015 & 0.0045 & - \\
\hline
\end{tabular}


Table C-3. (continued).

\begin{tabular}{|c|c|c|c|c|c|c|}
\hline Radionuclide & $\begin{array}{c}\text { Sample } \\
\text { Location }\end{array}$ & Date & $\begin{array}{c}\text { Concentrations } \\
(\mathrm{pCi} / \mathrm{g})\end{array}$ & $\begin{array}{l}\text { Uncertainty } \\
(\mathrm{pCi} / \mathrm{g})\end{array}$ & $\begin{array}{c}\mathrm{MDA} \\
(\mathrm{pCi} / \mathrm{g})\end{array}$ & Flag \\
\hline $\mathrm{Pu}-239 / 240$ & C79 & 1989 & 0.018 & 0.002 & 0.006 & 一 \\
\hline $\mathrm{Pu}-239 / 240$ & D30 & 1989 & 0.021 & 0.002 & 0.006 & 一 \\
\hline $\mathrm{Pu}-239 / 240$ & D96 & 1989 & 0.015 & 0.002 & 0.006 & 一 \\
\hline $\mathrm{Sb}-125$ & A15 & 2007 & 0.4699 & 0.0955 & 0.2865 & - \\
\hline $\mathrm{Sb}-125$ & A16 & 2007 & 0.387 & 0.059 & 0.177 & - \\
\hline $\mathrm{Sb}-125$ & A17 & 2007 & 0.3045 & 0.0505 & 0.1515 & 一 \\
\hline $\mathrm{Sb}-125$ & A 27 & 2007 & 0.58 & 0.0875 & 0.2625 & - \\
\hline $\mathrm{Sb}-125$ & A28 & 2007 & 0.1308 & 0.058 & 0.174 & $\mathrm{U}$ \\
\hline $\mathrm{Sb}-125$ & A37 & 2007 & 0.468 & 0.1095 & 0.3285 & - \\
\hline $\mathrm{Sb}-125$ & A38 & 2007 & 0.2803 & 0.062 & 0.186 & - \\
\hline $\mathrm{Sb}-125$ & $\mathrm{~A} 4$ & 2007 & 0.1576 & 0.04275 & 0.12825 & 一 \\
\hline $\mathrm{Sb}-125$ & A44 & 2007 & 0.5998 & 0.103 & 0.309 & - \\
\hline $\mathrm{Sb}-125$ & A45 & 2007 & 0.1683 & 0.04445 & 0.13335 & - \\
\hline $\mathrm{Sb}-125$ & A47 & 2007 & 0.3291 & 0.0525 & 0.1575 & - \\
\hline $\mathrm{Sb}-125$ & A48 & 2007 & 0.5109 & 0.083 & 0.249 & - \\
\hline $\mathrm{Sb}-125$ & A49 & 2007 & 0.3688 & 0.062 & 0.186 & - \\
\hline $\mathrm{Sb}-125$ & $\mathrm{~A} 5$ & 2007 & 0.2178 & 0.068 & 0.204 & - \\
\hline $\mathrm{Sb}-125$ & A50 & 2007 & 0.5153 & 0.0795 & 0.2385 & - \\
\hline $\mathrm{Sb}-125$ & A55 & 2007 & 0.3285 & 0.051 & 0.153 & - \\
\hline $\mathrm{Sb}-125$ & A58 & 2007 & 0.1921 & 0.0625 & 0.1875 & - \\
\hline $\mathrm{Sb}-125$ & A59 & 2007 & 0.3146 & 0.04025 & 0.12075 & - \\
\hline $\mathrm{Sb}-125$ & A6 & 2007 & 0.2959 & 0.0484 & 0.1452 & - \\
\hline $\mathrm{Sb}-125$ & A60 & 2007 & 0.4206 & 0.0835 & 0.2505 & - \\
\hline $\mathrm{Sb}-125$ & A61 & 2007 & 0.5059 & 0.1 & 0.3 & - \\
\hline $\mathrm{Sb}-125$ & A67 & 2007 & 0.09461 & 0.0735 & 0.2205 & $\mathrm{U}$ \\
\hline $\mathrm{Sb}-125$ & A69 & 2007 & 0.09751 & 0.055 & 0.165 & $\mathrm{U}$ \\
\hline $\mathrm{Sb}-125$ & A70 & 2007 & 0.4739 & 0.0705 & 0.2115 & - \\
\hline $\mathrm{Sb}-125$ & A71 & 2007 & 0.1516 & 0.04205 & 0.12615 & - \\
\hline $\mathrm{Sb}-125$ & A72 & 2007 & 0.2183 & 0.0585 & 0.1755 & - \\
\hline $\mathrm{Sb}-125$ & B101 & 2007 & 0.34 & 0.0555 & 0.1665 & - \\
\hline $\mathrm{Sb}-125$ & B102 & 2007 & 0.3989 & 0.078 & 0.234 & - \\
\hline $\mathrm{Sb}-125$ & B103 & 2007 & 0.3027 & 0.04375 & 0.13125 & - \\
\hline $\mathrm{Sb}-125$ & B104 & 2007 & 0.3399 & 0.051 & 0.153 & - \\
\hline Sb-125 & B105 & 2007 & 0.3386 & 0.054 & 0.162 & - \\
\hline $\mathrm{Sb}-125$ & B14 & 2007 & 0.4713 & 0.0825 & 0.2475 & - \\
\hline $\mathrm{Sb}-125$ & $\mathrm{~B} 17$ & 2007 & 0.2961 & 0.062 & 0.186 & 一 \\
\hline $\mathrm{Sb}-125$ & $\mathrm{~B} 25$ & 2007 & 0.2267 & 0.094 & 0.282 & $\mathrm{U}$ \\
\hline
\end{tabular}


Table C-3. (continued).

\begin{tabular}{|c|c|c|c|c|c|c|}
\hline Radionuclide & $\begin{array}{c}\text { Sample } \\
\text { Location }\end{array}$ & Date & $\begin{array}{c}\text { Concentrations } \\
(\mathrm{pCi} / \mathrm{g})\end{array}$ & $\begin{array}{l}\text { Uncertainty } \\
(\mathrm{pCi} / \mathrm{g})\end{array}$ & $\begin{array}{c}\mathrm{MDA} \\
(\mathrm{pCi} / \mathrm{g})\end{array}$ & Flag \\
\hline $\mathrm{Sb}-125$ & $\mathrm{~B} 26$ & 2007 & 0.3765 & 0.077 & 0.231 & - \\
\hline $\mathrm{Sb}-125$ & B28 & 2007 & 0.2839 & 0.0471 & 0.1413 & 一 \\
\hline $\mathrm{Sb}-125$ & B29 & 2007 & 0.1823 & 0.0745 & 0.2235 & $\mathrm{U}$ \\
\hline $\mathrm{Sb}-125$ & B3 & 2007 & 0.4507 & 0.0815 & 0.2445 & - \\
\hline $\mathrm{Sb}-125$ & B4 & 2007 & 0.3181 & 0.0545 & 0.1635 & - \\
\hline $\mathrm{Sb}-125$ & B40 & 2007 & 0.344 & 0.0685 & 0.2055 & - \\
\hline $\mathrm{Sb}-125$ & B47 & 2007 & 0.3501 & 0.093 & 0.279 & - \\
\hline $\mathrm{Sb}-125$ & B50 & 2007 & 0.1197 & 0.064 & 0.192 & $\mathrm{U}$ \\
\hline $\mathrm{Sb}-125$ & B51 & 2007 & 0.3114 & 0.0685 & 0.2055 & - \\
\hline $\mathrm{Sb}-125$ & B52 & 2007 & 0.1564 & 0.04425 & 0.13275 & - \\
\hline $\mathrm{Sb}-125$ & B59 & 2007 & 0.7193 & 0.162 & 0.486 & - \\
\hline $\mathrm{Sb}-125$ & B60 & 2007 & 1.075 & 0.168 & 0.504 & - \\
\hline $\mathrm{Sb}-125$ & B61 & 2007 & 0.3815 & 0.0605 & 0.1815 & - \\
\hline $\mathrm{Sb}-125$ & B62 & 2007 & 0.2139 & 0.04055 & 0.12165 & - \\
\hline $\mathrm{Sb}-125$ & B69 & 2007 & 1.492 & 0.3825 & 1.1475 & - \\
\hline $\mathrm{Sb}-125$ & B71 & 2007 & 0.7765 & 0.1225 & 0.3675 & - \\
\hline $\mathrm{Sb}-125$ & B73 & 2007 & 0.275 & 0.04605 & 0.13815 & - \\
\hline $\mathrm{Sb}-125$ & B80 & 2007 & 0.2825 & 0.098 & 0.294 & $\mathrm{U}$ \\
\hline $\mathrm{Sb}-125$ & B81 & 2007 & 0.2304 & 0.0665 & 0.1995 & - \\
\hline $\mathrm{Sb}-125$ & B82 & 2007 & 0.3594 & 0.0545 & 0.1635 & - \\
\hline $\mathrm{Sb}-125$ & B83 & 2007 & 0.2257 & 0.0488 & 0.1464 & - \\
\hline $\mathrm{Sb}-125$ & B88 & 2007 & 0.2338 & 0.0458 & 0.1374 & - \\
\hline $\mathrm{Sb}-125$ & B89 & 2007 & 0.3111 & 0.055 & 0.165 & - \\
\hline $\mathrm{Sb}-125$ & B90 & 2007 & 0.173 & 0.0615 & 0.1845 & $\mathrm{U}$ \\
\hline $\mathrm{Sb}-125$ & B92 & 2007 & 0.3521 & 0.0545 & 0.1635 & - \\
\hline $\mathrm{Sb}-125$ & B93 & 2007 & 0.2379 & 0.052 & 0.156 & 一 \\
\hline $\mathrm{Sb}-125$ & B94 & 2007 & 0.1729 & 0.0384 & 0.1152 & - \\
\hline $\mathrm{Sb}-125$ & B95 & 2007 & 0.06979 & 0.0314 & 0.0942 & $\mathrm{U}$ \\
\hline $\mathrm{Sb}-125$ & B99 & 2007 & 0.2918 & 0.066 & 0.198 & - \\
\hline $\mathrm{Sb}-125$ & $\mathrm{C} 14$ & 2007 & 0.187 & 0.0505 & 0.1515 & - \\
\hline $\mathrm{Sb}-125$ & $\mathrm{C} 15$ & 2007 & 0.3681 & 0.0755 & 0.2265 & - \\
\hline $\mathrm{Sb}-125$ & $\mathrm{C} 36$ & 2007 & 0.4606 & 0.1015 & 0.3045 & - \\
\hline $\mathrm{Sb}-125$ & C37 & 2007 & 0.4863 & 0.097 & 0.291 & - \\
\hline $\mathrm{Sb}-125$ & $\mathrm{C} 46$ & 2007 & 0.8255 & 0.163 & 0.489 & - \\
\hline $\mathrm{Sb}-125$ & $\mathrm{C} 47$ & 2007 & 0.3578 & 0.058 & 0.174 & - \\
\hline $\mathrm{Sb}-125$ & $\mathrm{C} 48$ & 2007 & 0.1973 & 0.058 & 0.174 & - \\
\hline $\mathrm{Sb}-125$ & C56 & 2007 & 0.3822 & 0.053 & 0.159 & - \\
\hline
\end{tabular}


Table C-3. (continued).

\begin{tabular}{|c|c|c|c|c|c|c|}
\hline Radionuclide & $\begin{array}{l}\text { Sample } \\
\text { Location }\end{array}$ & Date & $\begin{array}{c}\text { Concentrations } \\
(\mathrm{pCi} / \mathrm{g})\end{array}$ & $\begin{array}{l}\text { Uncertainty } \\
(\mathrm{pCi} / \mathrm{g})\end{array}$ & $\begin{array}{l}\text { MDA } \\
(\mathrm{pCi} / \mathrm{g})\end{array}$ & Flag \\
\hline $\mathrm{Sb}-125$ & $\mathrm{C} 57$ & 2007 & 0.3826 & 0.112 & 0.336 & - \\
\hline $\mathrm{Sb}-125$ & D58 & 2007 & 0.08303 & 0.03335 & 0.10005 & $\mathrm{U}$ \\
\hline $\mathrm{Sb}-125$ & D68 & 2007 & 0.5758 & 0.0795 & 0.2385 & 一 \\
\hline $\mathrm{Sb}-125$ & D69 & 2007 & 0.3163 & 0.054 & 0.162 & - \\
\hline $\mathrm{Sb}-125$ & D79 & 2007 & 0.3849 & 0.0655 & 0.1965 & - \\
\hline $\mathrm{Sb}-125$ & D80 & 2007 & 0.03939 & 0.0432 & 0.1296 & $\mathrm{U}$ \\
\hline $\mathrm{Sb}-125$ & D89 & 2007 & 0.259 & 0.07 & 0.21 & - \\
\hline $\mathrm{Sb}-125$ & D90 & 2007 & 0.2649 & 0.03575 & 0.10725 & - \\
\hline $\mathrm{Sb}-125$ & D91 & 2007 & 0.2252 & 0.062 & 0.186 & - \\
\hline $\mathrm{Sb}-125$ & A 15 & 2011 & 0.1502 & 0.0343 & 0.1029 & - \\
\hline $\mathrm{Sb}-125$ & A16 & 2011 & -0.04523 & 0.0444 & 0.1332 & $\mathrm{U}$ \\
\hline $\mathrm{Sb}-125$ & A17 & 2011 & 0.07291 & 0.0418 & 0.1254 & $\mathrm{U}$ \\
\hline $\mathrm{Sb}-125$ & A27 & 2011 & 0.07812 & 0.0448 & 0.1344 & $\mathrm{U}$ \\
\hline $\mathrm{Sb}-125$ & A28 & 2011 & 0.039 & 0.0438 & 0.1314 & $\mathrm{U}$ \\
\hline $\mathrm{Sb}-125$ & A38 & 2011 & -0.04674 & 0.0497 & 0.1491 & $\mathrm{U}$ \\
\hline $\mathrm{Sb}-125$ & A39 & 2011 & 0.06301 & 0.045 & 0.135 & $\mathrm{U}$ \\
\hline $\mathrm{Sb}-125$ & $\mathrm{~A} 4$ & 2011 & -0.06104 & 0.0449 & 0.1347 & $\mathrm{U}$ \\
\hline $\mathrm{Sb}-125$ & A44 & 2011 & 0.09478 & 0.0464 & 0.1392 & $\mathrm{U}$ \\
\hline $\mathrm{Sb}-125$ & A47 & 2011 & -0.06306 & 0.0603 & 0.1809 & $\mathrm{U}$ \\
\hline $\mathrm{Sb}-125$ & A49 & 2011 & 0.02381 & 0.0577 & 0.1731 & $\mathrm{U}$ \\
\hline $\mathrm{Sb}-125$ & A50 & 2011 & -0.02806 & 0.0546 & 0.1638 & $\mathrm{U}$ \\
\hline $\mathrm{Sb}-125$ & A55 & 2011 & -0.06385 & 0.0475 & 0.1425 & $\mathrm{U}$ \\
\hline $\mathrm{Sb}-125$ & A58 & 2011 & -0.09493 & 0.0482 & 0.1446 & $\mathrm{U}$ \\
\hline $\mathrm{Sb}-125$ & A59 & 2011 & -0.08614 & 0.0476 & 0.1428 & $\mathrm{U}$ \\
\hline $\mathrm{Sb}-125$ & A6 & 2011 & -0.08628 & 0.047 & 0.141 & $\mathrm{U}$ \\
\hline $\mathrm{Sb}-125$ & A61 & 2011 & -0.1221 & 0.0434 & 0.1302 & $\mathrm{U}$ \\
\hline $\mathrm{Sb}-125$ & A66 & 2011 & -0.04631 & 0.0488 & 0.1464 & $\mathrm{U}$ \\
\hline $\mathrm{Sb}-125$ & A69 & 2011 & 0.07454 & 0.0491 & 0.1473 & $\mathrm{U}$ \\
\hline $\mathrm{Sb}-125$ & A70 & 2011 & 0.7707 & 0.0451 & 0.1353 & - \\
\hline $\mathrm{Sb}-125$ & A72 & 2011 & 0.1031 & 0.0421 & 0.1263 & $\mathrm{U}$ \\
\hline $\mathrm{Sb}-125$ & B101 & 2011 & 0.05686 & 0.0412 & 0.1236 & $\mathrm{U}$ \\
\hline $\mathrm{Sb}-125$ & B102 & 2011 & 0.001085 & 0.0451 & 0.1353 & $\mathrm{U}$ \\
\hline Sb-125 & $\mathrm{B} 103$ & 2011 & 0.1289 & 0.0428 & 0.1284 & - \\
\hline Sb-125 & B104 & 2011 & -0.01511 & 0.0458 & 0.1374 & $\mathrm{U}$ \\
\hline $\mathrm{Sb}-125$ & B106 & 2011 & 0.03967 & 0.0426 & 0.1278 & $\mathrm{U}$ \\
\hline $\mathrm{Sb}-125$ & $\mathrm{~B} 14$ & 2011 & -0.0839 & 0.0523 & 0.1569 & $\mathrm{U}$ \\
\hline $\mathrm{Sb}-125$ & $\mathrm{~B} 15$ & 2011 & 0.06217 & 0.0524 & 0.1572 & $\mathrm{U}$ \\
\hline
\end{tabular}


Table C-3. (continued).

\begin{tabular}{|c|c|c|c|c|c|c|}
\hline Radionuclide & $\begin{array}{l}\text { Sample } \\
\text { Location }\end{array}$ & Date & $\begin{array}{c}\text { Concentrations } \\
(\mathrm{pCi} / \mathrm{g})\end{array}$ & $\begin{array}{l}\text { Uncertainty } \\
(\mathrm{pCi} / \mathrm{g})\end{array}$ & $\begin{array}{l}\text { MDA } \\
(\mathrm{pCi} / \mathrm{g})\end{array}$ & Flag \\
\hline $\mathrm{Sb}-125$ & $\mathrm{~B} 17$ & 2011 & -0.02418 & 0.0296 & 0.0888 & $\mathrm{U}$ \\
\hline $\mathrm{Sb}-125$ & $\mathrm{~B} 25$ & 2011 & -0.1133 & 0.062 & 0.186 & $\mathrm{U}$ \\
\hline $\mathrm{Sb}-125$ & $\mathrm{~B} 28$ & 2011 & 0.07748 & 0.0439 & 0.1317 & $\mathrm{U}$ \\
\hline $\mathrm{Sb}-125$ & B29 & 2011 & -0.02098 & 0.0445 & 0.1335 & $\mathrm{U}$ \\
\hline $\mathrm{Sb}-125$ & B3 & 2011 & 0.04709 & 0.0469 & 0.1407 & $\mathrm{U}$ \\
\hline $\mathrm{Sb}-125$ & B39 & 2011 & 0.06593 & 0.0298 & 0.0894 & $\mathrm{U}$ \\
\hline $\mathrm{Sb}-125$ & B4 & 2011 & 0.07309 & 0.0427 & 0.1281 & $\mathrm{U}$ \\
\hline $\mathrm{Sb}-125$ & B40 & 2011 & 0.1056 & 0.0293 & 0.0879 & - \\
\hline $\mathrm{Sb}-125$ & B47 & 2011 & 0.2983 & 0.0633 & 0.1899 & - \\
\hline $\mathrm{Sb}-125$ & B48 & 2011 & 0.1007 & 0.0586 & 0.1758 & $\mathrm{U}$ \\
\hline $\mathrm{Sb}-125$ & B50 & 2011 & -0.07444 & 0.0472 & 0.1416 & $\mathrm{U}$ \\
\hline $\mathrm{Sb}-125$ & B52 & 2011 & 0.1024 & 0.041 & 0.123 & $\mathrm{U}$ \\
\hline $\mathrm{Sb}-125$ & B61 & 2011 & -0.04446 & 0.0499 & 0.1497 & $\mathrm{U}$ \\
\hline $\mathrm{Sb}-125$ & B62 & 2011 & 0.0682 & 0.0421 & 0.1263 & $\mathrm{U}$ \\
\hline $\mathrm{Sb}-125$ & B71 & 2011 & 0.02447 & 0.0495 & 0.1485 & $\mathrm{U}$ \\
\hline $\mathrm{Sb}-125$ & B73 & 2011 & -0.06905 & 0.0504 & 0.1512 & $\mathrm{U}$ \\
\hline $\mathrm{Sb}-125$ & $\mathrm{~B} 80$ & 2011 & 0.0328 & 0.0802 & 0.2406 & $\mathrm{U}$ \\
\hline $\mathrm{Sb}-125$ & B83 & 2011 & 0.002016 & 0.0468 & 0.1404 & $\mathrm{U}$ \\
\hline $\mathrm{Sb}-125$ & B84 & 2011 & 0.1419 & 0.0498 & 0.1494 & $\mathrm{U}$ \\
\hline $\mathrm{Sb}-125$ & B89 & 2011 & 0.06551 & 0.045 & 0.135 & $\mathrm{U}$ \\
\hline $\mathrm{Sb}-125$ & B90 & 2011 & 0.08206 & 0.0507 & 0.1521 & $\mathrm{U}$ \\
\hline $\mathrm{Sb}-125$ & B91 & 2011 & 0.01732 & 0.0505 & 0.1515 & $\mathrm{U}$ \\
\hline $\mathrm{Sb}-125$ & B92 & 2011 & -0.03329 & 0.0459 & 0.1377 & $\mathrm{U}$ \\
\hline $\mathrm{Sb}-125$ & B93 & 2011 & -0.04603 & 0.0439 & 0.1317 & $\mathrm{U}$ \\
\hline $\mathrm{Sb}-125$ & B94 & 2011 & 0.1205 & 0.0491 & 0.1473 & $\mathrm{U}$ \\
\hline $\mathrm{Sb}-125$ & B99 & 2011 & 0.0895 & 0.039 & 0.117 & $\mathrm{U}$ \\
\hline $\mathrm{Sb}-125$ & $\mathrm{C} 15$ & 2011 & 0.1154 & 0.0412 & 0.1236 & $\mathrm{U}$ \\
\hline $\mathrm{Sb}-125$ & $\mathrm{C} 25$ & 2011 & -0.04459 & 0.0387 & 0.1161 & $\mathrm{U}$ \\
\hline $\mathrm{Sb}-125$ & C37 & 2011 & 0.07752 & 0.0384 & 0.1152 & $\mathrm{U}$ \\
\hline $\mathrm{Sb}-125$ & $\mathrm{C} 46$ & 2011 & -0.04241 & 0.0422 & 0.1266 & $\mathrm{U}$ \\
\hline $\mathrm{Sb}-125$ & $\mathrm{C} 48$ & 2011 & 0.1093 & 0.0371 & 0.1113 & $\mathrm{U}$ \\
\hline $\mathrm{Sb}-125$ & C56 & 2011 & -0.06699 & 0.0488 & 0.1464 & $\mathrm{U}$ \\
\hline $\mathrm{Sb}-125$ & C57 & 2011 & 0.04079 & 0.0403 & 0.1209 & $\mathrm{U}$ \\
\hline Sb-125 & D58 & 2011 & 0.03935 & 0.0462 & 0.1386 & $\mathrm{U}$ \\
\hline $\mathrm{Sb}-125$ & D68 & 2011 & 0.8069 & 0.0436 & 0.1308 & - \\
\hline $\mathrm{Sb}-125$ & D89 & 2011 & -0.0625 & 0.0454 & 0.1362 & $\mathrm{U}$ \\
\hline $\mathrm{Sb}-125$ & D91 & 2011 & -0.01864 & 0.0457 & 0.1371 & $\mathrm{U}$ \\
\hline
\end{tabular}


Table C-3. (continued).

\begin{tabular}{|c|c|c|c|c|c|c|}
\hline Radionuclide & $\begin{array}{l}\text { Sample } \\
\text { Location }\end{array}$ & Date & $\begin{array}{l}\text { Concentrations } \\
(\mathrm{pCi} / \mathrm{g})\end{array}$ & $\begin{array}{l}\text { Uncertainty } \\
(\mathrm{pCi} / \mathrm{g})\end{array}$ & $\begin{array}{l}\text { MDA } \\
(\mathrm{pCi} / \mathrm{g})\end{array}$ & Flag \\
\hline $\mathrm{Sb}-125$ & A15 & 2012 & -0.01218 & 0.0434 & 0.1302 & $\mathrm{U}$ \\
\hline $\mathrm{Sb}-125$ & A38 & 2012 & 0.0223 & 0.0468 & 0.1404 & $\mathrm{U}$ \\
\hline $\mathrm{Sb}-125$ & A44 & 2012 & 0.02173 & 0.0469 & 0.1407 & $\mathrm{U}$ \\
\hline $\mathrm{Sb}-125$ & A47 & 2012 & -0.002232 & 0.0441 & 0.1323 & $\mathrm{U}$ \\
\hline $\mathrm{Sb}-125$ & A59 & 2012 & -0.02327 & 0.0438 & 0.1314 & $\mathrm{U}$ \\
\hline $\mathrm{Sb}-125$ & A6 & 2012 & -0.01663 & 0.0448 & 0.1344 & $\mathrm{U}$ \\
\hline $\mathrm{Sb}-125$ & A69 & 2012 & 0.003527 & 0.0453 & 0.1359 & $\mathrm{U}$ \\
\hline $\mathrm{Sb}-125$ & A70 & 2012 & 0.02388 & 0.0413 & 0.1239 & $\mathrm{U}$ \\
\hline $\mathrm{Sb}-125$ & A72 & 2012 & -0.03608 & 0.0417 & 0.1251 & $\mathrm{U}$ \\
\hline $\mathrm{Sb}-125$ & B14 & 2012 & 0.04581 & 0.044 & 0.132 & $\mathrm{U}$ \\
\hline $\mathrm{Sb}-125$ & B48 & 2012 & 0.007959 & 0.0509 & 0.1527 & $\mathrm{U}$ \\
\hline $\mathrm{Sb}-125$ & B83 & 2012 & 0.02706 & 0.0367 & 0.1101 & $\mathrm{U}$ \\
\hline $\mathrm{Sb}-125$ & $\mathrm{C} 46$ & 2012 & -0.03563 & 0.0432 & 0.1296 & $\mathrm{U}$ \\
\hline $\mathrm{Sb}-125$ & C57 & 2012 & 0.01837 & 0.0397 & 0.1191 & $\mathrm{U}$ \\
\hline $\mathrm{Sb}-125$ & $\begin{array}{l}2 \text { INCH AIR } \\
\text { - INTEC }\end{array}$ & 2013 & 0.009235 & 0.038 & 0.114 & $\mathrm{U}$ \\
\hline $\mathrm{Sb}-125$ & A47 & 2013 & 0.2984 & 0.069666667 & 0.209 & - \\
\hline $\mathrm{Sb}-125$ & A49 & 2013 & 0.063 & 0.0172 & 0.0515 & - \\
\hline $\mathrm{Sb}-125$ & A59 & 2013 & 0.07963 & 0.043 & 0.129 & $\mathrm{U}$ \\
\hline $\mathrm{Sb}-125$ & A71 & 2013 & 0.4467 & 0.07 & 0.21 & - \\
\hline $\mathrm{Sb}-125$ & A72 & 2013 & 0.06063 & 0.0182 & 0.0545 & - \\
\hline $\mathrm{Sb}-125$ & $\begin{array}{c}2 \text { INCH AIR } \\
- \text { CPP }\end{array}$ & 2014 & -0.01203 & 0.058 & 0.174 & $\mathrm{U}$ \\
\hline $\mathrm{Sb}-125$ & $\begin{array}{l}2 \text { INCH AIR } \\
\text { - INTEC }\end{array}$ & 2014 & -0.1965 & 0.0474 & 0.1422 & $\mathrm{U}$ \\
\hline $\mathrm{Sb}-125$ & A38 & 2014 & -0.1559 & 0.0489 & 0.1467 & $\mathrm{U}$ \\
\hline $\mathrm{Sb}-125$ & A47 & 2014 & 0.004347 & 0.0648 & 0.1944 & $\mathrm{U}$ \\
\hline $\mathrm{Sb}-125$ & A58 & 2014 & -0.02004 & 0.0515 & 0.1545 & $\mathrm{U}$ \\
\hline $\mathrm{Sb}-125$ & A59 & 2014 & 0.001388 & 0.0609 & 0.1827 & $\mathrm{U}$ \\
\hline $\mathrm{Sb}-125$ & B15 & 2014 & -0.2591 & 0.0861 & 0.2583 & $\mathrm{U}$ \\
\hline $\mathrm{Sb}-125$ & B28 & 2014 & -0.01773 & 0.0465 & 0.1395 & $\mathrm{U}$ \\
\hline $\mathrm{Sb}-125$ & B29 & 2014 & -0.1412 & 0.0474 & 0.1422 & $\mathrm{U}$ \\
\hline $\mathrm{Sb}-125$ & B3 & 2014 & 0.02008 & 0.0316 & 0.0948 & $\mathrm{U}$ \\
\hline $\mathrm{Sb}-125$ & B3 & 2014 & -0.08612 & 0.0818 & 0.2454 & $\mathrm{U}$ \\
\hline $\mathrm{Sb}-125$ & B4 & 2014 & -0.04469 & 0.0312 & 0.0936 & $\mathrm{U}$ \\
\hline $\mathrm{Sb}-125$ & B40 & 2014 & -0.01734 & 0.0311 & 0.0933 & $\mathrm{U}$ \\
\hline $\mathrm{Sb}-125$ & B50 & 2014 & 0.001923 & 0.0492 & 0.1476 & $\mathrm{U}$ \\
\hline Sb-125 & B52 & 2014 & -0.0355 & 0.054 & 0.162 & $\mathrm{U}$ \\
\hline
\end{tabular}


Table C-3. (continued).

\begin{tabular}{|c|c|c|c|c|c|c|}
\hline Radionuclide & $\begin{array}{c}\text { Sample } \\
\text { Location }\end{array}$ & Date & $\begin{array}{l}\text { Concentrations } \\
(\mathrm{pCi} / \mathrm{g})\end{array}$ & $\begin{array}{l}\text { Uncertainty } \\
(\mathrm{pCi} / \mathrm{g})\end{array}$ & $\begin{array}{c}\mathrm{MDA} \\
(\mathrm{pCi} / \mathrm{g})\end{array}$ & Flag \\
\hline Sb-125 & B52 & 2014 & -0.03901 & 0.0616 & 0.1848 & $\mathrm{U}$ \\
\hline Sr-90 & B-52 & 1982 & 3 & 0.16 & 0.48 & 一 \\
\hline Sr-90 & A46 & 1989 & 10.6 & 0.3 & 0.9 & - \\
\hline Sr-90 & A52 & 1989 & 3.43 & 0.15 & 0.45 & - \\
\hline Sr-90 & A90 & 1989 & 1.04 & 0.08 & 0.24 & - \\
\hline Sr-90 & B119 & 1989 & 4.6 & 0.2 & 0.6 & - \\
\hline Sr-90 & B52 & 1989 & 1.35 & 0.09 & 0.27 & - \\
\hline Sr-90 & B70 & 1989 & 5.7 & 0.2 & 0.6 & - \\
\hline Sr-90 & $\mathrm{C} 40$ & 1989 & 0.48 & 0.05 & 0.15 & - \\
\hline Sr-90 & C96 & 1989 & 0.45 & 0.05 & 0.15 & - \\
\hline Sr-90 & D37 & 1989 & 1.59 & 0.09 & 0.27 & - \\
\hline Sr-90 & D63 & 1989 & 0.39 & 0.06 & 0.18 & - \\
\hline U-233/234 & A-17 & 1982 & 1.07 & 0.03 & 0.09 & - \\
\hline U-233/234 & A-48 & 1982 & 1.05 & 0.03 & 0.09 & - \\
\hline U-233/234 & B-27 & 1982 & 1.09 & 0.03 & 0.09 & - \\
\hline U-233/234 & B-48 & 1982 & 1.02 & 0.03 & 0.09 & - \\
\hline U-233/234 & D-57 & 1982 & 1.04 & 0.03 & 0.09 & - \\
\hline U-234 & A15 & 2007 & 27.41 & 16.15 & 48.45 & $\mathrm{U}$ \\
\hline U-234 & A16 & 2007 & 52.12 & 23.45 & 70.35 & $\mathrm{U}$ \\
\hline U-234 & A17 & 2007 & 34.55 & 23.95 & 71.85 & $\mathrm{U}$ \\
\hline U-234 & $\mathrm{A} 27$ & 2007 & 51.58 & 45 & 135 & $\mathrm{U}$ \\
\hline U-234 & A28 & 2007 & 49.08 & 27.75 & 83.25 & $\mathrm{U}$ \\
\hline U-234 & A38 & 2007 & 71.59 & 51.5 & 154.5 & $\mathrm{U}$ \\
\hline U-234 & A39 & 2007 & 146.8 & 4.2 & 12.6 & - \\
\hline U-234 & A4 & 2007 & 44.31 & 33.9 & 101.7 & $\mathrm{U}$ \\
\hline U-234 & A45 & 2007 & 15.64 & 15.1 & 45.3 & $\mathrm{U}$ \\
\hline U-234 & A47 & 2007 & 60.34 & 43.95 & 131.85 & $\mathrm{U}$ \\
\hline U-234 & A48 & 2007 & 45.9 & 22.15 & 66.45 & $\mathrm{U}$ \\
\hline U-234 & A49 & 2007 & 28.07 & 13.2 & 39.6 & $\mathrm{U}$ \\
\hline U-234 & A5 & 2007 & 24.24 & 12.35 & 37.05 & $\mathrm{U}$ \\
\hline U-234 & A50 & 2007 & 63.1 & 23.6 & 70.8 & $\mathrm{U}$ \\
\hline U-234 & A56 & 2007 & 118 & 4.76 & 14.28 & - \\
\hline U-234 & A58 & 2007 & 54.09 & 19.35 & 58.05 & $\mathrm{U}$ \\
\hline U-234 & A59 & 2007 & 0.1791 & 30.05 & 90.15 & $\mathrm{U}$ \\
\hline U-234 & A6 & 2007 & 43.09 & 20.1 & 60.3 & $\mathrm{U}$ \\
\hline U-234 & A60 & 2007 & 25.85 & 17.3 & 51.9 & $\mathrm{U}$ \\
\hline U-234 & A61 & 2007 & 56.17 & 23.85 & 71.55 & $\mathrm{U}$ \\
\hline
\end{tabular}


Table C-3. (continued).

\begin{tabular}{|c|c|c|c|c|c|c|}
\hline Radionuclide & $\begin{array}{c}\text { Sample } \\
\text { Location }\end{array}$ & Date & $\begin{array}{c}\text { Concentrations } \\
(\mathrm{pCi} / \mathrm{g})\end{array}$ & $\begin{array}{l}\text { Uncertainty } \\
(\mathrm{pCi} / \mathrm{g})\end{array}$ & $\begin{array}{c}\mathrm{MDA} \\
(\mathrm{pCi} / \mathrm{g})\end{array}$ & Flag \\
\hline $\mathrm{U}-234$ & A66 & 2007 & 91.86 & 3.82 & 11.46 & 一 \\
\hline U-234 & A67 & 2007 & 58.24 & 25.95 & 77.85 & $\mathrm{U}$ \\
\hline U-234 & A68 & 2007 & 45.75 & 2.24 & 6.72 & - \\
\hline U-234 & A69 & 2007 & 35.42 & 29.75 & 89.25 & $\mathrm{U}$ \\
\hline U-234 & A70 & 2007 & 11.38 & 14.55 & 43.65 & $\mathrm{U}$ \\
\hline U-234 & A71 & 2007 & 50.26 & 23.5 & 70.5 & $\mathrm{U}$ \\
\hline U-234 & A72 & 2007 & 21.97 & 16.25 & 48.75 & $\mathrm{U}$ \\
\hline U-234 & $\mathrm{B} 100$ & 2007 & 42.8 & 2.05 & 6.15 & - \\
\hline $\mathrm{U}-234$ & B102 & 2007 & 61.61 & 45.2 & 135.6 & $\mathrm{U}$ \\
\hline $\mathrm{U}-234$ & B103 & 2007 & 48.7 & 44 & 132 & $\mathrm{U}$ \\
\hline U-234 & B104 & 2007 & 39.13 & 27.7 & 83.1 & $\mathrm{U}$ \\
\hline U-234 & B105 & 2007 & 61.67 & 23.1 & 69.3 & $\mathrm{U}$ \\
\hline U-234 & $\mathrm{B} 106$ & 2007 & 146.6 & 4.32 & 12.96 & 一 \\
\hline U-234 & $\mathrm{B} 14$ & 2007 & 57.99 & 25.8 & 77.4 & $\mathrm{U}$ \\
\hline U-234 & B15 & 2007 & 153.9 & 4.41 & 13.23 & - \\
\hline U-234 & B17 & 2007 & 38.36 & 18.6 & 55.8 & $\mathrm{U}$ \\
\hline U-234 & $\mathrm{B} 25$ & 2007 & 43.57 & 32.9 & 98.7 & $\mathrm{U}$ \\
\hline U-234 & B28 & 2007 & 78.53 & 34.05 & 102.15 & $\mathrm{U}$ \\
\hline U-234 & B29 & 2007 & 85.05 & 45.7 & 137.1 & $\mathrm{U}$ \\
\hline U-234 & B3 & 2007 & 44.8 & 25 & 75 & $\mathrm{U}$ \\
\hline U-234 & B36 & 2007 & 669.5 & 9.38 & 28.14 & - \\
\hline U-234 & B37 & 2007 & 251.1 & 5.22 & 15.66 & - \\
\hline U-234 & B39 & 2007 & 49.86 & 2.16 & 6.48 & - \\
\hline U-234 & B40 & 2007 & -61.54 & 37.05 & 111.15 & $\mathrm{U}$ \\
\hline U-234 & B47 & 2007 & 24.6 & 26.2 & 78.6 & $\mathrm{U}$ \\
\hline U-234 & B48 & 2007 & 27.41 & 16.95 & 50.85 & $\mathrm{U}$ \\
\hline U-234 & B50 & 2007 & 66.73 & 31.4 & 94.2 & $\mathrm{U}$ \\
\hline U-234 & B51 & 2007 & 25.41 & 23.45 & 70.35 & $\mathrm{U}$ \\
\hline U-234 & B52 & 2007 & 65.7 & 23 & 69 & $\mathrm{U}$ \\
\hline U-234 & B59 & 2007 & 288.2 & 139 & 417 & $\mathrm{U}$ \\
\hline U-234 & B60 & 2007 & 38.75 & 22.25 & 66.75 & $\mathrm{U}$ \\
\hline U-234 & B61 & 2007 & 21.68 & 16.7 & 50.1 & $\mathrm{U}$ \\
\hline U-234 & B62 & 2007 & 30.02 & 14.55 & 43.65 & $\mathrm{U}$ \\
\hline U-234 & B71 & 2007 & 141.2 & 130.5 & 391.5 & $\mathrm{U}$ \\
\hline U-234 & B73 & 2007 & 32.34 & 17.65 & 52.95 & $\mathrm{U}$ \\
\hline U-234 & B80 & 2007 & 122.4 & 88.5 & 265.5 & $\mathrm{U}$ \\
\hline U-234 & B81 & 2007 & 186.2 & 66 & 198 & $\mathrm{U}$ \\
\hline
\end{tabular}


Table C-3. (continued).

\begin{tabular}{|c|c|c|c|c|c|c|}
\hline Radionuclide & $\begin{array}{c}\text { Sample } \\
\text { Location }\end{array}$ & Date & $\begin{array}{c}\text { Concentrations } \\
(\mathrm{pCi} / \mathrm{g})\end{array}$ & $\begin{array}{l}\text { Uncertainty } \\
(\mathrm{pCi} / \mathrm{g})\end{array}$ & $\begin{array}{c}\mathrm{MDA} \\
(\mathrm{pCi} / \mathrm{g})\end{array}$ & Flag \\
\hline $\mathrm{U}-234$ & B82 & 2007 & 62.02 & 35.35 & 106.05 & $\mathrm{U}$ \\
\hline U-234 & B83 & 2007 & 51.06 & 22.45 & 67.35 & $\mathrm{U}$ \\
\hline U-234 & B84 & 2007 & 48.14 & 2.77 & 8.31 & - \\
\hline U-234 & B88 & 2007 & 31.09 & 19.85 & 59.55 & $\mathrm{U}$ \\
\hline U-234 & B90 & 2007 & 31.06 & 17.6 & 52.8 & $\mathrm{U}$ \\
\hline U-234 & B91 & 2007 & 211.4 & 4.77 & 14.31 & - \\
\hline U-234 & B92 & 2007 & 73.92 & 47.95 & 143.85 & $\mathrm{U}$ \\
\hline U-234 & B93 & 2007 & 26.91 & 28.05 & 84.15 & $\mathrm{U}$ \\
\hline $\mathrm{U}-234$ & B94 & 2007 & 28.54 & 29.15 & 87.45 & $\mathrm{U}$ \\
\hline $\mathrm{U}-234$ & B95 & 2007 & 21.65 & 39.2 & 117.6 & $\mathrm{U}$ \\
\hline U-234 & B99 & 2007 & 67.56 & 23.85 & 71.55 & $\mathrm{U}$ \\
\hline U-234 & $\mathrm{C} 14$ & 2007 & 10.11 & 12.9 & 38.7 & $\mathrm{U}$ \\
\hline U-234 & $\mathrm{C} 15$ & 2007 & 42.23 & 15.45 & 46.35 & $\mathrm{U}$ \\
\hline U-234 & $\mathrm{C} 25$ & 2007 & 119.1 & 3.41 & 10.23 & - \\
\hline U-234 & $\mathrm{C} 36$ & 2007 & 54.47 & 46.15 & 138.45 & $\mathrm{U}$ \\
\hline U-234 & C37 & 2007 & 43.93 & 25.7 & 77.1 & $\mathrm{U}$ \\
\hline U-234 & $\mathrm{C} 46$ & 2007 & 17.89 & 11.95 & 35.85 & $\mathrm{U}$ \\
\hline U-234 & $\mathrm{C} 47$ & 2007 & 18.62 & 18 & 54 & $\mathrm{U}$ \\
\hline U-234 & $\mathrm{C} 48$ & 2007 & 38.4 & 17.85 & 53.55 & $\mathrm{U}$ \\
\hline U-234 & C56 & 2007 & 48.13 & 18.1 & 54.3 & $\mathrm{U}$ \\
\hline U-234 & C57 & 2007 & 11.89 & 10.65 & 31.95 & $\mathrm{U}$ \\
\hline U-234 & D57 & 2007 & 128.6 & 4.74 & 14.22 & - \\
\hline U-234 & D58 & 2007 & 19.2 & 18.3 & 54.9 & $\mathrm{U}$ \\
\hline U-234 & D68 & 2007 & 100.3 & 43.95 & 131.85 & $\mathrm{U}$ \\
\hline U-234 & D69 & 2007 & 37.55 & 21.2 & 63.6 & $\mathrm{U}$ \\
\hline U-234 & D80 & 2007 & 2.228 & 24.55 & 73.65 & $\mathrm{U}$ \\
\hline U-234 & D89 & 2007 & 91.56 & 46.95 & 140.85 & $\mathrm{U}$ \\
\hline U-234 & D90 & 2007 & 57.9 & 26.1 & 78.3 & $\mathrm{U}$ \\
\hline U-234 & D91 & 2007 & 13.79 & 26.5 & 79.5 & $\mathrm{U}$ \\
\hline U-234 & B104 & 2008 & 11.26 & 2.38 & 7.14 & - \\
\hline U-234 & A50 & 2009 & 3.374 & 2.92 & 8.76 & $\mathrm{U}$ \\
\hline U-234 & A56 & 2009 & 52.08 & 4.415 & 13.245 & - \\
\hline U-234 & A61 & 2009 & 6.501 & 2.135 & 6.405 & - \\
\hline U-234 & A27 & 2010 & 31.9 & 2.32 & 6.96 & - \\
\hline U-234 & A28 & 2010 & 11.18 & 2.44 & 7.32 & - \\
\hline U-234 & A38 & 2010 & 38.48 & 2.39 & 7.17 & - \\
\hline U-234 & A39 & 2010 & 10.61 & 2.44 & 7.32 & - \\
\hline
\end{tabular}


Table C-3. (continued).

\begin{tabular}{|c|c|c|c|c|c|c|}
\hline Radionuclide & $\begin{array}{c}\text { Sample } \\
\text { Location }\end{array}$ & Date & $\begin{array}{c}\text { Concentrations } \\
(\mathrm{pCi} / \mathrm{g})\end{array}$ & $\begin{array}{l}\text { Uncertainty } \\
(\mathrm{pCi} / \mathrm{g})\end{array}$ & $\begin{array}{c}\mathrm{MDA} \\
(\mathrm{pCi} / \mathrm{g})\end{array}$ & Flag \\
\hline $\mathrm{U}-234$ & A49 & 2010 & 171 & 4.71 & 14.13 & - \\
\hline U-234 & A50 & 2010 & 30.59 & 2.05 & 6.15 & 一 \\
\hline U-234 & A59 & 2010 & 169.7 & 4.65 & 13.95 & 一 \\
\hline U-234 & A60 & 2010 & 162.2 & 4.51 & 13.53 & - \\
\hline U-234 & A61 & 2010 & 21.53 & 2.01 & 6.03 & - \\
\hline U-234 & A71 & 2010 & 157.1 & 4.45 & 13.35 & - \\
\hline $\mathrm{U}-234$ & A72 & 2010 & 25.97 & 1.98 & 5.94 & - \\
\hline U-234 & A15 & 2011 & 33.04 & 68 & 204 & $\mathrm{U}$ \\
\hline $\mathrm{U}-234$ & A16 & 2011 & -25.38 & 86.2 & 258.6 & $\mathrm{U}$ \\
\hline U-234 & A17 & 2011 & 6.172 & 71.7 & 215.1 & $\mathrm{U}$ \\
\hline U-234 & A27 & 2011 & 51.44 & 88.9 & 266.7 & $\mathrm{U}$ \\
\hline U-234 & A28 & 2011 & 15.65 & 80.9 & 242.7 & $\mathrm{U}$ \\
\hline U-234 & A38 & 2011 & 62.58 & 95.8 & 287.4 & $\mathrm{U}$ \\
\hline U-234 & A39 & 2011 & -69.89 & 88.8 & 266.4 & $\mathrm{U}$ \\
\hline U-234 & $\mathrm{A} 4$ & 2011 & 0.02796 & 80.3 & 240.9 & $\mathrm{U}$ \\
\hline U-234 & A44 & 2011 & -56.46 & 108 & 324 & $\mathrm{U}$ \\
\hline U-234 & A47 & 2011 & 61.19 & 109 & 327 & $\mathrm{U}$ \\
\hline U-234 & A49 & 2011 & -18.9 & 104 & 312 & $\mathrm{U}$ \\
\hline U-234 & A50 & 2011 & -20.64 & 90.1 & 270.3 & $\mathrm{U}$ \\
\hline U-234 & A55 & 2011 & -45.07 & 85 & 255 & $\mathrm{U}$ \\
\hline U-234 & A58 & 2011 & -29.32 & 96.3 & 288.9 & $\mathrm{U}$ \\
\hline U-234 & A59 & 2011 & -9.235 & 102 & 306 & $\mathrm{U}$ \\
\hline U-234 & A6 & 2011 & -32.3 & 78.2 & 234.6 & $\mathrm{U}$ \\
\hline U-234 & A61 & 2011 & -11.62 & 90.1 & 270.3 & $\mathrm{U}$ \\
\hline U-234 & A66 & 2011 & -34.97 & 79 & 237 & $\mathrm{U}$ \\
\hline U-234 & A69 & 2011 & -42.61 & 95.8 & 287.4 & $\mathrm{U}$ \\
\hline U-234 & A70 & 2011 & 24.66 & 95.7 & 287.1 & $\mathrm{U}$ \\
\hline U-234 & A72 & 2011 & -91.68 & 89.4 & 268.2 & $\mathrm{U}$ \\
\hline U-234 & B101 & 2011 & -19.27 & 81 & 243 & $\mathrm{U}$ \\
\hline U-234 & B102 & 2011 & -0.8537 & 92.3 & 276.9 & $\mathrm{U}$ \\
\hline U-234 & B103 & 2011 & -20.79 & 84.7 & 254.1 & $\mathrm{U}$ \\
\hline U-234 & B104 & 2011 & -29.13 & 92.1 & 276.3 & $\mathrm{U}$ \\
\hline U-234 & B106 & 2011 & 4.515 & 80.5 & 241.5 & $\mathrm{U}$ \\
\hline U-234 & B14 & 2011 & -21.67 & 88.3 & 264.9 & $\mathrm{U}$ \\
\hline U-234 & B15 & 2011 & 20.8 & 96.7 & 290.1 & $\mathrm{U}$ \\
\hline U-234 & $\mathrm{B} 17$ & 2011 & -682.8 & 75.3 & 225.9 & $\mathrm{U}$ \\
\hline U-234 & $\mathrm{B} 25$ & 2011 & 9.596 & 105 & 315 & $\mathrm{U}$ \\
\hline
\end{tabular}


Table C-3. (continued).

\begin{tabular}{|c|c|c|c|c|c|c|}
\hline Radionuclide & $\begin{array}{c}\text { Sample } \\
\text { Location }\end{array}$ & Date & $\begin{array}{c}\text { Concentrations } \\
(\mathrm{pCi} / \mathrm{g})\end{array}$ & $\begin{array}{l}\text { Uncertainty } \\
(\mathrm{pCi} / \mathrm{g})\end{array}$ & $\begin{array}{c}\mathrm{MDA} \\
(\mathrm{pCi} / \mathrm{g})\end{array}$ & Flag \\
\hline $\mathrm{U}-234$ & $\mathrm{~B} 28$ & 2011 & -41.74 & 91.5 & 274.5 & $\mathrm{U}$ \\
\hline U-234 & B29 & 2011 & 8.661 & 77.4 & 232.2 & $\mathrm{U}$ \\
\hline U-234 & B3 & 2011 & 18.14 & 95.7 & 287.1 & $\mathrm{U}$ \\
\hline U-234 & B39 & 2011 & -656 & 75.2 & 225.6 & $\mathrm{U}$ \\
\hline U-234 & $\mathrm{B} 4$ & 2011 & -6.493 & 65.9 & 197.7 & $\mathrm{U}$ \\
\hline U-234 & B40 & 2011 & -609.7 & 72.4 & 217.2 & $\mathrm{U}$ \\
\hline U-234 & B47 & 2011 & 5.614 & 131 & 393 & $\mathrm{U}$ \\
\hline U-234 & B48 & 2011 & 4.054 & 93 & 279 & $\mathrm{U}$ \\
\hline $\mathrm{U}-234$ & B50 & 2011 & 45.95 & 90.7 & 272.1 & $\mathrm{U}$ \\
\hline $\mathrm{U}-234$ & B52 & 2011 & 5.59 & 89.6 & 268.8 & $\mathrm{U}$ \\
\hline U-234 & B61 & 2011 & -36.87 & 82.4 & 247.2 & $\mathrm{U}$ \\
\hline U-234 & B62 & 2011 & -82.5 & 64 & 192 & $\mathrm{U}$ \\
\hline U-234 & B71 & 2011 & -92.5 & 125 & 375 & $\mathrm{U}$ \\
\hline U-234 & B73 & 2011 & -33.58 & 82.4 & 247.2 & $\mathrm{U}$ \\
\hline U-234 & $\mathrm{B} 80$ & 2011 & 37.79 & 219 & 657 & $\mathrm{U}$ \\
\hline U-234 & B83 & 2011 & -13.68 & 87.1 & 261.3 & $\mathrm{U}$ \\
\hline U-234 & B84 & 2011 & 3.254 & 81.7 & 245.1 & $\mathrm{U}$ \\
\hline U-234 & B89 & 2011 & -57.88 & 96.2 & 288.6 & $\mathrm{U}$ \\
\hline U-234 & B90 & 2011 & -3.418 & 90.7 & 272.1 & $\mathrm{U}$ \\
\hline U-234 & B91 & 2011 & -6.68 & 111 & 333 & $\mathrm{U}$ \\
\hline U-234 & B92 & 2011 & 0 & 2.5 & 7.5 & $\mathrm{U}$ \\
\hline U-234 & B93 & 2011 & -54.37 & 96.5 & 289.5 & $\mathrm{U}$ \\
\hline U-234 & B94 & 2011 & -74.32 & 93.4 & 280.2 & $\mathrm{U}$ \\
\hline U-234 & B99 & 2011 & -21.49 & 77.1 & 231.3 & $\mathrm{U}$ \\
\hline U-234 & $\mathrm{C} 15$ & 2011 & -30.29 & 77.2 & 231.6 & $\mathrm{U}$ \\
\hline U-234 & $\mathrm{C} 25$ & 2011 & 0 & 2.5 & 7.5 & $\mathrm{U}$ \\
\hline U-234 & C37 & 2011 & 24.59 & 76 & 228 & $\mathrm{U}$ \\
\hline U-234 & $\mathrm{C} 46$ & 2011 & 51.02 & 78.8 & 236.4 & $\mathrm{U}$ \\
\hline U-234 & $\mathrm{C} 48$ & 2011 & -55.83 & 81.2 & 243.6 & $\mathrm{U}$ \\
\hline U-234 & C56 & 2011 & 4.68 & 79.7 & 239.1 & $\mathrm{U}$ \\
\hline U-234 & C57 & 2011 & -61.33 & 76.1 & 228.3 & $\mathrm{U}$ \\
\hline U-234 & D58 & 2011 & 81.29 & 86.5 & 259.5 & $\mathrm{U}$ \\
\hline U-234 & D68 & 2011 & 6.438 & 84.3 & 252.9 & $\mathrm{U}$ \\
\hline U-234 & D89 & 2011 & -57.67 & 81.6 & 244.8 & $\mathrm{U}$ \\
\hline U-234 & D91 & 2011 & 2.935 & 71.1 & 213.3 & $\mathrm{U}$ \\
\hline U-234 & A15 & 2012 & 2.155 & 28 & 84 & $\mathrm{U}$ \\
\hline U-234 & A38 & 2012 & 13.12 & 30 & 90 & $\mathrm{U}$ \\
\hline
\end{tabular}


Table C-3. (continued).

\begin{tabular}{|c|c|c|c|c|c|c|}
\hline Radionuclide & $\begin{array}{l}\text { Sample } \\
\text { Location }\end{array}$ & Date & $\begin{array}{c}\text { Concentrations } \\
(\mathrm{pCi} / \mathrm{g})\end{array}$ & $\begin{array}{l}\text { Uncertainty } \\
(\mathrm{pCi} / \mathrm{g})\end{array}$ & $\begin{array}{c}\mathrm{MDA} \\
(\mathrm{pCi} / \mathrm{g})\end{array}$ & Flag \\
\hline U-234 & A44 & 2012 & 5.236 & 37.5 & 112.5 & $\mathrm{U}$ \\
\hline U-234 & A47 & 2012 & 7.307 & 33.8 & 101.4 & $\mathrm{U}$ \\
\hline $\mathrm{U}-234$ & A59 & 2012 & -6.879 & 27.4 & 82.2 & $\mathrm{U}$ \\
\hline U-234 & A6 & 2012 & 8.129 & 29.5 & 88.5 & $\mathrm{U}$ \\
\hline U-234 & A69 & 2012 & -6.341 & 29.5 & 88.5 & $\mathrm{U}$ \\
\hline $\mathrm{U}-234$ & A70 & 2012 & 15.81 & 26.2 & 78.6 & $\mathrm{U}$ \\
\hline U-234 & A72 & 2012 & 3.75 & 19.2 & 57.6 & $\mathrm{U}$ \\
\hline U-234 & $\mathrm{B} 14$ & 2012 & 11.94 & 19.1 & 57.3 & $\mathrm{U}$ \\
\hline U-234 & B48 & 2012 & 11.9 & 37.7 & 113.1 & $\mathrm{U}$ \\
\hline U-234 & B83 & 2012 & 17.59 & 27 & 81 & $\mathrm{U}$ \\
\hline U-234 & $\mathrm{C} 46$ & 2012 & 29.36 & 31.4 & 94.2 & $\mathrm{U}$ \\
\hline U-234 & C57 & 2012 & 23.36 & 28.5 & 85.5 & $\mathrm{U}$ \\
\hline U-234 & D68 & 2012 & -11.79 & 141 & 423 & $\mathrm{U}$ \\
\hline U-234 & $\begin{array}{l}2 \text { INCH AIR } \\
\text { - INTEC }\end{array}$ & 2013 & 31.99 & 46.33 & 139 & $\mathrm{U}$ \\
\hline U-234 & A47 & 2013 & 30.39 & 53 & 159 & $\mathrm{U}$ \\
\hline U-234 & A49 & 2013 & -6.576 & 50 & 150 & $\mathrm{U}$ \\
\hline U-234 & A59 & 2013 & 38.79 & 49.67 & 149 & $\mathrm{U}$ \\
\hline U-234 & A71 & 2013 & 63.39 & 46.67 & 140 & $\mathrm{U}$ \\
\hline $\mathrm{U}-234$ & A72 & 2013 & -42.7 & 49.67 & 149 & $\mathrm{U}$ \\
\hline U-234 & $\begin{array}{c}2 \text { INCH AIR } \\
- \text { CPP }\end{array}$ & 2014 & -0.6581 & 92.3 & 276.9 & $\mathrm{U}$ \\
\hline U-234 & $\begin{array}{c}2 \text { INCH AIR } \\
\text { - INTEC }\end{array}$ & 2014 & -19.62 & 66.2 & 198.6 & $\mathrm{U}$ \\
\hline $\mathrm{U}-234$ & $\mathrm{~A} 38$ & 2014 & -23.89 & 54 & 162 & $\mathrm{U}$ \\
\hline U-234 & A47 & 2014 & -99.97 & 86.6 & 259.8 & $\mathrm{U}$ \\
\hline U-234 & A58 & 2014 & 1.449 & 64.8 & 194.4 & $\mathrm{U}$ \\
\hline U-234 & A59 & 2014 & -24.39 & 70.2 & 210.6 & $\mathrm{U}$ \\
\hline U-234 & $\mathrm{B} 15$ & 2014 & -34.51 & 127 & 381 & $\mathrm{U}$ \\
\hline U-234 & $\mathrm{B} 28$ & 2014 & -82.15 & 80.1 & 240.3 & $\mathrm{U}$ \\
\hline U-234 & B29 & 2014 & -66.69 & 81.6 & 244.8 & $\mathrm{U}$ \\
\hline U-234 & B3 & 2014 & 2.907 & 56.4 & 169.2 & $\mathrm{U}$ \\
\hline U-234 & B3 & 2014 & 1.222 & 128 & 384 & $\mathrm{U}$ \\
\hline U-234 & B4 & 2014 & -66.66 & 59.3 & 177.9 & $\mathrm{U}$ \\
\hline U-234 & B40 & 2014 & -16.41 & 48.6 & 145.8 & $\mathrm{U}$ \\
\hline U-234 & $\mathrm{B} 50$ & 2014 & -56.98 & 72.4 & 217.2 & $\mathrm{U}$ \\
\hline U-234 & B52 & 2014 & 44.45 & 101 & 303 & $\mathrm{U}$ \\
\hline U-234 & B52 & 2014 & -2.955 & 81.4 & 244.2 & $\mathrm{U}$ \\
\hline
\end{tabular}


Table C-3. (continued).

\begin{tabular}{|c|c|c|c|c|c|c|}
\hline Radionuclide & $\begin{array}{c}\text { Sample } \\
\text { Location }\end{array}$ & Date & $\begin{array}{c}\text { Concentrations } \\
(\mathrm{pCi} / \mathrm{g})\end{array}$ & $\begin{array}{l}\text { Uncertainty } \\
(\mathrm{pCi} / \mathrm{g})\end{array}$ & $\begin{array}{c}\mathrm{MDA} \\
(\mathrm{pCi} / \mathrm{g})\end{array}$ & Flag \\
\hline U-235 & A-17 & 1982 & 0.047 & 0.004 & 0.012 & - \\
\hline U-235 & A-48 & 1982 & 0.045 & 0.004 & 0.012 & 一 \\
\hline $\mathrm{U}-235$ & B-27 & 1982 & 0.044 & 0.004 & 0.012 & - \\
\hline $\mathrm{U}-235$ & B-48 & 1982 & 0.052 & 0.004 & 0.012 & - \\
\hline $\mathrm{U}-235$ & D-57 & 1982 & 0.05 & 0.004 & 0.012 & - \\
\hline U-235 & A15 & 2006 & 0.6971 & 0.3185 & 0.9555 & $\mathrm{U}$ \\
\hline U-235 & A16 & 2006 & 0.748 & 0.331 & 0.993 & $\mathrm{U}$ \\
\hline $\mathrm{U}-235$ & A17 & 2006 & 0.7792 & 0.29 & 0.87 & $\mathrm{U}$ \\
\hline $\mathrm{U}-235$ & $\mathrm{~A} 27$ & 2006 & 3.598 & 1.045 & 3.135 & \\
\hline U-235 & A38 & 2006 & 1.245 & 0.4905 & 1.4715 & $\mathrm{U}$ \\
\hline $\mathrm{U}-235$ & A39 & 2006 & 0.6763 & 0.256 & 0.768 & $\mathrm{U}$ \\
\hline U-235 & $\mathrm{A} 4$ & 2006 & 1.042 & 0.321 & 0.963 & - \\
\hline U-235 & A44 & 2006 & 3.213 & 0.67 & 2.01 & - \\
\hline U-235 & A45 & 2006 & 2.627 & 0.58 & 1.74 & - \\
\hline U-235 & A47 & 2006 & 0.5419 & 0.277 & 0.831 & $\mathrm{U}$ \\
\hline U-235 & A48 & 2006 & 0.5509 & 0.505 & 1.515 & $\mathrm{U}$ \\
\hline U-235 & A49 & 2006 & 0.7308 & 0.3245 & 0.9735 & $\mathrm{U}$ \\
\hline U-235 & $\mathrm{A} 5$ & 2006 & 1.809 & 0.4705 & 1.4115 & \\
\hline U-235 & A50 & 2006 & 0.667 & 0.3075 & 0.9225 & $\mathrm{U}$ \\
\hline U-235 & A55 & 2006 & 2.046 & 0.965 & 2.895 & $\mathrm{U}$ \\
\hline U-235 & A56 & 2006 & 0.6461 & 0.201 & 0.603 & - \\
\hline U-235 & A58 & 2006 & 0.5763 & 0.321 & 0.963 & $\mathrm{U}$ \\
\hline U-235 & A59 & 2006 & 1.293 & 0.77 & 2.31 & $\mathrm{U}$ \\
\hline U-235 & A6 & 2006 & 1.539 & 0.725 & 2.175 & $\mathrm{U}$ \\
\hline U-235 & A60 & 2006 & 0.5229 & 0.273 & 0.819 & $\mathrm{U}$ \\
\hline U-235 & A61 & 2006 & 0.7248 & 0.296 & 0.888 & $\mathrm{U}$ \\
\hline U-235 & A66 & 2006 & 1.076 & 0.309 & 0.927 & - \\
\hline U-235 & A67 & 2006 & 0.06303 & 0.252 & 0.756 & $\mathrm{U}$ \\
\hline U-235 & A68 & 2006 & 1.259 & 0.3695 & 1.1085 & - \\
\hline U-235 & A69 & 2006 & 1.874 & 0.4975 & 1.4925 & 一 \\
\hline U-235 & A70 & 2006 & 1.099 & 0.32 & 0.96 & - \\
\hline U-235 & A71 & 2006 & 0.7007 & 0.222 & 0.666 & - \\
\hline U-235 & A72 & 2006 & 2.065 & 0.74 & 2.22 & $\mathrm{U}$ \\
\hline U-235 & $\mathrm{B} 100$ & 2006 & 0.9297 & 0.55 & 1.65 & $\mathrm{U}$ \\
\hline U-235 & B101 & 2006 & 0.7183 & 0.343 & 1.029 & $\mathrm{U}$ \\
\hline U-235 & B102 & 2006 & 0.6917 & 0.2355 & 0.7065 & $\mathrm{U}$ \\
\hline U-235 & B103 & 2006 & 0.6311 & 0.205 & 0.615 & - \\
\hline
\end{tabular}


Table C-3. (continued).

\begin{tabular}{|c|c|c|c|c|c|c|}
\hline Radionuclide & $\begin{array}{c}\text { Sample } \\
\text { Location }\end{array}$ & Date & $\begin{array}{l}\text { Concentrations } \\
(\mathrm{pCi} / \mathrm{g})\end{array}$ & $\begin{array}{l}\text { Uncertainty } \\
(\mathrm{pCi} / \mathrm{g})\end{array}$ & $\begin{array}{c}\mathrm{MDA} \\
(\mathrm{pCi} / \mathrm{g})\end{array}$ & Flag \\
\hline U-235 & B104 & 2006 & 2.334 & 0.835 & 2.505 & $\mathrm{U}$ \\
\hline $\mathrm{U}-235$ & B105 & 2006 & 2.533 & 0.845 & 2.535 & $\mathrm{U}$ \\
\hline $\mathrm{U}-235$ & B106 & 2006 & 1.787 & 0.785 & 2.355 & $\mathrm{U}$ \\
\hline $\mathrm{U}-235$ & $\mathrm{~B} 14$ & 2006 & 0.3772 & 0.286 & 0.858 & $\mathrm{U}$ \\
\hline $\mathrm{U}-235$ & $\mathrm{~B} 15$ & 2006 & 0.8869 & 0.333 & 0.999 & $\mathrm{U}$ \\
\hline U-235 & $\mathrm{B} 17$ & 2006 & 0.4303 & 0.197 & 0.591 & $\mathrm{U}$ \\
\hline U-235 & B25 & 2006 & 0.8632 & 0.381 & 1.143 & $\mathrm{U}$ \\
\hline $\mathrm{U}-235$ & B26 & 2006 & 1.622 & 0.7 & 2.1 & $\mathrm{U}$ \\
\hline $\mathrm{U}-235$ & B28 & 2006 & 0.612 & 0.24 & 0.72 & $\mathrm{U}$ \\
\hline U-235 & B29 & 2006 & 0.2968 & 0.1755 & 0.5265 & $\mathrm{U}$ \\
\hline U-235 & B3 & 2006 & 0.3781 & 0.1715 & 0.5145 & $\mathrm{U}$ \\
\hline U-235 & $\mathrm{B} 37$ & 2006 & 0.9305 & 0.3165 & 0.9495 & $\mathrm{U}$ \\
\hline $\mathrm{U}-235$ & B39 & 2006 & 0.6913 & 0.431 & 1.293 & $\mathrm{U}$ \\
\hline $\mathrm{U}-235$ & B4 & 2006 & 0.7949 & 0.2965 & 0.8895 & $\mathrm{U}$ \\
\hline U-235 & B40 & 2006 & 0.8485 & 0.451 & 1.353 & $\mathrm{U}$ \\
\hline U-235 & B47 & 2006 & 0.5313 & 0.3935 & 1.1805 & $\mathrm{U}$ \\
\hline $\mathrm{U}-235$ & B48 & 2006 & 0.5135 & 0.269 & 0.807 & $\mathrm{U}$ \\
\hline U-235 & B50 & 2006 & 0.8828 & 0.267 & 0.801 & - \\
\hline U-235 & B51 & 2006 & 2.585 & 0.83 & 2.49 & - \\
\hline U-235 & B52 & 2006 & 0.3783 & 0.2225 & 0.6675 & $\mathrm{U}$ \\
\hline U-235 & B59 & 2006 & 1.38 & 0.625 & 1.875 & $\mathrm{U}$ \\
\hline U-235 & B60 & 2006 & 2.056 & 0.81 & 2.43 & $\mathrm{U}$ \\
\hline U-235 & B61 & 2006 & 0.7765 & 0.274 & 0.822 & $\mathrm{U}$ \\
\hline U-235 & B62 & 2006 & 2.087 & 0.615 & 1.845 & - \\
\hline U-235 & B68 & 2006 & 0.8642 & 0.269 & 0.807 & - \\
\hline U-235 & B71 & 2006 & 0.6706 & 0.2795 & 0.8385 & $\mathrm{U}$ \\
\hline U-235 & B73 & 2006 & 0.908 & 0.282 & 0.846 & - \\
\hline U-235 & B80 & 2006 & 0.9926 & 0.2585 & 0.7755 & - \\
\hline U-235 & B82 & 2006 & 0.542 & 0.213 & 0.639 & $\mathrm{U}$ \\
\hline U-235 & B83 & 2006 & 0.4498 & 0.1935 & 0.5805 & $\mathrm{U}$ \\
\hline U-235 & B84 & 2006 & 1.267 & 0.44 & 1.32 & $\mathrm{U}$ \\
\hline U-235 & B88 & 2006 & 0.6823 & 0.2345 & 0.7035 & $\mathrm{U}$ \\
\hline U-235 & B89 & 2006 & 0.9346 & 0.2255 & 0.6765 & - \\
\hline U-235 & B90 & 2006 & 0.9743 & 0.2615 & 0.7845 & - \\
\hline U-235 & B91 & 2006 & 0.7511 & 0.2425 & 0.7275 & - \\
\hline U-235 & B92 & 2006 & 0.3874 & 0.1385 & 0.4155 & $\mathrm{U}$ \\
\hline U-235 & B93 & 2006 & 0.3805 & 0.309 & 0.927 & $\mathrm{U}$ \\
\hline
\end{tabular}


Table C-3. (continued).

\begin{tabular}{|c|c|c|c|c|c|c|}
\hline Radionuclide & $\begin{array}{c}\text { Sample } \\
\text { Location }\end{array}$ & Date & $\begin{array}{c}\text { Concentrations } \\
(\mathrm{pCi} / \mathrm{g})\end{array}$ & $\begin{array}{l}\text { Uncertainty } \\
(\mathrm{pCi} / \mathrm{g})\end{array}$ & $\begin{array}{c}\mathrm{MDA} \\
(\mathrm{pCi} / \mathrm{g})\end{array}$ & Flag \\
\hline U-235 & B94 & 2006 & 0.5707 & 0.157 & 0.471 & 一 \\
\hline U-235 & B95 & 2006 & 0.873 & 0.478 & 1.434 & $\mathrm{U}$ \\
\hline U-235 & B99 & 2006 & 1.54 & 0.625 & 1.875 & $\mathrm{U}$ \\
\hline U-235 & $\mathrm{C} 14$ & 2006 & 1.46 & 0.419 & 1.257 & - \\
\hline U-235 & $\mathrm{C} 15$ & 2006 & 0.6277 & 0.237 & 0.711 & $\mathrm{U}$ \\
\hline U-235 & $\mathrm{C} 25$ & 2006 & 0.8578 & 0.239 & 0.717 & - \\
\hline U-235 & $\mathrm{C} 36$ & 2006 & 0.5823 & 0.274 & 0.822 & $\mathrm{U}$ \\
\hline $\mathrm{U}-235$ & C37 & 2006 & 0.5325 & 0.29 & 0.87 & $\mathrm{U}$ \\
\hline $\mathrm{U}-235$ & $\mathrm{C} 46$ & 2006 & 0.2893 & 0.1355 & 0.4065 & $\mathrm{U}$ \\
\hline $\mathrm{U}-235$ & $\mathrm{C} 47$ & 2006 & 0.9989 & 0.2975 & 0.8925 & - \\
\hline U-235 & $\mathrm{C} 48$ & 2006 & 1.941 & 0.3825 & 1.1475 & - \\
\hline U-235 & C56 & 2006 & 0.6166 & 0.2285 & 0.6855 & $\mathrm{U}$ \\
\hline U-235 & C57 & 2006 & 0.1177 & 0.1885 & 0.5655 & $\mathrm{U}$ \\
\hline U-235 & D57 & 2006 & 0.4149 & 0.1645 & 0.4935 & $\mathrm{U}$ \\
\hline U-235 & D58 & 2006 & 0.149 & 0.2945 & 0.8835 & $\mathrm{U}$ \\
\hline U-235 & D68 & 2006 & 0.3785 & 0.149 & 0.447 & $\mathrm{U}$ \\
\hline $\mathrm{U}-235$ & D79 & 2006 & 0.5198 & 0.271 & 0.813 & $\mathrm{U}$ \\
\hline U-235 & D89 & 2006 & 0.3561 & 0.223 & 0.669 & $\mathrm{U}$ \\
\hline U-235 & A15 & 2007 & 0.1771 & 0.1005 & 0.3015 & $\mathrm{U}$ \\
\hline U-235 & A16 & 2007 & 0.4949 & 0.089 & 0.267 & - \\
\hline U-235 & A17 & 2007 & 0.3208 & 0.065 & 0.195 & - \\
\hline U-235 & A27 & 2007 & 0.7617 & 0.1035 & 0.3105 & - \\
\hline U-235 & A28 & 2007 & 0.1407 & 0.0655 & 0.1965 & $\mathrm{U}$ \\
\hline U-235 & A37 & 2007 & 0.5521 & 0.095 & 0.285 & - \\
\hline U-235 & A38 & 2007 & 0.519 & 0.099 & 0.297 & - \\
\hline U-235 & A39 & 2007 & 2.445 & 0.775 & 2.325 & - \\
\hline U-235 & $\mathrm{A} 4$ & 2007 & 0.367 & 0.0635 & 0.1905 & - \\
\hline U-235 & A44 & 2007 & 0.446 & 0.089 & 0.267 & - \\
\hline U-235 & A45 & 2007 & 0.4142 & 0.107 & 0.321 & - \\
\hline U-235 & A47 & 2007 & 0.2877 & 0.078 & 0.234 & - \\
\hline U-235 & A48 & 2007 & 0.4143 & 0.0825 & 0.2475 & - \\
\hline U-235 & A49 & 2007 & 0.5015 & 0.1215 & 0.3645 & - \\
\hline U-235 & A5 & 2007 & 0.2732 & 0.0585 & 0.1755 & - \\
\hline U-235 & A50 & 2007 & 0.7695 & 0.1205 & 0.3615 & - \\
\hline U-235 & A55 & 2007 & 0.338 & 0.0765 & 0.2295 & - \\
\hline U-235 & A56 & 2007 & 0.2126 & 0.0745 & 0.2235 & $\mathrm{U}$ \\
\hline U-235 & A58 & 2007 & 0.4495 & 0.084 & 0.252 & - \\
\hline
\end{tabular}


Table C-3. (continued).

\begin{tabular}{|c|c|c|c|c|c|c|}
\hline Radionuclide & $\begin{array}{c}\text { Sample } \\
\text { Location }\end{array}$ & Date & $\begin{array}{c}\text { Concentrations } \\
(\mathrm{pCi} / \mathrm{g})\end{array}$ & $\begin{array}{l}\text { Uncertainty } \\
(\mathrm{pCi} / \mathrm{g})\end{array}$ & $\begin{array}{c}\mathrm{MDA} \\
(\mathrm{pCi} / \mathrm{g})\end{array}$ & Flag \\
\hline U-235 & A59 & 2007 & 0.4933 & 0.071 & 0.213 & - \\
\hline U-235 & A6 & 2007 & 0.3269 & 0.0935 & 0.2805 & 一 \\
\hline U-235 & A60 & 2007 & 0.395 & 0.092 & 0.276 & 一 \\
\hline U-235 & A61 & 2007 & 0.3648 & 0.076 & 0.228 & - \\
\hline U-235 & A66 & 2007 & 0.6074 & 0.126 & 0.378 & - \\
\hline U-235 & A67 & 2007 & 0.4883 & 0.0825 & 0.2475 & - \\
\hline $\mathrm{U}-235$ & A68 & 2007 & 0.2115 & 0.0561 & 0.1683 & - \\
\hline $\mathrm{U}-235$ & A69 & 2007 & 0.5061 & 0.0905 & 0.2715 & - \\
\hline $\mathrm{U}-235$ & A70 & 2007 & 0.3957 & 0.0885 & 0.2655 & - \\
\hline $\mathrm{U}-235$ & A71 & 2007 & 0.3662 & 0.06 & 0.18 & - \\
\hline $\mathrm{U}-235$ & A72 & 2007 & 0.5647 & 0.0945 & 0.2835 & - \\
\hline $\mathrm{U}-235$ & $\mathrm{~B} 100$ & 2007 & 0.4348 & 0.119 & 0.357 & - \\
\hline $\mathrm{U}-235$ & B101 & 2007 & 0.5545 & 0.0985 & 0.2955 & - \\
\hline $\mathrm{U}-235$ & B102 & 2007 & 0.2766 & 0.0765 & 0.2295 & - \\
\hline $\mathrm{U}-235$ & B103 & 2007 & 0.3226 & 0.0615 & 0.1845 & - \\
\hline $\mathrm{U}-235$ & B104 & 2007 & 0.2852 & 0.0675 & 0.2025 & - \\
\hline $\mathrm{U}-235$ & $\mathrm{~B} 105$ & 2007 & 0.4564 & 0.078 & 0.234 & - \\
\hline U-235 & B106 & 2007 & 0.2984 & 0.0902 & 0.2706 & - \\
\hline U-235 & B14 & 2007 & 0.3958 & 0.0805 & 0.2415 & - \\
\hline U-235 & B15 & 2007 & 0.08805 & 0.0863 & 0.2589 & $\mathrm{U}$ \\
\hline $\mathrm{U}-235$ & B17 & 2007 & 0.4698 & 0.1185 & 0.3555 & - \\
\hline U-235 & $\mathrm{B} 25$ & 2007 & 0.6958 & 0.1205 & 0.3615 & - \\
\hline U-235 & $\mathrm{B} 26$ & 2007 & 0.3198 & 0.0685 & 0.2055 & - \\
\hline U-235 & $\mathrm{B} 28$ & 2007 & 0.4174 & 0.082 & 0.246 & - \\
\hline U-235 & B29 & 2007 & 0.395 & 0.0665 & 0.1995 & - \\
\hline U-235 & B3 & 2007 & 0.2994 & 0.067 & 0.201 & 一 \\
\hline U-235 & B36 & 2007 & 0.1974 & 0.134 & 0.402 & $\mathrm{U}$ \\
\hline U-235 & B37 & 2007 & 0.1534 & 0.104 & 0.312 & $\mathrm{U}$ \\
\hline U-235 & B39 & 2007 & 0.5098 & 0.105 & 0.315 & - \\
\hline U-235 & B4 & 2007 & 0.3311 & 0.0635 & 0.1905 & - \\
\hline U-235 & B40 & 2007 & 0.2605 & 0.0725 & 0.2175 & - \\
\hline $\mathrm{U}-235$ & B47 & 2007 & 0.2554 & 0.105 & 0.315 & $\mathrm{U}$ \\
\hline $\mathrm{U}-235$ & B48 & 2007 & 0.4649 & 0.1005 & 0.3015 & - \\
\hline U-235 & B50 & 2007 & 0.4151 & 0.085 & 0.255 & - \\
\hline U-235 & B51 & 2007 & 0.3564 & 0.0915 & 0.2745 & - \\
\hline U-235 & B52 & 2007 & 0.3965 & 0.0825 & 0.2475 & - \\
\hline U-235 & B59 & 2007 & 0.7715 & 0.193 & 0.579 & - \\
\hline
\end{tabular}


Table C-3. (continued).

\begin{tabular}{|c|c|c|c|c|c|c|}
\hline Radionuclide & $\begin{array}{c}\text { Sample } \\
\text { Location }\end{array}$ & Date & $\begin{array}{c}\text { Concentrations } \\
(\mathrm{pCi} / \mathrm{g})\end{array}$ & $\begin{array}{l}\text { Uncertainty } \\
(\mathrm{pCi} / \mathrm{g})\end{array}$ & $\begin{array}{c}\mathrm{MDA} \\
(\mathrm{pCi} / \mathrm{g})\end{array}$ & Flag \\
\hline U-235 & B60 & 2007 & 0.3831 & 0.1355 & 0.4065 & $\mathrm{U}$ \\
\hline U-235 & B61 & 2007 & 0.335 & 0.069 & 0.207 & 一 \\
\hline U-235 & B62 & 2007 & 0.3771 & 0.076 & 0.228 & 一 \\
\hline U-235 & B69 & 2007 & 2.13 & 0.59 & 1.77 & - \\
\hline U-235 & B71 & 2007 & 1.155 & 0.306 & 0.918 & - \\
\hline U-235 & B73 & 2007 & 0.5803 & 0.0965 & 0.2895 & - \\
\hline U-235 & $\mathrm{B} 80$ & 2007 & 0.6156 & 0.188 & 0.564 & 一 \\
\hline $\mathrm{U}-235$ & B81 & 2007 & 0.8973 & 0.158 & 0.474 & - \\
\hline $\mathrm{U}-235$ & B82 & 2007 & 0.4686 & 0.112 & 0.336 & - \\
\hline $\mathrm{U}-235$ & B83 & 2007 & 0.478 & 0.0835 & 0.2505 & - \\
\hline $\mathrm{U}-235$ & B84 & 2007 & 0.3433 & 0.105 & 0.315 & - \\
\hline $\mathrm{U}-235$ & B88 & 2007 & 0.334 & 0.0885 & 0.2655 & - \\
\hline $\mathrm{U}-235$ & B89 & 2007 & 0.3594 & 0.063 & 0.189 & - \\
\hline $\mathrm{U}-235$ & B90 & 2007 & 0.847 & 0.1215 & 0.3645 & - \\
\hline $\mathrm{U}-235$ & B91 & 2007 & 0.1925 & 0.0976 & 0.2928 & $\mathrm{U}$ \\
\hline $\mathrm{U}-235$ & B92 & 2007 & 0.4645 & 0.0965 & 0.2895 & - \\
\hline $\mathrm{U}-235$ & B93 & 2007 & 0.2385 & 0.0755 & 0.2265 & - \\
\hline U-235 & B94 & 2007 & 0.4266 & 0.063 & 0.189 & - \\
\hline U-235 & B95 & 2007 & 0.4837 & 0.0735 & 0.2205 & - \\
\hline U-235 & B99 & 2007 & 0.4056 & 0.0615 & 0.1845 & - \\
\hline $\mathrm{U}-235$ & $\mathrm{C} 14$ & 2007 & 0.4413 & 0.0745 & 0.2235 & - \\
\hline U-235 & $\mathrm{C} 15$ & 2007 & 0.4921 & 0.084 & 0.252 & - \\
\hline U-235 & $\mathrm{C} 25$ & 2007 & 0.6816 & 0.0566 & 0.1698 & - \\
\hline U-235 & $\mathrm{C} 36$ & 2007 & 0.3463 & 0.061 & 0.183 & - \\
\hline U-235 & C37 & 2007 & 0.7227 & 0.136 & 0.408 & - \\
\hline U-235 & $\mathrm{C} 46$ & 2007 & 0.4238 & 0.0785 & 0.2355 & - \\
\hline U-235 & $\mathrm{C} 47$ & 2007 & 0.2272 & 0.03915 & 0.11745 & - \\
\hline U-235 & $\mathrm{C} 48$ & 2007 & 0.3434 & 0.061 & 0.183 & - \\
\hline U-235 & C56 & 2007 & 0.5037 & 0.091 & 0.273 & - \\
\hline U-235 & C57 & 2007 & 0.3116 & 0.0825 & 0.2475 & - \\
\hline U-235 & D57 & 2007 & 0.1511 & 0.093 & 0.279 & $\mathrm{U}$ \\
\hline $\mathrm{U}-235$ & D58 & 2007 & 0.2563 & 0.0735 & 0.2205 & - \\
\hline $\mathrm{U}-235$ & D68 & 2007 & 0.4013 & 0.0675 & 0.2025 & - \\
\hline U-235 & D69 & 2007 & 0.4855 & 0.082 & 0.246 & - \\
\hline U-235 & D79 & 2007 & 0.4144 & 0.079 & 0.237 & - \\
\hline U-235 & D80 & 2007 & 0.3145 & 0.051 & 0.153 & - \\
\hline U-235 & D89 & 2007 & 0.274 & 0.061 & 0.183 & - \\
\hline
\end{tabular}


Table C-3. (continued).

\begin{tabular}{|c|c|c|c|c|c|c|}
\hline Radionuclide & $\begin{array}{c}\text { Sample } \\
\text { Location }\end{array}$ & Date & $\begin{array}{c}\text { Concentrations } \\
(\mathrm{pCi} / \mathrm{g})\end{array}$ & $\begin{array}{l}\text { Uncertainty } \\
(\mathrm{pCi} / \mathrm{g})\end{array}$ & $\begin{array}{c}\mathrm{MDA} \\
(\mathrm{pCi} / \mathrm{g})\end{array}$ & Flag \\
\hline U-235 & D90 & 2007 & 0.3755 & 0.0645 & 0.1935 & - \\
\hline U-235 & D91 & 2007 & 0.4974 & 0.09 & 0.27 & 一 \\
\hline U-235 & B104 & 2008 & 0.3771 & 0.0539 & 0.1617 & 一 \\
\hline U-235 & $\mathrm{A} 48$ & 2009 & 0.3171 & 0.0555 & 0.1665 & - \\
\hline U-235 & A56 & 2009 & 0.2614 & 0.0545 & 0.1635 & - \\
\hline U-235 & A6 & 2009 & 0.1757 & 0.0337 & 0.1011 & - \\
\hline $\mathrm{U}-235$ & A61 & 2009 & 0.3313 & 0.0435 & 0.1305 & - \\
\hline $\mathrm{U}-235$ & B60 & 2009 & 0.1477 & 0.11 & 0.33 & $\mathrm{U}$ \\
\hline $\mathrm{U}-235$ & B61 & 2009 & 0.2297 & 0.0464 & 0.1392 & - \\
\hline U-235 & B91 & 2009 & 0.1949 & 0.11 & 0.33 & $\mathrm{U}$ \\
\hline U-235 & B95 & 2009 & 0.1025 & 0.0462 & 0.1386 & $\mathrm{U}$ \\
\hline U-235 & B99 & 2009 & 0.2436 & 0.1455 & 0.4365 & $\mathrm{U}$ \\
\hline U-235 & C57 & 2009 & 0.09418 & 0.0224 & 0.0672 & - \\
\hline U-235 & D69 & 2009 & 0.4454 & 0.3515 & 1.0545 & $\mathrm{U}$ \\
\hline U-235 & A27 & 2010 & 2.251 & 0.461 & 1.383 & 一 \\
\hline U-235 & A28 & 2010 & 0.3919 & 0.0624 & 0.1872 & 一 \\
\hline $\mathrm{U}-235$ & A38 & 2010 & 1.545 & 0.259 & 0.777 & - \\
\hline U-235 & A39 & 2010 & 0.4258 & 0.0629 & 0.1887 & - \\
\hline U-235 & A49 & 2010 & 1.654 & 0.32 & 0.96 & - \\
\hline U-235 & $\mathrm{A} 50$ & 2010 & 1.918 & 0.393 & 1.179 & - \\
\hline U-235 & A59 & 2010 & 1.253 & 0.355 & 1.065 & 一 \\
\hline U-235 & A60 & 2010 & 1.57 & 0.605 & 1.815 & $\mathrm{U}$ \\
\hline U-235 & A61 & 2010 & 0.3067 & 0.0577 & 0.1731 & - \\
\hline U-235 & A71 & 2010 & 1.977 & 0.299 & 0.897 & - \\
\hline U-235 & A72 & 2010 & 0.4088 & 0.0705 & 0.2115 & - \\
\hline U-235 & A15 & 2011 & 0.003743 & 0.152 & 0.456 & $\mathrm{U}$ \\
\hline U-235 & A16 & 2011 & -0.000168 & 0.197 & 0.591 & $\mathrm{U}$ \\
\hline U-235 & A17 & 2011 & -0.1077 & 0.193 & 0.579 & $\mathrm{U}$ \\
\hline U-235 & A27 & 2011 & 0.1241 & 0.204 & 0.612 & $\mathrm{U}$ \\
\hline U-235 & A28 & 2011 & -0.245 & 0.196 & 0.588 & $\mathrm{U}$ \\
\hline U-235 & A38 & 2011 & 0.1106 & 0.221 & 0.663 & $\mathrm{U}$ \\
\hline U-235 & A39 & 2011 & 0.3114 & 0.216 & 0.648 & $\mathrm{U}$ \\
\hline U-235 & A4 & 2011 & 0.1595 & 0.203 & 0.609 & $\mathrm{U}$ \\
\hline U-235 & A44 & 2011 & -0.1187 & 0.247 & 0.741 & $\mathrm{U}$ \\
\hline U-235 & A47 & 2011 & 0.05201 & 0.257 & 0.771 & $\mathrm{U}$ \\
\hline U-235 & A49 & 2011 & -0.07648 & 0.227 & 0.681 & $\mathrm{U}$ \\
\hline U-235 & A50 & 2011 & 0.06955 & 0.228 & 0.684 & $\mathrm{U}$ \\
\hline
\end{tabular}


Table C-3. (continued).

\begin{tabular}{|c|c|c|c|c|c|c|}
\hline Radionuclide & $\begin{array}{c}\text { Sample } \\
\text { Location }\end{array}$ & Date & $\begin{array}{c}\text { Concentrations } \\
(\mathrm{pCi} / \mathrm{g})\end{array}$ & $\begin{array}{l}\text { Uncertainty } \\
(\mathrm{pCi} / \mathrm{g})\end{array}$ & $\begin{array}{c}\mathrm{MDA} \\
(\mathrm{pCi} / \mathrm{g})\end{array}$ & Flag \\
\hline U-235 & A55 & 2011 & 0.1932 & 0.218 & 0.654 & $\mathrm{U}$ \\
\hline U-235 & A58 & 2011 & 0.03477 & 0.221 & 0.663 & $\mathrm{U}$ \\
\hline U-235 & A59 & 2011 & 0.07666 & 0.24 & 0.72 & $\mathrm{U}$ \\
\hline $\mathrm{U}-235$ & A6 & 2011 & 0.01434 & 0.193 & 0.579 & $\mathrm{U}$ \\
\hline U-235 & A61 & 2011 & 0.1075 & 0.205 & 0.615 & $\mathrm{U}$ \\
\hline U-235 & A66 & 2011 & 0.1333 & 0.196 & 0.588 & $\mathrm{U}$ \\
\hline U-235 & A69 & 2011 & 0.08206 & 0.225 & 0.675 & $\mathrm{U}$ \\
\hline $\mathrm{U}-235$ & A70 & 2011 & 0.3643 & 0.223 & 0.669 & $\mathrm{U}$ \\
\hline $\mathrm{U}-235$ & A72 & 2011 & -0.0497 & 0.191 & 0.573 & $\mathrm{U}$ \\
\hline U-235 & B101 & 2011 & -0.1143 & 0.196 & 0.588 & $\mathrm{U}$ \\
\hline U-235 & B102 & 2011 & 0.03978 & 0.199 & 0.597 & $\mathrm{U}$ \\
\hline U-235 & B103 & 2011 & 0.1057 & 0.209 & 0.627 & $\mathrm{U}$ \\
\hline U-235 & B104 & 2011 & 0.01241 & 0.211 & 0.633 & $\mathrm{U}$ \\
\hline U-235 & B106 & 2011 & 0.1003 & 0.197 & 0.591 & $\mathrm{U}$ \\
\hline U-235 & $\mathrm{B} 14$ & 2011 & 0.006411 & 0.219 & 0.657 & $\mathrm{U}$ \\
\hline U-235 & B15 & 2011 & 0.01087 & 0.227 & 0.681 & $\mathrm{U}$ \\
\hline $\mathrm{U}-235$ & $\mathrm{~B} 17$ & 2011 & -1.142 & 0.141 & 0.423 & $\mathrm{U}$ \\
\hline U-235 & $\mathrm{B} 25$ & 2011 & 0.049 & 0.276 & 0.828 & $\mathrm{U}$ \\
\hline U-235 & $\mathrm{B} 28$ & 2011 & 0.2206 & 0.215 & 0.645 & $\mathrm{U}$ \\
\hline U-235 & B29 & 2011 & 0.122 & 0.204 & 0.612 & $\mathrm{U}$ \\
\hline U-235 & B3 & 2011 & -0.06869 & 0.228 & 0.684 & $\mathrm{U}$ \\
\hline U-235 & B39 & 2011 & -0.03755 & 0.151 & 0.453 & $\mathrm{U}$ \\
\hline U-235 & $\mathrm{B} 4$ & 2011 & -0.0266 & 0.199 & 0.597 & $\mathrm{U}$ \\
\hline U-235 & B40 & 2011 & -0.7093 & 0.132 & 0.396 & $\mathrm{U}$ \\
\hline U-235 & B47 & 2011 & -0.123 & 0.321 & 0.963 & $\mathrm{U}$ \\
\hline U-235 & B48 & 2011 & -0.1567 & 0.274 & 0.822 & $\mathrm{U}$ \\
\hline U-235 & B50 & 2011 & 0.03868 & 0.22 & 0.66 & $\mathrm{U}$ \\
\hline U-235 & B52 & 2011 & 0.01064 & 0.21 & 0.63 & $\mathrm{U}$ \\
\hline U-235 & B61 & 2011 & 0.0907 & 0.211 & 0.633 & $\mathrm{U}$ \\
\hline U-235 & B62 & 2011 & -0.1407 & 0.181 & 0.543 & $\mathrm{U}$ \\
\hline U-235 & B71 & 2011 & 0.01922 & 0.273 & 0.819 & $\mathrm{U}$ \\
\hline U-235 & B73 & 2011 & -0.02559 & 0.208 & 0.624 & $\mathrm{U}$ \\
\hline U-235 & $\mathrm{B} 80$ & 2011 & -0.04135 & 0.457 & 1.371 & $\mathrm{U}$ \\
\hline U-235 & B83 & 2011 & 0.05741 & 0.205 & 0.615 & $\mathrm{U}$ \\
\hline U-235 & B84 & 2011 & 0.1127 & 0.193 & 0.579 & $\mathrm{U}$ \\
\hline U-235 & B89 & 2011 & 0.2615 & 0.228 & 0.684 & $\mathrm{U}$ \\
\hline U-235 & B90 & 2011 & 0.02923 & 0.223 & 0.669 & $\mathrm{U}$ \\
\hline
\end{tabular}


Table C-3. (continued).

\begin{tabular}{|c|c|c|c|c|c|c|}
\hline Radionuclide & $\begin{array}{l}\text { Sample } \\
\text { Location }\end{array}$ & Date & $\begin{array}{c}\text { Concentrations } \\
(\mathrm{pCi} / \mathrm{g})\end{array}$ & $\begin{array}{l}\text { Uncertainty } \\
(\mathrm{pCi} / \mathrm{g})\end{array}$ & $\begin{array}{c}\mathrm{MDA} \\
(\mathrm{pCi} / \mathrm{g})\end{array}$ & Flag \\
\hline U-235 & B91 & 2011 & -0.04882 & 0.237 & 0.711 & $\mathrm{U}$ \\
\hline U-235 & B92 & 2011 & 0.1684 & 0.243 & 0.729 & $\mathrm{U}$ \\
\hline U-235 & B93 & 2011 & 0.03608 & 0.207 & 0.621 & $\mathrm{U}$ \\
\hline $\mathrm{U}-235$ & B94 & 2011 & 0.07089 & 0.211 & 0.633 & $\mathrm{U}$ \\
\hline U-235 & B99 & 2011 & -0.09455 & 0.176 & 0.528 & $\mathrm{U}$ \\
\hline $\mathrm{U}-235$ & $\mathrm{C} 15$ & 2011 & 0.003122 & 0.197 & 0.591 & $\mathrm{U}$ \\
\hline U-235 & $\mathrm{C} 25$ & 2011 & -0.07595 & 0.17 & 0.51 & $\mathrm{U}$ \\
\hline U-235 & C37 & 2011 & -0.1713 & 0.19 & 0.57 & $\mathrm{U}$ \\
\hline U-235 & $\mathrm{C} 46$ & 2011 & 0.05409 & 0.2 & 0.6 & $\mathrm{U}$ \\
\hline $\mathrm{U}-235$ & $\mathrm{C} 48$ & 2011 & 0.0219 & 0.182 & 0.546 & $\mathrm{U}$ \\
\hline $\mathrm{U}-235$ & C56 & 2011 & 0.123 & 0.203 & 0.609 & $\mathrm{U}$ \\
\hline U-235 & C57 & 2011 & -0.1136 & 0.194 & 0.582 & $\mathrm{U}$ \\
\hline $\mathrm{U}-235$ & D58 & 2011 & 0.1753 & 0.209 & 0.627 & $\mathrm{U}$ \\
\hline $\mathrm{U}-235$ & D68 & 2011 & 0.2098 & 0.215 & 0.645 & $\mathrm{U}$ \\
\hline $\mathrm{U}-235$ & D89 & 2011 & -0.1083 & 0.201 & 0.603 & $\mathrm{U}$ \\
\hline $\mathrm{U}-235$ & D91 & 2011 & 0.03054 & 0.196 & 0.588 & $\mathrm{U}$ \\
\hline $\mathrm{U}-235$ & A 15 & 2012 & -0.2335 & 0.181 & 0.543 & $\mathrm{U}$ \\
\hline $\mathrm{U}-235$ & A38 & 2012 & 0.08405 & 0.205 & 0.615 & $\mathrm{U}$ \\
\hline U-235 & A44 & 2012 & 0.07117 & 0.212 & 0.636 & $\mathrm{U}$ \\
\hline U-235 & A47 & 2012 & 0.1659 & 0.202 & 0.606 & $\mathrm{U}$ \\
\hline U-235 & A59 & 2012 & 0.1268 & 0.18 & 0.54 & $\mathrm{U}$ \\
\hline U-235 & A6 & 2012 & 0.02305 & 0.197 & 0.591 & $\mathrm{U}$ \\
\hline U-235 & A69 & 2012 & 0.09558 & 0.186 & 0.558 & $\mathrm{U}$ \\
\hline U-235 & A70 & 2012 & -0.07803 & 0.157 & 0.471 & $\mathrm{U}$ \\
\hline U-235 & A72 & 2012 & 0.05924 & 0.172 & 0.516 & $\mathrm{U}$ \\
\hline U-235 & B14 & 2012 & 0.1552 & 0.182 & 0.546 & $\mathrm{U}$ \\
\hline U-235 & B48 & 2012 & -0.1167 & 0.227 & 0.681 & $\mathrm{U}$ \\
\hline U-235 & B83 & 2012 & 0.1371 & 0.172 & 0.516 & $\mathrm{U}$ \\
\hline U-235 & $\mathrm{C} 46$ & 2012 & -0.1986 & 0.185 & 0.555 & $\mathrm{U}$ \\
\hline U-235 & C57 & 2012 & 0.01066 & 0.179 & 0.537 & $\mathrm{U}$ \\
\hline U-235 & $\begin{array}{l}2 \text { INCH AIR } \\
\text { - INTEC }\end{array}$ & 2013 & 0.1353 & 0.0907 & 0.272 & $\mathrm{U}$ \\
\hline U-235 & A47 & 2013 & 0.3093 & 0.0923 & 0.277 & - \\
\hline U-235 & A49 & 2013 & 0.07798 & 0.118 & 0.354 & $\mathrm{U}$ \\
\hline U-235 & A59 & 2013 & 0.1169 & 0.0787 & 0.236 & $\mathrm{U}$ \\
\hline U-235 & A71 & 2013 & 0.226 & 0.097 & 0.291 & $\mathrm{U}$ \\
\hline $\mathrm{U}-235$ & A72 & 2013 & 0.04812 & 0.111 & 0.333 & $\mathrm{U}$ \\
\hline
\end{tabular}


Table C-3. (continued).

\begin{tabular}{|c|c|c|c|c|c|c|}
\hline Radionuclide & $\begin{array}{c}\text { Sample } \\
\text { Location }\end{array}$ & Date & $\begin{array}{c}\text { Concentrations } \\
(\mathrm{pCi} / \mathrm{g})\end{array}$ & $\begin{array}{l}\text { Uncertainty } \\
(\mathrm{pCi} / \mathrm{g})\end{array}$ & $\begin{array}{c}\mathrm{MDA} \\
(\mathrm{pCi} / \mathrm{g})\end{array}$ & Flag \\
\hline U-235 & $\begin{array}{c}2 \text { INCH AIR } \\
- \text { CPP }\end{array}$ & 2014 & 0.1003 & 0.21 & 0.63 & $\mathrm{U}$ \\
\hline $\mathrm{U}-235$ & $\begin{array}{l}2 \text { INCH AIR } \\
\text { - INTEC }\end{array}$ & 2014 & -0.0003003 & 0.157 & 0.471 & $\mathrm{U}$ \\
\hline U-235 & A38 & 2014 & 0.1309 & 0.168 & 0.504 & $\mathrm{U}$ \\
\hline U-235 & A47 & 2014 & 0.04593 & 0.201 & 0.603 & $\mathrm{U}$ \\
\hline U-235 & A58 & 2014 & -0.1163 & 0.16 & 0.48 & $\mathrm{U}$ \\
\hline U-235 & A59 & 2014 & 0.05051 & 0.175 & 0.525 & $\mathrm{U}$ \\
\hline U-235 & $\mathrm{B} 15$ & 2014 & -0.04836 & 0.281 & 0.843 & $\mathrm{U}$ \\
\hline U-235 & $\mathrm{B} 28$ & 2014 & 0.1246 & 0.18 & 0.54 & $\mathrm{U}$ \\
\hline $\mathrm{U}-235$ & B29 & 2014 & -0.2247 & 0.175 & 0.525 & $\mathrm{U}$ \\
\hline U-235 & B3 & 2014 & 0.007732 & 0.142 & 0.426 & $\mathrm{U}$ \\
\hline U-235 & B3 & 2014 & -0.1627 & 0.312 & 0.936 & $\mathrm{U}$ \\
\hline U-235 & B4 & 2014 & 0.007249 & 0.125 & 0.375 & $\mathrm{U}$ \\
\hline U-235 & B40 & 2014 & 0.06826 & 0.127 & 0.381 & $\mathrm{U}$ \\
\hline $\mathrm{U}-235$ & B50 & 2014 & -0.1199 & 0.191 & 0.573 & $\mathrm{U}$ \\
\hline $\mathrm{U}-235$ & B52 & 2014 & 0.06692 & 0.185 & 0.555 & $\mathrm{U}$ \\
\hline $\mathrm{U}-235$ & B52 & 2014 & -0.1665 & 0.23 & 0.69 & $\mathrm{U}$ \\
\hline $\mathrm{U}-238$ & A-17 & 1982 & 1.17 & 0.08 & 0.24 & - \\
\hline $\mathrm{U}-238$ & A-48 & 1982 & 1.07 & 0.03 & 0.09 & - \\
\hline U-238 & B-27 & 1982 & 1.12 & 0.03 & 0.09 & - \\
\hline U-238 & B-48 & 1982 & 1.12 & 0.03 & 0.09 & - \\
\hline U-238 & D-57 & 1982 & 1.06 & 0.03 & 0.09 & - \\
\hline U-238 & A15 & 2006 & 11.2 & 1.35 & 4.05 & - \\
\hline U-238 & A17 & 2006 & 14.85 & 1.705 & 5.115 & - \\
\hline U-238 & A27 & 2006 & 17.18 & 2.515 & 7.545 & - \\
\hline U-238 & A28 & 2006 & 6.302 & 1.685 & 5.055 & - \\
\hline U-238 & A38 & 2006 & 87.87 & 14.55 & 43.65 & - \\
\hline U-238 & A39 & 2006 & 6.107 & 1.195 & 3.585 & - \\
\hline U-238 & A4 & 2006 & 12.46 & 1.89 & 5.67 & - \\
\hline U-238 & A44 & 2006 & 10.58 & 2.59 & 7.77 & - \\
\hline U-238 & A45 & 2006 & 26.42 & 2.92 & 8.76 & - \\
\hline U-238 & A47 & 2006 & 7.643 & 1.125 & 3.375 & - \\
\hline U-238 & A48 & 2006 & 38.71 & 3.505 & 10.515 & - \\
\hline U-238 & A49 & 2006 & 3.597 & 1.12 & 3.36 & - \\
\hline U-238 & A50 & 2006 & 1.685 & 0.55 & 1.65 & - \\
\hline U-238 & A55 & 2006 & 4.132 & 0.885 & 2.655 & - \\
\hline
\end{tabular}


Table C-3. (continued).

\begin{tabular}{|c|c|c|c|c|c|c|}
\hline Radionuclide & $\begin{array}{c}\text { Sample } \\
\text { Location }\end{array}$ & Date & $\begin{array}{c}\text { Concentrations } \\
(\mathrm{pCi} / \mathrm{g})\end{array}$ & $\begin{array}{l}\text { Uncertainty } \\
(\mathrm{pCi} / \mathrm{g})\end{array}$ & $\begin{array}{c}\mathrm{MDA} \\
(\mathrm{pCi} / \mathrm{g})\end{array}$ & Flag \\
\hline $\mathrm{U}-238$ & A56 & 2006 & 6.358 & 1.805 & 5.415 & - \\
\hline $\mathrm{U}-238$ & A58 & 2006 & 28.32 & 3.755 & 11.265 & 一 \\
\hline $\mathrm{U}-238$ & A59 & 2006 & 30.02 & 4.245 & 12.735 & 一 \\
\hline $\mathrm{U}-238$ & A6 & 2006 & 29.2 & 4.57 & 13.71 & - \\
\hline $\mathrm{U}-238$ & A60 & 2006 & 11.13 & 2.97 & 8.91 & - \\
\hline $\mathrm{U}-238$ & A61 & 2006 & 4.273 & 1.13 & 3.39 & - \\
\hline U-238 & A66 & 2006 & 15.72 & 2.855 & 8.565 & - \\
\hline $\mathrm{U}-238$ & A67 & 2006 & 5.861 & 1.35 & 4.05 & - \\
\hline $\mathrm{U}-238$ & A68 & 2006 & 38.7 & 11.25 & 33.75 & - \\
\hline $\mathrm{U}-238$ & A69 & 2006 & 43.49 & 5.65 & 16.95 & - \\
\hline $\mathrm{U}-238$ & A70 & 2006 & 5.237 & 1.24 & 3.72 & - \\
\hline $\mathrm{U}-238$ & A71 & 2006 & 4.416 & 1.11 & 3.33 & - \\
\hline $\mathrm{U}-238$ & A72 & 2006 & 19.04 & 2.58 & 7.74 & - \\
\hline $\mathrm{U}-238$ & B100 & 2006 & 12.48 & 2.665 & 7.995 & - \\
\hline $\mathrm{U}-238$ & B101 & 2006 & 5.334 & 1.56 & 4.68 & - \\
\hline $\mathrm{U}-238$ & B102 & 2006 & 12.93 & 1.455 & 4.365 & - \\
\hline $\mathrm{U}-238$ & $\mathrm{~B} 103$ & 2006 & 3.208 & 0.83 & 2.49 & - \\
\hline U-238 & B104 & 2006 & 31.24 & 3.65 & 10.95 & - \\
\hline U-238 & B105 & 2006 & 30.38 & 3.815 & 11.445 & - \\
\hline U-238 & B106 & 2006 & 9.476 & 2.14 & 6.42 & - \\
\hline U-238 & B14 & 2006 & 10.92 & 2.065 & 6.195 & - \\
\hline U-238 & B15 & 2006 & 3.995 & 1.38 & 4.14 & $\mathrm{U}$ \\
\hline U-238 & B17 & 2006 & 7.839 & 1.615 & 4.845 & - \\
\hline U-238 & B25 & 2006 & 6.298 & 1.565 & 4.695 & - \\
\hline U-238 & B28 & 2006 & 2.588 & 1.02 & 3.06 & $\mathrm{U}$ \\
\hline U-238 & B29 & 2006 & 4.933 & 1.265 & 3.795 & - \\
\hline U-238 & B3 & 2006 & 3.777 & 1.065 & 3.195 & - \\
\hline U-238 & B36 & 2006 & 19.95 & 3.525 & 10.575 & - \\
\hline U-238 & B37 & 2006 & 3.374 & 1.11 & 3.33 & - \\
\hline U-238 & B39 & 2006 & 32.01 & 3.25 & 9.75 & - \\
\hline U-238 & B4 & 2006 & 4.82 & 1.065 & 3.195 & - \\
\hline U-238 & B40 & 2006 & 4.319 & 1.27 & 3.81 & - \\
\hline U-238 & B47 & 2006 & 3.95 & 0.89 & 2.67 & - \\
\hline U-238 & B48 & 2006 & 5.035 & 1.15 & 3.45 & - \\
\hline U-238 & B50 & 2006 & 5.029 & 1.26 & 3.78 & - \\
\hline U-238 & B51 & 2006 & 38.58 & 5.1 & 15.3 & - \\
\hline U-238 & B52 & 2006 & 4.2 & 1.27 & 3.81 & - \\
\hline
\end{tabular}


Table C-3. (continued).

\begin{tabular}{|c|c|c|c|c|c|c|}
\hline Radionuclide & $\begin{array}{c}\text { Sample } \\
\text { Location }\end{array}$ & Date & $\begin{array}{c}\text { Concentrations } \\
(\mathrm{pCi} / \mathrm{g})\end{array}$ & $\begin{array}{l}\text { Uncertainty } \\
(\mathrm{pCi} / \mathrm{g})\end{array}$ & $\begin{array}{c}\mathrm{MDA} \\
(\mathrm{pCi} / \mathrm{g})\end{array}$ & Flag \\
\hline $\mathrm{U}-238$ & B59 & 2006 & 20.11 & 2.74 & 8.22 & - \\
\hline U-238 & B60 & 2006 & 13.78 & 3.065 & 9.195 & 一 \\
\hline $\mathrm{U}-238$ & B61 & 2006 & 3.691 & 1.125 & 3.375 & - \\
\hline $\mathrm{U}-238$ & B62 & 2006 & 22.16 & 3.06 & 9.18 & - \\
\hline $\mathrm{U}-238$ & B68 & 2006 & 16.45 & 2.52 & 7.56 & - \\
\hline $\mathrm{U}-238$ & B71 & 2006 & 5.148 & 1.515 & 4.545 & - \\
\hline $\mathrm{U}-238$ & B73 & 2006 & 4.596 & 1.32 & 3.96 & - \\
\hline $\mathrm{U}-238$ & B80 & 2006 & 6.485 & 1.525 & 4.575 & - \\
\hline $\mathrm{U}-238$ & B81 & 2006 & 21.19 & 2.81 & 8.43 & - \\
\hline U-238 & B83 & 2006 & 1.43 & 0.75 & 2.25 & $\mathrm{U}$ \\
\hline $\mathrm{U}-238$ & B84 & 2006 & 8.415 & 2.225 & 6.675 & 一 \\
\hline $\mathrm{U}-238$ & B88 & 2006 & 7.901 & 1.355 & 4.065 & 一 \\
\hline $\mathrm{U}-238$ & B89 & 2006 & 6.846 & 1.41 & 4.23 & - \\
\hline $\mathrm{U}-238$ & B90 & 2006 & 3.056 & 0.895 & 2.685 & - \\
\hline $\mathrm{U}-238$ & B91 & 2006 & 4.118 & 1.04 & 3.12 & - \\
\hline $\mathrm{U}-238$ & B92 & 2006 & 15.15 & 4.885 & 14.655 & - \\
\hline $\mathrm{U}-238$ & B93 & 2006 & 10.28 & 2.295 & 6.885 & - \\
\hline U-238 & B94 & 2006 & 21.84 & 2.955 & 8.865 & - \\
\hline U-238 & B95 & 2006 & 17.62 & 2.81 & 8.43 & - \\
\hline U-238 & B99 & 2006 & 18.65 & 3.795 & 11.385 & - \\
\hline U-238 & C14 & 2006 & 17.79 & 2.61 & 7.83 & - \\
\hline U-238 & $\mathrm{C} 25$ & 2006 & 6.052 & 1.075 & 3.225 & - \\
\hline U-238 & C36 & 2006 & 2.649 & 0.955 & 2.865 & $\mathrm{U}$ \\
\hline U-238 & C37 & 2006 & 3.558 & 2.34 & 7.02 & $\mathrm{U}$ \\
\hline U-238 & $\mathrm{C} 46$ & 2006 & 7.97 & 1.65 & 4.95 & - \\
\hline U-238 & $\mathrm{C} 47$ & 2006 & 6.358 & 1.68 & 5.04 & - \\
\hline $\mathrm{U}-238$ & $\mathrm{C} 48$ & 2006 & 53.98 & 8.25 & 24.75 & - \\
\hline U-238 & C56 & 2006 & 12.72 & 1.8 & 5.4 & - \\
\hline U-238 & C57 & 2006 & 8.384 & 1.465 & 4.395 & - \\
\hline U-238 & D57 & 2006 & 10.4 & 1.385 & 4.155 & - \\
\hline U-238 & D58 & 2006 & 7 & 1.885 & 5.655 & - \\
\hline U-238 & D68 & 2006 & 6.265 & 1.465 & 4.395 & - \\
\hline U-238 & D69 & 2006 & 5.261 & 17.45 & 52.35 & $\mathrm{U}$ \\
\hline U-238 & D79 & 2006 & 2.224 & 0.835 & 2.505 & $\mathrm{U}$ \\
\hline U-238 & D80 & 2006 & 2.82 & 24.6 & 73.8 & $\mathrm{U}$ \\
\hline U-238 & D89 & 2006 & 3.012 & 1.315 & 3.945 & $\mathrm{U}$ \\
\hline U-238 & D90 & 2006 & 6.38 & 24.2 & 72.6 & $\mathrm{U}$ \\
\hline
\end{tabular}


Table C-3. (continued).

\begin{tabular}{|c|c|c|c|c|c|c|}
\hline Radionuclide & $\begin{array}{c}\text { Sample } \\
\text { Location }\end{array}$ & Date & $\begin{array}{c}\text { Concentrations } \\
(\mathrm{pCi} / \mathrm{g})\end{array}$ & $\begin{array}{l}\text { Uncertainty } \\
(\mathrm{pCi} / \mathrm{g})\end{array}$ & $\begin{array}{c}\mathrm{MDA} \\
(\mathrm{pCi} / \mathrm{g})\end{array}$ & Flag \\
\hline $\mathrm{U}-238$ & D91 & 2006 & 25.1 & 13.5 & 40.5 & $\mathrm{U}$ \\
\hline $\mathrm{U}-238$ & A15 & 2007 & 3.887 & 0.565 & 1.695 & 一 \\
\hline $\mathrm{U}-238$ & A16 & 2007 & 3.09 & 0.635 & 1.905 & 一 \\
\hline $\mathrm{U}-238$ & A17 & 2007 & 2.434 & 0.494 & 1.482 & - \\
\hline $\mathrm{U}-238$ & A27 & 2007 & 4.882 & 1.36 & 4.08 & - \\
\hline $\mathrm{U}-238$ & A28 & 2007 & 2.94 & 1.48 & 4.44 & $\mathrm{U}$ \\
\hline U-238 & A37 & 2007 & 5.6 & 0.68 & 2.04 & 一 \\
\hline $\mathrm{U}-238$ & A38 & 2007 & 5.457 & 0.66 & 1.98 & - \\
\hline $\mathrm{U}-238$ & $\mathrm{~A} 4$ & 2007 & 2.771 & 0.3915 & 1.1745 & - \\
\hline $\mathrm{U}-238$ & A44 & 2007 & 4.353 & 0.54 & 1.62 & - \\
\hline $\mathrm{U}-238$ & A45 & 2007 & 1.9 & 0.53 & 1.59 & - \\
\hline $\mathrm{U}-238$ & A47 & 2007 & 3.026 & 0.565 & 1.695 & - \\
\hline $\mathrm{U}-238$ & A48 & 2007 & 2.119 & 0.56 & 1.68 & - \\
\hline $\mathrm{U}-238$ & A49 & 2007 & 1.806 & 0.49 & 1.47 & - \\
\hline $\mathrm{U}-238$ & A5 & 2007 & 2.967 & 0.52 & 1.56 & - \\
\hline $\mathrm{U}-238$ & A50 & 2007 & 10.12 & 1.795 & 5.385 & - \\
\hline $\mathrm{U}-238$ & A55 & 2007 & 6.125 & 1.25 & 3.75 & - \\
\hline U-238 & A56 & 2007 & 196.5 & 6.81 & 20.43 & - \\
\hline U-238 & A58 & 2007 & 1.842 & 0.53 & 1.59 & - \\
\hline U-238 & A59 & 2007 & 1.93 & 0.413 & 1.239 & - \\
\hline U-238 & A6 & 2007 & 3.503 & 0.73 & 2.19 & - \\
\hline U-238 & A60 & 2007 & 1.491 & 0.505 & 1.515 & $\mathrm{U}$ \\
\hline U-238 & A61 & 2007 & 5.344 & 1.195 & 3.585 & - \\
\hline U-238 & A66 & 2007 & 27.79 & 5.88 & 17.64 & - \\
\hline U-238 & A67 & 2007 & 4.119 & 0.51 & 1.53 & 一 \\
\hline U-238 & A68 & 2007 & 0 & 3.22 & 9.66 & $\mathrm{U}$ \\
\hline U-238 & A69 & 2007 & 1.2 & 0.254 & 0.762 & - \\
\hline U-238 & A70 & 2007 & 3.607 & 0.555 & 1.665 & - \\
\hline U-238 & A71 & 2007 & 1.495 & 0.4465 & 1.3395 & - \\
\hline U-238 & A72 & 2007 & 5.69 & 1.19 & 3.57 & - \\
\hline U-238 & $\mathrm{B} 100$ & 2007 & 0 & 2.97 & 8.91 & $\mathrm{U}$ \\
\hline U-238 & B101 & 2007 & 4.368 & 0.625 & 1.875 & - \\
\hline U-238 & B102 & 2007 & 1.359 & 0.371 & 1.113 & - \\
\hline U-238 & B103 & 2007 & 4.958 & 1.135 & 3.405 & - \\
\hline U-238 & B104 & 2007 & 3.449 & 0.525 & 1.575 & - \\
\hline U-238 & B105 & 2007 & 1.98 & 0.492 & 1.476 & - \\
\hline U-238 & B106 & 2007 & 0 & 6.78 & 20.34 & $\mathrm{U}$ \\
\hline
\end{tabular}


Table C-3. (continued).

\begin{tabular}{|c|c|c|c|c|c|c|}
\hline Radionuclide & $\begin{array}{c}\text { Sample } \\
\text { Location }\end{array}$ & Date & $\begin{array}{c}\text { Concentrations } \\
(\mathrm{pCi} / \mathrm{g})\end{array}$ & $\begin{array}{l}\text { Uncertainty } \\
(\mathrm{pCi} / \mathrm{g})\end{array}$ & $\begin{array}{c}\mathrm{MDA} \\
(\mathrm{pCi} / \mathrm{g})\end{array}$ & Flag \\
\hline $\mathrm{U}-238$ & $\mathrm{~B} 14$ & 2007 & 2.096 & 0.525 & 1.575 & - \\
\hline $\mathrm{U}-238$ & B15 & 2007 & 0.2061 & 6.52 & 19.56 & $\mathrm{U}$ \\
\hline $\mathrm{U}-238$ & $\mathrm{~B} 17$ & 2007 & 2.285 & 0.429 & 1.287 & 一 \\
\hline $\mathrm{U}-238$ & $\mathrm{~B} 25$ & 2007 & 3.438 & 0.635 & 1.905 & - \\
\hline $\mathrm{U}-238$ & $\mathrm{~B} 26$ & 2007 & 6.299 & 1.015 & 3.045 & - \\
\hline $\mathrm{U}-238$ & B28 & 2007 & 2.303 & 0.464 & 1.392 & 一 \\
\hline $\mathrm{U}-238$ & B29 & 2007 & 3.029 & 0.74 & 2.22 & - \\
\hline $\mathrm{U}-238$ & B3 & 2007 & 1.978 & 0.479 & 1.437 & - \\
\hline $\mathrm{U}-238$ & B36 & 2007 & 0.2472 & 14.7 & 44.1 & $\mathrm{U}$ \\
\hline U-238 & B37 & 2007 & 0.06451 & 7.36 & 22.08 & $\mathrm{U}$ \\
\hline $\mathrm{U}-238$ & B39 & 2007 & 0 & 2.98 & 8.94 & $\mathrm{U}$ \\
\hline $\mathrm{U}-238$ & B4 & 2007 & 6.47 & 0.915 & 2.745 & - \\
\hline $\mathrm{U}-238$ & B40 & 2007 & 4.306 & 0.575 & 1.725 & - \\
\hline $\mathrm{U}-238$ & B47 & 2007 & 4.232 & 0.815 & 2.445 & - \\
\hline $\mathrm{U}-238$ & B48 & 2007 & 2.084 & 0.755 & 2.265 & $\mathrm{U}$ \\
\hline $\mathrm{U}-238$ & $\mathrm{~B} 50$ & 2007 & 3.523 & 0.595 & 1.785 & - \\
\hline $\mathrm{U}-238$ & B51 & 2007 & 2.084 & 0.4645 & 1.3935 & 一 \\
\hline U-238 & B52 & 2007 & 2.261 & 0.4725 & 1.4175 & - \\
\hline U-238 & B59 & 2007 & 7.509 & 1.2 & 3.6 & - \\
\hline U-238 & B60 & 2007 & 5.834 & 0.72 & 2.16 & - \\
\hline U-238 & B61 & 2007 & 2.245 & 0.615 & 1.845 & - \\
\hline U-238 & B62 & 2007 & 2.426 & 0.4475 & 1.3425 & - \\
\hline U-238 & B69 & 2007 & 26.07 & 4.4 & 13.2 & - \\
\hline U-238 & B71 & 2007 & 7.433 & 2.305 & 6.915 & - \\
\hline U-238 & B73 & 2007 & 2.963 & 0.4875 & 1.4625 & - \\
\hline U-238 & B80 & 2007 & 4.922 & 1.26 & 3.78 & - \\
\hline U-238 & B81 & 2007 & 5.663 & 1.175 & 3.525 & - \\
\hline U-238 & B82 & 2007 & 7.286 & 2.215 & 6.645 & - \\
\hline U-238 & B83 & 2007 & 1.688 & 0.468 & 1.404 & - \\
\hline U-238 & B84 & 2007 & 0 & 3.87 & 11.61 & $\mathrm{U}$ \\
\hline U-238 & B88 & 2007 & 2.322 & 0.54 & 1.62 & - \\
\hline U-238 & B89 & 2007 & 3.811 & 0.555 & 1.665 & - \\
\hline U-238 & B90 & 2007 & 4.314 & 0.56 & 1.68 & - \\
\hline U-238 & B91 & 2007 & 0.2158 & 7.11 & 21.33 & $\mathrm{U}$ \\
\hline U-238 & B92 & 2007 & 10.24 & 1.735 & 5.205 & - \\
\hline U-238 & B93 & 2007 & 2.346 & 0.423 & 1.269 & - \\
\hline U-238 & B94 & 2007 & 3.775 & 0.4535 & 1.3605 & - \\
\hline
\end{tabular}


Table C-3. (continued).

\begin{tabular}{|c|c|c|c|c|c|c|}
\hline Radionuclide & $\begin{array}{c}\text { Sample } \\
\text { Location }\end{array}$ & Date & $\begin{array}{c}\text { Concentrations } \\
(\mathrm{pCi} / \mathrm{g})\end{array}$ & $\begin{array}{l}\text { Uncertainty } \\
(\mathrm{pCi} / \mathrm{g})\end{array}$ & $\begin{array}{c}\mathrm{MDA} \\
(\mathrm{pCi} / \mathrm{g})\end{array}$ & Flag \\
\hline $\mathrm{U}-238$ & B95 & 2007 & 1.864 & 0.3895 & 1.1685 & - \\
\hline $\mathrm{U}-238$ & B99 & 2007 & 3.528 & 0.464 & 1.392 & 一 \\
\hline $\mathrm{U}-238$ & $\mathrm{C} 14$ & 2007 & 1.728 & 0.4575 & 1.3725 & 一 \\
\hline $\mathrm{U}-238$ & $\mathrm{C} 15$ & 2007 & 1.776 & 0.545 & 1.635 & - \\
\hline $\mathrm{U}-238$ & $\mathrm{C} 25$ & 2007 & 0 & 5.34 & 16.02 & $\mathrm{U}$ \\
\hline $\mathrm{U}-238$ & $\mathrm{C} 36$ & 2007 & 3.914 & 0.99 & 2.97 & - \\
\hline $\mathrm{U}-238$ & C37 & 2007 & 3.419 & 0.54 & 1.62 & - \\
\hline $\mathrm{U}-238$ & $\mathrm{C} 46$ & 2007 & 5.097 & 1.165 & 3.495 & - \\
\hline $\mathrm{U}-238$ & $\mathrm{C} 47$ & 2007 & 1.938 & 0.505 & 1.515 & - \\
\hline $\mathrm{U}-238$ & $\mathrm{C} 48$ & 2007 & 2.229 & 0.458 & 1.374 & - \\
\hline $\mathrm{U}-238$ & C56 & 2007 & 8.541 & 1.185 & 3.555 & - \\
\hline $\mathrm{U}-238$ & $\mathrm{C} 57$ & 2007 & 6.741 & 0.94 & 2.82 & - \\
\hline $\mathrm{U}-238$ & D57 & 2007 & 256.3 & 6.4 & 19.2 & - \\
\hline $\mathrm{U}-238$ & D58 & 2007 & 3.214 & 0.471 & 1.413 & - \\
\hline $\mathrm{U}-238$ & D68 & 2007 & 4.749 & 1.15 & 3.45 & - \\
\hline $\mathrm{U}-238$ & D69 & 2007 & 4.032 & 0.565 & 1.695 & - \\
\hline $\mathrm{U}-238$ & D79 & 2007 & 8.366 & 1.29 & 3.87 & - \\
\hline $\mathrm{U}-238$ & D80 & 2007 & 3.58 & 0.4205 & 1.2615 & - \\
\hline U-238 & D89 & 2007 & 0.7828 & 0.59 & 1.77 & $\mathrm{U}$ \\
\hline U-238 & D90 & 2007 & 5.648 & 1.175 & 3.525 & - \\
\hline U-238 & D91 & 2007 & 1.17 & 0.4295 & 1.2885 & $\mathrm{U}$ \\
\hline $\mathrm{U}-238$ & B104 & 2008 & 0.3476 & 0.352 & 1.056 & $\mathrm{U}$ \\
\hline U-238 & A56 & 2009 & 159.2 & 5.9 & 17.7 & - \\
\hline U-238 & A58 & 2009 & 227.9 & 6.4 & 19.2 & - \\
\hline U-238 & $\mathrm{B} 28$ & 2009 & 5.966 & 2.92 & 8.76 & $\mathrm{U}$ \\
\hline U-238 & B52 & 2009 & 168.7 & 5.7 & 17.1 & - \\
\hline U-238 & $\mathrm{C} 36$ & 2009 & 4.508 & 3.985 & 11.955 & $\mathrm{U}$ \\
\hline U-238 & D89 & 2009 & 201.9 & 5.95 & 17.85 & - \\
\hline U-238 & $\mathrm{A} 27$ & 2010 & 3.48 & 3.28 & 9.84 & $\mathrm{U}$ \\
\hline U-238 & A38 & 2010 & 72.4 & 29.9 & 89.7 & $\mathrm{U}$ \\
\hline $\mathrm{U}-238$ & A 15 & 2011 & -0.06192 & 0.667 & 2.001 & $\mathrm{U}$ \\
\hline $\mathrm{U}-238$ & A16 & 2011 & 1.119 & 0.895 & 2.685 & $\mathrm{U}$ \\
\hline $\mathrm{U}-238$ & A17 & 2011 & 0.8349 & 0.847 & 2.541 & $\mathrm{U}$ \\
\hline U-238 & A27 & 2011 & 2.694 & 0.818 & 2.454 & - \\
\hline U-238 & A28 & 2011 & 0.7497 & 0.9 & 2.7 & $\mathrm{U}$ \\
\hline U-238 & A38 & 2011 & 1.192 & 0.76 & 2.28 & $\mathrm{U}$ \\
\hline U-238 & A39 & 2011 & 1.049 & 0.765 & 2.295 & $\mathrm{U}$ \\
\hline
\end{tabular}


Table C-3. (continued).

\begin{tabular}{|c|c|c|c|c|c|c|}
\hline Radionuclide & $\begin{array}{c}\text { Sample } \\
\text { Location }\end{array}$ & Date & $\begin{array}{c}\text { Concentrations } \\
(\mathrm{pCi} / \mathrm{g})\end{array}$ & $\begin{array}{l}\text { Uncertainty } \\
(\mathrm{pCi} / \mathrm{g})\end{array}$ & $\begin{array}{c}\mathrm{MDA} \\
(\mathrm{pCi} / \mathrm{g})\end{array}$ & Flag \\
\hline U-238 & A4 & 2011 & 1.077 & 0.901 & 2.703 & $\mathrm{U}$ \\
\hline U-238 & A44 & 2011 & 0.9637 & 0.704 & 2.112 & $\mathrm{U}$ \\
\hline $\mathrm{U}-238$ & A47 & 2011 & 1.624 & 0.969 & 2.907 & $\mathrm{U}$ \\
\hline $\mathrm{U}-238$ & A49 & 2011 & 1.731 & 0.973 & 2.919 & $\mathrm{U}$ \\
\hline $\mathrm{U}-238$ & A50 & 2011 & 1.122 & 1.03 & 3.09 & $\mathrm{U}$ \\
\hline $\mathrm{U}-238$ & A55 & 2011 & 1.391 & 0.858 & 2.574 & $\mathrm{U}$ \\
\hline U-238 & A58 & 2011 & 3.608 & 0.973 & 2.919 & - \\
\hline $\mathrm{U}-238$ & A59 & 2011 & 0.06416 & 0.601 & 1.803 & $\mathrm{U}$ \\
\hline $\mathrm{U}-238$ & A6 & 2011 & 1.235 & 1.02 & 3.06 & $\mathrm{U}$ \\
\hline U-238 & A61 & 2011 & 1.536 & 0.679 & 2.037 & $\mathrm{U}$ \\
\hline $\mathrm{U}-238$ & A66 & 2011 & 2.165 & 1.02 & 3.06 & $\mathrm{U}$ \\
\hline $\mathrm{U}-238$ & A69 & 2011 & 0.06245 & 1.08 & 3.24 & $\mathrm{U}$ \\
\hline $\mathrm{U}-238$ & A70 & 2011 & -0.15 & 0.744 & 2.232 & $\mathrm{U}$ \\
\hline $\mathrm{U}-238$ & A72 & 2011 & 0.9638 & 0.704 & 2.112 & $\mathrm{U}$ \\
\hline $\mathrm{U}-238$ & B101 & 2011 & 0 & 0.165 & 0.495 & $\mathrm{U}$ \\
\hline U-238 & B102 & 2011 & 0.02632 & 0.837 & 2.511 & $\mathrm{U}$ \\
\hline $\mathrm{U}-238$ & B103 & 2011 & 1.652 & 0.993 & 2.979 & $\mathrm{U}$ \\
\hline U-238 & B104 & 2011 & 1.051 & 0.74 & 2.22 & $\mathrm{U}$ \\
\hline U-238 & B106 & 2011 & -0.4582 & 0.741 & 2.223 & $\mathrm{U}$ \\
\hline U-238 & B14 & 2011 & 2.242 & 0.989 & 2.967 & $\mathrm{U}$ \\
\hline U-238 & B15 & 2011 & 1.683 & 0.735 & 2.205 & $\mathrm{U}$ \\
\hline U-238 & $\mathrm{B} 17$ & 2011 & -0.1816 & 0.325 & 0.975 & $\mathrm{U}$ \\
\hline U-238 & B25 & 2011 & 2.469 & 1.03 & 3.09 & $\mathrm{U}$ \\
\hline U-238 & $\mathrm{B} 28$ & 2011 & 0.9426 & 0.764 & 2.292 & $\mathrm{U}$ \\
\hline U-238 & B29 & 2011 & 0.7047 & 0.923 & 2.769 & $\mathrm{U}$ \\
\hline U-238 & B3 & 2011 & 1.237 & 0.757 & 2.271 & $\mathrm{U}$ \\
\hline U-238 & B39 & 2011 & 0.07115 & 0.313 & 0.939 & $\mathrm{U}$ \\
\hline U-238 & B4 & 2011 & 1.208 & 0.89 & 2.67 & $\mathrm{U}$ \\
\hline U-238 & B40 & 2011 & 0.07269 & 0.309 & 0.927 & $\mathrm{U}$ \\
\hline U-238 & B47 & 2011 & 1.131 & 1.07 & 3.21 & $\mathrm{U}$ \\
\hline $\mathrm{U}-238$ & B48 & 2011 & -1.442 & 0.721 & 2.163 & $\mathrm{U}$ \\
\hline U-238 & B50 & 2011 & 1.169 & 0.754 & 2.262 & $\mathrm{U}$ \\
\hline U-238 & B52 & 2011 & 1.937 & 0.774 & 2.322 & $\mathrm{U}$ \\
\hline U-238 & B61 & 2011 & 0.5297 & 0.996 & 2.988 & $\mathrm{U}$ \\
\hline U-238 & B62 & 2011 & 1.644 & 0.856 & 2.568 & $\mathrm{U}$ \\
\hline U-238 & B71 & 2011 & 3.152 & 1.24 & 3.72 & $\mathrm{U}$ \\
\hline U-238 & B73 & 2011 & 0.8414 & 1.04 & 3.12 & $\mathrm{U}$ \\
\hline
\end{tabular}


Table C-3. (continued).

\begin{tabular}{|c|c|c|c|c|c|c|}
\hline Radionuclide & $\begin{array}{l}\text { Sample } \\
\text { Location }\end{array}$ & Date & $\begin{array}{c}\text { Concentrations } \\
(\mathrm{pCi} / \mathrm{g})\end{array}$ & $\begin{array}{l}\text { Uncertainty } \\
(\mathrm{pCi} / \mathrm{g})\end{array}$ & $\begin{array}{c}\mathrm{MDA} \\
(\mathrm{pCi} / \mathrm{g})\end{array}$ & Flag \\
\hline $\mathrm{U}-238$ & $\mathrm{~B} 80$ & 2011 & 1.174 & 0.918 & 2.754 & $\mathrm{U}$ \\
\hline U-238 & B83 & 2011 & 0.6996 & 0.848 & 2.544 & $\mathrm{U}$ \\
\hline $\mathrm{U}-238$ & B84 & 2011 & 1.652 & 1.05 & 3.15 & $\mathrm{U}$ \\
\hline $\mathrm{U}-238$ & B89 & 2011 & 1.008 & 0.987 & 2.961 & $\mathrm{U}$ \\
\hline $\mathrm{U}-238$ & B90 & 2011 & 0.1244 & 0.94 & 2.82 & $\mathrm{U}$ \\
\hline U-238 & B91 & 2011 & 4.457 & 1.26 & 3.78 & - \\
\hline U-238 & B92 & 2011 & 1.306 & 0.705 & 2.115 & $\mathrm{U}$ \\
\hline $\mathrm{U}-238$ & B93 & 2011 & 1.65 & 0.679 & 2.037 & $\mathrm{U}$ \\
\hline $\mathrm{U}-238$ & B94 & 2011 & 1.24 & 0.716 & 2.148 & $\mathrm{U}$ \\
\hline U-238 & B99 & 2011 & 0.4358 & 0.836 & 2.508 & $\mathrm{U}$ \\
\hline $\mathrm{U}-238$ & $\mathrm{C} 15$ & 2011 & 1.464 & 0.896 & 2.688 & $\mathrm{U}$ \\
\hline $\mathrm{U}-238$ & $\mathrm{C} 25$ & 2011 & 1.209 & 0.68 & 2.04 & $\mathrm{U}$ \\
\hline $\mathrm{U}-238$ & $\mathrm{C} 37$ & 2011 & 1.385 & 0.861 & 2.583 & $\mathrm{U}$ \\
\hline $\mathrm{U}-238$ & $\mathrm{C} 46$ & 2011 & 0.5959 & 0.849 & 2.547 & $\mathrm{U}$ \\
\hline U-238 & $\mathrm{C} 48$ & 2011 & 2.807 & 0.865 & 2.595 & - \\
\hline U-238 & C56 & 2011 & 2.205 & 1 & 3 & $\mathrm{U}$ \\
\hline $\mathrm{U}-238$ & C57 & 2011 & -0.1158 & 0.803 & 2.409 & $\mathrm{U}$ \\
\hline $\mathrm{U}-238$ & D58 & 2011 & 1.608 & 0.728 & 2.184 & $\mathrm{U}$ \\
\hline U-238 & D68 & 2011 & 0.8694 & 0.66 & 1.98 & $\mathrm{U}$ \\
\hline U-238 & D89 & 2011 & 1.87 & 0.898 & 2.694 & $\mathrm{U}$ \\
\hline $\mathrm{U}-238$ & D91 & 2011 & 1.771 & 0.985 & 2.955 & $\mathrm{U}$ \\
\hline $\mathrm{U}-238$ & A 15 & 2012 & 0.6126 & 0.77 & 2.31 & $\mathrm{U}$ \\
\hline U-238 & A38 & 2012 & 1.178 & 0.894 & 2.682 & $\mathrm{U}$ \\
\hline U-238 & A44 & 2012 & 1.052 & 0.831 & 2.493 & $\mathrm{U}$ \\
\hline U-238 & A47 & 2012 & 1.085 & 0.791 & 2.373 & $\mathrm{U}$ \\
\hline U-238 & A59 & 2012 & 5.564 & 1.44 & 4.32 & - \\
\hline U-238 & A6 & 2012 & 1.027 & 0.954 & 2.862 & $\mathrm{U}$ \\
\hline U-238 & A69 & 2012 & 1.349 & 0.927 & 2.781 & $\mathrm{U}$ \\
\hline U-238 & A70 & 2012 & 4.204 & 1.08 & 3.24 & - \\
\hline U-238 & A72 & 2012 & -0.003106 & 0.925 & 2.775 & $\mathrm{U}$ \\
\hline $\mathrm{U}-238$ & B14 & 2012 & 0.9848 & 0.844 & 2.532 & $\mathrm{U}$ \\
\hline $\mathrm{U}-238$ & B48 & 2012 & 1.024 & 0.847 & 2.541 & $\mathrm{U}$ \\
\hline $\mathrm{U}-238$ & B83 & 2012 & 1.701 & 0.743 & 2.229 & $\mathrm{U}$ \\
\hline U-238 & $\mathrm{C} 46$ & 2012 & 1.05 & 0.896 & 2.688 & $\mathrm{U}$ \\
\hline U-238 & C57 & 2012 & 1.666 & 0.84 & 2.52 & $\mathrm{U}$ \\
\hline U-238 & D68 & 2012 & 1.423 & 62.7 & 188.1 & $\mathrm{U}$ \\
\hline
\end{tabular}


Table C-3. (continued).

\begin{tabular}{|c|c|c|c|c|c|c|}
\hline Radionuclide & $\begin{array}{c}\text { Sample } \\
\text { Location }\end{array}$ & Date & $\begin{array}{c}\text { Concentrations } \\
(\mathrm{pCi} / \mathrm{g})\end{array}$ & $\begin{array}{l}\text { Uncertainty } \\
(\mathrm{pCi} / \mathrm{g})\end{array}$ & $\begin{array}{c}\mathrm{MDA} \\
(\mathrm{pCi} / \mathrm{g})\end{array}$ & Flag \\
\hline $\mathrm{U}-238$ & $\begin{array}{l}2 \text { INCH AIR } \\
\text { - INTEC }\end{array}$ & 2013 & 16.27 & 2.37 & 7.11 & - \\
\hline U-238 & A47 & 2013 & 18.51 & 2.58 & 7.74 & - \\
\hline U-238 & A49 & 2013 & 0.6332 & 0.393 & 1.18 & $\mathrm{U}$ \\
\hline U-238 & A59 & 2013 & 8.914 & 2.64 & 7.92 & - \\
\hline $\mathrm{U}-238$ & A71 & 2013 & 1.496 & 1.463 & 4.39 & $\mathrm{U}$ \\
\hline $\mathrm{U}-238$ & A72 & 2013 & 0 & 0.079 & 0.237 & $\mathrm{U}$ \\
\hline $\mathrm{U}-238$ & $\begin{array}{c}2 \text { INCH AIR } \\
- \text { CPP }\end{array}$ & 2014 & 1.469 & 0.751 & 2.253 & $\mathrm{U}$ \\
\hline U-238 & $\begin{array}{c}2 \text { INCH AIR } \\
\text { - INTEC }\end{array}$ & 2014 & 1.481 & 0.791 & 2.373 & $\mathrm{U}$ \\
\hline $\mathrm{U}-238$ & A38 & 2014 & 1.146 & 0.872 & 2.616 & $\mathrm{U}$ \\
\hline $\mathrm{U}-238$ & A47 & 2014 & 0.0555 & 0.795 & 2.385 & $\mathrm{U}$ \\
\hline $\mathrm{U}-238$ & A58 & 2014 & 1.242 & 0.846 & 2.538 & $\mathrm{U}$ \\
\hline U-238 & A59 & 2014 & 0.799 & 0.761 & 2.283 & $\mathrm{U}$ \\
\hline $\mathrm{U}-238$ & $\mathrm{~B} 15$ & 2014 & 0.4685 & 1.51 & 4.53 & $\mathrm{U}$ \\
\hline $\mathrm{U}-238$ & B28 & 2014 & 0.8406 & 0.722 & 2.166 & $\mathrm{U}$ \\
\hline $\mathrm{U}-238$ & B29 & 2014 & 0.8999 & 0.726 & 2.178 & $\mathrm{U}$ \\
\hline $\mathrm{U}-238$ & B3 & 2014 & 2.22 & 0.588 & 1.764 & - \\
\hline $\mathrm{U}-238$ & B3 & 2014 & 1.849 & 1.28 & 3.84 & $\mathrm{U}$ \\
\hline $\mathrm{U}-238$ & B4 & 2014 & 1.298 & 0.507 & 1.521 & $\mathrm{U}$ \\
\hline U-238 & B40 & 2014 & 0.1386 & 0.441 & 1.323 & $\mathrm{U}$ \\
\hline U-238 & B50 & 2014 & 0.6392 & 0.703 & 2.109 & $\mathrm{U}$ \\
\hline U-238 & B52 & 2014 & 1.79 & 1.02 & 3.06 & $\mathrm{U}$ \\
\hline U-238 & B52 & 2014 & 0.9798 & 0.898 & 2.694 & $\mathrm{U}$ \\
\hline
\end{tabular}

Table C-4. Radionuclides concentrations in surface soils associated with MFC.

\begin{tabular}{|c|c|c|c|c|c|c|}
\hline Radionuclide & $\begin{array}{c}\text { Sample } \\
\text { Location }\end{array}$ & Date & $\begin{array}{c}\text { Concentrations } \\
(\mathrm{pCi} / \mathrm{g})\end{array}$ & $\begin{array}{c}\text { Uncertainty } \\
(\mathrm{pCi} / \mathrm{g})\end{array}$ & $\begin{array}{c}\text { MDA } \\
(\mathrm{pCi} / \mathrm{g})\end{array}$ & Flag \\
\hline Am-241 & EBRII-11 & 1978 & 0.005 & 0.002 & 0.006 & $\mathrm{U}$ \\
\hline Am-241 & EBRII-15 & 1978 & 0.007 & 0.002 & 0.006 & - \\
\hline Am-241 & EBRII-17 & 1978 & 0.0038 & 0.0014 & 0.0042 & $\mathrm{U}$ \\
\hline Am-241 & EBRII-3 & 1978 & 0.006 & 0.002 & 0.006 & - \\
\hline Am-241 & EBRII-5 & 1978 & 0.008 & 0.002 & 0.006 & - \\
\hline Am-241 & EBRII-7 & 1978 & 0.003 & 0.002 & 0.006 & $\mathrm{U}$ \\
\hline Am-241 & EBRII-8 & 1978 & 0.008 & 0.002 & 0.006 & - \\
\hline Am-241 & TREAT-1 & 1978 & 0.003 & 0.002 & 0.006 & $\mathrm{U}$ \\
\hline Am-241 & TREAT-3 & 1978 & 0.003 & 0.002 & 0.006 & $\mathrm{U}$ \\
\hline
\end{tabular}


Table C-4. (continued).

\begin{tabular}{|c|c|c|c|c|c|c|}
\hline Radionuclide & $\begin{array}{c}\text { Sample } \\
\text { Location }\end{array}$ & Date & $\begin{array}{c}\text { Concentrations } \\
(\mathrm{pCi} / \mathrm{g})\end{array}$ & $\begin{array}{c}\text { Uncertainty } \\
(\mathrm{pCi} / \mathrm{g})\end{array}$ & $\begin{array}{c}\text { MDA } \\
(\mathrm{pCi} / \mathrm{g})\end{array}$ & Flag \\
\hline Co-60 & EBRII-10 & 2006 & 0.1576 & 0.01495 & 0.04485 & - \\
\hline Co-60 & EBRII-11 & 2006 & 0.05625 & 0.00975 & 0.02925 & 一 \\
\hline Co-60 & EBRII-12 & 2006 & 0.06537 & 0.0097 & 0.0291 & - \\
\hline Co-60 & EBRII-13 & 2006 & 0.1235 & 0.01875 & 0.05625 & - \\
\hline Co-60 & EBRII-15 & 2006 & 0.0942 & 0.0166 & 0.0498 & - \\
\hline Co-60 & EBRII-16 & 2006 & 0.03889 & 0.01015 & 0.03045 & - \\
\hline Co-60 & EBRII-17 & 2006 & 0.05783 & 0.01 & 0.03 & - \\
\hline Co-60 & EBRII-3 & 2006 & 0.04879 & 0.00975 & 0.02925 & - \\
\hline Co-60 & EBRII-4 & 2006 & 0.11 & 0.0165 & 0.0495 & - \\
\hline Co-60 & EBRII-5 & 2006 & 0.05757 & 0.01145 & 0.03435 & - \\
\hline Co-60 & EBRII-6 & 2006 & 0.1668 & 0.0226 & 0.0678 & - \\
\hline Co-60 & EBRII-9 & 2006 & 0.07287 & 0.0157 & 0.0471 & - \\
\hline Сo-60 & TRT-4 & 2006 & 0.1478 & 0.0238 & 0.0714 & - \\
\hline Co-60 & EBRII-1 & 2011 & 0.0001081 & 0.00323 & 0.00969 & $\mathrm{U}$ \\
\hline Co-60 & EBRII-10 & 2011 & -0.00458 & 0.00498 & 0.01494 & $\mathrm{U}$ \\
\hline Co-60 & EBRII-11 & 2011 & 0.000353 & 0.00343 & 0.01029 & $\mathrm{U}$ \\
\hline Co-60 & EBRII-12 & 2011 & 0.001386 & 0.00362 & 0.01086 & $\mathrm{U}$ \\
\hline Co-60 & EBRII-13 & 2011 & 0.003404 & 0.00395 & 0.01185 & $\mathrm{U}$ \\
\hline Co-60 & EBRII-15 & 2011 & 0.004332 & 0.00255 & 0.00765 & $\mathrm{U}$ \\
\hline Co-60 & EBRII-16 & 2011 & 0.001018 & 0.00273 & 0.00819 & $\mathrm{U}$ \\
\hline Co-60 & EBRII-17 & 2011 & -0.002603 & 0.00495 & 0.01485 & $\mathrm{U}$ \\
\hline Co-60 & EBRII-2 & 2011 & 0.002239 & 0.00384 & 0.01152 & $\mathrm{U}$ \\
\hline Co-60 & EBRII-3 & 2011 & -0.00319 & 0.00498 & 0.01494 & $\mathrm{U}$ \\
\hline Co-60 & EBRII-4 & 2011 & 0.003925 & 0.00358 & 0.01074 & $\mathrm{U}$ \\
\hline Co-60 & EBRII-6 & 2011 & -0.001269 & 0.00302 & 0.00906 & $\mathrm{U}$ \\
\hline Co-60 & TRT-1 & 2011 & 0.001164 & 0.00426 & 0.01278 & $\mathrm{U}$ \\
\hline Co-60 & TRT-2 & 2011 & 0.171 & 0.00914 & 0.02742 & - \\
\hline Co-60 & TRT-3 & 2011 & 0.006177 & 0.00431 & 0.01293 & $\mathrm{U}$ \\
\hline Co-60 & TRT-4 & 2011 & 0.002969 & 0.00381 & 0.01143 & $\mathrm{U}$ \\
\hline Co-60 & EBRII-10 & 2012 & 0.0004873 & 0.00461 & 0.01383 & $\mathrm{U}$ \\
\hline Co-60 & EBRII-11 & 2012 & 0.2029 & 0.00996 & 0.02988 & - \\
\hline Co-60 & EBRII-12 & 2012 & -0.001485 & 0.00402 & 0.01206 & $\mathrm{U}$ \\
\hline Co-60 & EBRII-16 & 2012 & -0.0002159 & 0.00373 & 0.01119 & $\mathrm{U}$ \\
\hline Co-60 & EBRII-2 & 2012 & 0.003298 & 0.0042 & 0.0126 & $\mathrm{U}$ \\
\hline Co-60 & EBRII-4 & 2012 & 0.005677 & 0.00431 & 0.01293 & $\mathrm{U}$ \\
\hline Co-60 & TRT-1 & 2012 & -0.003682 & 0.00417 & 0.01251 & $\mathrm{U}$ \\
\hline Co-60 & TRT-3 & 2012 & -0.001169 & 0.00476 & 0.01428 & $\mathrm{U}$ \\
\hline
\end{tabular}


Table C-4. (continued).

\begin{tabular}{|c|c|c|c|c|c|c|}
\hline Radionuclide & $\begin{array}{c}\text { Sample } \\
\text { Location }\end{array}$ & Date & $\begin{array}{c}\text { Concentrations } \\
(\mathrm{pCi} / \mathrm{g})\end{array}$ & $\begin{array}{l}\text { Uncertainty } \\
(\mathrm{pCi} / \mathrm{g})\end{array}$ & $\begin{array}{c}\text { MDA } \\
(\mathrm{pCi} / \mathrm{g})\end{array}$ & Flag \\
\hline Co-60 & EBRII-10 & 2013 & 0.002182 & 0.00203 & 0.00609 & $\mathrm{U}$ \\
\hline Co-60 & EBRII-11 & 2013 & -0.0008988 & 0.00298 & 0.00895 & $\mathrm{U}$ \\
\hline Co-60 & EBRII-13 & 2013 & -0.0005521 & 0.00291 & 0.00872 & $\mathrm{U}$ \\
\hline Co-60 & TRT-1 & 2013 & -0.00001731 & 0.000313 & 0.000939 & $\mathrm{U}$ \\
\hline Co-60 & $\begin{array}{c}2 \text { INCH AIR } \\
- \text { MFC }\end{array}$ & 2014 & 0.001358 & 0.00133 & 0.00399 & $\mathrm{U}$ \\
\hline Co-60 & EBRII-10 & 2014 & 0.0004599 & 0.001 & 0.00423 & $\mathrm{U}$ \\
\hline Co-60 & EBRII-11 & 2014 & 0.00004946 & 0.00104 & 0.00312 & $\mathrm{U}$ \\
\hline Co-60 & EBRII-2 & 2014 & -0.000839 & 0.00146 & 0.00438 & $\mathrm{U}$ \\
\hline Co-60 & EBRII-4 & 2014 & 0.0001278 & 0.001 & 0.003 & $\mathrm{U}$ \\
\hline Co-60 & EBRII-6 & 2014 & 0.0008236 & 0.00118 & 0.00354 & $\mathrm{U}$ \\
\hline Co-60 & TRT-1 & 2014 & 0.0009356 & 0.00104 & 0.00312 & $\mathrm{U}$ \\
\hline Co-60 & TRT-2 & 2014 & 0.04528 & 0.00673 & 0.02019 & - \\
\hline Co-60 & TRT-3 & 2014 & -0.0002747 & 0.00138 & 0.00414 & $\mathrm{U}$ \\
\hline Co-60 & TRT-4 & 2014 & -0.0008663 & 0.0014 & 0.0042 & $\mathrm{U}$ \\
\hline Cs-134 & EBRII-1 & 2007 & 0.08104 & 0.013 & 0.039 & - \\
\hline Cs-134 & EBRII-10 & 2007 & 0.05185 & 0.0116 & 0.0348 & - \\
\hline Cs-134 & EBRII-11 & 2007 & 0.05354 & 0.00978 & 0.02934 & 一 \\
\hline Cs-134 & EBRII-12 & 2007 & 0.05822 & 0.0101 & 0.0303 & - \\
\hline Cs-134 & EBRII-13 & 2007 & 0.06 & 0.0102 & 0.0306 & 一 \\
\hline Cs-134 & EBRII-15 & 2007 & 0.1154 & 0.0158 & 0.0474 & 一 \\
\hline Cs-134 & EBRII-16 & 2007 & 0.04471 & 0.00718 & 0.02154 & 一 \\
\hline Cs-134 & EBRII-17 & 2007 & 0.07171 & 0.0115 & 0.0345 & 一 \\
\hline Cs-134 & EBRII-2 & 2007 & 0.02023 & 0.0124 & 0.0372 & $\mathrm{U}$ \\
\hline Cs-134 & EBRII-3 & 2007 & -0.002703 & 0.00884 & 0.02652 & $\mathrm{U}$ \\
\hline Cs-134 & EBRII-4 & 2007 & 0.03837 & 0.0103 & 0.0309 & - \\
\hline Cs-134 & EBRII-5 & 2007 & 0.09657 & 0.0197 & 0.0591 & - \\
\hline Cs-134 & EBRII-6 & 2007 & 0.06696 & 0.0161 & 0.0483 & - \\
\hline Cs-134 & EBRII-9 & 2007 & 0.1089 & 0.0186 & 0.0558 & - \\
\hline Cs-134 & TRT-1 & 2007 & 0.002874 & 0.00532 & 0.01596 & $\mathrm{U}$ \\
\hline Cs-134 & TRT-2 & 2007 & 0.05962 & 0.00949 & 0.02847 & - \\
\hline Cs-134 & TRT-3 & 2007 & 0.08241 & 0.0133 & 0.0399 & 一 \\
\hline Cs-134 & TRT-4 & 2007 & 0.01644 & 0.00438 & 0.01314 & - \\
\hline Cs-134 & EBRII-10 & 2008 & 0.04136 & 0.0066 & 0.0198 & - \\
\hline Cs-134 & EBRII-11 & 2008 & 0.04345 & 0.00675 & 0.02025 & - \\
\hline Cs-134 & EBRII-12 & 2008 & 0.01091 & 0.00347 & 0.01041 & - \\
\hline Cs-134 & EBRII-13 & 2008 & 0.04279 & 0.0063 & 0.0189 & 一 \\
\hline
\end{tabular}


Table C-4. (continued).

\begin{tabular}{|c|c|c|c|c|c|c|}
\hline Radionuclide & $\begin{array}{c}\text { Sample } \\
\text { Location }\end{array}$ & Date & $\begin{array}{c}\text { Concentrations } \\
(\mathrm{pCi} / \mathrm{g})\end{array}$ & $\begin{array}{l}\text { Uncertainty } \\
(\mathrm{pCi} / \mathrm{g})\end{array}$ & $\begin{array}{c}\text { MDA } \\
(\mathrm{pCi} / \mathrm{g})\end{array}$ & Flag \\
\hline Cs-134 & EBRII-15 & 2008 & 0.01154 & 0.00374 & 0.01122 & - \\
\hline Cs-134 & EBRII-16 & 2008 & 0.02039 & 0.0066 & 0.0198 & 一 \\
\hline Cs-134 & EBRII-17 & 2008 & 0.03779 & 0.004985 & 0.014955 & - \\
\hline Cs-134 & EBRII-2 & 2008 & 0.03963 & 0.0062 & 0.0186 & - \\
\hline Cs-134 & EBRII-3 & 2008 & 0.03042 & 0.00373 & 0.01119 & - \\
\hline Cs-134 & EBRII-4 & 2008 & 0.05204 & 0.0075 & 0.0225 & - \\
\hline Cs-134 & EBRII-6 & 2008 & 0.007699 & 0.00415 & 0.01245 & $\mathrm{U}$ \\
\hline Cs-134 & TRT-1 & 2008 & 0.003791 & 0.003015 & 0.009045 & $\mathrm{U}$ \\
\hline Cs-134 & TRT-2 & 2008 & 0.02446 & 0.00855 & 0.02565 & $\mathrm{U}$ \\
\hline Cs-134 & TRT-3 & 2008 & 0.02797 & 0.00492 & 0.01476 & - \\
\hline Cs-134 & TRT-4 & 2008 & 0.005213 & 0.00318 & 0.00954 & $\mathrm{U}$ \\
\hline Cs-134 & EBRII-1 & 2009 & 0.03004 & 0.0115 & 0.0345 & $\mathrm{U}$ \\
\hline Cs-134 & EBRII-10 & 2009 & 0.02502 & 0.0106 & 0.0318 & $\mathrm{U}$ \\
\hline Cs-134 & EBRII-12 & 2009 & 0.01979 & 0.00695 & 0.02085 & $\mathrm{U}$ \\
\hline Cs-134 & EBRII-5 & 2009 & 0.02546 & 0.0099 & 0.0297 & $\mathrm{U}$ \\
\hline Cs-134 & EBRII-9 & 2009 & 0.01068 & 0.00795 & 0.02385 & $\mathrm{U}$ \\
\hline Cs-134 & EBRII-10 & 2010 & 0.08851 & 0.0117 & 0.0351 & 一 \\
\hline Cs-134 & EBRII-11 & 2010 & 0.07067 & 0.00894 & 0.02682 & - \\
\hline Cs-134 & EBRII-15 & 2010 & 0.1311 & 0.016 & 0.048 & - \\
\hline Cs-134 & EBRII-16 & 2010 & 0.02532 & 0.00316 & 0.00948 & - \\
\hline Cs-134 & EBRII-17 & 2010 & 0.01602 & 0.00468 & 0.01404 & - \\
\hline Cs-134 & EBRII-6 & 2010 & 0.07914 & 0.0103 & 0.0309 & - \\
\hline Cs-134 & EBRII-9 & 2010 & 0.05522 & 0.00963 & 0.02889 & - \\
\hline Cs-134 & TRT-1 & 2010 & 0.04768 & 0.00758 & 0.02274 & - \\
\hline Cs-134 & TRT-2 & 2010 & 0.04346 & 0.00607 & 0.01821 & 一 \\
\hline Cs-134 & TRT-3 & 2010 & 0.06777 & 0.0105 & 0.0315 & 一 \\
\hline Cs-134 & TRT-4 & 2010 & 0.007767 & 0.00351 & 0.01053 & $\mathrm{U}$ \\
\hline Cs-134 & EBRII-1 & 2011 & 0.006905 & 0.0126 & 0.0378 & $\mathrm{U}$ \\
\hline Cs-134 & EBRII-10 & 2011 & -0.015 & 0.0238 & 0.0714 & $\mathrm{U}$ \\
\hline Cs-134 & EBRII-11 & 2011 & -0.001254 & 0.0209 & 0.0627 & $\mathrm{U}$ \\
\hline Cs-134 & EBRII-12 & 2011 & -0.005638 & 0.021 & 0.0642 & $\mathrm{U}$ \\
\hline Cs-134 & EBRII-13 & 2011 & 0.007308 & 0.022 & 0.066 & $\mathrm{U}$ \\
\hline Cs-134 & EBRII-15 & 2011 & 0.01363 & 0.0178 & 0.0534 & $\mathrm{U}$ \\
\hline Cs-134 & EBRII-16 & 2011 & -0.002191 & 0.0189 & 0.0567 & $\mathrm{U}$ \\
\hline Cs-134 & EBRII-17 & 2011 & 0.006725 & 0.0235 & 0.0705 & $\mathrm{U}$ \\
\hline Cs-134 & EBRII-2 & 2011 & -0.002124 & 0.022 & 0.066 & $\mathrm{U}$ \\
\hline Cs-134 & EBRII-3 & 2011 & 0.0001688 & 0.0238 & 0.0714 & $\mathrm{U}$ \\
\hline
\end{tabular}


Table C-4. (continued).

\begin{tabular}{|c|c|c|c|c|c|c|}
\hline Radionuclide & $\begin{array}{l}\text { Sample } \\
\text { Location }\end{array}$ & Date & $\begin{array}{c}\text { Concentrations } \\
(\mathrm{pCi} / \mathrm{g})\end{array}$ & $\begin{array}{l}\text { Uncertainty } \\
(\mathrm{pCi} / \mathrm{g})\end{array}$ & $\begin{array}{c}\mathrm{MDA} \\
(\mathrm{pCi} / \mathrm{g})\end{array}$ & Flag \\
\hline Cs-134 & EBRII-4 & 2011 & -0.02237 & 0.0208 & 0.0624 & $\mathrm{U}$ \\
\hline Cs-134 & EBRII-6 & 2011 & 0.001182 & 0.0101 & 0.0303 & $\mathrm{U}$ \\
\hline Cs-134 & TRT-1 & 2011 & -0.07424 & 0.00944 & 0.02832 & $\mathrm{U}$ \\
\hline Cs-134 & TRT-2 & 2011 & 0.01309 & 0.0239 & 0.0717 & $\mathrm{U}$ \\
\hline Cs-134 & TRT-3 & 2011 & -0.004559 & 0.024 & 0.072 & $\mathrm{U}$ \\
\hline Cs-134 & TRT-4 & 2011 & -0.07314 & 0.00883 & 0.02649 & $\mathrm{U}$ \\
\hline Cs-134 & EBRII-10 & 2012 & 0.02174 & 0.00769 & 0.02307 & $\mathrm{U}$ \\
\hline Cs-134 & EBRII-11 & 2012 & 0.05849 & 0.0085 & 0.0255 & - \\
\hline Cs-134 & EBRII-12 & 2012 & 0.0584 & 0.00771 & 0.02313 & - \\
\hline Cs-134 & EBRII-16 & 2012 & 0.05815 & 0.00763 & 0.02289 & - \\
\hline Cs-134 & EBRII-2 & 2012 & 0.05079 & 0.00866 & 0.02598 & - \\
\hline Cs-134 & EBRII-4 & 2012 & 0.05636 & 0.008 & 0.024 & - \\
\hline Cs-134 & TRT-1 & 2012 & 0.0726 & 0.00927 & 0.02781 & - \\
\hline Cs-134 & TRT-3 & 2012 & 0.06514 & 0.00914 & 0.02742 & - \\
\hline Cs-134 & EBRII-10 & 2013 & 0.04883 & 0.00913 & 0.0274 & - \\
\hline Cs-134 & EBRII-11 & 2013 & 0.05736 & 0.0136 & 0.0408 & 一 \\
\hline Cs-134 & EBRII-13 & 2013 & 0.08978 & 0.0135 & 0.0406 & - \\
\hline Cs-134 & TRT-1 & 2013 & -0.003819 & 0.0109 & 0.0328 & $\mathrm{U}$ \\
\hline Cs-134 & $\begin{array}{l}2 \text { INCH AIR } \\
\text { - MFC }\end{array}$ & 2014 & 0.02482 & 0.0133 & 0.0399 & $\mathrm{U}$ \\
\hline Cs-134 & EBRII-10 & 2014 & 0.04194 & 0.0147 & 0.0441 & $\mathrm{U}$ \\
\hline Cs-134 & EBRII-11 & 2014 & 0.03639 & 0.0107 & 0.0321 & - \\
\hline Cs-134 & EBRII-2 & 2014 & 0.06079 & 0.0109 & 0.0327 & - \\
\hline Cs-134 & EBRII-4 & 2014 & 0.04228 & 0.0102 & 0.0306 & - \\
\hline Cs-134 & EBRII-6 & 2014 & 0.03755 & 0.0124 & 0.0372 & - \\
\hline Cs-134 & TRT-1 & 2014 & 0.04104 & 0.0081 & 0.0243 & - \\
\hline Cs-134 & TRT-2 & 2014 & 0.0462 & 0.0133 & 0.0399 & 一 \\
\hline Cs-134 & TRT-3 & 2014 & 0.04441 & 0.0132 & 0.0396 & 一 \\
\hline Cs-134 & TRT-4 & 2014 & 0.03858 & 0.0141 & 0.0423 & $\mathrm{U}$ \\
\hline Cs-137 & EBRII-1 & 1978 & 1.14 & 0.085 & 0.255 & - \\
\hline Cs-137 & EBRII-10 & 1978 & 1.31 & 0.089 & 0.267 & - \\
\hline Cs-137 & EBRII-11 & 1978 & 1.05 & 0.083 & 0.249 & - \\
\hline Cs-137 & EBRII-12 & 1978 & 0.601 & 0.057 & 0.171 & - \\
\hline Cs-137 & EBRII-13 & 1978 & 0.906 & 0.066 & 0.198 & 一 \\
\hline Cs-137 & EBRII-14 & 1978 & 0.872 & 0.072 & 0.216 & - \\
\hline Cs-137 & EBRII-15 & 1978 & 1.18 & 0.088 & 0.264 & 一 \\
\hline Cs-137 & EBRII-16 & 1978 & 1.19 & 0.075 & 0.225 & - \\
\hline
\end{tabular}


Table C-4. (continued).

\begin{tabular}{|c|c|c|c|c|c|c|}
\hline Radionuclide & $\begin{array}{c}\text { Sample } \\
\text { Location }\end{array}$ & Date & $\begin{array}{c}\text { Concentrations } \\
(\mathrm{pCi} / \mathrm{g})\end{array}$ & $\begin{array}{l}\text { Uncertainty } \\
(\mathrm{pCi} / \mathrm{g})\end{array}$ & $\begin{array}{l}\text { MDA } \\
(\mathrm{pCi} / \mathrm{g})\end{array}$ & Flag \\
\hline Cs-137 & EBRII-17 & 1978 & 0.671 & 0.0660 & 0.198 & - \\
\hline Cs-137 & EBRII-2 & 1978 & 0.85 & 0.074 & 0.222 & 一 \\
\hline Cs-137 & EBRII-3 & 1978 & 0.921 & 0.072 & 0.216 & - \\
\hline Cs-137 & EBRII-4 & 1978 & 0.944 & 0.080 & 0.24 & - \\
\hline Cs-137 & EBRII-5 & 1978 & 0.956 & 0.084 & 0.252 & - \\
\hline Cs-137 & EBRII-6 & 1978 & 0.828 & 0.065 & 0.195 & - \\
\hline Cs-137 & EBRII-7 & 1978 & 1.12 & 0.089 & 0.267 & - \\
\hline Cs-137 & EBRII-8 & 1978 & 1.21 & 0.085 & 0.255 & - \\
\hline Cs-137 & EBRII-9 & 1978 & 0.72 & 0.07 & 0.21 & - \\
\hline Cs-137 & TREAT-1 & 1978 & 0.737 & 0.073 & 0.219 & - \\
\hline Cs-137 & TREAT-2 & 1978 & 0.854 & 0.074 & 0.222 & - \\
\hline Cs-137 & TREAT-3 & 1978 & 0.732 & 0.063 & 0.189 & - \\
\hline Cs-137 & TREAT-4 & 1978 & 0.772 & 0.076 & 0.228 & - \\
\hline Cs-137 & EBRII-10 & 1986 & 0.78 & 0.08 & 0.24 & - \\
\hline Cs-137 & EBRII-11 & 1986 & 1.26 & 0.1 & 0.3 & - \\
\hline Cs-137 & EBRII-12 & 1986 & 0.57 & 0.07 & 0.21 & 一 \\
\hline Cs-137 & EBRII-13 & 1986 & 0.55 & 0.07 & 0.21 & 一 \\
\hline Cs-137 & EBRII-14 & 1986 & 1.13 & 0.09 & 0.27 & - \\
\hline Cs-137 & EBRII-15 & 1986 & 1.25 & 0.1 & 0.3 & - \\
\hline Cs-137 & EBRII-16 & 1986 & -0.02 & 0.05 & 0.15 & $\mathrm{U}$ \\
\hline Cs-137 & EBRII-17 & 1986 & 1.17 & 0.09 & 0.27 & - \\
\hline Cs-137 & EBRII-2 & 1986 & 0.81 & 0.08 & 0.24 & - \\
\hline Cs-137 & EBRII-3 & 1986 & 0.74 & 0.08 & 0.24 & - \\
\hline Cs-137 & EBRII-4 & 1986 & 0.15 & 0.05 & 0.15 & - \\
\hline Cs-137 & EBRII-5 & 1986 & 0.67 & 0.0700 & 0.21 & 一 \\
\hline Cs-137 & EBRII-6 & 1986 & 1.23 & 0.09 & 0.27 & 一 \\
\hline Cs-137 & EBRII-9 & 1986 & 0.62 & 0.07 & 0.21 & - \\
\hline Cs-137 & TREAT-1 & 1986 & 0.97 & 0.08 & 0.24 & - \\
\hline Cs-137 & TREAT-2 & 1986 & 0.21 & 0.05 & 0.15 & - \\
\hline Cs-137 & TREAT-3 & 1986 & 0.71 & 0.0700 & 0.21 & - \\
\hline Cs-137 & TREAT-4 & 1986 & 0.52 & 0.07 & 0.21 & - \\
\hline Cs-137 & ANL-10 & 1993 & 0.48 & 0.06 & 0.18 & - \\
\hline Cs-137 & ANL-11 & 1993 & 1.99 & 0.11 & 0.33 & 一 \\
\hline Cs-137 & ANL-12 & 1993 & -0.04 & 0.04 & 0.12 & $\mathrm{U}$ \\
\hline Cs-137 & ANL-13 & 1993 & 0.62 & 0.06 & 0.18 & - \\
\hline Cs-137 & ANL-14 & 1993 & 0.73 & 0.06 & 0.18 & - \\
\hline Cs-137 & ANL-15 & 1993 & 0.66 & 0.0600 & 0.18 & 一 \\
\hline
\end{tabular}


Table C-4. (continued).

\begin{tabular}{|c|c|c|c|c|c|c|}
\hline Radionuclide & $\begin{array}{c}\text { Sample } \\
\text { Location }\end{array}$ & Date & $\begin{array}{c}\text { Concentrations } \\
(\mathrm{pCi} / \mathrm{g})\end{array}$ & $\begin{array}{l}\text { Uncertainty } \\
(\mathrm{pCi} / \mathrm{g})\end{array}$ & $\begin{array}{l}\text { MDA } \\
(\mathrm{pCi} / \mathrm{g})\end{array}$ & Flag \\
\hline Cs-137 & ANL-16 & 1993 & 1.64 & 0.01 & 0.03 & - \\
\hline Cs-137 & ANL-17 & 1993 & 0.83 & 0.07 & 0.21 & 一 \\
\hline Cs-137 & ANL-2 & 1993 & 1.29 & 0.08 & 0.24 & - \\
\hline Cs-137 & ANL-3 & 1993 & 0.68 & 0.0600 & 0.18 & - \\
\hline Cs-137 & ANL-4 & 1993 & 0.34 & 0.05 & 0.15 & - \\
\hline Cs-137 & ANL-5 & 1993 & 0.95 & 0.07 & 0.21 & - \\
\hline Cs-137 & ANL-6 & 1993 & 0.6 & 0.06 & 0.18 & - \\
\hline Cs-137 & ANL-9 & 1993 & 0.55 & 0.06 & 0.18 & - \\
\hline Cs-137 & TREAT-1 & 1993 & 0.38 & 0.05 & 0.15 & - \\
\hline Cs-137 & TREAT-2 & 1993 & 0.09 & 0.04 & 0.12 & $\mathrm{U}$ \\
\hline Cs-137 & TREAT-3 & 1993 & 0.49 & 0.05 & 0.15 & - \\
\hline Cs-137 & TREAT-4 & 1993 & 0.41 & 0.05 & 0.15 & - \\
\hline Cs-137 & EBRII-1 & 2007 & 0.2268 & 0.00892 & 0.02676 & - \\
\hline Cs-137 & EBRII-10 & 2007 & 0.215 & 0.0132 & 0.0396 & - \\
\hline Cs-137 & EBRII-11 & 2007 & 0.2238 & 0.00822 & 0.02466 & - \\
\hline Cs-137 & EBRII-12 & 2007 & 0.1615 & 0.0141 & 0.0423 & 一 \\
\hline Cs-137 & EBRII-13 & 2007 & 0.2122 & 0.011 & 0.033 & 一 \\
\hline Cs-137 & EBRII-15 & 2007 & 0.2252 & 0.00835 & 0.02505 & - \\
\hline Cs-137 & EBRII-16 & 2007 & 0.1775 & 0.00611 & 0.01833 & - \\
\hline Cs-137 & EBRII-17 & 2007 & 0.1324 & 0.00538 & 0.01614 & 一 \\
\hline Cs-137 & EBRII-2 & 2007 & 0.1363 & 0.00845 & 0.02535 & - \\
\hline Cs-137 & EBRII-3 & 2007 & 0.1848 & 0.017 & 0.051 & - \\
\hline Cs-137 & EBRII-4 & 2007 & 0.1333 & 0.0144 & 0.0432 & - \\
\hline Cs-137 & EBRII-5 & 2007 & 0.239 & 0.0116 & 0.0348 & - \\
\hline Cs-137 & EBRII-6 & 2007 & 0.2255 & 0.0083 & 0.0249 & 一 \\
\hline Cs-137 & EBRII-9 & 2007 & 0.1289 & 0.00766 & 0.02298 & 一 \\
\hline Cs-137 & TRT-1 & 2007 & 0.1197 & 0.0105 & 0.0315 & - \\
\hline Cs-137 & TRT-2 & 2007 & 0.2437 & 0.00791 & 0.02373 & - \\
\hline Cs-137 & TRT-3 & 2007 & 0.2056 & 0.0143 & 0.0429 & - \\
\hline Cs-137 & TRT-4 & 2007 & 0.1577 & 0.00901 & 0.02703 & - \\
\hline Cs-137 & EBRII-1 & 2008 & 0.2014 & 0.00515 & 0.01545 & - \\
\hline Cs-137 & EBRII-10 & 2008 & 0.1926 & 0.0058 & 0.0174 & - \\
\hline Cs-137 & EBRII-11 & 2008 & 0.2647 & 0.0056 & 0.0168 & - \\
\hline Cs-137 & EBRII-12 & 2008 & 0.1411 & 0.004225 & 0.012675 & - \\
\hline Cs-137 & EBRII-13 & 2008 & 0.2148 & 0.00615 & 0.01845 & - \\
\hline Cs-137 & EBRII-15 & 2008 & 0.2112 & 0.006 & 0.0165 & - \\
\hline Cs-137 & EBRII-16 & 2008 & 0.191 & 0.00635 & 0.01905 & - \\
\hline
\end{tabular}


Table C-4. (continued).

\begin{tabular}{|c|c|c|c|c|c|c|}
\hline Radionuclide & $\begin{array}{c}\text { Sample } \\
\text { Location }\end{array}$ & Date & $\begin{array}{c}\text { Concentrations } \\
(\mathrm{pCi} / \mathrm{g})\end{array}$ & $\begin{array}{l}\text { Uncertainty } \\
(\mathrm{pCi} / \mathrm{g})\end{array}$ & $\begin{array}{l}\text { MDA } \\
(\mathrm{pCi} / \mathrm{g})\end{array}$ & Flag \\
\hline Cs-137 & EBRII-17 & 2008 & 0.1553 & 0.00464 & 0.01392 & - \\
\hline Cs-137 & EBRII-2 & 2008 & 0.1205 & 0.003515 & 0.010545 & 一 \\
\hline Cs-137 & EBRII-3 & 2008 & 0.1272 & 0.00414 & 0.01242 & - \\
\hline Cs-137 & EBRII-4 & 2008 & 0.1023 & 0.003295 & 0.009885 & - \\
\hline Cs-137 & EBRII-6 & 2008 & 0.2181 & 0.00705 & 0.02115 & - \\
\hline Cs-137 & EBRII-9 & 2008 & 0.1421 & 0.0056 & 0.0168 & - \\
\hline Cs-137 & TRT-1 & 2008 & 0.09886 & 0.002745 & 0.008235 & - \\
\hline Cs-137 & TRT-2 & 2008 & 0.2612 & 0.0082 & 0.0246 & - \\
\hline Cs-137 & TRT-3 & 2008 & 0.1974 & 0.0076 & 0.0228 & - \\
\hline Cs-137 & TRT-4 & 2008 & 0.1349 & 0.004095 & 0.012285 & - \\
\hline Cs-137 & EBRII-1 & 2009 & 0.2814 & 0.00585 & 0.01755 & - \\
\hline Cs-137 & EBRII-10 & 2009 & 0.2547 & 0.0095 & 0.0285 & - \\
\hline Cs-137 & EBRII-11 & 2009 & 0.3112 & 0.0098 & 0.0294 & - \\
\hline Cs-137 & EBRII-12 & 2009 & 0.1878 & 0.00865 & 0.02595 & - \\
\hline Cs-137 & EBRII-13 & 2009 & 0.2443 & 0.0059 & 0.0177 & 一 \\
\hline Cs-137 & EBRII-15 & 2009 & 0.2716 & 0.00575 & 0.01725 & 一 \\
\hline Cs-137 & EBRII-16 & 2009 & 0.2215 & 0.00805 & 0.02415 & 一 \\
\hline Cs-137 & EBRII-17 & 2009 & 0.1905 & 0.0083 & 0.0249 & - \\
\hline Cs-137 & EBRII-5 & 2009 & 0.2751 & 0.01005 & 0.03015 & - \\
\hline Cs-137 & EBRII-6 & 2009 & 0.2571 & 0.0059 & 0.0177 & 一 \\
\hline Cs-137 & EBRII-9 & 2009 & 0.2137 & 0.00995 & 0.02985 & - \\
\hline Cs-137 & TRT-1 & 2009 & 0.1619 & 0.0055 & 0.0165 & - \\
\hline Cs-137 & TRT-2 & 2009 & 0.4195 & 0.00705 & 0.02115 & - \\
\hline Cs-137 & TRT-3 & 2009 & 0.1975 & 0.00153 & 0.00459 & - \\
\hline Cs-137 & TRT-4 & 2009 & 0.1812 & 0.00635 & 0.01905 & 一 \\
\hline Cs-137 & EBRII-10 & 2010 & 0.3749 & 0.0166 & 0.0498 & 一 \\
\hline Cs-137 & EBRII-11 & 2010 & 0.3064 & 0.00935 & 0.02805 & - \\
\hline Cs-137 & EBRII-15 & 2010 & 0.2537 & 0.00875 & 0.02625 & - \\
\hline Cs-137 & EBRII-16 & 2010 & 0.2284 & 0.00666 & 0.01998 & - \\
\hline Cs-137 & EBRII-17 & 2010 & 0.1562 & 0.0074 & 0.0222 & - \\
\hline Cs-137 & EBRII-6 & 2010 & 0.2831 & 0.00553 & 0.01659 & - \\
\hline Cs-137 & EBRII-9 & 2010 & 0.2498 & 0.011 & 0.033 & - \\
\hline Cs-137 & TRT-1 & 2010 & 0.1833 & 0.0101 & 0.0303 & - \\
\hline Cs-137 & TRT-2 & 2010 & 0.3813 & 0.00872 & 0.02616 & - \\
\hline Cs-137 & TRT-3 & 2010 & 0.1407 & 0.011 & 0.0315 & - \\
\hline Cs-137 & TRT-4 & 2010 & 0.2135 & 0.00784 & 0.02352 & - \\
\hline Cs-137 & EBRII-1 & 2011 & 0.54 & 0.0154 & 0.0462 & - \\
\hline
\end{tabular}


Table C-4. (continued).

\begin{tabular}{|c|c|c|c|c|c|c|}
\hline Radionuclide & $\begin{array}{c}\text { Sample } \\
\text { Location }\end{array}$ & Date & $\begin{array}{c}\text { Concentrations } \\
(\mathrm{pCi} / \mathrm{g})\end{array}$ & $\begin{array}{l}\text { Uncertainty } \\
(\mathrm{pCi} / \mathrm{g})\end{array}$ & $\begin{array}{l}\text { MDA } \\
(\mathrm{pCi} / \mathrm{g})\end{array}$ & Flag \\
\hline Cs-137 & EBRII-10 & 2011 & 0.5708 & 0.0203 & 0.0609 & - \\
\hline Cs-137 & EBRII-11 & 2011 & 0.5376 & 0.0128 & 0.0384 & 一 \\
\hline Cs-137 & EBRII-12 & 2011 & 0.4818 & 0.0125 & 0.0375 & - \\
\hline Cs-137 & EBRII-13 & 2011 & 0.5337 & 0.011 & 0.033 & - \\
\hline Cs-137 & EBRII-15 & 2011 & 0.6565 & 0.0133 & 0.0399 & - \\
\hline Cs-137 & EBRII-16 & 2011 & 0.5303 & 0.0132 & 0.0396 & - \\
\hline Cs-137 & EBRII-17 & 2011 & 0.4457 & 0.0153 & 0.0459 & - \\
\hline Cs-137 & EBRII-2 & 2011 & 0.3065 & 0.0122 & 0.0366 & - \\
\hline Cs-137 & EBRII-3 & 2011 & 0.4099 & 0.0144 & 0.0432 & - \\
\hline Cs-137 & EBRII-4 & 2011 & 0.1374 & 0.0103 & 0.0309 & - \\
\hline Cs-137 & EBRII-6 & 2011 & 0.5188 & 0.0157 & 0.0471 & - \\
\hline Cs-137 & TRT-1 & 2011 & 0.4226 & 0.0122 & 0.0366 & - \\
\hline Cs-137 & TRT-2 & 2011 & 0.9707 & 0.0258 & 0.0774 & - \\
\hline Cs-137 & TRT-3 & 2011 & 0.2931 & 0.0125 & 0.0375 & - \\
\hline Cs-137 & TRT-4 & 2011 & 0.1712 & 0.0106 & 0.0318 & 一 \\
\hline Cs-137 & EBRII-10 & 2012 & 0.1389 & 0.00373 & 0.01119 & 一 \\
\hline Cs-137 & EBRII-11 & 2012 & 0.2248 & 0.00548 & 0.01644 & 一 \\
\hline Cs-137 & EBRII-12 & 2012 & 0.2016 & 0.00704 & 0.02112 & - \\
\hline Cs-137 & EBRII-16 & 2012 & 0.1457 & 0.00526 & 0.01578 & - \\
\hline Cs-137 & EBRII-2 & 2012 & 0.1393 & 0.006 & 0.01938 & - \\
\hline Cs-137 & EBRII-4 & 2012 & 0.09864 & 0.00432 & 0.01296 & - \\
\hline Cs-137 & TRT-1 & 2012 & 0.142 & 0.00786 & 0.02358 & - \\
\hline Cs-137 & TRT-3 & 2012 & 0.1926 & 0.00522 & 0.01566 & - \\
\hline Cs-137 & EBRII-10 & 2013 & 0.1459 & 0.00993 & 0.0298 & - \\
\hline Cs-137 & EBRII-11 & 2013 & 0.2687 & 0.0176 & 0.0528 & 一 \\
\hline Cs-137 & EBRII-13 & 2013 & 0.2252 & 0.0111 & 0.0332 & 一 \\
\hline Cs-137 & TRT-1 & 2013 & 0.05232 & 0.00383 & 0.0115 & - \\
\hline Cs-137 & $\begin{array}{c}2 \text { INCH AIR } \\
- \text { MFC }\end{array}$ & 2014 & 0.05601 & 0.00411 & 0.01233 & - \\
\hline Cs-137 & EBRII-10 & 2014 & 0.1451 & 0.0108 & 0.0324 & - \\
\hline Cs-137 & EBRII-11 & 2014 & 0.233 & 0.00916 & 0.02748 & - \\
\hline Cs-137 & EBRII-2 & 2014 & 0.1299 & 0.00839 & 0.02517 & 一 \\
\hline Cs-137 & EBRII-4 & 2014 & 0.1154 & 0.00578 & 0.01734 & - \\
\hline Cs-137 & EBRII-6 & 2014 & 0.1806 & 0.00471 & 0.01413 & - \\
\hline Cs-137 & TRT-1 & 2014 & 0.2261 & 0.0113 & 0.0339 & - \\
\hline Cs-137 & TRT-2 & 2014 & 0.4927 & 0.00771 & 0.02313 & - \\
\hline Cs-137 & TRT-3 & 2014 & 0.156 & 0.00475 & 0.01425 & - \\
\hline
\end{tabular}


Table C-4. (continued).

\begin{tabular}{|c|c|c|c|c|c|c|}
\hline Radionuclide & $\begin{array}{c}\text { Sample } \\
\text { Location }\end{array}$ & Date & $\begin{array}{c}\text { Concentrations } \\
(\mathrm{pCi} / \mathrm{g})\end{array}$ & $\begin{array}{l}\text { Uncertainty } \\
(\mathrm{pCi} / \mathrm{g})\end{array}$ & $\begin{array}{c}\text { MDA } \\
(\mathrm{pCi} / \mathrm{g})\end{array}$ & Flag \\
\hline Cs-137 & TRT-4 & 2014 & 0.1447 & 0.00915 & 0.02745 & \\
\hline Eu-152 & EBRII-1 & 2011 & -0.01225 & 0.0241 & 0.0723 & $\mathrm{U}$ \\
\hline Eu-152 & EBRII-10 & 2011 & 0.008298 & 0.0304 & 0.0912 & $\mathrm{U}$ \\
\hline $\mathrm{Eu}-152$ & EBRII-11 & 2011 & 0.01887 & 0.0255 & 0.0765 & $\mathrm{U}$ \\
\hline $\mathrm{Eu}-152$ & EBRII-12 & 2011 & 0.01672 & 0.0245 & 0.0735 & $\mathrm{U}$ \\
\hline $\mathrm{Eu}-152$ & EBRII-13 & 2011 & 0.02235 & 0.0257 & 0.0771 & $\mathrm{U}$ \\
\hline $\mathrm{Eu}-152$ & EBRII-15 & 2011 & 0.002671 & 0.0235 & 0.0705 & $\mathrm{U}$ \\
\hline Eu-152 & EBRII-16 & 2011 & 0.008703 & 0.0243 & 0.0729 & $\mathrm{U}$ \\
\hline Eu-152 & EBRII-17 & 2011 & 0.04236 & 0.0299 & 0.0897 & $\mathrm{U}$ \\
\hline Eu-152 & EBRII-2 & 2011 & 0.01011 & 0.0257 & 0.0771 & $\mathrm{U}$ \\
\hline Eu-152 & EBRII-3 & 2011 & 0.02089 & 0.0276 & 0.0828 & $\mathrm{U}$ \\
\hline Eu-152 & EBRII-4 & 2011 & 0.04107 & 0.0245 & 0.0735 & $\mathrm{U}$ \\
\hline $\mathrm{Eu}-152$ & EBRII-6 & 2011 & -0.0115 & 0.0273 & 0.0819 & $\mathrm{U}$ \\
\hline $\mathrm{Eu}-152$ & TRT-1 & 2011 & 0.0106 & 0.0254 & 0.0762 & $\mathrm{U}$ \\
\hline Eu-152 & TRT-2 & 2011 & 0.07093 & 0.0407 & 0.1221 & $\mathrm{U}$ \\
\hline Eu-152 & TRT-3 & 2011 & -0.000007952 & 0.0379 & 0.1137 & $\mathrm{U}$ \\
\hline Eu-152 & TRT-4 & 2011 & 0.02157 & 0.0453 & 0.1359 & $\mathrm{U}$ \\
\hline $\mathrm{Eu}-152$ & EBRII-10 & 2012 & 0.01293 & 0.0263 & 0.0789 & $\mathrm{U}$ \\
\hline Eu-152 & EBRII-11 & 2012 & 0.04599 & 0.0221 & 0.0663 & $\mathrm{U}$ \\
\hline Eu-152 & EBRII-12 & 2012 & 0.01712 & 0.0224 & 0.0672 & $\mathrm{U}$ \\
\hline Eu-152 & EBRII-16 & 2012 & 0.01687 & 0.0223 & 0.0669 & $\mathrm{U}$ \\
\hline Eu-152 & EBRII-2 & 2012 & 0.01452 & 0.0254 & 0.0762 & $\mathrm{U}$ \\
\hline Eu-152 & EBRII-4 & 2012 & 0.01045 & 0.022 & 0.066 & $\mathrm{U}$ \\
\hline Eu-152 & TRT-1 & 2012 & 0.02634 & 0.026 & 0.078 & $\mathrm{U}$ \\
\hline Eu-152 & TRT-3 & 2012 & 0.01165 & 0.0266 & 0.0798 & $\mathrm{U}$ \\
\hline Eu-152 & EBRII-10 & 2013 & 0.2761 & 0.036 & 0.108 & - \\
\hline Eu-152 & EBRII-11 & 2013 & 0.1451 & 0.0314 & 0.0941 & - \\
\hline Eu-152 & EBRII-13 & 2013 & 0.1446 & 0.0329 & 0.0987 & - \\
\hline Eu-152 & TRT-1 & 2013 & 0.005333 & 0.0150 & 0.0449 & $\mathrm{U}$ \\
\hline Eu-152 & $\begin{array}{c}2 \text { INCH AIR } \\
- \text { MFC }\end{array}$ & 2014 & -0.0235 & 0.0308 & 0.0924 & $\mathrm{U}$ \\
\hline Eu-152 & EBRII-10 & 2014 & -0.01835 & 0.0264 & 0.0792 & $\mathrm{U}$ \\
\hline Eu-152 & EBRII-11 & 2014 & -0.003838 & 0.0244 & 0.0732 & $\mathrm{U}$ \\
\hline Eu-152 & EBRII-2 & 2014 & -0.01183 & 0.0229 & 0.0687 & $\mathrm{U}$ \\
\hline Eu-152 & EBRII-4 & 2014 & 0.02117 & 0.024 & 0.072 & $\mathrm{U}$ \\
\hline Eu-152 & EBRII-6 & 2014 & 0.02967 & 0.0246 & 0.0738 & $\mathrm{U}$ \\
\hline Eu-152 & TRT-1 & 2014 & 0.01435 & 0.0224 & 0.0672 & $\mathrm{U}$ \\
\hline
\end{tabular}


Table C-4. (continued).

\begin{tabular}{|c|c|c|c|c|c|c|}
\hline Radionuclide & $\begin{array}{c}\text { Sample } \\
\text { Location }\end{array}$ & Date & $\begin{array}{c}\text { Concentrations } \\
(\mathrm{pCi} / \mathrm{g})\end{array}$ & $\begin{array}{l}\text { Uncertainty } \\
(\mathrm{pCi} / \mathrm{g})\end{array}$ & $\begin{array}{c}\text { MDA } \\
(\mathrm{pCi} / \mathrm{g})\end{array}$ & Flag \\
\hline Eu-152 & TRT-2 & 2014 & 0.02076 & 0.0254 & 0.0762 & $\mathrm{U}$ \\
\hline $\mathrm{Eu}-152$ & TRT-3 & 2014 & 0.01815 & 0.0274 & 0.0822 & $\mathrm{U}$ \\
\hline Eu-152 & TRT-4 & 2014 & -0.05088 & 0.0457 & 0.1371 & $\mathrm{U}$ \\
\hline $\mathrm{Pu}-238$ & EBRII-11 & 1978 & 0.0004 & 0.0009 & 0.0027 & $\mathrm{U}$ \\
\hline $\mathrm{Pu}-238$ & EBRII-15 & 1978 & 0.0013 & 0.001 & 0.003 & $\mathrm{U}$ \\
\hline $\mathrm{Pu}-238$ & EBRII-17 & 1978 & 0.01 & 0.002 & 0.006 & - \\
\hline $\mathrm{Pu}-238$ & EBRII-3 & 1978 & 0.0011 & 0.001 & 0.003 & $\mathrm{U}$ \\
\hline $\mathrm{Pu}-238$ & EBRII-5 & 1978 & 0.0007 & 0.0009 & 0.0027 & $\mathrm{U}$ \\
\hline $\mathrm{Pu}-238$ & EBRII-7 & 1978 & 0.0006 & 0.001 & 0.003 & $\mathrm{U}$ \\
\hline $\mathrm{Pu}-238$ & EBRII-8 & 1978 & 0.0018 & 0.001 & 0.003 & $\mathrm{U}$ \\
\hline $\mathrm{Pu}-238$ & TREAT-1 & 1978 & 0.0007 & 0.0007 & 0.0021 & $\mathrm{U}$ \\
\hline $\mathrm{Pu}-238$ & TREAT-3 & 1978 & 0.0008 & 0.0007 & 0.0021 & $\mathrm{U}$ \\
\hline $\mathrm{Pu}-239 / 240$ & EBRII-11 & 1978 & 0.019 & 0.003 & 0.009 & - \\
\hline $\mathrm{Pu}-239 / 240$ & EBRII-15 & 1978 & 0.026 & 0.003 & 0.009 & 一 \\
\hline $\mathrm{Pu}-239 / 240$ & EBRII-17 & 1978 & 0.009 & 0.002 & 0.006 & 一 \\
\hline $\mathrm{Pu}-239 / 240$ & EBRII-3 & 1978 & 0.019 & 0.003 & 0.009 & 一 \\
\hline $\mathrm{Pu}-239 / 240$ & EBRII-5 & 1978 & 0.022 & 0.003 & 0.009 & 一 \\
\hline $\mathrm{Pu}-239 / 240$ & EBRII-7 & 1978 & 0.023 & 0.003 & 0.009 & - \\
\hline $\mathrm{Pu}-239 / 240$ & EBRII-8 & 1978 & 0.032 & 0.003 & 0.009 & - \\
\hline $\mathrm{Pu}-239 / 240$ & TREAT-1 & 1978 & 0.015 & 0.002 & 0.006 & - \\
\hline $\mathrm{Pu}-239 / 240$ & TREAT-3 & 1978 & 0.013 & 0.002 & 0.006 & - \\
\hline Sb-125 & EBRII-1 & 2011 & -0.09004 & 0.0355 & 0.1065 & $\mathrm{U}$ \\
\hline $\mathrm{Sb}-125$ & EBRII-10 & 2011 & -0.06733 & 0.0437 & 0.1311 & $\mathrm{U}$ \\
\hline $\mathrm{Sb}-125$ & EBRII-11 & 2011 & 0.05013 & 0.0346 & 0.1038 & $\mathrm{U}$ \\
\hline $\mathrm{Sb}-125$ & EBRII-12 & 2011 & 0.03962 & 0.035 & 0.105 & $\mathrm{U}$ \\
\hline $\mathrm{Sb}-125$ & EBRII-13 & 2011 & 0.04783 & 0.0374 & 0.1122 & $\mathrm{U}$ \\
\hline $\mathrm{Sb}-125$ & EBRII-15 & 2011 & 0.1051 & 0.0264 & 0.0792 & - \\
\hline $\mathrm{Sb}-125$ & EBRII-16 & 2011 & 0.08654 & 0.0329 & 0.0987 & $\mathrm{U}$ \\
\hline $\mathrm{Sb}-125$ & EBRII-17 & 2011 & 0.06741 & 0.04 & 0.12 & $\mathrm{U}$ \\
\hline $\mathrm{Sb}-125$ & EBRII-2 & 2011 & 0.05414 & 0.0312 & 0.0936 & $\mathrm{U}$ \\
\hline $\mathrm{Sb}-125$ & EBRII-3 & 2011 & 0.03951 & 0.043 & 0.129 & $\mathrm{U}$ \\
\hline $\mathrm{Sb}-125$ & EBRII-4 & 2011 & 0.03633 & 0.0352 & 0.1056 & $\mathrm{U}$ \\
\hline $\mathrm{Sb}-125$ & EBRII-6 & 2011 & 0.214 & 0.0316 & 0.0948 & - \\
\hline $\mathrm{Sb}-125$ & TRT-1 & 2011 & 0.008143 & 0.0352 & 0.1056 & $\mathrm{U}$ \\
\hline $\mathrm{Sb}-125$ & TRT-2 & 2011 & 0.09314 & 0.0486 & 0.1458 & $\mathrm{U}$ \\
\hline $\mathrm{Sb}-125$ & TRT-3 & 2011 & -0.02426 & 0.0481 & 0.1443 & $\mathrm{U}$ \\
\hline $\mathrm{Sb}-125$ & TRT-4 & 2011 & -0.0157 & 0.0546 & 0.1638 & $\mathrm{U}$ \\
\hline
\end{tabular}


Table C-4. (continued).

\begin{tabular}{|c|c|c|c|c|c|c|}
\hline Radionuclide & $\begin{array}{l}\text { Sample } \\
\text { Location }\end{array}$ & Date & $\begin{array}{c}\text { Concentrations } \\
(\mathrm{pCi} / \mathrm{g})\end{array}$ & $\begin{array}{l}\text { Uncertainty } \\
(\mathrm{pCi} / \mathrm{g})\end{array}$ & $\begin{array}{c}\text { MDA } \\
(\mathrm{pCi} / \mathrm{g})\end{array}$ & Flag \\
\hline $\mathrm{Sb}-125$ & EBRII-10 & 2012 & 0.03198 & 0.0348 & 0.1044 & $\mathrm{U}$ \\
\hline $\mathrm{Sb}-125$ & EBRII-11 & 2012 & 0.001256 & 0.0348 & 0.1044 & $\mathrm{U}$ \\
\hline $\mathrm{Sb}-125$ & EBRII-12 & 2012 & -0.004069 & 0.0289 & 0.0867 & $\mathrm{U}$ \\
\hline $\mathrm{Sb}-125$ & EBRII-16 & 2012 & -0.01808 & 0.0285 & 0.0855 & $\mathrm{U}$ \\
\hline $\mathrm{Sb}-125$ & EBRII-2 & 2012 & -0.01158 & 0.033 & 0.099 & $\mathrm{U}$ \\
\hline $\mathrm{Sb}-125$ & EBRII-4 & 2012 & 0.01805 & 0.0252 & 0.0756 & $\mathrm{U}$ \\
\hline $\mathrm{Sb}-125$ & TRT-1 & 2012 & -0.01512 & 0.0361 & 0.1083 & $\mathrm{U}$ \\
\hline $\mathrm{Sb}-125$ & TRT-3 & 2012 & 0.00008154 & 0.0359 & 0.1077 & $\mathrm{U}$ \\
\hline $\mathrm{Sb}-125$ & EBRII-10 & 2013 & 0.1831 & 0.0403 & 0.121 & - \\
\hline $\mathrm{Sb}-125$ & EBRII-11 & 2013 & 0.3933 & 0.0613 & 0.184 & - \\
\hline $\mathrm{Sb}-125$ & EBRII-13 & 2013 & 0.2261 & 0.0443 & 0.133 & - \\
\hline $\mathrm{Sb}-125$ & TRT-1 & 2013 & 0.04844 & 0.0135 & 0.0405 & - \\
\hline $\mathrm{Sb}-125$ & $\begin{array}{c}2 \text { INCH AIR } \\
- \text { MFC }\end{array}$ & 2014 & 0.02261 & 0.0499 & 0.1497 & $\mathrm{U}$ \\
\hline $\mathrm{Sb}-125$ & EBRII-10 & 2014 & -0.01579 & 0.0505 & 0.1515 & $\mathrm{U}$ \\
\hline $\mathrm{Sb}-125$ & EBRII-11 & 2014 & -0.1543 & 0.0423 & 0.1269 & $\mathrm{U}$ \\
\hline $\mathrm{Sb}-125$ & EBRII-2 & 2014 & -0.1268 & 0.045 & 0.135 & $\mathrm{U}$ \\
\hline $\mathrm{Sb}-125$ & EBRII-4 & 2014 & -0.1258 & 0.0397 & 0.1191 & $\mathrm{U}$ \\
\hline $\mathrm{Sb}-125$ & EBRII-6 & 2014 & -0.03506 & 0.0448 & 0.1344 & $\mathrm{U}$ \\
\hline $\mathrm{Sb}-125$ & TRT-1 & 2014 & -0.1017 & 0.0415 & 0.1245 & $\mathrm{U}$ \\
\hline $\mathrm{Sb}-125$ & TRT-2 & 2014 & -0.1452 & 0.0484 & 0.1452 & $\mathrm{U}$ \\
\hline $\mathrm{Sb}-125$ & TRT-3 & 2014 & -0.1274 & 0.0495 & 0.1485 & $\mathrm{U}$ \\
\hline $\mathrm{Sb}-125$ & TRT-4 & 2014 & -0.003347 & 0.0637 & 0.1911 & $\mathrm{U}$ \\
\hline Sr-90 & EBRII-11 & 1978 & 0.66 & 0.05 & 0.15 & - \\
\hline Sr-90 & EBRII-15 & 1978 & 0.64 & 0.05 & 0.15 & - \\
\hline Sr-90 & EBRII-17 & 1978 & 0.48 & 0.04 & 0.12 & - \\
\hline Sr-90 & EBRII-3 & 1978 & 0.45 & 0.04 & 0.12 & 一 \\
\hline Sr-90 & EBRII-5 & 1978 & 0.53 & 0.05 & 0.15 & 一 \\
\hline Sr-90 & EBRII-7 & 1978 & 0.56 & 0.05 & 0.15 & - \\
\hline Sr-90 & EBRII-8 & 1978 & 0.58 & 0.05 & 0.15 & - \\
\hline Sr-90 & TREAT-1 & 1978 & 0.74 & 0.05 & 0.15 & - \\
\hline Sr-90 & TREAT-3 & 1978 & 0.56 & 0.05 & 0.15 & - \\
\hline U-234 & EBRII-1 & 2007 & 144.1 & 4.05 & 12.15 & - \\
\hline U-234 & EBRII-10 & 2007 & 123.3 & 3.75 & 11.25 & - \\
\hline U-234 & EBRII-11 & 2007 & 152.1 & 4.01 & 12.03 & - \\
\hline U-234 & EBRII-12 & 2007 & 34.9 & 3.65 & 10.95 & - \\
\hline U-234 & EBRII-13 & 2007 & 120.5 & 3.92 & 11.76 & - \\
\hline
\end{tabular}


Table C-4. (continued).

\begin{tabular}{|c|c|c|c|c|c|c|}
\hline Radionuclide & $\begin{array}{c}\text { Sample } \\
\text { Location }\end{array}$ & Date & $\begin{array}{c}\text { Concentrations } \\
(\mathrm{pCi} / \mathrm{g})\end{array}$ & $\begin{array}{l}\text { Uncertainty } \\
(\mathrm{pCi} / \mathrm{g})\end{array}$ & $\begin{array}{c}\text { MDA } \\
(\mathrm{pCi} / \mathrm{g})\end{array}$ & Flag \\
\hline U-234 & EBRII-15 & 2007 & 60.3 & 2.92 & 8.76 & - \\
\hline $\mathrm{U}-234$ & EBRII-16 & 2007 & 75.02 & 2.75 & 8.25 & 一 \\
\hline U-234 & EBRII-17 & 2007 & 0 & 3.42 & 10.26 & $\mathrm{U}$ \\
\hline U-234 & EBRII-2 & 2007 & 133.2 & 3.9 & 11.7 & - \\
\hline U-234 & EBRII-3 & 2007 & 123.3 & 3.84 & 11.52 & - \\
\hline U-234 & EBRII-4 & 2007 & 76.29 & 3.35 & 10.05 & - \\
\hline U-234 & EBRII-5 & 2007 & 192.2 & 4.62 & 13.86 & - \\
\hline U-234 & EBRII-6 & 2007 & 134.4 & 4.26 & 12.78 & - \\
\hline U-234 & EBRII-9 & 2007 & 134.3 & 3.72 & 11.16 & - \\
\hline U-234 & TRT-1 & 2007 & 133 & 3.93 & 11.79 & - \\
\hline U-234 & TRT-2 & 2007 & 186.2 & 4.68 & 14.04 & - \\
\hline U-234 & TRT-3 & 2007 & 238.8 & 5.23 & 15.69 & - \\
\hline $\mathrm{U}-234$ & TRT-4 & 2007 & 328.9 & 6.64 & 19.92 & - \\
\hline U-234 & EBRII-10 & 2008 & 28.1 & 1.71 & 5.13 & - \\
\hline $\mathrm{U}-234$ & EBRII-11 & 2008 & 27.18 & 1.75 & 5.25 & - \\
\hline $\mathrm{U}-234$ & EBRII-12 & 2008 & 15.59 & 2.565 & 7.695 & - \\
\hline U-234 & EBRII-13 & 2008 & 22.12 & 2.25 & 6.75 & 一 \\
\hline U-234 & EBRII-15 & 2008 & 9.163 & 2.635 & 7.905 & - \\
\hline U-234 & EBRII-16 & 2008 & 23.63 & 2.09 & 6.27 & - \\
\hline U-234 & EBRII-17 & 2008 & 7.772 & 2.5 & 7.5 & 一 \\
\hline U-234 & EBRII-2 & 2008 & 15.72 & 2.125 & 6.375 & - \\
\hline U-234 & EBRII-3 & 2008 & 3.874 & 2.345 & 7.035 & $\mathrm{U}$ \\
\hline U-234 & EBRII-4 & 2008 & 20.1 & 2.115 & 6.345 & - \\
\hline U-234 & EBRII-6 & 2008 & 30.19 & 1.805 & 5.415 & - \\
\hline U-234 & TRT-1 & 2008 & 15.61 & 2.595 & 7.785 & 一 \\
\hline U-234 & TRT-2 & 2008 & 37.03 & 2.395 & 7.185 & 一 \\
\hline U-234 & TRT-3 & 2008 & 50.14 & 2.74 & 8.22 & - \\
\hline U-234 & TRT-4 & 2008 & 0 & 3.865 & 11.595 & $\mathrm{U}$ \\
\hline U-234 & EBRII-1 & 2009 & -4.816 & 2.82 & 8.46 & $\mathrm{U}$ \\
\hline U-234 & EBRII-10 & 2009 & -0.5302 & 2.895 & 8.685 & $\mathrm{U}$ \\
\hline U-234 & EBRII-12 & 2009 & 2.552 & 5.95 & 17.85 & $\mathrm{U}$ \\
\hline U-234 & EBRII-13 & 2009 & 5.912 & 2.46 & 7.38 & $\mathrm{U}$ \\
\hline U-234 & EBRII-5 & 2009 & -1.419 & 7.3 & 21.9 & $\mathrm{U}$ \\
\hline U-234 & EBRII-9 & 2009 & 1.283 & 6.35 & 19.05 & U \\
\hline U-234 & TRT-2 & 2009 & 6.44 & 2.945 & 8.835 & $\mathrm{U}$ \\
\hline U-234 & TRT-4 & 2009 & 8.025 & 3.71 & 11.13 & $\mathrm{U}$ \\
\hline U-234 & EBRII-10 & 2010 & 40.25 & 2.33 & 6.99 & - \\
\hline
\end{tabular}


Table C-4. (continued).

\begin{tabular}{|c|c|c|c|c|c|c|}
\hline Radionuclide & $\begin{array}{l}\text { Sample } \\
\text { Location }\end{array}$ & Date & $\begin{array}{c}\text { Concentrations } \\
(\mathrm{pCi} / \mathrm{g})\end{array}$ & $\begin{array}{l}\text { Uncertainty } \\
(\mathrm{pCi} / \mathrm{g})\end{array}$ & $\begin{array}{c}\text { MDA } \\
(\mathrm{pCi} / \mathrm{g})\end{array}$ & Flag \\
\hline $\mathrm{U}-234$ & EBRII-11 & 2010 & 57.83 & 2.84 & 8.52 & - \\
\hline U-234 & EBRII-15 & 2010 & 12.3 & 2.31 & 6.93 & 一 \\
\hline $\mathrm{U}-234$ & EBRII-16 & 2010 & 40.33 & 2.17 & 6.51 & - \\
\hline U-234 & EBRII-17 & 2010 & 6.782 & 2.07 & 6.21 & - \\
\hline U-234 & EBRII-6 & 2010 & 40.06 & 2.4 & 7.2 & - \\
\hline $\mathrm{U}-234$ & EBRII-9 & 2010 & 92.51 & 3.28 & 9.84 & 一 \\
\hline U-234 & TRT-1 & 2010 & 95.38 & 4.44 & 13.32 & - \\
\hline U-234 & TRT-3 & 2010 & 44.39 & 3.04 & 9.12 & - \\
\hline $\mathrm{U}-234$ & TRT-4 & 2010 & 137.9 & 4.52 & 13.56 & - \\
\hline U-234 & EBRII-1 & 2011 & 10.31 & 73.6 & 220.8 & $\mathrm{U}$ \\
\hline U-234 & EBRII-10 & 2011 & -0.5349 & 72.4 & 217.2 & $\mathrm{U}$ \\
\hline U-234 & EBRII-11 & 2011 & -17.54 & 62.5 & 187.5 & $\mathrm{U}$ \\
\hline U-234 & EBRII-12 & 2011 & -21.25 & 66 & 198 & $\mathrm{U}$ \\
\hline $\mathrm{U}-234$ & EBRII-13 & 2011 & 31.99 & 66.5 & 199.5 & $\mathrm{U}$ \\
\hline U-234 & EBRII-15 & 2011 & 40.66 & 58.4 & 175.2 & $\mathrm{U}$ \\
\hline $\mathrm{U}-234$ & EBRII-16 & 2011 & -66.2 & 72.6 & 217.8 & $\mathrm{U}$ \\
\hline $\mathrm{U}-234$ & EBRII-17 & 2011 & -42.4 & 77.2 & 231.6 & $\mathrm{U}$ \\
\hline U-234 & EBRII-2 & 2011 & 22.77 & 67.2 & 201.6 & $\mathrm{U}$ \\
\hline U-234 & EBRII-3 & 2011 & -36.86 & 80.3 & 240.9 & $\mathrm{U}$ \\
\hline U-234 & EBRII-4 & 2011 & -2.58 & 67.4 & 202.2 & $\mathrm{U}$ \\
\hline U-234 & EBRII-6 & 2011 & 2.353 & 74 & 222 & $\mathrm{U}$ \\
\hline U-234 & TRT-1 & 2011 & -7.458 & 68.5 & 205.5 & $\mathrm{U}$ \\
\hline U-234 & TRT-3 & 2011 & -44.36 & 108 & 324 & $\mathrm{U}$ \\
\hline U-234 & EBRII-10 & 2012 & 9.61 & 26.1 & 78.3 & $\mathrm{U}$ \\
\hline U-234 & EBRII-11 & 2012 & 14.68 & 16.3 & 48.9 & $\mathrm{U}$ \\
\hline U-234 & EBRII-12 & 2012 & 12.45 & 22.6 & 67.8 & $\mathrm{U}$ \\
\hline U-234 & EBRII-16 & 2012 & -4.269 & 21.6 & 64.8 & $\mathrm{U}$ \\
\hline U-234 & EBRII-2 & 2012 & 17.34 & 24.2 & 72.6 & $\mathrm{U}$ \\
\hline U-234 & EBRII-4 & 2012 & 9.5 & 15.3 & 45.9 & $\mathrm{U}$ \\
\hline U-234 & TRT-1 & 2012 & 17.22 & 25.3 & 75.9 & $\mathrm{U}$ \\
\hline U-234 & TRT-3 & 2012 & -3.504 & 20.7 & 62.1 & $\mathrm{U}$ \\
\hline U-234 & EBRII-10 & 2013 & 3.689 & 31.13 & 93.4 & $\mathrm{U}$ \\
\hline U-234 & EBRII-11 & 2013 & 36.51 & 42.33 & 127 & $\mathrm{U}$ \\
\hline U-234 & EBRII-13 & 2013 & 84.44 & 35.67 & 107 & $\mathrm{U}$ \\
\hline U-234 & TRT-1 & 2013 & -0.7145 & 39.33 & 118 & $\mathrm{U}$ \\
\hline U-234 & $\begin{array}{c}2 \text { INCH AIR } \\
- \text { MFC }\end{array}$ & 2014 & -51.56 & 87.3 & 261.9 & $\mathrm{U}$ \\
\hline
\end{tabular}


Table C-4. (continued).

\begin{tabular}{|c|c|c|c|c|c|c|}
\hline Radionuclide & $\begin{array}{c}\text { Sample } \\
\text { Location }\end{array}$ & Date & $\begin{array}{c}\text { Concentrations } \\
(\mathrm{pCi} / \mathrm{g})\end{array}$ & $\begin{array}{l}\text { Uncertainty } \\
(\mathrm{pCi} / \mathrm{g})\end{array}$ & $\begin{array}{c}\text { MDA } \\
(\mathrm{pCi} / \mathrm{g})\end{array}$ & Flag \\
\hline $\mathrm{U}-234$ & EBRII-10 & 2014 & -26.91 & 84.6 & 253.8 & $\mathrm{U}$ \\
\hline $\mathrm{U}-234$ & EBRII-11 & 2014 & 66.28 & 62.4 & 187.2 & $\mathrm{U}$ \\
\hline $\mathrm{U}-234$ & EBRII-2 & 2014 & 25.73 & 59.1 & 177.3 & $\mathrm{U}$ \\
\hline U-234 & EBRII-4 & 2014 & 10.13 & 54.9 & 164.7 & $\mathrm{U}$ \\
\hline $\mathrm{U}-234$ & EBRII-6 & 2014 & 29.62 & 63.7 & 191.1 & $\mathrm{U}$ \\
\hline U-234 & TRT-1 & 2014 & -48.9 & 65.9 & 197.7 & $\mathrm{U}$ \\
\hline U-234 & TRT-2 & 2014 & -20.41 & 65.7 & 197.1 & $\mathrm{U}$ \\
\hline U-234 & TRT-3 & 2014 & -0.06759 & 64.5 & 193.5 & $\mathrm{U}$ \\
\hline $\mathrm{U}-235$ & EBRII-10 & 2006 & 0.1011 & 0.224 & 0.672 & $\mathrm{U}$ \\
\hline U-235 & EBRII-11 & 2006 & 1.118 & 0.318 & 0.954 & - \\
\hline U-235 & EBRII-12 & 2006 & 0.6175 & 0.237 & 0.711 & $\mathrm{U}$ \\
\hline U-235 & EBRII-13 & 2006 & 0.9659 & 0.755 & 2.265 & $\mathrm{U}$ \\
\hline U-235 & EBRII-15 & 2006 & 2.189 & 0.645 & 1.935 & \\
\hline U-235 & EBRII-16 & 2006 & 0.7855 & 0.277 & 0.831 & $\mathrm{U}$ \\
\hline U-235 & EBRII-17 & 2006 & 1.431 & 0.352 & 1.056 & - \\
\hline U-235 & EBRII-3 & 2006 & 3.29 & 0.71 & 2.13 & - \\
\hline U-235 & EBRII-4 & 2006 & 1.592 & 0.52 & 1.56 & - \\
\hline $\mathrm{U}-235$ & EBRII-5 & 2006 & 1.398 & 0.4225 & 1.2675 & - \\
\hline U-235 & EBRII-6 & 2006 & 1.885 & 0.74 & 2.22 & $\mathrm{U}$ \\
\hline U-235 & EBRII-9 & 2006 & 2.046 & 0.635 & 1.905 & - \\
\hline U-235 & EBRII-1 & 2007 & 0.1872 & 0.108 & 0.324 & $\mathrm{U}$ \\
\hline U-235 & EBRII-10 & 2007 & 0.4 & 0.107 & 0.321 & - \\
\hline U-235 & EBRII-11 & 2007 & 0.428 & 0.121 & 0.363 & - \\
\hline U-235 & EBRII-12 & 2007 & 0.1269 & 0.0658 & 0.1974 & $\mathrm{U}$ \\
\hline U-235 & EBRII-13 & 2007 & 0.3005 & 0.0977 & 0.2931 & - \\
\hline U-235 & EBRII-15 & 2007 & 0.2798 & 0.0881 & 0.2643 & - \\
\hline U-235 & EBRII-16 & 2007 & 0.07988 & 0.0605 & 0.1815 & $\mathrm{U}$ \\
\hline U-235 & EBRII-17 & 2007 & 0.06915 & 0.0585 & 0.1755 & $\mathrm{U}$ \\
\hline U-235 & EBRII-2 & 2007 & 0.2425 & 0.0967 & 0.2901 & $\mathrm{U}$ \\
\hline U-235 & EBRII-3 & 2007 & 0.2854 & 0.0978 & 0.2934 & $\mathrm{U}$ \\
\hline U-235 & EBRII-4 & 2007 & 0.2876 & 0.0942 & 0.2826 & - \\
\hline U-235 & EBRII-5 & 2007 & 0.3074 & 0.1 & 0.3 & - \\
\hline U-235 & EBRII-6 & 2007 & 0.3338 & 0.103 & 0.309 & 一 \\
\hline U-235 & EBRII-9 & 2007 & 0.2883 & 0.0623 & 0.1869 & - \\
\hline U-235 & TRT-1 & 2007 & 0.4693 & 0.108 & 0.324 & 一 \\
\hline U-235 & TRT-2 & 2007 & 0.1302 & 0.0942 & 0.2826 & $\mathrm{U}$ \\
\hline U-235 & TRT-3 & 2007 & 0.0951 & 0.0823 & 0.2469 & $\mathrm{U}$ \\
\hline
\end{tabular}


Table C-4. (continued).

\begin{tabular}{|c|c|c|c|c|c|c|}
\hline Radionuclide & $\begin{array}{c}\text { Sample } \\
\text { Location }\end{array}$ & Date & $\begin{array}{c}\text { Concentrations } \\
(\mathrm{pCi} / \mathrm{g})\end{array}$ & $\begin{array}{l}\text { Uncertainty } \\
(\mathrm{pCi} / \mathrm{g})\end{array}$ & $\begin{array}{c}\text { MDA } \\
(\mathrm{pCi} / \mathrm{g})\end{array}$ & Flag \\
\hline $\mathrm{U}-235$ & TRT-4 & 2007 & 0.5554 & 0.165 & 0.495 & - \\
\hline U-235 & EBRII-10 & 2008 & 0.09696 & 0.06 & 0.18 & $\mathrm{U}$ \\
\hline U-235 & EBRII-11 & 2008 & 0.1669 & 0.0725 & 0.2175 & $\mathrm{U}$ \\
\hline $\mathrm{U}-235$ & EBRII-12 & 2008 & 0.2367 & 0.057 & 0.171 & - \\
\hline U-235 & EBRII-13 & 2008 & 0.09426 & 0.0675 & 0.2025 & $\mathrm{U}$ \\
\hline U-235 & EBRII-15 & 2008 & 0.1744 & 0.0675 & 0.2025 & $\mathrm{U}$ \\
\hline U-235 & EBRII-16 & 2008 & 0.1173 & 0.055 & 0.165 & $\mathrm{U}$ \\
\hline U-235 & EBRII-17 & 2008 & 0.2603 & 0.055 & 0.165 & - \\
\hline $\mathrm{U}-235$ & EBRII-2 & 2008 & 0.1863 & 0.04245 & 0.12735 & - \\
\hline U-235 & EBRII-3 & 2008 & 0.2203 & 0.0645 & 0.1935 & - \\
\hline U-235 & EBRII-4 & 2008 & 0.1224 & 0.0535 & 0.1605 & $\mathrm{U}$ \\
\hline U-235 & EBRII-6 & 2008 & 0.1945 & 0.073 & 0.219 & $\mathrm{U}$ \\
\hline $\mathrm{U}-235$ & TRT-1 & 2008 & 0.09809 & 0.062 & 0.186 & $\mathrm{U}$ \\
\hline U-235 & TRT-2 & 2008 & 0.1318 & 0.0685 & 0.2055 & $\mathrm{U}$ \\
\hline U-235 & TRT-3 & 2008 & 0.1912 & 0.0885 & 0.2655 & $\mathrm{U}$ \\
\hline U-235 & TRT-4 & 2008 & 0.3587 & 0.1125 & 0.3375 & - \\
\hline U-235 & EBRII-1 & 2009 & 0.2908 & 0.063 & 0.189 & - \\
\hline U-235 & EBRII-10 & 2009 & 0.07384 & 0.062 & 0.186 & $\mathrm{U}$ \\
\hline U-235 & EBRII-11 & 2009 & 0.2166 & 0.04895 & 0.14685 & - \\
\hline U-235 & EBRII-12 & 2009 & 0.1495 & 0.0685 & 0.2055 & $\mathrm{U}$ \\
\hline U-235 & EBRII-5 & 2009 & 0.1936 & 0.077 & 0.231 & $\mathrm{U}$ \\
\hline U-235 & EBRII-9 & 2009 & 0.2331 & 0.0695 & 0.2085 & - \\
\hline U-235 & TRT-3 & 2009 & 0.1906 & 0.0625 & 0.1875 & - \\
\hline U-235 & EBRII-10 & 2010 & 0.9797 & 0.236 & 0.708 & - \\
\hline U-235 & EBRII-11 & 2010 & 2.279 & 0.763 & 2.289 & $\mathrm{U}$ \\
\hline U-235 & EBRII-15 & 2010 & 0.2182 & 0.0434 & 0.1302 & 一 \\
\hline U-235 & EBRII-16 & 2010 & 0.2051 & 0.0353 & 0.1059 & - \\
\hline U-235 & EBRII-17 & 2010 & 0.2279 & 0.0483 & 0.1449 & - \\
\hline U-235 & EBRII-6 & 2010 & 0.2819 & 0.0607 & 0.1821 & - \\
\hline U-235 & EBRII-9 & 2010 & 0.2655 & 0.0479 & 0.1437 & - \\
\hline U-235 & TRT-1 & 2010 & 0.9286 & 0.261 & 0.783 & - \\
\hline $\mathrm{U}-235$ & TRT-2 & 2010 & 1.471 & 0.337 & 1.011 & - \\
\hline $\mathrm{U}-235$ & TRT-3 & 2010 & 1.287 & 0.276 & 0.828 & - \\
\hline $\mathrm{U}-235$ & TRT-4 & 2010 & 2.998 & 0.818 & 2.454 & - \\
\hline U-235 & EBRII-1 & 2011 & 0.09718 & 0.167 & 0.501 & $\mathrm{U}$ \\
\hline U-235 & EBRII-10 & 2011 & 0.1761 & 0.189 & 0.567 & $\mathrm{U}$ \\
\hline U-235 & EBRII-11 & 2011 & -0.1681 & 0.169 & 0.507 & $\mathrm{U}$ \\
\hline
\end{tabular}


Table C-4. (continued).

\begin{tabular}{|c|c|c|c|c|c|c|}
\hline Radionuclide & $\begin{array}{c}\text { Sample } \\
\text { Location }\end{array}$ & Date & $\begin{array}{c}\text { Concentrations } \\
(\mathrm{pCi} / \mathrm{g})\end{array}$ & $\begin{array}{l}\text { Uncertainty } \\
(\mathrm{pCi} / \mathrm{g})\end{array}$ & $\begin{array}{c}\text { MDA } \\
(\mathrm{pCi} / \mathrm{g})\end{array}$ & Flag \\
\hline U-235 & EBRII-12 & 2011 & 0.08963 & 0.16 & 0.48 & $\mathrm{U}$ \\
\hline U-235 & EBRII-13 & 2011 & -0.008613 & 0.175 & 0.525 & $\mathrm{U}$ \\
\hline U-235 & EBRII-15 & 2011 & -0.03967 & 0.149 & 0.447 & $\mathrm{U}$ \\
\hline U-235 & EBRII-16 & 2011 & 0.1535 & 0.167 & 0.501 & $\mathrm{U}$ \\
\hline U-235 & EBRII-17 & 2011 & 0.2327 & 0.185 & 0.555 & $\mathrm{U}$ \\
\hline $\mathrm{U}-235$ & EBRII-2 & 2011 & 0.01813 & 0.166 & 0.498 & $\mathrm{U}$ \\
\hline U-235 & EBRII-3 & 2011 & -0.01375 & 0.172 & 0.516 & $\mathrm{U}$ \\
\hline U-235 & EBRII-4 & 2011 & 0.1078 & 0.17 & 0.51 & $\mathrm{U}$ \\
\hline U-235 & EBRII-6 & 2011 & -0.03526 & 0.158 & 0.474 & $\mathrm{U}$ \\
\hline U-235 & TRT-1 & 2011 & -0.1246 & 0.147 & 0.441 & $\mathrm{U}$ \\
\hline U-235 & TRT-2 & 2011 & 0.4271 & 0.226 & 0.678 & $\mathrm{U}$ \\
\hline U-235 & TRT-3 & 2011 & 0.0281 & 0.225 & 0.675 & $\mathrm{U}$ \\
\hline U-235 & TRT-4 & 2011 & -0.1017 & 0.327 & 0.981 & $\mathrm{U}$ \\
\hline U-235 & EBRII-10 & 2012 & -0.002216 & 0.164 & 0.492 & $\mathrm{U}$ \\
\hline U-235 & EBRII-11 & 2012 & 0.106 & 0.147 & 0.441 & $\mathrm{U}$ \\
\hline U-235 & EBRII-12 & 2012 & -0.05327 & 0.143 & 0.429 & $\mathrm{U}$ \\
\hline $\mathrm{U}-235$ & EBRII-16 & 2012 & 0.06877 & 0.14 & 0.42 & $\mathrm{U}$ \\
\hline U-235 & EBRII-2 & 2012 & -0.06067 & 0.16 & 0.48 & $\mathrm{U}$ \\
\hline U-235 & EBRII-4 & 2012 & 0.03299 & 0.136 & 0.408 & $\mathrm{U}$ \\
\hline U-235 & TRT-1 & 2012 & 0.07728 & 0.158 & 0.474 & $\mathrm{U}$ \\
\hline U-235 & TRT-3 & 2012 & 0.02424 & 0.168 & 0.504 & $\mathrm{U}$ \\
\hline U-235 & EBRII-10 & 2013 & 0.3074 & 0.089 & 0.267 & - \\
\hline U-235 & EBRII-11 & 2013 & 0.3676 & 0.0907 & 0.272 & - \\
\hline U-235 & EBRII-13 & 2013 & 0.1954 & 0.0877 & 0.263 & $\mathrm{U}$ \\
\hline $\mathrm{U}-235$ & TRT-1 & 2013 & 0.07505 & 0.096 & 0.289 & $\mathrm{U}$ \\
\hline U-235 & $\begin{array}{c}2 \text { INCH AIR } \\
\text { - MFC }\end{array}$ & 2014 & -0.04416 & 0.2 & 0.6 & $\mathrm{U}$ \\
\hline U-235 & EBRII-10 & 2014 & -0.05775 & 0.196 & 0.588 & $\mathrm{U}$ \\
\hline U-235 & EBRII-11 & 2014 & 0.042 & 0.146 & 0.438 & $\mathrm{U}$ \\
\hline U-235 & EBRII-2 & 2014 & 0.0553 & 0.139 & 0.417 & $\mathrm{U}$ \\
\hline U-235 & EBRII-4 & 2014 & -0.05136 & 0.149 & 0.447 & $\mathrm{U}$ \\
\hline U-235 & EBRII-6 & 2014 & 0.1013 & 0.152 & 0.456 & $\mathrm{U}$ \\
\hline U-235 & TRT-1 & 2014 & 0.02438 & 0.14 & 0.42 & $\mathrm{U}$ \\
\hline U-235 & TRT-2 & 2014 & 0.008722 & 0.16 & 0.48 & $\mathrm{U}$ \\
\hline U-235 & TRT-3 & 2014 & -0.155 & 0.177 & 0.531 & $\mathrm{U}$ \\
\hline U-235 & TRT-4 & 2014 & -0.03618 & 0.294 & 0.882 & $\mathrm{U}$ \\
\hline U-238 & EBRII-10 & 2006 & 19.24 & 3.21 & 9.63 & - \\
\hline
\end{tabular}


Table C-4. (continued).

\begin{tabular}{|c|c|c|c|c|c|c|}
\hline Radionuclide & $\begin{array}{c}\text { Sample } \\
\text { Location }\end{array}$ & Date & $\begin{array}{c}\text { Concentrations } \\
(\mathrm{pCi} / \mathrm{g})\end{array}$ & $\begin{array}{l}\text { Uncertainty } \\
(\mathrm{pCi} / \mathrm{g})\end{array}$ & $\begin{array}{l}\text { MDA } \\
(\mathrm{pCi} / \mathrm{g})\end{array}$ & Flag \\
\hline $\mathrm{U}-238$ & EBRII-11 & 2006 & 14.97 & 3.295 & 9.885 & - \\
\hline $\mathrm{U}-238$ & EBRII-12 & 2006 & 16.92 & 2.75 & 8.25 & 一 \\
\hline U-238 & EBRII-13 & 2006 & 21.59 & 2.82 & 8.46 & - \\
\hline $\mathrm{U}-238$ & EBRII-15 & 2006 & 40.33 & 4.665 & 13.995 & - \\
\hline U-238 & EBRII-16 & 2006 & 16.18 & 2.21 & 6.63 & - \\
\hline $\mathrm{U}-238$ & EBRII-17 & 2006 & 17.08 & 2.675 & 8.025 & - \\
\hline $\mathrm{U}-238$ & EBRII-3 & 2006 & 22.36 & 3.515 & 10.545 & - \\
\hline $\mathrm{U}-238$ & EBRII-4 & 2006 & 30.09 & 3.78 & 11.34 & - \\
\hline $\mathrm{U}-238$ & EBRII-5 & 2006 & 3.921 & 1.145 & 3.435 & - \\
\hline $\mathrm{U}-238$ & EBRII-6 & 2006 & 28.91 & 3.715 & 11.145 & - \\
\hline U-238 & EBRII-9 & 2006 & 14.78 & 2.965 & 8.895 & - \\
\hline $\mathrm{U}-238$ & TRT-4 & 2006 & 24.79 & 3.34 & 10.02 & - \\
\hline U-238 & EBRII-1 & 2007 & 0 & 6.3900 & 19.17 & $\mathrm{U}$ \\
\hline U-238 & EBRII-10 & 2007 & 0.1916 & 5.95 & 17.85 & $\mathrm{U}$ \\
\hline U-238 & EBRII-11 & 2007 & 0 & 5.98 & 17.94 & $\mathrm{U}$ \\
\hline U-238 & EBRII-12 & 2007 & 0 & 5.28 & 15.84 & $\mathrm{U}$ \\
\hline U-238 & EBRII-13 & 2007 & 0.06509 & 6.12 & 18.36 & $\mathrm{U}$ \\
\hline $\mathrm{U}-238$ & EBRII-15 & 2007 & 0.2582 & 4.49 & 13.47 & $\mathrm{U}$ \\
\hline U-238 & EBRII-16 & 2007 & 0 & 4.3 & 12.9 & $\mathrm{U}$ \\
\hline $\mathrm{U}-238$ & EBRII-17 & 2007 & 0 & 4.7 & 14.1 & $\mathrm{U}$ \\
\hline U-238 & EBRII-2 & 2007 & 0.0491 & 6.19 & 18.57 & $\mathrm{U}$ \\
\hline U-238 & EBRII-3 & 2007 & 0 & 6.02 & 18.06 & $\mathrm{U}$ \\
\hline $\mathrm{U}-238$ & EBRII-4 & 2007 & 2.036 & 4.79 & 14.37 & $\mathrm{U}$ \\
\hline U-238 & EBRII-5 & 2007 & 0.2443 & 6.89 & 20.67 & $\mathrm{U}$ \\
\hline U-238 & EBRII-6 & 2007 & 0 & 6.5 & 19.5 & $\mathrm{U}$ \\
\hline U-238 & EBRII-9 & 2007 & 0 & 5.58 & 16.74 & $\mathrm{U}$ \\
\hline U-238 & TRT-1 & 2007 & 0.219 & 6.24 & 18.72 & $\mathrm{U}$ \\
\hline U-238 & TRT-2 & 2007 & 0.1496 & 7.38 & 22.14 & $\mathrm{U}$ \\
\hline U-238 & TRT-3 & 2007 & 0.3932 & 8.28 & 24.84 & $\mathrm{U}$ \\
\hline U-238 & TRT-4 & 2007 & 0 & 10.4 & 31.2 & $\mathrm{U}$ \\
\hline U-238 & EBRII-10 & 2008 & 0 & 2.395 & 7.185 & $\mathrm{U}$ \\
\hline U-238 & EBRII-11 & 2008 & 0 & 2.4200 & 7.26 & $\mathrm{U}$ \\
\hline U-238 & EBRII-12 & 2008 & 0 & 3.6000 & 10.8 & $\mathrm{U}$ \\
\hline U-238 & EBRII-13 & 2008 & 45.31 & 3.035 & 9.105 & - \\
\hline U-238 & EBRII-15 & 2008 & 0.3261 & 3.66 & 10.98 & $\mathrm{U}$ \\
\hline U-238 & EBRII-16 & 2008 & 34.39 & 2.865 & 8.595 & - \\
\hline U-238 & EBRII-17 & 2008 & 5.383 & 3.415 & 10.245 & $\mathrm{U}$ \\
\hline
\end{tabular}


Table C-4. (continued).

\begin{tabular}{|c|c|c|c|c|c|c|}
\hline Radionuclide & $\begin{array}{c}\text { Sample } \\
\text { Location }\end{array}$ & Date & $\begin{array}{c}\text { Concentrations } \\
(\mathrm{pCi} / \mathrm{g})\end{array}$ & $\begin{array}{l}\text { Uncertainty } \\
(\mathrm{pCi} / \mathrm{g})\end{array}$ & $\begin{array}{c}\text { MDA } \\
(\mathrm{pCi} / \mathrm{g})\end{array}$ & Flag \\
\hline $\mathrm{U}-238$ & EBRII-2 & 2008 & 37.58 & 2.85 & 8.55 & 一 \\
\hline $\mathrm{U}-238$ & EBRII-3 & 2008 & 5.168 & 3.305 & 9.915 & $\mathrm{U}$ \\
\hline $\mathrm{U}-238$ & EBRII-4 & 2008 & 38.94 & 2.855 & 8.565 & - \\
\hline $\mathrm{U}-238$ & EBRII-6 & 2008 & 0 & 2.505 & 7.515 & $\mathrm{U}$ \\
\hline $\mathrm{U}-238$ & TRT-1 & 2008 & 7.391 & 3.72 & 11.16 & $\mathrm{U}$ \\
\hline $\mathrm{U}-238$ & TRT-2 & 2008 & 0 & 3.64 & 10.92 & $\mathrm{U}$ \\
\hline $\mathrm{U}-238$ & TRT-3 & 2008 & 0.1232 & 3.98 & 11.94 & $\mathrm{U}$ \\
\hline $\mathrm{U}-238$ & TRT-4 & 2008 & 0 & 5.45 & 16.35 & $\mathrm{U}$ \\
\hline $\mathrm{U}-238$ & EBRII-1 & 2009 & 5.703 & 4.47 & 13.41 & $\mathrm{U}$ \\
\hline $\mathrm{U}-238$ & EBRII-10 & 2009 & 0.3258 & 2.985 & 8.955 & $\mathrm{U}$ \\
\hline U-238 & EBRII-12 & 2009 & 5.237 & 8.5 & 25.5 & $\mathrm{U}$ \\
\hline U-238 & EBRII-5 & 2009 & -0.09104 & 10.4 & 31.2 & $\mathrm{U}$ \\
\hline U-238 & EBRII-9 & 2009 & -0.4916 & 8.9 & 26.7 & $\mathrm{U}$ \\
\hline U-238 & TRT-4 & 2009 & 9.657 & 5.05 & 15.15 & $\mathrm{U}$ \\
\hline U-238 & EBRII-10 & 2010 & 18.25 & 14.1 & 42.3 & $\mathrm{U}$ \\
\hline U-238 & TRT-1 & 2010 & 164.5 & 6.09 & 18.27 & - \\
\hline U-238 & TRT-2 & 2010 & 32.81 & 32.5 & 97.5 & $\mathrm{U}$ \\
\hline $\mathrm{U}-238$ & TRT-3 & 2010 & 87.7 & 4.1 & 12.3 & - \\
\hline U-238 & TRT-4 & 2010 & 123.6 & 46.1 & 138.3 & $\mathrm{U}$ \\
\hline U-238 & EBRII-1 & 2011 & -0.03709 & 0.642 & 1.926 & $\mathrm{U}$ \\
\hline U-238 & EBRII-10 & 2011 & 1.096 & 0.991 & 2.973 & $\mathrm{U}$ \\
\hline U-238 & EBRII-11 & 2011 & 0.8392 & 0.75 & 2.25 & $\mathrm{U}$ \\
\hline U-238 & EBRII-12 & 2011 & 1.204 & 0.733 & 2.199 & $\mathrm{U}$ \\
\hline U-238 & EBRII-13 & 2011 & -0.2632 & 0.788 & 2.364 & $\mathrm{U}$ \\
\hline U-238 & EBRII-15 & 2011 & 1.009 & 0.616 & 1.848 & $\mathrm{U}$ \\
\hline U-238 & EBRII-16 & 2011 & 1.456 & 0.621 & 1.863 & $\mathrm{U}$ \\
\hline U-238 & EBRII-17 & 2011 & 1.329 & 0.942 & 2.826 & $\mathrm{U}$ \\
\hline U-238 & EBRII-2 & 2011 & 0.973 & 0.822 & 2.466 & $\mathrm{U}$ \\
\hline U-238 & EBRII-3 & 2011 & 1.289 & 0.957 & 2.871 & $\mathrm{U}$ \\
\hline U-238 & EBRII-4 & 2011 & 0.8758 & 0.746 & 2.238 & $\mathrm{U}$ \\
\hline U-238 & EBRII-6 & 2011 & 0.02149 & 0.628 & 1.884 & $\mathrm{U}$ \\
\hline U-238 & TRT-1 & 2011 & 0.844 & 0.746 & 2.238 & $\mathrm{U}$ \\
\hline U-238 & TRT-2 & 2011 & 3.826 & 1.08 & 3.24 & - \\
\hline U-238 & TRT-3 & 2011 & 2.269 & 0.93 & 2.79 & $\mathrm{U}$ \\
\hline U-238 & TRT-4 & 2011 & 1.364 & 0.738 & 2.214 & $\mathrm{U}$ \\
\hline $\mathrm{U}-238$ & EBRII-10 & 2012 & 1.594 & 0.861 & 2.583 & $\mathrm{U}$ \\
\hline $\mathrm{U}-238$ & EBRII-11 & 2012 & 0.8505 & 0.847 & 2.541 & $\mathrm{U}$ \\
\hline
\end{tabular}


Table C-4. (continued).

\begin{tabular}{|c|c|c|c|c|c|c|}
\hline Radionuclide & $\begin{array}{c}\text { Sample } \\
\text { Location }\end{array}$ & Date & $\begin{array}{c}\text { Concentrations } \\
(\mathrm{pCi} / \mathrm{g})\end{array}$ & $\begin{array}{c}\text { Uncertainty } \\
(\mathrm{pCi} / \mathrm{g})\end{array}$ & $\begin{array}{c}\text { MDA } \\
(\mathrm{pCi} / \mathrm{g})\end{array}$ & Flag \\
\hline U-238 & EBRII-12 & 2012 & 2.582 & 0.659 & 1.977 & - \\
\hline $\mathrm{U}-238$ & EBRII-16 & 2012 & 0.74 & 0.67 & 2.01 & $\mathrm{U}$ \\
\hline $\mathrm{U}-238$ & EBRII-2 & 2012 & 1.634 & 0.786 & 2.358 & $\mathrm{U}$ \\
\hline $\mathrm{U}-238$ & EBRII-4 & 2012 & 0.3506 & 0.786 & 2.358 & $\mathrm{U}$ \\
\hline $\mathrm{U}-238$ & TRT-1 & 2012 & 1.213 & 0.832 & 2.496 & $\mathrm{U}$ \\
\hline $\mathrm{U}-238$ & TRT-3 & 2012 & 1.967 & 0.859 & 2.577 & $\mathrm{U}$ \\
\hline $\mathrm{U}-238$ & EBRII-10 & 2013 & 8.648 & 2.093 & 6.28 & - \\
\hline $\mathrm{U}-238$ & EBRII-11 & 2013 & 1.855 & 1.717 & 5.15 & $\mathrm{U}$ \\
\hline $\mathrm{U}-238$ & EBRII-13 & 2013 & 11.87 & 2.45 & 7.35 & - \\
\hline $\mathrm{U}-238$ & TRT-1 & 2013 & 0.4803 & 0.393 & 1.18 & $\mathrm{U}$ \\
\hline $\mathrm{U}-238$ & 2 INCH AIR & 2014 & 0.5867 & 0.851 & 2.553 & $\mathrm{U}$ \\
\hline $\mathrm{U}-238$ & EBRII-10 & 2014 & 1.052 & 0.934 & 2.802 & $\mathrm{U}$ \\
\hline $\mathrm{U}-238$ & EBRII-11 & 2014 & 1.361 & 0.72 & 2.16 & $\mathrm{U}$ \\
\hline $\mathrm{U}-238$ & EBRII-2 & 2014 & 0.8123 & 0.845 & 2.535 & $\mathrm{U}$ \\
\hline $\mathrm{U}-238$ & EBRII-4 & 2014 & 3.412 & 1.11 & 3.33 & - \\
\hline $\mathrm{U}-238$ & EBRII-6 & 2014 & 1.07 & 0.813 & 2.439 & $\mathrm{U}$ \\
\hline $\mathrm{U}-238$ & TRT-1 & 2014 & 1.229 & 0.688 & 2.064 & $\mathrm{U}$ \\
\hline $\mathrm{U}-238$ & TRT-2 & 2014 & 1.135 & 0.855 & 2.565 & $\mathrm{U}$ \\
\hline $\mathrm{U}-238$ & TRT-3 & 2014 & 1.824 & 0.864 & 2.592 & $\mathrm{U}$ \\
\hline $\mathrm{U}-238$ & TRT-4 & 2014 & 1.677 & 0.92 & 2.76 & $\mathrm{U}$ \\
\hline
\end{tabular}

Table C-5. Radionuclides concentrations in surface soils associated with RWMC.

\begin{tabular}{|c|c|c|c|l|l|c|}
\hline Radionuclide & $\begin{array}{c}\text { Sample } \\
\text { Location }\end{array}$ & Date & $\begin{array}{c}\text { Concentrations } \\
(\mathrm{pCi} / \mathrm{g})\end{array}$ & $\begin{array}{c}\text { Uncertainty } \\
(\mathrm{pCi} / \mathrm{g})\end{array}$ & $\begin{array}{c}\text { MDA } \\
(\mathrm{pCi} / \mathrm{g})\end{array}$ & Flag \\
\hline Am-241 & $2-3$ & 1972 & 1.84 & 0.09 & 0.27 & - \\
\hline Am-241 & $2-4$ & 1972 & 1.14 & 0.27 & 0.81 & - \\
\hline Am-241 & $2-5$ & 1972 & 0.0094 & 0.140 & 0.42 & U \\
\hline Am-241 & $3-1$ & 1972 & 0.025 & 0.003 & 0.009 & - \\
\hline Am-241 & $3-2$ & 1972 & 0.194 & 0.009 & 0.027 & - \\
\hline Am-241 & $3-3$ & 1972 & 0.716 & 0.036 & 0.108 & - \\
\hline Am-241 & $3-4$ & 1972 & 0.39 & 0.018 & 0.054 & - \\
\hline Am-241 & $3-5$ & 1972 & 0.185 & 0.00675 & 0.02025 & - \\
\hline Am-241 & $2-6$ & 1973 & 0.346 & 0.009 & 0.027 & - \\
\hline Am-241 & $2-7$ & 1973 & 0.019 & 0.002 & 0.006 & - \\
\hline Am-241 & $2-8$ & 1973 & 0.024 & 0.003 & 0.009 & - \\
\hline Am-241 & $3-6$ & 1973 & 0.095 & 0.00450 & 0.0135 & - \\
\hline
\end{tabular}


Table C-5. (continued).

\begin{tabular}{|c|c|c|c|c|c|c|}
\hline Radionuclide & $\begin{array}{c}\text { Sample } \\
\text { Location }\end{array}$ & Date & $\begin{array}{c}\text { Concentrations } \\
(\mathrm{pCi} / \mathrm{g})\end{array}$ & $\begin{array}{l}\text { Uncertainty } \\
(\mathrm{pCi} / \mathrm{g})\end{array}$ & $\begin{array}{c}\mathrm{MDA} \\
(\mathrm{pCi} / \mathrm{g})\end{array}$ & Flag \\
\hline Am-241 & $3-7$ & 1973 & 0.059 & 0.00225 & 0.00675 & - \\
\hline Am-241 & $3-8$ & 1973 & 0.009 & 0.0027 & 0.0081 & - \\
\hline Am-241 & 4-1 & 1973 & 0.176 & 0.0045 & 0.0135 & - \\
\hline Am-241 & $4-2$ & 1973 & 0.114 & 0.009 & 0.027 & - \\
\hline Am-241 & 4-3 & 1973 & 0.059 & 0.00225 & 0.00675 & - \\
\hline Am-241 & $4-4$ & 1973 & 0.021 & 0.00155 & 0.00465 & - \\
\hline Am-241 & $4-5$ & 1973 & 0.02 & 0.0027 & 0.0081 & - \\
\hline Am-241 & $5-1$ & 1973 & 8.4 & 0.315 & 0.945 & - \\
\hline Am-241 & $5-2$ & 1973 & 2.16 & 0.09 & 0.27 & - \\
\hline Am-241 & $5-3$ & 1973 & 0.33 & 0.009 & 0.027 & - \\
\hline Am-241 & $5-4$ & 1973 & 0.126 & 0.009 & 0.027 & - \\
\hline Am-241 & $10-1$ & 1974 & 0.0095 & 0.0018 & 0.0054 & - \\
\hline Am-241 & $5-10$ & 1974 & 0.009 & 0.0011 & 0.0033 & - \\
\hline Am-241 & $5-11$ & 1974 & 0.004 & 0.0018 & 0.0054 & $\mathrm{U}$ \\
\hline Am-241 & $5-12$ & 1974 & 0.0054 & 0.00045 & 0.00135 & - \\
\hline Am-241 & $5-13$ & 1974 & 0.121 & 0.0045 & 0.0135 & - \\
\hline Am-241 & $5-14$ & 1974 & 0.027 & 0.0018 & 0.0054 & - \\
\hline Am-241 & $5-5$ & 1974 & 0.207 & 0.018 & 0.054 & - \\
\hline Am-241 & $5-6$ & 1974 & 0.049 & 0.00225 & 0.00675 & - \\
\hline Am-241 & $5-7$ & 1974 & 0.034 & 0.004 & 0.012 & - \\
\hline Am-241 & $5-8$ & 1974 & 0.086 & 0.0045 & 0.0135 & - \\
\hline Am-241 & $6-1$ & 1974 & 0.04 & 0.009 & 0.027 & - \\
\hline Am-241 & $6-2$ & 1974 & 0.037 & 0.004 & 0.012 & - \\
\hline Am-241 & $6-3$ & 1974 & 0.144 & 0.014 & 0.042 & - \\
\hline Am-241 & $6-4$ & 1974 & 0.037 & 0.0036 & 0.0108 & 一 \\
\hline Am-241 & $6-5$ & 1974 & 0.0072 & 0.0018 & 0.0054 & 一 \\
\hline Am-241 & $6-6$ & 1974 & 0.004 & 0.0007 & 0.0021 & - \\
\hline Am-241 & $7-1$ & 1974 & 0.018 & 0.0045 & 0.0135 & - \\
\hline Am-241 & $7-2$ & 1974 & 0.014 & 0.0018 & 0.0054 & - \\
\hline Am-241 & $7-3$ & 1974 & 0.104 & 0.00225 & 0.00675 & - \\
\hline Am-241 & $7-5$ & 1974 & 0.0117 & 0.003 & 0.009 & - \\
\hline Am-241 & $7-6$ & 1974 & 0.006 & 0.0009 & 0.0027 & - \\
\hline Am-241 & $8-1$ & 1974 & 0.059 & 0.00225 & 0.00675 & - \\
\hline Am-241 & $8-2$ & 1974 & 0.022 & 0.0009 & 0.0027 & - \\
\hline Am-241 & $8-3$ & 1974 & 0.012 & 0.0007 & 0.0021 & - \\
\hline Am-241 & $8-4$ & 1974 & 0.006 & 0.0009 & 0.0027 & - \\
\hline Am-241 & $9-1$ & 1974 & 0.0049 & 0.0009 & 0.0027 & - \\
\hline
\end{tabular}


Table C-5. (continued).

\begin{tabular}{|c|c|c|c|c|c|c|}
\hline Radionuclide & $\begin{array}{l}\text { Sample } \\
\text { Location }\end{array}$ & Date & $\begin{array}{l}\text { Concentrations } \\
(\mathrm{pCi} / \mathrm{g})\end{array}$ & $\begin{array}{l}\text { Uncertainty } \\
\text { (pCi/g) }\end{array}$ & $\begin{array}{l}\mathrm{MDA} \\
(\mathrm{pCi} / \mathrm{g})\end{array}$ & Flag \\
\hline Am-241 & $9-7$ & 1974 & 0.011 & 0.0011 & 0.0033 & - \\
\hline Am-241 & $10-1$ & 1978 & 0.009 & 0.002 & 0.006 & 一 \\
\hline Am-241 & $2-3$ & 1978 & 0.283 & 0.007 & 0.021 & - \\
\hline Am-241 & $2-5$ & 1978 & 0.065 & 0.004 & 0.012 & - \\
\hline Am-241 & $2-7$ & 1978 & 0.011 & 0.001 & 0.003 & - \\
\hline Am-241 & $3-2$ & 1978 & 0.02 & 0.01 & 0.03 & $\mathrm{U}$ \\
\hline Am-241 & $3-6$ & 1978 & 0.013 & 0.002 & 0.006 & - \\
\hline Am-241 & $3-8$ & 1978 & 0.009 & 0.001 & 0.003 & - \\
\hline Am-241 & $4-1$ & 1978 & 0.141 & 0.007 & 0.021 & - \\
\hline Am-241 & $4-3$ & 1978 & 0.02 & 0.003 & 0.009 & - \\
\hline Am-241 & $4-5$ & 1978 & 0.009 & 0.003 & 0.009 & - \\
\hline Am-241 & 4-A & 1978 & 0.007 & 0.0005 & 0.0015 & - \\
\hline Am-241 & 4-B & 1978 & 0.022 & 0.004 & 0.012 & - \\
\hline Am-241 & $4-\mathrm{C}$ & 1978 & 0.022 & 0.003 & 0.009 & - \\
\hline Am-241 & $5-11$ & 1978 & 0.005 & 0.0005 & 0.0015 & - \\
\hline Am-241 & $5-13$ & 1978 & 0.125 & 0.003 & 0.009 & - \\
\hline Am-241 & $5-15$ & 1978 & 0.0003 & 0.00065 & 0.00195 & $\mathrm{U}$ \\
\hline Am-241 & $5-5$ & 1978 & 0.177 & 0.004 & 0.012 & - \\
\hline Am-241 & $5-7$ & 1978 & 0.0066 & 0.001 & 0.003 & - \\
\hline Am-241 & $5-8$ & 1978 & 0.055 & 0.002 & 0.006 & - \\
\hline Am-241 & $6-1$ & 1978 & 0.013 & 0.002 & 0.006 & - \\
\hline Am-241 & $6-3$ & 1978 & 0.026 & 0.003 & 0.009 & - \\
\hline Am-241 & $6-5$ & 1978 & 0.008 & 0.002 & 0.006 & - \\
\hline Am-241 & $6-7$ & 1978 & 0.004 & 0.0005 & 0.0015 & - \\
\hline Am-241 & $7-2$ & 1978 & 0.026 & 0.003 & 0.009 & - \\
\hline Am-241 & $7-4$ & 1978 & 0.026 & 0.003 & 0.009 & - \\
\hline Am-241 & $7-6$ & 1978 & 0.005 & 0.001 & 0.003 & - \\
\hline Am-241 & 7-A & 1978 & 0.02 & 0.003 & 0.009 & - \\
\hline Am-241 & $8-1$ & 1978 & 0.002 & 0.001 & 0.003 & $\mathrm{U}$ \\
\hline Am-241 & $8-3$ & 1978 & 0.019 & 0.003 & 0.009 & - \\
\hline Am-241 & $8-5$ & 1978 & 0.006 & 0.0005 & 0.0015 & - \\
\hline Am-241 & 8-A & 1978 & 0.032 & 0.003 & 0.009 & - \\
\hline Am-241 & 8-B & 1978 & 0.011 & 0.0015 & 0.0045 & - \\
\hline Am-241 & $8-\mathrm{C}$ & 1978 & 0.004 & 0.001 & 0.003 & - \\
\hline Am-241 & $9-A$ & 1978 & 0.003 & 0.001 & 0.003 & - \\
\hline Am-241 & $10-1$ & 1985 & 0.014 & 0.003 & 0.009 & - \\
\hline Am-241 & $2-4$ & 1985 & 0.63 & 0.03 & 0.09 & - \\
\hline
\end{tabular}


Table C-5. (continued).

\begin{tabular}{|c|c|c|c|c|c|c|}
\hline Radionuclide & $\begin{array}{l}\text { Sample } \\
\text { Location }\end{array}$ & Date & $\begin{array}{l}\text { Concentrations } \\
(\mathrm{pCi} / \mathrm{g})\end{array}$ & $\begin{array}{l}\text { Uncertainty } \\
(\mathrm{pCi} / \mathrm{g})\end{array}$ & $\begin{array}{c}\mathrm{MDA} \\
(\mathrm{pCi} / \mathrm{g})\end{array}$ & Flag \\
\hline Am-241 & $3-1$ & 1985 & 0.017 & 0.003 & 0.009 & - \\
\hline Am-241 & $3-4$ & 1985 & 0.47 & 0.02 & 0.06 & - \\
\hline Am-241 & $3-7$ & 1985 & 0.016 & 0.003 & 0.009 & - \\
\hline Am-241 & $4-2$ & 1985 & 0.056 & 0.0025 & 0.0075 & - \\
\hline Am-241 & $4-4$ & 1985 & 0.018 & 0.003 & 0.009 & - \\
\hline Am-241 & 4-A & 1985 & 0 & 0.001 & 0.003 & $\mathrm{U}$ \\
\hline Am-241 & $4-B$ & 1985 & 0.01 & 0.003 & 0.009 & - \\
\hline Am-241 & $4-\mathrm{C}$ & 1985 & 0.012 & 0.003 & 0.009 & - \\
\hline Am-241 & $5-10$ & 1985 & 0.009 & 0.0015 & 0.0045 & - \\
\hline Am-241 & $5-15$ & 1985 & 0.009 & 0.0015 & 0.0045 & - \\
\hline Am-241 & $5-5$ & 1985 & 0.289 & 0.0065 & 0.0195 & 一 \\
\hline Am-241 & $5-7$ & 1985 & 0.024 & 0.003 & 0.009 & 一 \\
\hline Am-241 & $6-1$ & 1985 & 0.046 & 0.004 & 0.012 & - \\
\hline Am-241 & $7-3$ & 1985 & 0.037 & 0.004 & 0.012 & 一 \\
\hline Am-241 & $7-5$ & 1985 & 0.002 & 0.002 & 0.006 & $\mathrm{U}$ \\
\hline Am-241 & $7-7$ & 1985 & 0.004 & 0.001 & 0.003 & - \\
\hline Am-241 & 7-A & 1985 & 0.007 & 0.0015 & 0.0045 & - \\
\hline Am-241 & $8-1$ & 1985 & 0.004 & 0.0020 & 0.006 & $\mathrm{U}$ \\
\hline Am-241 & $8-2$ & 1985 & 0.009 & 0.0015 & 0.0045 & - \\
\hline Am-241 & $8-5$ & 1985 & 0.005 & 0.00100 & 0.003 & - \\
\hline Am-241 & $8-\mathrm{C}$ & 1985 & 0.002 & 0.002 & 0.006 & $\mathrm{U}$ \\
\hline Am-241 & 9-A & 1985 & 0.004 & 0.000666667 & 0.002 & - \\
\hline Am-241 & RWMC 10-1 & 2007 & 0.09503 & 0.0055 & 0.0165 & - \\
\hline Am-241 & RWMC 3-1 & 2007 & 0.5608 & 0.0061 & 0.0183 & - \\
\hline Am-241 & RWMC 5-12 & 2007 & 0.2003 & 0.00458 & 0.01374 & 一 \\
\hline Am-241 & RWMC 5-15 & 2007 & 0.121 & 0.03865 & 0.11595 & 一 \\
\hline Am-241 & RWMC 5-4 & 2007 & 0.2734 & 0.004735 & 0.014205 & - \\
\hline Am-241 & RWMC 6-1 & 2007 & 0.1107 & 0.0403 & 0.1209 & $\mathrm{U}$ \\
\hline Am-241 & RWMC 6-9 & 2007 & 0.008619 & 0.00462 & 0.01386 & $\mathrm{U}$ \\
\hline Am-241 & RWMC 8-1 & 2007 & 0.01002 & 0.0283 & 0.0849 & $\mathrm{U}$ \\
\hline Am-241 & RWMC 10-1 & 2011 & 0.005838 & 0.0997 & 0.2991 & $\mathrm{U}$ \\
\hline Am-241 & RWMC 2-4 & 2011 & 0.8083 & 0.138 & 0.414 & - \\
\hline Am-241 & RWMC 2-6 & 2011 & 0.4427 & 0.107 & 0.321 & - \\
\hline Am-241 & RWMC 2-8 & 2011 & -0.00124 & 0.0939 & 0.2817 & $\mathrm{U}$ \\
\hline Am-241 & RWMC 3-4 & 2011 & 0.3027 & 0.077 & 0.231 & - \\
\hline Am-241 & RWMC 3-6 & 2011 & 0.0676 & 0.095 & 0.2862 & $\mathrm{U}$ \\
\hline Am-241 & RWMC 3-8 & 2011 & 0.000711 & 0.0908 & 0.2724 & $\mathrm{U}$ \\
\hline
\end{tabular}


Table C-5. (continued).

\begin{tabular}{|c|c|c|c|c|c|c|}
\hline Radionuclide & $\begin{array}{l}\text { Sample } \\
\text { Location }\end{array}$ & Date & $\begin{array}{l}\text { Concentrations } \\
(\mathrm{pCi} / \mathrm{g})\end{array}$ & $\begin{array}{l}\text { Uncertainty } \\
(\mathrm{pCi} / \mathrm{g})\end{array}$ & $\begin{array}{c}\mathrm{MDA} \\
(\mathrm{pCi} / \mathrm{g})\end{array}$ & Flag \\
\hline Am-241 & RWMC 4-1 & 2011 & 0.3328 & 0.124 & 0.372 & $\mathrm{U}$ \\
\hline Am-241 & RWMC 4-5 & 2011 & 0.0893 & 0.09260 & 0.2778 & $\mathrm{U}$ \\
\hline Am-241 & RWMC 5-12 & 2011 & -0.09897 & 0.0788 & 0.2364 & $\mathrm{U}$ \\
\hline Am-241 & RWMC 5-4 & 2011 & 1.141 & 0.118 & 0.354 & - \\
\hline Am-241 & RWMC 5-7 & 2011 & 0.06677 & 0.0952 & 0.2856 & $\mathrm{U}$ \\
\hline Am-241 & RWMC 6-1 & 2011 & 0.02223 & 0.0915 & 0.2745 & $\mathrm{U}$ \\
\hline Am-241 & RWMC 6-3 & 2011 & 0.0541 & 0.0931 & 0.2793 & $\mathrm{U}$ \\
\hline Am-241 & RWMC 6-7 & 2011 & 0.04536 & 0.101 & 0.303 & $\mathrm{U}$ \\
\hline Am-241 & RWMC 7-2 & 2011 & 0.2367 & 0.0938 & 0.2814 & $\mathrm{U}$ \\
\hline Am-241 & RWMC 8-1 & 2011 & 0.06851 & 0.0983 & 0.2949 & $\mathrm{U}$ \\
\hline Am-241 & RWMC 8-5 & 2011 & 0.01418 & 0.0936 & 0.2808 & $\mathrm{U}$ \\
\hline Am-241 & RWMC 9-1 & 2011 & 0.04978 & 0.0944 & 0.2832 & $\mathrm{U}$ \\
\hline Am-241 & $5-14$ & 2012 & 0.0018 & 0.069 & 0.207 & $\mathrm{U}$ \\
\hline Am-241 & RWMC 2-4 & 2012 & 0.72 & 0.129 & 0.387 & - \\
\hline Am-241 & RWMC 3-4 & 2012 & 0.9458 & 0.128 & 0.384 & - \\
\hline Am-241 & RWMC 4-1 & 2012 & 0.4169 & 0.123 & 0.369 & - \\
\hline Am-241 & RWMC 5-4 & 2012 & 0.003658 & 0.0468 & 0.1404 & $\mathrm{U}$ \\
\hline Am-241 & RWMC 6-1 & 2012 & 0.01275 & 0.0423 & 0.1269 & $\mathrm{U}$ \\
\hline Am-241 & RWMC 8-1 & 2012 & 0.04549 & 0.0714 & 0.2142 & $\mathrm{U}$ \\
\hline Am-241 & $\begin{array}{l}2 \text { INCH AIR } \\
\text { - RWMC }\end{array}$ & 2013 & 0.02098 & 0.228 & 0.684 & $\mathrm{U}$ \\
\hline Am-241 & $\begin{array}{c}2 \text { INCH AIR } \\
\text { - RWMC }\end{array}$ & 2013 & 0.02098 & 0.076 & 0.228 & $\mathrm{U}$ \\
\hline Am-241 & RWMC 2-4 & 2013 & 15.27 & 0.249 & 0.747 & - \\
\hline Am-241 & RWMC 3-5 & 2013 & 0.04307 & 0.072666667 & 0.218 & $\mathrm{U}$ \\
\hline Am-241 & RWMC 5-7 & 2013 & 0.05765 & 0.0301 & 0.0903 & $\mathrm{U}$ \\
\hline Am-241 & $5-9$ & 2014 & 0.0023 & 0.0156 & 0.0468 & $\mathrm{U}$ \\
\hline Am-241 & 7-B & 2014 & 0.003 & 0.000735 & 0.002205 & - \\
\hline Am-241 & RWMC 2-4 & 2014 & 2.809 & 0.355 & 1.065 & 一 \\
\hline Am-241 & RWMC 2-4 & 2014 & 0.5722 & 0.127 & 0.381 & 一 \\
\hline Am-241 & RWMC 3-4 & 2014 & 0.9339 & 0.168 & 0.504 & 一 \\
\hline Am-241 & RWMC 4-1 & 2014 & 0.8144 & 0.228 & 0.684 & - \\
\hline Am-241 & RWMC 5-4 & 2014 & 4.152 & 0.144 & 0.432 & - \\
\hline Am-241 & RWMC 6-1 & 2014 & 0.7373 & 0.228 & 0.684 & - \\
\hline Am-241 & RWMC 6-3 & 2014 & 0.09979 & 0.102 & 0.306 & $\mathrm{U}$ \\
\hline Co-60 & $2-3$ & 1972 & 0.75 & 0.020 & 0.06 & - \\
\hline Co-60 & $2-4$ & 1972 & 0.33 & 0.005 & 0.015 & - \\
\hline
\end{tabular}


Table C-5. (continued).

\begin{tabular}{|c|c|c|c|c|c|c|}
\hline Radionuclide & $\begin{array}{c}\text { Sample } \\
\text { Location }\end{array}$ & Date & $\begin{array}{c}\text { Concentrations } \\
(\mathrm{pCi} / \mathrm{g})\end{array}$ & $\begin{array}{l}\text { Uncertainty } \\
(\mathrm{pCi} / \mathrm{g})\end{array}$ & $\begin{array}{c}\mathrm{MDA} \\
(\mathrm{pCi} / \mathrm{g})\end{array}$ & Flag \\
\hline Co-60 & $2-5$ & 1972 & 0.04 & 0.0100 & 0.03 & - \\
\hline Co-60 & $3-1$ & 1972 & 0.05 & 0.004 & 0.012 & - \\
\hline Co-60 & $3-2$ & 1972 & 53 & 0.01 & 0.03 & - \\
\hline Co-60 & $3-3$ & 1972 & 0.45 & 0.01 & 0.03 & - \\
\hline Co-60 & $3-4$ & 1972 & 2.1 & 0.1 & 0.3 & - \\
\hline Co-60 & $5-1$ & 1973 & 0.131 & 0.008 & 0.024 & - \\
\hline Co-60 & $5-2$ & 1973 & 0.28 & 0.005 & 0.015 & - \\
\hline Co-60 & $5-3$ & 1973 & 0.113 & 0.0035 & 0.0105 & - \\
\hline Co-60 & $5-4$ & 1973 & 0.31 & 0.005 & 0.015 & - \\
\hline Co-60 & $10-1$ & 1978 & 0 & 0.004 & 0.012 & $\mathrm{U}$ \\
\hline Co-60 & $2-3$ & 1978 & 0.175 & 0.014 & 0.042 & - \\
\hline Co-60 & $2-5$ & 1978 & 0.00913 & 0.00445 & 0.01335 & $\mathrm{U}$ \\
\hline Co-60 & $2-7$ & 1978 & 0.0262 & 0.0092 & 0.0276 & $\mathrm{U}$ \\
\hline Co-60 & $3-2$ & 1978 & 0.0365 & 0.011 & 0.033 & 一 \\
\hline Co-60 & $3-4$ & 1978 & 0.0373 & 0.0085 & 0.0255 & - \\
\hline Co-60 & $3-6$ & 1978 & 0.00596 & 0.011 & 0.033 & $\mathrm{U}$ \\
\hline Co-60 & $3-8$ & 1978 & 0.0441 & 0.0097 & 0.0291 & - \\
\hline Co-60 & $4-3$ & 1978 & -0.0132 & 0.0089 & 0.0267 & $\mathrm{U}$ \\
\hline $\mathrm{Co}-60$ & $4-5$ & 1978 & -0.0137 & 0.00445 & 0.01335 & $\mathrm{U}$ \\
\hline Co-60 & 4-A & 1978 & -0.00422 & 0.00455 & 0.01365 & $\mathrm{U}$ \\
\hline Co-60 & $4-B$ & 1978 & 0.0014 & 0.008 & 0.0243 & $\mathrm{U}$ \\
\hline Co-60 & 4-C & 1978 & 0.0217 & 0.0092 & 0.0276 & $\mathrm{U}$ \\
\hline Co-60 & $5-11$ & 1978 & 0.06 & 0.01 & 0.03 & - \\
\hline Co-60 & $5-13$ & 1978 & 0.00228 & 0.0089 & 0.0267 & $\mathrm{U}$ \\
\hline Co-60 & $5-15$ & 1978 & -0.00181 & 0.0046 & 0.0138 & $\mathrm{U}$ \\
\hline Co-60 & $5-5$ & 1978 & 0.0327 & 0.0092 & 0.0276 & - \\
\hline Co-60 & $5-7$ & 1978 & 0.00334 & 0.0097 & 0.0291 & $\mathrm{U}$ \\
\hline Co-60 & $5-8$ & 1978 & 0.0241 & 0.00055 & 0.00165 & - \\
\hline Co-60 & $6-1$ & 1978 & 0.00844 & 0.0042 & 0.0126 & $\mathrm{U}$ \\
\hline Co-60 & $6-3$ & 1978 & 0.000193 & 0.00485 & 0.01455 & $\mathrm{U}$ \\
\hline Co-60 & $6-5$ & 1978 & 0.00691 & 0.0082 & 0.0246 & $\mathrm{U}$ \\
\hline Co-60 & $6-7$ & 1978 & 0.00838 & 0.003033333 & 0.0091 & $\mathrm{U}$ \\
\hline Co-60 & $7-2$ & 1978 & 0.0084 & 0.0055 & 0.0165 & $\mathrm{U}$ \\
\hline Co-60 & $7-4$ & 1978 & -0.00594 & 0.0045 & 0.0135 & $\mathrm{U}$ \\
\hline Co-60 & $7-6$ & 1978 & -0.0177 & 0.005 & 0.015 & $\mathrm{U}$ \\
\hline Co-60 & $7-\mathrm{A}$ & 1978 & 0.0307 & 0.00425 & 0.01275 & - \\
\hline Co-60 & 7-B & 1978 & 0.00826 & 0.0095 & 0.0285 & $\mathrm{U}$ \\
\hline
\end{tabular}


Table C-5. (continued).

\begin{tabular}{|c|c|c|c|c|c|c|}
\hline Radionuclide & $\begin{array}{c}\text { Sample } \\
\text { Location }\end{array}$ & Date & $\begin{array}{c}\text { Concentrations } \\
(\mathrm{pCi} / \mathrm{g})\end{array}$ & $\begin{array}{l}\text { Uncertainty } \\
(\mathrm{pCi} / \mathrm{g})\end{array}$ & $\begin{array}{c}\mathrm{MDA} \\
(\mathrm{pCi} / \mathrm{g})\end{array}$ & Flag \\
\hline Co-60 & $8-1$ & 1978 & 0.176 & 0.002666667 & 0.008 & - \\
\hline Co-60 & $8-3$ & 1978 & 0.0284 & 0.00475 & 0.01425 & - \\
\hline Co-60 & $8-5$ & 1978 & 0.00408 & 0.0097 & 0.0291 & $\mathrm{U}$ \\
\hline Co-60 & $8-\mathrm{A}$ & 1978 & -0.00436 & 0.002733333 & 0.0082 & $\mathrm{U}$ \\
\hline Co-60 & 8 -B & 1978 & -0.00999 & 0.0045 & 0.0135 & $\mathrm{U}$ \\
\hline Co-60 & $8-\mathrm{C}$ & 1978 & 0.00301 & 0.01 & 0.03 & $\mathrm{U}$ \\
\hline Co-60 & $9-\mathrm{A}$ & 1978 & -0.00985 & 0.006 & 0.018 & $\mathrm{U}$ \\
\hline Co-60 & RWMC 2-4 & 2005 & 0.1362 & 0.004785 & 0.014355 & - \\
\hline Co-60 & RWMC 10-1 & 2006 & 0.03508 & 1.44 & 4.32 & $\mathrm{U}$ \\
\hline Co-60 & RWMC 2-5 & 2006 & 0.04393 & 2.56 & 7.68 & $\mathrm{U}$ \\
\hline Co-60 & RWMC 2-6 & 2006 & 0.02273 & 1.38 & 4.14 & $\mathrm{U}$ \\
\hline Co-60 & RWMC 2-7 & 2006 & 0.0547 & 2.2950 & 6.885 & $\mathrm{U}$ \\
\hline Co-60 & RWMC 2-8 & 2006 & 0.0413 & 1.275 & 3.825 & $\mathrm{U}$ \\
\hline Co-60 & RWMC 2-8 & 2006 & 0.006542 & 0.0189 & 0.0567 & $\mathrm{U}$ \\
\hline Co-60 & RWMC 3-1 & 2006 & 0.03609 & 1.63 & 4.89 & $\mathrm{U}$ \\
\hline Co-60 & RWMC 3-4 & 2006 & 0.06792 & 2.005 & 6.015 & $\mathrm{U}$ \\
\hline Co-60 & RWMC 3-5 & 2006 & 0.0851 & 1.725 & 5.175 & $\mathrm{U}$ \\
\hline Co-60 & RWMC 3-7 & 2006 & 0.09807 & 1.735 & 5.205 & $\mathrm{U}$ \\
\hline $\mathrm{Co}-60$ & RWMC 3-8 & 2006 & 0.05174 & 0.01005 & 0.03015 & - \\
\hline Co-60 & RWMC 4-1 & 2006 & 0.0407 & 16.55 & 49.65 & $\mathrm{U}$ \\
\hline Co-60 & RWMC 4-2 & 2006 & 0.027 & 4.97 & 14.91 & $\mathrm{U}$ \\
\hline Co-60 & RWMC 4-3 & 2006 & 0.02876 & 2.955 & 8.865 & $\mathrm{U}$ \\
\hline Co-60 & RWMC 4-4 & 2006 & 0.02849 & 2.75 & 8.25 & $\mathrm{U}$ \\
\hline Co-60 & RWMC 4-5 & 2006 & 0.02432 & 2.75 & 8.25 & $\mathrm{U}$ \\
\hline Co-60 & RWMC 5-10 & 2006 & 0.05465 & 2.01 & 6.03 & $\mathrm{U}$ \\
\hline Co-60 & RWMC 5-11 & 2006 & 0.09864 & 1.845 & 5.535 & $\mathrm{U}$ \\
\hline Co-60 & RWMC 5-12 & 2006 & 0.01871 & 1.305 & 3.915 & $\mathrm{U}$ \\
\hline Co-60 & RWMC 5-14 & 2006 & 0.109 & 2.695 & 8.085 & $\mathrm{U}$ \\
\hline Co-60 & RWMC 5-15 & 2006 & 0.03386 & 1.57 & 4.71 & $\mathrm{U}$ \\
\hline Co-60 & RWMC 5-4 & 2006 & 0.05753 & 1.49 & 4.47 & $\mathrm{U}$ \\
\hline Co-60 & RWMC 5-6 & 2006 & 0.02629 & 1.39 & 4.17 & $\mathrm{U}$ \\
\hline Co-60 & RWMC 5-7 & 2006 & 0.04342 & 0.0091 & 0.02715 & - \\
\hline Co-60 & RWMC 5-8 & 2006 & 0.01908 & 3.36 & 10.08 & $\mathrm{U}$ \\
\hline Co-60 & RWMC 5-9 & 2006 & 0.04441 & 2.385 & 7.155 & $\mathrm{U}$ \\
\hline Co-60 & RWMC 6-1 & 2006 & 0.04392 & 1.79 & 5.37 & $\mathrm{U}$ \\
\hline Co-60 & RWMC 6-2 & 2006 & 0.04129 & 0.003005 & 0.009015 & - \\
\hline Co-60 & RWMC 6-4 & 2006 & 0.04745 & 0.0062 & 0.01845 & - \\
\hline
\end{tabular}


Table C-5. (continued).

\begin{tabular}{|c|c|c|c|c|c|c|}
\hline Radionuclide & $\begin{array}{c}\text { Sample } \\
\text { Location }\end{array}$ & Date & $\begin{array}{c}\text { Concentrations } \\
(\mathrm{pCi} / \mathrm{g})\end{array}$ & $\begin{array}{l}\text { Uncertainty } \\
(\mathrm{pCi} / \mathrm{g})\end{array}$ & $\begin{array}{c}\text { MDA } \\
(\mathrm{pCi} / \mathrm{g})\end{array}$ & Flag \\
\hline Co-60 & RWMC 6-5 & 2006 & 0.06728 & 2.95 & 8.85 & $\mathrm{U}$ \\
\hline Co-60 & RWMC 6-6 & 2006 & 0.04451 & 9.4 & 28.2 & $\mathrm{U}$ \\
\hline Co-60 & RWMC 6-7 & 2006 & 0.01338 & 0.985 & 2.955 & $\mathrm{U}$ \\
\hline Co-60 & RWMC 7-3 & 2006 & 0.03879 & 1.935 & 5.805 & $\mathrm{U}$ \\
\hline Co-60 & RWMC 7-4 & 2006 & 0.06145 & 1.455 & 4.365 & $\mathrm{U}$ \\
\hline Co-60 & RWMC 7-5 & 2006 & 0.03095 & 2.355 & 7.065 & $\mathrm{U}$ \\
\hline Co-60 & RWMC 7-6 & 2006 & 0.07822 & 2.54 & 7.62 & $\mathrm{U}$ \\
\hline $\mathrm{Co}-60$ & RWMC 7-7 & 2006 & 0.02347 & 1.755 & 5.265 & $\mathrm{U}$ \\
\hline $\mathrm{Co}-60$ & RWMC 9-1 & 2006 & 0.1309 & 1.34 & 4.02 & $\mathrm{U}$ \\
\hline Co-60 & RWMC 10-1 & 2007 & 0.02181 & 0.525 & 1.575 & $\mathrm{U}$ \\
\hline Co-60 & RWMC 2-4 & 2007 & 0.02785 & 0.04865 & 0.14595 & $\mathrm{U}$ \\
\hline Co-60 & RWMC 2-5 & 2007 & 0.02016 & 0.0715 & 0.2145 & $\mathrm{U}$ \\
\hline Co-60 & RWMC 2-6 & 2007 & 0.01592 & 0.04255 & 0.12765 & $\mathrm{U}$ \\
\hline Co-60 & RWMC 2-7 & 2007 & 0.02003 & 0.0408 & 0.1224 & $\mathrm{U}$ \\
\hline Co-60 & RWMC 3-1 & 2007 & 0.02415 & 0.6 & 1.8 & $\mathrm{U}$ \\
\hline Co-60 & RWMC 3-4 & 2007 & 0.02131 & 0.0482 & 0.1446 & $\mathrm{U}$ \\
\hline Co-60 & RWMC 3-5 & 2007 & 0.03854 & 0.056 & 0.168 & $\mathrm{U}$ \\
\hline Co-60 & RWMC 3-6 & 2007 & 0.01411 & 0.0685 & 0.2055 & $\mathrm{U}$ \\
\hline Co-60 & RWMC 3-7 & 2007 & 0.004402 & 0.068 & 0.204 & $\mathrm{U}$ \\
\hline Co-60 & RWMC 3-8 & 2007 & 0.03349 & 0.0835 & 0.2505 & $\mathrm{U}$ \\
\hline Co-60 & RWMC 4-1 & 2007 & 0.02433 & 0.052 & 0.156 & $\mathrm{U}$ \\
\hline Co-60 & RWMC 4-2 & 2007 & 0.03199 & 0.105 & 0.315 & $\mathrm{U}$ \\
\hline Co-60 & RWMC 4-3 & 2007 & 0.01336 & 0.0815 & 0.2445 & $\mathrm{U}$ \\
\hline Co-60 & RWMC 4-4 & 2007 & 0.01228 & 0.03005 & 0.09015 & $\mathrm{U}$ \\
\hline Co-60 & RWMC 4-5 & 2007 & 0.0101 & 0.056 & 0.168 & $\mathrm{U}$ \\
\hline Co-60 & RWMC 5-10 & 2007 & 0.0109 & 0.01155 & 0.03465 & $\mathrm{U}$ \\
\hline Co-60 & RWMC 5-11 & 2007 & 0.002606 & 0.013 & 0.039 & $\mathrm{U}$ \\
\hline Co-60 & RWMC 5-12 & 2007 & 0.02219 & 0.191 & 0.573 & $\mathrm{U}$ \\
\hline Co-60 & RWMC 5-14 & 2007 & 0.05446 & 0.091 & 0.273 & $\mathrm{U}$ \\
\hline Co-60 & RWMC 5-15 & 2007 & 0.02435 & 0.0535 & 0.1605 & $\mathrm{U}$ \\
\hline Co-60 & RWMC 5-4 & 2007 & 0.01527 & 3.65 & 10.95 & $\mathrm{U}$ \\
\hline Co-60 & RWMC 5-6 & 2007 & 0.0185 & 0.011 & 0.033 & $\mathrm{U}$ \\
\hline Co-60 & RWMC 5-8 & 2007 & 0.009057 & 0.089 & 0.267 & $\mathrm{U}$ \\
\hline Co-60 & RWMC 5-9 & 2007 & 0.0234 & 0.0965 & 0.2895 & $\mathrm{U}$ \\
\hline Co-60 & RWMC 6-1 & 2007 & 0.04542 & 0.02155 & 0.06465 & $\mathrm{U}$ \\
\hline Co-60 & RWMC 6-2 & 2007 & 0.01604 & 0.0357 & 0.1071 & $\mathrm{U}$ \\
\hline Co-60 & RWMC 6-3 & 2007 & 0.01973 & 0.051 & 0.153 & $\mathrm{U}$ \\
\hline
\end{tabular}


Table C-5. (continued).

\begin{tabular}{|c|c|c|c|c|c|c|}
\hline Radionuclide & $\begin{array}{c}\text { Sample } \\
\text { Location }\end{array}$ & Date & $\begin{array}{c}\text { Concentrations } \\
(\mathrm{pCi} / \mathrm{g})\end{array}$ & $\begin{array}{l}\text { Uncertainty } \\
(\mathrm{pCi} / \mathrm{g})\end{array}$ & $\begin{array}{c}\mathrm{MDA} \\
(\mathrm{pCi} / \mathrm{g})\end{array}$ & Flag \\
\hline Co-60 & RWMC 6-4 & 2007 & 0.007687 & 0.146 & 0.4365 & $\mathrm{U}$ \\
\hline Co-60 & RWMC 6-5 & 2007 & 0.01272 & 0.128 & 0.384 & $\mathrm{U}$ \\
\hline Co-60 & RWMC 6-6 & 2007 & 0.03056 & 0.0408 & 0.1224 & $\mathrm{U}$ \\
\hline Co-60 & RWMC 6-7 & 2007 & 0.01421 & 0.0239 & 0.0717 & $\mathrm{U}$ \\
\hline Co-60 & RWMC 6-9 & 2007 & 0.02392 & 0.95 & 2.85 & $\mathrm{U}$ \\
\hline Co-60 & RWMC 7-2 & 2007 & 0.01376 & 0.00875 & 0.02625 & $\mathrm{U}$ \\
\hline Co-60 & RWMC 7-3 & 2007 & 0.02547 & 0.07 & 0.21 & $\mathrm{U}$ \\
\hline $\mathrm{Co}-60$ & RWMC 7-4 & 2007 & 0.02588 & 0.0555 & 0.1665 & $\mathrm{U}$ \\
\hline Co-60 & RWMC 7-5 & 2007 & 0.02864 & 0.0505 & 0.1515 & $\mathrm{U}$ \\
\hline Co-60 & RWMC 7-6 & 2007 & 0.008029 & 0.01305 & 0.03915 & $\mathrm{U}$ \\
\hline Co-60 & RWMC 7-7 & 2007 & 0.02255 & 0.03695 & 0.11085 & $\mathrm{U}$ \\
\hline Co-60 & RWMC 8-1 & 2007 & 0.02336 & 0.086 & 0.258 & $\mathrm{U}$ \\
\hline Co-60 & RWMC 8-4 & 2007 & 0.02238 & 0.0715 & 0.2145 & $\mathrm{U}$ \\
\hline Co-60 & RWMC 8-5 & 2007 & 0.0203 & 0.0413 & 0.1239 & $\mathrm{U}$ \\
\hline Co-60 & RWMC 9-1 & 2007 & 0.01631 & 0.03665 & 0.10995 & $\mathrm{U}$ \\
\hline Co-60 & RWMC 5-4 & 2010 & 0.002166 & 0.00468 & 0.01404 & $\mathrm{U}$ \\
\hline Co-60 & RWMC 10-1 & 2011 & -0.0001377 & 0.00454 & 0.01362 & $\mathrm{U}$ \\
\hline Co-60 & RWMC 2-4 & 2011 & 0.003653 & 0.00486 & 0.01458 & $\mathrm{U}$ \\
\hline Co-60 & RWMC 2-6 & 2011 & -0.002406 & 0.00418 & 0.01254 & $\mathrm{U}$ \\
\hline Co-60 & RWMC 2-8 & 2011 & -0.0003484 & 0.00379 & 0.01137 & $\mathrm{U}$ \\
\hline Co-60 & RWMC 3-4 & 2011 & 0.002874 & 0.00457 & 0.01371 & $\mathrm{U}$ \\
\hline Co-60 & RWMC 3-6 & 2011 & -0.0009032 & 0.00421 & 0.01263 & $\mathrm{U}$ \\
\hline Co-60 & RWMC 3-8 & 2011 & 0.0007645 & 0.00463 & 0.01389 & $\mathrm{U}$ \\
\hline Co-60 & RWMC 4-1 & 2011 & -0.000137 & 0.00472 & 0.01416 & $\mathrm{U}$ \\
\hline Co-60 & RWMC 4-1 & 2011 & -0.0009063 & 0.00368 & 0.01104 & $\mathrm{U}$ \\
\hline Co-60 & RWMC 4-5 & 2011 & -0.001505 & 0.00456 & 0.01368 & $\mathrm{U}$ \\
\hline Co-60 & RWMC 5-12 & 2011 & 0.004576 & 0.00367 & 0.01101 & $\mathrm{U}$ \\
\hline Co-60 & RWMC 5-7 & 2011 & -0.006008 & 0.00485 & 0.01455 & $\mathrm{U}$ \\
\hline Co-60 & RWMC 6-1 & 2011 & 0.0000146 & 0.00322 & 0.00966 & $\mathrm{U}$ \\
\hline Co-60 & RWMC 6-3 & 2011 & 0.002186 & 0.00435 & 0.01305 & $\mathrm{U}$ \\
\hline Co-60 & RWMC 6-7 & 2011 & -0.001768 & 0.0052 & 0.0156 & $\mathrm{U}$ \\
\hline Co-60 & RWMC 7-2 & 2011 & 0.003495 & 0.00459 & 0.01377 & $\mathrm{U}$ \\
\hline Co-60 & RWMC 8-1 & 2011 & 0.003824 & 0.00448 & 0.01344 & $\mathrm{U}$ \\
\hline Co-60 & RWMC 8-5 & 2011 & 0.001562 & 0.00509 & 0.01527 & $\mathrm{U}$ \\
\hline Co-60 & RWMC 9-1 & 2011 & 0.0005726 & 0.0030 & 0.00888 & $\mathrm{U}$ \\
\hline Co-60 & RWMC 2-4 & 2012 & 0.002558 & 0.00401 & 0.01203 & $\mathrm{U}$ \\
\hline Co-60 & RWMC 3-4 & 2012 & -0.0005804 & 0.00501 & 0.01503 & $\mathrm{U}$ \\
\hline
\end{tabular}


Table C-5. (continued).

\begin{tabular}{|c|c|c|c|c|c|c|}
\hline Radionuclide & $\begin{array}{l}\text { Sample } \\
\text { Location }\end{array}$ & Date & $\begin{array}{l}\text { Concentrations } \\
(\mathrm{pCi} / \mathrm{g})\end{array}$ & $\begin{array}{l}\text { Uncertainty } \\
(\mathrm{pCi} / \mathrm{g})\end{array}$ & $\begin{array}{c}\mathrm{MDA} \\
(\mathrm{pCi} / \mathrm{g})\end{array}$ & Flag \\
\hline $\mathrm{Co}-60$ & RWMC 5-4 & 2012 & 0.004007 & 0.0046 & 0.01383 & $\mathrm{U}$ \\
\hline $\mathrm{Co}-60$ & RWMC 6-1 & 2012 & -0.004479 & 0.00444 & 0.01332 & $\mathrm{U}$ \\
\hline Co-60 & RWMC 8-1 & 2012 & 0.002582 & 0.00382 & 0.01146 & $\mathrm{U}$ \\
\hline Co-60 & $\begin{array}{c}2 \text { INCH AIR } \\
\text { - RWMC }\end{array}$ & 2013 & 0.0008422 & 0.00915 & 0.02745 & $\mathrm{U}$ \\
\hline Co-60 & $\begin{array}{l}2 \text { INCH AIR } \\
\text { - RWMC }\end{array}$ & 2013 & 0.0008422 & 0.00305 & 0.00915 & $\mathrm{U}$ \\
\hline Co-60 & RWMC 2-4 & 2013 & 0.0008699 & 0.003283333 & 0.00985 & $\mathrm{U}$ \\
\hline Co-60 & RWMC 3-5 & 2013 & 0.009989 & 0.002733333 & 0.0082 & - \\
\hline Co-60 & RWMC 5-7 & 2013 & 0.0003676 & 0.00311 & 0.00932 & $\mathrm{U}$ \\
\hline Co-60 & RWMC 2-4 & 2014 & 0.001877 & 0.00208 & 0.00624 & $\mathrm{U}$ \\
\hline $\mathrm{Co}-60$ & RWMC 2-4 & 2014 & -0.0007302 & 0.00167 & 0.00501 & $\mathrm{U}$ \\
\hline Co-60 & RWMC 3-4 & 2014 & -0.000008105 & 0.000872 & 0.002616 & $\mathrm{U}$ \\
\hline Co-60 & RWMC 4-1 & 2014 & -0.001646 & 0.00213 & 0.00639 & $\mathrm{U}$ \\
\hline Co-60 & RWMC 5-4 & 2014 & -0.0001379 & 0.00119 & 0.00357 & $\mathrm{U}$ \\
\hline Co-60 & RWMC 6-1 & 2014 & 0.001075 & 0.000807 & 0.002421 & $\mathrm{U}$ \\
\hline $\mathrm{Co}-60$ & RWMC 6-3 & 2014 & -0.0005297 & 0.00224 & 0.00672 & $\mathrm{U}$ \\
\hline Cs-134 & $3-3$ & 1972 & 0.069 & 0.008 & 0.024 & - \\
\hline Cs-134 & RWMC 5-7 & 2007 & 0.0687 & 0.00988 & 0.02964 & - \\
\hline Cs-134 & RWMC 2-5 & 2009 & 0.02457 & 0.002545 & 0.007635 & - \\
\hline Cs-134 & RWMC 2-8 & 2009 & 0.0375 & 0.00414 & 0.01242 & - \\
\hline Cs-134 & RWMC 3-8 & 2009 & 0.006642 & 0.00525 & 0.01575 & $\mathrm{U}$ \\
\hline Cs-134 & RWMC 4-3 & 2009 & 0.01694 & 0.00351 & 0.01053 & - \\
\hline Cs-134 & RWMC 5-15 & 2009 & 0.02807 & 0.00695 & 0.02085 & - \\
\hline Cs-134 & RWMC 10-1 & 2010 & 0.02369 & 0.00662 & 0.01986 & 一 \\
\hline Cs-134 & RWMC 2-4 & 2010 & 0.06453 & 0.005 & 0.015 & - \\
\hline Cs-134 & RWMC 2-6 & 2010 & 0.01575 & 0.00201 & 0.00603 & - \\
\hline Cs-134 & RWMC 3-1 & 2010 & 0.05565 & 0.00846 & 0.02538 & - \\
\hline Cs-134 & RWMC 3-5 & 2010 & 0.04439 & 0.00603 & 0.01809 & 一 \\
\hline Cs-134 & RWMC 3-6 & 2010 & 0.05433 & 0.0103 & 0.0309 & 一 \\
\hline Cs-134 & RWMC 3-7 & 2010 & 0.07855 & 0.0111 & 0.0333 & 一 \\
\hline Cs-134 & RWMC 3-8 & 2010 & 0.0691 & 0.0369 & 0.1107 & $\mathrm{U}$ \\
\hline Cs-134 & RWMC 4-1 & 2010 & 0.0477 & 0.0073 & 0.0219 & - \\
\hline Cs-134 & RWMC 4-2 & 2010 & 0.0279 & 0.00447 & 0.01341 & - \\
\hline Cs-134 & RWMC 4-3 & 2010 & 0.06064 & 0.00982 & 0.02946 & - \\
\hline Cs-134 & RWMC 4-4 & 2010 & 0.0281 & 0.00839 & 0.02517 & - \\
\hline Cs-134 & RWMC 4-5 & 2010 & 0.036 & 0.00731 & 0.02193 & - \\
\hline
\end{tabular}


Table C-5. (continued).

\begin{tabular}{|c|c|c|c|c|c|c|}
\hline Radionuclide & $\begin{array}{c}\text { Sample } \\
\text { Location }\end{array}$ & Date & $\begin{array}{c}\text { Concentrations } \\
(\mathrm{pCi} / \mathrm{g})\end{array}$ & $\begin{array}{l}\text { Uncertainty } \\
(\mathrm{pCi} / \mathrm{g})\end{array}$ & $\begin{array}{c}\mathrm{MDA} \\
(\mathrm{pCi} / \mathrm{g})\end{array}$ & Flag \\
\hline Cs-134 & RWMC 5-10 & 2010 & 0.07294 & 0.015 & 0.045 & - \\
\hline Cs-134 & RWMC 5-11 & 2010 & 0.05173 & 0.00857 & 0.02571 & 一 \\
\hline Cs-134 & RWMC 5-12 & 2010 & 0.03843 & 0.00634 & 0.01902 & - \\
\hline Cs-134 & RWMC 5-14 & 2010 & 0.1417 & 0.0209 & 0.0627 & - \\
\hline Cs-134 & RWMC 5-15 & 2010 & 0.01744 & 0.00411 & 0.01233 & - \\
\hline Cs-134 & RWMC 5-4 & 2010 & 0.05223 & 0.00721 & 0.02163 & - \\
\hline Cs-134 & RWMC 5-6 & 2010 & 0.0513 & 0.0129 & 0.0387 & - \\
\hline Cs-134 & RWMC 5-7 & 2010 & 0.05173 & 0.00857 & 0.02571 & - \\
\hline Cs-134 & RWMC 5-8 & 2010 & 0.1081 & 0.0173 & 0.0519 & - \\
\hline Cs-134 & RWMC 5-9 & 2010 & 0.09421 & 0.021 & 0.063 & - \\
\hline Cs-134 & RWMC 6-1 & 2010 & 0.0847 & 0.0123 & 0.0369 & 一 \\
\hline Cs-134 & RWMC 6-2 & 2010 & 0.01519 & 0.00367 & 0.01101 & - \\
\hline Cs-134 & RWMC 6-3 & 2010 & 0.05039 & 0.0147 & 0.0441 & - \\
\hline Cs-134 & RWMC 6-4 & 2010 & 0.04721 & 0.00802 & 0.02406 & - \\
\hline Cs-134 & RWMC 6-5 & 2010 & 0.05718 & 0.00753 & 0.02259 & - \\
\hline Cs-134 & RWMC 6-6 & 2010 & 0.03894 & 0.00548 & 0.01644 & - \\
\hline Cs-134 & RWMC 6-7 & 2010 & 0.08709 & 0.0104 & 0.0312 & - \\
\hline Cs-134 & RWMC 7-2 & 2010 & 0.05179 & 0.00716 & 0.02148 & - \\
\hline Cs-134 & RWMC 7-3 & 2010 & 0.03439 & 0.0069 & 0.0207 & - \\
\hline Cs-134 & RWMC 7-4 & 2010 & 0.05554 & 0.0086 & 0.0258 & - \\
\hline Cs-134 & RWMC 7-5 & 2010 & 0.04534 & 0.00722 & 0.02166 & - \\
\hline Cs-134 & RWMC 7-6 & 2010 & 0.06628 & 0.00806 & 0.02418 & - \\
\hline Cs-134 & RWMC 7-7 & 2010 & 0.1019 & 0.0127 & 0.0381 & - \\
\hline Cs-134 & RWMC 8-1 & 2010 & 0.05419 & 0.00949 & 0.02847 & - \\
\hline Cs-134 & RWMC 8-4 & 2010 & 0.04242 & 0.00631 & 0.01893 & 一 \\
\hline Cs-134 & RWMC 8-5 & 2010 & 0.05722 & 0.00878 & 0.02634 & 一 \\
\hline Cs-134 & RWMC 9-1 & 2010 & 0.03898 & 0.00503 & 0.01509 & - \\
\hline Cs-134 & RWMC 10-1 & 2011 & -0.02065 & 0.025 & 0.075 & $\mathrm{U}$ \\
\hline Cs-134 & RWMC 2-4 & 2011 & -0.01753 & 0.0133 & 0.0399 & $\mathrm{U}$ \\
\hline Cs-134 & RWMC 2-6 & 2011 & -0.0003842 & 0.0129 & 0.0387 & $\mathrm{U}$ \\
\hline Cs-134 & RWMC 2-8 & 2011 & 0.008597 & 0.0131 & 0.0393 & $\mathrm{U}$ \\
\hline Cs-134 & RWMC 3-4 & 2011 & -0.04696 & 0.0153 & 0.0459 & $\mathrm{U}$ \\
\hline Cs-134 & RWMC 3-6 & 2011 & -0.00247 & 0.017 & 0.051 & $\mathrm{U}$ \\
\hline Cs-134 & RWMC 3-8 & 2011 & 0.008029 & 0.0119 & 0.0357 & $\mathrm{U}$ \\
\hline Cs-134 & RWMC 4-1 & 2011 & -0.07023 & 0.0158 & 0.0474 & $\mathrm{U}$ \\
\hline Cs-134 & RWMC 4-5 & 2011 & -0.008205 & 0.0119 & 0.0357 & $\mathrm{U}$ \\
\hline Cs-134 & RWMC 5-12 & 2011 & -0.0008898 & 0.0153 & 0.0459 & $\mathrm{U}$ \\
\hline
\end{tabular}


Table C-5. (continued).

\begin{tabular}{|c|c|c|c|c|c|c|}
\hline Radionuclide & $\begin{array}{l}\text { Sample } \\
\text { Location }\end{array}$ & Date & $\begin{array}{l}\text { Concentrations } \\
(\mathrm{pCi} / \mathrm{g})\end{array}$ & $\begin{array}{l}\text { Uncertainty } \\
(\mathrm{pCi} / \mathrm{g})\end{array}$ & $\begin{array}{c}\mathrm{MDA} \\
(\mathrm{pCi} / \mathrm{g})\end{array}$ & Flag \\
\hline Cs-134 & RWMC 5-4 & 2011 & -0.008972 & 0.0252 & 0.0756 & $\mathrm{U}$ \\
\hline Cs-134 & RWMC 5-7 & 2011 & 0.005653 & 0.0238 & 0.0714 & $\mathrm{U}$ \\
\hline Cs-134 & RWMC 6-1 & 2011 & -0.01617 & 0.0102 & 0.0306 & $\mathrm{U}$ \\
\hline Cs-134 & RWMC 6-3 & 2011 & 0.00171 & 0.0213 & 0.0639 & $\mathrm{U}$ \\
\hline Cs-134 & RWMC 6-7 & 2011 & -0.004355 & 0.0251 & 0.0753 & $\mathrm{U}$ \\
\hline Cs-134 & RWMC 7-2 & 2011 & 0.009518 & 0.0228 & 0.0684 & $\mathrm{U}$ \\
\hline Cs-134 & RWMC 8-1 & 2011 & 0.00233 & 0.0245 & 0.0735 & $\mathrm{U}$ \\
\hline Cs-134 & RWMC 8-5 & 2011 & -0.004243 & 0.0241 & 0.0723 & $\mathrm{U}$ \\
\hline Cs-134 & RWMC 9-1 & 2011 & -0.009371 & 0.0242 & 0.0726 & $\mathrm{U}$ \\
\hline Cs-134 & RWMC 2-4 & 2012 & 0.07999 & 0.0084 & 0.0252 & - \\
\hline Cs-134 & RWMC 3-4 & 2012 & 0.06727 & 0.00978 & 0.02934 & 一 \\
\hline Cs-134 & RWMC 4-1 & 2012 & 0.0733 & 0.00855 & 0.02565 & 一 \\
\hline Cs-134 & RWMC 5-4 & 2012 & 0.07012 & 0.00922 & 0.02766 & - \\
\hline Cs-134 & RWMC 6-1 & 2012 & 0.06552 & 0.00865 & 0.02595 & - \\
\hline Cs-134 & RWMC 8-1 & 2012 & 0.01995 & 0.00724 & 0.02172 & $\mathrm{U}$ \\
\hline Cs-134 & $\begin{array}{l}2 \text { INCH AIR } \\
\text { - RWMC }\end{array}$ & 2013 & 0.06654 & 0.0341 & 0.1023 & $\mathrm{U}$ \\
\hline Cs-134 & $\begin{array}{l}2 \text { INCH AIR } \\
\text { - RWMC }\end{array}$ & 2013 & 0.06654 & 0.011366667 & 0.0341 & 一 \\
\hline Cs-134 & RWMC 2-4 & 2013 & 0.05356 & 0.011733333 & 0.0352 & - \\
\hline Cs-134 & RWMC 3-5 & 2013 & 0.06052 & 0.012633333 & 0.0379 & - \\
\hline Cs-134 & RWMC 5-7 & 2013 & 0.07587 & 0.0133 & 0.0399 & - \\
\hline Cs-134 & RWMC 2-4 & 2014 & 0.06338 & 0.0194 & 0.0582 & - \\
\hline Cs-134 & RWMC 2-4 & 2014 & -0.005712 & 0.013 & 0.039 & $\mathrm{U}$ \\
\hline Cs-134 & RWMC 3-4 & 2014 & 0.03204 & 0.0083 & 0.0249 & 一 \\
\hline Cs-134 & RWMC 4-1 & 2014 & 0.07438 & 0.0178 & 0.0534 & - \\
\hline Cs-134 & RWMC 5-4 & 2014 & -0.01806 & 0.0112 & 0.0336 & $\mathrm{U}$ \\
\hline Cs-134 & RWMC 6-1 & 2014 & 0.03509 & 0.00909 & 0.02727 & - \\
\hline Cs-134 & RWMC 6-3 & 2014 & 0.08769 & 0.0177 & 0.0531 & 一 \\
\hline Cs-137 & $2-3$ & 1972 & 3.76 & 0.03 & 0.09 & 一 \\
\hline Cs-137 & $2-4$ & 1972 & 2.45 & 0.02 & 0.06 & 一 \\
\hline Cs-137 & $2-5$ & 1972 & 2.8 & 0.02 & 0.06 & - \\
\hline Cs-137 & $3-1$ & 1972 & 2.04 & 0.02 & 0.06 & - \\
\hline Cs-137 & $3-2$ & 1972 & 2.48 & 0.02 & 0.06 & - \\
\hline Cs-137 & $3-3$ & 1972 & 1.76 & 0.01 & 0.03 & - \\
\hline Cs-137 & $3-4$ & 1972 & 1.43 & 0.01 & 0.03 & - \\
\hline Cs-137 & $4-3$ & 1973 & 1.31 & 0.02 & 0.06 & - \\
\hline
\end{tabular}


Table C-5. (continued).

\begin{tabular}{|c|c|c|c|c|c|c|}
\hline Radionuclide & $\begin{array}{c}\text { Sample } \\
\text { Location }\end{array}$ & Date & $\begin{array}{c}\text { Concentrations } \\
(\mathrm{pCi} / \mathrm{g})\end{array}$ & $\begin{array}{l}\text { Uncertainty } \\
(\mathrm{pCi} / \mathrm{g})\end{array}$ & $\begin{array}{c}\mathrm{MDA} \\
(\mathrm{pCi} / \mathrm{g})\end{array}$ & Flag \\
\hline Cs-137 & $5-1$ & 1973 & 1.42 & 0.02 & 0.06 & - \\
\hline Cs-137 & $5-2$ & 1973 & 2.16 & 0.02 & 0.06 & - \\
\hline Cs-137 & $5-3$ & 1973 & 2.04 & 0.02 & 0.06 & - \\
\hline Cs-137 & $5-4$ & 1973 & 2.39 & 0.02 & 0.06 & - \\
\hline Cs-137 & $10-1$ & 1978 & 0.793 & 0.029 & 0.087 & - \\
\hline Cs-137 & $2-3$ & 1978 & 1.37 & 0.048 & 0.144 & - \\
\hline Cs-137 & $2-5$ & 1978 & 0.0525 & 0.011 & 0.033 & - \\
\hline Cs-137 & $2-7$ & 1978 & 0.585 & 0.023 & 0.069 & - \\
\hline Cs-137 & $3-2$ & 1978 & 2.03 & 0.068 & 0.204 & - \\
\hline Cs-137 & $3-4$ & 1978 & 0.92 & 0.034 & 0.102 & - \\
\hline Cs-137 & $3-6$ & 1978 & 0.471 & 0.0021 & 0.0063 & - \\
\hline Cs-137 & $3-8$ & 1978 & 0.727 & 0.027 & 0.081 & - \\
\hline Cs-137 & $4-1$ & 1978 & 1.42 & 0.046 & 0.138 & - \\
\hline Cs-137 & $4-3$ & 1978 & 0.972 & 0.033 & 0.099 & - \\
\hline Cs-137 & $4-5$ & 1978 & 0.714 & 0.026 & 0.078 & - \\
\hline Cs-137 & $4-\mathrm{A}$ & 1978 & 0.82 & 0.029 & 0.087 & - \\
\hline Cs-137 & 4-B & 1978 & 0.63 & 0.024 & 0.072 & - \\
\hline Cs-137 & 4-C & 1978 & 0.803 & 0.03 & 0.09 & - \\
\hline Cs-137 & $5-11$ & 1978 & 0.403 & 0.019 & 0.057 & - \\
\hline Cs-137 & $5-13$ & 1978 & 3.54 & 0.1 & 0.3 & - \\
\hline Cs-137 & $5-15$ & 1978 & 0.205 & 0.014 & 0.042 & - \\
\hline Cs-137 & $5-5$ & 1978 & 1.12 & 0.039 & 0.117 & - \\
\hline Cs-137 & $5-7$ & 1978 & 0.73 & 0.027 & 0.081 & - \\
\hline Cs-137 & $5-8$ & 1978 & 1.17 & 0.041 & 0.123 & - \\
\hline Cs-137 & $6-1$ & 1978 & 0.739 & 0.028 & 0.084 & 一 \\
\hline Cs-137 & $6-3$ & 1978 & 0.736 & 0.028 & 0.084 & 一 \\
\hline Cs-137 & $6-5$ & 1978 & 0.703 & 0.026 & 0.078 & - \\
\hline Cs-137 & $6-7$ & 1978 & 0.782 & 0.03 & 0.087 & - \\
\hline Cs-137 & $7-2$ & 1978 & 0.821 & 0.03 & 0.09 & - \\
\hline Cs-137 & $7-4$ & 1978 & 0.896 & 0.031 & 0.093 & - \\
\hline Cs-137 & $7-6$ & 1978 & 0.775 & 0.028 & 0.084 & - \\
\hline Cs- 137 & $7-\mathrm{A}$ & 1978 & 0.814 & 0.029 & 0.087 & - \\
\hline Cs-137 & 7-B & 1978 & 0.57 & 0.023 & 0.069 & - \\
\hline Cs-137 & $8-1$ & 1978 & 0.721 & 0.026 & 0.078 & - \\
\hline Cs-137 & $8-3$ & 1978 & 0.897 & 0.033 & 0.099 & - \\
\hline Cs- 137 & $8-5$ & 1978 & 1.12 & 0.038 & 0.114 & - \\
\hline Cs-137 & 8-A & 1978 & 0.939 & 0.032 & 0.096 & - \\
\hline
\end{tabular}


Table C-5. (continued).

\begin{tabular}{|c|c|c|c|c|c|c|}
\hline Radionuclide & $\begin{array}{c}\text { Sample } \\
\text { Location }\end{array}$ & Date & $\begin{array}{c}\text { Concentrations } \\
(\mathrm{pCi} / \mathrm{g})\end{array}$ & $\begin{array}{l}\text { Uncertainty } \\
(\mathrm{pCi} / \mathrm{g})\end{array}$ & $\begin{array}{c}\mathrm{MDA} \\
(\mathrm{pCi} / \mathrm{g})\end{array}$ & Flag \\
\hline Cs-137 & $8-\mathrm{B}$ & 1978 & 0.833 & 0.03 & 0.087 & - \\
\hline Cs-137 & $8-\mathrm{C}$ & 1978 & 0.911 & 0.032 & 0.096 & - \\
\hline Cs-137 & 9-A & 1978 & 0.41 & 0.019 & 0.057 & - \\
\hline Cs-137 & $10-1$ & 1985 & 0.5 & 0.06 & 0.18 & - \\
\hline Cs-137 & $2-4$ & 1985 & 0.86 & 0.08 & 0.24 & - \\
\hline Cs-137 & $3-1$ & 1985 & 0.66 & 0.07 & 0.21 & - \\
\hline Cs-137 & $3-7$ & 1985 & 0.56 & 0.07 & 0.21 & - \\
\hline Cs-137 & $4-2$ & 1985 & 0.65 & 0.06 & 0.18 & - \\
\hline Cs-137 & $4-\mathrm{A}$ & 1985 & 0.6 & 0.07 & 0.21 & - \\
\hline Cs-137 & $4-\mathrm{C}$ & 1985 & 0.35 & 0.025 & 0.075 & - \\
\hline Cs-137 & $5-10$ & 1985 & 0.72 & 0.1 & 0.21 & - \\
\hline Cs-137 & $5-15$ & 1985 & 0.76 & 0.07 & 0.21 & - \\
\hline Cs-137 & $5-5$ & 1985 & 0.66 & 0.07 & 0.21 & - \\
\hline Cs-137 & $5-7$ & 1985 & 1.04 & 0.09 & 0.27 & - \\
\hline Cs-137 & $6-1$ & 1985 & 0.75 & 0.08 & 0.24 & - \\
\hline Cs-137 & $7-3$ & 1985 & 0.92 & 0.1 & 0.24 & - \\
\hline Cs-137 & $7-5$ & 1985 & 0.66 & 0.07 & 0.21 & - \\
\hline Cs-137 & $7-7$ & 1985 & 0.59 & 0.1 & 0.21 & - \\
\hline Cs-137 & 7-A & 1985 & 0.87 & 0.070 & 0.21 & - \\
\hline Cs-137 & 8-1 & 1985 & 0.61 & 0.06 & 0.18 & - \\
\hline Cs-137 & $8-2$ & 1985 & 0.79 & 0.07 & 0.21 & - \\
\hline Cs-137 & $8-5$ & 1985 & 0.057 & 0.03 & 0.09 & $\mathrm{U}$ \\
\hline Cs- 137 & 8-C & 1985 & 0.53 & 0.07 & 0.21 & - \\
\hline Cs-137 & 9-A & 1985 & 0.052 & 0.03 & 0.09 & $\mathrm{U}$ \\
\hline Cs-137 & $10-1$ & 1993 & 0.35 & 0.025 & 0.075 & 一 \\
\hline Cs-137 & $2-4$ & 1993 & 0.51 & 0.06 & 0.18 & 一 \\
\hline Cs-137 & $2-5$ & 1993 & 0.46 & 0.06 & 0.18 & - \\
\hline Cs-137 & $3-4$ & 1993 & 0.74 & 0.06 & 0.18 & - \\
\hline Cs-137 & $3-6$ & 1993 & 0.68 & 0.06 & 0.18 & - \\
\hline Cs-137 & 4-1 & 1993 & 0.7 & 0.06 & 0.18 & - \\
\hline Cs-137 & $5-12$ & 1993 & 0.58 & 0.06 & 0.18 & - \\
\hline Cs- 137 & $5-15$ & 1993 & 0.76 & 0.06 & 0.18 & - \\
\hline Cs-137 & $5-4$ & 1993 & 1.34 & 0.09 & 0.27 & - \\
\hline Cs-137 & $5-7$ & 1993 & 0.69 & 0.06 & 0.18 & - \\
\hline Cs-137 & $5-9$ & 1993 & 0.98 & 0.07 & 0.21 & - \\
\hline Cs- 137 & $6-4$ & 1993 & 0.63 & 0.06 & 0.18 & - \\
\hline Cs-137 & $7-2$ & 1993 & 0.51 & 0.05 & 0.15 & - \\
\hline
\end{tabular}


Table C-5. (continued).

\begin{tabular}{|c|c|c|c|c|c|c|}
\hline Radionuclide & $\begin{array}{c}\text { Sample } \\
\text { Location }\end{array}$ & Date & $\begin{array}{c}\text { Concentrations } \\
(\mathrm{pCi} / \mathrm{g})\end{array}$ & $\begin{array}{l}\text { Uncertainty } \\
(\mathrm{pCi} / \mathrm{g})\end{array}$ & $\begin{array}{c}\mathrm{MDA} \\
(\mathrm{pCi} / \mathrm{g})\end{array}$ & Flag \\
\hline Cs-137 & 7-B & 1993 & 0.47 & 0.06 & 0.18 & - \\
\hline Cs-137 & $8-1$ & 1993 & 0.69 & 0.06 & 0.18 & - \\
\hline Cs-137 & $8-4$ & 1993 & 0.52 & 0.06 & 0.18 & - \\
\hline Cs-137 & $8-5$ & 1993 & 0.52 & 0.06 & 0.18 & - \\
\hline Cs-137 & RWMC 2-4 & 2005 & 0.1453 & 0.00575 & 0.01725 & - \\
\hline Cs-137 & RWMC 8-1 & 2005 & 0.1002 & 0.005 & 0.01515 & - \\
\hline Cs-137 & RWMC 8-4 & 2005 & 0.1095 & 0.00957 & 0.02871 & - \\
\hline Cs-137 & RWMC 8-5 & 2005 & 0.08006 & 0.0115 & 0.0345 & - \\
\hline Cs-137 & RWMC 10-1 & 2006 & 0.08418 & 0.2245 & 0.6735 & $\mathrm{U}$ \\
\hline Cs-137 & RWMC 2-6 & 2006 & 0.1336 & 0.1925 & 0.5775 & $\mathrm{U}$ \\
\hline Cs-137 & RWMC 2-7 & 2006 & 0.1226 & 0.387 & 1.161 & $\mathrm{U}$ \\
\hline Cs-137 & RWMC 2-8 & 2006 & 0.2834 & 0.01185 & 0.03555 & - \\
\hline Cs-137 & RWMC 2-8 & 2006 & 0.1187 & 0.186 & 0.558 & $\mathrm{U}$ \\
\hline Cs-137 & RWMC 3-1 & 2006 & 0.1485 & 0.202 & 0.606 & $\mathrm{U}$ \\
\hline Cs-137 & RWMC 3-4 & 2006 & 0.1266 & 0.322 & 0.966 & $\mathrm{U}$ \\
\hline Cs-137 & RWMC 3-5 & 2006 & 0.1266 & 0.2305 & 0.6915 & $\mathrm{U}$ \\
\hline Cs-137 & RWMC 3-6 & 2006 & 0.1188 & 0.0064 & 0.0192 & - \\
\hline Cs-137 & RWMC 3-7 & 2006 & 0.1244 & 0.2825 & 0.8475 & $\mathrm{U}$ \\
\hline Cs-137 & RWMC 4-2 & 2006 & 0.1075 & 0.635 & 1.905 & $\mathrm{U}$ \\
\hline Cs-137 & RWMC 4-3 & 2006 & 0.1081 & 0.79 & 2.37 & $\mathrm{U}$ \\
\hline Cs-137 & RWMC 4-4 & 2006 & 0.1004 & 0.2085 & 0.6255 & $\mathrm{U}$ \\
\hline Cs-137 & RWMC 4-5 & 2006 & 0.09192 & 0.327 & 0.981 & $\mathrm{U}$ \\
\hline Cs-137 & RWMC 5-10 & 2006 & 0.1233 & 0.2685 & 0.8055 & $\mathrm{U}$ \\
\hline Cs-137 & RWMC 5-11 & 2006 & 0.09832 & 0.427 & 1.281 & $\mathrm{U}$ \\
\hline Cs-137 & RWMC 5-12 & 2006 & 0.1068 & 0.259 & 0.777 & $\mathrm{U}$ \\
\hline Cs-137 & RWMC 5-14 & 2006 & 0.1225 & 0.2215 & 0.6645 & $\mathrm{U}$ \\
\hline Cs-137 & RWMC 5-15 & 2006 & 0.1244 & 0.3355 & 1.0065 & $\mathrm{U}$ \\
\hline Cs-137 & RWMC 5-4 & 2006 & 0.1232 & 0.2785 & 0.8355 & $\mathrm{U}$ \\
\hline Cs-137 & RWMC 5-6 & 2006 & 0.1169 & 0.3625 & 1.0875 & $\mathrm{U}$ \\
\hline Cs-137 & RWMC 5-7 & 2006 & 0.1287 & 0.00595 & 0.01785 & - \\
\hline Cs-137 & RWMC 5-8 & 2006 & 0.08303 & 0.54 & 1.62 & $\mathrm{U}$ \\
\hline Cs-137 & RWMC 5-9 & 2006 & 0.09614 & 0.3125 & 0.9375 & $\mathrm{U}$ \\
\hline Cs-137 & RWMC 6-1 & 2006 & 0.1297 & 0.194 & 0.582 & $\mathrm{U}$ \\
\hline Cs-137 & RWMC 6-2 & 2006 & 0.1333 & 0.52 & 1.56 & $\mathrm{U}$ \\
\hline Cs-137 & RWMC 6-5 & 2006 & 0.0899 & 0.57 & 1.71 & $\mathrm{U}$ \\
\hline Cs-137 & RWMC 6-6 & 2006 & 0.07244 & 0.2785 & 0.8355 & $\mathrm{U}$ \\
\hline Cs-137 & RWMC 6-7 & 2006 & 0.1031 & 0.3715 & 1.1145 & $\mathrm{U}$ \\
\hline
\end{tabular}


Table C-5. (continued).

\begin{tabular}{|c|c|c|c|c|c|c|}
\hline Radionuclide & $\begin{array}{c}\text { Sample } \\
\text { Location }\end{array}$ & Date & $\begin{array}{c}\text { Concentrations } \\
(\mathrm{pCi} / \mathrm{g})\end{array}$ & $\begin{array}{l}\text { Uncertainty } \\
(\mathrm{pCi} / \mathrm{g})\end{array}$ & $\begin{array}{c}\mathrm{MDA} \\
(\mathrm{pCi} / \mathrm{g})\end{array}$ & Flag \\
\hline Cs-137 & RWMC 7-3 & 2006 & 0.08038 & 0.3285 & 0.9855 & $\mathrm{U}$ \\
\hline Cs-137 & RWMC 7-4 & 2006 & 0.0981 & 0.278 & 0.834 & $\mathrm{U}$ \\
\hline Cs-137 & RWMC 7-5 & 2006 & 0.09665 & 0.262 & 0.786 & $\mathrm{U}$ \\
\hline Cs-137 & RWMC 7-6 & 2006 & 0.1274 & 0.2785 & 0.8355 & $\mathrm{U}$ \\
\hline Cs-137 & RWMC 7-7 & 2006 & 0.1016 & 0.352 & 1.056 & $\mathrm{U}$ \\
\hline Cs-137 & RWMC 9-1 & 2006 & 0.1196 & 0.1985 & 0.5955 & $\mathrm{U}$ \\
\hline Cs-137 & RWMC 10-1 & 2007 & 0.2073 & 0.0975 & 0.2925 & $\mathrm{U}$ \\
\hline Cs-137 & RWMC 2-4 & 2007 & 0.2107 & 0.084 & 0.252 & $\mathrm{U}$ \\
\hline Cs-137 & RWMC 2-5 & 2007 & 0.3009 & 0.04735 & 0.14205 & - \\
\hline Cs-137 & RWMC 2-6 & 2007 & 0.2624 & 0.038 & 0.114 & - \\
\hline Cs-137 & RWMC 2-7 & 2007 & 0.3293 & 0.051 & 0.153 & 一 \\
\hline Cs-137 & RWMC 3-1 & 2007 & 0.3137 & 0.091 & 0.273 & 一 \\
\hline Cs-137 & RWMC 3-4 & 2007 & 0.2628 & 0.09 & 0.27 & $\mathrm{U}$ \\
\hline Cs-137 & RWMC 3-5 & 2007 & 0.3221 & 0.086 & 0.258 & 一 \\
\hline Cs-137 & RWMC 3-6 & 2007 & 0.2874 & 0.054 & 0.162 & - \\
\hline Cs-137 & RWMC 3-7 & 2007 & 0.26 & 0.078 & 0.234 & - \\
\hline Cs-137 & RWMC 3-8 & 2007 & 0.2743 & 0.065 & 0.195 & - \\
\hline Cs-137 & RWMC 4-1 & 2007 & 0.5191 & 0.0675 & 0.2025 & - \\
\hline Cs-137 & RWMC 4-2 & 2007 & 0.2438 & 0.079 & 0.237 & - \\
\hline Cs-137 & RWMC 4-2 & 2007 & 0.2085 & 0.0106 & 0.0318 & - \\
\hline Cs-137 & RWMC 4-3 & 2007 & 0.2856 & 0.056 & 0.168 & 一 \\
\hline Cs-137 & RWMC 4-4 & 2007 & 0.2217 & 0.0705 & 0.2115 & - \\
\hline Cs-137 & RWMC 4-5 & 2007 & 0.2277 & 0.0675 & 0.2025 & - \\
\hline Cs-137 & RWMC 5-10 & 2007 & 0.3782 & 0.03505 & 0.10515 & - \\
\hline Cs-137 & RWMC 5-11 & 2007 & 0.2219 & 0.106 & 0.318 & $\mathrm{U}$ \\
\hline Cs-137 & RWMC 5-12 & 2007 & 0.2869 & 0.3895 & 1.1685 & $\mathrm{U}$ \\
\hline Cs-137 & RWMC 5-14 & 2007 & 1.012 & 0.106 & 0.318 & - \\
\hline Cs-137 & RWMC 5-15 & 2007 & 0.2935 & 0.53 & 1.59 & $\mathrm{U}$ \\
\hline Cs-137 & RWMC 5-4 & 2007 & 0.364 & 0.1525 & 0.4575 & $\mathrm{U}$ \\
\hline Cs-137 & RWMC 5-6 & 2007 & 0.2216 & 0.0354 & 0.1062 & - \\
\hline Cs-137 & RWMC 5-7 & 2007 & 0.2778 & 0.0103 & 0.0309 & - \\
\hline Cs-137 & RWMC 5-8 & 2007 & 0.2955 & 0.0308 & 0.0924 & - \\
\hline Cs-137 & RWMC 5-9 & 2007 & 0.2758 & 0.04415 & 0.13245 & - \\
\hline Cs-137 & RWMC 6-1 & 2007 & 0.2724 & 0.04775 & 0.14325 & - \\
\hline Cs-137 & RWMC 6-2 & 2007 & 0.2241 & 0.0535 & 0.1605 & 一 \\
\hline Cs-137 & RWMC 6-3 & 2007 & 0.2695 & 0.0845 & 0.2535 & - \\
\hline Cs-137 & RWMC 6-4 & 2007 & 0.2518 & 0.03615 & 0.10845 & - \\
\hline
\end{tabular}


Table C-5. (continued).

\begin{tabular}{|c|c|c|c|c|c|c|}
\hline Radionuclide & $\begin{array}{c}\text { Sample } \\
\text { Location }\end{array}$ & Date & $\begin{array}{c}\text { Concentrations } \\
(\mathrm{pCi} / \mathrm{g})\end{array}$ & $\begin{array}{l}\text { Uncertainty } \\
(\mathrm{pCi} / \mathrm{g})\end{array}$ & $\begin{array}{c}\mathrm{MDA} \\
(\mathrm{pCi} / \mathrm{g})\end{array}$ & Flag \\
\hline Cs-137 & RWMC 6-5 & 2007 & 0.2423 & 0.0765 & 0.2295 & - \\
\hline Cs-137 & RWMC 6-6 & 2007 & 0.1793 & 0.039 & 0.117 & - \\
\hline Cs-137 & RWMC 6-7 & 2007 & 0.1884 & 0.069 & 0.207 & $\mathrm{U}$ \\
\hline Cs-137 & RWMC 6-9 & 2007 & 0.2913 & 0.0635 & 0.1905 & - \\
\hline Cs-137 & RWMC 7-2 & 2007 & 0.3112 & 0.1415 & 0.4245 & $\mathrm{U}$ \\
\hline Cs-137 & RWMC 7-3 & 2007 & 0.1505 & 0.065 & 0.195 & $\mathrm{U}$ \\
\hline Cs-137 & RWMC 7-4 & 2007 & 0.2336 & 0.0327 & 0.0981 & - \\
\hline Cs-137 & RWMC 7-5 & 2007 & 0.2454 & 0.0494 & 0.1482 & - \\
\hline Cs-137 & RWMC 7-6 & 2007 & 0.2613 & 0.079 & 0.237 & - \\
\hline Cs-137 & RWMC 7-7 & 2007 & 0.2146 & 0.1405 & 0.4215 & $\mathrm{U}$ \\
\hline Cs-137 & RWMC 8-1 & 2007 & 0.2961 & 1.055 & 3.165 & $\mathrm{U}$ \\
\hline Cs-137 & RWMC 8-4 & 2007 & 0.2721 & 0.0384 & 0.1152 & 一 \\
\hline Cs-137 & RWMC 9-1 & 2007 & 0.2242 & 0.06 & 0.18 & - \\
\hline Cs-137 & RWMC 2-4 & 2009 & 0.2285 & 0.00805 & 0.02415 & 一 \\
\hline Cs-137 & RWMC 2-5 & 2009 & 0.1598 & 0.695 & 2.085 & $\mathrm{U}$ \\
\hline Cs-137 & RWMC 2-6 & 2009 & 0.1845 & 0.00825 & 0.02475 & - \\
\hline Cs-137 & RWMC 2-7 & 2009 & 0.2029 & 0.0045 & 0.0135 & - \\
\hline Cs-137 & RWMC 2-8 & 2009 & 0.155 & 0.0064 & 0.01905 & - \\
\hline Cs-137 & RWMC 2-8 & 2009 & 0.1418 & 0.00845 & 0.02535 & - \\
\hline Cs-137 & RWMC 3-1 & 2009 & 0.1831 & 0.0625 & 0.1875 & $\mathrm{U}$ \\
\hline Cs-137 & RWMC 3-4 & 2009 & 0.1923 & 0.0362 & 0.1086 & 一 \\
\hline Cs-137 & RWMC 3-5 & 2009 & 0.208 & 0.00725 & 0.02175 & - \\
\hline Cs-137 & RWMC 3-6 & 2009 & 0.177 & 0.00645 & 0.01935 & - \\
\hline Cs-137 & RWMC 3-7 & 2009 & 0.1835 & 0.0065 & 0.0195 & - \\
\hline Cs-137 & RWMC 3-8 & 2009 & 0.1845 & 0.0052 & 0.0156 & 一 \\
\hline Cs-137 & RWMC 4-1 & 2009 & 0.1844 & 0.0227 & 0.0681 & 一 \\
\hline Cs-137 & RWMC 4-3 & 2009 & 0.1785 & 0.00715 & 0.02145 & - \\
\hline Cs-137 & RWMC 4-4 & 2009 & 0.1885 & 0.0875 & 0.2625 & $\mathrm{U}$ \\
\hline Cs-137 & RWMC 4-5 & 2009 & 0.1016 & 0.0055 & 0.0165 & - \\
\hline Cs-137 & RWMC 5-10 & 2009 & 0.2035 & 0.0057 & 0.0171 & - \\
\hline Cs-137 & RWMC 5-11 & 2009 & 0.1233 & 0.00725 & 0.02175 & - \\
\hline Cs-137 & RWMC 5-12 & 2009 & 0.1785 & 0.007 & 0.021 & - \\
\hline Cs-137 & RWMC 5-14 & 2009 & 0.1925 & 0.006 & 0.0192 & - \\
\hline Cs-137 & RWMC 5-15 & 2009 & 0.1597 & 0.0052 & 0.0156 & - \\
\hline Cs-137 & RWMC 5-4 & 2009 & 0.201 & 0.00585 & 0.01755 & 一 \\
\hline Cs-137 & RWMC 5-6 & 2009 & 0.1712 & 0.0048 & 0.0144 & - \\
\hline Cs-137 & RWMC 5-7 & 2009 & 0.2041 & 0.00815 & 0.02445 & - \\
\hline
\end{tabular}


Table C-5. (continued).

\begin{tabular}{|c|c|c|c|c|c|c|}
\hline Radionuclide & $\begin{array}{c}\text { Sample } \\
\text { Location }\end{array}$ & Date & $\begin{array}{c}\text { Concentrations } \\
(\mathrm{pCi} / \mathrm{g})\end{array}$ & $\begin{array}{l}\text { Uncertainty } \\
(\mathrm{pCi} / \mathrm{g})\end{array}$ & $\begin{array}{c}\mathrm{MDA} \\
(\mathrm{pCi} / \mathrm{g})\end{array}$ & Flag \\
\hline Cs-137 & RWMC 5-8 & 2009 & 0.1676 & 0.0068 & 0.0204 & - \\
\hline Cs-137 & RWMC 6-1 & 2009 & 0.1832 & 0.03645 & 0.10935 & - \\
\hline Cs-137 & RWMC 6-2 & 2009 & 0.1948 & 0.0081 & 0.0243 & - \\
\hline Cs-137 & RWMC 6-3 & 2009 & 0.2105 & 0.004825 & 0.014475 & - \\
\hline Cs-137 & RWMC 6-4 & 2009 & 0.1752 & 0.0062 & 0.0186 & - \\
\hline Cs-137 & RWMC 6-5 & 2009 & 0.1444 & 0.00479 & 0.01437 & - \\
\hline Cs-137 & RWMC 6-6 & 2009 & 0.1261 & 0.00383 & 0.01149 & - \\
\hline Cs-137 & RWMC 6-7 & 2009 & 0.1506 & 0.0062 & 0.0186 & - \\
\hline Cs-137 & RWMC 7-2 & 2009 & 0.2019 & 0.00645 & 0.01935 & - \\
\hline Cs-137 & RWMC 7-5 & 2009 & 0.168 & 0.0431 & 0.1293 & - \\
\hline Cs-137 & RWMC 7-6 & 2009 & 0.1759 & 0.00795 & 0.02385 & 一 \\
\hline Cs-137 & RWMC 7-7 & 2009 & 0.1634 & 0.00476 & 0.01428 & 一 \\
\hline Cs-137 & RWMC 8-1 & 2009 & 0.1742 & 0.00715 & 0.02145 & - \\
\hline Cs-137 & RWMC 8-4 & 2009 & 0.1706 & 0.004275 & 0.012825 & - \\
\hline Cs-137 & RWMC 8-5 & 2009 & 0.1818 & 0.00805 & 0.02415 & - \\
\hline Cs-137 & RWMC 9-1 & 2009 & 0.1538 & 0.004715 & 0.014145 & - \\
\hline Cs-137 & RWMC 10-1 & 2010 & 0.1668 & 0.00644 & 0.01932 & - \\
\hline Cs-137 & RWMC 2-4 & 2010 & 0.2321 & 0.00538 & 0.01614 & - \\
\hline Cs-137 & RWMC 2-6 & 2010 & 0.1106 & 0.00495 & 0.01485 & - \\
\hline Cs-137 & RWMC 3-1 & 2010 & 0.2105 & 0.00785 & 0.02355 & - \\
\hline Cs-137 & RWMC 3-5 & 2010 & 0.1758 & 0.0029 & 0.0087 & - \\
\hline Cs-137 & RWMC 3-6 & 2010 & 0.1628 & 0.00629 & 0.01887 & - \\
\hline Cs-137 & RWMC 3-7 & 2010 & 0.1876 & 0.00806 & 0.02418 & - \\
\hline Cs-137 & RWMC 3-8 & 2010 & 0.1843 & 0.00432 & 0.01296 & - \\
\hline Cs-137 & RWMC 4-1 & 2010 & 0.1988 & 0.0101 & 0.0303 & 一 \\
\hline Cs-137 & RWMC 4-2 & 2010 & 0.2276 & 0.0112 & 0.0336 & 一 \\
\hline Cs-137 & RWMC 4-3 & 2010 & 0.1596 & 0.00392 & 0.01176 & - \\
\hline Cs-137 & RWMC 4-4 & 2010 & 0.1685 & 0.00374 & 0.01122 & - \\
\hline Cs-137 & RWMC 4-5 & 2010 & 0.1405 & 0.0071 & 0.0213 & - \\
\hline Cs-137 & RWMC 5-10 & 2010 & 0.2085 & 0.00932 & 0.02796 & - \\
\hline Cs-137 & RWMC 5-11 & 2010 & 0.2222 & 0.00992 & 0.02976 & - \\
\hline Cs-137 & RWMC 5-12 & 2010 & 0.1621 & 0.00681 & 0.02043 & - \\
\hline Cs-137 & RWMC 5-14 & 2010 & 0.1779 & 0.0101 & 0.0303 & - \\
\hline Cs-137 & RWMC 5-15 & 2010 & 0.2112 & 0.00994 & 0.02982 & - \\
\hline Cs-137 & RWMC 5-4 & 2010 & 0.7561 & 0.0194 & 0.0582 & 一 \\
\hline Cs-137 & RWMC 5-4 & 2010 & 0.1841 & 0.00524 & 0.01572 & - \\
\hline Cs-137 & RWMC 5-6 & 2010 & 0.1703 & 0.00896 & 0.02688 & - \\
\hline
\end{tabular}


Table C-5. (continued).

\begin{tabular}{|c|c|c|c|c|c|c|}
\hline Radionuclide & $\begin{array}{c}\text { Sample } \\
\text { Location }\end{array}$ & Date & $\begin{array}{c}\text { Concentrations } \\
(\mathrm{pCi} / \mathrm{g})\end{array}$ & $\begin{array}{l}\text { Uncertainty } \\
(\mathrm{pCi} / \mathrm{g})\end{array}$ & $\begin{array}{c}\mathrm{MDA} \\
(\mathrm{pCi} / \mathrm{g})\end{array}$ & Flag \\
\hline Cs-137 & RWMC 5-7 & 2010 & 0.2222 & 0.00992 & 0.02976 & - \\
\hline Cs-137 & RWMC 5-8 & 2010 & 0.1661 & 0.00773 & 0.02319 & - \\
\hline Cs-137 & RWMC 5-9 & 2010 & 0.1608 & 0.00858 & 0.02574 & - \\
\hline Cs-137 & RWMC 6-1 & 2010 & 0.2015 & 0.00852 & 0.02556 & - \\
\hline Cs-137 & RWMC 6-2 & 2010 & 0.1996 & 0.00653 & 0.01959 & - \\
\hline Cs-137 & RWMC 6-3 & 2010 & 0.2039 & 0.00981 & 0.02943 & - \\
\hline Cs-137 & RWMC 6-4 & 2010 & 0.1557 & 0.0034 & 0.01032 & - \\
\hline Cs-137 & RWMC 6-5 & 2010 & 0.1474 & 0.00719 & 0.02157 & - \\
\hline Cs-137 & RWMC 6-6 & 2010 & 0.111 & 0.00228 & 0.00684 & - \\
\hline Cs-137 & RWMC 6-7 & 2010 & 0.1599 & 0.00615 & 0.01845 & - \\
\hline Cs-137 & RWMC 7-2 & 2010 & 0.2223 & 0.00945 & 0.02835 & 一 \\
\hline Cs-137 & RWMC 7-3 & 2010 & 0.1457 & 0.00258 & 0.00774 & 一 \\
\hline Cs-137 & RWMC 7-4 & 2010 & 0.1718 & 0.00654 & 0.01962 & - \\
\hline Cs-137 & RWMC 7-5 & 2010 & 0.2047 & 0.0135 & 0.0405 & - \\
\hline Cs-137 & RWMC 7-6 & 2010 & 0.2081 & 0.00857 & 0.02571 & - \\
\hline Cs-137 & RWMC 7-7 & 2010 & 0.2213 & 0.0137 & 0.0411 & - \\
\hline Cs-137 & RWMC 8-1 & 2010 & 0.2081 & 0.00988 & 0.02964 & - \\
\hline Cs-137 & RWMC 8-4 & 2010 & 0.07565 & 0.0054 & 0.0162 & - \\
\hline Cs-137 & RWMC 8-5 & 2010 & 0.2002 & 0.00715 & 0.02145 & - \\
\hline Cs-137 & RWMC 9-1 & 2010 & 0.1529 & 0.00557 & 0.01671 & - \\
\hline Cs-137 & RWMC 10-1 & 2011 & 0.4309 & 0.0223 & 0.0669 & - \\
\hline Cs-137 & RWMC 2-4 & 2011 & 0.4077 & 0.0157 & 0.0471 & - \\
\hline Cs-137 & RWMC 2-6 & 2011 & 0.5338 & 0.02 & 0.054 & - \\
\hline Cs-137 & RWMC 2-8 & 2011 & 0.2298 & 0.0141 & 0.0423 & - \\
\hline Cs-137 & RWMC 3-4 & 2011 & 0.25 & 0.0157 & 0.0471 & 一 \\
\hline Cs-137 & RWMC 3-6 & 2011 & 0.5359 & 0.02 & 0.0651 & 一 \\
\hline Cs-137 & RWMC 3-8 & 2011 & 0.3541 & 0.0146 & 0.0438 & - \\
\hline Cs-137 & RWMC 4-1 & 2011 & 0.5888 & 0.0155 & 0.0465 & - \\
\hline Cs-137 & RWMC 4-5 & 2011 & 0.4451 & 0.0181 & 0.0543 & - \\
\hline Cs-137 & RWMC 5-12 & 2011 & 0.2245 & 0.0123 & 0.0369 & - \\
\hline Cs-137 & RWMC 5-7 & 2011 & 0.5076 & 0.0205 & 0.0615 & - \\
\hline Cs-137 & RWMC 6-1 & 2011 & 0.1841 & 0.0162 & 0.0486 & - \\
\hline Cs-137 & RWMC 6-3 & 2011 & 0.127 & 0.0126 & 0.0378 & - \\
\hline Cs-137 & RWMC 6-7 & 2011 & 0.4131 & 0.0117 & 0.0351 & - \\
\hline Cs-137 & RWMC 7-2 & 2011 & 0.5668 & 0.0231 & 0.0693 & 一 \\
\hline Cs-137 & RWMC 8-1 & 2011 & 0.1295 & 0.0124 & 0.0372 & - \\
\hline Cs-137 & RWMC 8-5 & 2011 & 0.512 & 0.024 & 0.072 & - \\
\hline
\end{tabular}


Table C-5. (continued).

\begin{tabular}{|c|c|c|c|c|c|c|}
\hline Radionuclide & $\begin{array}{l}\text { Sample } \\
\text { Location }\end{array}$ & Date & $\begin{array}{l}\text { Concentrations } \\
(\mathrm{pCi} / \mathrm{g})\end{array}$ & $\begin{array}{l}\text { Uncertainty } \\
(\mathrm{pCi} / \mathrm{g})\end{array}$ & $\begin{array}{c}\mathrm{MDA} \\
(\mathrm{pCi} / \mathrm{g})\end{array}$ & Flag \\
\hline Cs-137 & RWMC 9-1 & 2011 & 0.4914 & 0.0149 & 0.0447 & - \\
\hline Cs-137 & RWMC 2-4 & 2012 & 0.2167 & 0.00671 & 0.02013 & - \\
\hline Cs-137 & RWMC 3-4 & 2012 & 0.2237 & 0.01 & 0.03 & - \\
\hline Cs-137 & RWMC 4-1 & 2012 & 0.2311 & 0.011 & 0.033 & - \\
\hline Cs-137 & RWMC 5-4 & 2012 & 0.1029 & 0.00458 & 0.01374 & - \\
\hline Cs-137 & RWMC 6-1 & 2012 & 0.08017 & 0.0041 & 0.0123 & - \\
\hline Cs-137 & RWMC 8-1 & 2012 & 0.1529 & 0.00724 & 0.02172 & 一 \\
\hline Cs-137 & $\begin{array}{l}2 \text { INCH AIR } \\
\text { - RWMC }\end{array}$ & 2013 & 0.1761 & 0.0319 & 0.0957 & 一 \\
\hline Cs-137 & $\begin{array}{c}2 \text { INCH AIR } \\
\text { - RWMC }\end{array}$ & 2013 & 0.1761 & 0.010633333 & 0.0319 & - \\
\hline Cs-137 & RWMC 2-4 & 2013 & 0.7373 & 0.0127 & 0.0381 & - \\
\hline Cs-137 & RWMC 3-5 & 2013 & 0.2238 & 0.011866667 & 0.0356 & - \\
\hline Cs-137 & RWMC 5-7 & 2013 & 0.2232 & 0.011233333 & 0.0337 & - \\
\hline Cs-137 & RWMC 2-4 & 2014 & 0.3187 & 0.0202 & 0.0606 & - \\
\hline Cs-137 & RWMC 2-4 & 2014 & 0.1439 & 0.0144 & 0.0432 & 一 \\
\hline Cs-137 & RWMC 3-4 & 2014 & 0.1547 & 0.00318 & 0.00954 & - \\
\hline Cs-137 & RWMC 4-1 & 2014 & 0.4513 & 0.0186 & 0.0558 & - \\
\hline Cs-137 & RWMC 5-4 & 2014 & 0.1928 & 0.0090 & 0.02709 & - \\
\hline Cs-137 & RWMC 6-1 & 2014 & 0.1204 & 0.00366 & 0.01098 & - \\
\hline Cs-137 & RWMC 6-3 & 2014 & 0.4386 & 0.0263 & 0.0789 & - \\
\hline Eu-152 & RWMC 10-1 & 2007 & 0.32 & 0.097 & 0.291 & - \\
\hline Eu-152 & RWMC 2-4 & 2007 & 0.1453 & 0.0088 & 0.0264 & - \\
\hline Eu-152 & RWMC 2-5 & 2007 & 0.2397 & 0.0187 & 0.0561 & - \\
\hline Eu-152 & RWMC 2-6 & 2007 & 0.517 & 0.0117 & 0.0351 & 一 \\
\hline Eu-152 & RWMC 2-7 & 2007 & 0.4407 & 0.0107 & 0.0321 & - \\
\hline Eu-152 & RWMC 3-1 & 2007 & 0.263 & 0.0555 & 0.1665 & 一 \\
\hline Eu-152 & RWMC 3-4 & 2007 & 0.3533 & 0.00965 & 0.02895 & - \\
\hline $\mathrm{Eu}-152$ & RWMC 3-5 & 2007 & 0.8876 & 0.0147 & 0.0441 & 一 \\
\hline $\mathrm{Eu}-152$ & RWMC 3-6 & 2007 & 0.2783 & 0.01265 & 0.03795 & 一 \\
\hline Eu-152 & RWMC 3-7 & 2007 & 0.4788 & 0.0139 & 0.0417 & 一 \\
\hline Eu-152 & RWMC 3-8 & 2007 & 0.2 & 0.0116 & 0.0348 & - \\
\hline Eu-152 & RWMC 4-1 & 2007 & 0.3108 & 0.00505 & 0.01515 & - \\
\hline Eu-152 & RWMC 4-2 & 2007 & 0.2353 & 0.02885 & 0.08655 & - \\
\hline Eu-152 & RWMC 4-3 & 2007 & 0.4745 & 0.01005 & 0.03015 & - \\
\hline Eu-152 & RWMC 4-4 & 2007 & 0.1989 & 0.0119 & 0.0357 & - \\
\hline Eu-152 & RWMC 4-5 & 2007 & 0.3626 & 0.0124 & 0.0372 & - \\
\hline
\end{tabular}


Table C-5. (continued).

\begin{tabular}{|c|c|c|c|c|c|c|}
\hline Radionuclide & $\begin{array}{c}\text { Sample } \\
\text { Location }\end{array}$ & Date & $\begin{array}{c}\text { Concentrations } \\
(\mathrm{pCi} / \mathrm{g})\end{array}$ & $\begin{array}{l}\text { Uncertainty } \\
(\mathrm{pCi} / \mathrm{g})\end{array}$ & $\begin{array}{c}\mathrm{MDA} \\
(\mathrm{pCi} / \mathrm{g})\end{array}$ & Flag \\
\hline Eu-152 & RWMC 5-10 & 2007 & 1.403 & 0.0088 & 0.0264 & - \\
\hline Eu-152 & RWMC 5-11 & 2007 & 0.2959 & 0.02415 & 0.07245 & 一 \\
\hline Eu-152 & RWMC 5-12 & 2007 & 0.2881 & 0.0154 & 0.0462 & - \\
\hline Eu-152 & RWMC 5-14 & 2007 & 1.057 & 0.01415 & 0.04245 & - \\
\hline Eu-152 & RWMC 5-15 & 2007 & 0.2922 & 0.03375 & 0.10125 & - \\
\hline Eu-152 & RWMC 5-4 & 2007 & 1.187 & 0.071 & 0.213 & - \\
\hline Eu-152 & RWMC 5-6 & 2007 & 0.5347 & 0.0136 & 0.0408 & - \\
\hline Eu-152 & RWMC 5-8 & 2007 & 0.282 & 0.00945 & 0.02835 & - \\
\hline Eu-152 & RWMC 5-9 & 2007 & 0.1749 & 0.01755 & 0.05265 & - \\
\hline Eu-152 & RWMC 6-1 & 2007 & 0.2431 & 0.01845 & 0.05535 & - \\
\hline Eu-152 & RWMC 6-2 & 2007 & 0.2854 & 0.01135 & 0.03405 & 一 \\
\hline Eu-152 & RWMC 6-3 & 2007 & 0.3213 & 0.012 & 0.036 & - \\
\hline Eu-152 & RWMC 6-4 & 2007 & 0.1973 & 0.0139 & 0.0417 & - \\
\hline Eu-152 & RWMC 6-5 & 2007 & 0.2106 & 0.0056 & 0.0168 & - \\
\hline Eu-152 & RWMC 6-6 & 2007 & 0.3139 & 0.0153 & 0.0459 & - \\
\hline $\mathrm{Eu}-152$ & RWMC 6-7 & 2007 & 0.4628 & 0.00645 & 0.01935 & - \\
\hline Eu-152 & RWMC 6-9 & 2007 & 0.3908 & 0.1305 & 0.3915 & $\mathrm{U}$ \\
\hline Eu-152 & RWMC 7-2 & 2007 & 0.3048 & 0.0063 & 0.0189 & - \\
\hline Eu-152 & RWMC 7-3 & 2007 & 0.5589 & 0.00825 & 0.02475 & - \\
\hline Eu-152 & RWMC 7-4 & 2007 & 0.2693 & 0.0113 & 0.0339 & - \\
\hline $\mathrm{Eu}-152$ & RWMC 7-5 & 2007 & 0.2192 & 0.01125 & 0.03375 & 一 \\
\hline Eu-152 & RWMC 7-6 & 2007 & 0.2317 & 0.0183 & 0.0549 & - \\
\hline Eu-152 & RWMC 7-7 & 2007 & 0.2397 & 0.00945 & 0.02835 & - \\
\hline Eu-152 & RWMC 8-1 & 2007 & 0.1524 & 0.0086 & 0.0258 & - \\
\hline Eu-152 & RWMC 8-4 & 2007 & 0.4579 & 0.0132 & 0.0396 & 一 \\
\hline Eu-152 & RWMC 8-5 & 2007 & 0.5985 & 0.064 & 0.192 & 一 \\
\hline Eu-152 & RWMC 9-1 & 2007 & 0.2081 & 0.00775 & 0.02325 & - \\
\hline Eu-152 & RWMC 5-4 & 2010 & 0.004001 & 0.0314 & 0.0942 & $\mathrm{U}$ \\
\hline Eu-152 & RWMC 10-1 & 2011 & 0.02199 & 0.0298 & 0.0894 & $\mathrm{U}$ \\
\hline Eu-152 & RWMC 2-4 & 2011 & -0.009919 & 0.0327 & 0.0981 & $\mathrm{U}$ \\
\hline Eu-152 & RWMC 2-6 & 2011 & 0.01882 & 0.0266 & 0.0798 & $\mathrm{U}$ \\
\hline Eu-152 & RWMC 2-8 & 2011 & -0.02089 & 0.0284 & 0.0852 & $\mathrm{U}$ \\
\hline Eu-152 & RWMC 3-4 & 2011 & 0.01484 & 0.0307 & 0.0921 & $\mathrm{U}$ \\
\hline Eu-152 & RWMC 3-6 & 2011 & 0.0008288 & 0.0271 & 0.0813 & $\mathrm{U}$ \\
\hline Eu-152 & RWMC 3-8 & 2011 & -0.009544 & 0.03 & 0.09 & $\mathrm{U}$ \\
\hline Eu-152 & RWMC 4-1 & 2011 & 0.01425 & 0.0294 & 0.0882 & $\mathrm{U}$ \\
\hline Eu-152 & RWMC 4-1 & 2011 & -0.002518 & 0.0219 & 0.0657 & $\mathrm{U}$ \\
\hline
\end{tabular}


Table C-5. (continued).

\begin{tabular}{|c|c|c|c|c|c|c|}
\hline Radionuclide & $\begin{array}{l}\text { Sample } \\
\text { Location }\end{array}$ & Date & $\begin{array}{l}\text { Concentrations } \\
(\mathrm{pCi} / \mathrm{g})\end{array}$ & $\begin{array}{l}\text { Uncertainty } \\
(\mathrm{pCi} / \mathrm{g})\end{array}$ & $\begin{array}{c}\mathrm{MDA} \\
(\mathrm{pCi} / \mathrm{g})\end{array}$ & Flag \\
\hline Eu-152 & RWMC 4-5 & 2011 & 0.02435 & 0.0279 & 0.0837 & $\mathrm{U}$ \\
\hline $\mathrm{Eu}-152$ & RWMC 5-12 & 2011 & -0.01587 & 0.0257 & 0.0771 & $\mathrm{U}$ \\
\hline Eu-152 & RWMC 5-7 & 2011 & 0.03169 & 0.0276 & 0.0828 & $\mathrm{U}$ \\
\hline Eu-152 & RWMC 6-1 & 2011 & 0.01291 & 0.0264 & 0.0792 & $\mathrm{U}$ \\
\hline Eu-152 & RWMC 6-3 & 2011 & -0.01289 & 0.0259 & 0.0777 & $\mathrm{U}$ \\
\hline Eu-152 & RWMC 6-7 & 2011 & -0.0001385 & 0.0317 & 0.0951 & $\mathrm{U}$ \\
\hline Eu-152 & RWMC 7-2 & 2011 & -0.01145 & 0.0293 & 0.0879 & $\mathrm{U}$ \\
\hline Eu-152 & RWMC 8-1 & 2011 & 0.01273 & 0.03 & 0.09 & $\mathrm{U}$ \\
\hline Eu-152 & RWMC 8-5 & 2011 & 0.04982 & 0.0313 & 0.0939 & $\mathrm{U}$ \\
\hline Eu-152 & RWMC 9-1 & 2011 & 0.008186 & 0.0306 & 0.0918 & $\mathrm{U}$ \\
\hline Eu-152 & RWMC 2-4 & 2012 & 0.01825 & 0.0246 & 0.0738 & $\mathrm{U}$ \\
\hline $\mathrm{Eu}-152$ & RWMC 3-4 & 2012 & 0.03389 & 0.0277 & 0.0831 & $\mathrm{U}$ \\
\hline Eu-152 & RWMC 3-5 & 2012 & 0.1302 & 0.0329 & 0.0987 & - \\
\hline Eu-152 & RWMC 5-4 & 2012 & 0.02051 & 0.0234 & 0.0702 & $\mathrm{U}$ \\
\hline Eu-152 & RWMC 6-1 & 2012 & 0.03139 & 0.0219 & 0.0657 & $\mathrm{U}$ \\
\hline $\mathrm{Eu}-152$ & RWMC 8-1 & 2012 & 0.02745 & 0.0233 & 0.0699 & $\mathrm{U}$ \\
\hline Eu-152 & $\begin{array}{l}2 \text { INCH AIR } \\
\text { - RWMC }\end{array}$ & 2013 & 0.1113 & 0.0277 & 0.0831 & - \\
\hline Eu-152 & RWMC 2-4 & 2013 & 0.1201 & 0.039666667 & 0.119 & - \\
\hline Eu-152 & RWMC 3-4 & 2013 & 0.007423 & 0.0209 & 0.0627 & $\mathrm{U}$ \\
\hline Eu-152 & RWMC 5-7 & 2013 & 0.1697 & 0.034 & 0.102 & - \\
\hline Eu-152 & RWMC 2-4 & 2014 & 0.00355 & 0.0381 & 0.1143 & $\mathrm{U}$ \\
\hline Eu-152 & RWMC 2-4 & 2014 & -0.001385 & 0.0248 & 0.0744 & $\mathrm{U}$ \\
\hline $\mathrm{Eu}-152$ & RWMC 4-1 & 2014 & 0.05386 & 0.0346 & 0.1038 & $\mathrm{U}$ \\
\hline Eu-152 & RWMC 5-4 & 2014 & 0.02324 & 0.0292 & 0.0876 & $\mathrm{U}$ \\
\hline Eu-152 & RWMC 6-1 & 2014 & 0.003263 & 0.0219 & 0.0657 & $\mathrm{U}$ \\
\hline Eu-152 & RWMC 6-3 & 2014 & 0.04793 & 0.0299 & 0.0897 & $\mathrm{U}$ \\
\hline $\mathrm{Pu}-238$ & $2-3$ & 1972 & 0.018 & 0.002 & 0.006 & - \\
\hline $\mathrm{Pu}-238$ & $2-4$ & 1972 & 0.0072 & 0.0007 & 0.0021 & - \\
\hline $\mathrm{Pu}-238$ & $2-5$ & 1972 & 0.0031 & 0.0045 & 0.0135 & $\mathrm{U}$ \\
\hline $\mathrm{Pu}-238$ & $3-1$ & 1972 & 0.0036 & 0.00014 & 0.00042 & 一 \\
\hline $\mathrm{Pu}-238$ & $3-2$ & 1972 & 0.0086 & 0.00135 & 0.00405 & 一 \\
\hline $\mathrm{Pu}-238$ & $3-3$ & 1972 & 0.0067 & 0.0013 & 0.0039 & - \\
\hline $\mathrm{Pu}-238$ & $3-4$ & 1972 & 0.011 & 0.00112 & 0.00336 & - \\
\hline $\mathrm{Pu}-238$ & $3-5$ & 1972 & 0.0045 & 0.00045 & 0.00135 & - \\
\hline $\mathrm{Pu}-238$ & $2-6$ & 1973 & 0.0076 & 0.0007 & 0.0021 & 一 \\
\hline $\mathrm{Pu}-238$ & $2-7$ & 1973 & 0.0031 & 0.0014 & 0.0042 & $\mathrm{U}$ \\
\hline
\end{tabular}


Table C-5. (continued).

\begin{tabular}{|c|c|c|c|c|c|c|}
\hline Radionuclide & $\begin{array}{c}\text { Sample } \\
\text { Location }\end{array}$ & Date & $\begin{array}{c}\text { Concentrations } \\
(\mathrm{pCi} / \mathrm{g})\end{array}$ & $\begin{array}{l}\text { Uncertainty } \\
(\mathrm{pCi} / \mathrm{g})\end{array}$ & $\begin{array}{c}\text { MDA } \\
(\mathrm{pCi} / \mathrm{g})\end{array}$ & Flag \\
\hline $\mathrm{Pu}-238$ & $2-8$ & 1973 & 0.0049 & 0.00045 & 0.00135 & - \\
\hline $\mathrm{Pu}-238$ & $3-6$ & 1973 & 0.0018 & 0.003 & 0.009 & $\mathrm{U}$ \\
\hline $\mathrm{Pu}-238$ & $3-7$ & 1973 & 0.0049 & 0.000675 & 0.002025 & - \\
\hline $\mathrm{Pu}-238$ & $3-8$ & 1973 & 0.0049 & 0.0009 & 0.0027 & - \\
\hline $\mathrm{Pu}-238$ & $4-1$ & 1973 & 0.0049 & 0.0009 & 0.0027 & - \\
\hline $\mathrm{Pu}-238$ & $4-2$ & 1973 & 0.0022 & 0.0014 & 0.0042 & $\mathrm{U}$ \\
\hline $\mathrm{Pu}-238$ & $4-3$ & 1973 & 0.0027 & 0.0014 & 0.0042 & $\mathrm{U}$ \\
\hline $\mathrm{Pu}-238$ & $4-4$ & 1973 & 0.0045 & 0.00045 & 0.00135 & - \\
\hline $\mathrm{Pu}-238$ & $4-5$ & 1973 & 0.004 & 0.0009 & 0.0027 & - \\
\hline $\mathrm{Pu}-238$ & $5-1$ & 1973 & 0.058 & 0.002 & 0.006 & - \\
\hline $\mathrm{Pu}-238$ & $5-2$ & 1973 & 0.013 & 0.00135 & 0.00405 & 一 \\
\hline $\mathrm{Pu}-238$ & $5-3$ & 1973 & 0.005 & 0.0007 & 0.0021 & 一 \\
\hline $\mathrm{Pu}-238$ & $5-4$ & 1973 & 0.00816 & 0.0009 & 0.0027 & - \\
\hline $\mathrm{Pu}-238$ & $10-1$ & 1974 & 0.0027 & 0.009 & 0.027 & $\mathrm{U}$ \\
\hline $\mathrm{Pu}-238$ & $5-10$ & 1974 & 0.0045 & 0.00045 & 0.00135 & - \\
\hline $\mathrm{Pu}-238$ & $5-11$ & 1974 & 0.0014 & 0.0009 & 0.0027 & $\mathrm{U}$ \\
\hline $\mathrm{Pu}-238$ & $5-12$ & 1974 & 0.0036 & 0.0014 & 0.0042 & $\mathrm{U}$ \\
\hline $\mathrm{Pu}-238$ & $5-13$ & 1974 & 0.0031 & 0.0014 & 0.0042 & $\mathrm{U}$ \\
\hline $\mathrm{Pu}-238$ & $5-14$ & 1974 & 0.0072 & 0.0014 & 0.0042 & - \\
\hline $\mathrm{Pu}-238$ & $5-14$ & 1974 & 0.0009 & 0.0014 & 0.0042 & $\mathrm{U}$ \\
\hline $\mathrm{Pu}-238$ & $5-5$ & 1974 & 0.0068 & 0.0009 & 0.0027 & \\
\hline $\mathrm{Pu}-238$ & $5-6$ & 1974 & 0.0022 & 0.0009 & 0.0027 & $\mathrm{U}$ \\
\hline $\mathrm{Pu}-238$ & $5-7$ & 1974 & 0.004 & 0.0009 & 0.0027 & - \\
\hline $\mathrm{Pu}-238$ & $5-8$ & 1974 & 0.0063 & 0.0009 & 0.0027 & - \\
\hline $\mathrm{Pu}-238$ & $5-9$ & 1974 & 0.0059 & 0.0009 & 0.0027 & - \\
\hline $\mathrm{Pu}-238$ & $6-1$ & 1974 & 0.0022 & 0.0022 & 0.0066 & $\mathrm{U}$ \\
\hline $\mathrm{Pu}-238$ & $6-2$ & 1974 & 0.0036 & 0.0014 & 0.0042 & $\mathrm{U}$ \\
\hline $\mathrm{Pu}-238$ & $6-3$ & 1974 & 0.009 & 0.00225 & 0.00675 & - \\
\hline $\mathrm{Pu}-238$ & $6-4$ & 1974 & 0.0095 & 0.0007 & 0.0021 & - \\
\hline $\mathrm{Pu}-238$ & $6-5$ & 1974 & 0.0014 & 0.0009 & 0.0027 & $\mathrm{U}$ \\
\hline $\mathrm{Pu}-238$ & $6-6$ & 1974 & 0.0009 & 0.0014 & 0.0042 & $\mathrm{U}$ \\
\hline $\mathrm{Pu}-238$ & $7-1$ & 1974 & 0.005 & 0.0007 & 0.0021 & - \\
\hline $\mathrm{Pu}-238$ & $7-2$ & 1974 & 0.009 & 0.00225 & 0.00675 & - \\
\hline $\mathrm{Pu}-238$ & $7-3$ & 1974 & 0.058 & 0.0018 & 0.0054 & - \\
\hline $\mathrm{Pu}-238$ & $7-4$ & 1974 & 0.0022 & 0.0014 & 0.0042 & $\mathrm{U}$ \\
\hline $\mathrm{Pu}-238$ & $7-5$ & 1974 & 0.0022 & 0.0022 & 0.0066 & $\mathrm{U}$ \\
\hline $\mathrm{Pu}-238$ & $7-6$ & 1974 & 0.0018 & 0.0018 & 0.0054 & $\mathrm{U}$ \\
\hline
\end{tabular}


Table C-5. (continued).

\begin{tabular}{|c|c|c|c|c|c|c|}
\hline Radionuclide & $\begin{array}{c}\text { Sample } \\
\text { Location }\end{array}$ & Date & $\begin{array}{c}\text { Concentrations } \\
(\mathrm{pCi} / \mathrm{g})\end{array}$ & $\begin{array}{l}\text { Uncertainty } \\
(\mathrm{pCi} / \mathrm{g})\end{array}$ & $\begin{array}{c}\mathrm{MDA} \\
(\mathrm{pCi} / \mathrm{g})\end{array}$ & Flag \\
\hline $\mathrm{Pu}-238$ & $8-1$ & 1974 & 0.018 & 0.0045 & 0.0135 & - \\
\hline $\mathrm{Pu}-238$ & $8-2$ & 1974 & 0.0018 & 0.0018 & 0.0054 & $\mathrm{U}$ \\
\hline $\mathrm{Pu}-238$ & $8-3$ & 1974 & 0.0049 & 0.0009 & 0.0027 & - \\
\hline $\mathrm{Pu}-238$ & $8-4$ & 1974 & 0.0095 & 0.00135 & 0.00405 & - \\
\hline $\mathrm{Pu}-238$ & $9-1$ & 1974 & 0.0009 & 0.0009 & 0.0027 & $\mathrm{U}$ \\
\hline $\mathrm{Pu}-238$ & $9-7$ & 1974 & 0.0049 & 0.00045 & 0.00135 & - \\
\hline $\mathrm{Pu}-238$ & $10-1$ & 1978 & 0.0009 & 0.0007 & 0.0021 & $\mathrm{U}$ \\
\hline $\mathrm{Pu}-238$ & $2-3$ & 1978 & 0.007 & 0.006 & 0.018 & $\mathrm{U}$ \\
\hline $\mathrm{Pu}-238$ & $2-5$ & 1978 & 0 & 0.00025 & 0.00075 & $\mathrm{U}$ \\
\hline $\mathrm{Pu}-238$ & $2-7$ & 1978 & 0.002 & 0.001 & 0.003 & $\mathrm{U}$ \\
\hline $\mathrm{Pu}-238$ & $3-2$ & 1978 & 0.003 & 0.001 & 0.003 & - \\
\hline $\mathrm{Pu}-238$ & $3-6$ & 1978 & 0.002 & 0.001 & 0.003 & $\mathrm{U}$ \\
\hline $\mathrm{Pu}-238$ & $3-8$ & 1978 & 0.0008 & 0.0007 & 0.0021 & $\mathrm{U}$ \\
\hline $\mathrm{Pu}-238$ & $4-1$ & 1978 & 0.0018 & 0.0009 & 0.0027 & $\mathrm{U}$ \\
\hline $\mathrm{Pu}-238$ & $4-3$ & 1978 & 0.0011 & 0.0007 & 0.0021 & $\mathrm{U}$ \\
\hline $\mathrm{Pu}-238$ & $4-5$ & 1978 & 0.0004 & 0.0006 & 0.0018 & $\mathrm{U}$ \\
\hline $\mathrm{Pu}-238$ & 4-A & 1978 & 0.005 & 0.001 & 0.003 & - \\
\hline $\mathrm{Pu}-238$ & 4-B & 1978 & 0.0006 & 0.0004 & 0.0012 & $\mathrm{U}$ \\
\hline $\mathrm{Pu}-238$ & 4-C & 1978 & 0.02 & - & 0 & - \\
\hline $\mathrm{Pu}-238$ & $5-11$ & 1978 & 0.0002 & 0.0003 & 0.0009 & $\mathrm{U}$ \\
\hline $\mathrm{Pu}-238$ & $5-13$ & 1978 & 0.003 & 0.001 & 0.003 & - \\
\hline $\mathrm{Pu}-238$ & $5-15$ & 1978 & 0.0002 & 0.000166667 & 0.0005 & $\mathrm{U}$ \\
\hline $\mathrm{Pu}-238$ & $5-5$ & 1978 & 0.0009 & 0.0009 & 0.0027 & $\mathrm{U}$ \\
\hline $\mathrm{Pu}-238$ & $5-7$ & 1978 & 0.0005 & 0.0008 & 0.0024 & $\mathrm{U}$ \\
\hline $\mathrm{Pu}-238$ & $5-8$ & 1978 & 0.0022 & 0.0009 & 0.0027 & $\mathrm{U}$ \\
\hline $\mathrm{Pu}-238$ & $6-1$ & 1978 & 0.0002 & 0.00035 & 0.00105 & $\mathrm{U}$ \\
\hline $\mathrm{Pu}-238$ & $6-3$ & 1978 & 0.0005 & 0.0007 & 0.0021 & $\mathrm{U}$ \\
\hline $\mathrm{Pu}-238$ & $6-5$ & 1978 & 0.001 & 0.001 & 0.003 & $\mathrm{U}$ \\
\hline $\mathrm{Pu}-238$ & $6-7$ & 1978 & 0.0008 & 0.0009 & 0.0027 & $\mathrm{U}$ \\
\hline $\mathrm{Pu}-238$ & $7-2$ & 1978 & 0.004 & 0.0005 & 0.0015 & - \\
\hline $\mathrm{Pu}-238$ & $7-4$ & 1978 & 0.003 & 0.001 & 0.003 & - \\
\hline $\mathrm{Pu}-238$ & $7-6$ & 1978 & 0.00059 & 0.0012 & 0.0036 & $\mathrm{U}$ \\
\hline $\mathrm{Pu}-238$ & $7-\mathrm{A}$ & 1978 & 0.0002 & 0.0003 & 0.0009 & $\mathrm{U}$ \\
\hline $\mathrm{Pu}-238$ & 7-B & 1978 & 0.0006 & 0.0006 & 0.0018 & $\mathrm{U}$ \\
\hline $\mathrm{Pu}-238$ & $8-1$ & 1978 & 0.0016 & 0.0009 & 0.0027 & $\mathrm{U}$ \\
\hline $\mathrm{Pu}-238$ & $8-3$ & 1978 & 0.0024 & 0.0009 & 0.0027 & $\mathrm{U}$ \\
\hline $\mathrm{Pu}-238$ & $8-5$ & 1978 & 0.003 & 0.001 & 0.003 & - \\
\hline
\end{tabular}


Table C-5. (continued).

\begin{tabular}{|c|c|c|c|c|c|c|}
\hline Radionuclide & $\begin{array}{c}\text { Sample } \\
\text { Location }\end{array}$ & Date & $\begin{array}{c}\text { Concentrations } \\
(\mathrm{pCi} / \mathrm{g})\end{array}$ & $\begin{array}{l}\text { Uncertainty } \\
(\mathrm{pCi} / \mathrm{g})\end{array}$ & $\begin{array}{c}\mathrm{MDA} \\
(\mathrm{pCi} / \mathrm{g})\end{array}$ & Flag \\
\hline $\mathrm{Pu}-238$ & $8-A$ & 1978 & 0.0016 & 0.0009 & 0.0027 & $\mathrm{U}$ \\
\hline $\mathrm{Pu}-238$ & $8-B$ & 1978 & 0.001 & 0.0009 & 0.0027 & $\mathrm{U}$ \\
\hline $\mathrm{Pu}-238$ & $8-\mathrm{C}$ & 1978 & 0.0004 & 0.0006 & 0.0018 & $\mathrm{U}$ \\
\hline $\mathrm{Pu}-238$ & $9-\mathrm{A}$ & 1978 & 0.0005 & 0.0006 & 0.0018 & $\mathrm{U}$ \\
\hline $\mathrm{Pu}-238$ & $10-1$ & 1985 & 0.0015 & 0.0017 & 0.0051 & $\mathrm{U}$ \\
\hline $\mathrm{Pu}-238$ & $2-4$ & 1985 & 0 & 0.0015 & 0.0045 & $\mathrm{U}$ \\
\hline $\mathrm{Pu}-238$ & $3-1$ & 1985 & 0.002 & 0.0015 & 0.0045 & $\mathrm{U}$ \\
\hline $\mathrm{Pu}-238$ & $3-4$ & 1985 & 0.009 & 0.001 & 0.003 & - \\
\hline $\mathrm{Pu}-238$ & $3-7$ & 1985 & 0.0011 & 0.0015 & 0.0045 & $\mathrm{U}$ \\
\hline $\mathrm{Pu}-238$ & $4-2$ & 1985 & 0.0031 & 0.00075 & 0.00225 & - \\
\hline $\mathrm{Pu}-238$ & $4-4$ & 1985 & 0.0033 & 0.0017 & 0.0051 & $\mathrm{U}$ \\
\hline $\mathrm{Pu}-238$ & $4-\mathrm{A}$ & 1985 & 0.0021 & 0.0016 & 0.0048 & $\mathrm{U}$ \\
\hline $\mathrm{Pu}-238$ & 4-B & 1985 & 0.0005 & 0.0016 & 0.0048 & $\mathrm{U}$ \\
\hline $\mathrm{Pu}-238$ & 4-C & 1985 & 0.0022 & 0.0015 & 0.0045 & $\mathrm{U}$ \\
\hline $\mathrm{Pu}-238$ & $5-10$ & 1985 & 0.0035 & 0.0016 & 0.0048 & $\mathrm{U}$ \\
\hline $\mathrm{Pu}-238$ & $5-15$ & 1985 & 0.0021 & 0.0016 & 0.0048 & $\mathrm{U}$ \\
\hline $\mathrm{Pu}-238$ & $5-5$ & 1985 & 0.0021 & 0.0015 & 0.0045 & $\mathrm{U}$ \\
\hline $\mathrm{Pu}-238$ & $5-7$ & 1985 & 0.003 & 0.002 & 0.006 & $\mathrm{U}$ \\
\hline $\mathrm{Pu}-238$ & $6-1$ & 1985 & 0.005 & 0.001 & 0.003 & - \\
\hline $\mathrm{Pu}-238$ & $7-3$ & 1985 & 0.0002 & 0.0016 & 0.0048 & $\mathrm{U}$ \\
\hline $\mathrm{Pu}-238$ & $7-5$ & 1985 & 0.0025 & 0.0015 & 0.0045 & $\mathrm{U}$ \\
\hline $\mathrm{Pu}-238$ & $7-7$ & 1985 & 0.0029 & 0.0016 & 0.0048 & $\mathrm{U}$ \\
\hline $\mathrm{Pu}-238$ & $7-\mathrm{A}$ & 1985 & 0.0033 & 0.0015 & 0.0045 & $\mathrm{U}$ \\
\hline $\mathrm{Pu}-238$ & $8-1$ & 1985 & 0.0024 & 0.0015 & 0.0045 & $\mathrm{U}$ \\
\hline $\mathrm{Pu}-238$ & $8-2$ & 1985 & 0.001 & 0.002 & 0.006 & $\mathrm{U}$ \\
\hline $\mathrm{Pu}-238$ & $8-5$ & 1985 & 0.003 & 0.002 & 0.006 & $\mathrm{U}$ \\
\hline $\mathrm{Pu}-238$ & $8-\mathrm{C}$ & 1985 & 0.003 & 0.002 & 0.006 & $\mathrm{U}$ \\
\hline $\mathrm{Pu}-238$ & 9-A & 1985 & 0 & 0.001 & 0.003 & $\mathrm{U}$ \\
\hline $\mathrm{Pu}-239 / 240$ & $2-3$ & 1972 & 0.644 & 0.018 & 0.054 & - \\
\hline $\mathrm{Pu}-239 / 240$ & $2-4$ & 1972 & 0.4 & 0.014 & 0.042 & - \\
\hline $\mathrm{Pu}-239 / 240$ & $2-5$ & 1972 & 0.0054 & 0.0007 & 0.0021 & - \\
\hline $\mathrm{Pu}-239 / 240$ & $3-1$ & 1972 & 0.0027 & 0.032 & 0.096 & $\mathrm{U}$ \\
\hline $\mathrm{Pu}-239 / 240$ & $3-2$ & 1972 & 0.207 & 0.009 & 0.027 & - \\
\hline $\mathrm{Pu}-239 / 240$ & $3-3$ & 1972 & 0.171 & 0.000433333 & 0.0013 & - \\
\hline $\mathrm{Pu}-239 / 240$ & $3-4$ & 1972 & 0.166 & 0.004 & 0.012 & - \\
\hline $\mathrm{Pu}-239 / 240$ & $3-5$ & 1972 & 0.059 & 0.0018 & 0.0054 & - \\
\hline $\mathrm{Pu}-239 / 240$ & $2-6$ & 1973 & 0.055 & 0.0165 & 0.0495 & - \\
\hline
\end{tabular}


Table C-5. (continued).

\begin{tabular}{|c|c|c|c|c|c|c|}
\hline Radionuclide & $\begin{array}{c}\text { Sample } \\
\text { Location }\end{array}$ & Date & $\begin{array}{c}\text { Concentrations } \\
(\mathrm{pCi} / \mathrm{g})\end{array}$ & $\begin{array}{l}\text { Uncertainty } \\
(\mathrm{pCi} / \mathrm{g})\end{array}$ & $\begin{array}{c}\mathrm{MDA} \\
(\mathrm{pCi} / \mathrm{g})\end{array}$ & Flag \\
\hline $\mathrm{Pu}-239 / 240$ & $2-7$ & 1973 & 0.017 & 0.018 & 0.054 & $\mathrm{U}$ \\
\hline $\mathrm{Pu}-239 / 240$ & $2-8$ & 1973 & 0.038 & 0.003 & 0.009 & 一 \\
\hline $\mathrm{Pu}-239 / 240$ & $3-6$ & 1973 & 0.022 & 0.0045 & 0.0135 & - \\
\hline $\mathrm{Pu}-239 / 240$ & $3-7$ & 1973 & 0.527 & 0.0036 & 0.0108 & - \\
\hline $\mathrm{Pu}-239 / 240$ & $3-8$ & 1973 & 0.027 & 0.0018 & 0.0054 & - \\
\hline $\mathrm{Pu}-239 / 240$ & $4-1$ & 1973 & 0.063 & 0.0045 & 0.0135 & - \\
\hline $\mathrm{Pu}-239 / 240$ & $4-2$ & 1973 & 0.043 & 0.0031 & 0.0093 & - \\
\hline $\mathrm{Pu}-239 / 240$ & $4-3$ & 1973 & 0.031 & 0.0027 & 0.0081 & - \\
\hline $\mathrm{Pu}-239 / 240$ & $4-4$ & 1973 & 0.024 & 0.0022 & 0.0066 & - \\
\hline $\mathrm{Pu}-239 / 240$ & $4-5$ & 1973 & 0.028 & 0.0011 & 0.0033 & - \\
\hline $\mathrm{Pu}-239 / 240$ & $5-1$ & 1973 & 2.57 & 0.045 & 0.135 & - \\
\hline $\mathrm{Pu}-239 / 240$ & $5-2$ & 1973 & 0.405 & 0.014 & 0.042 & 一 \\
\hline $\mathrm{Pu}-239 / 240$ & $5-3$ & 1973 & 0.144 & 0.0045 & 0.0135 & - \\
\hline $\mathrm{Pu}-239 / 240$ & $5-4$ & 1973 & 0.0086 & 0.0009 & 0.0027 & - \\
\hline $\mathrm{Pu}-239 / 240$ & $10-1$ & 1974 & 0.0095 & 0.0018 & 0.0054 & - \\
\hline $\mathrm{Pu}-239 / 240$ & $5-10$ & 1974 & 0.04 & 0.0027 & 0.0081 & - \\
\hline $\mathrm{Pu}-239 / 240$ & $5-11$ & 1974 & 0.011 & 0.0007 & 0.0021 & - \\
\hline $\mathrm{Pu}-239 / 240$ & $5-12$ & 1974 & 0.014 & 0.0022 & 0.0066 & - \\
\hline $\mathrm{Pu}-239 / 240$ & $5-13$ & 1974 & 0.077 & 0.00225 & 0.00675 & - \\
\hline $\mathrm{Pu}-239 / 240$ & $5-14$ & 1974 & 0.083 & 0.002 & 0.006 & - \\
\hline $\mathrm{Pu}-239 / 240$ & $5-14$ & 1974 & 0.0018 & 0.0009 & 0.0027 & $\mathrm{U}$ \\
\hline $\mathrm{Pu}-239 / 240$ & $5-5$ & 1974 & 0.117 & 0.0045 & 0.0135 & - \\
\hline $\mathrm{Pu}-239 / 240$ & $5-6$ & 1974 & 0.0441 & 0.0027 & 0.0081 & - \\
\hline $\mathrm{Pu}-239 / 240$ & $5-7$ & 1974 & 0.0441 & 0.0009 & 0.0027 & - \\
\hline $\mathrm{Pu}-239 / 240$ & $5-8$ & 1974 & 0.108 & 0.002 & 0.006 & 一 \\
\hline $\mathrm{Pu}-239 / 240$ & $5-9$ & 1974 & 0.036 & 0.0027 & 0.0081 & 一 \\
\hline $\mathrm{Pu}-239 / 240$ & $6-1$ & 1974 & 0.049 & 0.003 & 0.009 & - \\
\hline $\mathrm{Pu}-239 / 240$ & $6-2$ & 1974 & 0.029 & 0.0027 & 0.0081 & - \\
\hline $\mathrm{Pu}-239 / 240$ & $6-3$ & 1974 & 0.126 & 0.009 & 0.027 & - \\
\hline $\mathrm{Pu}-239 / 240$ & $6-4$ & 1974 & 0.365 & 0.009 & 0.027 & - \\
\hline $\mathrm{Pu}-239 / 240$ & $6-5$ & 1974 & 0.0117 & 0.0007 & 0.0021 & - \\
\hline $\mathrm{Pu}-239 / 240$ & $6-6$ & 1974 & 0.149 & 0.0022 & 0.0066 & - \\
\hline $\mathrm{Pu}-239 / 240$ & $7-1$ & 1974 & 0.0049 & 0.0045 & 0.0135 & $\mathrm{U}$ \\
\hline $\mathrm{Pu}-239 / 240$ & $7-2$ & 1974 & 0.03 & 0.009 & 0.027 & - \\
\hline $\mathrm{Pu}-239 / 240$ & $7-3$ & 1974 & 0.049 & 0.0045 & 0.0135 & 一 \\
\hline $\mathrm{Pu}-239 / 240$ & $7-4$ & 1974 & 0.104 & 0.00225 & 0.00675 & - \\
\hline $\mathrm{Pu}-239 / 240$ & $7-5$ & 1974 & 0.027 & 0.0045 & 0.0135 & - \\
\hline
\end{tabular}


Table C-5. (continued).

\begin{tabular}{|c|c|c|c|c|c|c|}
\hline Radionuclide & $\begin{array}{c}\text { Sample } \\
\text { Location }\end{array}$ & Date & $\begin{array}{c}\text { Concentrations } \\
(\mathrm{pCi} / \mathrm{g})\end{array}$ & $\begin{array}{l}\text { Uncertainty } \\
(\mathrm{pCi} / \mathrm{g})\end{array}$ & $\begin{array}{c}\mathrm{MDA} \\
(\mathrm{pCi} / \mathrm{g})\end{array}$ & Flag \\
\hline $\mathrm{Pu}-239 / 240$ & $7-6$ & 1974 & 0.031 & 0.0045 & 0.0135 & - \\
\hline $\mathrm{Pu}-239 / 240$ & $8-1$ & 1974 & 0.185 & 0.007 & 0.021 & - \\
\hline $\mathrm{Pu}-239 / 240$ & $8-2$ & 1974 & 0.027 & 0.0045 & 0.0135 & - \\
\hline $\mathrm{Pu}-239 / 240$ & $8-3$ & 1974 & 0.042 & 0.0036 & 0.0108 & - \\
\hline $\mathrm{Pu}-239 / 240$ & $8-4$ & 1974 & 0.036 & 0.0045 & 0.0135 & - \\
\hline $\mathrm{Pu}-239 / 240$ & $9-1$ & 1974 & 0.011 & 0.0014 & 0.0042 & - \\
\hline $\mathrm{Pu}-239 / 240$ & $9-7$ & 1974 & 0.052 & 0.0018 & 0.0054 & - \\
\hline $\mathrm{Pu}-239 / 240$ & $10-1$ & 1978 & 0.012 & 0.001 & 0.003 & - \\
\hline $\mathrm{Pu}-239 / 240$ & $2-3$ & 1978 & 0.17 & 0.01 & 0.03 & - \\
\hline $\mathrm{Pu}-239 / 240$ & $2-5$ & 1978 & 0.022 & 0.003 & 0.009 & - \\
\hline $\mathrm{Pu}-239 / 240$ & $2-7$ & 1978 & 0.009 & 0.0005 & 0.0015 & 一 \\
\hline $\mathrm{Pu}-239 / 240$ & $3-2$ & 1978 & 0.053 & 0.002 & 0.006 & 一 \\
\hline $\mathrm{Pu}-239 / 240$ & $3-6$ & 1978 & 0.015 & 0.002 & 0.006 & - \\
\hline $\mathrm{Pu}-239 / 240$ & $3-8$ & 1978 & 0.018 & 0.002 & 0.006 & - \\
\hline $\mathrm{Pu}-239 / 240$ & $4-1$ & 1978 & 0.042 & 0.003 & 0.009 & - \\
\hline $\mathrm{Pu}-239 / 240$ & $4-3$ & 1978 & 0.017 & 0.002 & 0.006 & - \\
\hline $\mathrm{Pu}-239 / 240$ & $4-5$ & 1978 & 0.013 & 0.002 & 0.006 & - \\
\hline $\mathrm{Pu}-239 / 240$ & 4-A & 1978 & 0.018 & 0.002 & 0.006 & - \\
\hline $\mathrm{Pu}-239 / 240$ & 4-B & 1978 & 0.018 & 0.002 & 0.006 & - \\
\hline $\mathrm{Pu}-239 / 240$ & 4-C & 1978 & 0.015 & 0.002 & 0.006 & - \\
\hline $\mathrm{Pu}-239 / 240$ & $5-11$ & 1978 & 0.011 & 0.002 & 0.006 & - \\
\hline $\mathrm{Pu}-239 / 240$ & $5-13$ & 1978 & 0.081 & 0.0025 & 0.0075 & - \\
\hline $\mathrm{Pu}-239 / 240$ & $5-15$ & 1978 & 0.004 & 0.0005 & 0.0015 & - \\
\hline $\mathrm{Pu}-239 / 240$ & $5-5$ & 1978 & 0.078 & 0.0025 & 0.0075 & - \\
\hline $\mathrm{Pu}-239 / 240$ & $5-7$ & 1978 & 0.016 & 0.002 & 0.006 & 一 \\
\hline $\mathrm{Pu}-239 / 240$ & $5-8$ & 1978 & 0.042 & 0.003 & 0.009 & 一 \\
\hline $\mathrm{Pu}-239 / 240$ & $6-1$ & 1978 & 0.019 & 0.002 & 0.006 & - \\
\hline $\mathrm{Pu}-239 / 240$ & $6-3$ & 1978 & 0.02 & 0.002 & 0.006 & - \\
\hline $\mathrm{Pu}-239 / 240$ & $6-5$ & 1978 & 0.013 & 0.001 & 0.003 & - \\
\hline $\mathrm{Pu}-239 / 240$ & $6-7$ & 1978 & 0.013 & 0.002 & 0.006 & - \\
\hline $\mathrm{Pu}-239 / 240$ & $7-2$ & 1978 & 0.063 & 0.004 & 0.012 & - \\
\hline $\mathrm{Pu}-239 / 240$ & $7-4$ & 1978 & 0.129 & 0.006 & 0.018 & - \\
\hline $\mathrm{Pu}-239 / 240$ & $7-6$ & 1978 & 0.022 & 0.0015 & 0.0045 & - \\
\hline $\mathrm{Pu}-239 / 240$ & $7-A$ & 1978 & 0.016 & 0.002 & 0.006 & - \\
\hline $\mathrm{Pu}-239 / 240$ & 7-B & 1978 & 0.012 & 0.001 & 0.003 & - \\
\hline $\mathrm{Pu}-239 / 240$ & $8-1$ & 1978 & 0.015 & 0.002 & 0.006 & - \\
\hline $\mathrm{Pu}-239 / 240$ & $8-3$ & 1978 & 0.087 & 0.0025 & 0.0075 & - \\
\hline
\end{tabular}


Table C-5. (continued).

\begin{tabular}{|c|c|c|c|c|c|c|}
\hline Radionuclide & $\begin{array}{c}\text { Sample } \\
\text { Location }\end{array}$ & Date & $\begin{array}{c}\text { Concentrations } \\
(\mathrm{pCi} / \mathrm{g})\end{array}$ & $\begin{array}{c}\text { Uncertainty } \\
(\mathrm{pCi} / \mathrm{g})\end{array}$ & $\begin{array}{c}\mathrm{MDA} \\
(\mathrm{pCi} / \mathrm{g})\end{array}$ & Flag \\
\hline $\mathrm{Pu}-239 / 240$ & $8-5$ & 1978 & 0.025 & 0.002 & 0.006 & - \\
\hline $\mathrm{Pu}-239 / 240$ & $8-A$ & 1978 & 0.018 & 0.002 & 0.006 & - \\
\hline $\mathrm{Pu}-239 / 240$ & $8-\mathrm{B}$ & 1978 & 0.031 & 0.0015 & 0.0045 & - \\
\hline $\mathrm{Pu}-239 / 240$ & $8-\mathrm{C}$ & 1978 & 0.014 & 0.002 & 0.006 & - \\
\hline $\mathrm{Pu}-239 / 240$ & 9-A & 1978 & 0.006 & 0.0005 & 0.0015 & - \\
\hline $\mathrm{Pu}-239 / 240$ & $10-1$ & 1985 & 0.014 & 0.002 & 0.006 & - \\
\hline $\mathrm{Pu}-239 / 240$ & $2-4$ & 1985 & 0.152 & 0.012 & 0.036 & - \\
\hline $\mathrm{Pu}-239 / 240$ & $3-1$ & 1985 & 0.017 & 0.002 & 0.006 & - \\
\hline $\mathrm{Pu}-239 / 240$ & $3-4$ & 1985 & 0.351 & 0.0085 & 0.0255 & - \\
\hline $\mathrm{Pu}-239 / 240$ & $3-7$ & 1985 & 0.017 & 0.002 & 0.006 & - \\
\hline $\mathrm{Pu}-239 / 240$ & $4-2$ & 1985 & 0.023 & 0.0015 & 0.0045 & 一 \\
\hline $\mathrm{Pu}-239 / 240$ & $4-4$ & 1985 & 0.025 & 0.0015 & 0.0045 & 一 \\
\hline $\mathrm{Pu}-239 / 240$ & 4-A & 1985 & 0.017 & 0.002 & 0.006 & - \\
\hline $\mathrm{Pu}-239 / 240$ & $4-\mathrm{B}$ & 1985 & 0.008 & 0.001 & 0.003 & - \\
\hline $\mathrm{Pu}-239 / 240$ & $4-\mathrm{C}$ & 1985 & 0.007 & 0.001 & 0.003 & - \\
\hline $\mathrm{Pu}-239 / 240$ & $5-10$ & 1985 & 0.047 & 0.004 & 0.012 & - \\
\hline $\mathrm{Pu}-239 / 240$ & $5-15$ & 1985 & 0.016 & 0.002 & 0.006 & - \\
\hline $\mathrm{Pu}-239 / 240$ & $5-5$ & 1985 & 0.062 & 0.005 & 0.015 & - \\
\hline $\mathrm{Pu}-239 / 240$ & $5-7$ & 1985 & 0.03 & 0.0015 & 0.0045 & - \\
\hline $\mathrm{Pu}-239 / 240$ & $6-1$ & 1985 & 0.047 & 0.004 & 0.012 & - \\
\hline $\mathrm{Pu}-239 / 240$ & $7-3$ & 1985 & 0.025 & 0.003 & 0.009 & - \\
\hline $\mathrm{Pu}-239 / 240$ & $7-5$ & 1985 & 0.021 & 0.0015 & 0.0045 & - \\
\hline $\mathrm{Pu}-239 / 240$ & $7-7$ & 1985 & 0.024 & 0.0015 & 0.0045 & - \\
\hline $\mathrm{Pu}-239 / 240$ & $7-A$ & 1985 & 0.021 & 0.003 & 0.009 & - \\
\hline $\mathrm{Pu}-239 / 240$ & $8-1$ & 1985 & 0.018 & 0.003 & 0.009 & 一 \\
\hline $\mathrm{Pu}-239 / 240$ & $8-2$ & 1985 & 0.025 & 0.0015 & 0.0045 & 一 \\
\hline $\mathrm{Pu}-239 / 240$ & $8-5$ & 1985 & 0.015 & 0.003 & 0.009 & - \\
\hline $\mathrm{Pu}-239 / 240$ & $8-\mathrm{C}$ & 1985 & 0.018 & 0.002 & 0.006 & - \\
\hline $\mathrm{Pu}-239 / 240$ & 9-A & 1985 & 0.016 & 0.003 & 0.009 & - \\
\hline $\mathrm{Sb}-125$ & $2-4$ & 1972 & 0.18 & 0.02 & 0.06 & - \\
\hline $\mathrm{Sb}-125$ & $3-1$ & 1972 & 0.14 & 0.02 & 0.06 & - \\
\hline $\mathrm{Sb}-125$ & $3-2$ & 1972 & 0.14 & 0.02 & 0.06 & - \\
\hline $\mathrm{Sb}-125$ & $3-3$ & 1972 & 0.14 & 0.02 & 0.06 & - \\
\hline $\mathrm{Sb}-125$ & $4-3$ & 1973 & 0.12 & 0.01 & 0.03 & - \\
\hline $\mathrm{Sb}-125$ & $5-1$ & 1973 & 0.12 & 0.01 & 0.03 & - \\
\hline $\mathrm{Sb}-125$ & $5-2$ & 1973 & 0.16 & 0.02 & 0.06 & - \\
\hline $\mathrm{Sb}-125$ & $5-3$ & 1973 & 0.13 & 0.02 & 0.06 & - \\
\hline
\end{tabular}


Table C-5. (continued).

\begin{tabular}{|c|c|c|c|c|c|c|}
\hline Radionuclide & $\begin{array}{l}\text { Sample } \\
\text { Location }\end{array}$ & Date & $\begin{array}{l}\text { Concentrations } \\
(\mathrm{pCi} / \mathrm{g})\end{array}$ & $\begin{array}{l}\text { Uncertainty } \\
(\mathrm{pCi} / \mathrm{g})\end{array}$ & $\begin{array}{l}\mathrm{MDA} \\
(\mathrm{pCi} / \mathrm{g})\end{array}$ & Flag \\
\hline Sb-125 & $5-4$ & 1973 & 0.15 & 0.02 & 0.06 & - \\
\hline Sb-125 & $5-13$ & 1978 & 0.176 & 0.02 & 0.06 & 一 \\
\hline $\mathrm{Sb}-125$ & $8-3$ & 1978 & 0.0894 & 0.032 & 0.096 & $\mathrm{U}$ \\
\hline $\mathrm{Sb}-125$ & RWMC 10-1 & 2007 & 0.4207 & 0.04215 & 0.12645 & - \\
\hline $\mathrm{Sb}-125$ & RWMC 2-4 & 2007 & 0.2333 & 0.0605 & 0.1815 & - \\
\hline $\mathrm{Sb}-125$ & RWMC 2-5 & 2007 & 0.2016 & 0.0675 & 0.2025 & $\mathrm{U}$ \\
\hline $\mathrm{Sb}-125$ & RWMC 2-6 & 2007 & 0.2536 & 0.0493 & 0.1479 & - \\
\hline $\mathrm{Sb}-125$ & RWMC 2-7 & 2007 & 0.4349 & 0.067 & 0.201 & - \\
\hline $\mathrm{Sb}-125$ & RWMC 2-8 & 2007 & 0.2987 & 0.0685 & 0.2055 & - \\
\hline $\mathrm{Sb}-125$ & RWMC 3-1 & 2007 & 0.2187 & 0.0328 & 0.0984 & - \\
\hline $\mathrm{Sb}-125$ & RWMC 3-4 & 2007 & 0.1545 & 0.073 & 0.219 & $\mathrm{U}$ \\
\hline $\mathrm{Sb}-125$ & RWMC 3-5 & 2007 & 0.5879 & 0.1215 & 0.3645 & - \\
\hline $\mathrm{Sb}-125$ & RWMC 3-6 & 2007 & 0.3984 & 0.069 & 0.207 & - \\
\hline $\mathrm{Sb}-125$ & RWMC 3-7 & 2007 & 0.3575 & 0.0725 & 0.2175 & - \\
\hline $\mathrm{Sb}-125$ & RWMC 3-8 & 2007 & 0.2807 & 0.068 & 0.204 & - \\
\hline $\mathrm{Sb}-125$ & RWMC 4-1 & 2007 & 0.2706 & 0.072 & 0.216 & - \\
\hline $\mathrm{Sb}-125$ & RWMC 4-2 & 2007 & 0.4213 & 0.0685 & 0.2055 & - \\
\hline $\mathrm{Sb}-125$ & RWMC 4-3 & 2007 & 0.06891 & 0.0895 & 0.2685 & $\mathrm{U}$ \\
\hline $\mathrm{Sb}-125$ & RWMC 4-4 & 2007 & 0.3558 & 0.076 & 0.228 & - \\
\hline $\mathrm{Sb}-125$ & RWMC 4-5 & 2007 & 0.2787 & 0.069 & 0.207 & - \\
\hline $\mathrm{Sb}-125$ & RWMC 5-10 & 2007 & 0.6717 & 0.086 & 0.258 & - \\
\hline $\mathrm{Sb}-125$ & RWMC 5-11 & 2007 & 0.3248 & 0.086 & 0.258 & - \\
\hline $\mathrm{Sb}-125$ & RWMC 5-12 & 2007 & 0.27 & 0.0286 & 0.0858 & - \\
\hline $\mathrm{Sb}-125$ & RWMC 5-4 & 2007 & 0.4895 & 0.0965 & 0.2895 & - \\
\hline $\mathrm{Sb}-125$ & RWMC 5-8 & 2007 & 0.1844 & 0.0685 & 0.2055 & $\mathrm{U}$ \\
\hline $\mathrm{Sb}-125$ & RWMC 5-9 & 2007 & 0.3107 & 0.0855 & 0.2565 & - \\
\hline $\mathrm{Sb}-125$ & RWMC 6-2 & 2007 & 0.2622 & 0.059 & 0.177 & - \\
\hline $\mathrm{Sb}-125$ & RWMC 6-3 & 2007 & 0.3561 & 0.0635 & 0.1905 & - \\
\hline $\mathrm{Sb}-125$ & RWMC 6-4 & 2007 & 0.2309 & 0.065 & 0.195 & - \\
\hline $\mathrm{Sb}-125$ & RWMC 6-5 & 2007 & 0.1829 & 0.0495 & 0.1485 & - \\
\hline $\mathrm{Sb}-125$ & RWMC 6-6 & 2007 & 0.405 & 0.0705 & 0.2115 & - \\
\hline $\mathrm{Sb}-125$ & RWMC 6-7 & 2007 & 0.262 & 0.061 & 0.183 & - \\
\hline $\mathrm{Sb}-125$ & RWMC 6-9 & 2007 & 0.2987 & 0.03725 & 0.11175 & - \\
\hline Sb-125 & RWMC 7-2 & 2007 & 0.2667 & 0.067 & 0.201 & - \\
\hline $\mathrm{Sb}-125$ & RWMC 7-3 & 2007 & 0.5242 & 0.0625 & 0.1875 & - \\
\hline $\mathrm{Sb}-125$ & RWMC 7-4 & 2007 & 0.2316 & 0.0505 & 0.1515 & - \\
\hline $\mathrm{Sb}-125$ & RWMC 7-5 & 2007 & 0.2017 & 0.0715 & 0.2145 & $\mathrm{U}$ \\
\hline
\end{tabular}


Table C-5. (continued).

\begin{tabular}{|c|c|c|c|c|c|c|}
\hline Radionuclide & $\begin{array}{l}\text { Sample } \\
\text { Location }\end{array}$ & Date & $\begin{array}{l}\text { Concentrations } \\
(\mathrm{pCi} / \mathrm{g})\end{array}$ & $\begin{array}{l}\text { Uncertainty } \\
(\mathrm{pCi} / \mathrm{g})\end{array}$ & $\begin{array}{c}\mathrm{MDA} \\
(\mathrm{pCi} / \mathrm{g})\end{array}$ & Flag \\
\hline $\mathrm{Sb}-125$ & RWMC 7-6 & 2007 & 0.2763 & 0.0605 & 0.1815 & - \\
\hline $\mathrm{Sb}-125$ & RWMC 7-7 & 2007 & 0.0552 & 0.061 & 0.183 & $\mathrm{U}$ \\
\hline $\mathrm{Sb}-125$ & RWMC 8-4 & 2007 & 0.3383 & 0.048 & 0.144 & - \\
\hline $\mathrm{Sb}-125$ & RWMC 9-1 & 2007 & 0.2676 & 0.0545 & 0.1635 & - \\
\hline $\mathrm{Sb}-125$ & RWMC 10-1 & 2011 & 0.07524 & 0.041 & 0.123 & $\mathrm{U}$ \\
\hline $\mathrm{Sb}-125$ & RWMC 2-4 & 2011 & 0.001423 & 0.0424 & 0.1272 & $\mathrm{U}$ \\
\hline $\mathrm{Sb}-125$ & RWMC 2-6 & 2011 & -0.05513 & 0.0392 & 0.1176 & $\mathrm{U}$ \\
\hline $\mathrm{Sb}-125$ & RWMC 2-8 & 2011 & 0.01756 & 0.0404 & 0.1212 & $\mathrm{U}$ \\
\hline $\mathrm{Sb}-125$ & RWMC 3-4 & 2011 & 0.02215 & 0.043 & 0.129 & $\mathrm{U}$ \\
\hline $\mathrm{Sb}-125$ & RWMC 3-6 & 2011 & -0.01932 & 0.0391 & 0.1173 & $\mathrm{U}$ \\
\hline $\mathrm{Sb}-125$ & RWMC 3-8 & 2011 & 0.02143 & 0.0416 & 0.1248 & $\mathrm{U}$ \\
\hline $\mathrm{Sb}-125$ & RWMC 4-1 & 2011 & 0.1051 & 0.0438 & 0.1314 & $\mathrm{U}$ \\
\hline $\mathrm{Sb}-125$ & RWMC 4-5 & 2011 & -0.03099 & 0.0378 & 0.1134 & $\mathrm{U}$ \\
\hline $\mathrm{Sb}-125$ & RWMC 5-12 & 2011 & -0.05389 & 0.037 & 0.111 & $\mathrm{U}$ \\
\hline $\mathrm{Sb}-125$ & RWMC 5-4 & 2011 & 0.02386 & 0.0437 & 0.1311 & $\mathrm{U}$ \\
\hline $\mathrm{Sb}-125$ & RWMC 5-7 & 2011 & -0.007075 & 0.0418 & 0.1254 & $\mathrm{U}$ \\
\hline $\mathrm{Sb}-125$ & RWMC 6-1 & 2011 & -0.05623 & 0.0335 & 0.1005 & $\mathrm{U}$ \\
\hline $\mathrm{Sb}-125$ & RWMC 6-3 & 2011 & 0.008085 & 0.0398 & 0.1194 & $\mathrm{U}$ \\
\hline $\mathrm{Sb}-125$ & RWMC 6-7 & 2011 & 0.1629 & 0.0437 & 0.1311 & - \\
\hline $\mathrm{Sb}-125$ & RWMC 7-2 & 2011 & 0.1022 & 0.0391 & 0.1173 & $\mathrm{U}$ \\
\hline $\mathrm{Sb}-125$ & RWMC 8-1 & 2011 & -0.005367 & 0.0411 & 0.1233 & $\mathrm{U}$ \\
\hline $\mathrm{Sb}-125$ & RWMC 8-5 & 2011 & 0.06898 & 0.0432 & 0.1296 & $\mathrm{U}$ \\
\hline $\mathrm{Sb}-125$ & RWMC 9-1 & 2011 & 0.0683 & 0.0405 & 0.1215 & $\mathrm{U}$ \\
\hline $\mathrm{Sb}-125$ & RWMC 2-4 & 2012 & 0.01468 & 0.0328 & 0.0984 & $\mathrm{U}$ \\
\hline $\mathrm{Sb}-125$ & RWMC 3-4 & 2012 & 0.0008926 & 0.0379 & 0.1137 & $\mathrm{U}$ \\
\hline $\mathrm{Sb}-125$ & RWMC 4-1 & 2012 & -0.01497 & 0.0321 & 0.0963 & $\mathrm{U}$ \\
\hline $\mathrm{Sb}-125$ & RWMC 5-4 & 2012 & -0.0009813 & 0.035 & 0.105 & $\mathrm{U}$ \\
\hline $\mathrm{Sb}-125$ & RWMC 6-1 & 2012 & -0.02884 & 0.0309 & 0.0927 & $\mathrm{U}$ \\
\hline $\mathrm{Sb}-125$ & RWMC 8-1 & 2012 & -0.007087 & 0.0299 & 0.0897 & $\mathrm{U}$ \\
\hline $\mathrm{Sb}-125$ & $\begin{array}{l}2 \text { INCH AIR } \\
\text { - RWMC }\end{array}$ & 2013 & 0.2918 & 0.166 & 0.498 & $\mathrm{U}$ \\
\hline $\mathrm{Sb}-125$ & $\begin{array}{c}2 \text { INCH AIR } \\
\text { - RWMC }\end{array}$ & 2013 & 0.2918 & 0.055333333 & 0.166 & - \\
\hline $\mathrm{Sb}-125$ & RWMC 2-4 & 2013 & 0.4115 & 0.077666667 & 0.233 & - \\
\hline $\mathrm{Sb}-125$ & RWMC 3-5 & 2013 & 0.01406 & 0.039 & 0.117 & $\mathrm{U}$ \\
\hline $\mathrm{Sb}-125$ & RWMC 5-7 & 2013 & 0.1517 & 0.047333333 & 0.142 & - \\
\hline $\mathrm{Sb}-125$ & RWMC 2-4 & 2014 & 0.001747 & 0.0658 & 0.1974 & $\mathrm{U}$ \\
\hline
\end{tabular}


Table C-5. (continued).

\begin{tabular}{|c|c|c|c|c|c|c|}
\hline Radionuclide & $\begin{array}{c}\text { Sample } \\
\text { Location }\end{array}$ & Date & $\begin{array}{c}\text { Concentrations } \\
(\mathrm{pCi} / \mathrm{g})\end{array}$ & $\begin{array}{c}\text { Uncertainty } \\
(\mathrm{pCi} / \mathrm{g})\end{array}$ & $\begin{array}{c}\mathrm{MDA} \\
(\mathrm{pCi} / \mathrm{g})\end{array}$ & Flag \\
\hline $\mathrm{Sb}-125$ & RWMC 2-4 & 2014 & -0.03223 & 0.0441 & 0.1323 & $\mathrm{U}$ \\
\hline $\mathrm{Sb}-125$ & RWMC 3-4 & 2014 & -0.01001 & 0.034 & 0.102 & $\mathrm{U}$ \\
\hline $\mathrm{Sb}-125$ & RWMC 4-1 & 2014 & 0.002262 & 0.0516 & 0.1548 & $\mathrm{U}$ \\
\hline $\mathrm{Sb}-125$ & RWMC 5-4 & 2014 & -0.02792 & 0.0452 & 0.1356 & $\mathrm{U}$ \\
\hline $\mathrm{Sb}-125$ & RWMC 6-1 & 2014 & -0.01593 & 0.0348 & 0.1044 & $\mathrm{U}$ \\
\hline $\mathrm{Sb}-125$ & RWMC 6-3 & 2014 & 0.03284 & 0.0508 & 0.1524 & $\mathrm{U}$ \\
\hline Sr-90 & $2-3$ & 1972 & 1.17 & 0.14 & 0.42 & - \\
\hline Sr-90 & $2-4$ & 1972 & 1.08 & 0.13 & 0.39 & - \\
\hline Sr-90 & $2-5$ & 1972 & 0.72 & 0.09 & 0.27 & - \\
\hline Sr-90 & $3-1$ & 1972 & 0.991 & 0.09 & 0.27 & - \\
\hline Sr-90 & $3-2$ & 1972 & 0.486 & 0.064 & 0.192 & 一 \\
\hline Sr-90 & $3-3$ & 1972 & 0.693 & 0.099 & 0.297 & 一 \\
\hline Sr-90 & $3-4$ & 1972 & 0.99 & 0.09 & 0.27 & - \\
\hline Sr-90 & $3-5$ & 1972 & 0.387 & 0.039 & 0.117 & - \\
\hline Sr-90 & $5-1$ & 1973 & 1.31 & 0.09 & 0.27 & - \\
\hline Sr-90 & $5-3$ & 1973 & 0.765 & 0.09 & 0.27 & - \\
\hline Sr-90 & $5-4$ & 1973 & 1.3 & 0.09 & 0.27 & - \\
\hline Sr-90 & $2-3$ & 1978 & 0.71 & 0.05 & 0.15 & - \\
\hline Sr-90 & $3-2$ & 1978 & 2.52 & 0.12 & 0.36 & - \\
\hline Sr-90 & $4-1$ & 1978 & 0.71 & 0.05 & 0.15 & - \\
\hline Sr-90 & $5-13$ & 1978 & 0.8 & 0.1 & 0.3 & - \\
\hline Sr-90 & $5-5$ & 1978 & 0.83 & 0.05 & 0.15 & - \\
\hline Sr-90 & $7-2$ & 1978 & 0.55 & 0.05 & 0.15 & - \\
\hline Sr-90 & $7-4$ & 1978 & 0.48 & 0.05 & 0.15 & - \\
\hline Sr-90 & $5-5$ & 1985 & 0.37 & 0.08 & 0.24 & 一 \\
\hline Sr-90 & $7-3$ & 1985 & 0.36 & 0.03 & 0.09 & 一 \\
\hline U-233/234 & $2-4$ & 1972 & 1.16 & 0.02 & 0.06 & - \\
\hline U-233/234 & $3-6$ & 1978 & 1.1 & 0.03 & 0.09 & - \\
\hline U-233/234 & $4-3$ & 1978 & 0.86 & 0.02 & 0.06 & - \\
\hline U-233/234 & $6-1$ & 1978 & 1.03 & 0.02 & 0.06 & - \\
\hline U-233/234 & $7-4$ & 1978 & 1.13 & 0.02 & 0.06 & - \\
\hline U-233/234 & 8-A & 1978 & 1.01 & 0.02 & 0.06 & - \\
\hline U-233/234 & $10-1$ & 1985 & 1.03 & 0.04 & 0.12 & - \\
\hline U-233/234 & $2-4$ & 1985 & 1.01 & 0.04 & 0.12 & - \\
\hline U-233/234 & $3-1$ & 1985 & 1.05 & 0.04 & 0.12 & 一 \\
\hline U-233/234 & $3-4$ & 1985 & 1.11 & 0.04 & 0.12 & - \\
\hline U-233/234 & $3-7$ & 1985 & 1.04 & 0.04 & 0.12 & - \\
\hline
\end{tabular}


Table C-5. (continued).

\begin{tabular}{|c|c|c|c|c|c|c|}
\hline Radionuclide & $\begin{array}{c}\text { Sample } \\
\text { Location }\end{array}$ & Date & $\begin{array}{c}\text { Concentrations } \\
(\mathrm{pCi} / \mathrm{g})\end{array}$ & $\begin{array}{l}\text { Uncertainty } \\
(\mathrm{pCi} / \mathrm{g})\end{array}$ & $\begin{array}{c}\mathrm{MDA} \\
(\mathrm{pCi} / \mathrm{g})\end{array}$ & Flag \\
\hline U-233/234 & $4-2$ & 1985 & 1.12 & 0.04 & 0.12 & - \\
\hline U-233/234 & $4-4$ & 1985 & 1.18 & 0.04 & 0.12 & - \\
\hline U-233/234 & 4-A & 1985 & 0.99 & 0.04 & 0.12 & - \\
\hline U-233/234 & $4-B$ & 1985 & 0.99 & 0.04 & 0.12 & - \\
\hline U-233/234 & $4-\mathrm{C}$ & 1985 & 0.97 & 0.04 & 0.12 & - \\
\hline U-233/234 & $5-15$ & 1985 & 0.96 & 0.04 & 0.12 & - \\
\hline U-233/234 & $5-5$ & 1985 & 1.07 & 0.04 & 0.12 & - \\
\hline U-233/234 & $5-7$ & 1985 & 1.07 & 0.04 & 0.12 & - \\
\hline U-233/234 & $6-1$ & 1985 & 1.04 & 0.04 & 0.12 & - \\
\hline U-233/234 & $7-3$ & 1985 & 1.03 & 0.04 & 0.12 & - \\
\hline U-233/234 & $7-5$ & 1985 & 1.05 & 0.04 & 0.12 & 一 \\
\hline U-233/234 & $7-7$ & 1985 & 1.04 & 0.04 & 0.12 & 一 \\
\hline U-233/234 & 7-A & 1985 & 0.95 & 0.03 & 0.09 & - \\
\hline U-233/234 & $8-1$ & 1985 & 1.06 & 0.04 & 0.12 & - \\
\hline U-233/234 & $8-2$ & 1985 & 1.08 & 0.04 & 0.12 & - \\
\hline U-233/234 & $8-5$ & 1985 & 1.12 & 0.04 & 0.12 & - \\
\hline U-233/234 & $8-\mathrm{C}$ & 1985 & 1.04 & 0.04 & 0.12 & - \\
\hline $\mathrm{U}-233 / 234$ & 9-A & 1985 & 1.02 & 0.04 & 0.12 & - \\
\hline U-234 & RWMC 2-8 & 2006 & 20.23 & 4.265 & 12.795 & - \\
\hline $\mathrm{U}-234$ & RWMC 10-1 & 2007 & 16.51 & 0.04295 & 0.12885 & - \\
\hline U-234 & RWMC 2-4 & 2007 & 7.199 & 0.00935 & 0.02805 & 一 \\
\hline U-234 & RWMC 2-5 & 2007 & 35.45 & 0.0131 & 0.0393 & - \\
\hline U-234 & RWMC 2-6 & 2007 & 40.39 & 0.01365 & 0.04095 & - \\
\hline U-234 & RWMC 2-7 & 2007 & 27.73 & 0.0141 & 0.0423 & - \\
\hline U-234 & RWMC 3-1 & 2007 & 104.2 & 0.0491 & 0.1473 & 一 \\
\hline U-234 & RWMC 3-4 & 2007 & 42.99 & 0.003815 & 0.011445 & 一 \\
\hline U-234 & RWMC 3-5 & 2007 & 26.3 & 0.01425 & 0.04275 & - \\
\hline $\mathrm{U}-234$ & RWMC 3-6 & 2007 & 23.05 & 0.0119 & 0.0357 & - \\
\hline $\mathrm{U}-234$ & RWMC 3-7 & 2007 & 24.85 & 0.0152 & 0.0456 & - \\
\hline $\mathrm{U}-234$ & RWMC 3-8 & 2007 & 112.2 & 0.01775 & 0.05325 & - \\
\hline $\mathrm{U}-234$ & RWMC 4-1 & 2007 & 68.35 & 0.01795 & 0.05385 & - \\
\hline $\mathrm{U}-234$ & RWMC 4-2 & 2007 & 43.83 & 0.0185 & 0.0555 & - \\
\hline $\mathrm{U}-234$ & RWMC 4-3 & 2007 & 77.21 & 0.0052 & 0.0156 & - \\
\hline U-234 & RWMC 4-4 & 2007 & 20.21 & 0.01215 & 0.03645 & - \\
\hline U-234 & RWMC 4-5 & 2007 & 25.79 & 0.00595 & 0.01785 & 一 \\
\hline U-234 & RWMC 5-10 & 2007 & 47.07 & 0.0051 & 0.0153 & - \\
\hline U-234 & RWMC 5-11 & 2007 & 29.16 & 0.004565 & 0.013695 & - \\
\hline
\end{tabular}


Table C-5. (continued).

\begin{tabular}{|c|c|c|c|c|c|c|}
\hline Radionuclide & $\begin{array}{c}\text { Sample } \\
\text { Location }\end{array}$ & Date & $\begin{array}{c}\text { Concentrations } \\
(\mathrm{pCi} / \mathrm{g})\end{array}$ & $\begin{array}{l}\text { Uncertainty } \\
(\mathrm{pCi} / \mathrm{g})\end{array}$ & $\begin{array}{c}\mathrm{MDA} \\
(\mathrm{pCi} / \mathrm{g})\end{array}$ & Flag \\
\hline $\mathrm{U}-234$ & RWMC 5-12 & 2007 & 14.29 & 0.02245 & 0.06735 & - \\
\hline U-234 & RWMC 5-15 & 2007 & 19.24 & 0.01545 & 0.04635 & - \\
\hline U-234 & RWMC 5-4 & 2007 & 67.99 & 0.061 & 0.183 & - \\
\hline U-234 & RWMC 5-7 & 2007 & 132.6 & 4.57 & 13.71 & - \\
\hline U-234 & RWMC 5-8 & 2007 & 33.93 & 0.01145 & 0.03435 & - \\
\hline U-234 & RWMC 5-9 & 2007 & 13.98 & 0.0165 & 0.0495 & - \\
\hline $\mathrm{U}-234$ & RWMC 6-2 & 2007 & 27.3 & 0.0085 & 0.0255 & - \\
\hline $\mathrm{U}-234$ & RWMC 6-3 & 2007 & 54.22 & 0.01055 & 0.03165 & - \\
\hline $\mathrm{U}-234$ & RWMC 6-4 & 2007 & 10.14 & 0.01055 & 0.03165 & - \\
\hline $\mathrm{U}-234$ & RWMC 6-5 & 2007 & 79.23 & 0.00955 & 0.02865 & - \\
\hline $\mathrm{U}-234$ & RWMC 6-6 & 2007 & 11.1 & 0.0059 & 0.0177 & - \\
\hline $\mathrm{U}-234$ & RWMC 6-7 & 2007 & 47.66 & 0.0054 & 0.0162 & 一 \\
\hline $\mathrm{U}-234$ & RWMC 6-9 & 2007 & 24.1 & 0.088 & 0.264 & - \\
\hline $\mathrm{U}-234$ & RWMC 7-2 & 2007 & 19.32 & 0.00555 & 0.01665 & - \\
\hline $\mathrm{U}-234$ & RWMC 7-3 & 2007 & 41.15 & 0.01215 & 0.03645 & - \\
\hline U-234 & RWMC 7-4 & 2007 & 49.35 & 0.00585 & 0.01755 & - \\
\hline U-234 & RWMC 7-5 & 2007 & 55.01 & 0.0126 & 0.0378 & - \\
\hline $\mathrm{U}-234$ & RWMC 7-6 & 2007 & 14.4 & 0.004405 & 0.013215 & - \\
\hline U-234 & RWMC 7-7 & 2007 & 23.98 & 0.0117 & 0.0351 & - \\
\hline $\mathrm{U}-234$ & RWMC 8-1 & 2007 & 35.94 & 0.0051 & 0.0153 & - \\
\hline $\mathrm{U}-234$ & RWMC 8-4 & 2007 & 38.48 & 0.0132 & 0.0396 & - \\
\hline $\mathrm{U}-234$ & RWMC 8-5 & 2007 & 188.2 & 0.0146 & 0.0438 & - \\
\hline $\mathrm{U}-234$ & RWMC 9-1 & 2007 & 22.57 & 0.0123 & 0.0369 & - \\
\hline U-234 & RWMC 2-5 & 2009 & 0.509 & 1.075 & 3.225 & $\mathrm{U}$ \\
\hline U-234 & RWMC 2-8 & 2009 & 1.187 & 1.01 & 3.03 & $\mathrm{U}$ \\
\hline U-234 & RWMC 4-4 & 2009 & 10.78 & 0.00885 & 0.02655 & - \\
\hline U-234 & RWMC 6-4 & 2009 & 2.081 & 2.575 & 7.725 & $\mathrm{U}$ \\
\hline $\mathrm{U}-234$ & RWMC 7-6 & 2009 & 4.158 & 0.04675 & 0.14025 & - \\
\hline $\mathrm{U}-234$ & RWMC 8-5 & 2009 & 4.863 & 0.00715 & 0.02145 & - \\
\hline $\mathrm{U}-234$ & RWMC 9-1 & 2009 & 4.555 & 0.00695 & 0.02085 & - \\
\hline $\mathrm{U}-234$ & RWMC 10-1 & 2010 & 26.1 & 1.88 & 5.64 & - \\
\hline $\mathrm{U}-234$ & RWMC 2-6 & 2010 & 7.071 & 4 & 12 & $\mathrm{U}$ \\
\hline $\mathrm{U}-234$ & RWMC 3-1 & 2010 & 22.84 & 2.19 & 6.57 & - \\
\hline U-234 & RWMC 3-6 & 2010 & 66.58 & 3.69 & 11.07 & - \\
\hline U-234 & RWMC 3-7 & 2010 & 21.57 & 1.94 & 5.82 & 一 \\
\hline U-234 & RWMC 3-8 & 2010 & 29.62 & 1.96 & 5.88 & - \\
\hline U-234 & RWMC 4-1 & 2010 & 25.3 & 2.24 & 6.72 & - \\
\hline
\end{tabular}


Table C-5. (continued).

\begin{tabular}{|c|c|c|c|c|c|c|}
\hline Radionuclide & $\begin{array}{c}\text { Sample } \\
\text { Location }\end{array}$ & Date & $\begin{array}{c}\text { Concentrations } \\
(\mathrm{pCi} / \mathrm{g})\end{array}$ & $\begin{array}{l}\text { Uncertainty } \\
(\mathrm{pCi} / \mathrm{g})\end{array}$ & $\begin{array}{c}\mathrm{MDA} \\
(\mathrm{pCi} / \mathrm{g})\end{array}$ & Flag \\
\hline $\mathrm{U}-234$ & RWMC 4-2 & 2010 & 26.94 & 2.07 & 6.21 & - \\
\hline U-234 & RWMC 4-3 & 2010 & 18.6 & 1.7 & 5.1 & - \\
\hline U-234 & RWMC 4-4 & 2010 & 27.89 & 1.98 & 5.94 & - \\
\hline U-234 & RWMC 4-5 & 2010 & 38.09 & 2.18 & 6.54 & - \\
\hline U-234 & RWMC 5-10 & 2010 & 16.6 & 2.42 & 7.26 & - \\
\hline U-234 & RWMC 5-11 & 2010 & 27.11 & 1.94 & 5.82 & - \\
\hline $\mathrm{U}-234$ & RWMC 5-12 & 2010 & 38.96 & 2.15 & 6.45 & - \\
\hline $\mathrm{U}-234$ & RWMC 5-14 & 2010 & 11.49 & 1.76 & 5.28 & - \\
\hline $\mathrm{U}-234$ & RWMC 5-15 & 2010 & 27.04 & 1.96 & 5.88 & - \\
\hline $\mathrm{U}-234$ & RWMC 5-6 & 2010 & 20.27 & 1.66 & 4.98 & - \\
\hline $\mathrm{U}-234$ & RWMC 5-7 & 2010 & 27.11 & 1.94 & 5.82 & 一 \\
\hline $\mathrm{U}-234$ & RWMC 5-8 & 2010 & 16.5 & 1.95 & 5.85 & 一 \\
\hline $\mathrm{U}-234$ & RWMC 5-9 & 2010 & 24.22 & 1.63 & 4.89 & - \\
\hline $\mathrm{U}-234$ & RWMC 6-1 & 2010 & 17.57 & 1.7 & 5.1 & - \\
\hline $\mathrm{U}-234$ & RWMC 6-2 & 2010 & 39.62 & 2.17 & 6.51 & - \\
\hline $\mathrm{U}-234$ & RWMC 6-3 & 2010 & 7.86 & 1.43 & 4.29 & - \\
\hline U-234 & RWMC 6-4 & 2010 & 10.74 & 1.74 & 5.22 & - \\
\hline $\mathrm{U}-234$ & RWMC 6-5 & 2010 & 14.66 & 1.7 & 5.1 & - \\
\hline U-234 & RWMC 6-6 & 2010 & 9.179 & 1.9 & 5.7 & - \\
\hline $\mathrm{U}-234$ & RWMC 6-7 & 2010 & 5.614 & 2.3 & 6.9 & $\mathrm{U}$ \\
\hline $\mathrm{U}-234$ & RWMC 7-2 & 2010 & 9.86 & 1.6 & 4.8 & - \\
\hline $\mathrm{U}-234$ & RWMC 7-3 & 2010 & 38.45 & 2.15 & 6.45 & - \\
\hline $\mathrm{U}-234$ & RWMC 7-4 & 2010 & 18.61 & 1.55 & 4.65 & - \\
\hline U-234 & RWMC 7-5 & 2010 & 25.79 & 1.91 & 5.73 & - \\
\hline U-234 & RWMC 7-6 & 2010 & 22.98 & 1.87 & 5.61 & 一 \\
\hline U-234 & RWMC 7-7 & 2010 & 29.14 & 2.04 & 6.12 & 一 \\
\hline U-234 & RWMC 8-1 & 2010 & 27.58 & 2.05 & 6.15 & - \\
\hline $\mathrm{U}-234$ & RWMC 8-4 & 2010 & 61.63 & 3.55 & 10.65 & - \\
\hline $\mathrm{U}-234$ & RWMC 8-5 & 2010 & 28.13 & 2.03 & 6.09 & - \\
\hline $\mathrm{U}-234$ & RWMC 9-1 & 2010 & 5.095 & 1.89 & 5.67 & $\mathrm{U}$ \\
\hline $\mathrm{U}-234$ & RWMC 4-1 & 2011 & -7.214 & 19.8 & 59.4 & $\mathrm{U}$ \\
\hline $\mathrm{U}-234$ & RWMC 2-4 & 2012 & 32.25 & 25 & 75 & $\mathrm{U}$ \\
\hline $\mathrm{U}-234$ & RWMC 3-4 & 2012 & 30.59 & 26.7 & 80.1 & $\mathrm{U}$ \\
\hline U-234 & RWMC 3-5 & 2012 & 11.65 & 42.33333333 & 127 & $\mathrm{U}$ \\
\hline U-234 & RWMC 5-4 & 2012 & -6.716 & 17.1 & 51.3 & $\mathrm{U}$ \\
\hline U-234 & RWMC 6-1 & 2012 & 19.69 & 14.2 & 42.6 & $\mathrm{U}$ \\
\hline U-234 & RWMC 8-1 & 2012 & 29.29 & 22.5 & 67.5 & $\mathrm{U}$ \\
\hline
\end{tabular}


Table C-5. (continued).

\begin{tabular}{|c|c|c|c|c|c|c|}
\hline Radionuclide & $\begin{array}{l}\text { Sample } \\
\text { Location }\end{array}$ & Date & $\begin{array}{l}\text { Concentrations } \\
(\mathrm{pCi} / \mathrm{g})\end{array}$ & $\begin{array}{l}\text { Uncertainty } \\
(\mathrm{pCi} / \mathrm{g})\end{array}$ & $\begin{array}{l}\mathrm{MDA} \\
(\mathrm{pCi} / \mathrm{g})\end{array}$ & Flag \\
\hline U-234 & $\begin{array}{l}2 \text { INCH AIR } \\
- \text { RWMC }\end{array}$ & 2013 & 22.36 & 33.26666667 & 99.8 & $\mathrm{U}$ \\
\hline U-234 & RWMC 3-4 & 2013 & -37.91 & 57.2 & 171.6 & $\mathrm{U}$ \\
\hline U-234 & RWMC 5-7 & 2013 & 44.72 & 39.33333333 & 118 & $\mathrm{U}$ \\
\hline $\mathrm{U}-234$ & RWMC 2-4 & 2014 & -63.02 & 55.9 & 167.7 & $\mathrm{U}$ \\
\hline $\mathrm{U}-234$ & RWMC 6-1 & 2014 & -57.17 & 57 & 171 & $\mathrm{U}$ \\
\hline $\mathrm{U}-235$ & $10-1$ & 1978 & 0.098 & 0.009 & 0.027 & - \\
\hline $\mathrm{U}-235$ & $2-3$ & 1978 & 0.0963 & 0.0095 & 0.0285 & - \\
\hline $\mathrm{U}-235$ & $3-2$ & 1978 & 0.111 & 0.01 & 0.03 & - \\
\hline $\mathrm{U}-235$ & $3-6$ & 1978 & 0.109 & 0.021 & 0.063 & - \\
\hline $\mathrm{U}-235$ & $4-3$ & 1978 & 0.043 & 0.004 & 0.012 & - \\
\hline $\mathrm{U}-235$ & $4-5$ & 1978 & 0.111 & 0.008 & 0.024 & - \\
\hline U-235 & $4-\mathrm{A}$ & 1978 & 0.0827 & 0.0095 & 0.0285 & - \\
\hline U-235 & $5-13$ & 1978 & 0.124 & 0.0085 & 0.0255 & - \\
\hline U-235 & $5-15$ & 1978 & 0.0742 & 0.0075 & 0.0225 & - \\
\hline $\mathrm{U}-235$ & $5-5$ & 1978 & 0.0745 & 0.008 & 0.024 & - \\
\hline $\mathrm{U}-235$ & $5-8$ & 1978 & 11.9 & 0.021 & 0.063 & - \\
\hline U-235 & $6-1$ & 1978 & 0.101 & 0.016 & 0.048 & - \\
\hline $\mathrm{U}-235$ & $6-3$ & 1978 & 0.118 & 0.016 & 0.048 & - \\
\hline U-235 & $6-7$ & 1978 & 0.093 & 0.0075 & 0.0225 & - \\
\hline U-235 & $7-6$ & 1978 & 0.125 & 0.01 & 0.03 & - \\
\hline U-235 & $8-1$ & 1978 & 0.0823 & 0.0008 & 0.0024 & - \\
\hline U-235 & $8-3$ & 1978 & 0.101 & 0.016 & 0.048 & - \\
\hline U-235 & $8-5$ & 1978 & 0.0991 & 0.069 & 0.207 & $\mathrm{U}$ \\
\hline U-235 & 8-A & 1978 & 0.0815 & 0.008 & 0.024 & - \\
\hline U-235 & $8-\mathrm{C}$ & 1978 & 0.112 & 0.016 & 0.048 & - \\
\hline U-235 & $10-1$ & 1985 & 0.053 & 0.013 & 0.039 & - \\
\hline U-235 & $2-4$ & 1985 & 0.05 & 0.0125 & 0.0375 & - \\
\hline U-235 & $3-1$ & 1985 & 0.081 & 0.015 & 0.045 & - \\
\hline U-235 & $3-4$ & 1985 & 0.056 & 0.014 & 0.042 & - \\
\hline U-235 & $3-7$ & 1985 & 0.053 & 0.013 & 0.039 & - \\
\hline U-235 & $4-2$ & 1985 & 0.055 & 0.014 & 0.042 & - \\
\hline U-235 & 4-4 & 1985 & 0.058 & 0.0145 & 0.0435 & - \\
\hline U-235 & 4-A & 1985 & 0.052 & 0.013 & 0.039 & - \\
\hline U-235 & 4-B & 1985 & 0.053 & 0.013 & 0.039 & - \\
\hline U-235 & 4-C & 1985 & 0.051 & 0.0125 & 0.0375 & - \\
\hline U-235 & $5-10$ & 1985 & 0.055 & 0.0135 & 0.0405 & - \\
\hline
\end{tabular}


Table C-5. (continued).

\begin{tabular}{|c|c|c|c|c|c|c|}
\hline Radionuclide & $\begin{array}{c}\text { Sample } \\
\text { Location }\end{array}$ & Date & $\begin{array}{c}\text { Concentrations } \\
(\mathrm{pCi} / \mathrm{g})\end{array}$ & $\begin{array}{l}\text { Uncertainty } \\
(\mathrm{pCi} / \mathrm{g})\end{array}$ & $\begin{array}{c}\text { MDA } \\
(\mathrm{pCi} / \mathrm{g})\end{array}$ & Flag \\
\hline U-235 & $5-15$ & 1985 & 0.042 & 0.021 & 0.063 & $\mathrm{U}$ \\
\hline U-235 & $5-5$ & 1985 & 0.052 & 0.013 & 0.039 & - \\
\hline U-235 & $5-7$ & 1985 & 0.063 & 0.031 & 0.093 & $\mathrm{U}$ \\
\hline U-235 & $6-1$ & 1985 & 0.055 & 0.014 & 0.042 & - \\
\hline U-235 & $7-3$ & 1985 & 0.041 & 0.021 & 0.063 & $\mathrm{U}$ \\
\hline U-235 & $7-5$ & 1985 & 0.049 & 0.0125 & 0.0375 & - \\
\hline $\mathrm{U}-235$ & $7-7$ & 1985 & 0.057 & 0.0145 & 0.0435 & - \\
\hline $\mathrm{U}-235$ & $7-A$ & 1985 & 0.049 & 0.0125 & 0.0375 & - \\
\hline $\mathrm{U}-235$ & $8-1$ & 1985 & 0.05 & 0.0125 & 0.0375 & - \\
\hline $\mathrm{U}-235$ & $8-2$ & 1985 & 0.055 & 0.014 & 0.042 & - \\
\hline U-235 & $8-5$ & 1985 & 0.064 & 0.032 & 0.096 & $\mathrm{U}$ \\
\hline U-235 & $8-\mathrm{C}$ & 1985 & 0.053 & 0.013 & 0.039 & - \\
\hline U-235 & 9-A & 1985 & 0.058 & 0.0145 & 0.0435 & - \\
\hline U-235 & RWMC 2-4 & 2005 & 2.717 & 1.65 & 4.95 & $\mathrm{U}$ \\
\hline U-235 & RWMC 2-5 & 2005 & 0.6396 & 0.00635 & 0.01905 & - \\
\hline U-235 & RWMC 10-1 & 2006 & 0.7105 & 0.004635 & 0.013905 & - \\
\hline $\mathrm{U}-235$ & RWMC 2-6 & 2006 & 0.5868 & 0.00645 & 0.01935 & - \\
\hline $\mathrm{U}-235$ & RWMC 2-7 & 2006 & 0.3089 & 0.0065 & 0.0195 & - \\
\hline $\mathrm{U}-235$ & RWMC 2-8 & 2006 & 0.4887 & 0.00805 & 0.02415 & - \\
\hline $\mathrm{U}-235$ & RWMC 2-8 & 2006 & 0.4111 & 0.00905 & 0.02715 & - \\
\hline U-235 & RWMC 3-1 & 2006 & 0.8473 & 0.0037 & 0.0111 & 一 \\
\hline U-235 & RWMC 3-4 & 2006 & 1.534 & 0.0055 & 0.0165 & - \\
\hline U-235 & RWMC 3-5 & 2006 & 0.7895 & 0.00605 & 0.01815 & - \\
\hline U-235 & RWMC 3-6 & 2006 & 5.797 & 0.02045 & 0.06135 & - \\
\hline U-235 & RWMC 3-7 & 2006 & 1.954 & 0.00775 & 0.02325 & 一 \\
\hline U-235 & RWMC 3-8 & 2006 & 0.7999 & 0.0159 & 0.0477 & 一 \\
\hline U-235 & RWMC 4-1 & 2006 & 0.2936 & 0.00625 & 0.01875 & - \\
\hline $\mathrm{U}-235$ & RWMC 4-2 & 2006 & 0.9281 & 0.0069 & 0.0207 & - \\
\hline $\mathrm{U}-235$ & RWMC 4-3 & 2006 & 1.276 & 0.00555 & 0.01665 & - \\
\hline $\mathrm{U}-235$ & RWMC 4-4 & 2006 & 1.022 & 0.0057 & 0.0171 & - \\
\hline $\mathrm{U}-235$ & RWMC 4-5 & 2006 & 0.3951 & 0.00875 & 0.02625 & - \\
\hline $\mathrm{U}-235$ & RWMC 5-10 & 2006 & 0.8833 & 0.003925 & 0.011775 & - \\
\hline $\mathrm{U}-235$ & RWMC 5-11 & 2006 & 2.309 & 0.0058 & 0.0174 & - \\
\hline $\mathrm{U}-235$ & RWMC 5-12 & 2006 & 0.2709 & 0.0058 & 0.0174 & - \\
\hline U-235 & RWMC 5-14 & 2006 & 2.41 & 0.0051 & 0.0153 & 一 \\
\hline $\mathrm{U}-235$ & RWMC 5-15 & 2006 & 0.2295 & 0.00725 & 0.02175 & - \\
\hline $\mathrm{U}-235$ & RWMC 5-4 & 2006 & 0.7732 & 0.004325 & 0.012975 & - \\
\hline
\end{tabular}


Table C-5. (continued).

\begin{tabular}{|c|c|c|c|c|c|c|}
\hline Radionuclide & $\begin{array}{c}\text { Sample } \\
\text { Location }\end{array}$ & Date & $\begin{array}{c}\text { Concentrations } \\
(\mathrm{pCi} / \mathrm{g})\end{array}$ & $\begin{array}{l}\text { Uncertainty } \\
(\mathrm{pCi} / \mathrm{g})\end{array}$ & $\begin{array}{c}\mathrm{MDA} \\
(\mathrm{pCi} / \mathrm{g})\end{array}$ & Flag \\
\hline U-235 & RWMC 5-6 & 2006 & 0.7903 & 0.004 & 0.012 & - \\
\hline U-235 & RWMC 5-7 & 2006 & 0.8109 & 0.2615 & 0.7845 & - \\
\hline U-235 & RWMC 5-8 & 2006 & 0.5668 & 0.00635 & 0.01905 & - \\
\hline U-235 & RWMC 5-9 & 2006 & 0.6516 & 0.0055 & 0.0165 & - \\
\hline U-235 & RWMC 6-1 & 2006 & 1.132 & 0.00875 & 0.02625 & - \\
\hline U-235 & RWMC 6-2 & 2006 & 1.223 & 0.00555 & 0.01665 & - \\
\hline $\mathrm{U}-235$ & RWMC 6-3 & 2006 & 1.007 & 0.01 & 0.03 & - \\
\hline $\mathrm{U}-235$ & RWMC 6-4 & 2006 & 2.293 & 0.0087 & 0.0261 & - \\
\hline $\mathrm{U}-235$ & RWMC 6-5 & 2006 & 0.5059 & 0.0128 & 0.0384 & - \\
\hline $\mathrm{U}-235$ & RWMC 6-6 & 2006 & 0.8452 & 0.00605 & 0.01815 & - \\
\hline $\mathrm{U}-235$ & RWMC 6-7 & 2006 & 0.3118 & 0.01115 & 0.03345 & 一 \\
\hline $\mathrm{U}-235$ & RWMC 7-3 & 2006 & 1.197 & 0.00615 & 0.01845 & 一 \\
\hline $\mathrm{U}-235$ & RWMC 7-4 & 2006 & 0.4268 & 0.003765 & 0.011295 & - \\
\hline $\mathrm{U}-235$ & RWMC 7-5 & 2006 & 0.649 & 0.0053 & 0.0159 & - \\
\hline $\mathrm{U}-235$ & RWMC 7-6 & 2006 & 3.631 & 0.00334 & 0.01002 & - \\
\hline U-235 & RWMC 7-7 & 2006 & 1 & 0.004875 & 0.014625 & - \\
\hline $\mathrm{U}-235$ & RWMC 9-1 & 2006 & 1.344 & 0.00456 & 0.01368 & - \\
\hline $\mathrm{U}-235$ & RWMC 10-1 & 2007 & 0.3846 & 0.01335 & 0.04005 & - \\
\hline $\mathrm{U}-235$ & RWMC 2-4 & 2007 & 0.3813 & 0.00439 & 0.01317 & - \\
\hline $\mathrm{U}-235$ & RWMC 2-5 & 2007 & 0.3425 & 0.0054 & 0.0162 & - \\
\hline U-235 & RWMC 2-6 & 2007 & 0.2928 & 0.00431 & 0.01293 & 一 \\
\hline U-235 & RWMC 2-7 & 2007 & 0.4357 & 0.00261 & 0.00783 & - \\
\hline U-235 & RWMC 3-1 & 2007 & 0.3329 & 0.0106 & 0.0318 & - \\
\hline U-235 & RWMC 3-4 & 2007 & 0.5184 & 0.00545 & 0.01635 & - \\
\hline U-235 & RWMC 3-5 & 2007 & 0.6104 & 0.0052 & 0.0156 & 一 \\
\hline U-235 & RWMC 3-6 & 2007 & 0.2392 & 0.0064 & 0.0192 & 一 \\
\hline U-235 & RWMC 3-7 & 2007 & 0.2851 & 0.0054 & 0.0162 & - \\
\hline $\mathrm{U}-235$ & RWMC 3-8 & 2007 & 0.135 & 0.00233 & 0.00699 & - \\
\hline $\mathrm{U}-235$ & RWMC 4-1 & 2007 & 0.3902 & 0.0079 & 0.0237 & - \\
\hline $\mathrm{U}-235$ & RWMC 4-2 & 2007 & 0.4573 & 0.009 & 0.027 & - \\
\hline $\mathrm{U}-235$ & RWMC 4-3 & 2007 & 0.5227 & 0.0083 & 0.0249 & - \\
\hline $\mathrm{U}-235$ & RWMC 4-4 & 2007 & 0.422 & 0.00326 & 0.00978 & - \\
\hline $\mathrm{U}-235$ & RWMC 4-5 & 2007 & 0.3474 & 0.003415 & 0.010245 & - \\
\hline $\mathrm{U}-235$ & RWMC 5-10 & 2007 & 0.4406 & 0.0635 & 0.1905 & - \\
\hline U-235 & RWMC 5-11 & 2007 & 0.3162 & 0.084 & 0.252 & - \\
\hline U-235 & RWMC 5-12 & 2007 & 0.335 & 0.0091 & 0.0273 & - \\
\hline $\mathrm{U}-235$ & RWMC 5-14 & 2007 & 0.9801 & 0.00605 & 0.01815 & - \\
\hline
\end{tabular}


Table C-5. (continued).

\begin{tabular}{|c|c|c|c|c|c|c|}
\hline Radionuclide & $\begin{array}{c}\text { Sample } \\
\text { Location }\end{array}$ & Date & $\begin{array}{c}\text { Concentrations } \\
(\mathrm{pCi} / \mathrm{g})\end{array}$ & $\begin{array}{l}\text { Uncertainty } \\
(\mathrm{pCi} / \mathrm{g})\end{array}$ & $\begin{array}{c}\mathrm{MDA} \\
(\mathrm{pCi} / \mathrm{g})\end{array}$ & Flag \\
\hline U-235 & RWMC 5-15 & 2007 & 0.3725 & 0.01645 & 0.04935 & - \\
\hline U-235 & RWMC 5-4 & 2007 & 0.6284 & 0.0135 & 0.0405 & - \\
\hline U-235 & RWMC 5-6 & 2007 & 0.4301 & 0.00277 & 0.00831 & - \\
\hline U-235 & RWMC 5-7 & 2007 & 0.1493 & 0.0838 & 0.2514 & $\mathrm{U}$ \\
\hline U-235 & RWMC 5-8 & 2007 & 0.2418 & 0.00457 & 0.01371 & - \\
\hline U-235 & RWMC 5-9 & 2007 & 0.4254 & 0.00191 & 0.00573 & - \\
\hline $\mathrm{U}-235$ & RWMC 6-1 & 2007 & 0.2371 & 0.01075 & 0.03225 & - \\
\hline $\mathrm{U}-235$ & RWMC 6-2 & 2007 & 0.3431 & 0.00228 & 0.00684 & - \\
\hline $\mathrm{U}-235$ & RWMC 6-3 & 2007 & 0.2954 & 0.003385 & 0.010155 & - \\
\hline $\mathrm{U}-235$ & RWMC 6-4 & 2007 & 0.2656 & 0.0052 & 0.0156 & - \\
\hline $\mathrm{U}-235$ & RWMC 6-5 & 2007 & 0.2898 & 0.007 & 0.021 & 一 \\
\hline $\mathrm{U}-235$ & RWMC 6-6 & 2007 & 0.3599 & 0.002665 & 0.007995 & 一 \\
\hline $\mathrm{U}-235$ & RWMC 6-7 & 2007 & 0.258 & 0.00515 & 0.01545 & - \\
\hline $\mathrm{U}-235$ & RWMC 6-9 & 2007 & 0.2871 & 0.0093 & 0.0279 & - \\
\hline $\mathrm{U}-235$ & RWMC 7-2 & 2007 & 0.3766 & 0.051 & 0.153 & - \\
\hline U-235 & RWMC 7-3 & 2007 & 0.3719 & 0.0052 & 0.0156 & - \\
\hline $\mathrm{U}-235$ & RWMC 7-4 & 2007 & 0.2595 & 0.00585 & 0.01755 & - \\
\hline $\mathrm{U}-235$ & RWMC 7-5 & 2007 & 0.3736 & 0.003085 & 0.009255 & - \\
\hline $\mathrm{U}-235$ & RWMC 7-6 & 2007 & 0.3634 & 0.0565 & 0.1695 & - \\
\hline $\mathrm{U}-235$ & RWMC 7-7 & 2007 & 0.3239 & 0.00685 & 0.02055 & - \\
\hline U-235 & RWMC 8-1 & 2007 & 0.2923 & 0.00456 & 0.01368 & - \\
\hline U-235 & RWMC 8-4 & 2007 & 0.267 & 0.00322 & 0.00966 & - \\
\hline U-235 & RWMC 8-5 & 2007 & 0.3337 & 0.01325 & 0.03975 & - \\
\hline U-235 & RWMC 9-1 & 2007 & 0.2699 & 0.00885 & 0.02655 & - \\
\hline U-235 & RWMC 2-5 & 2009 & 0.2668 & 0.00605 & 0.01815 & 一 \\
\hline U-235 & RWMC 2-6 & 2009 & 0.05966 & 0.00725 & 0.02175 & 一 \\
\hline U-235 & RWMC 2-7 & 2009 & 0.03935 & 0.0377 & 0.1131 & $\mathrm{U}$ \\
\hline $\mathrm{U}-235$ & RWMC 2-8 & 2009 & 0.7081 & 0.185 & 0.555 & - \\
\hline $\mathrm{U}-235$ & RWMC 5-6 & 2009 & 0.1774 & 0.0199 & 0.0597 & - \\
\hline $\mathrm{U}-235$ & RWMC 5-7 & 2009 & 0.1445 & 0.0283 & 0.0849 & - \\
\hline $\mathrm{U}-235$ & RWMC 5-8 & 2009 & 0.1703 & 0.0073 & 0.0219 & - \\
\hline $\mathrm{U}-235$ & RWMC 6-3 & 2009 & 0.2004 & 0.00481 & 0.01443 & - \\
\hline $\mathrm{U}-235$ & RWMC 6-7 & 2009 & 0.2485 & 0.0053 & 0.0159 & - \\
\hline U-235 & RWMC 7-6 & 2009 & 0.2094 & 0.0069 & 0.0207 & - \\
\hline U-235 & RWMC 10-1 & 2010 & 0.4033 & 0.0599 & 0.1797 & 一 \\
\hline U-235 & RWMC 2-4 & 2010 & 2.531 & 0.727 & 2.181 & - \\
\hline U-235 & RWMC 2-6 & 2010 & 0.5375 & 0.136 & 0.408 & - \\
\hline
\end{tabular}


Table C-5. (continued).

\begin{tabular}{|c|c|c|c|c|c|c|}
\hline Radionuclide & $\begin{array}{c}\text { Sample } \\
\text { Location }\end{array}$ & Date & $\begin{array}{c}\text { Concentrations } \\
(\mathrm{pCi} / \mathrm{g})\end{array}$ & $\begin{array}{l}\text { Uncertainty } \\
(\mathrm{pCi} / \mathrm{g})\end{array}$ & $\begin{array}{c}\mathrm{MDA} \\
(\mathrm{pCi} / \mathrm{g})\end{array}$ & Flag \\
\hline U-235 & RWMC 3-1 & 2010 & 0.2493 & 0.0586 & 0.1758 & - \\
\hline U-235 & RWMC 3-5 & 2010 & 1.89 & 0.44 & 1.32 & - \\
\hline U-235 & RWMC 3-6 & 2010 & 1.049 & 0.255 & 0.765 & - \\
\hline U-235 & RWMC 3-7 & 2010 & 0.288 & 0.0466 & 0.1398 & - \\
\hline U-235 & RWMC 3-8 & 2010 & 0.7177 & 0.217 & 0.651 & - \\
\hline U-235 & RWMC 4-1 & 2010 & 0.157 & 0.0432 & 0.1296 & - \\
\hline $\mathrm{U}-235$ & RWMC 4-2 & 2010 & 0.3715 & 0.0502 & 0.1506 & - \\
\hline $\mathrm{U}-235$ & RWMC 4-3 & 2010 & 0.3154 & 0.0529 & 0.1587 & - \\
\hline $\mathrm{U}-235$ & RWMC 4-4 & 2010 & 0.2099 & 0.0423 & 0.1269 & - \\
\hline $\mathrm{U}-235$ & RWMC 4-5 & 2010 & 1.018 & 0.19 & 0.57 & - \\
\hline $\mathrm{U}-235$ & RWMC 5-10 & 2010 & 0.8474 & 0.19 & 0.57 & 一 \\
\hline $\mathrm{U}-235$ & RWMC 5-11 & 2010 & 0.8656 & 0.204 & 0.612 & - \\
\hline $\mathrm{U}-235$ & RWMC 5-12 & 2010 & 0.3724 & 0.0569 & 0.1707 & - \\
\hline $\mathrm{U}-235$ & RWMC 5-14 & 2010 & 2.47 & 0.604 & 1.812 & - \\
\hline $\mathrm{U}-235$ & RWMC 5-15 & 2010 & 1.132 & 0.235 & 0.705 & - \\
\hline U-235 & RWMC 5-4 & 2010 & 1.126 & 0.227 & 0.681 & - \\
\hline $\mathrm{U}-235$ & RWMC 5-4 & 2010 & 0.1112 & 0.183 & 0.549 & $\mathrm{U}$ \\
\hline U-235 & RWMC 5-6 & 2010 & 0.4285 & 0.0576 & 0.1728 & - \\
\hline U-235 & RWMC 5-7 & 2010 & 0.8656 & 0.204 & 0.612 & - \\
\hline U-235 & RWMC 5-8 & 2010 & 0.3753 & 0.0585 & 0.1755 & - \\
\hline U-235 & RWMC 5-9 & 2010 & 1.028 & 0.233 & 0.699 & - \\
\hline U-235 & RWMC 6-1 & 2010 & 4.118 & 0.62 & 1.86 & - \\
\hline U-235 & RWMC 6-2 & 2010 & 0.2718 & 0.0484 & 0.1452 & - \\
\hline U-235 & RWMC 6-3 & 2010 & 1.191 & 0.231 & 0.693 & - \\
\hline U-235 & RWMC 6-4 & 2010 & 0.3013 & 0.0598 & 0.1794 & 一 \\
\hline U-235 & RWMC 6-5 & 2010 & 0.182 & 0.0476 & 0.1428 & 一 \\
\hline U-235 & RWMC 6-6 & 2010 & 0.2986 & 0.0555 & 0.1665 & - \\
\hline U-235 & RWMC 6-7 & 2010 & 0.7493 & 0.184 & 0.552 & - \\
\hline U-235 & RWMC 7-2 & 2010 & 2.479 & 0.595 & 1.785 & - \\
\hline U-235 & RWMC 7-3 & 2010 & 0.3401 & 0.0519 & 0.1557 & - \\
\hline U-235 & RWMC 7-4 & 2010 & 0.9975 & 0.229 & 0.687 & - \\
\hline $\mathrm{U}-235$ & RWMC 7-5 & 2010 & 0.2192 & 0.0465 & 0.1395 & - \\
\hline $\mathrm{U}-235$ & RWMC 7-6 & 2010 & 3.341 & 0.639 & 1.917 & - \\
\hline U-235 & RWMC 7-7 & 2010 & 0.4722 & 0.071 & 0.213 & - \\
\hline U-235 & RWMC 8-1 & 2010 & 0.4099 & 0.0655 & 0.1965 & 一 \\
\hline U-235 & RWMC 8-4 & 2010 & 0.9419 & 0.316 & 0.948 & $\mathrm{U}$ \\
\hline U-235 & RWMC 8-5 & 2010 & 1.092 & 0.21 & 0.63 & - \\
\hline
\end{tabular}


Table C-5. (continued).

\begin{tabular}{|c|c|c|c|c|c|c|}
\hline Radionuclide & $\begin{array}{c}\text { Sample } \\
\text { Location }\end{array}$ & Date & $\begin{array}{c}\text { Concentrations } \\
(\mathrm{pCi} / \mathrm{g})\end{array}$ & $\begin{array}{l}\text { Uncertainty } \\
(\mathrm{pCi} / \mathrm{g})\end{array}$ & $\begin{array}{c}\mathrm{MDA} \\
(\mathrm{pCi} / \mathrm{g})\end{array}$ & Flag \\
\hline U-235 & RWMC 9-1 & 2010 & 0.2049 & 0.0429 & 0.1287 & - \\
\hline U-235 & RWMC 10-1 & 2011 & -0.0711 & 0.186 & 0.558 & $\mathrm{U}$ \\
\hline $\mathrm{U}-235$ & RWMC 2-4 & 2011 & 0.08319 & 0.195 & 0.585 & $\mathrm{U}$ \\
\hline $\mathrm{U}-235$ & RWMC 2-6 & 2011 & 0.05189 & 0.177 & 0.531 & $\mathrm{U}$ \\
\hline U-235 & RWMC 2-8 & 2011 & 0.0233 & 0.179 & 0.537 & $\mathrm{U}$ \\
\hline U-235 & RWMC 3-4 & 2011 & -0.01841 & 0.183 & 0.549 & $\mathrm{U}$ \\
\hline $\mathrm{U}-235$ & RWMC 3-6 & 2011 & 0.03848 & 0.178 & 0.534 & $\mathrm{U}$ \\
\hline U-235 & RWMC 3-8 & 2011 & -0.08745 & 0.184 & 0.552 & $\mathrm{U}$ \\
\hline U-235 & RWMC 4-1 & 2011 & 0.04411 & 0.151 & 0.453 & $\mathrm{U}$ \\
\hline U-235 & RWMC 4-1 & 2011 & 0.01584 & 0.192 & 0.576 & $\mathrm{U}$ \\
\hline U-235 & RWMC 4-5 & 2011 & 0.02357 & 0.186 & 0.558 & $\mathrm{U}$ \\
\hline U-235 & RWMC 5-12 & 2011 & 0.09666 & 0.145 & 0.435 & $\mathrm{U}$ \\
\hline U-235 & RWMC 5-7 & 2011 & 0.08005 & 0.179 & 0.537 & $\mathrm{U}$ \\
\hline U-235 & RWMC 6-1 & 2011 & 0.09207 & 0.167 & 0.501 & $\mathrm{U}$ \\
\hline U-235 & RWMC 6-3 & 2011 & 0.2295 & 0.179 & 0.537 & $\mathrm{U}$ \\
\hline $\mathrm{U}-235$ & RWMC 6-7 & 2011 & 0.007271 & 0.199 & 0.597 & $\mathrm{U}$ \\
\hline $\mathrm{U}-235$ & RWMC 7-2 & 2011 & -0.1075 & 0.175 & 0.525 & $\mathrm{U}$ \\
\hline U-235 & RWMC 8-1 & 2011 & -0.02463 & 0.177 & 0.531 & $\mathrm{U}$ \\
\hline U-235 & RWMC 8-5 & 2011 & -0.2323 & 0.196 & 0.588 & $\mathrm{U}$ \\
\hline U-235 & RWMC 9-1 & 2011 & -0.0229 & 0.185 & 0.555 & $\mathrm{U}$ \\
\hline U-235 & RWMC 2-4 & 2012 & 0.1215 & 0.154 & 0.462 & $\mathrm{U}$ \\
\hline U-235 & RWMC 3-4 & 2012 & 0.02388 & 0.146 & 0.438 & $\mathrm{U}$ \\
\hline U-235 & RWMC 3-5 & 2012 & 0.203 & 0.055666667 & 0.167 & - \\
\hline U-235 & RWMC 5-4 & 2012 & 0.1115 & 0.15 & 0.45 & $\mathrm{U}$ \\
\hline U-235 & RWMC 6-1 & 2012 & 0.1412 & 0.142 & 0.426 & $\mathrm{U}$ \\
\hline U-235 & RWMC 8-1 & 2012 & 0.07348 & 0.148 & 0.444 & $\mathrm{U}$ \\
\hline U-235 & $\begin{array}{l}2 \text { INCH AIR } \\
\text { - RWMC }\end{array}$ & 2013 & 0.2514 & 0.251 & 0.753 & $\mathrm{U}$ \\
\hline U-235 & $\begin{array}{l}2 \text { INCH AIR } \\
\text { - RWMC }\end{array}$ & 2013 & 0.2514 & 0.083666667 & 0.251 & - \\
\hline U-235 & RWMC 2-4 & 2013 & 0.4814 & 0.129333333 & 0.388 & - \\
\hline U-235 & RWMC 3-4 & 2013 & -0.01217 & 0.141 & 0.423 & $\mathrm{U}$ \\
\hline U-235 & RWMC 5-7 & 2013 & 0.4637 & 0.092 & 0.276 & - \\
\hline U-235 & RWMC 2-4 & 2014 & -0.0419 & 0.245 & 0.735 & $\mathrm{U}$ \\
\hline U-235 & RWMC 2-4 & 2014 & -0.1396 & 0.149 & 0.447 & $\mathrm{U}$ \\
\hline U-235 & RWMC 4-1 & 2014 & -0.02984 & 0.191 & 0.573 & $\mathrm{U}$ \\
\hline $\mathrm{U}-235$ & RWMC 5-4 & 2014 & -0.01218 & 0.18 & 0.54 & $\mathrm{U}$ \\
\hline
\end{tabular}


Table C-5. (continued).

\begin{tabular}{|c|c|c|c|c|c|c|}
\hline Radionuclide & $\begin{array}{c}\text { Sample } \\
\text { Location }\end{array}$ & Date & $\begin{array}{c}\text { Concentrations } \\
(\mathrm{pCi} / \mathrm{g})\end{array}$ & $\begin{array}{c}\text { Uncertainty } \\
(\mathrm{pCi} / \mathrm{g})\end{array}$ & $\begin{array}{c}\text { MDA } \\
(\mathrm{pCi} / \mathrm{g})\end{array}$ & Flag \\
\hline U-235 & RWMC 6-1 & 2014 & -0.1239 & 0.137 & 0.411 & $\mathrm{U}$ \\
\hline U-235 & RWMC 6-3 & 2014 & 0.1895 & 0.203 & 0.609 & $\mathrm{U}$ \\
\hline $\mathrm{U}-238$ & $2-4$ & 1972 & 1.12 & 0.022 & 0.066 & - \\
\hline $\mathrm{U}-238$ & $3-6$ & 1978 & 1.23 & 0.03 & 0.09 & - \\
\hline $\mathrm{U}-238$ & 4-3 & 1978 & 0.88 & 0.02 & 0.06 & - \\
\hline $\mathrm{U}-238$ & $6-1$ & 1978 & 1.11 & 0.03 & 0.09 & - \\
\hline $\mathrm{U}-238$ & $7-4$ & 1978 & 1.22 & 0.03 & 0.09 & - \\
\hline $\mathrm{U}-238$ & $8-\mathrm{A}$ & 1978 & 1.1 & 0.02 & 0.06 & - \\
\hline $\mathrm{U}-238$ & $10-1$ & 1985 & 1.1 & 0.04 & 0.12 & - \\
\hline $\mathrm{U}-238$ & $2-4$ & 1985 & 1.05 & 0.04 & 0.12 & - \\
\hline $\mathrm{U}-238$ & $3-1$ & 1985 & 1.12 & 0.04 & 0.12 & 一 \\
\hline $\mathrm{U}-238$ & $3-4$ & 1985 & 1.18 & 0.04 & 0.12 & - \\
\hline $\mathrm{U}-238$ & $3-7$ & 1985 & 1.18 & 0.04 & 0.12 & - \\
\hline $\mathrm{U}-238$ & $4-2$ & 1985 & 1.16 & 0.04 & 0.12 & - \\
\hline $\mathrm{U}-238$ & $4-4$ & 1985 & 1.07 & 0.04 & 0.12 & - \\
\hline $\mathrm{U}-238$ & $4-\mathrm{A}$ & 1985 & 1.06 & 0.04 & 0.12 & - \\
\hline $\mathrm{U}-238$ & 4-B & 1985 & 1.08 & 0.04 & 0.12 & - \\
\hline $\mathrm{U}-238$ & 4-C & 1985 & 1.06 & 0.04 & 0.12 & - \\
\hline U-238 & $5-10$ & 1985 & 1.13 & 0.04 & 0.12 & - \\
\hline U-238 & $5-15$ & 1985 & 1.02 & 0.04 & 0.12 & - \\
\hline $\mathrm{U}-238$ & $5-5$ & 1985 & 1.02 & 0.04 & 0.12 & - \\
\hline $\mathrm{U}-238$ & $5-7$ & 1985 & 1.14 & 0.04 & 0.12 & - \\
\hline U-238 & $6-1$ & 1985 & 1.11 & 0.04 & 0.12 & - \\
\hline U-238 & $7-3$ & 1985 & 1.13 & 0.04 & 0.12 & - \\
\hline U-238 & $7-5$ & 1985 & 1.08 & 0.04 & 0.12 & 一 \\
\hline U-238 & $7-7$ & 1985 & 1.13 & 0.04 & 0.12 & 一 \\
\hline U-238 & 7-A & 1985 & 1.02 & 0.04 & 0.12 & - \\
\hline U-238 & $8-1$ & 1985 & 1.12 & 0.04 & 0.12 & - \\
\hline U-238 & $8-2$ & 1985 & 1.14 & 0.04 & 0.12 & - \\
\hline U-238 & $8-5$ & 1985 & 1.15 & 0.04 & 0.12 & - \\
\hline $\mathrm{U}-238$ & $8-\mathrm{C}$ & 1985 & 1.06 & 0.04 & 0.12 & - \\
\hline $\mathrm{U}-238$ & $9-\mathrm{A}$ & 1985 & 1.09 & 0.04 & 0.12 & - \\
\hline $\mathrm{U}-238$ & RWMC 2-4 & 2005 & 9.604 & 0.002525 & 0.007575 & - \\
\hline U-238 & RWMC 2-5 & 2005 & 4.826 & 3.855 & 11.565 & $\mathrm{U}$ \\
\hline U-238 & RWMC 7-2 & 2005 & 16.41 & 7.71 & 23.13 & $\mathrm{U}$ \\
\hline U-238 & RWMC 8-1 & 2005 & 5.884 & 2.89 & 8.67 & $\mathrm{U}$ \\
\hline U-238 & RWMC 8-4 & 2005 & 6.662 & 3.3 & 9.9 & $\mathrm{U}$ \\
\hline
\end{tabular}


Table C-5. (continued).

\begin{tabular}{|c|c|c|c|c|c|c|}
\hline Radionuclide & $\begin{array}{c}\text { Sample } \\
\text { Location }\end{array}$ & Date & $\begin{array}{c}\text { Concentrations } \\
(\mathrm{pCi} / \mathrm{g})\end{array}$ & $\begin{array}{l}\text { Uncertainty } \\
(\mathrm{pCi} / \mathrm{g})\end{array}$ & $\begin{array}{c}\mathrm{MDA} \\
(\mathrm{pCi} / \mathrm{g})\end{array}$ & Flag \\
\hline $\mathrm{U}-238$ & RWMC 10-1 & 2006 & 5.529 & 0.0105 & 0.0315 & - \\
\hline $\mathrm{U}-238$ & RWMC 2-6 & 2006 & 5.229 & 0.023 & 0.069 & - \\
\hline $\mathrm{U}-238$ & RWMC 2-7 & 2006 & 8.393 & 0.00615 & 0.01845 & - \\
\hline $\mathrm{U}-238$ & RWMC 2-8 & 2006 & 6.062 & 0.0123 & 0.0369 & - \\
\hline $\mathrm{U}-238$ & RWMC 2-8 & 2006 & 1.597 & 0.021 & 0.063 & - \\
\hline $\mathrm{U}-238$ & RWMC 3-1 & 2006 & 14.25 & 0.0086 & 0.0258 & - \\
\hline $\mathrm{U}-238$ & RWMC 3-4 & 2006 & 21.65 & 0.01475 & 0.04425 & - \\
\hline $\mathrm{U}-238$ & RWMC 3-5 & 2006 & 14.81 & 0.00855 & 0.02565 & - \\
\hline $\mathrm{U}-238$ & RWMC 3-7 & 2006 & 15.98 & 0.83 & 2.49 & - \\
\hline $\mathrm{U}-238$ & RWMC 4-1 & 2006 & 4.281 & 0.01405 & 0.04215 & - \\
\hline $\mathrm{U}-238$ & RWMC 4-2 & 2006 & 6.298 & 0.01125 & 0.03375 & 一 \\
\hline $\mathrm{U}-238$ & RWMC 4-3 & 2006 & 4.22 & 0.0073 & 0.0219 & - \\
\hline $\mathrm{U}-238$ & RWMC 4-4 & 2006 & 12.25 & 0.0069 & 0.0207 & - \\
\hline $\mathrm{U}-238$ & RWMC 4-5 & 2006 & 15.93 & 0.0082 & 0.0246 & - \\
\hline $\mathrm{U}-238$ & RWMC 5-10 & 2006 & 8.179 & 0.004145 & 0.012435 & - \\
\hline U-238 & RWMC 5-11 & 2006 & 21.1 & 0.0075 & 0.0225 & - \\
\hline $\mathrm{U}-238$ & RWMC 5-12 & 2006 & 15.82 & 0.01595 & 0.04785 & - \\
\hline $\mathrm{U}-238$ & RWMC 5-14 & 2006 & 17.77 & 0.0106 & 0.0318 & - \\
\hline $\mathrm{U}-238$ & RWMC 5-15 & 2006 & 18.08 & 0.0087 & 0.0261 & - \\
\hline $\mathrm{U}-238$ & RWMC 5-4 & 2006 & 22.46 & 0.00855 & 0.02565 & - \\
\hline $\mathrm{U}-238$ & RWMC 5-6 & 2006 & 5.74 & 0.0068 & 0.0204 & - \\
\hline $\mathrm{U}-238$ & RWMC 5-7 & 2006 & 6.927 & 1.475 & 4.425 & - \\
\hline $\mathrm{U}-238$ & RWMC 5-8 & 2006 & 10.99 & 0.00965 & 0.02895 & - \\
\hline $\mathrm{U}-238$ & RWMC 5-9 & 2006 & 5.362 & 0.0074 & 0.0222 & - \\
\hline U-238 & RWMC 6-1 & 2006 & 7.519 & 0.01165 & 0.03495 & 一 \\
\hline U-238 & RWMC 6-2 & 2006 & 12.47 & 0.298 & 0.894 & 一 \\
\hline $\mathrm{U}-238$ & RWMC 6-3 & 2006 & 21.25 & 0.0136 & 0.0408 & - \\
\hline $\mathrm{U}-238$ & RWMC 6-4 & 2006 & 20.16 & 0.4275 & 1.2825 & - \\
\hline $\mathrm{U}-238$ & RWMC 6-5 & 2006 & 7.588 & 0.525 & 1.575 & - \\
\hline $\mathrm{U}-238$ & RWMC 6-6 & 2006 & 5.223 & 0.00875 & 0.02625 & - \\
\hline $\mathrm{U}-238$ & RWMC 6-7 & 2006 & 5.369 & 0.0153 & 0.0459 & - \\
\hline $\mathrm{U}-238$ & RWMC 7-3 & 2006 & 52.98 & 0.01615 & 0.04845 & - \\
\hline $\mathrm{U}-238$ & RWMC 7-4 & 2006 & 10.56 & 0.00595 & 0.01785 & - \\
\hline $\mathrm{U}-238$ & RWMC 7-5 & 2006 & 11.73 & 0.0068 & 0.0204 & - \\
\hline U-238 & RWMC 7-6 & 2006 & 46.49 & 0.00925 & 0.02775 & 一 \\
\hline U-238 & RWMC 7-7 & 2006 & 11.11 & 0.00545 & 0.01635 & - \\
\hline U-238 & RWMC 9-1 & 2006 & 12.1 & 0.0119 & 0.0357 & - \\
\hline
\end{tabular}


Table C-5. (continued).

\begin{tabular}{|c|c|c|c|c|c|c|}
\hline Radionuclide & $\begin{array}{c}\text { Sample } \\
\text { Location }\end{array}$ & Date & $\begin{array}{c}\text { Concentrations } \\
(\mathrm{pCi} / \mathrm{g})\end{array}$ & $\begin{array}{l}\text { Uncertainty } \\
(\mathrm{pCi} / \mathrm{g})\end{array}$ & $\begin{array}{c}\mathrm{MDA} \\
(\mathrm{pCi} / \mathrm{g})\end{array}$ & Flag \\
\hline $\mathrm{U}-238$ & RWMC 10-1 & 2007 & 1.177 & 0.0144 & 0.0432 & - \\
\hline $\mathrm{U}-238$ & RWMC 2-4 & 2007 & 1.231 & 0.073 & 0.219 & - \\
\hline $\mathrm{U}-238$ & RWMC 2-5 & 2007 & 4.66 & 0.062 & 0.186 & - \\
\hline $\mathrm{U}-238$ & RWMC 2-6 & 2007 & 6.019 & 0.031 & 0.093 & - \\
\hline $\mathrm{U}-238$ & RWMC 2-7 & 2007 & 3.595 & 0.027 & 0.081 & - \\
\hline $\mathrm{U}-238$ & RWMC 3-1 & 2007 & 2.962 & 0.01675 & 0.05025 & - \\
\hline $\mathrm{U}-238$ & RWMC 3-4 & 2007 & 4.184 & 0.02185 & 0.06555 & - \\
\hline $\mathrm{U}-238$ & RWMC 3-5 & 2007 & 8.003 & 0.0306 & 0.0918 & - \\
\hline $\mathrm{U}-238$ & RWMC 3-6 & 2007 & 1.886 & 0.0244 & 0.0732 & - \\
\hline $\mathrm{U}-238$ & RWMC 3-7 & 2007 & 3.897 & 0.0363 & 0.1089 & - \\
\hline $\mathrm{U}-238$ & RWMC 3-8 & 2007 & 2.718 & 0.0525 & 0.1575 & 一 \\
\hline $\mathrm{U}-238$ & RWMC 4-1 & 2007 & 3.99 & 0.052 & 0.156 & 一 \\
\hline $\mathrm{U}-238$ & RWMC 4-2 & 2007 & 7.533 & 0.0595 & 0.1785 & - \\
\hline $\mathrm{U}-238$ & RWMC 4-3 & 2007 & 3.974 & 0.03155 & 0.09465 & - \\
\hline $\mathrm{U}-238$ & RWMC 4-4 & 2007 & 3.305 & 0.04105 & 0.12315 & - \\
\hline U-238 & RWMC 4-5 & 2007 & 6.338 & 0.0404 & 0.1212 & - \\
\hline $\mathrm{U}-238$ & RWMC 5-10 & 2007 & 11.06 & 0.079 & 0.237 & - \\
\hline $\mathrm{U}-238$ & RWMC 5-11 & 2007 & 1.828 & 21 & 63 & $\mathrm{U}$ \\
\hline $\mathrm{U}-238$ & RWMC 5-12 & 2007 & 2.755 & 0.0945 & 0.2835 & - \\
\hline $\mathrm{U}-238$ & RWMC 5-14 & 2007 & 5.105 & 0.0059 & 0.0177 & - \\
\hline $\mathrm{U}-238$ & RWMC 5-15 & 2007 & 2.718 & 0.0056 & 0.0168 & - \\
\hline $\mathrm{U}-238$ & RWMC 5-4 & 2007 & 7.722 & 0.01265 & 0.03795 & - \\
\hline $\mathrm{U}-238$ & RWMC 5-6 & 2007 & 3.852 & 0.03155 & 0.09465 & - \\
\hline $\mathrm{U}-238$ & RWMC 5-7 & 2007 & 0 & 6.77 & 20.31 & $\mathrm{U}$ \\
\hline U-238 & RWMC 5-8 & 2007 & 3.804 & 0.053 & 0.159 & 一 \\
\hline U-238 & RWMC 5-9 & 2007 & 1.467 & 0.03315 & 0.09945 & 一 \\
\hline $\mathrm{U}-238$ & RWMC 6-1 & 2007 & 5.868 & 0.02425 & 0.07275 & - \\
\hline $\mathrm{U}-238$ & RWMC 6-2 & 2007 & 1.291 & 0.0381 & 0.1143 & - \\
\hline $\mathrm{U}-238$ & RWMC 6-3 & 2007 & 2.299 & 0.02355 & 0.07065 & - \\
\hline $\mathrm{U}-238$ & RWMC 6-4 & 2007 & 4.17 & 0.052 & 0.156 & - \\
\hline $\mathrm{U}-238$ & RWMC 6-5 & 2007 & 1.371 & 0.03755 & 0.11265 & - \\
\hline $\mathrm{U}-238$ & RWMC 6-6 & 2007 & 2.052 & 0.0326 & 0.0978 & - \\
\hline $\mathrm{U}-238$ & RWMC 6-7 & 2007 & 7.089 & 0.0246 & 0.0738 & - \\
\hline U-238 & RWMC 6-9 & 2007 & 1.864 & 0.0186 & 0.0558 & - \\
\hline $\mathrm{U}-238$ & RWMC 7-2 & 2007 & 1.706 & 26.35 & 79.05 & $\mathrm{U}$ \\
\hline U-238 & RWMC 7-3 & 2007 & 2.012 & 0.0355 & 0.1065 & - \\
\hline U-238 & RWMC 7-4 & 2007 & 1.189 & 0.02495 & 0.07485 & - \\
\hline
\end{tabular}


Table C-5. (continued).

\begin{tabular}{|c|c|c|c|c|c|c|}
\hline Radionuclide & $\begin{array}{c}\text { Sample } \\
\text { Location }\end{array}$ & Date & $\begin{array}{c}\text { Concentrations } \\
(\mathrm{pCi} / \mathrm{g})\end{array}$ & $\begin{array}{l}\text { Uncertainty } \\
(\mathrm{pCi} / \mathrm{g})\end{array}$ & $\begin{array}{c}\mathrm{MDA} \\
(\mathrm{pCi} / \mathrm{g})\end{array}$ & Flag \\
\hline $\mathrm{U}-238$ & RWMC 7-5 & 2007 & 2.886 & 0.03115 & 0.09345 & - \\
\hline $\mathrm{U}-238$ & RWMC 7-6 & 2007 & 4.432 & 43 & 129 & $\mathrm{U}$ \\
\hline $\mathrm{U}-238$ & RWMC 7-7 & 2007 & 2.451 & 0.0314 & 0.0942 & - \\
\hline $\mathrm{U}-238$ & RWMC 8-1 & 2007 & 3.711 & 0.081 & 0.243 & - \\
\hline $\mathrm{U}-238$ & RWMC 8-4 & 2007 & 7.274 & 0.03335 & 0.10005 & - \\
\hline $\mathrm{U}-238$ & RWMC 8-5 & 2007 & 5.196 & 0.0525 & 0.1575 & - \\
\hline $\mathrm{U}-238$ & RWMC 9-1 & 2007 & 3.975 & 0.03045 & 0.09135 & - \\
\hline $\mathrm{U}-238$ & RWMC 2-5 & 2009 & -4 & 2.225 & 6.675 & $\mathrm{U}$ \\
\hline $\mathrm{U}-238$ & RWMC 2-6 & 2009 & 21.15 & 5.23 & 15.69 & - \\
\hline $\mathrm{U}-238$ & RWMC 2-8 & 2009 & 24.24 & 20.2 & 60.6 & $\mathrm{U}$ \\
\hline $\mathrm{U}-238$ & RWMC 8-4 & 2009 & 4.936 & 0.0078 & 0.0234 & 一 \\
\hline $\mathrm{U}-238$ & RWMC 3-6 & 2010 & 112.1 & 5.05 & 15.15 & 一 \\
\hline $\mathrm{U}-238$ & RWMC 3-7 & 2010 & 36.75 & 2.58 & 7.74 & - \\
\hline $\mathrm{U}-238$ & RWMC 4-1 & 2010 & 47.76 & 3.05 & 9.15 & - \\
\hline U-238 & RWMC 4-2 & 2010 & 63.61 & 24.2 & 72.6 & $\mathrm{U}$ \\
\hline U-238 & RWMC 4-4 & 2010 & 32.11 & 28.2 & 84.6 & $\mathrm{U}$ \\
\hline $\mathrm{U}-238$ & RWMC 4-5 & 2010 & 16.97 & 16.6 & 49.8 & $\mathrm{U}$ \\
\hline $\mathrm{U}-238$ & RWMC 5-10 & 2010 & 47.37 & 3.18 & 9.54 & - \\
\hline $\mathrm{U}-238$ & RWMC 5-4 & 2010 & 207.8 & 47.4 & 142.2 & - \\
\hline $\mathrm{U}-238$ & RWMC 5-4 & 2010 & 1.215 & 1 & 3 & $\mathrm{U}$ \\
\hline U-238 & RWMC 6-4 & 2010 & 23.37 & 22.8 & 68.4 & $\mathrm{U}$ \\
\hline $\mathrm{U}-238$ & RWMC 7-2 & 2010 & 43.66 & 24.6 & 73.8 & $\mathrm{U}$ \\
\hline $\mathrm{U}-238$ & RWMC 7-4 & 2010 & 31.56 & 21.6 & 64.8 & $\mathrm{U}$ \\
\hline $\mathrm{U}-238$ & RWMC 8-1 & 2010 & 146.2 & 27.8 & 83.4 & - \\
\hline U-238 & RWMC 9-1 & 2010 & 2.685 & 2.59 & 7.77 & $\mathrm{U}$ \\
\hline U-238 & RWMC 10-1 & 2011 & 1.416 & 0.945 & 2.835 & $\mathrm{U}$ \\
\hline $\mathrm{U}-238$ & RWMC 2-4 & 2011 & 0.004092 & 0.968 & 2.904 & $\mathrm{U}$ \\
\hline $\mathrm{U}-238$ & RWMC 2-6 & 2011 & 1.139 & 0.803 & 2.409 & $\mathrm{U}$ \\
\hline $\mathrm{U}-238$ & RWMC 2-8 & 2011 & 0.7944 & 0.849 & 2.547 & $\mathrm{U}$ \\
\hline $\mathrm{U}-238$ & RWMC 3-4 & 2011 & 0.7071 & 0.909 & 2.727 & $\mathrm{U}$ \\
\hline $\mathrm{U}-238$ & RWMC 3-6 & 2011 & 3.071 & 0.959 & 2.877 & - \\
\hline $\mathrm{U}-238$ & RWMC 3-8 & 2011 & 0 & 0.19 & 0.57 & $\mathrm{U}$ \\
\hline $\mathrm{U}-238$ & RWMC 4-1 & 2011 & 1.374 & 1 & 3 & $\mathrm{U}$ \\
\hline U-238 & RWMC 4-1 & 2011 & 0.8743 & 0.774 & 2.322 & $\mathrm{U}$ \\
\hline U-238 & RWMC 4-5 & 2011 & 1.39 & 0.851 & 2.553 & $\mathrm{U}$ \\
\hline $\mathrm{U}-238$ & RWMC 5-12 & 2011 & 0.8115 & 0.731 & 2.193 & $\mathrm{U}$ \\
\hline $\mathrm{U}-238$ & RWMC 5-7 & 2011 & -0.539 & 0.885 & 2.655 & $\mathrm{U}$ \\
\hline
\end{tabular}


Table C-5. (continued).

\begin{tabular}{|c|c|c|c|c|c|c|}
\hline Radionuclide & $\begin{array}{l}\text { Sample } \\
\text { Location }\end{array}$ & Date & $\begin{array}{l}\text { Concentrations } \\
(\mathrm{pCi} / \mathrm{g})\end{array}$ & $\begin{array}{l}\text { Uncertainty } \\
\text { (pCi/g) }\end{array}$ & $\begin{array}{l}\mathrm{MDA} \\
(\mathrm{pCi} / \mathrm{g})\end{array}$ & Flag \\
\hline $\mathrm{U}-238$ & RWMC 6-1 & 2011 & 0.03709 & 0.611 & 1.833 & $\mathrm{U}$ \\
\hline $\mathrm{U}-238$ & RWMC 6-3 & 2011 & 1.23 & 0.873 & 2.619 & $\mathrm{U}$ \\
\hline $\mathrm{U}-238$ & RWMC 6-7 & 2011 & 2.819 & 1.01 & 3.03 & $\mathrm{U}$ \\
\hline $\mathrm{U}-238$ & RWMC 7-2 & 2011 & 1.247 & 0.9 & 2.7 & $\mathrm{U}$ \\
\hline $\mathrm{U}-238$ & RWMC 8-1 & 2011 & 4.462 & 1.18 & 3.54 & \\
\hline $\mathrm{U}-238$ & RWMC 8-5 & 2011 & 1.505 & 0.98 & 2.94 & $\mathrm{U}$ \\
\hline $\mathrm{U}-238$ & RWMC 9-1 & 2011 & 2.659 & 0.715 & 2.145 & - \\
\hline $\mathrm{U}-238$ & RWMC 2-4 & 2012 & 1.216 & 0.758 & 2.274 & $\mathrm{U}$ \\
\hline $\mathrm{U}-238$ & RWMC 3-4 & 2012 & 0.9934 & 0.885 & 2.655 & $\mathrm{U}$ \\
\hline $\mathrm{U}-238$ & RWMC 3-5 & 2012 & 11.31 & 2.306666667 & 6.92 & - \\
\hline $\mathrm{U}-238$ & RWMC 5-4 & 2012 & 1.332 & 0.825 & 2.475 & $\mathrm{U}$ \\
\hline $\mathrm{U}-238$ & RWMC 6-1 & 2012 & 0.9122 & 0.789 & 2.367 & $\mathrm{U}$ \\
\hline $\mathrm{U}-238$ & RWMC 8-1 & 2012 & 1.937 & 0.739 & 2.217 & $\mathrm{U}$ \\
\hline U-238 & $\begin{array}{l}2 \text { INCH AIR } \\
\text { - RWMC }\end{array}$ & 2013 & 4.919 & 2.85 & 8.55 & $\mathrm{U}$ \\
\hline U-238 & $\begin{array}{l}2 \text { INCH AIR } \\
\text { - RWMC }\end{array}$ & 2013 & 4.919 & 0.95 & 2.85 & - \\
\hline U-238 & RWMC 2-4 & 2013 & 19.08 & 4.2 & 12.6 & - \\
\hline $\mathrm{U}-238$ & RWMC 3-4 & 2013 & 0.8292 & 0.533 & 1.599 & $\mathrm{U}$ \\
\hline $\mathrm{U}-238$ & RWMC 5-7 & 2013 & 2.147 & 1.356666667 & 4.07 & $\mathrm{U}$ \\
\hline $\mathrm{U}-238$ & RWMC 2-4 & 2014 & 0.2858 & 1.22 & 3.66 & $\mathrm{U}$ \\
\hline $\mathrm{U}-238$ & RWMC 2-4 & 2014 & -0.5831 & 0.963 & 2.889 & $\mathrm{U}$ \\
\hline $\mathrm{U}-238$ & RWMC 4-1 & 2014 & 2.258 & 1.17 & 3.51 & $\mathrm{U}$ \\
\hline $\mathrm{U}-238$ & RWMC 5-4 & 2014 & 1.503 & 0.685 & 2.055 & $\mathrm{U}$ \\
\hline $\mathrm{U}-238$ & RWMC 6-1 & 2014 & 0.5855 & 0.545 & 1.635 & $\mathrm{U}$ \\
\hline $\mathrm{U}-238$ & RWMC 6-3 & 2014 & 2.071 & 1.19 & 3.57 & $\mathrm{U}$ \\
\hline
\end{tabular}

Table C-6. Radionuclides concentrations in surface soils associated with TAN/SMC.

\begin{tabular}{|c|c|c|c|c|c|c|}
\hline Radionuclide & $\begin{array}{c}\text { Sample } \\
\text { Location }\end{array}$ & Date & $\begin{array}{c}\text { Concentrations } \\
(\mathrm{pCi} / \mathrm{g})\end{array}$ & $\begin{array}{c}\text { Uncertainty } \\
(\mathrm{pCi} / \mathrm{g})\end{array}$ & $\begin{array}{c}\text { MDA } \\
(\mathrm{pCi} / \mathrm{g})\end{array}$ & Flag \\
\hline Am-241 & LOFT 1-76 & 1976 & 0.005 & 0.002 & 0.006 & $\mathrm{U}$ \\
\hline Am-241 & LOFT 2-76 & 1976 & 0.007 & 0.002 & 0.006 & - \\
\hline Am-241 & LOFT 3-76 & 1976 & 0.005 & 0.002 & 0.006 & $\mathrm{U}$ \\
\hline Am-241 & LOFT 4-76 & 1976 & 0.002 & 0.001 & 0.003 & $\mathrm{U}$ \\
\hline Am-241 & LOFT 5-75 & 1976 & 0.003 & 0.001 & 0.003 & - \\
\hline Am-241 & TSF-1 & 1981 & 0.004 & 0.002 & 0.006 & $\mathrm{U}$ \\
\hline Am-241 & TSF-3 & 1981 & 0.002 & 0.002 & 0.006 & $\mathrm{U}$ \\
\hline Am-241 & TSF-7 & 1981 & 0.0014 & 0.002 & 0.006 & $\mathrm{U}$ \\
\hline
\end{tabular}


Table C-6. (continued).

\begin{tabular}{|c|c|c|c|c|c|c|}
\hline Radionuclide & $\begin{array}{c}\text { Sample } \\
\text { Location }\end{array}$ & Date & $\begin{array}{c}\text { Concentrations } \\
(\mathrm{pCi} / \mathrm{g})\end{array}$ & $\begin{array}{l}\text { Uncertainty } \\
(\mathrm{pCi} / \mathrm{g})\end{array}$ & $\begin{array}{c}\mathrm{MDA} \\
(\mathrm{pCi} / \mathrm{g})\end{array}$ & Flag \\
\hline Am-241 & TSF-1 & 1988 & 0.007 & 0.004 & 0.012 & $\mathrm{U}$ \\
\hline Am-241 & TSF-2 & 1988 & 0.029 & 0.005 & 0.015 & - \\
\hline Am-241 & TSF-6 & 1988 & 0.086 & 0.011 & 0.033 & - \\
\hline Co-60 & TSF-3 & 1981 & 0.21 & 0.05600 & 0.168 & - \\
\hline Co-60 & IET-6 & 2006 & 0.124 & 0.0179 & 0.0537 & - \\
\hline Co-60 & IET-7 & 2006 & 0.04831 & 0.00815 & 0.02445 & - \\
\hline Co-60 & IET-8 & 2006 & 0.09975 & 0.0177 & 0.0531 & - \\
\hline Co-60 & IET-9 & 2006 & 0.03735 & 0.00995 & 0.02985 & - \\
\hline Co-60 & L1-76 & 2006 & 0.06147 & 0.0094 & 0.0282 & - \\
\hline Co-60 & L2-76 & 2006 & 0.0846 & 0.01475 & 0.04425 & - \\
\hline Co-60 & L3-76 & 2006 & 0.1309 & 0.02405 & 0.07215 & 一 \\
\hline Co-60 & L4-76 & 2006 & -0.0004739 & 0.00505 & 0.01515 & $\mathrm{U}$ \\
\hline Co-60 & L5-76 & 2006 & -0.0001839 & 0.004025 & 0.012075 & $\mathrm{U}$ \\
\hline Co-60 & TSF-1 & 2006 & 0.006942 & 0.0054 & 0.0162 & $\mathrm{U}$ \\
\hline Co-60 & TSF-6 & 2006 & 0.004388 & 0.0054 & 0.0162 & $\mathrm{U}$ \\
\hline Co-60 & TSF-7 & 2006 & 0.04713 & 0.00945 & 0.02835 & - \\
\hline Co-60 & TSF-8 & 2006 & 0.06725 & 0.0145 & 0.0435 & - \\
\hline Co-60 & TSF-9 & 2006 & 0.009961 & 0.0051 & 0.0153 & $\mathrm{U}$ \\
\hline $\mathrm{Co}-60$ & WRRTF-5 & 2006 & 0.004234 & 0.004755 & 0.014265 & $\mathrm{U}$ \\
\hline Co-60 & WRRTF-6 & 2006 & 0.08303 & 0.0128 & 0.0384 & - \\
\hline Co-60 & WRRTF-7 & 2006 & 0.1173 & 0.0201 & 0.0603 & 一 \\
\hline Co-60 & WRRTF-8 & 2006 & 0.002501 & 0.00396 & 0.01188 & $\mathrm{U}$ \\
\hline Co-60 & IET-6 & 2011 & 0.002296 & 0.00375 & 0.01125 & $\mathrm{U}$ \\
\hline Co-60 & IET-7 & 2011 & -0.0009331 & 0.003 & 0.00978 & $\mathrm{U}$ \\
\hline Co-60 & IET-8 & 2011 & 0.002898 & 0.00316 & 0.00948 & $\mathrm{U}$ \\
\hline Co-60 & IET-9 & 2011 & -0.00211 & 0.00379 & 0.01137 & $\mathrm{U}$ \\
\hline Co-60 & $\mathrm{L} 2-76$ & 2011 & 0.003492 & 0.00363 & 0.01089 & $\mathrm{U}$ \\
\hline Co-60 & L3-76 & 2011 & -0.0004918 & 0.00279 & 0.00837 & $\mathrm{U}$ \\
\hline Co-60 & L4-76 & 2011 & 0.002939 & 0.00458 & 0.01374 & $\mathrm{U}$ \\
\hline Co-60 & L5-76 & 2011 & -0.0001879 & 0.00333 & 0.00999 & $\mathrm{U}$ \\
\hline Co-60 & TSF-1 & 2011 & 0.00006648 & 0.00304 & 0.00912 & $\mathrm{U}$ \\
\hline Co-60 & TSF-6 & 2011 & 0.003802 & 0.00426 & 0.01278 & $\mathrm{U}$ \\
\hline Co-60 & TSF-7 & 2011 & -0.000726 & 0.00402 & 0.01206 & $\mathrm{U}$ \\
\hline Co-60 & TSF-8 & 2011 & 0.003547 & 0.00458 & 0.01374 & $\mathrm{U}$ \\
\hline Co-60 & TSF-9 & 2011 & 0.0001377 & 0.00295 & 0.00885 & $\mathrm{U}$ \\
\hline Co-60 & WRRTF-5 & 2011 & -0.0004112 & 0.00349 & 0.01047 & $\mathrm{U}$ \\
\hline Co-60 & WRRTF-6 & 2011 & 0.004265 & 0.00459 & 0.01377 & $\mathrm{U}$ \\
\hline
\end{tabular}


Table C-6. (continued).

\begin{tabular}{|c|c|c|c|c|c|c|}
\hline Radionuclide & $\begin{array}{l}\text { Sample } \\
\text { Location }\end{array}$ & Date & $\begin{array}{l}\text { Concentrations } \\
(\mathrm{pCi} / \mathrm{g})\end{array}$ & $\begin{array}{l}\text { Uncertainty } \\
(\mathrm{pCi} / \mathrm{g})\end{array}$ & $\begin{array}{c}\mathrm{MDA} \\
(\mathrm{pCi} / \mathrm{g})\end{array}$ & Flag \\
\hline Co-60 & WRRTF-7 & 2011 & -0.004101 & 0.0045 & 0.0135 & $\mathrm{U}$ \\
\hline Co-60 & WRRTF-8 & 2011 & 0.0002946 & 0.0028 & 0.0084 & $\mathrm{U}$ \\
\hline Co-60 & $\mathrm{L} 1-76$ & 2012 & 0.001256 & 0.0032 & 0.0096 & $\mathrm{U}$ \\
\hline Co-60 & WRRTF-5 & 2012 & -0.0003805 & 0.0030 & 0.00897 & $\mathrm{U}$ \\
\hline Co-60 & WRRTF-7 & 2012 & 0.002655 & 0.00412 & 0.01236 & $\mathrm{U}$ \\
\hline Co-60 & TSF-6 & 2013 & 0.01402 & 0.00407 & 0.0122 & - \\
\hline Co-60 & $\begin{array}{c}2 \text { INCH AIR } \\
- \text { SMC }\end{array}$ & 2014 & -0.0006793 & 0.00117 & 0.00351 & $\mathrm{U}$ \\
\hline Co-60 & TSF-1 & 2014 & 0.0004809 & 0.0014 & 0.0042 & $\mathrm{U}$ \\
\hline $\mathrm{Co}-60$ & TSF-6 & 2014 & 0.001506 & 0.00146 & 0.00438 & $\mathrm{U}$ \\
\hline Co-60 & TSF-7 & 2014 & 0.00003378 & 0.00102 & 0.00306 & $\mathrm{U}$ \\
\hline Co-60 & TSF-8 & 2014 & -0.0006068 & 0.00167 & 0.00501 & $\mathrm{U}$ \\
\hline Co-60 & TSF-9 & 2014 & 0.001732 & 0.00137 & 0.00411 & $\mathrm{U}$ \\
\hline Co-60 & WRRTF-6 & 2014 & 0.0007879 & 0.00145 & 0.00435 & $\mathrm{U}$ \\
\hline Cs-134 & IET-6 & 2007 & 0.06877 & 0.011 & 0.033 & 一 \\
\hline Cs-134 & IET-7 & 2007 & 0.07063 & 0.0135 & 0.0405 & - \\
\hline Cs-134 & IET-8 & 2007 & 0.03044 & 0.0048 & 0.0144 & - \\
\hline Cs-134 & IET-9 & 2007 & 0.07361 & 0.0139 & 0.0417 & 一 \\
\hline Cs-134 & L1-76 & 2007 & 0.08852 & 0.0129 & 0.0387 & - \\
\hline Cs-134 & L2-76 & 2007 & 0.1084 & 0.015 & 0.045 & - \\
\hline Cs-134 & L3-76 & 2007 & 0.08464 & 0.0163 & 0.0489 & - \\
\hline Cs-134 & L4-76 & 2007 & 0.002981 & 0.00488 & 0.01464 & $\mathrm{U}$ \\
\hline Cs-134 & L5-76 & 2007 & 0.008246 & 0.00481 & 0.01443 & $\mathrm{U}$ \\
\hline Cs-134 & TSF-1 & 2007 & 0.06158 & 0.0103 & 0.0309 & 一 \\
\hline Cs-134 & TSF-6 & 2007 & 0.0475 & 0.00593 & 0.01779 & 一 \\
\hline Cs-134 & TSF-7 & 2007 & 0.09618 & 0.0241 & 0.0723 & 一 \\
\hline Cs-134 & TSF-8 & 2007 & 0.08889 & 0.0135 & 0.0405 & - \\
\hline Cs-134 & TSF-9 & 2007 & 0.06317 & 0.0105 & 0.0315 & - \\
\hline Cs-134 & WRRTF-5 & 2007 & 0.02612 & 0.00886 & 0.02658 & $\mathrm{U}$ \\
\hline Cs-134 & WRRTF-6 & 2007 & 0.09996 & 0.0182 & 0.0546 & - \\
\hline Cs-134 & WRRTF-7 & 2007 & 0.07175 & 0.0115 & 0.0345 & - \\
\hline Cs-134 & WRRTF-8 & 2007 & 0.0377 & 0.00616 & 0.01848 & 一 \\
\hline Cs-134 & IET-6 & 2010 & 0.04428 & 0.00625 & 0.01875 & - \\
\hline Cs-134 & IET-7 & 2010 & 0.02757 & 0.00515 & 0.01545 & - \\
\hline Cs-134 & IET-8 & 2010 & 0.02161 & 0.0024 & 0.00726 & - \\
\hline Cs-134 & IET-9 & 2010 & 0.03903 & 0.00944 & 0.02832 & 一 \\
\hline Cs-134 & L1-76 & 2010 & 0.03515 & 0.0046 & 0.0138 & - \\
\hline
\end{tabular}


Table C-6. (continued).

\begin{tabular}{|c|c|c|c|c|c|c|}
\hline Radionuclide & $\begin{array}{c}\text { Sample } \\
\text { Location }\end{array}$ & Date & $\begin{array}{c}\text { Concentrations } \\
(\mathrm{pCi} / \mathrm{g})\end{array}$ & $\begin{array}{l}\text { Uncertainty } \\
(\mathrm{pCi} / \mathrm{g})\end{array}$ & $\begin{array}{l}\text { MDA } \\
(\mathrm{pCi} / \mathrm{g})\end{array}$ & Flag \\
\hline Cs-134 & L2-76 & 2010 & 0.02731 & 0.00623 & 0.01869 & - \\
\hline Cs-134 & L3-76 & 2010 & 0.05578 & 0.0059 & 0.0177 & - \\
\hline Cs-134 & L4-76 & 2010 & 0.059 & 0.0168 & 0.0504 & - \\
\hline Cs-134 & L5-76 & 2010 & 0.04237 & 0.00722 & 0.02166 & - \\
\hline Cs-134 & TSF-6 & 2010 & 0.05551 & 0.00745 & 0.02235 & - \\
\hline Cs-134 & TSF-7 & 2010 & 0.049 & 0.0101 & 0.0303 & - \\
\hline Cs-134 & TSF-8 & 2010 & 0.07905 & 0.0102 & 0.0306 & - \\
\hline Cs-134 & TSF-9 & 2010 & 0.06294 & 0.0104 & 0.0312 & - \\
\hline Cs-134 & WRRTF-6 & 2010 & 0.05511 & 0.00781 & 0.02343 & - \\
\hline Cs-134 & WRRTF-7 & 2010 & 0.05268 & 0.00873 & 0.02619 & - \\
\hline Cs-134 & WRRTF-8 & 2010 & 0.02755 & 0.00613 & 0.01839 & - \\
\hline Cs-134 & IET-6 & 2011 & -0.007257 & 0.0208 & 0.0624 & $\mathrm{U}$ \\
\hline Cs-134 & IET-7 & 2011 & -0.00533 & 0.0148 & 0.0444 & $\mathrm{U}$ \\
\hline Cs-134 & IET-8 & 2011 & -0.0003889 & 0.019 & 0.057 & $\mathrm{U}$ \\
\hline Cs-134 & IET-9 & 2011 & -0.005299 & 0.00895 & 0.02685 & $\mathrm{U}$ \\
\hline Cs-134 & L2-76 & 2011 & 0.04522 & 0.0097 & 0.0291 & - \\
\hline Cs-134 & L3-76 & 2011 & 0.00193 & 0.0149 & 0.0447 & $\mathrm{U}$ \\
\hline Cs-134 & L4-76 & 2011 & -0.007949 & 0.0223 & 0.0669 & $\mathrm{U}$ \\
\hline Cs-134 & L5-76 & 2011 & -0.007383 & 0.0116 & 0.0348 & $\mathrm{U}$ \\
\hline Cs-134 & TSF-1 & 2011 & -0.01346 & 0.0173 & 0.0519 & $\mathrm{U}$ \\
\hline Cs-134 & TSF-6 & 2011 & 0.04498 & 0.0114 & 0.0342 & - \\
\hline Cs-134 & TSF-7 & 2011 & 0.01823 & 0.01390 & 0.0417 & $\mathrm{U}$ \\
\hline Cs-134 & TSF-8 & 2011 & 0.0319 & 0.0134 & 0.0402 & $\mathrm{U}$ \\
\hline Cs-134 & TSF-9 & 2011 & -0.05739 & 0.00955 & 0.02865 & $\mathrm{U}$ \\
\hline Cs-134 & WRRTF-5 & 2011 & 0.02073 & 0.00898 & 0.02694 & $\mathrm{U}$ \\
\hline Cs-134 & WRRTF-6 & 2011 & -0.01216 & 0.0232 & 0.0696 & $\mathrm{U}$ \\
\hline Cs-134 & WRRTF-7 & 2011 & 0.007082 & 0.023 & 0.069 & $\mathrm{U}$ \\
\hline Cs-134 & WRRTF-8 & 2011 & -0.002754 & 0.00854 & 0.02562 & $\mathrm{U}$ \\
\hline Cs-134 & L1-76 & 2012 & 0.04116 & 0.00642 & 0.01926 & - \\
\hline Cs-134 & WRRTF-5 & 2012 & 0.05597 & 0.007 & 0.021 & - \\
\hline Cs-134 & WRRTF-7 & 2012 & 0.04962 & 0.00827 & 0.02481 & - \\
\hline Cs-134 & TSF-6 & 2013 & 0.1081 & 0.0161 & 0.0482 & - \\
\hline Cs-134 & $\begin{array}{c}2 \text { INCH AIR } \\
- \text { SMC }\end{array}$ & 2014 & 0.02458 & 0.00958 & 0.02874 & $\mathrm{U}$ \\
\hline Cs-134 & TSF-1 & 2014 & 0.03193 & 0.0147 & 0.0441 & $\mathrm{U}$ \\
\hline Cs-134 & TSF-6 & 2014 & 0.04533 & 0.0117 & 0.0351 & 一 \\
\hline Cs-134 & TSF-7 & 2014 & 0.04341 & 0.0105 & 0.0315 & - \\
\hline
\end{tabular}


Table C-6. (continued).

\begin{tabular}{|c|c|c|c|c|c|c|}
\hline Radionuclide & $\begin{array}{c}\text { Sample } \\
\text { Location }\end{array}$ & Date & $\begin{array}{c}\text { Concentrations } \\
(\mathrm{pCi} / \mathrm{g})\end{array}$ & $\begin{array}{l}\text { Uncertainty } \\
(\mathrm{pCi} / \mathrm{g})\end{array}$ & $\begin{array}{c}\mathrm{MDA} \\
(\mathrm{pCi} / \mathrm{g})\end{array}$ & Flag \\
\hline Cs-134 & TSF-8 & 2014 & 0.05834 & 0.0122 & 0.0366 & - \\
\hline Cs-134 & TSF-9 & 2014 & 0.02573 & 0.0146 & 0.0438 & $\mathrm{U}$ \\
\hline Cs-134 & WRRTF-6 & 2014 & 0.03145 & 0.0116 & 0.0348 & $\mathrm{U}$ \\
\hline Cs-137 & LOFT $1-76$ & 1976 & 1.17 & 0.04 & 0.12 & - \\
\hline Cs-137 & LOFT 2-76 & 1976 & 1.41 & 0.05 & 0.15 & - \\
\hline Cs-137 & LOFT 3-76 & 1976 & 0.77 & 0.03 & 0.09 & - \\
\hline Cs-137 & LOFT 4-76 & 1976 & 0.73 & 0.020 & 0.06 & - \\
\hline Cs-137 & LOFT 5-75 & 1976 & 0.86 & 0.03 & 0.09 & - \\
\hline Cs-137 & LOFT $1-76$ & 1981 & 0.815 & 0.068 & 0.204 & - \\
\hline Cs-137 & LOFT 2-76 & 1981 & 0.838 & 0.069 & 0.207 & - \\
\hline Cs-137 & LOFT 3-76 & 1981 & 0.55 & 0.069 & 0.207 & 一 \\
\hline Cs-137 & LOFT 4-76 & 1981 & 0.471 & 0.054 & 0.162 & 一 \\
\hline Cs-137 & LOFT 5-75 & 1981 & 0.798 & 0.071 & 0.213 & - \\
\hline Cs-137 & TSF-1 & 1981 & 0.963 & 0.77 & 2.31 & $\mathrm{U}$ \\
\hline Cs-137 & TSF-2 & 1981 & 8.06 & 0.03 & 0.09 & - \\
\hline Cs-137 & TSF-3 & 1981 & 23.8 & 0.71 & 2.13 & - \\
\hline Cs-137 & TSF-4 & 1981 & 4.22 & 0.17 & 0.51 & - \\
\hline Cs-137 & TSF-5 & 1981 & 2.08 & 0.12 & 0.36 & - \\
\hline Cs-137 & TSF-6 & 1981 & 1.47 & 0.13 & 0.39 & - \\
\hline Cs-137 & TSF-7 & 1981 & 1.16 & 0.081 & 0.243 & - \\
\hline Cs-137 & TSF-8 & 1981 & 3.75 & 0.1600 & 0.48 & 一 \\
\hline Cs-137 & TSF-9 & 1981 & 1.23 & 0.087 & 0.261 & - \\
\hline Cs-137 & IET-6 & 1988 & 0.68 & 0.09 & 0.27 & - \\
\hline Cs-137 & IET-7 & 1988 & 1.05 & 0.07 & 0.21 & - \\
\hline Cs-137 & IET-8 & 1988 & 0.74 & 0.07 & 0.21 & 一 \\
\hline Cs-137 & IET-9 & 1988 & 0.9 & 0.06 & 0.18 & 一 \\
\hline Cs-137 & LOFT 1-76 & 1988 & 1.09 & 0.09 & 0.27 & - \\
\hline Cs-137 & LOFT 2-76 & 1988 & 1.16 & 0.08 & 0.24 & - \\
\hline Cs-137 & LOFT 3-76 & 1988 & 0.49 & 0.07 & 0.21 & - \\
\hline Cs-137 & LOFT 4-76 & 1988 & 0.48 & 0.06 & 0.18 & - \\
\hline Cs-137 & LOFT 5-75 & 1988 & 0.64 & 0.07 & 0.21 & - \\
\hline Cs-137 & TSF-1 & 1988 & 1.69 & 0.11 & 0.33 & - \\
\hline Cs-137 & TSF-2 & 1988 & 12.7 & 0.500 & 1.5 & - \\
\hline Cs-137 & TSF-6 & 1988 & 1.6 & 0.11 & 0.33 & - \\
\hline Cs-137 & TSF-7 & 1988 & 2.66 & 0.15 & 0.45 & - \\
\hline Cs-137 & TSF-8 & 1988 & 3.75 & 0.20000 & 0.6 & - \\
\hline Cs-137 & TSF-9 & 1988 & 1.02 & 0.09 & 0.27 & - \\
\hline
\end{tabular}


Table C-6. (continued).

\begin{tabular}{|c|c|c|c|c|c|c|}
\hline Radionuclide & $\begin{array}{c}\text { Sample } \\
\text { Location }\end{array}$ & Date & $\begin{array}{c}\text { Concentrations } \\
(\mathrm{pCi} / \mathrm{g})\end{array}$ & $\begin{array}{l}\text { Uncertainty } \\
(\mathrm{pCi} / \mathrm{g})\end{array}$ & $\begin{array}{c}\mathrm{MDA} \\
(\mathrm{pCi} / \mathrm{g})\end{array}$ & Flag \\
\hline Cs-137 & WRRTF-5 & 1988 & 0.24 & 0.06 & 0.18 & - \\
\hline Cs-137 & WRRTF-6 & 1988 & 0.52 & 0.08 & 0.24 & - \\
\hline Cs-137 & WRRTF-7 & 1988 & 0.57 & 0.08 & 0.24 & - \\
\hline Cs-137 & WRRTF-8 & 1988 & 0.84 & 0.08 & 0.24 & - \\
\hline Cs-137 & IET-6 & 2006 & 0.1194 & 0.00745 & 0.02235 & - \\
\hline Cs-137 & IET-7 & 2006 & 0.1154 & 0.00605 & 0.01815 & - \\
\hline Cs-137 & IET-8 & 2006 & 0.4308 & 0.0925 & 0.2775 & - \\
\hline Cs-137 & IET-9 & 2006 & 0.1163 & 0.0059 & 0.0177 & - \\
\hline Cs-137 & L1-76 & 2006 & 0.3422 & 0.1005 & 0.3015 & - \\
\hline Cs-137 & L2-76 & 2006 & 0.1215 & 0.0065 & 0.0195 & - \\
\hline Cs-137 & L3-76 & 2006 & 0.9995 & 0.18 & 0.54 & 一 \\
\hline Cs-137 & L4-76 & 2006 & 0.1269 & 0.01265 & 0.03795 & - \\
\hline Cs-137 & L5-76 & 2006 & 0.1208 & 0.0088 & 0.0264 & - \\
\hline Cs-137 & TSF-1 & 2006 & 0.1837 & 0.00775 & 0.02325 & - \\
\hline Cs-137 & TSF-6 & 2006 & 0.1693 & 0.00935 & 0.02805 & - \\
\hline Cs-137 & TSF-7 & 2006 & 0.548 & 0.0755 & 0.2265 & - \\
\hline Cs-137 & TSF-8 & 2006 & 0.4919 & 0.0097 & 0.0291 & - \\
\hline Cs-137 & TSF-9 & 2006 & 0.09393 & 0.00945 & 0.02835 & - \\
\hline Cs-137 & WRRTF-5 & 2006 & 0.07391 & 0.00545 & 0.01635 & - \\
\hline Cs-137 & WRRTF-6 & 2006 & 0.594 & 0.122 & 0.366 & - \\
\hline Cs-137 & WRRTF-7 & 2006 & 0.07164 & 0.00235 & 0.00705 & - \\
\hline Cs-137 & WRRTF-8 & 2006 & 0.07061 & 0.00715 & 0.02145 & - \\
\hline Cs-137 & IET-6 & 2007 & 0.2077 & 0.00418 & 0.01254 & - \\
\hline Cs-137 & IET-7 & 2007 & 0.2031 & 0.0186 & 0.0558 & - \\
\hline Cs-137 & IET-8 & 2007 & 0.2191 & 0.00982 & 0.02946 & 一 \\
\hline Cs-137 & IET-9 & 2007 & 0.2781 & 0.0169 & 0.0507 & 一 \\
\hline Cs-137 & L1-76 & 2007 & 0.2518 & 0.0149 & 0.0447 & - \\
\hline Cs-137 & L2-76 & 2007 & 0.2252 & 0.0209 & 0.0627 & - \\
\hline Cs-137 & L3-76 & 2007 & 0.2511 & 0.0229 & 0.0687 & - \\
\hline Cs-137 & L4-76 & 2007 & 0.1453 & 0.00426 & 0.01278 & - \\
\hline Cs-137 & L5-76 & 2007 & 0.2353 & 0.0185 & 0.0555 & - \\
\hline Cs-137 & TSF-1 & 2007 & 0.4386 & 0.0109 & 0.0327 & - \\
\hline Cs-137 & TSF-6 & 2007 & 0.3705 & 0.023 & 0.0675 & - \\
\hline Cs-137 & TSF-7 & 2007 & 0.5965 & 0.0144 & 0.0432 & - \\
\hline Cs-137 & TSF-8 & 2007 & 1.204 & 0.0409 & 0.1227 & - \\
\hline Cs-137 & TSF-9 & 2007 & 0.2542 & 0.0125 & 0.0375 & - \\
\hline Cs-137 & WRRTF-5 & 2007 & 0.1518 & 0.0101 & 0.0303 & - \\
\hline
\end{tabular}


Table C-6. (continued).

\begin{tabular}{|c|c|c|c|c|c|c|}
\hline Radionuclide & $\begin{array}{c}\text { Sample } \\
\text { Location }\end{array}$ & Date & $\begin{array}{c}\text { Concentrations } \\
(\mathrm{pCi} / \mathrm{g})\end{array}$ & $\begin{array}{l}\text { Uncertainty } \\
(\mathrm{pCi} / \mathrm{g})\end{array}$ & $\begin{array}{c}\mathrm{MDA} \\
(\mathrm{pCi} / \mathrm{g})\end{array}$ & Flag \\
\hline Cs-137 & WRRTF-6 & 2007 & 0.2041 & 0.0141 & 0.0423 & - \\
\hline Cs-137 & WRRTF-7 & 2007 & 0.2122 & 0.0133 & 0.0399 & - \\
\hline Cs-137 & WRRTF-8 & 2007 & 0.1625 & 0.0169 & 0.0507 & - \\
\hline Cs-137 & IET-6 & 2009 & 0.2274 & 0.0098 & 0.0294 & - \\
\hline Cs-137 & IET-7 & 2009 & 0.1524 & 0.00178 & 0.00534 & - \\
\hline Cs-137 & IET-8 & 2009 & 0.1813 & 0.00565 & 0.01695 & - \\
\hline Cs-137 & IET-9 & 2009 & 0.1883 & 0.00685 & 0.02055 & - \\
\hline Cs-137 & L1-76 & 2009 & 0.1909 & 0.0064 & 0.0192 & - \\
\hline Cs-137 & L2-76 & 2009 & 0.2164 & 0.006 & 0.018 & - \\
\hline Cs-137 & L3-76 & 2009 & 0.1397 & 0.00215 & 0.00645 & - \\
\hline Cs-137 & L4-76 & 2009 & 0.1383 & 0.0051 & 0.0153 & - \\
\hline Cs-137 & L5-76 & 2009 & 0.1774 & 0.00805 & 0.02415 & 一 \\
\hline Cs-137 & TSF-1 & 2009 & 0.3903 & 0.0095 & 0.0285 & - \\
\hline Cs-137 & TSF-6 & 2009 & 0.3387 & 0.00825 & 0.02475 & - \\
\hline Cs-137 & TSF-7 & 2009 & 0.1769 & 0.001755 & 0.005265 & - \\
\hline Cs-137 & TSF-8 & 2009 & 1.085 & 0.01095 & 0.03285 & - \\
\hline Cs-137 & WRRTF-5 & 2009 & 0.1296 & 0.0076 & 0.0228 & - \\
\hline Cs-137 & WRRTF-6 & 2009 & 0.1636 & 0.0088 & 0.0264 & - \\
\hline Cs-137 & WRRTF-7 & 2009 & 0.1623 & 0.0093 & 0.0279 & - \\
\hline Cs-137 & WRRTF-8 & 2009 & 0.1029 & 0.0065 & 0.0195 & - \\
\hline Cs-137 & IET-6 & 2010 & 0.2614 & 0.00875 & 0.02625 & - \\
\hline Cs-137 & IET-7 & 2010 & 0.1617 & 0.00735 & 0.02205 & - \\
\hline Cs-137 & IET-8 & 2010 & 0.1525 & 0.0053 & 0.0159 & - \\
\hline Cs-137 & IET-9 & 2010 & 0.1748 & 0.00814 & 0.02442 & - \\
\hline Cs-137 & L1-76 & 2010 & 0.1917 & 0.00722 & 0.02166 & 一 \\
\hline Cs-137 & L2-76 & 2010 & 0.1933 & 0.0107 & 0.0321 & 一 \\
\hline Cs-137 & L3-76 & 2010 & 0.1575 & 0.0074 & 0.02205 & - \\
\hline Cs-137 & L4-76 & 2010 & 0.1202 & 0.00354 & 0.01062 & - \\
\hline Cs-137 & L5-76 & 2010 & 0.2109 & 0.0108 & 0.0324 & - \\
\hline Cs-137 & TSF-6 & 2010 & 0.3695 & 0.011 & 0.033 & - \\
\hline Cs-137 & TSF-7 & 2010 & 0.325 & 0.00502 & 0.01506 & - \\
\hline Cs-137 & TSF-8 & 2010 & 0.9118 & 0.0164 & 0.0492 & - \\
\hline Cs-137 & TSF-9 & 2010 & 0.2037 & 0.00839 & 0.02517 & - \\
\hline Cs-137 & WRRTF-6 & 2010 & 0.1374 & 0.00418 & 0.01254 & - \\
\hline Cs-137 & WRRTF-7 & 2010 & 0.1362 & 0.00423 & 0.01269 & - \\
\hline Cs-137 & WRRTF-8 & 2010 & 0.1213 & 0.00934 & 0.02802 & - \\
\hline Cs-137 & IET-6 & 2011 & 0.4204 & 0.0122 & 0.0366 & - \\
\hline
\end{tabular}


Table C-6. (continued).

\begin{tabular}{|c|c|c|c|c|c|c|}
\hline Radionuclide & $\begin{array}{l}\text { Sample } \\
\text { Location }\end{array}$ & Date & $\begin{array}{c}\text { Concentrations } \\
(\mathrm{pCi} / \mathrm{g})\end{array}$ & $\begin{array}{l}\text { Uncertainty } \\
(\mathrm{pCi} / \mathrm{g})\end{array}$ & $\begin{array}{c}\mathrm{MDA} \\
(\mathrm{pCi} / \mathrm{g})\end{array}$ & Flag \\
\hline Cs-137 & IET-7 & 2011 & 0.3688 & 0.0118 & 0.0354 & - \\
\hline Cs-137 & IET-8 & 2011 & 0.31 & 0.0106 & 0.0318 & - \\
\hline Cs-137 & IET-9 & 2011 & 0.4697 & 0.0172 & 0.0516 & - \\
\hline Cs-137 & $\mathrm{L} 2-76$ & 2011 & 0.4193 & 0.0119 & 0.0357 & - \\
\hline Cs-137 & L3-76 & 2011 & 0.2805 & 0.00754 & 0.02262 & - \\
\hline Cs-137 & L4-76 & 2011 & 0.3124 & 0.0123 & 0.0369 & - \\
\hline Cs-137 & L5-76 & 2011 & 0.3693 & 0.0121 & 0.0363 & - \\
\hline Cs-137 & TSF-1 & 2011 & 0.838 & 0.0115 & 0.0345 & - \\
\hline Cs-137 & TSF-6 & 2011 & 0.6579 & 0.0144 & 0.0432 & - \\
\hline Cs-137 & TSF-7 & 2011 & 1.005 & 0.0184 & 0.0552 & - \\
\hline Cs-137 & TSF-8 & 2011 & 2.569 & 0.0294 & 0.0882 & - \\
\hline Cs-137 & TSF-9 & 2011 & 0.4512 & 0.0111 & 0.0333 & - \\
\hline Cs-137 & WRRTF-5 & 2011 & 0.2464 & 0.00846 & 0.02538 & - \\
\hline Cs-137 & WRRTF-6 & 2011 & 0.3832 & 0.0148 & 0.0444 & - \\
\hline Cs-137 & WRRTF-7 & 2011 & 0.3435 & 0.0168 & 0.0504 & - \\
\hline Cs-137 & WRRTF-8 & 2011 & 0.2056 & 0.00936 & 0.02808 & 一 \\
\hline Cs-137 & L1-76 & 2012 & 0.1446 & 0.0042 & 0.01263 & - \\
\hline Cs-137 & WRRTF-5 & 2012 & 0.1077 & 0.00787 & 0.02361 & - \\
\hline Cs-137 & WRRTF-7 & 2012 & 0.1302 & 0.00498 & 0.01494 & - \\
\hline Cs-137 & TSF-6 & 2013 & 0.2592 & 0.0048 & 0.0144 & - \\
\hline Cs-137 & $\begin{array}{c}2 \text { INCH AIR } \\
- \text { SMC }\end{array}$ & 2014 & 0.0164 & 0.00322 & 0.00966 & 一 \\
\hline Cs-137 & TSF-1 & 2014 & 0.3043 & 0.013 & 0.039 & - \\
\hline Cs-137 & TSF-6 & 2014 & 0.3656 & 0.0206 & 0.0618 & - \\
\hline Cs-137 & TSF-7 & 2014 & 0.2194 & 0.00438 & 0.01314 & - \\
\hline Cs-137 & TSF-8 & 2014 & 0.9604 & 0.00982 & 0.02946 & - \\
\hline Cs-137 & TSF-9 & 2014 & 0.1081 & 0.00481 & 0.01443 & - \\
\hline Cs-137 & WRRTF-6 & 2014 & 0.1391 & 0.00514 & 0.01542 & - \\
\hline Eu-152 & IET-6 & 2011 & 0.006742 & 0.023 & 0.069 & $\mathrm{U}$ \\
\hline Eu-152 & IET-7 & 2011 & 0.01517 & 0.0211 & 0.0633 & $\mathrm{U}$ \\
\hline Eu-152 & IET-8 & 2011 & 0.01255 & 0.0214 & 0.0642 & $\mathrm{U}$ \\
\hline Eu-152 & IET-9 & 2011 & 0.008969 & 0.0255 & 0.0765 & $\mathrm{U}$ \\
\hline Eu-152 & L2-76 & 2011 & 0.00296 & 0.0224 & 0.0672 & $\mathrm{U}$ \\
\hline Eu-152 & L3-76 & 2011 & 0.0175 & 0.0236 & 0.0708 & $\mathrm{U}$ \\
\hline Eu-152 & L4-76 & 2011 & 0.03248 & 0.0294 & 0.0882 & $\mathrm{U}$ \\
\hline Eu-152 & L5-76 & 2011 & 0.0177 & 0.0286 & 0.0858 & $\mathrm{U}$ \\
\hline $\mathrm{Eu}-152$ & TSF-1 & 2011 & 0.03444 & 0.0212 & 0.0636 & $\mathrm{U}$ \\
\hline
\end{tabular}


Table C-6. (continued).

\begin{tabular}{|c|c|c|c|c|c|c|}
\hline Radionuclide & $\begin{array}{l}\text { Sample } \\
\text { Location }\end{array}$ & Date & $\begin{array}{c}\text { Concentrations } \\
(\mathrm{pCi} / \mathrm{g})\end{array}$ & $\begin{array}{l}\text { Uncertainty } \\
(\mathrm{pCi} / \mathrm{g})\end{array}$ & $\begin{array}{c}\mathrm{MDA} \\
(\mathrm{pCi} / \mathrm{g})\end{array}$ & Flag \\
\hline Eu-152 & TSF-6 & 2011 & 0.014 & 0.0272 & 0.0816 & $\mathrm{U}$ \\
\hline Eu-152 & TSF-7 & 2011 & 0.02032 & 0.028 & 0.084 & $\mathrm{U}$ \\
\hline Eu-152 & TSF-8 & 2011 & 0.03855 & 0.0302 & 0.0906 & $\mathrm{U}$ \\
\hline Eu-152 & TSF-9 & 2011 & 0.01254 & 0.0233 & 0.0699 & $\mathrm{U}$ \\
\hline Eu-152 & WRRTF-5 & 2011 & 0.01726 & 0.0219 & 0.0657 & $\mathrm{U}$ \\
\hline Eu-152 & WRRTF-6 & 2011 & 0.096 & 0.0291 & 0.0873 & - \\
\hline Eu-152 & WRRTF-7 & 2011 & 0.02072 & 0.0288 & 0.0864 & $\mathrm{U}$ \\
\hline Eu-152 & WRRTF-8 & 2011 & 0.01433 & 0.024 & 0.072 & $\mathrm{U}$ \\
\hline Eu-152 & L1-76 & 2012 & 0.03755 & 0.0161 & 0.0483 & $\mathrm{U}$ \\
\hline Eu-152 & WRRTF-5 & 2012 & 0.01112 & 0.0205 & 0.0615 & $\mathrm{U}$ \\
\hline Eu-152 & WRRTF-7 & 2012 & 0.02151 & 0.0185 & 0.0555 & $\mathrm{U}$ \\
\hline Eu-152 & TSF-6 & 2013 & 0.155 & 0.030066667 & 0.0902 & - \\
\hline Eu-152 & $\begin{array}{c}2 \text { INCH AIR } \\
- \text { SMC }\end{array}$ & 2014 & 0.01554 & 0.019 & 0.0573 & $\mathrm{U}$ \\
\hline Eu-152 & TSF-1 & 2014 & 0.004745 & 0.0295 & 0.0885 & $\mathrm{U}$ \\
\hline Eu-152 & TSF-6 & 2014 & -0.01031 & 0.0218 & 0.0654 & $\mathrm{U}$ \\
\hline Eu-152 & TSF-7 & 2014 & 0.03123 & 0.0192 & 0.0576 & $\mathrm{U}$ \\
\hline Eu-152 & TSF-8 & 2014 & 0.02054 & 0.0235 & 0.0705 & $\mathrm{U}$ \\
\hline Eu-152 & TSF-9 & 2014 & -0.05029 & 0.0296 & 0.0888 & $\mathrm{U}$ \\
\hline Eu-152 & WRRTF-6 & 2014 & -0.006819 & 0.021 & 0.0615 & $\mathrm{U}$ \\
\hline $\mathrm{Pu}-238$ & LOFT $1-76$ & 1976 & 0.0015 & 0.0011 & 0.0033 & $\mathrm{U}$ \\
\hline $\mathrm{Pu}-238$ & LOFT 2-76 & 1976 & 0.003 & 0.0013 & 0.0039 & $\mathrm{U}$ \\
\hline $\mathrm{Pu}-238$ & LOFT 3-76 & 1976 & 0.001 & 0.001 & 0.003 & $\mathrm{U}$ \\
\hline $\mathrm{Pu}-238$ & LOFT 4-76 & 1976 & 0.001 & 0.001 & 0.003 & $\mathrm{U}$ \\
\hline $\mathrm{Pu}-238$ & LOFT 5-75 & 1976 & 0.014 & 0.001 & 0.003 & - \\
\hline $\mathrm{Pu}-238$ & TSF-1 & 1981 & 0 & 0.002 & 0.006 & $\mathrm{U}$ \\
\hline $\mathrm{Pu}-238$ & TSF-3 & 1981 & 0.002 & 0.002 & 0.006 & $\mathrm{U}$ \\
\hline $\mathrm{Pu}-238$ & TSF-7 & 1981 & -0.002 & 0.002 & 0.006 & $\mathrm{U}$ \\
\hline $\mathrm{Pu}-238$ & TSF-1 & 1988 & 0.003 & 0.002 & 0.006 & $\mathrm{U}$ \\
\hline $\mathrm{Pu}-238$ & TSF-2 & 1988 & 0.002 & 0.002 & 0.006 & $\mathrm{U}$ \\
\hline $\mathrm{Pu}-238$ & TSF-6 & 1988 & 0.003 & 0.001 & 0.003 & 一 \\
\hline $\mathrm{Pu}-239 / 240$ & LOFT $1-76$ & 1976 & 0.023 & 0.002 & 0.006 & - \\
\hline $\mathrm{Pu}-239 / 240$ & LOFT 2-76 & 1976 & 0.026 & 0.002 & 0.006 & - \\
\hline $\mathrm{Pu}-239 / 240$ & LOFT 3-76 & 1976 & 0.011 & 0.002 & 0.006 & - \\
\hline $\mathrm{Pu}-239 / 240$ & LOFT 4-76 & 1976 & 0.014 & 0.002 & 0.006 & - \\
\hline $\mathrm{Pu}-239 / 240$ & LOFT 5-75 & 1976 & 0.013 & 0.002 & 0.006 & 一 \\
\hline $\mathrm{Pu}-239 / 240$ & TSF-1 & 1981 & 0.011 & 0.003 & 0.009 & - \\
\hline
\end{tabular}


Table C-6. (continued).

\begin{tabular}{|c|c|c|c|c|c|c|}
\hline Radionuclide & $\begin{array}{l}\text { Sample } \\
\text { Location }\end{array}$ & Date & $\begin{array}{c}\text { Concentrations } \\
(\mathrm{pCi} / \mathrm{g})\end{array}$ & $\begin{array}{l}\text { Uncertainty } \\
(\mathrm{pCi} / \mathrm{g})\end{array}$ & $\begin{array}{c}\mathrm{MDA} \\
(\mathrm{pCi} / \mathrm{g})\end{array}$ & Flag \\
\hline $\mathrm{Pu}-239 / 240$ & TSF-3 & 1981 & 0.011 & 0.002 & 0.006 & - \\
\hline $\mathrm{Pu}-239 / 240$ & TSF-7 & 1981 & 0.01 & 0.0020 & 0.006 & - \\
\hline $\mathrm{Pu}-239 / 240$ & TSF-1 & 1988 & 0.008 & 0.002 & 0.006 & - \\
\hline $\mathrm{Pu}-239 / 240$ & TSF-2 & 1988 & 0.014 & 0.003 & 0.009 & - \\
\hline $\mathrm{Pu}-239 / 240$ & TSF-6 & 1988 & 0.029 & 0.003 & 0.009 & - \\
\hline $\mathrm{Sb}-125$ & IET-6 & 2011 & 0.02715 & 0.0337 & 0.1011 & $\mathrm{U}$ \\
\hline $\mathrm{Sb}-125$ & IET-7 & 2011 & -0.004162 & 0.0312 & 0.0936 & $\mathrm{U}$ \\
\hline $\mathrm{Sb}-125$ & IET-8 & 2011 & 0.02346 & 0.02920 & 0.0876 & $\mathrm{U}$ \\
\hline $\mathrm{Sb}-125$ & IET-9 & 2011 & -0.00724 & 0.0369 & 0.1107 & $\mathrm{U}$ \\
\hline $\mathrm{Sb}-125$ & L2-76 & 2011 & 0.6859 & 0.0333 & 0.0999 & - \\
\hline $\mathrm{Sb}-125$ & L3-76 & 2011 & -0.03426 & 0.0328 & 0.0984 & $\mathrm{U}$ \\
\hline $\mathrm{Sb}-125$ & L4-76 & 2011 & 0.05626 & 0.0391 & 0.1173 & $\mathrm{U}$ \\
\hline $\mathrm{Sb}-125$ & L5-76 & 2011 & -0.05746 & 0.0412 & 0.1236 & $\mathrm{U}$ \\
\hline $\mathrm{Sb}-125$ & TSF-1 & 2011 & -0.002396 & 0.0318 & 0.0954 & $\mathrm{U}$ \\
\hline $\mathrm{Sb}-125$ & TSF-6 & 2011 & 0.04945 & 0.03830 & 0.1149 & $\mathrm{U}$ \\
\hline $\mathrm{Sb}-125$ & TSF-7 & 2011 & -0.09995 & 0.0443 & 0.1329 & $\mathrm{U}$ \\
\hline $\mathrm{Sb}-125$ & TSF- 8 & 2011 & 0.06987 & 0.0445 & 0.1335 & $\mathrm{U}$ \\
\hline $\mathrm{Sb}-125$ & TSF-9 & 2011 & -0.02397 & 0.0308 & 0.0924 & $\mathrm{U}$ \\
\hline $\mathrm{Sb}-125$ & WRRTF-5 & 2011 & -0.0192 & 0.0336 & 0.1008 & $\mathrm{U}$ \\
\hline $\mathrm{Sb}-125$ & WRRTF-6 & 2011 & -0.05755 & 0.0405 & 0.1215 & $\mathrm{U}$ \\
\hline $\mathrm{Sb}-125$ & WRRTF-7 & 2011 & 0.0169 & 0.0392 & 0.1176 & $\mathrm{U}$ \\
\hline $\mathrm{Sb}-125$ & WRRTF-8 & 2011 & 0.03347 & 0.0301 & 0.0903 & $\mathrm{U}$ \\
\hline $\mathrm{Sb}-125$ & L1-76 & 2012 & 0.01427 & 0.0249 & 0.0747 & $\mathrm{U}$ \\
\hline $\mathrm{Sb}-125$ & WRRTF-5 & 2012 & 0.01423 & 0.0279 & 0.0837 & $\mathrm{U}$ \\
\hline $\mathrm{Sb}-125$ & WRRTF-7 & 2012 & -0.002 & 0.0323 & 0.0969 & $\mathrm{U}$ \\
\hline $\mathrm{Sb}-125$ & TSF-6 & 2013 & 0.2667 & 0.0623 & 0.187 & - \\
\hline $\mathrm{Sb}-125$ & $\begin{array}{c}2 \text { INCH AIR } \\
- \text { SMC }\end{array}$ & 2014 & -0.007939 & 0.0424 & 0.1272 & $\mathrm{U}$ \\
\hline $\mathrm{Sb}-125$ & TSF-1 & 2014 & 0.00856 & 0.0524 & 0.1572 & $\mathrm{U}$ \\
\hline $\mathrm{Sb}-125$ & TSF-6 & 2014 & 0.01042 & 0.0473 & 0.1419 & $\mathrm{U}$ \\
\hline $\mathrm{Sb}-125$ & TSF-7 & 2014 & 0.02055 & 0.0362 & 0.1086 & $\mathrm{U}$ \\
\hline $\mathrm{Sb}-125$ & TSF-8 & 2014 & -0.1013 & 0.0465 & 0.1395 & $\mathrm{U}$ \\
\hline $\mathrm{Sb}-125$ & TSF-9 & 2014 & 0.8039 & 0.0527 & 0.1581 & - \\
\hline $\mathrm{Sb}-125$ & WRRTF-6 & 2014 & -0.1639 & 0.0402 & 0.1206 & $\mathrm{U}$ \\
\hline Sr-90 & LOFT 1-76 & 1976 & 0.65 & 0.05 & 0.15 & - \\
\hline Sr-90 & LOFT 2-76 & 1976 & 0.66 & 0.05 & 0.15 & 一 \\
\hline Sr-90 & LOFT 3-76 & 1976 & 0.47 & 0.05 & 0.15 & - \\
\hline
\end{tabular}


Table C-6. (continued).

\begin{tabular}{|c|c|c|c|c|c|c|}
\hline Radionuclide & $\begin{array}{c}\text { Sample } \\
\text { Location }\end{array}$ & Date & $\begin{array}{c}\text { Concentrations } \\
(\mathrm{pCi} / \mathrm{g})\end{array}$ & $\begin{array}{l}\text { Uncertainty } \\
(\mathrm{pCi} / \mathrm{g})\end{array}$ & $\begin{array}{c}\mathrm{MDA} \\
(\mathrm{pCi} / \mathrm{g})\end{array}$ & Flag \\
\hline Sr-90 & LOFT 4-76 & 1976 & 0.47 & 0.05 & 0.15 & - \\
\hline Sr-90 & LOFT 5-75 & 1976 & 0.45 & 0.06 & 0.18 & - \\
\hline Sr-90 & TSF-1 & 1981 & 0.85 & 0.14 & 0.42 & - \\
\hline Sr-90 & TSF-3 & 1981 & 1.15 & 0.09 & 0.27 & - \\
\hline Sr-90 & TSF-7 & 1981 & 0.39 & 0.06 & 0.18 & - \\
\hline U-234 & IET-6 & 2007 & 52.88 & 2.18 & 6.54 & - \\
\hline $\mathrm{U}-234$ & IET-7 & 2007 & 78.28 & 2.61 & 7.83 & - \\
\hline $\mathrm{U}-234$ & IET-8 & 2007 & 67.87 & 3.32 & 9.96 & - \\
\hline $\mathrm{U}-234$ & IET-9 & 2007 & 98.24 & 3.27 & 9.81 & - \\
\hline $\mathrm{U}-234$ & L1-76 & 2007 & 81.26 & 3 & 9 & - \\
\hline $\mathrm{U}-234$ & L2-76 & 2007 & 102.3 & 3.34 & 10.02 & 一 \\
\hline $\mathrm{U}-234$ & L3-76 & 2007 & 103.1 & 3.44 & 10.32 & 一 \\
\hline $\mathrm{U}-234$ & L4-76 & 2007 & 106.5 & 3.49 & 10.47 & - \\
\hline $\mathrm{U}-234$ & L5-76 & 2007 & 27.55 & 2.2700 & 6.81 & - \\
\hline $\mathrm{U}-234$ & TSF-1 & 2007 & 28.85 & 2.3 & 6.9 & - \\
\hline $\mathrm{U}-234$ & TSF-6 & 2007 & 27.87 & 2.16 & 6.48 & - \\
\hline U-234 & TSF-7 & 2007 & 90.89 & 3.25 & 9.75 & - \\
\hline U-234 & TSF-8 & 2007 & 413.9 & 7.06 & 21.18 & - \\
\hline U-234 & TSF-9 & 2007 & 28.05 & 2.31 & 6.93 & - \\
\hline U-234 & WRRTF-5 & 2007 & 30.41 & 2.1 & 6.3 & - \\
\hline U-234 & WRRTF-6 & 2007 & 21.47 & 2.06 & 6.18 & - \\
\hline U-234 & WRRTF-7 & 2007 & 101.5 & 3.45 & 10.35 & - \\
\hline U-234 & WRRTF-8 & 2007 & 94.83 & 3.36 & 10.08 & - \\
\hline U-234 & L3-76 & 2009 & 5.891 & 0.825 & 2.475 & - \\
\hline U-234 & IET-6 & 2010 & 8.034 & 1.5400 & 4.62 & 一 \\
\hline U-234 & IET-7 & 2010 & 11.79 & 1.39 & 4.17 & 一 \\
\hline U-234 & IET-8 & 2010 & 28.94 & 1.88 & 5.64 & - \\
\hline U-234 & IET-9 & 2010 & 80.45 & 3.28 & 9.84 & - \\
\hline U-234 & L1-76 & 2010 & 39.53 & 2.18 & 6.54 & - \\
\hline U-234 & L2-76 & 2010 & 18.65 & 1.63 & 4.89 & - \\
\hline U-234 & L3-76 & 2010 & 45.24 & 2.41 & 7.23 & - \\
\hline U-234 & L4-76 & 2010 & 107.8 & 3.68 & 11.04 & - \\
\hline U-234 & TSF-6 & 2010 & 16.69 & 1.98 & 5.94 & - \\
\hline U-234 & TSF-7 & 2010 & 111.3 & 3.82 & 11.46 & - \\
\hline U-234 & TSF-8 & 2010 & 96.3 & 3.76 & 11.28 & 一 \\
\hline U-234 & TSF-9 & 2010 & 17.32 & 1.69 & 5.07 & - \\
\hline U-234 & WRRTF-6 & 2010 & 15.65 & 1.5 & 4.5 & - \\
\hline
\end{tabular}


Table C-6. (continued).

\begin{tabular}{|c|c|c|c|c|c|c|}
\hline Radionuclide & $\begin{array}{l}\text { Sample } \\
\text { Location }\end{array}$ & Date & $\begin{array}{c}\text { Concentrations } \\
(\mathrm{pCi} / \mathrm{g})\end{array}$ & $\begin{array}{l}\text { Uncertainty } \\
(\mathrm{pCi} / \mathrm{g})\end{array}$ & $\begin{array}{c}\mathrm{MDA} \\
(\mathrm{pCi} / \mathrm{g})\end{array}$ & Flag \\
\hline U-234 & WRRTF-7 & 2010 & 18.5 & 1.57 & 4.71 & - \\
\hline U-234 & WRRTF-8 & 2010 & 20.49 & 1.69 & 5.07 & - \\
\hline U-234 & IET-6 & 2011 & -43.45 & 63.7 & 191.1 & $\mathrm{U}$ \\
\hline U-234 & IET-7 & 2011 & -2.464 & 50.1 & 150.3 & $\mathrm{U}$ \\
\hline U-234 & IET-8 & 2011 & -3.025 & 53.3 & 159.9 & $\mathrm{U}$ \\
\hline $\mathrm{U}-234$ & IET-9 & 2011 & -19.94 & 61 & 183 & $\mathrm{U}$ \\
\hline $\mathrm{U}-234$ & L2-76 & 2011 & 0.3156 & 62.2 & 186.6 & $\mathrm{U}$ \\
\hline $\mathrm{U}-234$ & L3-76 & 2011 & -29.14 & 60.7 & 182.1 & $\mathrm{U}$ \\
\hline $\mathrm{U}-234$ & L4-76 & 2011 & 90.94 & 75.8 & 227.4 & $\mathrm{U}$ \\
\hline U-234 & L5-76 & 2011 & -44.42 & 80.7 & 242.1 & $\mathrm{U}$ \\
\hline U-234 & TSF-1 & 2011 & -50.73 & 67.1 & 201.3 & $\mathrm{U}$ \\
\hline U-234 & TSF-6 & 2011 & 29.86 & 70.2 & 210.6 & $\mathrm{U}$ \\
\hline U-234 & TSF-7 & 2011 & -0.01494 & 69.5 & 208.5 & $\mathrm{U}$ \\
\hline $\mathrm{U}-234$ & TSF-8 & 2011 & 71.47 & 84.9 & 254.7 & $\mathrm{U}$ \\
\hline $\mathrm{U}-234$ & TSF-9 & 2011 & 9.588 & 66.2 & 198.6 & $\mathrm{U}$ \\
\hline $\mathrm{U}-234$ & WRRTF-5 & 2011 & 63.75 & 59.4 & 178.2 & $\mathrm{U}$ \\
\hline $\mathrm{U}-234$ & WRRTF-6 & 2011 & -66.71 & 71.4 & 214.2 & $\mathrm{U}$ \\
\hline U-234 & WRRTF-7 & 2011 & 3.563 & 70.7 & 212.1 & $\mathrm{U}$ \\
\hline U-234 & WRRTF-8 & 2011 & 39.82 & 69.6 & 208.8 & $\mathrm{U}$ \\
\hline U-234 & L1-76 & 2012 & -2.587 & 11.8 & 35.4 & $\mathrm{U}$ \\
\hline U-234 & WRRTF-5 & 2012 & 5.637 & 20 & 60 & $\mathrm{U}$ \\
\hline U-234 & WRRTF-7 & 2012 & 1.553 & 14.5 & 43.5 & $\mathrm{U}$ \\
\hline U-234 & TSF-6 & 2013 & 63.58 & 42.33 & 127 & $\mathrm{U}$ \\
\hline U-234 & $\begin{array}{c}2 \text { INCH AIR } \\
- \text { SMC }\end{array}$ & 2014 & -7.101 & 53.3 & 159.9 & $\mathrm{U}$ \\
\hline U-234 & TSF-1 & 2014 & 48.62 & 78.8 & 236.4 & $\mathrm{U}$ \\
\hline U-234 & TSF-6 & 2014 & 26.31 & 64.8 & 194.4 & $\mathrm{U}$ \\
\hline U-234 & TSF-7 & 2014 & 13.51 & 43.3 & 129.9 & $\mathrm{U}$ \\
\hline U-234 & TSF-8 & 2014 & -12.11 & 59.8 & 179.4 & $\mathrm{U}$ \\
\hline U-234 & TSF-9 & 2014 & -46.01 & 82.9 & 248.7 & $\mathrm{U}$ \\
\hline U-234 & WRRTF-6 & 2014 & -26.57 & 52.5 & 157.5 & $\mathrm{U}$ \\
\hline U-235 & IET-6 & 2006 & 1.415 & 0.575 & 1.725 & $\mathrm{U}$ \\
\hline U-235 & IET-7 & 2006 & 1.624 & 0.55 & 1.65 & $\mathrm{U}$ \\
\hline U-235 & IET-8 & 2006 & 1.988 & 0.4845 & 1.4535 & - \\
\hline U-235 & IET-9 & 2006 & 1.433 & 0.376 & 1.128 & - \\
\hline U-235 & L1-76 & 2006 & 3.193 & 0.685 & 2.055 & - \\
\hline $\mathrm{U}-235$ & L2-76 & 2006 & 0.9748 & 0.4005 & 1.2015 & $\mathrm{U}$ \\
\hline
\end{tabular}


Table C-6. (continued).

\begin{tabular}{|c|c|c|c|c|c|c|}
\hline Radionuclide & $\begin{array}{c}\text { Sample } \\
\text { Location }\end{array}$ & Date & $\begin{array}{c}\text { Concentrations } \\
(\mathrm{pCi} / \mathrm{g})\end{array}$ & $\begin{array}{l}\text { Uncertainty } \\
(\mathrm{pCi} / \mathrm{g})\end{array}$ & $\begin{array}{c}\mathrm{MDA} \\
(\mathrm{pCi} / \mathrm{g})\end{array}$ & Flag \\
\hline U-235 & L3-76 & 2006 & 2.675 & 0.715 & 2.145 & - \\
\hline U-235 & L4-76 & 2006 & -0.1144 & 0.2565 & 0.7695 & $\mathrm{U}$ \\
\hline U-235 & L5-76 & 2006 & 0.184 & 0.2305 & 0.6915 & $\mathrm{U}$ \\
\hline U-235 & TSF-1 & 2006 & -0.1096 & 0.27 & 0.81 & $\mathrm{U}$ \\
\hline U-235 & TSF-6 & 2006 & -0.06985 & 0.277 & 0.831 & $\mathrm{U}$ \\
\hline U-235 & TSF-7 & 2006 & 3.181 & 0.86 & 2.58 & - \\
\hline U-235 & TSF-8 & 2006 & 1.083 & 0.585 & 1.755 & $\mathrm{U}$ \\
\hline $\mathrm{U}-235$ & TSF-9 & 2006 & -0.01044 & 0.227 & 0.681 & $\mathrm{U}$ \\
\hline $\mathrm{U}-235$ & WRRTF-5 & 2006 & 0.1964 & 0.229 & 0.687 & $\mathrm{U}$ \\
\hline U-235 & WRRTF-6 & 2006 & 0.3743 & 0.17 & 0.51 & $\mathrm{U}$ \\
\hline U-235 & WRRTF-7 & 2006 & 0.4855 & 0.975 & 2.925 & $\mathrm{U}$ \\
\hline U-235 & WRRTF-8 & 2006 & -0.0999 & 0.229 & 0.687 & $\mathrm{U}$ \\
\hline U-235 & IET-6 & 2007 & 0.1656 & 0.07 & 0.1956 & $\mathrm{U}$ \\
\hline U-235 & IET-7 & 2007 & 0.2993 & 0.0797 & 0.2391 & - \\
\hline U-235 & IET-8 & 2007 & 0.4681 & 0.0932 & 0.2796 & - \\
\hline U-235 & IET-9 & 2007 & 0.2641 & 0.068 & 0.204 & - \\
\hline $\mathrm{U}-235$ & L1-76 & 2007 & 0.1488 & 0.0712 & 0.2136 & $\mathrm{U}$ \\
\hline U-235 & L2-76 & 2007 & 0.3363 & 0.085 & 0.255 & - \\
\hline U-235 & L3-76 & 2007 & 0.3923 & 0.104 & 0.312 & - \\
\hline U-235 & L4-76 & 2007 & 0.2734 & 0.0934 & 0.2802 & $\mathrm{U}$ \\
\hline U-235 & L5-76 & 2007 & 0.4409 & 0.0958 & 0.2874 & - \\
\hline U-235 & TSF-1 & 2007 & 0.211 & 0.0736 & 0.2208 & $\mathrm{U}$ \\
\hline U-235 & TSF-6 & 2007 & 0.2143 & 0.0975 & 0.2925 & $\mathrm{U}$ \\
\hline U-235 & TSF-7 & 2007 & 0.1753 & 0.0764 & 0.2292 & $\mathrm{U}$ \\
\hline U-235 & TSF-8 & 2007 & 0.3882 & 0.0938 & 0.2814 & - \\
\hline U-235 & TSF-9 & 2007 & 0.3686 & 0.0884 & 0.2652 & - \\
\hline U-235 & WRRTF-5 & 2007 & 0.2481 & 0.0717 & 0.2151 & - \\
\hline U-235 & WRRTF-6 & 2007 & 0.3854 & 0.0907 & 0.2721 & - \\
\hline U-235 & WRRTF-7 & 2007 & 0.3015 & 0.0759 & 0.2277 & \\
\hline U-235 & WRRTF-8 & 2007 & 0.04745 & 0.1 & 0.2049 & $\mathrm{U}$ \\
\hline U-235 & IET-7 & 2009 & 0.1267 & 0.051 & 0.153 & $\mathrm{U}$ \\
\hline U-235 & L2-76 & 2009 & 0.1969 & 0.02755 & 0.08265 & - \\
\hline U-235 & L3-76 & 2009 & 0.1025 & 0.1 & 0.18 & $\mathrm{U}$ \\
\hline U-235 & IET-6 & 2010 & 0.2751 & 0.0514 & 0.1542 & - \\
\hline U-235 & IET-7 & 2010 & 0.129 & 0.0391 & 0.1173 & - \\
\hline U-235 & IET-8 & 2010 & 0.5844 & 0.159 & 0.477 & - \\
\hline $\mathrm{U}-235$ & IET-9 & 2010 & 1.008 & 0.245 & 0.735 & - \\
\hline
\end{tabular}


Table C-6. (continued).

\begin{tabular}{|c|c|c|c|c|c|c|}
\hline Radionuclide & $\begin{array}{l}\text { Sample } \\
\text { Location }\end{array}$ & Date & $\begin{array}{c}\text { Concentrations } \\
(\mathrm{pCi} / \mathrm{g})\end{array}$ & $\begin{array}{l}\text { Uncertainty } \\
(\mathrm{pCi} / \mathrm{g})\end{array}$ & $\begin{array}{c}\mathrm{MDA} \\
(\mathrm{pCi} / \mathrm{g})\end{array}$ & Flag \\
\hline U-235 & $\mathrm{L} 1-76$ & 2010 & 0.2612 & 0.0462 & 0.1386 & - \\
\hline U-235 & L2-76 & 2010 & 0.8529 & 0.174 & 0.522 & - \\
\hline U-235 & L3-76 & 2010 & 0.2926 & 0.0468 & 0.1404 & - \\
\hline $\mathrm{U}-235$ & L4-76 & 2010 & 0.9235 & 0.276 & 0.828 & - \\
\hline U-235 & L5-76 & 2010 & 1.444 & 0.217 & 0.651 & - \\
\hline U-235 & TSF-6 & 2010 & 1.139 & 0.233 & 0.699 & - \\
\hline U-235 & TSF-7 & 2010 & 1.15 & 0.256 & 0.768 & - \\
\hline U-235 & TSF-8 & 2010 & 0.7747 & 0.256 & 0.768 & - \\
\hline U-235 & TSF-9 & 2010 & 1.088 & 0.212 & 0.636 & - \\
\hline U-235 & WRRTF-6 & 2010 & 0.2521 & 0.0457 & 0.1371 & - \\
\hline $\mathrm{U}-235$ & WRRTF-7 & 2010 & 0.2422 & 0.05 & 0.1512 & - \\
\hline U-235 & WRRTF-8 & 2010 & 0.8924 & 0.183 & 0.549 & 一 \\
\hline $\mathrm{U}-235$ & IET-6 & 2011 & 0.02033 & 0.16 & 0.48 & $\mathrm{U}$ \\
\hline $\mathrm{U}-235$ & IET-7 & 2011 & 0.05071 & 0.14 & 0.426 & $\mathrm{U}$ \\
\hline $\mathrm{U}-235$ & IET-8 & 2011 & -0.07102 & 0.127 & 0.381 & $\mathrm{U}$ \\
\hline $\mathrm{U}-235$ & IET-9 & 2011 & -0.06927 & 0.14 & 0.42 & $\mathrm{U}$ \\
\hline $\mathrm{U}-235$ & L2-76 & 2011 & -0.009221 & 0.151 & 0.453 & $\mathrm{U}$ \\
\hline $\mathrm{U}-235$ & L3-76 & 2011 & -0.1428 & 0.15 & 0.45 & $\mathrm{U}$ \\
\hline U-235 & L4-76 & 2011 & 0.1672 & 0.184 & 0.552 & $\mathrm{U}$ \\
\hline U-235 & L5-76 & 2011 & 0.2332 & 0.193 & 0.579 & $\mathrm{U}$ \\
\hline U-235 & TSF-1 & 2011 & 0.01161 & 0.1 & 0.429 & $\mathrm{U}$ \\
\hline U-235 & TSF-6 & 2011 & -0.1635 & 0.17 & 0.51 & $\mathrm{U}$ \\
\hline U-235 & TSF-7 & 2011 & 0.1027 & 0.178 & 0.534 & $\mathrm{U}$ \\
\hline U-235 & TSF-8 & 2011 & 0.2009 & 0.195 & 0.585 & $\mathrm{U}$ \\
\hline $\mathrm{U}-235$ & TSF-9 & 2011 & 0.1048 & 0.151 & 0.453 & $\mathrm{U}$ \\
\hline $\mathrm{U}-235$ & WRRTF-5 & 2011 & 0.009377 & 0.159 & 0.477 & $\mathrm{U}$ \\
\hline U-235 & WRRTF-6 & 2011 & 0.131 & 0.173 & 0.519 & $\mathrm{U}$ \\
\hline $\mathrm{U}-235$ & WRRTF-7 & 2011 & -0.06129 & 0.177 & 0.531 & $\mathrm{U}$ \\
\hline $\mathrm{U}-235$ & WRRTF-8 & 2011 & 0.1379 & 0.162 & 0.486 & $\mathrm{U}$ \\
\hline U-235 & L1-76 & 2012 & 0.0707 & 0.113 & 0.339 & $\mathrm{U}$ \\
\hline U-235 & WRRTF-5 & 2012 & 0.1557 & 0.129 & 0.387 & $\mathrm{U}$ \\
\hline U-235 & WRRTF-7 & 2012 & 0.06636 & 0.128 & 0.384 & $\mathrm{U}$ \\
\hline U-235 & TSF-6 & 2013 & 0.1144 & 0.0577 & 0.173 & $\mathrm{U}$ \\
\hline U-235 & $\begin{array}{c}2 \text { INCH AIR } \\
- \text { SMC }\end{array}$ & 2014 & 0.06871 & 0.12 & 0.36 & $\mathrm{U}$ \\
\hline U-235 & TSF-1 & 2014 & -0.09704 & 0.198 & 0.594 & $\mathrm{U}$ \\
\hline U-235 & TSF-6 & 2014 & -0.05248 & 0.148 & 0.444 & $\mathrm{U}$ \\
\hline
\end{tabular}


Table C-6. (continued).

\begin{tabular}{|c|c|c|c|c|c|c|}
\hline Radionuclide & $\begin{array}{c}\text { Sample } \\
\text { Location }\end{array}$ & Date & $\begin{array}{c}\text { Concentrations } \\
(\mathrm{pCi} / \mathrm{g})\end{array}$ & $\begin{array}{l}\text { Uncertainty } \\
(\mathrm{pCi} / \mathrm{g})\end{array}$ & $\begin{array}{c}\mathrm{MDA} \\
(\mathrm{pCi} / \mathrm{g})\end{array}$ & Flag \\
\hline U-235 & TSF-7 & 2014 & -0.001988 & 0.126 & 0.378 & $\mathrm{U}$ \\
\hline U-235 & TSF-8 & 2014 & -0.01483 & 0.15 & 0.441 & $\mathrm{U}$ \\
\hline U-235 & TSF-9 & 2014 & -0.06305 & 0.196 & 0.588 & $\mathrm{U}$ \\
\hline U-235 & WRRTF-6 & 2014 & -0.1091 & 0.135 & 0.405 & $\mathrm{U}$ \\
\hline $\mathrm{U}-238$ & IET-6 & 2006 & 45.64 & 7.45 & 22.35 & - \\
\hline U-238 & IET-7 & 2006 & 31.34 & 3.995 & 11.985 & - \\
\hline $\mathrm{U}-238$ & IET-8 & 2006 & 15.87 & 2.425 & 7.275 & - \\
\hline $\mathrm{U}-238$ & IET-9 & 2006 & 28.89 & 4.055 & 12.165 & - \\
\hline $\mathrm{U}-238$ & L1-76 & 2006 & 33.75 & 4.41 & 13.23 & - \\
\hline $\mathrm{U}-238$ & L2-76 & 2006 & 20.3 & 3.24 & 9.72 & - \\
\hline $\mathrm{U}-238$ & L3-76 & 2006 & 26.54 & 3.53 & 10.59 & 一 \\
\hline $\mathrm{U}-238$ & L4-76 & 2006 & -0.1236 & 1.12 & 3.36 & $\mathrm{U}$ \\
\hline $\mathrm{U}-238$ & L5-76 & 2006 & 5.717 & 1.13 & 3.39 & - \\
\hline $\mathrm{U}-238$ & TSF-1 & 2006 & -0.1868 & 1.19 & 3.57 & $\mathrm{U}$ \\
\hline U-238 & TSF-6 & 2006 & 3.65 & 1.14 & 3.42 & - \\
\hline U-238 & TSF-7 & 2006 & 28.73 & 3.665 & 10.995 & - \\
\hline $\mathrm{U}-238$ & TSF-8 & 2006 & 35.01 & 4.465 & 13.395 & - \\
\hline $\mathrm{U}-238$ & TSF-9 & 2006 & 0.2955 & 1.205 & 3.615 & $\mathrm{U}$ \\
\hline U-238 & WRRTF-5 & 2006 & 1.126 & 0.99 & 2.97 & $\mathrm{U}$ \\
\hline U-238 & WRRTF-6 & 2006 & 26.33 & 3.42 & 10.26 & - \\
\hline U-238 & WRRTF-7 & 2006 & 31.55 & 4.715 & 14.145 & 一 \\
\hline U-238 & WRRTF-8 & 2006 & 16.69 & 2.91 & 8.73 & - \\
\hline U-238 & IET-6 & 2007 & 0.06112 & 3.45 & 10.35 & $\mathrm{U}$ \\
\hline U-238 & IET-7 & 2007 & 0.001383 & 4.12 & 12.36 & $\mathrm{U}$ \\
\hline U-238 & IET-8 & 2007 & 111.4 & 4.76 & 14.28 & - \\
\hline U-238 & IET-9 & 2007 & 0.1675 & 4.88 & 14.64 & $\mathrm{U}$ \\
\hline U-238 & L1-76 & 2007 & 0.2617 & 4.79 & 14.37 & $\mathrm{U}$ \\
\hline U-238 & L2-76 & 2007 & 0.05993 & 4.98 & 14.94 & $\mathrm{U}$ \\
\hline U-238 & L3-76 & 2007 & 0.001831 & 5.42 & 16.26 & $\mathrm{U}$ \\
\hline U-238 & L4-76 & 2007 & 0.08309 & 5.54 & 16.62 & $\mathrm{U}$ \\
\hline U-238 & L5-76 & 2007 & 0.0002811 & 3.21 & 9.63 & $\mathrm{U}$ \\
\hline U-238 & TSF-1 & 2007 & 0 & 3.17 & 9.51 & $\mathrm{U}$ \\
\hline U-238 & TSF-6 & 2007 & 0 & 3.16 & 9.48 & $\mathrm{U}$ \\
\hline U-238 & TSF-7 & 2007 & 0.1446 & 5.15 & 15.45 & $\mathrm{U}$ \\
\hline U-238 & TSF-8 & 2007 & 0 & 10.7 & 32.1 & $\mathrm{U}$ \\
\hline U-238 & TSF-9 & 2007 & 0.4285 & 3.14 & 9.42 & $\mathrm{U}$ \\
\hline U-238 & WRRTF-5 & 2007 & 0 & 2.94 & 8.82 & $\mathrm{U}$ \\
\hline
\end{tabular}


Table C-6. (continued).

\begin{tabular}{|c|c|c|c|c|c|c|}
\hline Radionuclide & $\begin{array}{l}\text { Sample } \\
\text { Location }\end{array}$ & Date & $\begin{array}{l}\text { Concentrations } \\
(\mathrm{pCi} / \mathrm{g})\end{array}$ & $\begin{array}{l}\text { Uncertainty } \\
(\mathrm{pCi} / \mathrm{g})\end{array}$ & $\begin{array}{c}\text { MDA } \\
(\mathrm{pCi} / \mathrm{g})\end{array}$ & Flag \\
\hline U-238 & WRRTF-6 & 2007 & 0 & 2.93 & 8.79 & $\mathrm{U}$ \\
\hline $\mathrm{U}-238$ & WRRTF-7 & 2007 & 0.058 & 5.42 & 16.26 & $\mathrm{U}$ \\
\hline $\mathrm{U}-238$ & WRRTF-8 & 2007 & 0.179 & 5.25 & 15.75 & $\mathrm{U}$ \\
\hline $\mathrm{U}-238$ & $\mathrm{~L} 1-76$ & 2009 & 86.8 & 5.45 & 16.35 & - \\
\hline $\mathrm{U}-238$ & IET-6 & 2010 & 57.68 & 23.9 & 71.7 & $\mathrm{U}$ \\
\hline $\mathrm{U}-238$ & IET-9 & 2010 & 33.25 & 18.8 & 56.4 & $\mathrm{U}$ \\
\hline $\mathrm{U}-238$ & L4-76 & 2010 & 23.94 & 21.2 & 63.6 & $\mathrm{U}$ \\
\hline $\mathrm{U}-238$ & WRRTF-6 & 2010 & 2.28 & 2.1 & 6.3 & $\mathrm{U}$ \\
\hline $\mathrm{U}-238$ & IET-6 & 2011 & 1.146 & 0.752 & 2.256 & $\mathrm{U}$ \\
\hline $\mathrm{U}-238$ & IET-7 & 2011 & 0.5476 & 0.645 & 1.935 & $\mathrm{U}$ \\
\hline $\mathrm{U}-238$ & IET-8 & 2011 & 0.5605 & 0.655 & 1.965 & $\mathrm{U}$ \\
\hline U-238 & IET-9 & 2011 & 1.63 & 0.801 & 2.403 & $\mathrm{U}$ \\
\hline $\mathrm{U}-238$ & L2-76 & 2011 & 0.5252 & 0.712 & 2.136 & $\mathrm{U}$ \\
\hline $\mathrm{U}-238$ & L3-76 & 2011 & 0.6755 & 0.617 & 1.851 & $\mathrm{U}$ \\
\hline U-238 & L4-76 & 2011 & 1.374 & 0.922 & 2.766 & $\mathrm{U}$ \\
\hline $\mathrm{U}-238$ & L5-76 & 2011 & 3.019 & 0.918 & 2.754 & - \\
\hline $\mathrm{U}-238$ & TSF-1 & 2011 & 2.358 & 0.669 & 2.007 & - \\
\hline U-238 & TSF-6 & 2011 & 1.187 & 0.832 & 2.496 & $\mathrm{U}$ \\
\hline U-238 & TSF-7 & 2011 & 1.374 & 0.969 & 2.907 & $\mathrm{U}$ \\
\hline U-238 & TSF-8 & 2011 & 1.713 & 0.961 & 2.883 & $\mathrm{U}$ \\
\hline U-238 & TSF-9 & 2011 & 1.714 & 0.553 & 1.659 & - \\
\hline $\mathrm{U}-238$ & WRRTF-5 & 2011 & 0.9822 & 0.724 & 2.172 & $\mathrm{U}$ \\
\hline $\mathrm{U}-238$ & WRRTF-6 & 2011 & 1.193 & 0.92 & 2.76 & $\mathrm{U}$ \\
\hline U-238 & WRRTF-7 & 2011 & -0.5428 & 0.75 & 2.25 & $\mathrm{U}$ \\
\hline $\mathrm{U}-238$ & WRRTF-8 & 2011 & 1.055 & 0.567 & 1.701 & $\mathrm{U}$ \\
\hline $\mathrm{U}-238$ & L1-76 & 2012 & 0.6917 & 0.619 & 1.857 & $\mathrm{U}$ \\
\hline $\mathrm{U}-238$ & WRRTF-5 & 2012 & 4.332 & 1.01 & 3.03 & - \\
\hline $\mathrm{U}-238$ & WRRTF-7 & 2012 & 1.16 & 0.774 & 2.322 & $\mathrm{U}$ \\
\hline $\mathrm{U}-238$ & TSF-6 & 2013 & 3.329 & 1.913 & 5.74 & $\mathrm{U}$ \\
\hline U-238 & $\begin{array}{c}2 \text { INCH AIR } \\
- \text { SMC }\end{array}$ & 2014 & -0.3254 & 0.456 & 1.368 & $\mathrm{U}$ \\
\hline U-238 & TSF-1 & 2014 & 1.267 & 0.91 & 2.73 & $\mathrm{U}$ \\
\hline U-238 & TSF-6 & 2014 & 0.9815 & 0.733 & 2.199 & $\mathrm{U}$ \\
\hline U-238 & TSF-7 & 2014 & 0.7491 & 0.683 & 2.049 & $\mathrm{U}$ \\
\hline U-238 & TSF-8 & 2014 & 1.607 & 0.835 & 2.505 & $\mathrm{U}$ \\
\hline U-238 & TSF-9 & 2014 & 1.441 & 0.912 & 2.736 & $\mathrm{U}$ \\
\hline U-238 & WRRTF-6 & 2014 & 1.461 & 0.786 & 2.358 & $\mathrm{U}$ \\
\hline
\end{tabular}


Table C-7. Radionuclides concentrations in surface soils associated with Atomic City.

\begin{tabular}{|c|c|c|c|c|c|}
\hline Radionuclide & Year & $\begin{array}{l}\text { Concentration } \\
(\mathrm{pCi} / \mathrm{g})\end{array}$ & $\begin{array}{l}\text { Uncertainty } \\
(\mathrm{pCi} / \mathrm{g})\end{array}$ & $\begin{array}{l}\text { MDA } \\
(\mathrm{pCi} / \mathrm{g})\end{array}$ & Flag \\
\hline Am-241 & 1994 & 0.006 & 0.002 & 0.006 & - \\
\hline Am-241 & 1996 & 0.009 & 0.003 & 0.009 & - \\
\hline Am-241 & 1998 & 0.02 & 0.0045 & 0.0135 & - \\
\hline Am-241 & 2000 & 0.00569 & 0.00075 & 0.00225 & - \\
\hline Am-241 & 2002 & 0.00569 & 0.00075 & 0.00225 & - \\
\hline Am-241 & 2004 & 0.0062 & 0.0062 & 0.0186 & - \\
\hline Am-241 & 2006 & 0.00117 & 0.0072 & 0.0216 & $\mathrm{U}$ \\
\hline Am-241 & 2008 & 0.004645 & 0.01694 & 0.05082 & $\mathrm{U}$ \\
\hline Am-241 & 2010 & -0.00904 & 0.00524 & 0.01572 & $\mathrm{U}$ \\
\hline Am-241 & 2012 & 0.00396 & 0.00709 & 0.02127 & $\mathrm{U}$ \\
\hline Am-241 & 2014 & 0.0133 & 0.00843 & 0.02529 & - \\
\hline Cs-137 & 1994 & 0.81 & 0.0205 & 0.0615 & - \\
\hline Cs-137 & 1996 & 0.44 & 0.01 & 0.03 & - \\
\hline Cs-137 & 1998 & 0.3 & 0.045 & 0.135 & - \\
\hline Cs-137 & 2000 & 0.613 & 0.0131 & 0.0393 & - \\
\hline Cs-137 & 2000 & 0.345 & 0.00852 & 0.02556 & - \\
\hline Cs-137 & 2002 & 0.544 & 0.0178 & 0.0534 & - \\
\hline Cs-137 & 2002 & 0.535 & 0.0176 & 0.0528 & - \\
\hline Cs-137 & 2002 & 0.118 & 0.00755 & 0.02265 & - \\
\hline Cs-137 & 2002 & 0.119 & 0.00438 & 0.01314 & - \\
\hline Cs-137 & 2004 & 0.557 & 0.0122 & 0.0366 & - \\
\hline Cs-137 & 2004 & 0.161 & 0.00797 & 0.02391 & - \\
\hline Cs-137 & 2006 & 0.367 & 0.0116 & 0.0348 & - \\
\hline Cs- 137 & 2006 & -0.575 & 0.271 & 0.813 & $\mathrm{U}$ \\
\hline Cs-137 & 2008 & 0.5249 & 0.0158 & 0.0474 & - \\
\hline Cs-137 & 2008 & 0.1741 & 0.0048 & 0.0144 & - \\
\hline Cs-137 & 2010 & 0.329 & 0.00859 & 0.02577 & - \\
\hline Cs-137 & 2010 & 0.0944 & 0.00276 & 0.00828 & - \\
\hline Cs-137 & 2012 & 0.447 & 0.0257 & 0.0771 & - \\
\hline Cs-137 & 2012 & 0.0928 & 0.00777 & 0.02331 & - \\
\hline Cs-137 & 2014 & 0.323 & 0.0186 & 0.0558 & - \\
\hline Cs-137 & 2014 & 0.0788 & 0.00575 & 0.01725 & - \\
\hline $\mathrm{Pu}-238$ & 1994 & 0.004 & 0.002 & 0.006 & - \\
\hline $\mathrm{Pu}-238$ & 1996 & 0 & 0.001 & 0.003 & $\mathrm{U}$ \\
\hline $\mathrm{Pu}-238$ & 1998 & 0.003 & 0.003 & 0.009 & - \\
\hline $\mathrm{Pu}-238$ & 2000 & 0.00235 & 0.00039 & 0.00117 & - \\
\hline $\mathrm{Pu}-238$ & 2002 & 0.00235 & 0.00039 & 0.00117 & - \\
\hline
\end{tabular}


Table C-7. (continued).

\begin{tabular}{|c|c|c|c|c|c|}
\hline Radionuclide & Year & $\begin{array}{l}\text { Concentration } \\
(\mathrm{pCi} / \mathrm{g})\end{array}$ & $\begin{array}{l}\text { Uncertainty } \\
(\mathrm{pCi} / \mathrm{g})\end{array}$ & $\begin{array}{l}\text { MDA } \\
(\mathrm{pCi} / \mathrm{g})\end{array}$ & Flag \\
\hline $\mathrm{Pu}-238$ & 2004 & 0.00211 & 0.000455 & 0.001365 & 一 \\
\hline $\mathrm{Pu}-238$ & 2006 & 0.014 & 0.0102 & 0.0306 & 一 \\
\hline $\mathrm{Pu}-238$ & 2008 & 0.001644 & 0.005995 & 0.017985 & $\mathrm{U}$ \\
\hline $\mathrm{Pu}-238$ & 2010 & 0.00452 & 0.0276 & 0.0828 & $\mathrm{U}$ \\
\hline $\mathrm{Pu}-238$ & 2012 & 0.009 & 0.00374 & 0.01122 & - \\
\hline $\mathrm{Pu}-238$ & 2014 & 0.0092 & 0.00456 & 0.01368 & - \\
\hline $\mathrm{Pu}-239 / 240$ & 1994 & 0.02 & 0.003 & 0.009 & - \\
\hline $\mathrm{Pu}-239 / 240$ & 1996 & 0.02 & 0.004 & 0.012 & - \\
\hline $\mathrm{Pu}-239 / 240$ & 1998 & 0.02 & 0.005 & 0.015 & - \\
\hline $\mathrm{Pu}-239 / 240$ & 2000 & 0.0174 & 0.0015 & 0.0045 & - \\
\hline $\mathrm{Pu}-239 / 240$ & 2002 & 0.0174 & 0.0015 & 0.0045 & 一 \\
\hline $\mathrm{Pu}-239 / 240$ & 2004 & 0.0204 & 0.0018 & 0.0054 & - \\
\hline $\mathrm{Pu}-239 / 240$ & 2006 & 0.011 & 0.0106 & 0.0318 & - \\
\hline $\mathrm{Pu}-239 / 240$ & 2008 & 0.04095 & 0.016635 & 0.049905 & 一 \\
\hline $\mathrm{Pu}-239 / 240$ & 2010 & -0.0126 & 0.0217 & 0.0651 & $\mathrm{U}$ \\
\hline $\mathrm{Pu}-239 / 240$ & 2012 & 0.0315 & 0.00759 & 0.02277 & 一 \\
\hline $\mathrm{Pu}-239 / 240$ & 2014 & 0.0349 & 0.00845 & 0.02535 & - \\
\hline Sr-90 & 1994 & 0.42 & 0.06 & 0.18 & - \\
\hline Sr-90 & 1996 & 0.22 & 0.025 & 0.075 & - \\
\hline Sr-90 & 1998 & 0.3 & 0.045 & 0.135 & 一 \\
\hline Sr-90 & 2000 & 0.215 & 0.36 & 1.08 & $\mathrm{U}$ \\
\hline Sr-90 & 2002 & 0.215 & 0.36 & 1.08 & $\mathrm{U}$ \\
\hline Sr-90 & 2004 & 0.199 & 0.034 & 0.102 & - \\
\hline Sr-90 & 2006 & 0.0928 & 0.0108 & 0.0324 & - \\
\hline Sr-90 & 2008 & 0.241 & 0.02345 & 0.07035 & 一 \\
\hline Sr-90 & 2010 & 0.0453 & 0.0114 & 0.0342 & 一 \\
\hline Sr-90 & 2012 & 0.141 & 0.0381 & 0.1143 & - \\
\hline Sr-90 & 2014 & 0.364 & 0.0539 & 0.1617 & - \\
\hline
\end{tabular}

Table C-8. Radionuclides concentrations in surface soils associated with Blackfoot.

\begin{tabular}{|c|c|c|c|c|c|}
\hline Radionuclide & Year & $\begin{array}{c}\text { Concentration } \\
(\mathrm{pCi} / \mathrm{g})\end{array}$ & $\begin{array}{c}\text { Uncertainty } \\
(\mathrm{pCi} / \mathrm{g})\end{array}$ & $\begin{array}{c}\text { MDA } \\
(\mathrm{pCi} / \mathrm{g})\end{array}$ & Flag \\
\hline Am-241 & 1994 & 0.006 & 0.002 & 0.006 & - \\
\hline Am-241 & 1996 & 0.01 & 0.0035 & 0.0105 & - \\
\hline Am-241 & 1998 & 0.007 & 0.0025 & 0.0075 & - \\
\hline Am-241 & 2000 & 0.00663 & 0.00088 & 0.00264 & - \\
\hline Am-241 & 2002 & 0.00663 & 0.00088 & 0.00264 & - \\
\hline
\end{tabular}


Table C-8. (continued).

\begin{tabular}{|c|c|c|c|c|c|}
\hline Radionuclide & Year & $\begin{array}{c}\text { Concentration } \\
(\mathrm{pCi} / \mathrm{g})\end{array}$ & $\begin{array}{l}\text { Uncertainty } \\
(\mathrm{pCi} / \mathrm{g})\end{array}$ & $\begin{array}{l}\text { MDA } \\
(\mathrm{pCi} / \mathrm{g})\end{array}$ & Flag \\
\hline Am-241 & 2002 & 0.00611 & 0.00072 & 0.00216 & 一 \\
\hline Am-241 & 2004 & 0.00423 & 0.00064 & 0.00192 & 一 \\
\hline Am-241 & 2006 & 0.0209 & 0.00814 & 0.02442 & - \\
\hline Am-241 & 2008 & -0.006237 & 0.004416 & 0.01325 & $\mathrm{U}$ \\
\hline Am-241 & 2010 & 0.00418 & 0.0108 & 0.0324 & $\mathrm{U}$ \\
\hline Am-241 & 2012 & 0.0158 & 0.0104 & 0.0312 & - \\
\hline Am-241 & 2014 & 0.0114 & 0.0102 & 0.0306 & - \\
\hline Cs-137 & 1994 & 1.01 & 0.01 & 0.03 & - \\
\hline Cs-137 & 1996 & 1.3 & 0.045 & 0.135 & - \\
\hline Cs-137 & 1998 & 0.6 & 0.015 & 0.045 & - \\
\hline Cs-137 & 2000 & 0.649 & 0.0174 & 0.0522 & 一 \\
\hline Cs-137 & 2002 & 0.61 & 0.0154 & 0.0462 & - \\
\hline Cs-137 & 2002 & 0.593 & 0.0127 & 0.0381 & - \\
\hline Cs-137 & 2002 & 0.3240 & 0.0109 & 0.0327 & - \\
\hline Cs-137 & 2002 & 0.3460 & 0.0088 & 0.0264 & - \\
\hline Cs-137 & 2004 & 0.229 & 0.0064 & 0.0192 & 一 \\
\hline Cs-137 & 2004 & 0.194 & 0.00524 & 0.01572 & - \\
\hline Cs-137 & 2006 & 0.226 & 0.00583 & 0.01749 & - \\
\hline Cs-137 & 2006 & 0.27 & 0.00991 & 0.02973 & - \\
\hline Cs- 137 & 2008 & 0.6020 & 0.0141 & 0.0423 & - \\
\hline Cs-137 & 2008 & 0.2082 & 0.0052 & 0.0155 & - \\
\hline Cs-137 & 2010 & 0.369 & 0.00937 & 0.02811 & - \\
\hline Cs-137 & 2010 & 0.139 & 0.00385 & 0.01155 & - \\
\hline Cs-137 & 2012 & 0.0522 & 0.00587 & 0.01761 & - \\
\hline Cs-137 & 2012 & 0.239 & 0.0144 & 0.0432 & 一 \\
\hline Cs-137 & 2014 & 0.167 & 0.0108 & 0.0324 & 一 \\
\hline Cs-137 & 2014 & 0.0347 & 0.00298 & 0.00894 & - \\
\hline $\mathrm{Pu}-238$ & 1994 & 0.002 & 0.0015 & 0.0045 & - \\
\hline $\mathrm{Pu}-238$ & 1996 & 0.002 & 0.0025 & 0.0075 & $\mathrm{U}$ \\
\hline $\mathrm{Pu}-238$ & 1998 & 0.01 & 0.005 & 0.015 & - \\
\hline $\mathrm{Pu}-238$ & 2000 & 0.0023 & 0.00043 & 0.00129 & - \\
\hline $\mathrm{Pu}-238$ & 2002 & 0.019 & 0.0017 & 0.0051 & - \\
\hline $\mathrm{Pu}-238$ & 2002 & 0.0153 & 0.0015 & 0.0045 & - \\
\hline $\mathrm{Pu}-238$ & 2004 & 0.00984 & 0.0017 & 0.0051 & - \\
\hline $\mathrm{Pu}-238$ & 2006 & 0.154 & 0.0294 & 0.0882 & 一 \\
\hline $\mathrm{Pu}-238$ & 2008 & -0.005634 & 0.0088 & 0.0264 & $\mathrm{U}$ \\
\hline $\mathrm{Pu}-238$ & 2010 & -0.0373 & 0.0113 & 0.0339 & $\mathrm{U}$ \\
\hline
\end{tabular}


Table C-8. (continued).

\begin{tabular}{|c|l|l|l|l|c|}
\hline Radionuclide & Year & $\begin{array}{c}\text { Concentration } \\
(\mathrm{pCi} / \mathrm{g})\end{array}$ & $\begin{array}{c}\text { Uncertainty } \\
(\mathrm{pCi} / \mathrm{g})\end{array}$ & $\begin{array}{c}\text { MDA } \\
(\mathrm{pCi} / \mathrm{g})\end{array}$ & Flag \\
\hline $\mathrm{Pu}-238$ & 2012 & 0.0118 & 0.00405 & 0.01215 & - \\
\hline $\mathrm{Pu}-238$ & 2014 & 0 & 0.0097 & 0.0291 & $\mathrm{U}$ \\
\hline $\mathrm{Pu}-239 / 240$ & 1994 & 0.03 & 0.003 & 0.009 & - \\
\hline $\mathrm{Pu}-239 / 240$ & 1996 & 0.03 & 0.01 & 0.03 & - \\
\hline $\mathrm{Pu}-239 / 240$ & 1998 & 0.03 & 0.005 & 0.015 & - \\
\hline $\mathrm{Pu}-239 / 240$ & 2000 & 0.019 & 0.0017 & 0.0051 & - \\
\hline $\mathrm{Pu}-239 / 240$ & 2002 & 0.0023 & 0.00043 & 0.00129 & - \\
\hline $\mathrm{Pu}-239 / 240$ & 2002 & 0.0019 & 0.0004 & 0.0012 & - \\
\hline $\mathrm{Pu}-239 / 240$ & 2004 & 0.00842 & 0.0016 & 0.0048 & - \\
\hline $\mathrm{Pu}-239 / 240$ & 2006 & 0.0885 & 0.0198 & 0.0594 & - \\
\hline $\mathrm{Pu}-239 / 240$ & 2008 & 0.01829 & 0.010185 & 0.0306 & - \\
\hline $\mathrm{Pu}-239 / 240$ & 2010 & 0.00936 & 0.015 & 0.045 & $\mathrm{U}$ \\
\hline $\mathrm{Pu}-239 / 240$ & 2012 & 0.0144 & 0.00486 & 0.01458 & - \\
\hline $\mathrm{Pu}-239 / 240$ & 2014 & 0.0153 & 0.00819 & 0.02457 & - \\
\hline $\mathrm{Sr}-90$ & 1994 & 0.27 & 0.05 & 0.15 & - \\
\hline $\mathrm{Sr}-90$ & 1996 & 0.21 & 0.025 & 0.075 & - \\
\hline $\mathrm{Sr}-90$ & 1998 & 0.11 & 0.3 & 0.9 & - \\
\hline $\mathrm{Sr}-90$ & 2000 & 0.0979 & 0.03 & 0.09 & - \\
\hline $\mathrm{Sr}-90$ & 2002 & 0.0979 & 0.03 & 0.09 & - \\
\hline $\mathrm{Sr}-90$ & 2002 & 0.0634 & 0.022 & 0.066 & - \\
\hline $\mathrm{Sr}-90$ & 2004 & 0.0625 & 0.019 & 0.057 & - \\
\hline $\mathrm{Sr}-90$ & 2006 & 0.0115 & 0.0095 & 0.0285 & - \\
\hline $\mathrm{Sr}-90$ & 2008 & 0.000718 & 0.00635 & 0.01905 & - \\
\hline $\mathrm{Sr}-90$ & 2010 & 0.0126 & 0.0121 & 0.0363 & - \\
\hline $\mathrm{Sr}-90$ & 2012 & 0.0977 & 0.0366 & 0.1098 & - \\
\hline $\mathrm{Sr}-90$ & 2014 & 0.125 & 0.0391 & 0.1173 & - \\
\hline & & & & - & - \\
\hline & & & & - \\
\hline
\end{tabular}

Table C-9. Radionuclides concentrations in surface soils associated with Butte City.

\begin{tabular}{|c|c|c|c|c|c|}
\hline Radionuclide & Year & $\begin{array}{c}\text { Concentration } \\
(\mathrm{pCi} / \mathrm{g})\end{array}$ & $\begin{array}{c}\text { Uncertainty } \\
(\mathrm{pCi} / \mathrm{g})\end{array}$ & $\begin{array}{c}\text { MDA } \\
(\mathrm{pCi} / \mathrm{g})\end{array}$ & Flag \\
\hline Am-241 & 1994 & 0.005 & 0.002 & 0.006 & - \\
\hline Am-241 & 1996 & 0.008 & 0.0035 & 0.0105 & - \\
\hline Am-241 & 1998 & 0.002 & 0.0015 & 0.0045 & - \\
\hline Am-241 & 2000 & 0.00725 & 0.0009 & 0.0027 & - \\
\hline Am-241 & 2002 & 0.00725 & 0.0009 & 0.0027 & - \\
\hline Am-241 & 2004 & 0.0054 & 0.0007 & 0.0021 & - \\
\hline Am-241 & 2006 & 0.00463 & 0.00858 & 0.0257 & U \\
\hline
\end{tabular}


Table C-9. (continued).

\begin{tabular}{|c|c|c|c|c|c|}
\hline Radionuclide & Year & $\begin{array}{c}\text { Concentration } \\
(\mathrm{pCi} / \mathrm{g})\end{array}$ & $\begin{array}{c}\text { Uncertainty } \\
(\mathrm{pCi} / \mathrm{g})\end{array}$ & $\begin{array}{c}\text { MDA } \\
(\mathrm{pCi} / \mathrm{g})\end{array}$ & Flag \\
\hline Am-241 & 2008 & 0.09415 & 0.044325 & 0.1330 & - \\
\hline Am-241 & 2010 & 0.0184 & 0.0277 & 0.0831 & $\mathrm{U}$ \\
\hline Am-241 & 2012 & 0.0126 & 0.00816 & 0.0245 & - \\
\hline Am-241 & 2014 & 0.0144 & 0.00988 & 0.0296 & - \\
\hline Cs-137 & 1994 & 0.8 & 0.02 & 0.06 & - \\
\hline Cs-137 & 1996 & 0.96 & 0.035 & 0.105 & - \\
\hline Cs-137 & 1998 & 0.2 & 0.045 & 0.135 & - \\
\hline Cs-137 & 2000 & 0.815 & 0.0212 & 0.0636 & - \\
\hline Cs-137 & 2000 & 0.162 & 0.00813 & 0.02439 & - \\
\hline Cs-137 & 2002 & 0.651 & 0.0179 & 0.0537 & - \\
\hline Cs-137 & 2002 & 0.603 & 0.0171 & 0.0513 & - \\
\hline Cs-137 & 2002 & 0.2140 & 0.0085 & 0.0254 & - \\
\hline Cs-137 & 2002 & 0.2240 & 0.0067 & 0.0202 & - \\
\hline Cs-137 & 2004 & 0.466 & 0.0104 & 0.0312 & - \\
\hline Cs-137 & 2004 & 0.143 & 0.00467 & 0.01401 & - \\
\hline Cs-137 & 2006 & 0.306 & 0.00791 & 0.02373 & - \\
\hline Cs-137 & 2006 & 0.0894 & 0.00283 & 0.00849 & - \\
\hline Cs-137 & 2008 & 0.06976 & 0.00586 & 0.01759 & - \\
\hline Cs-137 & 2008 & 0.35190 & 0.00812 & 0.02435 & - \\
\hline Cs-137 & 2010 & 0.4960 & 0.0108 & 0.0324 & - \\
\hline Cs-137 & 2010 & 0.1790 & 0.0048 & 0.0143 & - \\
\hline Cs-137 & 2012 & 0.444 & 0.0255 & 0.0765 & - \\
\hline Cs-137 & 2012 & 0.086 & 0.00599 & 0.01797 & - \\
\hline Cs-137 & 2014 & 0.476 & 0.027 & 0.081 & - \\
\hline Cs-137 & 2014 & 0.102 & 0.00692 & 0.02076 & - \\
\hline $\mathrm{Pu}-238$ & 1994 & 0.006 & 0.002 & 0.006 & - \\
\hline $\mathrm{Pu}-238$ & 1996 & 0.003 & 0.0015 & 0.0045 & - \\
\hline $\mathrm{Pu}-238$ & 1998 & 0.006 & 0.003 & 0.009 & - \\
\hline $\mathrm{Pu}-238$ & 2000 & 0.00261 & 0.00048 & 0.00144 & - \\
\hline $\mathrm{Pu}-238$ & 2002 & 0.0192 & 0.0018 & 0.0054 & - \\
\hline $\mathrm{Pu}-238$ & 2004 & 0.00166 & 0.00041 & 0.00123 & - \\
\hline $\mathrm{Pu}-238$ & 2006 & -0.0054 & 0.0118 & 0.0354 & $\mathrm{U}$ \\
\hline $\mathrm{Pu}-238$ & 2008 & 0.01623 & 0.01317 & 0.03951 & - \\
\hline $\mathrm{Pu}-238$ & 2010 & 0.0166 & 0.0118 & 0.0354 & - \\
\hline $\mathrm{Pu}-238$ & 2012 & 0.00234 & 0.00287 & 0.00861 & $\mathrm{U}$ \\
\hline $\mathrm{Pu}-238$ & 2014 & -0.00578 & 0.00709 & 0.02127 & $\mathrm{U}$ \\
\hline $\mathrm{Pu}-239 / 240$ & 1994 & 0.02 & 0.003 & 0.009 & - \\
\hline
\end{tabular}


Table C-9. (continued).

\begin{tabular}{|c|l|l|l|l|c|}
\hline Radionuclide & Year & $\begin{array}{c}\text { Concentration } \\
(\mathrm{pCi} / \mathrm{g})\end{array}$ & $\begin{array}{c}\text { Uncertainty } \\
(\mathrm{pCi} / \mathrm{g})\end{array}$ & $\begin{array}{c}\text { MDA } \\
(\mathrm{pCi} / \mathrm{g})\end{array}$ & Flag \\
\hline $\mathrm{Pu}-239 / 240$ & 1996 & 0.03 & 0.01 & 0.03 & - \\
\hline $\mathrm{Pu}-239 / 240$ & 1998 & 0.02 & 0.005 & 0.015 & - \\
\hline $\mathrm{Pu}-239 / 240$ & 2000 & 0.0192 & 0.0018 & 0.0054 & - \\
\hline $\mathrm{Pu}-239 / 240$ & 2002 & 0.00261 & 0.00048 & 0.00144 & - \\
\hline $\mathrm{Pu}-239 / 240$ & 2004 & 0.0171 & 0.00155 & 0.00465 & - \\
\hline $\mathrm{Pu}-239 / 240$ & 2006 & 0.000674 & 0.00634 & 0.01902 & $\mathrm{U}$ \\
\hline $\mathrm{Pu}-239 / 240$ & 2008 & 0.008112 & 0.00931 & 0.0279 & $\mathrm{U}$ \\
\hline $\mathrm{Pu}-239 / 240$ & 2010 & 0.0189 & 0.0195 & 0.0585 & $\mathrm{U}$ \\
\hline $\mathrm{Pu}-239 / 240$ & 2012 & 0.021 & 0.00571 & 0.01713 & - \\
\hline $\mathrm{Pu}-239 / 240$ & 2014 & 0.0231 & 0.0102 & 0.0306 & - \\
\hline $\mathrm{Sr}-90$ & 1994 & 0.31 & 0.07 & 0.21 & - \\
\hline $\mathrm{Sr}-90$ & 1996 & 0.31 & 0.035 & 0.105 & - \\
\hline $\mathrm{Sr}-90$ & 1998 & 0.2 & 0.045 & 0.135 & - \\
\hline $\mathrm{Sr}-90$ & 2000 & 0.194 & 0.36 & 1.08 & $\mathrm{U}$ \\
\hline $\mathrm{Sr}-90$ & 2002 & 0.194 & 0.36 & 1.08 & $\mathrm{U}$ \\
\hline $\mathrm{Sr}-90$ & 2004 & 0.195 & 0.0255 & 0.0765 & - \\
\hline $\mathrm{Sr}-90$ & 2006 & 0.106 & 0.0153 & 0.0459 & - \\
\hline $\mathrm{Sr}-90$ & 2008 & 0.091 & 0.01255 & 0.03765 & - \\
\hline $\mathrm{Sr}-90$ & 2010 & 0.0448 & 0.0128 & 0.0384 & - \\
\hline $\mathrm{Sr}-90$ & 2012 & 0.201 & 0.0412 & 0.1236 & - \\
\hline $\mathrm{Sr}-90$ & 2014 & 0.139 & 0.0387 & 0.1161 & - \\
\hline
\end{tabular}

Table C-10. Radionuclides concentrations in surface soils associated with Carey.

\begin{tabular}{|c|l|l|l|l|c|}
\hline Radionuclide & Year & $\begin{array}{c}\text { Concentration } \\
(\mathrm{pCi} / \mathrm{g})\end{array}$ & $\begin{array}{c}\text { Uncertainty } \\
(\mathrm{pCi} / \mathrm{g})\end{array}$ & $\begin{array}{c}\text { MDA } \\
(\mathrm{pCi} / \mathrm{g})\end{array}$ & Flag \\
\hline Am-241 & 1994 & 0 & 0.002 & 0.006 & $\mathrm{U}$ \\
\hline Am-241 & 1996 & 0.01 & 0.0035 & 0.0105 & - \\
\hline Am-241 & 1998 & 0.01 & 0.0045 & 0.0135 & - \\
\hline Am-241 & 2000 & 0.00913 & 0.00099 & 0.00297 & - \\
\hline Am-241 & 2002 & 0.00913 & 0.00099 & 0.0030 & - \\
\hline Am-241 & 2006 & 0.0556 & 0.0149 & 0.0447 & - \\
\hline Am-241 & 2008 & 0.02859 & 0.01675 & 0.05025 & - \\
\hline Am-241 & 2010 & -0.00716 & 0.0123 & 0.0369 & $\mathrm{U}$ \\
\hline Am-241 & 2012 & -0.00721 & 0.00541 & 0.0162 & $\mathrm{U}$ \\
\hline Am-241 & 2014 & 0.00442 & 0.00776 & 0.02328 & $\mathrm{U}$ \\
\hline Cs-137 & 1994 & 0.42 & 0.01 & 0.0300 & - \\
\hline Cs-137 & 1996 & 0.38 & 0.01 & 0.03 & - \\
\hline
\end{tabular}


Table C-10. (continued).

\begin{tabular}{|c|c|c|c|c|c|}
\hline Radionuclide & Year & $\begin{array}{c}\text { Concentration } \\
(\mathrm{pCi} / \mathrm{g})\end{array}$ & $\begin{array}{l}\text { Uncertainty } \\
(\mathrm{pCi} / \mathrm{g})\end{array}$ & $\begin{array}{c}\mathrm{MDA} \\
(\mathrm{pCi} / \mathrm{g})\end{array}$ & Flag \\
\hline Cs-137 & 1998 & 0.3 & 0.001 & 0.003 & - \\
\hline Cs-137 & 2000 & 0.2830 & 0.0086 & 0.0259 & - \\
\hline Cs-137 & 2000 & 0.416 & 0.0131 & 0.0393 & - \\
\hline Cs-137 & 2002 & 0.759 & 0.016 & 0.048 & - \\
\hline Cs-137 & 2002 & 0.733 & 0.0229 & 0.0687 & - \\
\hline Cs-137 & 2002 & 0.34 & 0.00952 & 0.02856 & - \\
\hline Cs-137 & 2002 & 0.32700 & 0.00762 & 0.02286 & - \\
\hline Cs-137 & 2004 & 0.50600 & 0.01130 & 0.03390 & - \\
\hline Cs-137 & 2004 & 0.0876 & 0.0067 & 0.0202 & - \\
\hline Cs-137 & 2006 & 0.554 & 0.0132 & 0.0396 & - \\
\hline Cs-137 & 2006 & 0.206 & 0.00535 & 0.01605 & - \\
\hline Cs-137 & 2008 & 0.4459 & 0.0117 & 0.0350 & - \\
\hline Cs-137 & 2008 & 0.1689 & 0.0053 & 0.0158 & - \\
\hline Cs-137 & 2010 & 0.186 & 0.00495 & 0.01485 & - \\
\hline Cs-137 & 2010 & 0.1190 & 0.0048 & 0.0145 & - \\
\hline Cs-137 & 2012 & 0.4450 & 0.0256 & 0.0768 & - \\
\hline Cs-137 & 2012 & 0.151 & 0.00958 & 0.02874 & - \\
\hline Cs-137 & 2014 & 0.35 & 0.0201 & 0.0603 & - \\
\hline Cs-137 & 2014 & 0.119 & 0.00804 & 0.02412 & - \\
\hline Pu-238 & 1994 & 0.002 & 0.0015 & 0.0045 & - \\
\hline $\mathrm{Pu}-238$ & 1996 & 0.005 & 0.0025 & 0.0075 & - \\
\hline $\mathrm{Pu}-238$ & 1998 & 0.01 & 0.01 & 0.03 & - \\
\hline $\mathrm{Pu}-238$ & 2000 & 0.00206 & 0.00038 & 0.00114 & - \\
\hline $\mathrm{Pu}-238$ & 2002 & 0.00206 & 0.00038 & 0.00114 & - \\
\hline $\mathrm{Pu}-238$ & 2004 & 0.00618 & 0.00085 & 0.00255 & - \\
\hline $\mathrm{Pu}-238$ & 2006 & -0.00138 & 0.00872 & 0.02616 & $\mathrm{U}$ \\
\hline $\mathrm{Pu}-238$ & 2008 & 0.03364 & 0.01684 & 0.05052 & - \\
\hline $\mathrm{Pu}-238$ & 2010 & 0.00428 & 0.00781 & 0.02343 & $\mathrm{U}$ \\
\hline $\mathrm{Pu}-238$ & 2012 & 0.0147 & 0.00502 & 0.01506 & - \\
\hline $\mathrm{Pu}-238$ & 2014 & 0.0156 & 0.00834 & 0.02502 & - \\
\hline $\mathrm{Pu}-239 / 240$ & 1994 & 0.02 & 0.002 & 0.006 & - \\
\hline $\mathrm{Pu}-239 / 240$ & 1996 & 0.02 & 0.005 & 0.015 & - \\
\hline $\mathrm{Pu}-239 / 240$ & 1998 & 0.04 & 0.015 & 0.045 & - \\
\hline $\mathrm{Pu}-239 / 240$ & 2000 & 0.0268 & 0.0022 & 0.0066 & - \\
\hline $\mathrm{Pu}-239 / 240$ & 2002 & 0.0268 & 0.0022 & 0.0066 & - \\
\hline $\mathrm{Pu}-239 / 240$ & 2004 & 0.0188 & 0.00175 & 0.00525 & - \\
\hline $\mathrm{Pu}-239 / 240$ & 2006 & 0.017 & 0.00862 & 0.0259 & - \\
\hline
\end{tabular}


Table C-10. (continued).

\begin{tabular}{|c|c|c|c|c|c|}
\hline Radionuclide & Year & $\begin{array}{c}\text { Concentration } \\
(\mathrm{pCi} / \mathrm{g})\end{array}$ & $\begin{array}{c}\text { Uncertainty } \\
(\mathrm{pCi} / \mathrm{g})\end{array}$ & $\begin{array}{c}\text { MDA } \\
(\mathrm{pCi} / \mathrm{g})\end{array}$ & Flag \\
\hline $\mathrm{Pu}-239 / 240$ & 2008 & -0.00395 & 0.00280 & 0.00839 & $\mathrm{U}$ \\
\hline $\mathrm{Pu}-239 / 240$ & 2010 & 0.00867 & 0.00606 & 0.0182 & - \\
\hline $\mathrm{Pu}-239 / 240$ & 2012 & 0.0342 & 0.0076 & 0.0228 & - \\
\hline $\mathrm{Pu}-239 / 240$ & 2014 & 0.0442 & 0.0113 & 0.0339 & - \\
\hline $\mathrm{Sr}-90$ & 1994 & 0.36 & 0.06 & 0.18 & - \\
\hline $\mathrm{Sr}-90$ & 1996 & 0.13 & 0.02 & 0.06 & - \\
\hline $\mathrm{Sr}-90$ & 1998 & 0.1 & 0.035 & 0.105 & - \\
\hline $\mathrm{Sr}-90$ & 2000 & 0.213 & 0.037 & 0.111 & - \\
\hline $\mathrm{Sr}-90$ & 2002 & 0.213 & 0.037 & 0.111 & - \\
\hline $\mathrm{Sr}-90$ & 2004 & 0.263 & 0.0325 & 0.0975 & - \\
\hline $\mathrm{Sr}-90$ & 2006 & 0.129 & 0.0164 & 0.0492 & - \\
\hline $\mathrm{Sr}-90$ & 2008 & 0.0447 & 0.0192 & 0.0576 & - \\
\hline $\mathrm{Sr}-90$ & 2010 & 0.00515 & 0.0107 & 0.0321 & $\mathrm{U}$ \\
\hline $\mathrm{Sr}-90$ & 2012 & 0.0495 & 0.0354 & 0.1062 & - \\
\hline $\mathrm{Sr}-90$ & 2014 & 0.0123 & 0.0357 & 0.1071 & $\mathrm{U}$ \\
\hline
\end{tabular}

Table C-11. Radionuclides concentrations in surface soils associated with FAA.

\begin{tabular}{|c|c|c|c|c|c|}
\hline & Year & $\begin{array}{c}\text { Concentration } \\
\text { Radionuclide }\end{array}$ & $\begin{array}{c}\text { Uncertainty } \\
(\mathrm{pCi} / \mathrm{g})\end{array}$ & MDA (pCi/g) & Flag \\
\hline Am-241 & 1994 & 0.011 & 0.003 & 0.009 & - \\
\hline Am-241 & 1996 & 0.02 & 0.004 & 0.012 & - \\
\hline Am-241 & 1998 & 0.01 & 0.005 & 0.015 & - \\
\hline Am-241 & 2000 & 0.00781 & 0.00091 & 0.00273 & - \\
\hline Am-241 & 2004 & 0.00864 & 0.00094 & 0.00282 & - \\
\hline Am-241 & 2006 & 0.0227 & 0.00762 & 0.02286 & - \\
\hline Am-241 & 2008 & 0.01378 & 0.01291 & 0.0387 & - \\
\hline Am-241 & 2010 & 0.0239 & 0.0275 & 0.0825 & U \\
\hline Am-241 & 2012 & 0.00517 & 0.00653 & 0.01959 & U \\
\hline Am-241 & 2014 & -0.00141 & 0.00771 & 0.0231 & U \\
\hline Cs-137 & 1994 & 1.38 & 0.02 & 0.0600 & - \\
\hline Cs-137 & 1996 & 1.26 & 0.025 & 0.075 & - \\
\hline Cs-137 & 1998 & 1.01 & 0.02 & 0.06 & - \\
\hline Cs-137 & 2000 & 0.714 & 0.022 & 0.066 & - \\
\hline Cs-137 & 2000 & 0.32600 & 0.00837 & 0.02511 & - \\
\hline Cs-137 & 2002 & 0.58200 & 0.01640 & 0.04920 & - \\
\hline Cs-137 & 2002 & 0.5900 & 0.0136 & 0.0408 & - \\
\hline Cs-137 & 2002 & 0.125 & 0.00428 & 0.01284 & - \\
\hline
\end{tabular}


Table C-11. (continued).

\begin{tabular}{|c|c|c|c|c|c|}
\hline Radionuclide & Year & $\begin{array}{c}\text { Concentration } \\
(\mathrm{pCi} / \mathrm{g})\end{array}$ & $\begin{array}{l}\text { Uncertainty } \\
(\mathrm{pCi} / \mathrm{g})\end{array}$ & $\operatorname{MDA}(\mathrm{pCi} / \mathrm{g})$ & Flag \\
\hline Cs-137 & 2002 & 0.109 & 0.0066 & 0.0198 & - \\
\hline Cs-137 & 2004 & 0.7170 & 0.0156 & 0.0468 & - \\
\hline Cs-137 & 2004 & 0.3570 & 0.0083 & 0.0249 & - \\
\hline Cs-137 & 2004 & 0.373 & 0.0123 & 0.0369 & - \\
\hline Cs-137 & 2006 & 0.268 & 0.297 & 0.891 & $\mathrm{U}$ \\
\hline Cs-137 & 2006 & 0.311 & 0.00729 & 0.02187 & - \\
\hline Cs-137 & 2008 & 0.4576 & 0.0118 & 0.0354 & - \\
\hline Cs-137 & 2008 & 0.1511 & 0.0045 & 0.0134 & - \\
\hline Cs-137 & 2010 & 0.519 & 0.0114 & 0.0342 & - \\
\hline Cs-137 & 2010 & 0.208 & 0.00549 & 0.01647 & - \\
\hline Cs-137 & 2012 & 0.7040 & 0.0411 & 0.1233 & - \\
\hline Cs-137 & 2012 & 0.1940 & 0.0118 & 0.0354 & - \\
\hline Cs-137 & 2014 & 0.494 & 0.0289 & 0.0867 & - \\
\hline Cs-137 & 2014 & 0.163 & 0.00985 & 0.02955 & - \\
\hline $\mathrm{Pu}-238$ & 1994 & 0.004 & 0.0015 & 0.0045 & - \\
\hline $\mathrm{Pu}-238$ & 1996 & 0.008 & 0.004 & 0.012 & - \\
\hline $\mathrm{Pu}-238$ & 1998 & 0.03 & 0.015 & 0.045 & - \\
\hline $\mathrm{Pu}-238$ & 2000 & 0.0017 & 0.00035 & 0.00105 & - \\
\hline $\mathrm{Pu}-238$ & 2004 & 0.0115 & 0.00135 & 0.00405 & - \\
\hline $\mathrm{Pu}-238$ & 2006 & 0.0517 & 0.0251 & 0.0753 & - \\
\hline $\mathrm{Pu}-238$ & 2008 & 0.02017 & 0.01465 & 0.04395 & - \\
\hline $\mathrm{Pu}-238$ & 2010 & -0.00166 & 0.00581 & 0.01743 & $\mathrm{U}$ \\
\hline $\mathrm{Pu}-238$ & 2012 & 0.00364 & 0.00313 & 0.00939 & - \\
\hline $\mathrm{Pu}-238$ & 2014 & 0.0036 & 0.00441 & 0.01323 & $\mathrm{U}$ \\
\hline $\mathrm{Pu}-239 / 240$ & 1994 & 0.05 & 0.004 & 0.012 & - \\
\hline $\mathrm{Pu}-239 / 240$ & 1996 & 0.05 & 0.01 & 0.03 & - \\
\hline $\mathrm{Pu}-239 / 240$ & 1998 & 0.04 & 0.015 & 0.045 & - \\
\hline $\mathrm{Pu}-239 / 240$ & 2000 & 0.0225 & 0.002 & 0.006 & - \\
\hline $\mathrm{Pu}-239 / 240$ & 2004 & 0.0245 & 0.0023 & 0.0069 & - \\
\hline $\mathrm{Pu}-239 / 240$ & 2006 & 0.0248 & 0.00971 & 0.0291 & - \\
\hline $\mathrm{Pu}-239 / 240$ & 2008 & 0.01425 & 0.01114 & 0.03342 & - \\
\hline $\mathrm{Pu}-239 / 240$ & 2010 & 0.0124 & 0.00862 & 0.02586 & - \\
\hline $\mathrm{Pu}-239 / 240$ & 2012 & 0.0185 & 0.00505 & 0.0152 & - \\
\hline $\mathrm{Pu}-239 / 240$ & 2014 & 0.02690 & 0.00769 & 0.02307 & - \\
\hline Sr-90 & 1994 & 0.57 & 0.07 & 0.21 & - \\
\hline Sr-90 & 1996 & 0.4 & 0.04 & 0.12 & - \\
\hline Sr-90 & 1998 & 0.4 & 0.05 & 0.15 & - \\
\hline
\end{tabular}


Table C-11. (continued).

\begin{tabular}{|c|c|c|c|c|c|}
\hline Radionuclide & Year & $\begin{array}{c}\text { Concentration } \\
(\mathrm{pCi} / \mathrm{g})\end{array}$ & $\begin{array}{c}\text { Uncertainty } \\
(\mathrm{pCi} / \mathrm{g})\end{array}$ & MDA (pCi/g) & Flag \\
\hline Sr-90 & 2000 & 0.243 & 0.038 & 0.114 & - \\
\hline Sr-90 & 2002 & 0.243 & 0.038 & 0.114 & - \\
\hline Sr-90 & 2004 & 0.187 & 0.026 & 0.078 & - \\
\hline Sr-90 & 2006 & 0.116 & 0.0153 & 0.0459 & - \\
\hline Sr-90 & 2008 & 0.199 & 0.02095 & 0.06285 & - \\
\hline Sr-90 & 2010 & 0.00968 & 0.014 & 0.042 & U \\
\hline Sr-90 & 2012 & 0.159 & 0.0405 & 0.1215 & - \\
\hline Sr-90 & 2014 & 0.157 & 0.0408 & 0.1224 & - \\
\hline
\end{tabular}

Table C-12. Radionuclides concentrations in surface soils associated with Frenchman's Cabin.

\begin{tabular}{|c|c|c|c|c|c|}
\hline Radionuclide & Year & $\begin{array}{c}\text { Concentration } \\
(\mathrm{pCi} / \mathrm{g})\end{array}$ & $\begin{array}{c}\text { Uncertainty } \\
(\mathrm{pCi} / \mathrm{g})\end{array}$ & $\begin{array}{c}\text { MDA } \\
(\mathrm{pCi} / \mathrm{g})\end{array}$ & Flag \\
\hline $\mathrm{Am}-241$ & 2010 & 0.0175 & 0.0142 & 0.0426 & - \\
\hline $\mathrm{Am}-241$ & 2012 & 0.000118 & 0.00678 & 0.02034 & $\mathrm{U}$ \\
\hline $\mathrm{Am}-241$ & 2014 & 0.0052 & 0.0107 & 0.0321 & $\mathrm{U}$ \\
\hline $\mathrm{Cs}-137$ & 2010 & 0.359 & 0.00801 & 0.02403 & - \\
\hline $\mathrm{Cs}-137$ & 2010 & 0.126 & 0.00347 & 0.01041 & - \\
\hline $\mathrm{Cs}-137$ & 2012 & 0.339 & 0.0206 & 0.0618 & - \\
\hline $\mathrm{Cs}-137$ & 2012 & 0.0991 & 0.00652 & 0.0196 & - \\
\hline $\mathrm{Cs}-137$ & 2014 & 0.275 & 0.0158 & 0.0474 & - \\
\hline $\mathrm{Cs}-137$ & 2014 & 0.0679 & 0.00489 & 0.01467 & - \\
\hline $\mathrm{Pu}-238$ & 2010 & 0.11 & 0.0486 & 0.1458 & - \\
\hline $\mathrm{Pu}-238$ & 2012 & 0.00762 & 0.00378 & 0.0113 & - \\
\hline $\mathrm{Pu}-238$ & 2014 & 0.00555 & 0.00491 & 0.01473 & - \\
\hline $\mathrm{Pu}-239 / 240$ & 2010 & 0.0167 & 0.0278 & 0.0834 & $\mathrm{U}$ \\
\hline $\mathrm{Pu}-239 / 240$ & 2012 & 0.0228 & 0.00687 & 0.02061 & - \\
\hline $\mathrm{Pu}-239 / 240$ & 2014 & 0.00923 & 0.00458 & 0.01374 & - \\
\hline $\mathrm{Sr}-90$ & 2010 & 0.04320 & 0.01100 & 0.03300 & - \\
\hline $\mathrm{Sr}-90$ & 2012 & 0.0816 & 0.0436 & 0.1308 & - \\
\hline $\mathrm{Sr}-90$ & 2014 & 0.0877 & 0.0339 & 0.1017 & - \\
\hline
\end{tabular}

Table C-13. Radionuclides concentrations in surface soils associated with Howe.

\begin{tabular}{|c|c|c|c|c|c|}
\hline & Year & $\begin{array}{c}\text { Concentration } \\
(\mathrm{pCi} / \mathrm{g})\end{array}$ & $\begin{array}{c}\text { Uncertainty } \\
(\mathrm{pCi} / \mathrm{g})\end{array}$ & MDA (pCi/g) & Flag \\
\hline Am-241 & 1994 & 0 & 0.002 & 0.006 & $\mathrm{U}$ \\
\hline Am-241 & 1996 & 0.01 & 0.0035 & 0.0105 & - \\
\hline Am-241 & 1998 & 0.01 & 0.0035 & 0.0105 & - \\
\hline Am-241 & 2004 & 0.00424 & 0.00065 & 0.00195 & - \\
\hline
\end{tabular}


Table C-13. (continued).

\begin{tabular}{|c|c|c|c|c|c|}
\hline Radionuclide & Year & $\begin{array}{c}\text { Concentration } \\
(\mathrm{pCi} / \mathrm{g})\end{array}$ & $\begin{array}{l}\text { Uncertainty } \\
(\mathrm{pCi} / \mathrm{g})\end{array}$ & MDA (pCi/g) & Flag \\
\hline Am-241 & 2006 & -0.0028 & 0.0106 & 0.0318 & $\mathrm{U}$ \\
\hline Am-241 & 2008 & 0.0042 & 0.0067 & 0.0202 & $\mathrm{U}$ \\
\hline Am-241 & 2010 & 0.00996 & 0.0115 & 0.0345 & $\mathrm{U}$ \\
\hline Am-241 & 2012 & 0.00908 & 0.00959 & 0.0288 & $\mathrm{U}$ \\
\hline Am-241 & 2014 & -0.00425 & 0.00685 & 0.0206 & $\mathrm{U}$ \\
\hline Cs-137 & 1994 & 0.5 & 0.01 & 0.0300 & - \\
\hline Cs-137 & 1996 & 0.38 & 0.015 & 0.0450 & - \\
\hline Cs-137 & 1998 & 0.2 & 0.035 & 0.105 & - \\
\hline Cs-137 & 2000 & 0.5250 & 0.0120 & 0.0360 & - \\
\hline Cs-137 & 2000 & 0.248 & 0.00595 & 0.01785 & - \\
\hline Cs-137 & 2000 & 0.235 & 0.00924 & 0.02772 & - \\
\hline Cs-137 & 2002 & 0.399 & 0.00971 & 0.02913 & - \\
\hline Cs-137 & 2002 & 0.409 & 0.0099 & 0.0297 & - \\
\hline Cs-137 & 2002 & 0.196 & 0.00613 & 0.01839 & - \\
\hline Cs-137 & 2002 & 0.193 & 0.00586 & 0.01758 & - \\
\hline Cs-137 & 2004 & 0.39 & 0.00879 & 0.02637 & - \\
\hline Cs-137 & 2004 & 0.124 & 0.00416 & 0.01248 & - \\
\hline Cs-137 & 2006 & 0.412 & 0.0122 & 0.0366 & - \\
\hline Cs-137 & 2006 & 0.181 & 0.00456 & 0.01368 & - \\
\hline Cs-137 & 2008 & 0.3077 & 0.0072 & 0.0216 & - \\
\hline Cs-137 & 2008 & 0.1039 & 0.0034 & 0.0101 & - \\
\hline Cs-137 & 2010 & 0.26600 & 0.00713 & 0.02139 & - \\
\hline Cs-137 & 2010 & 0.08730 & 0.00277 & 0.00831 & - \\
\hline Cs-137 & 2012 & 0.179 & 0.0121 & 0.0363 & - \\
\hline Cs-137 & 2012 & 0.109 & 0.00711 & 0.02133 & - \\
\hline Cs-137 & 2014 & 0.279 & 0.0166 & 0.0498 & - \\
\hline Cs-137 & 2014 & 0.074 & 0.00531 & 0.01593 & - \\
\hline $\mathrm{Pu}-238$ & 1994 & 0 & 0.001 & 0.003 & - \\
\hline $\mathrm{Pu}-238$ & 1996 & 0.001 & 0.001 & 0.003 & - \\
\hline $\mathrm{Pu}-238$ & 1998 & 0.0001 & 0.0035 & 0.0105 & - \\
\hline $\mathrm{Pu}-238$ & 2004 & 0.0010 & 0.0004 & 0.0011 & - \\
\hline $\mathrm{Pu}-238$ & 2006 & -0.0187 & 0.00728 & 0.02184 & $\mathrm{U}$ \\
\hline $\mathrm{Pu}-238$ & 2008 & -0.005163 & 0.008885 & 0.026655 & $\mathrm{U}$ \\
\hline $\mathrm{Pu}-238$ & 2010 & -0.00726 & 0.00685 & 0.02055 & $\mathrm{U}$ \\
\hline $\mathrm{Pu}-238$ & 2012 & 0.00749 & 0.00462 & 0.01386 & - \\
\hline $\mathrm{Pu}-238$ & 2014 & 0.0119 & 0.00588 & 0.01764 & - \\
\hline $\mathrm{Pu}-239 / 240$ & 1994 & 0.02 & 0.002 & 0.006 & - \\
\hline
\end{tabular}


Table C-13. (continued).

\begin{tabular}{|c|c|c|c|c|c|}
\hline Radionuclide & Year & $\begin{array}{c}\text { Concentration } \\
(\mathrm{pCi} / \mathrm{g})\end{array}$ & $\begin{array}{c}\text { Uncertainty } \\
(\mathrm{pCi} / \mathrm{g})\end{array}$ & MDA (pCi/g) & Flag \\
\hline $\mathrm{Pu}-239 / 240$ & 1996 & 0.02 & 0.004 & 0.012 & - \\
\hline $\mathrm{Pu}-239 / 240$ & 1998 & 0.01 & 0.004 & 0.012 & - \\
\hline $\mathrm{Pu}-239 / 240$ & 2004 & 0.0158 & 0.00185 & 0.00555 & - \\
\hline $\mathrm{Pu}-239 / 240$ & 2006 & 0.00631 & 0.0042 & 0.0126 & - \\
\hline $\mathrm{Pu}-239 / 240$ & 2008 & -0.004871 & 0.00345 & 0.01035 & $\mathrm{U}$ \\
\hline $\mathrm{Pu}-239 / 240$ & 2010 & 0.00783 & 0.00609 & 0.01827 & - \\
\hline $\mathrm{Pu}-239 / 240$ & 2012 & 0.00561 & 0.0046 & 0.0138 & - \\
\hline $\mathrm{Pu}-239 / 240$ & 2014 & 0.0166 & 0.00722 & 0.02166 & - \\
\hline $\mathrm{Sr}-90$ & 1994 & 0.35 & 0.06 & 0.18 & - \\
\hline $\mathrm{Sr}-90$ & 1996 & 0.35 & 0.1 & 0.3 & - \\
\hline $\mathrm{Sr}-90$ & 1998 & 0.2 & 0.035 & 0.105 & - \\
\hline $\mathrm{Sr}-90$ & 2000 & 0.0734 & 0.35 & 1.05 & $\mathrm{U}$ \\
\hline $\mathrm{Sr}-90$ & 2002 & 0.0734 & 0.35 & 1.05 & $\mathrm{U}$ \\
\hline $\mathrm{Sr}-90$ & 2004 & 0.154 & 0.024 & 0.072 & - \\
\hline $\mathrm{Sr}-90$ & 2006 & 0.0487 & 0.0097 & 0.0291 & - \\
\hline $\mathrm{Sr}-90$ & 2008 & 0.123 & 0.0139 & 0.0417 & - \\
\hline $\mathrm{Sr}-90$ & 2010 & 0.0204 & 0.0114 & 0.0342 & - \\
\hline $\mathrm{Sr}-90$ & 2012 & 0.123 & 0.0518 & 0.1554 & - \\
\hline $\mathrm{Sr}-90$ & 2014 & 0.0404 & 0.0316 & 0.0948 & - \\
\hline & & & & & - \\
\hline
\end{tabular}

Table C-14. Radionuclides concentrations in surface soils associated with Monteview.

\begin{tabular}{|c|c|c|c|c|c|}
\hline Radionuclide & Year & $\begin{array}{c}\text { Concentration } \\
(\mathrm{pCi} / \mathrm{g})\end{array}$ & $\begin{array}{c}\text { Uncertainty } \\
(\mathrm{pCi} / \mathrm{g})\end{array}$ & MDA (pCi/g) & Flag \\
\hline Am-241 & 1994 & 0.008 & 0.002 & 0.006 & - \\
\hline Am-241 & 1996 & 0.01 & 0.0035 & 0.0105 & - \\
\hline Am-241 & 1998 & 0.02 & 0.03 & 0.09 & $\mathrm{U}$ \\
\hline Am-241 & 2000 & 0.00335 & 0.00063 & 0.00189 & - \\
\hline Am-241 & 2002 & 0.00335 & 0.00063 & 0.0019 & - \\
\hline Am-241 & 2004 & 0.0194 & 0.0098 & 0.0294 & - \\
\hline Am-241 & 2006 & 0.00589 & 0.0133 & 0.0399 & $\mathrm{U}$ \\
\hline Am-241 & 2008 & 0.0085 & 0.0112 & 0.0336 & $\mathrm{U}$ \\
\hline Am-241 & 2010 & 0.0018 & 0.0107 & 0.0321 & $\mathrm{U}$ \\
\hline Am-241 & 2012 & 0.0106 & 0.0068 & 0.0204 & - \\
\hline Am-241 & 2014 & -0.00554 & 0.00821 & 0.0246 & $\mathrm{U}$ \\
\hline Cs-137 & 1994 & 1.11 & 0.01 & 0.03 & - \\
\hline Cs-137 & 1996 & 0.8200 & 0.0200 & 0.0600 & - \\
\hline Cs-137 & 1998 & 0.1 & 0.03 & 0.09 & - \\
\hline
\end{tabular}


Table C-14. (continued).

\begin{tabular}{|c|c|c|c|c|c|}
\hline Radionuclide & Year & $\begin{array}{c}\text { Concentration } \\
(\mathrm{pCi} / \mathrm{g})\end{array}$ & $\begin{array}{l}\text { Uncertainty } \\
(\mathrm{pCi} / \mathrm{g})\end{array}$ & MDA (pCi/g) & Flag \\
\hline Cs-137 & 2000 & 0.432 & 0.0149 & 0.0447 & - \\
\hline Cs-137 & 2000 & 0.373 & 0.0103 & 0.0309 & - \\
\hline Cs-137 & 2000 & 0.348 & 0.0127 & 0.0381 & - \\
\hline Cs-137 & 2002 & 0.362 & 0.0111 & 0.0333 & - \\
\hline Cs-137 & 2002 & 0.372 & 0.0105 & 0.0315 & - \\
\hline Cs-137 & 2002 & 0.3960 & 0.0097 & 0.0290 & - \\
\hline Cs-137 & 2002 & 0.3990 & 0.0112 & 0.0336 & - \\
\hline Cs-137 & 2004 & 0.32600 & 0.00790 & 0.02370 & - \\
\hline Cs-137 & 2004 & 0.20100 & 0.00498 & 0.01494 & - \\
\hline Cs-137 & 2006 & 0.32 & 0.00721 & 0.02163 & - \\
\hline Cs-137 & 2006 & 0.206 & 0.00766 & 0.02298 & - \\
\hline Cs-137 & 2008 & 0.3600 & 0.0090 & 0.0269 & - \\
\hline Cs-137 & 2008 & 0.3012 & 0.0112 & 0.0335 & - \\
\hline Cs-137 & 2010 & 0.264 & 0.00616 & 0.01848 & - \\
\hline Cs-137 & 2010 & 0.166 & 0.00509 & 0.01527 & - \\
\hline Cs-137 & 2012 & 0.285 & 0.0166 & 0.0498 & - \\
\hline Cs-137 & 2012 & 0.227 & 0.0141 & 0.0423 & - \\
\hline Cs-137 & 2014 & 0.288 & 0.0171 & 0.0513 & - \\
\hline Cs-137 & 2014 & 0.196 & 0.0114 & 0.0342 & - \\
\hline $\mathrm{Pu}-238$ & 1994 & 0 & 0.001 & 0.003 & $\mathrm{U}$ \\
\hline $\mathrm{Pu}-238$ & 1996 & 0.0010 & 0.0010 & 0.0030 & - \\
\hline $\mathrm{Pu}-238$ & 1998 & 0.002 & 0.0005 & 0.0015 & - \\
\hline $\mathrm{Pu}-238$ & 2000 & 0.000619 & 0.00025 & 0.00075 & - \\
\hline $\mathrm{Pu}-238$ & 2002 & 0.0111 & 0.0012 & 0.0036 & - \\
\hline $\mathrm{Pu}-238$ & 2004 & 0.00148 & 0.000375 & 0.001125 & - \\
\hline $\mathrm{Pu}-238$ & 2006 & -0.0104 & 0.0136 & 0.0408 & $\mathrm{U}$ \\
\hline $\mathrm{Pu}-238$ & 2008 & 0.0227 & 0.02104 & 0.06312 & - \\
\hline $\mathrm{Pu}-238$ & 2010 & -0.00269 & 0.0053 & 0.0159 & $\mathrm{U}$ \\
\hline $\mathrm{Pu}-238$ & 2012 & 0.0115 & 0.00392 & 0.01176 & - \\
\hline $\mathrm{Pu}-238$ & 2014 & 0.0117 & 0.00509 & 0.01527 & - \\
\hline $\mathrm{Pu}-239 / 240$ & 1994 & 0.03 & 0.003 & 0.009 & - \\
\hline $\mathrm{Pu}-239 / 240$ & 1996 & 0.02 & 0.004 & 0.012 & - \\
\hline $\mathrm{Pu}-239 / 240$ & 1998 & 0.02 & 0.005 & 0.015 & - \\
\hline $\mathrm{Pu}-239 / 240$ & 2000 & 0.0111 & 0.0012 & 0.0036 & - \\
\hline $\mathrm{Pu}-239 / 240$ & 2002 & 0.000619 & 0.00025 & 0.00075 & - \\
\hline $\mathrm{Pu}-239 / 240$ & 2004 & 0.0113 & 0.00012 & 0.00036 & - \\
\hline $\mathrm{Pu}-239 / 240$ & 2006 & 0.00421 & 0.00861 & 0.02583 & $\mathrm{U}$ \\
\hline
\end{tabular}


Table C-14. (continued).

\begin{tabular}{|c|l|l|l|l|c|}
\hline Radionuclide & Year & $\begin{array}{c}\text { Concentration } \\
(\mathrm{pCi} / \mathrm{g})\end{array}$ & $\begin{array}{c}\text { Uncertainty } \\
(\mathrm{pCi} / \mathrm{g})\end{array}$ & MDA (pCi/g) & Flag \\
\hline $\mathrm{Pu}-239 / 240$ & 2008 & 0.002412 & 0.008795 & 0.026385 & $\mathrm{U}$ \\
\hline $\mathrm{Pu}-239 / 240$ & 2010 & 0.00549 & 0.0067 & 0.0201 & $\mathrm{U}$ \\
\hline $\mathrm{Pu}-239 / 240$ & 2012 & 0.0242 & 0.00687 & 0.02061 & - \\
\hline $\mathrm{Pu}-239 / 240$ & 2014 & 0.00999 & 0.00477 & 0.01431 & - \\
\hline $\mathrm{Sr}-90$ & 1994 & 0.17 & 0.06 & 0.18 & - \\
\hline $\mathrm{Sr}-90$ & 1996 & 0.14 & 0.015 & 0.045 & - \\
\hline $\mathrm{Sr}-90$ & 1998 & 0.1 & 0.03 & 0.09 & - \\
\hline $\mathrm{Sr}-90$ & 2000 & 0.0557 & 0.35 & 1.05 & $\mathrm{U}$ \\
\hline $\mathrm{Sr}-90$ & 2002 & 0.0557 & 0.35 & 1.05 & $\mathrm{U}$ \\
\hline $\mathrm{Sr}-90$ & 2004 & 0.069 & 0.0165 & 0.0495 & - \\
\hline $\mathrm{Sr}-90$ & 2006 & 0.0297 & 0.0093 & 0.0279 & - \\
\hline $\mathrm{Sr}-90$ & 2008 & 0.0566 & 0.01 & 0.03 & - \\
\hline $\mathrm{Sr}-90$ & 2010 & 0.0844 & 0.0128 & 0.0384 & - \\
\hline $\mathrm{Sr}-90$ & 2012 & 0.0387 & 0.033 & 0.099 & - \\
\hline $\mathrm{Sr}-90$ & 2014 & -0.00386 & 0.0313 & 0.0939 & $\mathrm{U}$ \\
\hline
\end{tabular}

Table C-15. Radionuclides concentrations in surface soils associated with Mud Lake.

\begin{tabular}{|c|c|c|c|c|c|}
\hline Radionuclide & Year & $\begin{array}{c}\text { Concentration } \\
(\mathrm{pCi} / \mathrm{g})\end{array}$ & $\begin{array}{c}\text { Uncertainty } \\
(\mathrm{pCi} / \mathrm{g})\end{array}$ & MDA (pCi/g) & Flag \\
\hline Am-241 & 1994 & 0.002 & 0.002 & 0.006 & - \\
\hline Am-241 & 1996 & 0.0040 & 0.0025 & 0.0075 & - \\
\hline Am-241 & 1996 & 0.009 & 0.0035 & 0.0105 & - \\
\hline Am-241 & 1998 & 0.003 & 0.0015 & 0.0045 & - \\
\hline Am-241 & 1998 & 0.007 & 0.0025 & 0.0075 & - \\
\hline Am-241 & 2000 & 0.00393 & 0.00059 & 0.0018 & - \\
\hline Am-241 & 2000 & 0.00376 & 0.00058 & 0.00174 & - \\
\hline Am-241 & 2000 & 0.00166 & 0.00037 & 0.00111 & - \\
\hline Am-241 & 2002 & 0.0039 & 0.0006 & 0.0018 & - \\
\hline Am-241 & 2002 & 0.00376 & 0.00058 & 0.00174 & - \\
\hline Am-241 & 2002 & 0.00166 & 0.00037 & 0.00111 & - \\
\hline Am-241 & 2004 & 0.0035 & 0.00054 & 0.0016 & - \\
\hline Am-241 & 2004 & 0.0022 & 0.00041 & 0.00123 & - \\
\hline Am-241 & 2006 & 0.00478 & 0.00462 & 0.01386 & - \\
\hline Am-241 & 2006 & 0.00698 & 0.00675 & 0.02025 & - \\
\hline Am-241 & 2008 & 0.0875 & 0.0426 & 0.1277 & - \\
\hline Am-241 & 2008 & 0.00900 & 0.0232 & 0.0696 & U \\
\hline Am-241 & 2010 & 0.0197 & 0.0179 & 0.0537 & - \\
\hline
\end{tabular}


Table C-15. (continued).

\begin{tabular}{|c|c|c|c|c|c|}
\hline Radionuclide & Year & $\begin{array}{c}\text { Concentration } \\
(\mathrm{pCi} / \mathrm{g})\end{array}$ & $\begin{array}{l}\text { Uncertainty } \\
(\mathrm{pCi} / \mathrm{g})\end{array}$ & MDA (pCi/g) & Flag \\
\hline Am-241 & 2010 & 0.00173 & 0.0106 & 0.0318 & $\mathrm{U}$ \\
\hline Am-241 & 2012 & 0.00729 & 0.00978 & 0.02934 & $\mathrm{U}$ \\
\hline Am-241 & 2012 & 0.00967 & 0.00712 & 0.02136 & - \\
\hline Am-241 & 2014 & 0.00741 & 0.00793 & 0.02379 & $\mathrm{U}$ \\
\hline Am-241 & 2014 & -0.00355 & 0.00719 & 0.02157 & $\mathrm{U}$ \\
\hline Cs-137 & 1994 & 0.44 & 0.01 & 0.03 & - \\
\hline Cs-137 & 1996 & 0.59000 & 0.01500 & 0.04500 & - \\
\hline Cs-137 & 1996 & 0.14 & 0.005 & 0.015 & - \\
\hline Cs-137 & 1998 & 0.07000 & 0.02500 & 0.07500 & - \\
\hline Cs-137 & 1998 & 0.2 & 0.005 & 0.015 & - \\
\hline Cs-137 & 2000 & 0.471 & 0.012 & 0.036 & - \\
\hline Cs-137 & 2000 & 0.196 & 0.00887 & 0.02661 & - \\
\hline Cs-137 & 2000 & 0.311 & 0.00735 & 0.02205 & - \\
\hline Cs-137 & 2000 & 0.402 & 0.0137 & 0.0411 & - \\
\hline Cs-137 & 2002 & 0.4350 & 0.0105 & 0.0315 & - \\
\hline Cs-137 & 2002 & 0.4260 & 0.0129 & 0.0387 & - \\
\hline Cs-137 & 2002 & 0.41 & 0.00975 & 0.02925 & - \\
\hline Cs-137 & 2002 & 0.385 & 0.0116 & 0.0348 & - \\
\hline Cs-137 & 2002 & 0.0907 & 0.00592 & 0.01776 & - \\
\hline Cs-137 & 2002 & 0.102 & 0.00389 & 0.01167 & - \\
\hline Cs-137 & 2002 & 0.114 & 0.00408 & 0.01224 & - \\
\hline Cs-137 & 2002 & 0.0926 & 0.006 & 0.018 & - \\
\hline Cs-137 & 2002 & 0.0976 & 0.0062 & 0.0186 & - \\
\hline Cs-137 & 2002 & 0.114 & 0.00398 & 0.01194 & - \\
\hline Cs-137 & 2002 & 0.131 & 0.00669 & 0.02007 & - \\
\hline Cs-137 & 2002 & 0.121 & 0.0065 & 0.0195 & - \\
\hline Cs-137 & 2004 & 0.198 & 0.00505 & 0.01515 & - \\
\hline Cs-137 & 2004 & 0.1000 & 0.0036 & 0.0107 & - \\
\hline Cs-137 & 2004 & 0.0904 & 0.00301 & 0.00903 & - \\
\hline Cs-137 & 2004 & 0.0881 & 0.00572 & 0.01716 & - \\
\hline Cs-137 & 2006 & 0.244 & 0.00579 & 0.01737 & - \\
\hline Cs-137 & 2006 & 0.126 & 0.00621 & 0.01863 & - \\
\hline Cs-137 & 2006 & 0.487 & 0.0106 & 0.0318 & - \\
\hline Cs-137 & 2006 & 0.261 & 0.00938 & 0.02814 & - \\
\hline Cs-137 & 2008 & 0.3412 & 0.00776 & 0.02329 & - \\
\hline Cs-137 & 2008 & 0.2594 & 0.00626 & 0.01877 & - \\
\hline Cs-137 & 2008 & 0.3168 & 0.01069 & 0.03206 & - \\
\hline
\end{tabular}


Table C-15. (continued).

\begin{tabular}{|c|c|c|c|c|c|}
\hline Radionuclide & Year & $\begin{array}{l}\text { Concentration } \\
(\mathrm{pCi} / \mathrm{g})\end{array}$ & $\begin{array}{l}\text { Uncertainty } \\
\text { (pCi } / \mathrm{g})\end{array}$ & MDA (pCi/g) & Flag \\
\hline Cs-137 & 2008 & 0.2817 & 0.00676 & 0.02028 & - \\
\hline Cs-137 & 2010 & 0.3430 & 0.0078 & 0.0233 & - \\
\hline Cs-137 & 2010 & 0.1620 & 0.0042 & 0.0126 & 一 \\
\hline Cs-137 & 2010 & 0.238 & 0.00578 & 0.01734 & - \\
\hline Cs-137 & 2010 & 0.229 & 0.00644 & 0.01932 & - \\
\hline Cs-137 & 2012 & 0.114 & 0.00861 & 0.02583 & - \\
\hline Cs-137 & 2012 & 0.0504 & 0.00408 & 0.01224 & - \\
\hline Cs-137 & 2012 & 0.256 & 0.015 & 0.045 & - \\
\hline Cs-137 & 2012 & 0.223 & 0.0132 & 0.0396 & - \\
\hline Cs-137 & 2014 & 0.295 & 0.0176 & 0.0528 & - \\
\hline Cs-137 & 2014 & 0.161 & 0.00958 & 0.02874 & - \\
\hline Cs-137 & 2014 & 0.216 & 0.0132 & 0.0396 & - \\
\hline Cs-137 & 2014 & 0.192 & 0.0114 & 0.0342 & - \\
\hline $\mathrm{Pu}-238$ & 1994 & 0 & 0.001 & 0.003 & $\mathrm{U}$ \\
\hline $\mathrm{Pu}-238$ & 1996 & 0.001 & 0.0025 & 0.0075 & $\mathrm{U}$ \\
\hline $\mathrm{Pu}-238$ & 1996 & 0.002 & 0.0015 & 0.0045 & - \\
\hline $\mathrm{Pu}-238$ & 1998 & 0.008 & 0.0035 & 0.0105 & - \\
\hline $\mathrm{Pu}-238$ & 1998 & 0.01 & 0.004 & 0.012 & - \\
\hline $\mathrm{Pu}-238$ & 2000 & 0.00256 & 0.00045 & 0.00135 & - \\
\hline $\mathrm{Pu}-238$ & 2000 & 0.0000809 & 0.000081 & 0.000243 & $\mathrm{U}$ \\
\hline $\mathrm{Pu}-238$ & 2002 & 0.0118 & 0.0012 & 0.0036 & - \\
\hline $\mathrm{Pu}-238$ & 2002 & 0.0119 & 0.0012 & 0.0036 & - \\
\hline $\mathrm{Pu}-238$ & 2002 & 0.0000809 & 0.000081 & 0.000243 & $\mathrm{U}$ \\
\hline $\mathrm{Pu}-238$ & 2004 & 0.00388 & 0.0008 & 0.0024 & - \\
\hline $\mathrm{Pu}-238$ & 2004 & 0.00673 & 0.00105 & 0.00315 & - \\
\hline $\mathrm{Pu}-238$ & 2006 & -0.0102 & 0.00713 & 0.02139 & $\mathrm{U}$ \\
\hline $\mathrm{Pu}-238$ & 2006 & -0.0294 & 0.015 & 0.045 & $\mathrm{U}$ \\
\hline $\mathrm{Pu}-238$ & 2008 & 0.0205 & 0.0167 & 0.0500 & - \\
\hline $\mathrm{Pu}-238$ & 2008 & -0.0261 & 0.0088 & 0.0264 & $\mathrm{U}$ \\
\hline $\mathrm{Pu}-238$ & 2010 & 0.00151 & 0.00923 & 0.02769 & $\mathrm{U}$ \\
\hline $\mathrm{Pu}-238$ & 2010 & 0.044 & 0.032 & 0.096 & - \\
\hline $\mathrm{Pu}-238$ & 2012 & 0.00506 & 0.00402 & 0.01206 & - \\
\hline $\mathrm{Pu}-238$ & 2012 & 0.002 & 0.00489 & 0.01467 & $\mathrm{U}$ \\
\hline $\mathrm{Pu}-238$ & 2014 & 0.012 & 0.00641 & 0.01923 & - \\
\hline $\mathrm{Pu}-238$ & 2014 & 0.00493 & 0.00605 & 0.01815 & $\mathrm{U}$ \\
\hline $\mathrm{Pu}-239 / 240$ & 1994 & 0.01 & 0.0015 & 0.0045 & - \\
\hline $\mathrm{Pu}-239 / 240$ & 1996 & 0.02 & 0.01 & 0.03 & - \\
\hline
\end{tabular}


Table C-15. (continued).

\begin{tabular}{|c|c|c|c|c|c|}
\hline Radionuclide & Year & $\begin{array}{l}\text { Concentration } \\
(\mathrm{pCi} / \mathrm{g})\end{array}$ & $\begin{array}{l}\text { Uncertainty } \\
(\mathrm{pCi} / \mathrm{g})\end{array}$ & MDA (pCi/g) & Flag \\
\hline $\mathrm{Pu}-239 / 240$ & 1996 & 0.008 & 0.004 & 0.012 & - \\
\hline $\mathrm{Pu}-239 / 240$ & 1998 & 0.05 & 0.01 & 0.03 & - \\
\hline $\mathrm{Pu}-239 / 240$ & 1998 & 0.01 & 0.0045 & 0.0135 & - \\
\hline $\mathrm{Pu}-239 / 240$ & 2000 & 0.0118 & 0.0012 & 0.0036 & - \\
\hline $\mathrm{Pu}-239 / 240$ & 2000 & 0.0119 & 0.0012 & 0.0036 & - \\
\hline $\mathrm{Pu}-239 / 240$ & 2000 & 0.00307 & 0.00056 & 0.00168 & - \\
\hline $\mathrm{Pu}-239 / 240$ & 2002 & 0.00183 & 0.00038 & 0.00114 & - \\
\hline $\mathrm{Pu}-239 / 240$ & 2002 & 0.00256 & 0.00045 & 0.00135 & - \\
\hline $\mathrm{Pu}-239 / 240$ & 2002 & 0.00307 & 0.00056 & 0.00168 & - \\
\hline $\mathrm{Pu}-239 / 240$ & 2004 & 0.00744 & 0.0012 & 0.0036 & - \\
\hline $\mathrm{Pu}-239 / 240$ & 2004 & 0.00379 & 0.0008 & 0.0024 & - \\
\hline $\mathrm{Pu}-239 / 240$ & 2006 & 0.0104 & 0.00426 & 0.01278 & - \\
\hline $\mathrm{Pu}-239 / 240$ & 2006 & 0.00875 & 0.00938 & 0.02814 & $\mathrm{U}$ \\
\hline $\mathrm{Pu}-239 / 240$ & 2008 & 0.04173 & 0.019735 & 0.059205 & - \\
\hline $\mathrm{Pu}-239 / 240$ & 2008 & 0.01092 & 0.01253 & 0.03759 & $\mathrm{U}$ \\
\hline $\mathrm{Pu}-239 / 240$ & 2010 & 0.0209 & 0.0117 & 0.0351 & - \\
\hline $\mathrm{Pu}-239 / 240$ & 2010 & 0.00218 & 0.0195 & 0.0585 & $\mathrm{U}$ \\
\hline $\mathrm{Pu}-239 / 240$ & 2012 & 0.00252 & 0.00565 & 0.01695 & $\mathrm{U}$ \\
\hline $\mathrm{Pu}-239 / 240$ & 2012 & 0.0339 & 0.00951 & 0.02853 & - \\
\hline $\mathrm{Pu}-239 / 240$ & 2014 & 0.012 & 0.00806 & 0.02418 & - \\
\hline $\mathrm{Pu}-239 / 240$ & 2014 & 0.00983 & 0.00607 & 0.01821 & - \\
\hline Sr-90 & 1994 & 0.32 & 0.06 & 0.18 & - \\
\hline Sr-90 & 1996 & 0.1 & 0.015 & 0.045 & - \\
\hline Sr-90 & 1996 & 0.2 & 0.025 & 0.075 & - \\
\hline Sr-90 & 1998 & 0.07 & 0.025 & 0.075 & - \\
\hline Sr-90 & 1998 & 0.1 & 0.03 & 0.09 & - \\
\hline Sr-90 & 2000 & 0.00394 & 0.35 & 1.05 & $\mathrm{U}$ \\
\hline Sr-90 & 2000 & 0.0203 & 0.35 & 1.05 & $\mathrm{U}$ \\
\hline Sr-90 & 2002 & 0.00394 & 0.35 & 1.05 & $\mathrm{U}$ \\
\hline Sr-90 & 2002 & -0.163 & 0.33 & 0.99 & $\mathrm{U}$ \\
\hline Sr-90 & 2002 & 0.0203 & 0.35 & 1.05 & $\mathrm{U}$ \\
\hline Sr-90 & 2004 & 0.0593 & 0.0165 & 0.0495 & - \\
\hline Sr-90 & 2004 & 0.0345 & 0.0145 & 0.0435 & - \\
\hline Sr-90 & 2006 & 0.0448 & 0.0122 & 0.0366 & - \\
\hline Sr-90 & 2006 & 0.11 & 0.0177 & 0.0531 & - \\
\hline Sr-90 & 2008 & 0.0243 & 0.01125 & 0.03375 & - \\
\hline Sr-90 & 2008 & 0.0813 & 0.01465 & 0.04395 & - \\
\hline
\end{tabular}


Table C-15. (continued).

\begin{tabular}{|c|c|c|c|c|c|}
\hline Radionuclide & Year & $\begin{array}{c}\text { Concentration } \\
(\mathrm{pCi} / \mathrm{g})\end{array}$ & $\begin{array}{c}\text { Uncertainty } \\
(\mathrm{pCi} / \mathrm{g})\end{array}$ & MDA (pCi/g) & Flag \\
\hline Sr-90 & 2010 & 0.0417 & 0.0131 & 0.0393 & - \\
\hline Sr-90 & 2010 & -0.0289 & 0.0128 & 0.0384 & $\mathrm{U}$ \\
\hline Sr-90 & 2012 & 0.0138 & 0.035 & 0.105 & $\mathrm{U}$ \\
\hline Sr-90 & 2012 & 0.123 & 0.0411 & 0.1233 & - \\
\hline Sr-90 & 2014 & 0.0303 & 0.0292 & 0.0876 & - \\
\hline Sr-90 & 2014 & 0.0579 & 0.0307 & 0.0921 & - \\
\hline
\end{tabular}

Table C-16. Radionuclides concentrations in surface soils associated with Blue Dome/Birch Creek Hydro.

\begin{tabular}{|c|c|c|c|c|c|}
\hline Radionuclide & Year & $\begin{array}{l}\text { Concentration } \\
(\mathrm{pCi} / \mathrm{g})\end{array}$ & $\begin{array}{l}\text { Uncertainty } \\
(\mathrm{pCi} / \mathrm{g})\end{array}$ & $\begin{array}{l}\mathrm{MDA} \\
(\mathrm{pCi} / \mathrm{g})\end{array}$ & Flag \\
\hline Am-241 & 1994 & 0.007 & 0.002 & 0.006 & - \\
\hline Am-241 & 1996 & 0.01 & 0.0035 & 0.0105 & - \\
\hline Am-241 & 1998 & 0.009 & 0.003 & 0.009 & - \\
\hline Am-241 & 2000 & 0.00831 & 0.00098 & 0.00294 & - \\
\hline Am-241 & 2004 & 0.00757 & 0.00091 & 0.00273 & - \\
\hline Am-241 & 2004 & 0.0089 & 0.0010 & 0.0030 & - \\
\hline Am-241 & 2006 & 0.0168 & 0.0131 & 0.0393 & - \\
\hline Am-241 & 2008 & 0.02682 & 0.016755 & 0.0503 & - \\
\hline Am-241 & 2010 & -0.00777 & 0.0122 & 0.0366 & $\mathrm{U}$ \\
\hline Am-241 & 2012 & 0.0213 & 0.0084 & 0.0253 & - \\
\hline Am-241 & 2014 & 0.0108 & 0.0105 & 0.0315 & - \\
\hline Cs-137 & 1994 & 1.3 & 0.01 & 0.0300 & - \\
\hline Cs-137 & 1996 & 0.68 & 0.015 & 0.045 & - \\
\hline Cs-137 & 1998 & 0.4 & 0.05 & 0.15 & - \\
\hline Cs-137 & 2000 & 0.935 & 0.0194 & 0.0582 & - \\
\hline Cs-137 & 2000 & 0.211 & 0.00988 & 0.02964 & - \\
\hline Cs-137 & 2004 & 0.653 & 0.0152 & 0.0456 & - \\
\hline Cs-137 & 2004 & 0.264 & 0.00654 & 0.01962 & - \\
\hline Cs-137 & 2006 & 0.464 & 0.0111 & 0.0333 & - \\
\hline Cs- 137 & 2006 & 0.294 & 0.00694 & 0.02082 & - \\
\hline Cs-137 & 2008 & 0.6643 & 0.0195 & 0.0584 & - \\
\hline Cs-137 & 2008 & 0.0839 & 0.0035 & 0.0104 & - \\
\hline Cs-137 & 2010 & 0.57300 & 0.0125 & 0.0375 & - \\
\hline Cs-137 & 2010 & 0.132 & 0.0038 & 0.0114 & - \\
\hline Cs-137 & 2012 & 0.663 & 0.0376 & 0.1128 & - \\
\hline Cs-137 & 2012 & 0.162 & 0.0115 & 0.0345 & - \\
\hline Cs-137 & 2014 & 0.64100 & 0.03610 & 0.10830 & - \\
\hline
\end{tabular}


Table C-16. (continued).

\begin{tabular}{|c|c|c|c|c|c|}
\hline Radionuclide & Year & $\begin{array}{l}\text { Concentration } \\
(\mathrm{pCi} / \mathrm{g})\end{array}$ & $\begin{array}{l}\text { Uncertainty } \\
(\mathrm{pCi} / \mathrm{g})\end{array}$ & $\begin{array}{l}\text { MDA } \\
(\mathrm{pCi} / \mathrm{g})\end{array}$ & Flag \\
\hline Cs-137 & 2014 & 0.274 & 0.0165 & 0.0495 & - \\
\hline $\mathrm{Pu}-238$ & 1994 & 0.001 & 0.0015 & 0.0045 & $\mathrm{U}$ \\
\hline $\mathrm{Pu}-238$ & 1996 & 0.001 & 0.002 & 0.006 & $\mathrm{U}$ \\
\hline $\mathrm{Pu}-238$ & 1998 & 0.002 & 0.0015 & 0.0045 & - \\
\hline $\mathrm{Pu}-238$ & 2000 & 0.0013 & 0.0003 & 0.0010 & - \\
\hline $\mathrm{Pu}-238$ & 2004 & $2.36 \mathrm{E}-08$ & 0.000285 & 0.000855 & $\mathrm{U}$ \\
\hline $\mathrm{Pu}-238$ & 2004 & 0.000617 & 0.000295 & 0.000885 & - \\
\hline $\mathrm{Pu}-238$ & 2006 & -0.0274 & 0.0144 & 0.0432 & $\mathrm{U}$ \\
\hline $\mathrm{Pu}-238$ & 2008 & 0.004464 & 0.01151 & 0.03453 & $\mathrm{U}$ \\
\hline $\mathrm{Pu}-238$ & 2010 & -0.0016 & 0.0099 & 0.0297 & $\mathrm{U}$ \\
\hline $\mathrm{Pu}-238$ & 2012 & 0.0144 & 0.0051 & 0.0153 & - \\
\hline $\mathrm{Pu}-238$ & 2014 & 0.00737 & 0.00394 & 0.01182 & - \\
\hline $\mathrm{Pu}-239 / 240$ & 1994 & 0.04 & 0.003 & 0.009 & - \\
\hline $\mathrm{Pu}-239 / 240$ & 1996 & 0.012 & 0.003 & 0.009 & - \\
\hline $\mathrm{Pu}-239 / 240$ & 1998 & 0.02 & 0.005 & 0.015 & - \\
\hline $\mathrm{Pu}-239 / 240$ & 2000 & 0.0266 & 0.0024 & 0.0072 & - \\
\hline $\mathrm{Pu}-239 / 240$ & 2004 & 0.0222 & 0.0022 & 0.0066 & - \\
\hline $\mathrm{Pu}-239 / 240$ & 2006 & 0.00437 & 0.0101 & 0.0303 & $\mathrm{U}$ \\
\hline $\mathrm{Pu}-239 / 240$ & 2008 & 0.01654 & 0.01569 & 0.04707 & - \\
\hline $\mathrm{Pu}-239 / 240$ & 2010 & 0.00573 & 0.00591 & 0.01773 & $\mathrm{U}$ \\
\hline $\mathrm{Pu}-239 / 240$ & 2012 & 0.0430 & 0.0092 & 0.0277 & - \\
\hline $\mathrm{Pu}-239 / 240$ & 2014 & 0.0368 & 0.00789 & 0.02367 & - \\
\hline Sr-90 & 1994 & 0.52 & 0.08 & 0.24 & - \\
\hline Sr-90 & 1996 & 0.4 & 0.04 & 0.12 & - \\
\hline Sr-90 & 1998 & 0.4 & 0.05 & 0.15 & - \\
\hline Sr-90 & 2000 & 0.3130 & 0.37000 & 1.11000 & $\mathrm{U}$ \\
\hline Sr-90 & 2004 & 0.2640 & 0.03150 & 0.09450 & - \\
\hline Sr-90 & 2006 & 0.1010 & 0.0129 & 0.0387 & - \\
\hline Sr-90 & 2008 & 0.2330 & 0.01430 & 0.04290 & - \\
\hline Sr-90 & 2010 & 0.0230 & 0.01390 & 0.04170 & - \\
\hline Sr-90 & 2012 & 0.2650 & 0.0463 & 0.1389 & - \\
\hline Sr-90 & 2014 & 0.19 & 0.0366 & 0.1098 & - \\
\hline
\end{tabular}


Table C-17. Radionuclides concentrations in surface soils associated with St. Anthony.

\begin{tabular}{|c|c|c|c|c|c|}
\hline Radionuclide & Year & $\begin{array}{c}\text { Concentration } \\
(\mathrm{pCi} / \mathrm{g})\end{array}$ & $\begin{array}{l}\text { Uncertainty } \\
(\mathrm{pCi} / \mathrm{g})\end{array}$ & $\operatorname{MDA}(\mathrm{pCi} / \mathrm{g})$ & Flag \\
\hline Am-241 & 1994 & 0.008 & 0.003 & 0.0090 & - \\
\hline Am-241 & 1996 & 0.006 & 0.0025 & 0.0075 & - \\
\hline Am-241 & 1998 & 0.02 & 0.005 & 0.015 & - \\
\hline Am-241 & 2000 & 0.0146 & 0.0015 & 0.0045 & - \\
\hline Am-241 & 2002 & 0.0146 & 0.0015 & 0.0045 & - \\
\hline Am-241 & 2004 & 0.00587 & 0.00075 & 0.00225 & - \\
\hline Am-241 & 2006 & 0.00543 & 0.00504 & 0.01512 & - \\
\hline Am-241 & 2008 & 0.0132 & 0.01084 & 0.03252 & - \\
\hline Am-241 & 2010 & -0.0056 & 0.0142 & 0.0426 & $\mathrm{U}$ \\
\hline Am-241 & 2012 & 0.00851 & 0.00735 & 0.02205 & - \\
\hline Am-241 & 2014 & 0.0287 & 0.0113 & 0.0339 & - \\
\hline Cs- 137 & 1994 & 1.11 & 0.01 & 0.03 & - \\
\hline Cs-137 & 1996 & 1.1 & 0.025 & 0.075 & - \\
\hline Cs-137 & 1998 & 1 & 0.02 & 0.06 & - \\
\hline Cs-137 & 2000 & 0.6440 & 0.0210 & 0.0630 & - \\
\hline Cs-137 & 2000 & 0.7130 & 0.0158 & 0.0474 & - \\
\hline Cs-137 & 2002 & 1.27000 & 0.0303 & 0.0909 & - \\
\hline Cs-137 & 2002 & 1.27 & 0.0303 & 0.0909 & - \\
\hline Cs-137 & 2002 & 0.490 & 0.0117 & 0.0351 & - \\
\hline Cs-137 & 2002 & 0.492 & 0.0146 & 0.0438 & - \\
\hline Cs-137 & 2004 & 0.55100 & 0.01210 & 0.03630 & - \\
\hline Cs-137 & 2004 & 0.281 & 0.0103 & 0.0309 & - \\
\hline Cs-137 & 2006 & 0.0752 & 0.00326 & 0.0098 & - \\
\hline Cs-137 & 2006 & 0.0769 & 0.00388 & 0.01164 & - \\
\hline Cs-137 & 2008 & 0.4875 & 0.0109 & 0.0326 & - \\
\hline Cs-137 & 2008 & 0.5141 & 0.0128 & 0.0385 & - \\
\hline Cs-137 & 2010 & 0.71 & 0.0151 & 0.0453 & - \\
\hline Cs-137 & 2010 & 0.607 & 0.0133 & 0.0399 & - \\
\hline Cs-137 & 2012 & 0.746 & 0.0432 & 0.1296 & - \\
\hline Cs-137 & 2012 & 0.624 & 0.0353 & 0.1059 & - \\
\hline Cs-137 & 2014 & 0.616 & 0.0354 & 0.1062 & - \\
\hline Cs-137 & 2014 & 0.4260 & 0.0242 & 0.0726 & - \\
\hline $\mathrm{Pu}-238$ & 1994 & 0.002 & 0.0015 & 0.0045 & - \\
\hline $\mathrm{Pu}-238$ & 1996 & -0.0004 & 0.00035 & 0.00105 & $\mathrm{U}$ \\
\hline $\mathrm{Pu}-238$ & 1998 & 0.006 & 0.003 & 0.009 & - \\
\hline $\mathrm{Pu}-238$ & 2000 & 0.00147 & 0.00035 & 0.00105 & - \\
\hline
\end{tabular}


Table C-17. (continued).

\begin{tabular}{|c|c|c|c|c|c|}
\hline Radionuclide & Year & $\begin{array}{c}\text { Concentration } \\
(\mathrm{pCi} / \mathrm{g})\end{array}$ & $\begin{array}{l}\text { Uncertainty } \\
(\mathrm{pCi} / \mathrm{g})\end{array}$ & MDA (pCi/g) & Flag \\
\hline $\mathrm{Pu}-238$ & 2002 & 0.0326 & 0.0028 & 0.0084 & - \\
\hline $\mathrm{Pu}-238$ & 2004 & 0.00717 & 0.0009 & 0.0027 & - \\
\hline $\mathrm{Pu}-238$ & 2006 & -0.00371 & 0.00672 & 0.02016 & $\mathrm{U}$ \\
\hline $\mathrm{Pu}-238$ & 2008 & 0.01899 & 0.01557 & 0.04671 & - \\
\hline $\mathrm{Pu}-238$ & 2010 & 0.0657 & 0.0345 & 0.1035 & - \\
\hline $\mathrm{Pu}-238$ & 2012 & 0.0112 & 0.00491 & 0.01473 & - \\
\hline $\mathrm{Pu}-238$ & 2014 & 0.0105 & 0.00437 & 0.01311 & - \\
\hline $\mathrm{Pu}-239 / 240$ & 1994 & 0.03 & 0.003 & 0.009 & - \\
\hline $\mathrm{Pu}-239 / 240$ & 1996 & 0.02 & 0.005 & 0.015 & - \\
\hline $\mathrm{Pu}-239 / 240$ & 1998 & 0.06 & 0.005 & 0.015 & - \\
\hline $\mathrm{Pu}-239 / 240$ & 2000 & 0.0326 & 0.00280 & 0.00840 & - \\
\hline $\mathrm{Pu}-239 / 240$ & 2002 & 0.0015 & 0.00035 & 0.00105 & - \\
\hline $\mathrm{Pu}-239 / 240$ & 2004 & 0.019 & 0.00175 & 0.00525 & - \\
\hline $\mathrm{Pu}-239 / 240$ & 2006 & 0.00699 & 0.00415 & 0.01245 & - \\
\hline $\mathrm{Pu}-239 / 240$ & 2008 & 0.01179 & 0.008345 & 0.025035 & - \\
\hline $\mathrm{Pu}-239 / 240$ & 2010 & -0.0059 & 0.0256 & 0.0768 & $\mathrm{U}$ \\
\hline $\mathrm{Pu}-239 / 240$ & 2012 & 0.0404 & 0.00903 & 0.02709 & - \\
\hline $\mathrm{Pu}-239 / 240$ & 2014 & 0.035 & 0.00828 & 0.02484 & - \\
\hline Sr-90 & 1994 & 0.5600 & 0.07000 & 0.21000 & - \\
\hline Sr-90 & 1996 & 0.4200 & 0.05000 & 0.15000 & - \\
\hline Sr-90 & 1998 & 0.3400 & 0.0450 & 0.1350 & - \\
\hline Sr-90 & 2000 & 0.277 & 0.37 & 1.11 & $\mathrm{U}$ \\
\hline Sr-90 & 2002 & 0.277 & 0.37 & 1.11 & $\mathrm{U}$ \\
\hline Sr-90 & 2004 & 0.199 & 0.034 & 0.102 & - \\
\hline Sr-90 & 2006 & 0.0547 & 0.0146 & 0.0438 & - \\
\hline Sr-90 & 2008 & 0.174 & 0.0114 & 0.0342 & - \\
\hline Sr-90 & 2010 & 0.0432 & 0.011 & 0.033 & - \\
\hline Sr-90 & 2012 & 0.238 & 0.0436 & 0.1308 & - \\
\hline Sr-90 & 2014 & 0.15 & 0.0348 & 0.1044 & - \\
\hline
\end{tabular}

